\title{
Pharmacologic and Nonpharmacologic Therapies in Adult Patients With Exacerbation of COPD: A Systematic Review
}




\title{
Comparative Effectiveness Review
}

Number 221

\section{Pharmacologic and Nonpharmacologic Therapies in Adult Patients With Exacerbation of COPD: A Systematic Review}

\author{
Prepared for: \\ Agency for Healthcare Research and Quality \\ U.S. Department of Health and Human Services \\ 5600 Fishers Lane \\ Rockville, MD 20857 \\ www.ahrq.gov
}

Contract No. 290-2015-00013-I

Prepared by:

Mayo Clinic Evidence-based Practice Center

Rochester, MN

Investigators:

Claudia C. Dobler, M.D., Ph.D.

Allison S. Morrow, B.A.

Magdoleen H. Farah, M.B.B.S.

Bradley Beuschel, B.S.P.H.

Abdul M. Majzoub, M.D.

Michael E. Wilson, M.D.

Bashar Hasan, M.D.

Mohamed O. Seisa, M.D.

Lubna Daraz, Ph.D.

Larry J. Prokop, M.L.S.

M. Hassan Murad, M.D., M.P.H.

Zhen Wang, Ph.D.

AHRQ Publication No. 19(20)-EHC024-EF

October 2019 


\section{Key Messages}

\section{Purpose of Review}

To evaluate the effectiveness and harms of pharmacologic and nonpharmacologic treatments for exacerbations of chronic obstructive pulmonary disease.

\section{Key Messages}

- Antibiotic therapy increases the clinical cure rate and reduces the clinical failure rate.

- Oral and intravenous corticosteroids improve dyspnea and reduce the clinical failure rate.

- Antibiotics and corticosteroids are not associated with increase in serious adverse events.

- The evidence is insufficient to support the effect of aminophyllines, magnesium sulfate, mucolytics, inhaled corticosteroids, inhaled antibiotics, 5-lipoxygenase inhibitor, and statins on mortality, dyspnea, need for intubation, clinical failure, or hospital admission.

- Titrated oxygen reduces mortality compared with high flow oxygen.

- The evidence suggests benefits of some nonpharmacologic interventions such as chest physiotherapy using vibration/percussion/massage or using breathing technique (on dyspnea), resistance training (on dyspnea and quality of life), early pulmonary rehabilitation commenced before hospital discharge during the initial most acute phase of exacerbation rather than the convalescence period (on dyspnea), and whole body vibration training (on quality of life).

- Vitamin D supplementation may improve quality of life.

- The evidence is insufficient for comparative effectiveness of different regimens of antibiotics and corticosteroids based on type of agents, delivery modes, and duration of treatments.

- The evidence is insufficient for effectiveness of combinations of treatments that are each individually effective.

- Serious adverse events were not found to be different between most evaluated interventions. 
This report is based on research conducted by the Mayo Clinic Evidence-based Practice Center (EPC) under contract to the Agency for Healthcare Research and Quality (AHRQ), Rockville, MD (Contract No. 290-2015-00013-I). The findings and conclusions in this document are those of the authors, who are responsible for its contents; the findings and conclusions do not necessarily represent the views of AHRQ. Therefore, no statement in this report should be construed as an official position of AHRQ or of the U.S. Department of Health and Human Services.

\section{None of the investigators have any affiliations or financial involvement that conflicts with the material presented in this report.}

The information in this report is intended to help healthcare decision makers-patients and clinicians, health system leaders, and policymakers, among others-make well-informed decisions and thereby improve the quality of healthcare services. This report is not intended to be a substitute for the application of clinical judgment. Anyone who makes decisions concerning the provision of clinical care should consider this report in the same way as any medical reference and in conjunction with all other pertinent information, i.e., in the context of available resources and circumstances presented by individual patients.

This report is made available to the public under the terms of a licensing agreement between the author and the Agency for Healthcare Research and Quality. This report may be used and reprinted without permission except those copyrighted materials that are clearly noted in the report. Further reproduction of those copyrighted materials is prohibited without the express permission of copyright holders.

AHRQ or U.S. Department of Health and Human Services endorsement of any derivative products that may be developed from this report, such as clinical practice guidelines, other quality enhancement tools, or reimbursement or coverage policies, may not be stated or implied.

This report may periodically be assessed for the currency of conclusions. If an assessment is done, the resulting surveillance report describing the methodology and findings will be found on the Effective Health Care Program website at www.effectivehealthcare.ahrq.gov. Search on the title of the report.

People using assistive technology may not be able to fully access information in this report. For assistance contact EPC@ahrq.gov.

Suggested citation: Dobler CC, Morrow AS, Farah MH, Beuschel B, Majzoub AM, Wilson ME, Hasan B, Seisa MO, Daraz L, Prokop LJ, Murad MH, Wang Z. Pharmacologic and Nonpharmacologic Therapies in Adult Patients With Exacerbation of COPD: A Systematic Review. Comparative Effectiveness Review No. 221. (Prepared by the Mayo Clinic Evidencebased Practice Center under Contract No. 290-2015-00013-I.) AHRQ Publication No. 19(20)EHC024-EF. Rockville, MD: Agency for Healthcare Research and Quality; October 2019. Posted final reports are located on the Effective Health Care Program search page.

DOI: https://doi.org/10.23970/AHRQEPCCER221. 


\section{Preface}

The Agency for Healthcare Research and Quality (AHRQ), through its Evidence-based Practice Centers (EPCs), sponsors the development of systematic reviews to assist public- and private-sector organizations in their efforts to improve the quality of healthcare in the United States. These reviews provide comprehensive, science-based information on common, costly medical conditions, and new healthcare technologies and strategies.

Systematic reviews are the building blocks underlying evidence-based practice; they focus attention on the strength and limits of evidence from research studies about the effectiveness and safety of a clinical intervention. In the context of developing recommendations for practice, systematic reviews can help clarify whether assertions about the value of the intervention are based on strong evidence from clinical studies. For more information about AHRQ EPC systematic reviews, see www.effectivehealthcare.ahrq.gov/reference/purpose.cfm.

AHRQ expects that these systematic reviews will be helpful to health plans, providers, purchasers, government programs, and the healthcare system as a whole. Transparency and stakeholder input are essential to the Effective Health Care Program. Please visit the website (www.effectivehealthcare.ahrq.gov) to see draft research questions and reports or to join an email list to learn about new program products and opportunities for input.

If you have comments on this systematic review, they may be sent by mail to the Task Order Officer named below at: Agency for Healthcare Research and Quality, 5600 Fishers Lane, Rockville, MD 20857, or by email to epc@ahrq.hhs.gov.

Gopal Khanna, M.B.A.

Director

Agency for Healthcare Research and Quality

Stephanie Chang, M.D., M.P.H.

Director

Evidence-based Practice Center Program

Center for Evidence and Practice Improvement Agency for Healthcare Research and Quality
Arlene S. Bierman, M.D., M.S.

Director

Center for Evidence and Practice

Improvement

Agency for Healthcare Research and Quality

Lionel L. Bañez, M.D.

Task Order Officer

Center for Evidence and Practice

Improvement

Agency for Healthcare Research and Quality 


\section{Acknowledgments}

The authors gratefully acknowledge the following individuals for their contributions to this project: from the Agency for Healthcare Research and Quality, our Task Order Officer, Lionel Bañez, M.D., and from the American Academy of Family Physicians, Melanie Bird, Ph.D.

\section{Key Informants}

In designing the study questions, the EPC consulted several Key Informants who represent the end-users of research. The EPC sought the Key Informant input on the priority areas for research and synthesis. Key Informants are not involved in the analysis of the evidence or the writing of the report. Therefore, in the end, study questions, design, methodological approaches, and/or conclusions do not necessarily represent the views of individual Key Informants.

Key Informants must disclose any financial conflicts of interest greater than $\$ 5,000$ and any other relevant business or professional conflicts of interest. Because of their role as end-users, individuals with potential conflicts may be retained. The TOO and the EPC work to balance, manage, or mitigate any conflicts of interest.

The list of Key Informants who provided input to this report follows:

MeiLan K. Han, M.D., M.S.

University of Michigan

Ann Arbor, MI

Clare Hawkins, M.D.

Aspire Health Care

Houston, TX

Cara Pasquale, M.P.H.

COPD Patient Powered Research Network

Washington, DC

Antonello Punturieri, M.D., Ph.D.

National Heart, Lung, and Blood Institute

for Chronic Obstructive Pulmonary Disease

(COPD)/Environment

Bethesda, MD
Mark Steffen, M.D., M.P.H.

Blue Cross and Blue Shield of Minnesota

Eagan, MN

James J. Stevermer, M.D., M.S.P.H.

Department of Family and Community

Medicine

University of Missouri Health

Columbia, MO 


\section{Technical Expert Panel}

In designing the study questions and methodology at the outset of this report, the EPC consulted several technical and content experts. Broad expertise and perspectives were sought. Divergent and conflicted opinions are common and perceived as healthy scientific discourse that results in a thoughtful, relevant systematic review. Therefore, in the end, study questions, design, methodologic approaches, and/or conclusions do not necessarily represent the views of individual technical and content experts.

Technical Experts must disclose any financial conflicts of interest greater than $\$ 5,000$ and any other relevant business or professional conflicts of interest. Because of their unique clinical or content expertise, individuals with potential conflicts may be retained. The TOO and the EPC work to balance, manage, or mitigate any potential conflicts of interest identified.

The list of Technical Experts who provided input to this report follows:

Richard Albert, M.D.*

Department of Medicine, University of Colorado

Aurora, CO

MeiLan K. Han, M.D., M.S.*

University of Michigan

Ann Arbor, MI

Clare Hawkins, M.D.

Aspire Health Care

Houston, TX
James J. Stevermer, M.D., M.S.P.H.*

Department of Family and Community

Medicine

University of Missouri Health

Columbia, MO

James Stoller, M.D., M.S.*

Cleveland Clinic

Cleveland, $\mathrm{OH}$

*Provided input on Draft Report. 


\section{Peer Reviewers}

Prior to publication of the final evidence report, EPCs sought input from independent Peer Reviewers without financial conflicts of interest. However, the conclusions and synthesis of the scientific literature presented in this report do not necessarily represent the views of individual reviewers.

Peer Reviewers must disclose any financial conflicts of interest greater than $\$ 5,000$ and any other relevant business or professional conflicts of interest. Because of their unique clinical or content expertise, individuals with potential nonfinancial conflicts may be retained. The TOO and the EPC work to balance, manage, or mitigate any potential nonfinancial conflicts of interest identified.

The list of Peer Reviewers follows:

Jerry A. Krishnan, M.D., Ph.D.

Breathe Chicago Center

University of Illinois

Chicago, IL

Tianjing Li, M.D., M.H.S, Ph.D.

Johns Hopkins Bloomberg School of Public Health

Baltimore, MD 


\section{Pharmacologic and Nonpharmacologic Therapies in Adult Patients With Exacerbation of COPD: A Systematic Review}

\section{Structured Abstract}

Objectives. To synthesize existing knowledge about the effectiveness and harms of pharmacologic and nonpharmacologic treatments for exacerbations of chronic obstructive pulmonary disease (ECOPD).

Data sources. Embase ${ }^{\circledR}$, Epub Ahead of Print, In-Process \& Other Non-Indexed Citations, MEDLINE ${ }^{\circledR}$ Daily, MEDLINE, Cochrane Central Registrar of Controlled Trials, Ovid Cochrane Database of Systematic Reviews, and Scopus from database inception to January 2, 2019.

Review methods. We included randomized controlled trials (RCTs) that evaluated pharmacologic intervention or nonpharmacologic interventions for ECOPD. The strength of evidence (SOE) was graded for critical final health outcomes.

Results. We included 98 RCTs (13,401 patients, mean treatment duration 9.9 days, mean followup 3.7 months). Final health outcomes, including mortality, resolution of exacerbation, hospital readmissions, repeat exacerbations, and need for intubation, were infrequently evaluated and often showed no statistically significant differences between groups. Antibiotic therapy increases the clinical cure rate and reduces the clinical failure rate regardless of the severity of ECOPD (moderate SOE). There is insufficient evidence to support a particular antibiotic regimen. Oral and intravenous corticosteroids improve dyspnea and reduce the clinical failure rate (low SOE). Despite the ubiquitous use of inhaled bronchodilators in ECOPD, we found only a small number of trials that assessed lung function tests, and not final health outcomes. The evidence is insufficient to support the effect of aminophyllines, magnesium sulfate, mucolytics, inhaled corticosteroids, inhaled antibiotics, 5-lipoxygenase inhibitor, and statins on final health outcomes. Titrated oxygen reduces mortality compared with high flow oxygen (low SOE). Low SOE suggested benefit from some nonpharmacologic interventions such as chest physiotherapy using vibration/percussion/massage or breathing technique (on dyspnea), resistance training (on dyspnea and quality of life), early pulmonary rehabilitation commenced before hospital discharge during the initial most acute phase of exacerbation rather than the convalescence period (on dyspnea) and whole body vibration training (on quality of life). Vitamin D supplementation may improve quality of life (low SOE).

Conclusions. Although chronic obstructive pulmonary disease is a common condition, the evidence base for most interventions in ECOPD remains limited. Systemic antibiotics and corticosteroids are associated with improved outcomes in mild and moderate to severe ECOPD. Titrated oxygen reduces mortality. Future research is required to assess the effectiveness of several emerging nonpharmacologic and dietary treatments. 


\section{Contents}

Evidence Summary ............................................................................................................................. ES-1

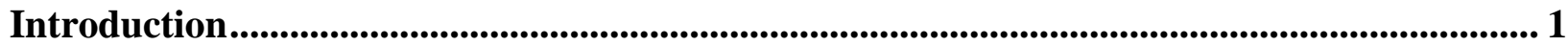

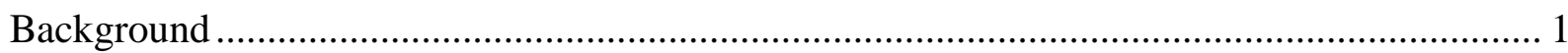

Key Questions ..................................................................................................... 3

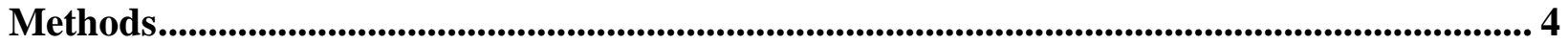

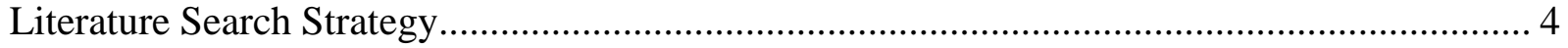

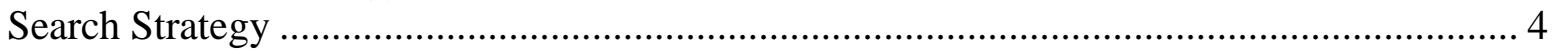

Inclusion and Exclusion Criteria................................................................................... 5

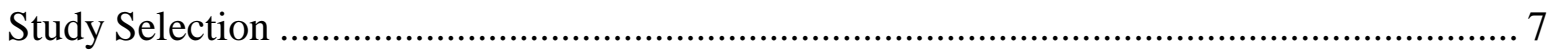

Data Abstraction and Data Management ............................................................................ 7

Assessment of the Risk of Bias of Individual Studies .............................................................. 7

Data Synthesis..................................................................................................................... 7

Grading the Strength of Evidence (SOE) for Major Comparisons and Outcomes .................. 9

Assessing Applicability .................................................................................................. 10

Peer Review and Public Commentary ................................................................................ 10

Results .......................................................................................................................................... 11

Literature Searches and Evidence Base ......................................................................... 11

KQ1. In adult patients with exacerbation of COPD, what are the benefits and harms of systemic corticosteroids and antibiotics compared with placebo or standard care? ................... 11

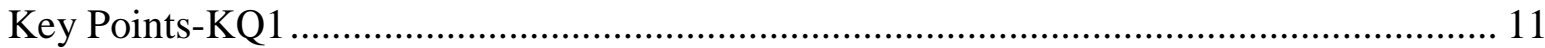

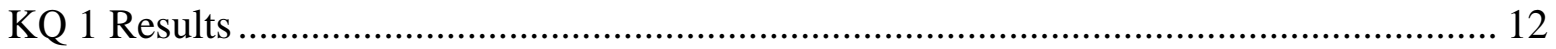

Systemic Antibiotics Versus Placebo or Management Without Systemic Antibiotics......... 12

Systemic Corticosteroids Versus Placebo or Management Without Systemic Corticosteroids

KQ2. In adult patients with exacerbation of COPD, what are the benefits and harms of emerging and other pharmacologic and nonpharmacologic therapies compared with placebo or

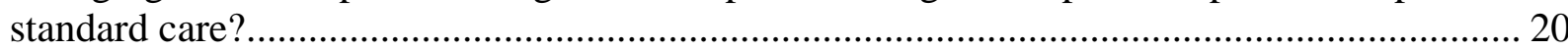

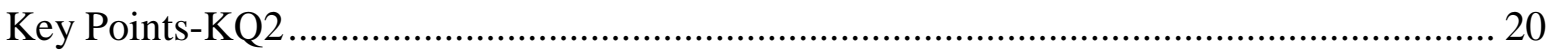

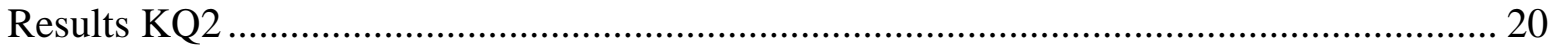

Pharmacologic Therapies ................................................................................................... 21

Nonpharmacologic Therapies .............................................................................................. 27

KQ3. In adult patients with exacerbation of COPD, what are the benefits and harms of combinations of treatments that are individually effective (based on empirical evidence in

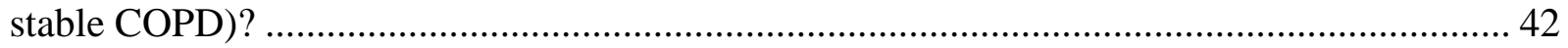

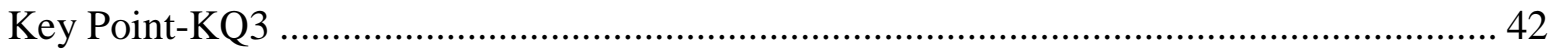

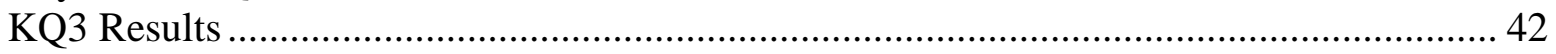

KQ4. In adult patients with exacerbation of COPD, what is the comparative effectiveness of different regimens of antibiotics and systemic corticosteroids based on type of agents (e.g.. broad-spectrum vs. narrow-spectrum antibiotics), delivery modes (e.g., intravenous, oral), and

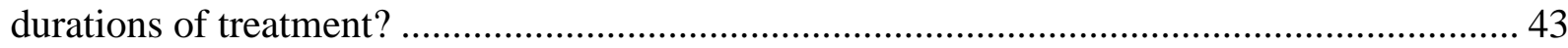

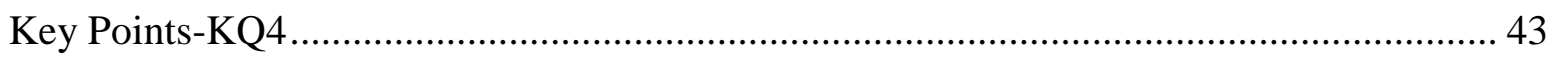

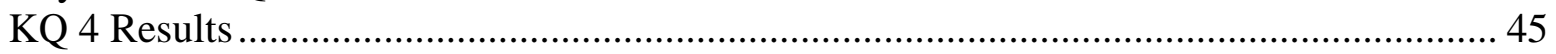

Fluoroquinolone Versus Aminopenicillin Plus Beta-Lactamase Inhibitor............................. 46

Ciprofloxacin (Fluoroquinolone) Versus Amoxicillin (Aminopenicillin)............................. 48 
Levofloxacin (Fluoroquinolone) Versus "Standard” Antibiotic Therapy (Clarithromycin or Cefuroxime or Amoxicillin + Clavulanic Acid) ................................................................... 48

Azithromycin (Macrolide) Versus Amoxicillin (Aminopenicillin) ........................................ 49

Cefaclor (Cephalosporin) Versus Ampicillin + Sulbactam (Aminopenicillin Plus Beta-

Lactamase Inhibitor) …………………………………................................................ 50

Fluoroquinolone Versus Cephalosporin ............................................................................. 51

Azithromycin (Macrolide) Versus Cefaclor (Cephalosporin) ................................................. 51

Azithromycin (Macrolide) Versus Ciprofloxacin (Fluoroquinolone).................................... 52

Amoxicillin (Aminopenicillin) Versus Amoxicillin Plus Clavulanic Acid............................ 52

Telithromycin (Ketolide) Versus Amoxicillin Plus Clavulanic Acid (Aminopenicillin Plus

Beta-Lactamase Inhibitor) .............................................................................................. 53

Azithromycin (Macrolide) Versus Aminopenicillin Plus Beta-Lactamase Inhibitor ............ 53

Prulifloxacin ( $4^{\text {th }}$ Generation Fluoroquinolone) Versus Levofloxacin $\left(2^{\text {nd }}\right.$ Generation

Fluoroquinolone)...................................................................................................... 54

Zabofloxacin (Next Generation Fluoroquinolone) Versus Moxifloxacin ( $4^{\text {th }}$ Generation

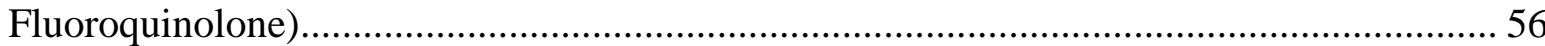

Cefpodoxime ( ${ }^{\text {rd }}$ Generation Cephalosporin) Versus Cefaclor $\left(2^{\text {nd }}\right.$ Generation

Cephalosporin) ............................................................................................................ 56

Meropenem (Carbapenem) Versus Imipenem (Carbapenem) ……………………............ 57

Comparative Effectiveness of Different Dosages of the Same Antibiotic ............................ 58

Comparative Effectiveness of Different Application Routes for Antibiotics ........................ 59

Comparative Effectiveness of Different Durations of Treatment With Antibiotics .............. 59

Corticosteroids-Comparative Effectiveness Studies .................................................................. 60

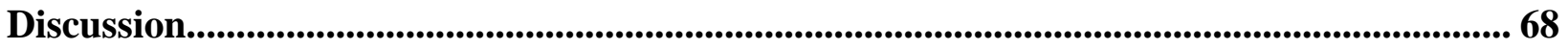

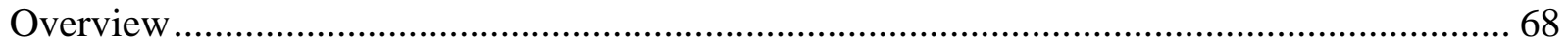

Findings in Relation to What Is Known ........................................................................... 71

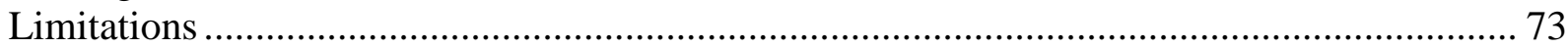

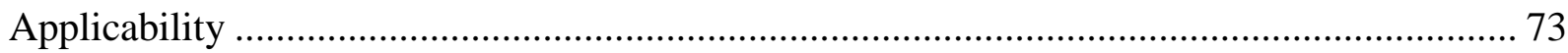

Suggestions for Future Research ...................................................................................... 74

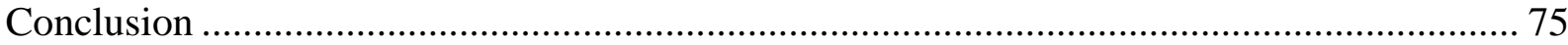

References........................................................................................................................................... 76

Abbreviations and Acronyms ................................................................................................................... 87

\section{Tables}

Table 1. PICOTS (population, interventions, comparisons, outcomes, timing, and setting).......... 6

Table 2. Categories of severity of exacerbation......................................................................... 8

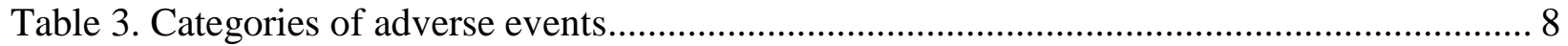

Table 4. Comparison of systemic antibiotics versus control, critical outcomes ........................... 14

Table 5. Comparison of systemic antibiotics versus control, additional outcomes ........................ 16

Table 6. Comparison of systemic corticosteroids versus control, critical outcomes ...................... 18

Table 7. Comparison of systemic corticosteroids versus control, additional outcomes ................ 19

Table 8. Comparison of intravenous aminophyllines versus placebo, critical outcomes .............. 21

Table 9. Comparison of intravenous aminophyllines versus placebo, additional outcomes ......... 21

Table 10. Comparison of intravenous magnesium sulfate versus placebo, critical outcomes...... 22

Table 11. Comparison of magnesium sulfate versus placebo, additional outcomes ...................... 22

Table 12. Comparison of oral mucolytics versus control, critical outcomes................................. 23 
Table 13. Comparison of oral mucolytics versus control, additional outcomes

Table 14. Comparison of inhaled corticosteroids with or without inhaled short- and long-acting bronchodilators versus placebo, critical outcomes

Table 15. Comparison of inhaled corticosteroids with or without inhaled short- and long-acting bronchodilators versus placebo, additional outcomes ....................................................... 25

Table 16. Comparison of inhaled antibiotics versus placebo .............................................. 26

Table 17. Long-acting muscarinic antagonists versus placebo............................................... 26

Table 18. Comparison of 5-lipoxygenase inhibitor (zileuton) versus placebo, critical outcomes 26

Table 19. Comparison of 5-lipoxygenase inhibitor (zileuton) versus placebo, additional outcomes

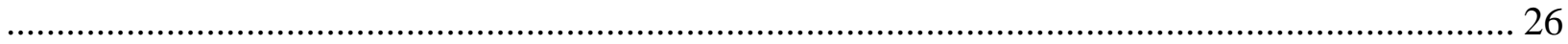

Table 20. Comparison of statin versus management without statin ........................................ 27

Table 21. Comparison of chest physiotherapy using breathing technique versus management without chest physiotherapy, critical outcomes ...

Table 22. Comparison of chest physiotherapy using breathing technique versus management without chest physiotherapy, additional outcomes .....

Table 23. Comparison of chest physiotherapy using vibration, percussion, or massage versus management without chest physiotherapy, critical outcomes.

Table 24. Comparison of chest physiotherapy using vibration, percussion, or massage versus management without chest physiotherapy, additional outcomes.

Table 25. Comparison of chest physiotherapy using positive expiratory pressure versus management without positive expiratory pressure, critical outcomes

Table 26. Comparison of chest physiotherapy using positive expiratory pressure versus management without positive expiratory pressure, additional outcomes 30

Table 27. Comparison of exercise using resistance training versus management without resistance training, critical outcomes

Table 28. Comparison of exercise using resistance training versus management without resistance training, additional outcomes

Table 29. Comparison of exercise using aerobic training versus management without aerobic training, critical outcomes.

Table 30. Comparison of exercise using aerobic training versus management without aerobic training, additional outcomes....

Table 31. Comparison of exercise using combined aerobic + resistance training versus management without exercise training

Table 32. Comparison of chest physiotherapy + exercise (breathing technique + range of motion exercises) combined versus management without exercise training, critical outcomes..... 34 Table 33. Comparison of chest physiotherapy + exercise (breathing technique + range of motion exercises) combined versus management without exercise training, additional outcomes .......... 34 Table 34. Comparison of early pulmonary rehabilitation versus management without early

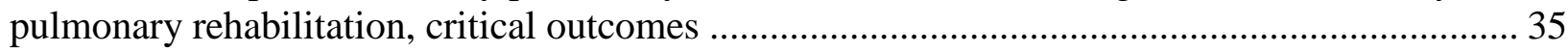
Table 35. Comparison of early pulmonary rehabilitation versus management without early pulmonary rehabilitation, additional outcomes. 35

Table 36. Comparison of whole body vibration training during ECOPD versus management without whole body vibration, critical outcomes. 36 Table 37. Comparison of whole body vibration training during ECOPD versus management without whole body vibration, additional outcomes. 
Table 38. Comparison of transcutaneous electrical nerve stimulation (TENS) during ECOPD versus management without TENS, critical outcomes ..................................................... 36 Table 39. Comparison of transcutaneous electrical nerve stimulation (TENS) during ECOPD

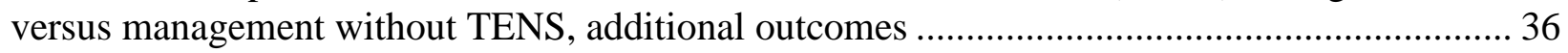
Table 40. Supplemental oxygen versus supplemental air.................................................. 37

Table 41. Titrated oxygen versus high flow oxygen, critical outcomes .................................. 38

Table 42. Titrated oxygen versus free flow oxygen, critical outcomes ................................... 38 Table 43. Comparison of a dietary intervention using a caloric supplement during ECOPD versus

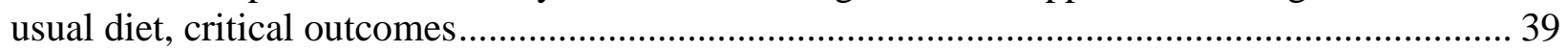
Table 44. Comparison of a dietary intervention using a caloric supplement during ECOPD versus

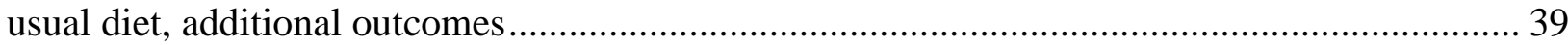

Table 45. Comparison of a dietary intervention using a caloric and a protein supplement during ECOPD versus placebo (non-caloric fluid, vanilla flavored water), critical outcomes............... 39 Table 46. Comparison of a dietary intervention using a caloric and a protein supplement during ECOPD versus placebo (non-caloric fluid, vanilla flavored water), additional outcomes ........... 40 Table 47. Comparison of a dietary intervention using a high fat low carbohydrate diet during

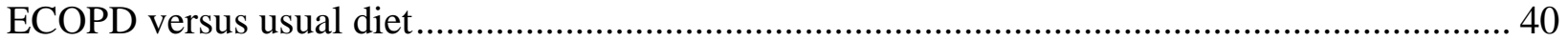
Table 48. Comparison of a dietary intervention using omega-3 fatty acid versus usual diet ....... 40 Table 49. Comparison of a dietary intervention using vitamin D during ECOPD versus placebo,

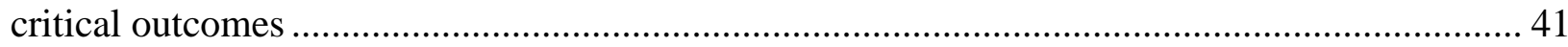
Table 50. Comparison of a dietary intervention using vitamin D during ECOPD versus placebo,

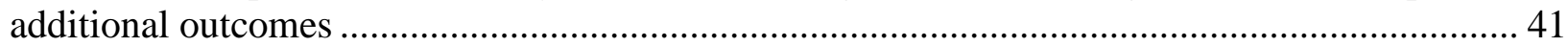
Table 51. Comparative effectiveness of inhalation treatments, critical outcomes ..................... 42 Table 52. Comparative effectiveness of inhalation treatments, additional outcomes.................. 43 Table 53. Comparison of fluoroquinolone versus aminopenicillin plus beta-lactamase inhibitor,

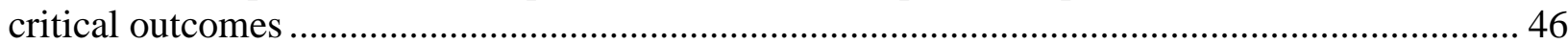
Table 54. Comparison of fluoroquinolone versus aminopenicillin plus beta-lactamase inhibitor,

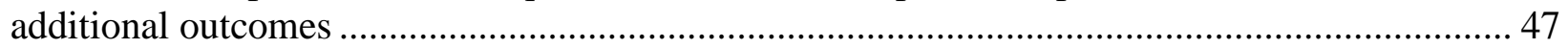

Table 55. Comparison of ciprofloxacin versus amoxicillin................................................. 48 Table 56. Comparison of levofloxacin versus "standard” antibiotic therapy (clarithromycin or cefuroxime or amoxicillin + clavulanic acid), critical outcomes............................................ 49 Table 57. Comparison of levofloxacin versus "standard” antibiotic therapy (clarithromycin or cefuroxime or amoxicillin + clavulanic acid), additional outcomes....................................... 49

Table 58. Comparison of azithromycin versus amoxicillin, critical outcomes.......................... 50

Table 59. Comparison of azithromycin versus amoxicillin, additional outcomes....................... 50

Table 60. Comparison of cefaclor versus ampicillin + sulbactam, critical outcomes ................ 50

Table 61. Comparison of cefaclor versus ampicillin + sulbactam, additional outcomes ............. 50

Table 62. Comparison of fluoroquinolone versus cephalosporin, critical outcomes................... 51

Table 63. Comparison of fluoroquinolone versus cephalosporin, additional outcomes.............. 51

Table 64. Comparison of azithromycin versus cefaclor, critical outcomes .............................. 52

Table 65. Comparison of azithromycin versus cefaclor, additional outcomes .......................... 52

Table 66. Comparison of azithromycin versus ciprofloxacin, critical outcomes ....................... 52

Table 67. Comparison of azithromycin versus ciprofloxacin, additional outcomes................... 52

Table 68. Comparison of amoxicillin versus amoxicillin plus clavulanic acid ......................... 53

Table 69. Comparison of telithromycin versus amoxicillin plus clavulanic acid....................... 53 
Table 70. Comparison of macrolide versus aminopenicillin plus beta-lactamase inhibitor, critical

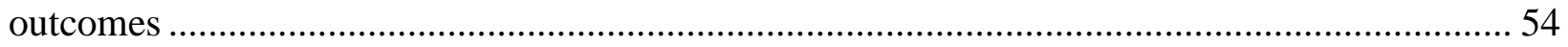

Table 71. Comparison of macrolide versus aminopenicillin plus beta-lactamase inhibitor,

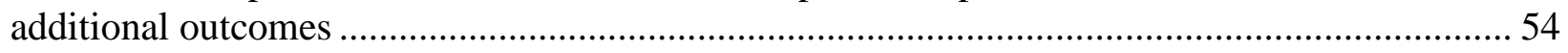

Table 72. Comparison of prulifloxacin versus levofloxacin, critical outcomes ........................ 54

Table 73. Comparison of prulifloxacin versus levofloxacin, additional outcomes ..................... 55

Table 74. Comparison of zabofloxacin versus moxifloxacin ................................................ 56

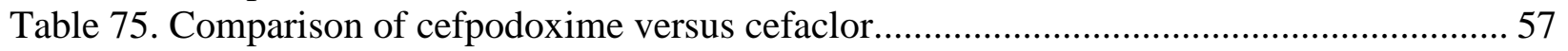

Table 76. Comparison of meropenem versus imipenem ..................................................... 57

Table 77. Comparison of trovafloxacin $200 \mathrm{mg}$ versus trovafloxacin $100 \mathrm{mg}$.......................... 58

Table 78. Comparison of intermittent intravenous cefotaxime versus continuous intravenous

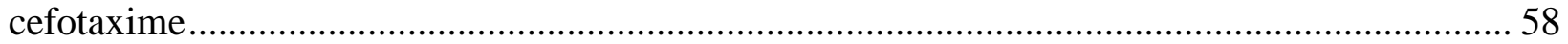

Table 79. Comparison of amoxicillin + clavulanic for 3 days versus 10 days ......................... 59

Table 80. Comparison of different systemic corticosteroid agents, critical outcomes ................. 60

Table 81. Comparison of different systemic corticosteroid agents, additional outcomes ............ 61

Table 82. Systemic corticosteroids versus inhaled corticosteroids, critical outcomes ................ 62

Table 83. Systemic corticosteroids versus inhaled corticosteroids, additional outcomes ........... 63

Table 84. Comparison of oral corticosteroids versus intravenous corticosteroids, critical outcomes ....

Table 85. Comparison of oral corticosteroids versus intravenous corticosteroids, additional

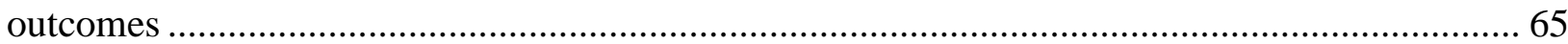

Table 86. Comparison of different durations of treatment with corticosteroids, critical outcomes

Table 87. Comparison of different durations of treatment with corticosteroids, additional outcomes

\section{Figures}

Figure 1. Analytic framework for Key Questions 1, 2, 3, and 4.......................................... 4

Figure 2. Summary of comparisons between different antibiotic agents................................. 45

Figure 3. Evidence map showing distribution of trials in mild (or mild/moderate) ECOPD (Key

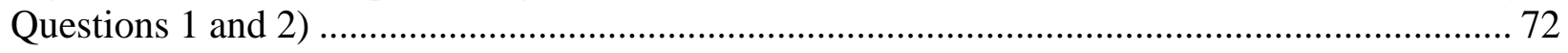

Figure 4. Evidence map showing distribution of trials in moderate to severe ECOPD (Key

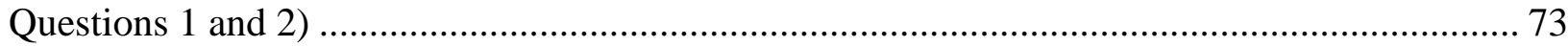

\section{Appendixes}

Appendix A. Flow Chart

Appendix B. Search Strategy

Appendix C. Excluded Studies

Appendix D. Characteristics of Included Studies

Appendix E. Risk of Bias

Appendix F. Results From Included Studies

Appendix G. Results by Severity

Appendix H. Adverse Events

Appendix I. Inclusion and Exclusion Criteria of Included Studies

Appendix J. Sensitivity Analysis

Appendix K. Appendix References 


\section{Evidence Summary}

\section{Background and Objectives}

Chronic obstructive pulmonary disease (COPD) is a common respiratory disease characterized by airflow limitation and chronic respiratory symptoms. The global prevalence is estimated to be greater than 10 percent, impacting approximately 380 million people worldwide. ${ }^{1}$ In the United States, COPD affects approximately 15 million people; chronic lower respiratory diseases, of which COPD is the largest contributing condition, are the fourth leading cause of death; ${ }^{2}$ and COPD costs more than $\$ 32$ billion annually. ${ }^{3,4}$ Patients with COPD experience chronic respiratory symptoms (including shortness of breath and cough), and have decreased quality of life, and premature mortality.

Patients with COPD are at risk of experiencing exacerbations of COPD (ECOPD). There have been various definitions of what constitutes an ECOPD. The Global Initiative for chronic obstructive lung disease (GOLD) defines ECOPD in its 2019 report as "acute worsening of respiratory symptoms that result in additional therapy." 5 ECOPD is generally characterized by increased dyspnea, increased frequency and severity of cough, and/or increased sputum production. ${ }^{6}$ ECOPD is a leading independent cause of increased mortality and morbidity among patients with COPD. ECOPD is associated with a higher risk of dying during or shortly after the exacerbation, lower quality of life, more hospital admission, depletion of financial resources, and a progressive decline in lung function. ${ }^{7-14}$ Hospitalizations for ECOPD account for more than half of all costs associated with COPD. ${ }^{10,15}$

In recent years, a number of clinical trials of treatments to prevent ECOPD have shown promising results, but the evidence for acute treatments during an episode of ECOPD appears to be surprisingly scarce, given how relatively common the condition is. ${ }^{16,17}$

Goals of management of ECOPD include relieving symptoms and hastening the recovery from ECOPD by addressing precipitating factors (e.g. antibiotic treatment for infections), improving expiratory airflow and gas exchange (and thus improving breathing) by using inhaled bronchodilators, and reducing lung inflammation with corticosteroids. Nonpharmacologic treatments include supplemental oxygen, nutritional support, and others. ${ }^{18-20}$ In addition, several new pharmacologic agents with novel mechanisms of action in early stages of development may be of potential benefit to COPD patients including those in acute exacerbation. ${ }^{21}$

The area of ECOPD management has several uncertainties and necessitates an up to date evidence synthesis. These uncertainties include the benefits and harms of emerging pharmacologic and nonpharmacologic treatments, the benefits and harms of treatments for ECOPD that have been found efficacious in stable COPD , the benefits and harms of antibiotics and systemic corticosteroids in mild ECOPD, the benefits and harms of combinations of treatments that have been found to be individually effective, and-for antibiotics and systemic corticosteroids-the comparative effectiveness of different types of agents (e.g. broad-spectrum versus narrow-spectrum antibiotics), delivery modes (e.g. intravenous, oral), and durations of treatment.

Examples of potentially emerging treatments for ECOPD, include immune-modulatory drugs and novel applications of treatments primarily used in stable COPD including mucolytics, aminophyllines, long-acting bronchodilators, inhaled corticosteroids, and others. Mucolytics may have a small effect on reducing the frequency of ECOPD. ${ }^{22,23}$ In clinical practice, they are also used during an ECOPD, where the evidence for their effectiveness appears less clear. 
For nonpharmacological treatments, there are a number of areas in which an update of the evidence is required to inform best practice management of ECOPD. There is increasing recognition that too much oxygen might do more harm than good, and not just in patients with chronic hypercapnic respiratory who are at risk of iatrogenic worsening of respiratory failure due to oversupply of oxygen. ${ }^{24,25}$ Titrated oxygen with a target saturation rate as opposed to high flow oxygen has therefore been used in patients with ECOPD. ${ }^{26}$ Historically, pulmonary rehabilitation programs have focused on enrolling patients with stable COPD or patients who had stabilized after an episode of ECOPD, but in more recent times, a number of trials have explored the role of exercise/early pulmonary rehabilitation during an episode of ECOPD. ${ }^{27,}{ }^{28}$ Chest physiotherapy using airway clearance techniques (including breathing technique, vibration/percussion, and autogenic drainage) are used routinely in many patients hospitalized with ECOPD. A Cochrane review published in 2012 found evidence that airway clearance techniques may reduce the need for hospital admission and improve health-related quality of life based on single studies with small study populations. ${ }^{29}$ An update of this evidence is indicated. Furthermore, many patients with COPD are in a state of hyper-metabolism in which their body consumes more calories per kilogram on calorimetric measures compared with a person without COPD, likely because of the increased work of breathing. ${ }^{30}$ This hyper-metabolic state is even more pronounced during episodes of ECOPD, posing questions about the optimal nutritional support for patients with ECOPD. ${ }^{31,32}$

Established treatments for ECOPD, such as antibiotics and systemic corticosteroids, may not be indicated in every single episode of an ECOPD. One uncertainty relates to the need for antibiotics in mild and moderately severe ECOPD, especially in an outpatient setting. ${ }^{33}$ While antibiotics for treatment of severe ECOPD have been shown to be beneficial in some studies, the need for antibiotics in less severe forms of COPD is unclear. ${ }^{34}$ Uncertainty remains regarding the use of systemic (oral, intravenous) corticosteroids relate to whether all patients stand to benefit from this treatment of ECOPD. ${ }^{34}$ These questions are important to address in view of trying to reduce prescriptions of antibiotics, where safely possible, to reduce potential adverse events including development of antibiotic resistance, and to reduce potentially significant adverse events from systemic corticosteroids, in particular hyperglycemia, in patients with glucose intolerance and diabetes. $^{35}$

Short-acting beta adrenergic agonists (SABAs) and short-acting muscarinic antagonists (SAMAs) are established treatments to relieve dyspnea and improve airflow obstruction during ECOPD, but the benefits of combining SABAs and SAMAs compared with using SABAs or SAMAs alone are unclear. ${ }^{36}$ Long-acting bronchodilators and inhaled corticosteroids have historically only be used in stable COPD, but there is emerging evidence that an increase in dosage of inhalation therapy with inhaled corticosteroids and long-acting beta agonists (LABAs) may be beneficial in early treatment of ECOPD when patients experience mild to moderate dyspnea and may result in no requirement of systemic corticosteroids in a large proportion of patients presenting with mild-to-moderate worsening of dyspnea. ${ }^{37}$ The benefit of using LABAs and long-acting muscarinic antagonists (LAMAs) in the treatment of manifest ECOPD is unclear. For antibiotics and systemic corticosteroids, the comparative effectiveness of different agents (e.g. broad-spectrum versus narrow-spectrum antibiotics), delivery modes and durations of treatment needs to be established. .8-41 $^{38}$

In summary, determining the optimal treatment plan for patients with ECOPD requires 1) a synthesis of existing knowledge regarding the effectiveness of treatment options and 2) a synthesis of existing knowledge regarding the harms of treatment options. Currently, the 
comparative benefits and harms of these varied treatment approaches including the optimal combination of these treatments to mitigate COPD exacerbation are unclear. A systematic review of current evidence assists clinicians in understanding and determining optimal management for ECOPD. This review focuses on evidence from randomized controlled trials (RCTs) as the gold standard design for evaluating a therapeutic intervention. In terms of adverse events, they are also likely to be captured in RCTs because of the acute nature of condition being studied.

\section{Scope and Key Questions}

\section{Scope of Review}

The systematic review assessed the effectiveness of systemic antibiotics, systemic corticosteroids and other pharmacologic and nonpharmacologic therapies stratified by severity of ECOPD. The study also evaluated the effectiveness of combinations of treatments, and compared different regimens (different agents, routes of administration, and duration of treatment) of antibiotics and corticosteroids. Health service interventions (e.g. hospital in the home as alternative to hospitalization) and interventions during the convalescence period were not included.

\section{Key Questions}

Key Question (KQ) 1. In adult patients with exacerbation of COPD, what are the benefits and harms of systemic corticosteroids and antibiotics compared with placebo or standard care?

KQ2. In adult patients with exacerbation of COPD, what are the benefits and harms of emerging and other pharmacologic and nonpharmacologic therapies compared with placebo or standard care?

KQ3. In adult patients with exacerbation of COPD, what are the benefits and harms of combinations of treatments that are individually effective (based on empirical evidence in stable COPD)?

KQ4. In adult patients with exacerbation of COPD, what is the comparative effectiveness of different regimens of antibiotics and systemic corticosteroids based on type of agents (e.g., broad-spectrum vs. narrowspectrum antibiotics), delivery modes (e.g., intravenous, oral), and durations of treatment?

\section{Methods}

We developed an analytic framework to guide the process of the systematic review. We followed the established methodologies of systematic reviews as outlined in the Agency for Healthcare Research and Quality (AHRQ) Methods Guide for Comparative Effectiveness Reviews. ${ }^{42}$ The reporting complies with the Preferred Reporting Items for Systematic Reviews 
and Meta-Analyses (PRISMA) statements. ${ }^{43}$ The study protocol is registered in the international prospective register of systematic reviews (PROSPERO \#: 42018111609) and published on the AHRQ website (https://effectivehealthcare.ahrq.gov/topics/copd/protocol). The full report details our literature search strategy, inclusion and exclusion criteria, data synthesis, assessments of risk of bias, and strength of evidence (SOE). We graded SOE for final health outcomes deemed to be most important or critical, including mortality, dyspnea, quality of life (QoL), need for intubation, repeat exacerbation and/or hospital readmissions and ECOPD resolution (clinical cure, failure). SOE was rated as high when we were very confident that the estimate of effect lies close to the true effect (the body of evidence has few or no deficiencies and is judged to be stable). SOE was rated as moderate when we were moderately confident that the estimate of effect lies close to the true effect (the body of evidence has some deficiencies and is judged to be likely stable). SOE was rated as low when we had limited confidence that the estimate of effect lies close to the true effect (the body of evidence has major or numerous deficiencies and is likely unstable). SOE was rated as insufficient when we had no evidence, were unable to estimate an effect, or had no confidence in the estimate of effect.

\section{Results}

The literature search identified 8,916 citations. An additional 36 references were identified through reference mining, grey literature search; and from Key Informants and Technical Experts. There were 98 original studies with a total of 13,401 patients that met inclusion criteria and were included in the systematic review. Most studies were conducted in hospitalized patients with moderate to severe COPD. The mean treatment duration was 9.9 days and there was a mean 3.7 months of reported followup.

KQ1. In adult patients with exacerbation of COPD, what are the benefits and harms of systemic corticosteroids and antibiotics compared with placebo or standard care?

\section{Systemic Antibiotics Versus Placebo or Management Without Systemic Antibiotics}

Antibiotics increased clinical cure of ECOPD compared with placebo or management without antibiotics at the end of the intervention and at the longest followup (moderate SOE).

Antibiotics reduced clinical failure rate compared with placebo at the end of the intervention (moderate SOE), but not at the longest followup (Low SOE).

Antibiotics did not change 30-day hospital readmission, repeat exacerbation, and quality of life, compared with placebo (low SOE).

No statistically significant difference in adverse events (AEs) was observed between antibiotics and placebo or management without antibiotics.

\section{Systemic Corticosteroids Versus Placebo or Management Without Systemic Corticosteroids}

Systemic corticosteroids improved dyspnea (Low SOE) and reduced clinical failure rate (low $\mathrm{SOE}$ ) at the end of the intervention, compared with placebo. 
No statistically significant difference in serious adverse events was found between systemic corticosteroids and placebo or management without systemic corticosteroids. Systemic corticosteroids were associated with fewer withdrawals but more endocrine related adverse events.

KQ2. In adult patients with exacerbation of COPD, what are the benefits and harms of emerging and other pharmacologic and nonpharmacologic therapies compared with placebo or standard care?

\section{Pharmacologic Therapies Versus Placebo or Management Without Pharmacologic Therapies}

The evidence was insufficient for the effect of aminophyllines, magnesium sulfate, mucolytics, inhaled corticosteroids, inhaled antibiotics, 5-lipoxygenase inhibitor and statins on mortality, dyspnea, need for intubation, clinical failure, or hospital admission.

Aminophyllines were associated with more gastrointestinal adverse events than placebo. No other statistically significant difference was found in adverse events between the remaining pharmacologic therapies and placebo or management without pharmacologic therapies.

\section{Nonpharmacologic Therapies Versus Management Without Nonpharmacologic Therapies}

Chest physiotherapy using vibration/percussion, breathing technique, or positive expiratory pressure did not improve dyspnea and other symptoms, quality of life, 6-minute walking distance, repeat exacerbations, or mortality (low SOE).

Resistance training improved dyspnea, and quality of life, compared with management without nonpharmacological therapies (low SOE).

Early pulmonary rehabilitation, commenced before hospital discharge during the initial most acute phase of exacerbation rather than the convalescence period, improved dyspnea, compared with management without rehabilitation (low SOE).

Whole body vibration training improved QoL compared with management without nonpharmacologic therapies (low SOE).

Titrated oxygen reduced mortality compared with high flow oxygen at the longest followup (low SOE).

Vitamin D supplementation improved quality of life compared with placebo (low SOE).

Omega-3 fatty acid enriched diet did not change quality of life, need for intubation, and dyspnea at the end of intervention compared with usual diet (low SOE).

Few adverse events were reported in studies of nonpharmacologic therapies. There was no statistically significant difference found in adverse events between nonpharmacologic therapies and management without nonpharmacologic therapies.

KQ3. In adult patients with exacerbation of COPD, what are the benefits and harms of combinations of treatments that are individually effective (based on empirical evidence in stable COPD)?

No statistically significant difference in adverse events was found between any of the combined treatments and individual treatments. 
KQ4. In adult patients with exacerbation of COPD, what is the comparative effectiveness of different regimens of antibiotics and systemic corticosteroids based on type of agents (e.g., broad-spectrum vs. narrowspectrum antibiotics), delivery modes (e.g., intravenous, oral), and durations of treatment?

\section{Comparative Effectiveness of Different Antibiotics}

Numerous antibiotics, given as empirical initial therapy for ECOPD (in the absence of pneumonia), were compared against each other, but the evidence was insufficient to estimate an effect on final health outcomes; except that levofloxacin reduced repeat exacerbations at 3 months of followup, compared with prulifloxacin (low SOE).

The only differences in adverse events that were statistically significantly were for higher rates of adverse events with amoxicillin plus clavulanic acid compared with telithromycin; and for higher rate of adverse events with imipenem plus cilastatin compared with meropenem.

\section{Comparative Effectiveness of Different Dosages of the Same} Antibiotic

The evidence comparing different dosages of the same antibiotic was insufficient for mortality, clinical cure and clinical failure.

No statistically significant difference in adverse events was found between trovafloxacin 200 milligrams (mg) and trovafloxacin $100 \mathrm{mg}$.

\section{Comparative Effectiveness of Different Application Routes for Antibiotics}

No studies were found.

\section{Comparative Effectiveness of Different Durations of Treatment With Antibiotics}

The evidence was insufficient when comparing 3 day versus 10 day regimens of amoxicillin plus clavulanic acid.

No statistically significant difference of AEs was found between 3 day and 10 day regimens of amoxicillin plus clavulanic acid.

\section{Comparative Effectiveness of Different Corticosteroids}

The evidence was insufficient when comparing the different corticosteroids for mortality, need for intubation, clinical failures, and dyspnea.

There was no statistically significant difference in AEs found between the different systemic corticosteroids. 


\section{Comparative Effectiveness of Different Routes of Application for Corticosteroids}

No difference between intravenous methylprednisolone and inhaled budesonide $40 \mathrm{mg}$ was found in quality of life and repeat exacerbations (low SOE). The evidence was insufficient when comparing the different routes of administration of corticosteroids for mortality, dyspnea, quality of life, repeat exacerbation, clinical failures, hospital admission, and intensive care unit (ICU) admission.

Inhaled Budesonide $40 \mathrm{mg}$ was associated with statistically significantly less endocrinerelated AEs than methylprednisolone.

\section{Comparative Effectiveness of Different Durations of Treatment With Corticosteroids}

The evidence was insufficient when comparing the different durations of corticosteroid treatment for mortality, hospital admission, need for intubation, clinical failure, quality of life, repeat exacerbation, and dyspnea.

There was no statistically significant difference found in AEs between the different systemic corticosteroid durations.

\section{Discussion}

We conducted a systematic review to assess the effectiveness of pharmacologic and nonpharmacologic therapies in adults with ECOPD. We assessed the effectiveness of systemic antibiotics, systemic corticosteroids and emerging and other pharmacologic and nonpharmacologic therapies stratified by severity of ECOPD. Further, we assessed the effectiveness of combinations of treatments, and we compared different regimens (different agents, routes of administration, and duration of treatment) of antibiotics and corticosteroids.

The majority of studies were conducted in hospitalized patients with moderate or severe ECOPD with only a small number of studies conducted in outpatients with mild or mild to moderate ECOPD.

Lung function was the most frequently assessed outcome, and often studies did not measure final health outcomes, such as mortality, resolution of exacerbation, hospital readmission etc., to allow for assessment of the correlation between this physiological surrogate outcome and final health outcome.

The findings of the systematic review highlight that in addition to standard therapy with antibiotics, systemic steroids and bronchodilators, some nonpharmacologic interventions hold promise for improving clinically important outcomes, in particular they might improve functional capacity and thus mitigate the deconditioning associated with ECOPD.

\section{Findings in Relation to What Is Known}

This review provides a comprehensive overview of pharmacologic and nonpharmacologic interventions in ECOPD. The literature on interventions for COPD and ECOPD has proliferated substantially in recent years with numerous published systematic reviews on different interventions for the management of COPD. For clinicians, health policy makers and other end users of the evidence it has become an almost impossible task to keep up with the ever increasing body of evidence on the management of ECOPD. This review therefore addresses an urgent need 
to provide an up-to-date summary of the current state of evidence for the management of ECOPD.

One of the main findings of this systematic review is that despite a proliferation of the COPD literature, the evidence base for most interventions in ECOPD remains low. While significant progress has been made in recent years in assessing interventions to prevent ECOPD (during stable COPD), the same cannot be said for acute interventions used during ECOPD.

For the standard therapy of ECOPD with systemic antibiotics, corticosteroids and bronchodilators, many questions remain unanswered, based on the findings of our review. While the discussion of COPD phenotypes (and ECOPD phenotypes) has taken center stage on the COPD research agenda, very limited information on ECOPD phenotypes (e.g. infective versus non-infective, high versus low eosinophil count) has been included in trials of intervention for ECOPD. In particular, whether a response to systemic corticosteroid treatment of ECOPD depends on the blood eosinophil level remains unexplored. Studies on inhaled corticosteroid (ICS) for prevention of ECOPD in stable COPD suggest that patients with higher blood eosinophil levels might be more likely to benefit from ICS treatment in terms of reducing the risk of ECOPD. ${ }^{44}$

Despite the ubiquitous use of SABAs and SAMAs in ECOPD, we found only two studies (KQ3) that studied their effectiveness. The role of LABAs and LAMAs in ECOPD remains largely unexplored with only one crossover trial identified in our review that assessed a LAMA versus placebo.

An important insight from our systemic review is that some nonpharmacologic interventions (resistance training, early pulmonary rehabilitation, whole body vibration training transcutaneous electrical nerve stimulation, caloric supplementation, and vitamin D) show promise, but the current evidence is largely based on single, relatively small RCTs. In stable COPD, pulmonary rehabilitation is one of the most effective (though underused) interventions. In recent years, there has been a significant interest in exploring the effects of pulmonary rehabilitation in patients who have recently experienced an ECOPD or even in patients who are in the acute phase of ECOPD (e.g. before hospital discharge).

Our review indicated that pulmonary rehabilitation during ECOPD may increase functional capacity (based on 6-minute walking distance). A potential risk for increased mortality associated with pulmonary rehabilitation commenced during hospitalization for ECOPD has previously been flagged in the guidelines on management of COPD exacerbations by the European Respiratory Society and the American Thoracic Society, published in $2017 .{ }^{45}$ We did not find a significant association with increased mortality for pulmonary rehabilitation or any form of exercise commenced during hospitalization. Our review did not include studies conducted in an ICU, chronic ventilator unit, or respiratory care unit, which might have contributed to the discrepancy in the findings. Also, a trial of rehabilitation commenced within 48 hours of hospital admission in 389 patients with exacerbations of different chronic respiratory conditions found an increase in mortality in the intervention group at one year (odds ratio: 1.74, 95\% confidence interval: 1.05 to 2.88). ${ }^{46}$ Mortality was, however, not reported in the subgroup of patients with COPD and is therefore not included in our review. Given the potential of exercise programs during hospitalization for ECOPD to ameliorate deconditioning and improve functional status, further research in this area is urgently needed. Other nonpharmacologic interventions during ECOPD that may improve functional capacity included resistance training, whole body vibration training and transcutaneous electrical nerve stimulation. As these findings were based on single, relatively small studies, evidence from well-conducted large RCTs will be 
required to confirm these findings. Similarly, caloric supplementation and vitamin D may improve quality of life in patients with ECOPD, but confirmation from well-conducted large RCTs is required before any definite conclusions can be drawn.

\section{Limitations}

For most interventions, only one RCT was available per outcome (KQ1-4), which limits inferences from the quantitative synthesis. Failure to detect statistical significance for most of the outcomes may have resulted from type II error. There was some heterogeneity in the definition of the severity of ECOPD, although in general mild ECOPD referred to patients that could be treated in an outpatient setting, whereas moderate to severe ECOPD was used for hospitalized patients. A number of studies included patients assessed in an emergency department with a broad range of severity of ECOPD. We used the definition of serious AEs listed by the original studies, which could have varied between studies.

Defining resolution of ECOPD and differentiating poor resolution from re-exacerbation can be challenging. We used outcomes as described in the original studies, which might have resulted in heterogeneity of definitions of ECOPD resolution and overlap between clinical failure and re-exacerbation between studies.

Very limited information on ECOPD phenotypes (e.g. infective versus non-infective, high versus low eosinophil count) has been included in trials of intervention. We could therefore not draw any conclusions about interventions for different ECOPD phenotypes. In particular, whether a response to systemic corticosteroids depends on the blood eosinophil levels remains unexplored.

Studies were overall at high risk of bias. This, together with the low number of studies per intervention/outcome, makes interpretation of the body of evidence challenging. We were unable to statistically evaluate publication bias and only included studies published in English. An evaluation of completed clinical trials registered in clinicaltrials.gov showed that 62 percent (24 out of 39) studies were not published.

\section{Applicability}

Most studies were conducted in hospitalized patients with moderate to severe ECOPD, and the results of these studies may not be applicable to patients with milder forms of ECOPD treated in an outpatient setting. KQ1 and KQ2 were stratified by severity of ECOPD, which allows determination of the generalizability of the results based on the severity of ECOPD. For KQ2, almost all studies were conducted in hospitalized patients. As we excluded studies conducted in an ICU setting, some of our findings may not be extrapolated to the most severely sick patients who require ICU admission for ECOPD.

The results of comparisons of different antibiotic agents/classes are context-specific, as the optimal antibiotic choice depends on local antimicrobial resistance patterns, which can change over time. The results of these comparisons (KQ4) are therefore not necessarily applicable to patients in different geographic locations and at different points in time.

COPD terminology has not been used consistently in the past with some older studies referring to chronic bronchitis without airflow obstruction as COPD. We excluded studies in patients with chronic bronchitis but no evidence of chronic airflow obstruction to increase applicability of the results to patients with chronic airflow obstruction.

Not all studies explicitly excluded patients with potential asthma or asthma-COPD overlap syndrome (ACOS), and there is therefore a potential for misclassification. 
Pulmonary rehabilitation is a complex (multi-component) intervention, which consists of exercise training, patient education and behavior change. The detailed interventions for pulmonary rehabilitation were reported in the included studies, which should facilitate reproducibility and applicability. While there are published standards for pulmonary rehabilitation programs, ${ }^{47}$ these have been developed in the context of pulmonary rehabilitation in patients with stable COPD (as opposed to patients with ECOPD).

\section{Suggestions for Future Research}

Lung function (forced expiratory volume in 1 second) was the most commonly assessed outcome in studies of interventions to manage ECOPD, while final health outcomes, such as resolution of ECOPD (clinical cure, clinical failure) and repeat exacerbation (with or without hospital admission), were rarely assessed. Future studies in ECOPD should focus on final health outcomes and include clinical resolution of ECOPD and risk of repeat exacerbation in addition to other final health outcomes, such as dyspnea and quality of life.

The response to antibiotic therapy as well as corticosteroid therapy in ECOPD likely differs based on the phenotype of the exacerbation episode. A number of studies that used procalcitonin-guided treatment algorithms have been conducted on antibiotic therapy versus placebo in ECOPD, ${ }^{48}$ but identification of responders to systemic corticosteroid treatment of ECOPD based on blood eosinophils remains unexplored. This contrasts with the increasing recognition of eosinophilic phenotypes in stable COPD patients who appear to be more likely to benefit from long-term ICS. ${ }^{44}$ Future studies on systemic corticosteroids in ECOPD should assess the treatment effect stratified by blood eosinophil count.

Chest physiotherapy using breathing technique and/or vibration/percussions and/or positive expiratory pressure is commonly prescribed in patients hospitalized for ECOPD, but there was insufficient evidence (from relatively small, low quality trials) that these interventions improve outcomes. As these are resource-intensive interventions, large well-designed trials with final health outcomes including clinical resolution of ECOPD and repeat exacerbations should be conducted to assess the role of chest physiotherapy for airway clearance in ECOPD and inform clinical practice.

It is currently unclear whether pulmonary rehabilitation commenced during hospitalization for ECOPD is associated with increased mortality. An increased mortality was found in the review conducted for the guidelines on management of COPD exacerbations by the European Respiratory Society and the American Thoracic Society but was not found in our systematic review. Given the potential benefit of pulmonary rehabilitation to counteract the deconditioning associated with ECOPD, we believe that conducting high-quality RCTs to answer this question should be a priority.

The relatively new treatment options of whole body vibration, transcutaneous electrical nerve stimulation (TENS), dietary interventions with caloric supplements and vitamin D need to be assessed in large high quality RCTs to inform recommendations about these treatments. Such literature (e.g., on vitamin D) is notorious for contradictory findings over time.

Further research is required to determine the optimal route of administration for systemic corticosteroids, i.e. to determine whether oral corticosteroids are generally not inferior to intravenous corticosteroids and to determine a potential role of inhaled corticosteroids (possibly as alternative to systemic corticosteroids) in ECOPD.

Patients hospitalized with COPD exacerbations are at high risk for hospital readmissions and death after hospital discharge, which emphasizes the importance of improving the hospital-to- 
home continuum of care. Our systematic review only focused on the acute episode of an exacerbation and did not include health service interventions, but there is an urgent need for research that assesses interventions to reduce the risk of adverse outcomes following hospital discharge. Much of the recent debate on adverse outcomes following hospital discharge has focused on reducing 30-day hospital readmissions in ECOPD, as the Medicare's Hospital Readmissions Reduction Program (HRRP) lowered payments to Inpatient Prospective Payment System hospitals with too many readmissions within 30 days. Recent evidence, however, showed that implementation of the HRRP was associated with a significant increase in trends in 30-day post-discharge mortality among patients hospitalized for heart failure and pneumonia. ${ }^{49}$ It is therefore evident that future research that aims to improve post-hospital discharge for any disease with frequent hospital readmissions including ECOPD should not focus on reducing 30day hospital admissions in isolation but only in conjunction with final health outcomes such as QoL and mortality.

\section{Conclusion}

Despite a proliferation of the COPD literature, the evidence base for most interventions in ECOPD remains limited. Systemic antibiotics and corticosteroids are associated with improved outcomes in mild and moderate to severe ECOPD. Titrated oxygen reduces mortality. Future research is required to assess the effectiveness of several emerging nonpharmacologic and dietary treatments. 


\section{References}

1. Adeloye D, Chua S, Lee C, et al. Global and regional estimates of COPD prevalence: Systematic review and meta-analysis. J Glob Health. 2015 Dec;5(2):020415. doi: 10.7189/jogh.05-020415. PMID: 26755942.

2. Murphy SL, Xu J, Kochanek KD, et al. Mortality in the United States, 2017. NCHS Data Brief. 2018 Nov(328):1-8. PMID: 30500322.

3. Kochanek KD, Murphy SL, Xu J, et al. Deaths: Final Data for 2014. Natl Vital Stat Rep. 2016 Jun;65(4):1-122. PMID: 27378572.

4. Ford ES, Murphy LB, Khavjou O, et al. Total and state-specific medical and absenteeism costs of COPD among adults aged $>/=18$ years in the United States for 2010 and projections through 2020. Chest. 2015 Jan;147(1):31-45. doi: 10.1378/chest.14-0972. PMID: 25058738.

5. Disease GIfCOL. Global Strategy for the Diagnosis, Management, and Prevention of Chronic Obstructive Lung Disease. 2019.

6. Anthonisen NR, Manfreda J, Warren CP, et al. Antibiotic therapy in exacerbations of chronic obstructive pulmonary disease. Annals of Internal Medicine. 1987 Feb;106(2):196-204. PMID: 3492164.

7. Connors AF, Jr., Dawson NV, Thomas C, et al. Outcomes following acute exacerbation of severe chronic obstructive lung disease. The SUPPORT investigators (Study to Understand Prognoses and Preferences for Outcomes and Risks of Treatments). Am J Respir Crit Care Med. 1996 Oct;154(4 Pt 1):959-67. doi: 10.1164/ajrccm.154.4.8887592. PMID: 8887592.

8. Seemungal TA, Donaldson GC, Paul EA, et al. Effect of exacerbation on quality of life in patients with chronic obstructive pulmonary disease. Am J Respir Crit Care Med. 1998 May;157(5 Pt 1):1418-22. doi: 10.1164/ajrccm.157.5.9709032. PMID: 9603117.
9. Miravitlles M, Murio C, Guerrero T, et al. Pharmacoeconomic evaluation of acute exacerbations of chronic bronchitis and COPD. Chest. 2002 May;121(5):1449-55. PMID: 12006427.

10. Miravitlles M, Garcia-Polo C, Domenech A, et al. Clinical outcomes and cost analysis of exacerbations in chronic obstructive pulmonary disease. Lung. 2013 Oct;191(5):523-30. doi: 10.1007/s00408013-9487-z. PMID: 23842641.

11. Seemungal TA, Donaldson GC, Bhowmik A, et al. Time course and recovery of exacerbations in patients with chronic obstructive pulmonary disease. Am J Respir Crit Care Med. 2000 May;161(5):1608-13. doi: 10.1164/ajrccm.161.5.9908022. PMID: 10806163.

12. Donaldson GC, Seemungal TA, Bhowmik A, et al. Relationship between exacerbation frequency and lung function decline in chronic obstructive pulmonary disease. Thorax. 2002 Oct;57(10):847-52. PMID: 12324669.

13. Doll H, Miravitlles M. Health-related QOL in acute exacerbations of chronic bronchitis and chronic obstructive pulmonary disease: a review of the literature. Pharmacoeconomics. 2005;23(4):345-63. PMID: 15853435.

14. Patil SP, Krishnan JA, Lechtzin N, et al. Inhospital mortality following acute exacerbations of chronic obstructive pulmonary disease. Arch Intern Med. 2003 May 26;163(10):1180-6. doi: 10.1001/archinte.163.10.1180. PMID: 12767954.

15. Wouters EF. Economic analysis of the Confronting COPD survey: an overview of results. Respir Med. 2003 Mar;97 Suppl C:S3-14. PMID: 12647938.

16. Aaron SD. Management and prevention of exacerbations of COPD. Bmj. 2014 Sep 22;349:g5237. doi: 10.1136/bmj.g5237. PMID: 25245156. 
17. Welte T. Acute exacerbation in COPD: we must do more. Eur Respir J. 2016 Jan;47(1):14-5. doi: 10.1183/13993003.01981-2015. PMID: 26721956.

18. Mackay AJ, Hurst JR. COPD exacerbations: causes, prevention, and treatment. Immunol Allergy Clin North Am. 2013 Feb;33(1):95115. doi: 10.1016/j.iac.2012.10.006. PMID: 23337067.

19. Calverley P. Current drug treatment, chronic and acute. Clin Chest Med. 2014

Mar;35(1):177-89. doi:

10.1016/j.ccm.2013.09.009. PMID: 24507845.

20. Cabrini L, Landoni G, Oriani A, et al. Noninvasive ventilation and survival in acute care settings: a comprehensive systematic review and metaanalysis of randomized controlled trials. Crit Care Med. 2015 Apr;43(4):880-8. doi: 10.1097/CCM.0000000000000819. PMID: 25565461.

21. Barjaktarevic IZ, Arredondo AF, Cooper CB. Positioning new pharmacotherapies for COPD. Int J Chron Obstruct Pulmon Dis. 2015;10:1427-42. doi: 10.2147/COPD.S83758. PMID: 26244017.

22. Poole PJ. Role of mucolytics in the management of COPD. Int J Chron Obstruct Pulmon Dis. 2006;1(2):123-8. PMID: 18046889.

23. Poole P, Black PN, Cates CJ. Mucolytic agents for chronic bronchitis or chronic obstructive pulmonary disease. Cochrane Database of Systematic Reviews. 2012 Aug 15(8):CD001287. doi: https://dx.doi.org/10.1002/14651858.CD001 287.pub4. PMID: 22895919.

24. Wijesinghe M, Perrin K, Healy B, et al. Prehospital oxygen therapy in acute exacerbations of chronic obstructive pulmonary disease. Intern Med J. 2011 Aug;41(8):618-22. doi: 10.1111/j.14455994.2010.02207.x. PMID: 20214690.

25. Austin MA, Wills KE, Blizzard L, et al. Effect of high flow oxygen on mortality in chronic obstructive pulmonary disease patients in prehospital setting: randomised controlled trial. BMJ. 2010 Oct 18;341:c5462. PMID: 20959284.
26. O'Driscoll BR, Howard LS, Earis J, et al. British Thoracic Society Guideline for oxygen use in adults in healthcare and emergency settings. BMJ Open Respir Res. 2017;4(1):e000170. doi: 10.1136/bmjresp2016-000170. PMID: 28883921.

27. Holland AE. Physiotherapy management of acute exacerbations of chronic obstructive pulmonary disease. J Physiother. 2014 Dec;60(4):181-8. doi: 10.1016/j.jphys.2014.08.018. PMID: 25450482.

28. Spencer L. Pulmonary rehabilitation for patients with acute chronic obstructive pulmonary disease exacerbations: is the evidence strengthening? Curr Opin Pulm Med. 2018 Mar;24(2):147-51. doi: 10.1097/mcp.0000000000000453. PMID: 29210749.

29. Osadnik CR, McDonald CF, Jones AP, et al. Airway clearance techniques for chronic obstructive pulmonary disease. Cochrane Database Syst Rev. 2012 Mar

14(3):CD008328. doi: 10.1002/14651858.CD008328.pub2. PMID: 22419331.

30. Creutzberg EC, Schols AM, BothmerQuaedvlieg FC, et al. Prevalence of an elevated resting energy expenditure in patients with chronic obstructive pulmonary disease in relation to body composition and lung function. Eur J Clin Nutr. 1998 Jun;52(6):396-401. PMID: 9683390.

31. Law S, Kumar P, Woods S, et al. Malnutrition screening in patients admitted to hospital with an exacerbation of chronic obstructive pulmonary disease and its association with patient outcomes. Hosp Pract (1995). 2016 Oct;44(4):207-12. doi: 10.1080/21548331.2016.1224007. PMID: 27560297.

32. Calder PC, Laviano A, Lonnqvist F, et al. Targeted medical nutrition for cachexia in chronic obstructive pulmonary disease: a randomized, controlled trial. J Cachexia Sarcopenia Muscle. 2018 Feb;9(1):28-40. doi: 10.1002/jcsm.12228. PMID: 28891198. 
33. Manalan K, Rashid T, Singanayagam A. Antibiotic treatment in exacerbations of chronic obstructive pulmonary disease: Recent trial results. Clinical Investigation. 201501 Feb;5(2):189-204. doi: http://dx.doi.org/10.4155/cli.14.113. PMID: 602447476.

34. Laue J, Reierth E, Melbye H. When should acute exacerbations of COPD be treated with systemic corticosteroids and antibiotics in primary care: a systematic review of current COPD guidelines. NPJ Prim Care Respir Med. 2015 Feb 19;25:15002. doi: 10.1038/npjpcrm.2015.2. PMID: 25695630.

35. Baker EH, Bell D. Blood glucose: of emerging importance in COPD exacerbations. Thorax. 2009 Oct;64(10):830-2. doi: 10.1136/thx.2009.118638. PMID: 19786710 .

36. Qureshi H, Sharafkhaneh A, Hanania NA. Chronic obstructive pulmonary disease exacerbations: latest evidence and clinical implications. Ther Adv Chronic Dis. 2014 Sep;5(5):212-27. doi: 10.1177/2040622314532862. PMID: 25177479.

37. Bourbeau J, Sedeno MF, Metz K, et al. Early COPD Exacerbation Treatment with Combination of ICS and LABA for Patients Presenting with Mild-to-Moderate Worsening of Dyspnea. Copd. 2016 Aug;13(4):439-47. doi: 10.3109/15412555.2015.1101435. PMID: 26752024.

38. Woods JA, Wheeler JS, Finch CK, et al. Corticosteroids in the treatment of acute exacerbations of chronic obstructive pulmonary disease. Int J Chron Obstruct Pulmon Dis. 2014;9:421-30. doi: 10.2147/copd.s51012. PMID: 24833897.

39. Bonilla Arcos D, Krishnan JA, Vandivier RW, et al. High-Dose Versus Low-Dose Systemic Steroids in the Treatment of Acute Exacerbations of Chronic Obstructive Pulmonary Disease: Systematic Review. Chronic Obstructive Pulmonary Diseases. 2016 Feb 17;3(2):580-8. doi: https://dx.doi.org/10.15326/jcopdf.3.2.2015. 0178. PMID: 28848882.
40. Walters JA, Tan DJ, White CJ, et al. Different durations of corticosteroid therapy for exacerbations of chronic obstructive pulmonary disease. Cochrane Database Syst Rev. 2018 Mar 19;3:Cd006897. doi: 10.1002/14651858.CD006897.pub4. PMID: 29553157.

41. Zhang HL, Tan M, Qiu AM, et al. Antibiotics for treatment of acute exacerbation of chronic obstructive pulmonary disease: a network meta-analysis. BMC Pulm Med. 2017 Dec 12;17(1):196. doi: 10.1186/s12890-017-0541-0. PMID: 29233130.

42. Methods Guide for Effectiveness and Comparative Effectiveness Reviews. AHRQ Publication No. 10(14)-EHC063-EF. Rockville, MD: Agency for Healthcare Research and Quality; January 2014. Chapters available at: www.effectivehealthcare.ahrq.gov.

43. Moher D, Liberati A, Tetzlaff J, et al. Preferred reporting items for systematic reviews and meta-analyses: the PRISMA statement. PLoS Med. 2009 Jul 21;6(7):e1000097. doi: 10.1371/journal.pmed.1000097. PMID: 19621072.

44. Barnes NC, Sharma R, Lettis S, et al. Blood eosinophils as a marker of response to inhaled corticosteroids in COPD. Eur Respir J. 2016 May;47(5):1374-82. doi: 10.1183/13993003.01370-2015. PMID: 26917606.

45. Wedzicha JA, Miravitlles M, Hurst JR, et al. Management of COPD exacerbations: A European respiratory society/American thoracic society guideline. European Respiratory Journal. 2017;49(3):1600791.

46. Greening NJ, Williams JEA, Hussain SF, et al. An early rehabilitation intervention to enhance recovery during hospital admission for an exacerbation of chronic respiratory disease: randomised controlled trial. BMJ : British Medical Journal. 2014;349:g4315. doi: 10.1136/bmj.g4315. 
47. Spruit MA, Singh SJ, Garvey C, et al. An official American Thoracic Society/European Respiratory Society statement: key concepts and advances in pulmonary rehabilitation. Am J Respir Crit Care Med. 2013 Oct 15;188(8):e13-64. doi: 10.1164/rccm.201309-1634ST. PMID: 24127811.

48. Mathioudakis AG, ChatzimavridouGrigoriadou V, Corlateanu A, et al. Procalcitonin to guide antibiotic administration in COPD exacerbations: a meta-analysis. Eur Respir Rev. 2017 Jan;26(143). doi: 10.1183/16000617.00732016. PMID: 28143877.
49. Wadhera RK, Joynt Maddox KE, Wasfy JH, et al. Association of the Hospital Readmissions Reduction Program With Mortality Among Medicare Beneficiaries Hospitalized for Heart Failure, Acute Myocardial Infarction, and Pneumonia. JAMA. 2018 Dec 25;320(24):2542-52. doi: 10.1001/jama.2018.19232. PMID: 3057588 


\section{Introduction}

\section{Background}

Chronic obstructive pulmonary disease (COPD) is a common respiratory disease characterized by airflow limitation and chronic respiratory symptoms. The global prevalence is estimated to be greater than 10 percent, impacting approximately 380 million people worldwide. ${ }^{1}$ In the United States, COPD affects approximately 15 million people; chronic lower respiratory diseases, of which COPD is the largest contributing condition, are the fourth leading cause of death; ${ }^{2}$ and COPD costs more than $\$ 32$ billion annually. ${ }^{3,4}$ Patients with COPD experience chronic respiratory symptoms (including shortness of breath and cough), have decreased quality of life, and premature mortality.

Patients with COPD are at risk of experiencing exacerbations of COPD (ECOPD). There have been various definitions of what constitutes an ECOPD. The Global Initiative for Chronic Obstructive Lung Disease (GOLD) defines ECOPD in its 2019 report as "acute worsening of respiratory symptoms that result in additional therapy." 5 ECOPD is generally characterized by increased dyspnea, increased frequency and severity of cough, and/or increased sputum production. ${ }^{6}$ ECOPD is a leading independent cause of increased mortality and morbidity among patients with COPD. ECOPD is associated with a higher risk of dying during or shortly after the exacerbation, lower quality of life, hospital admission, depletion of financial resources, and a progressive decline in lung function. ${ }^{7-14}$ Hospitalizations for ECOPD account for more than half of all costs associated with COPD. ${ }^{10,15}$

In recent years, a number of clinical trials of treatments to prevent ECOPD have shown promising results, but the evidence for acute treatments during an episode of ECOPD appears to be surprisingly scarce, given how relatively common the condition is. ${ }^{16,17}$

Goals of management of ECOPD include relieving symptoms and hastening the recovery from ECOPD by addressing precipitating factors (e.g. antibiotic treatment for infections), improving expiratory airflow and gas exchange (and thus improving breathing) by using inhaled bronchodilators, and reducing lung inflammation with corticosteroids. Nonpharmacologic treatments include supplemental oxygen, nutritional support, and others. ${ }^{18-20}$ In addition, several new pharmacologic agents with novel mechanisms of action in early stages of development may be of potential benefit to COPD patients including those in acute exacerbation. ${ }^{21}$

The area of ECOPD management has several uncertainties and necessitates an up to date evidence synthesis. These uncertainties include the benefits and harms of emerging pharmacologic and nonpharmacologic treatments, the benefits and harms of treatments for ECOPD that have been found efficacious in stable COPD, the benefits and harms of antibiotics and systemic corticosteroids in mild ECOPD, the benefits and harms of combinations of treatments that have been found to be individually effective, and for antibiotics and systemic corticosteroids the comparative effectiveness of different types of agents (e.g. broad-spectrum versus narrow-spectrum antibiotics), delivery modes (e.g. intravenous, oral), and durations of treatment.

Examples of potentially emerging treatments for ECOPD, include immune-modulatory drugs and novel applications of treatments primarily used in stable COPD including mucolytics, aminophyllines, long-acting bronchodilators, inhaled corticosteroids, and others. Mucolytics 
appear to have a small effect on reducing the frequency of ECOPD. ${ }^{22,23}$ In clinical practice, they are also used during an ECOPD, where the evidence for their effectiveness appears less clear.

For nonpharmacological treatments there are a number of areas in which an update of the evidence is required to inform best practice management of ECOPD. There is increasing recognition that too much oxygen might do more harm than good, and not just in patients with chronic hypercapnic respiratory who are at risk of iatrogenic worsening of respiratory failure due to oversupply of oxygen. ${ }^{24,25}$ Titrated oxygen with a target saturation rate as opposed to high flow oxygen has therefore been used in patients with ECOPD. ${ }^{26}$ Historically, pulmonary rehabilitation programs have focused on enrolling patients with stable COPD or patients who had stabilized after an episode of ECOPD, but in more recent times, a number of trials have explored the role of exercise/early pulmonary rehabilitation during an episode of ECOPD. ${ }^{27,}{ }^{28}$ Chest physiotherapy using airway clearance techniques (including breathing technique, vibration/percussion, and autogenic drainage) are used routinely in many hospitalized patients with ECOPD. A Cochrane review published in 2012 found evidence that airway clearance techniques may reduce the need for hospital admission and improve health-related quality of life based on single-center studies with small study populations. ${ }^{29}$ An update of this evidence is indicated. Furthermore, many patients with COPD are in a state of hyper-metabolism in which their body consumes more calories per kilogram on calorimetric measures compared with a person without COPD, likely because of the increased work of breathing. ${ }^{30}$ This hyper-metabolic state is even more pronounced during episodes of ECOPD, posing questions about the optimal nutritional support for patients with ECOPD. ${ }^{31,32}$

Established treatments for ECOPD, such as antibiotics and systemic corticosteroids, may not be indicated in every single episode of an ECOPD. One uncertainty relates to the need for antibiotics in mild and moderately severe ECOPD, especially in an outpatient setting. ${ }^{33}$ While antibiotics for treatment of severe ECOPD have been shown to be beneficial in some studies, the need for antibiotics in less severe forms of COPD is unclear. ${ }^{34}$ Uncertainty remains regarding the use of systemic (oral, intravenous) corticosteroids relate to whether all patients stand to benefit from this treatment of ECOPD. ${ }^{34}$ These questions are important to address in view of trying to reduce prescriptions of antibiotics, where safely possible, to reduce potential adverse events including development of antibiotic resistance, and to reduce potentially significant adverse events from systemic corticosteroids, in particular hyperglycemia, in patients with glucose intolerance and diabetes. ${ }^{35}$

Short-acting beta adrenergic agonists (SABAs) and short-acting muscarinic antagonists (SAMAs) are established treatments to relieve dyspnea and improve airflow obstruction during ECOPD, but the benefits of combining SABAs and SAMAs compared with using SABAs or SAMAs alone are unclear. ${ }^{36}$ Long-acting bronchodilators and inhaled corticosteroids have historically only be used in stable COPD, but there is emerging evidence that an increase in dosage of inhalation therapy with inhaled corticosteroids and long-acting beta agonists (LABAs) may be beneficial in early treatment of ECOPD when patients experience mild to moderate dyspnea and may result in no requirement of systemic corticosteroids in a large proportion of patients presenting with mild-to-moderate worsening of dyspnea. ${ }^{37}$ The benefit of using LABAs and long-acting muscarinic antagonists (LAMAs) in the treatment of manifest ECOPD is unclear. For antibiotics and systemic corticosteroids, the comparative effectiveness of different agents (e.g. broad-spectrum versus narrow-spectrum antibiotics), delivery modes and durations of treatment needs to be established. . $^{38-41}$ 
In summary, determining the optimal treatment plan for patients with ECOPD requires 1) a synthesis of existing knowledge regarding the effectiveness of treatment options and 2) a synthesis of existing knowledge regarding the harms of treatment options. Currently, the comparative benefits and harms of these varied treatment approaches including the optimal combination of these treatments to mitigate COPD exacerbation are unclear. A systematic review of current evidence assists clinicians in understanding and determining optimal management for ECOPD. This review focuses on evidence from randomized controlled trials (RCTs) as the gold standard design for evaluating a therapeutic intervention. In terms of adverse events, they are also likely to be captured in RCTs because of the acute nature of condition being studied.

\section{Key Questions}

The following Key Questions (KQs) were determined based on input from multiple key informants, and the public (drafted KQs were posted for public comment from January 26, 2018,to February 16, 2018. The related PICOTS (population, interventions, comparisons, outcomes, timing, and setting) are listed in Table 1.

KQ1. In adult patients with exacerbation of COPD, what are the benefits and harms of systemic corticosteroids and antibiotics compared with placebo or standard care?

KQ2. In adult patients with exacerbation of COPD, what are the benefits and harms of emerging and other pharmacologic and nonpharmacologic therapies compared with placebo or standard care?

KQ3. In adult patients with exacerbation of COPD, what are the benefits and harms of combinations of treatments that are individually effective (based on empirical evidence in stable COPD)?

KQ4. In adult patients with exacerbation of COPD, what is the comparative effectiveness of different regimens of antibiotics and systemic corticosteroids based on type of agents (e.g., broad-spectrum vs. narrowspectrum antibiotics), delivery modes (e.g., intravenous, oral), and durations of treatment? 


\section{Methods}

We developed an analytic framework to guide the process of the systematic review (Figure 1). We followed the established methodologies of systematic reviews as outlined in the Agency for Healthcare Research and Quality (AHRQ) Methods Guide for Comparative Effectiveness Reviews. $^{42}$ The reporting complies with the Preferred Reporting Items for Systematic Reviews and Meta-Analyses (PRISMA) statements. ${ }^{43}$ The study protocol is registered in the international prospective register of systematic reviews (PROSPERO \#: 42018111609) and published on the AHRQ website (https://effectivehealthcare.ahrq.gov/topics/copd/protocol).

\section{Figure 1. Analytic framework for Key Questions 1, 2, 3, and 4}

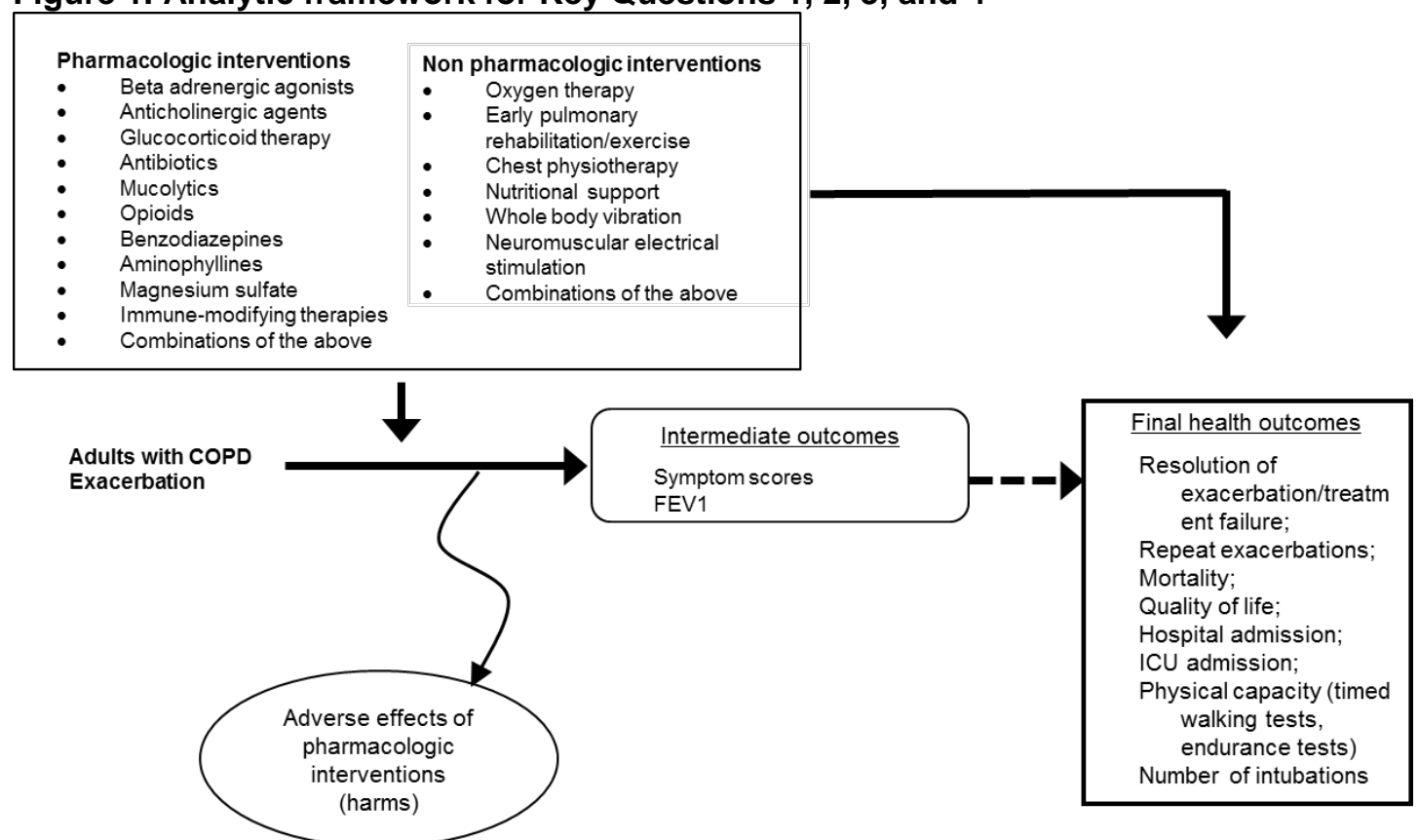

COPD: chronic obstructive pulmonary disease; FEV1 = forced expiratory value in 1 second; ICU = intensive care unit

\section{Literature Search Strategy}

\section{Search Strategy}

We conducted a comprehensive database search, including Embase, Epub Ahead of Print, InProcess \& Other Non-Indexed Citations, MEDLINE Daily, MEDLINE, Cochrane Central Registrar of Controlled Trials, Ovid Cochrane Database of Systematic Reviews, and Scopus from database inception to January 2, 2019. We searched Food and Drug Administration, ClinicalTrials.gov, Health Canada, Medicines and Healthcare Products Regulatory Agency (MHRA), AHRQ’s Horizon Scanning System, conference proceedings, patient advocate group websites, and medical society websites. Reference mining of relevant publications, completed trials identified through ClinicalTrials.gov, relevant systematic reviews, and meta-analysis was used to identify additional existing and new literature. The search strategy was developed by an experienced medical librarian and further peer-reviewed by an independent information 
specialist. The same librarian conducted the literature search. The detailed search strategy is listed in Appendix B.

\section{Inclusion and Exclusion Criteria}

The eligible studies had to meet all of the following criteria: 1 ) adult 18 years and older with exacerbations of chronic obstructive pulmonary disease (ECOPD); 2) received pharmacologic intervention or nonpharmacologic interventions; 3) compared with placebo, standard care, for antibiotics and systemic corticosteroids: different types of agents, different delivery modes, and different durations of treatments; 4) reported outcomes of interest; 5) conducted in outpatient, inpatients, and emergency department; 6) randomized controlled trials (RCTs); and 7) published in English. We excluded studies conducted in the intensive care unit, or chronic ventilator unit or respiratory care unit; studies of patients with exacerbation of chronic bronchitis if they did not have any evidence of airflow limitation on spirometry (at any time, including during a stable state); and studies of health service interventions (e.g. hospital in the home as alternative to hospitalization). We focused only on interventions during the initial acute phase of an exacerbation of COPD and not during the convalescence period. We did not restrict study location or sample size. The detailed inclusion and exclusion criteria are listed in Table 1.

All outcomes were final health outcomes except for the intermediate outcome, "forced expiratory volume in one second" (FEV1). FEV1 was included because it is a commonly used outcome in COPD studies and has been shown to be highly predictive of final health outcomes during ECOPD (including mortality, need for intubation, or hospital admission for COPD). ${ }^{50}$ 
Table 1. PICOTS (population, interventions, comparisons, outcomes, timing, and setting)

\begin{tabular}{|c|c|c|}
\hline PICOTS Elements & Inclusion Criteria & Exclusion Criteria \\
\hline Populations & $\begin{array}{l}\text { Patients with exacerbation of COPD } \\
\text { Adults } 18 \text { years and older }\end{array}$ & $\begin{array}{l}\text { Animals } \\
\text { Children (age }<18 \text { years) } \\
\text { Patients with stable COPD not in a } \\
\text { current exacerbation }\end{array}$ \\
\hline Interventions & $\begin{array}{l}\text { KQ 1,4: } \\
\text { Antibiotics } \\
\text { Systemic corticosteroids } \\
\text { KQ 2,3; Pharmacologic interventions } \\
\text { include: } \\
\text { Beta adrenergic agonists } \\
\text { Anticholinergic agents } \\
\text { Glucocorticoid therapy } \\
\text { Antibiotics } \\
\text { Mucolytics } \\
\text { PDE4 Inhibitors } \\
\text { Opioids } \\
\text { Benzodiazepines } \\
\text { Aminophyllines } \\
\text { Magnesium sulfate } \\
\text { Immune-modifying therapies } \\
\text { Combinations of the above } \\
\text { KQ 2,3; Nonpharmacologic } \\
\text { interventions include: } \\
\text { Oxygen therapy } \\
\text { Early pulmonary } \\
\text { rehabilitation/exercise } \\
\text { Chest physiotherapy } \\
\text { Nutritional support } \\
\text { Whole body vibration } \\
\text { Neuromuscular electrical stimulation } \\
\text { Combinations of the above }\end{array}$ & $\begin{array}{l}\text { Invasive and noninvasive mechanical } \\
\text { ventilations } \\
\text { Complementary and alternative } \\
\text { medicine interventions } \\
\text { Investigational drugs and drugs that } \\
\text { are only available outside of the U.S. } \\
\text { Interventions to prevent future COPD } \\
\text { exacerbations }\end{array}$ \\
\hline Comparators & $\begin{array}{l}\text { KQ 1: Placebo or standard care } \\
\text { KQ 2: Placebo or standard care } \\
\text { KQ 3: Placebo, standard care or } \\
\text { active individual intervention } \\
\text { KQ 4: Different types of antibiotics and } \\
\text { systemic corticosteroids, different } \\
\text { delivery modes and durations of } \\
\text { treatment }\end{array}$ & None \\
\hline Outcomes & $\begin{array}{ll}\text { Intermediate outcomes } \\
\text { - } & \text { Symptom scores } \\
\text { Final health outcomes } \\
\text { - } & \text { Resolution of } \\
& \text { exacerbation/treatment } \\
& \text { failure; } \\
\text { - } & \text { Repeat exacerbations; } \\
\text { - } & \text { Mortality; } \\
\text { - } & \text { Quality of life; } \\
\text { - } & \text { Hospital admission; } \\
\text { - } & \text { PCU admission; } \\
& \text { walking tests, endurance } \\
\text { - } & \text { Nests) } \\
\end{array}$ & None \\
\hline
\end{tabular}




\begin{tabular}{|c|c|c|}
\hline PICOTS Elements & Inclusion Criteria & Exclusion Criteria \\
\hline Timing & All & None \\
\hline Settings & $\begin{array}{l}\text { Outpatient, hospital, emergency } \\
\text { department }\end{array}$ & $\begin{array}{l}\mathrm{ICU} \text {, chronic ventilator unit or } \\
\text { respiratory care unit (RCU) }\end{array}$ \\
\hline Study design & $\begin{array}{l}\text { Original data } \\
\text { Any sample size } \\
\text { RCTs } \\
\text { Relevant systematic reviews, or meta- } \\
\text { analyses (used for identifying } \\
\text { additional studies) }\end{array}$ & $\begin{array}{l}\text { In vitro studies } \\
\text { Non-original data (e.g. narrative } \\
\text { reviews, editorials, letters, or erratum) } \\
\text { Observational studies } \\
\text { Case series } \\
\text { Qualitative studies } \\
\text { Cost-benefit analysis } \\
\text { Cross-sectional (i.e., non-longitudinal) } \\
\text { studies } \\
\text { Before-after studies } \\
\text { Survey }\end{array}$ \\
\hline Publications & Studies published in English only. & Foreign language studies \\
\hline
\end{tabular}

Abbreviations: COPD = chronic obstructive pulmonary disease; FEV1 = Forced Expiratory Volume in One Second; ICU = intensive care unit; KQ = Key Question; PICOTS = population, interventions, comparators, outcomes, timing, and settings; RCT $=$ randomized controlled trial

\section{Study Selection}

Independent reviewers, working in pairs, screened the titles and abstracts of all references. Studies included by either reviewer were retrieved for full-text screening. Independent reviewers, working in pairs, screened the full-text version of eligible references. Discrepancies between the reviewers were resolved through discussions and consensus.

\section{Data Abstraction and Data Management}

We developed a standardized data extraction form to extract study characteristics (author, study design, inclusion and exclusion criteria, patient characteristics, intervention, comparisons, outcomes, and related items for assessing study quality and applicability). The standardized form was pilot-tested by all study team members using five randomly selected studies. Reviewers worked independently to extract study details. A third reviewer reviewed data extraction, and resolve conflicts.

\section{Assessment of the Risk of Bias of Individual Studies}

We evaluated the risk of bias of the included study using the Cochrane Collaboration's Risk of Bias tool to assess sequence generation; allocation concealment; participant, personnel, and outcome assessor blinding; attrition bias; incomplete outcome data; selective outcome reporting; and other sources of bias. ${ }^{51}$

\section{Data Synthesis}

We summarized key features/characteristics (e.g. patient characteristics, intervention, comparison, outcomes, and conclusions) of the included studies and present in the tables by Key Questions (KQs). Table 2 presents the rules we used to categorize the severity of exacerbation. 
Table 2. Categories of severity of exacerbation

\begin{tabular}{|l|l|}
\hline $\begin{array}{c}\text { Severity of } \\
\text { Exacerbations }\end{array}$ & \\
\hline Mild & $\begin{array}{l}\text { Outpatient treatment, possibly ED visit, but less likely than for moderate ECOPD } \\
\text { OR classification as mild by the authors of the original study }\end{array}$ \\
\hline Moderate & $\begin{array}{l}\text { Requiring ED visit or hospitalization, but no mention of ventilatory failure (respiratory } \\
\text { acidosis)/subsequent ICU admissions } \\
\text { OR classification as moderate by the authors of the original study (including outpatients) }\end{array}$ \\
\hline Severe & $\begin{array}{l}\text { Requiring hospitalization, ventilatory failure (respiratory acidosis) and/or substantial rates of } \\
\text { subsequent ICU admissions and deaths (studies with main location in ICU were, however, } \\
\text { excluded) } \\
\text { OR classification as severe by the authors of the original study }\end{array}$ \\
\hline
\end{tabular}

ECOPD = exacerbation of chronic obstructive pulmonary disease; ED = emergency department; ICU = intensive care unit

Table 3 lists the categories of adverse events and examples. Mortality was reported as a primary effectiveness outcome and not reported as serious adverse events in this review. We used the definition of serious adverse events listed by the original studies.

Table 3. Categories of adverse events

\begin{tabular}{|l|l|}
\hline \multicolumn{1}{|c|}{ Type of Adverse Events } & \multicolumn{1}{c|}{ Example } \\
\hline Allergy and Immunology Adverse Event & Dermatitis \\
\hline Cardiovascular Adverse Event & $\begin{array}{l}\text { Paroxysmal atrial fibrillation, atrial fibrillation, palpitations, } \\
\text { arrhythmia, symptomatic sinus, tachycardia }\end{array}$ \\
\hline Dermatological Adverse event & Rash, urticaria, exanthema, pruritus \\
\hline Ear, Nose and Throat Adverse Event & Transient episode of vocal cord dysfunction \\
\hline Endocrine Adverse Event & Hyperglycemia, metabolism and nutrition disorders \\
\hline Gastrointestinal Adverse Event & $\begin{array}{l}\text { Gastrointestinal bleeding, diarrhea, nausea, stomachache, } \\
\text { epigastric pain, heartburn, vomiting, constipation }\end{array}$ \\
\hline General Internal Medicine Adverse Event & $\begin{array}{l}\text { Facial puffiness, dizziness, new or worse hypertension, fatigue, } \\
\text { chills, insomnia,, flushing, confusion, fever }\end{array}$ \\
\hline Hepatic Adverse Event & Increased aspartate aminotransferase \\
\hline Infectious (Non-Respiratory) Adverse Event & Vaginitis, urinary tract infection, influenza \\
\hline Musculoskeletal Adverse Event & $\begin{array}{l}\text { Muscle cramps, myalgia, tendonitis, rigors, musculoskeletal pain, } \\
\text { muscle soreness }\end{array}$ \\
\hline Neurological Adverse Event & Tremor, headache, seizure, rigors \\
\hline Ocular Adverse Event & Blurred vision \\
\hline Oncological Adverse Event & $\begin{array}{l}\text { Classified as malignancy-related AEs by authors of the original } \\
\text { study }\end{array}$ \\
\hline Psychiatric Adverse Event & Mood change, psychosis, nervousness \\
\hline Respiratory Adverse Event & $\begin{array}{l}\text { Shortness of breath, respiratory acidosis, requiring noninvasive } \\
\text { mechanical ventilation, dyspnea, bronchitis, wheezing, } \\
\text { pneumothorax and pneumomediastinum, pneumonia, worsening } \\
\text { exacerbation of COPD, bronchospasm }\end{array}$ \\
\hline Urogenital Adverse Event & Hematuria \\
\hline
\end{tabular}

AEs = adverse events; COPD = chronic obstructive pulmonary disease

For outcome definitions, we defined as a resolution or clinical cure of ECOPD a complete improvement of clinical signs and symptoms, and as a clinical failure the lack of a significant improvement of clinical signs and symptoms and/or the requirement for additional or alternate treatment for an ECOPD. A repeat exacerbation (or repeat exacerbation) was defined as a new ECOPD following the initial ECOPD. For dyspnea, we differentiated between dyspnea measured with a numeric scale that represented different severity levels of breathlessness (e.g. visual 
analogue scale or Borg scale) and dyspnea based on a questionnaire that assessed the functional limitation caused by dyspnea (e.g. medical research council dyspnea scale).

For crossover RCTs, we chose to qualitatively synthesize outcomes (i.e., not included in meta-analyses), as the included crossover RCTs suffered reporting and methodological issues, such as missing data, failure to control within-individual difference, and inhibited pooling with other studies. ${ }^{52}$

For other RCTs, we conducted meta-analyses to quantitatively summarize study findings. All statistical analyses were based on the intention-to-treat principle. Odds ratio and corresponding 95 percent confidence intervals were extracted or calculated for binary outcomes. For continuous outcomes, we calculated standardized mean difference when different measures for the same outcome were reported (e.g. St. George's Respiratory Questionnaire and 36-Item Short Form Survey (SF-36) for quality of life) and converted the direction of all measures (e.g. higher score represents better outcome). We calculated weighted mean difference when the included studies used the same outcome measure. For adverse events, we calculated rate ratio (i.e. ratio of the incidence rate of events within a given time between the intervention and the comparison).

For most of the outcomes, we extracted and pooled effect size by the end of the intervention and at the longest followup. For repeat exacerbation, we extracted at the end of intervention, 1month, 3 month, 6-month, 12-month, and longest followup, which were commonly used followup time points in studies that measured exacerbations. For hospital admission, we extracted 30-day admission and at the longest followup. Thirty-day hospital admissions were chosen because hospital admissions within 30 days of an index hospitalization count as a readmission in Medicare's Hospital Readmissions Reduction Program, which lowers payments to Inpatient Prospective Payment System hospitals with too many readmissions. ${ }^{53}$

We used the DerSimonian and Laird (D-L) random effect method to combine direct comparisons between treatments if the number of studies included in the analysis was larger than 3. ${ }^{54}$ The D-L method was chosen over alternative methods, such as the D-L with the HartungKnapp-Sidik-Jonkman variance correction (HKSJ) method and the profile likelihood (PL) method, because, when heterogeneity between the studies are low, the D-L method performs as well as alternative methods and the HKSJ method and PL method suffer methodological issues. 55-62 The HKSJ method and the PL method were used as sensitivity analyses for the D-L method (Appendix Table J.1.). When the number of studies was 3 or less, we used the fixed effect method based on the Mantel and Haenszel method due to instability of between-study variance. ${ }^{63}$ We evaluated heterogeneity between studies using $\mathrm{I}^{2}$ indicator. We were unable to use statistical methods to evaluate potential publication bias as the number of studies included in a direct comparison was small $(\mathrm{n}<20) .{ }^{64}$ All statistical analyses were conducted using Stata/SE version 15.1 (StataCorp LLC, College Station, TX).

\section{Grading the Strength of Evidence (SOE) for Major Comparisons and Outcomes}

We graded the strength of evidence (SOE) as per the AHRQ Methods Guide on assessing SOE. ${ }^{42}$ We graded SOE for most important or critical health outcomes, including mortality, dyspnea, quality of life (QoL), need for intubation, repeat exacerbation and/or hospital admissions and ECOPD resolution (clinical cure, failure). These outcomes were chosen because 
they are clinically important from a patient's perspective and are highly relevant for decision making.

RCTs started as high SOE. The domains to be used for all KQs were: the methodological limitations of the studies (i.e., risk of bias); precision (based on the size of the body of evidence, number of events, and confidence intervals); directness of the evidence to the KQs (focusing on whether the outcomes were important to patients vs. surrogates); consistency of results (based on qualitative and statistical approaches to evaluate for heterogeneity); and the likelihood of reporting and publication bias. In most cases, sensitivity analyses based on risk of bias were not feasible due to the small number of studies per analysis.

Based on this assessment, we assigned SOE rating as high, moderate, low, or "insufficient evidence to estimate an effect". SOE was rated as high when we were very confident that the estimate of effect lies close to the true effect (the body of evidence has few or no deficiencies and is judged to be stable). SOE was rated as moderate when we were moderately confident that the estimate of effect lies close to the true effect (the body of evidence has some deficiencies and is judged to be likely stable). SOE was rated as low when we had limited confidence that the estimate of effect lies close to the true effect (the body of evidence has major or numerous deficiencies and is likely unstable). SOE was rated as insufficient when we had no evidence, were unable to estimate an effect, or had no confidence in the estimate of effect. We produced summary of evidence tables for each comparison and for each outcome: data source, effect size, SOE rating; and rationale for judgments made on each domain of evidence rating.

\section{Assessing Applicability}

We followed the procedures outlined in the AHRQ Methods Guide for Effectiveness and Comparative Effectiveness Reviews to assess the applicability of the findings within and across studies. ${ }^{42}$ We focused on whether the populations, interventions, and comparisons in existing studies were representative of current practice. For studies to have good applicability, the interventions used in research need to be available, accessible, acceptable and feasible to implement; and patients enrolled in the studies should be similar to typical patients with ECOPD, in particular in relation to underlying severity of COPD and co-morbidities. This congruence between research and practice as it relates to applicability was evaluated qualitatively and reported narratively. We reported limitations of applicability of the whole body of evidence in the discussion.

\section{Peer Review and Public Commentary}

A draft report was posted for peer review between March 29th and May 6th, 2019 and public comments between March 28th and May 6th, 2019. We revised and finalized the draft report in response to comments. However, the findings and conclusions are those of the authors, who are responsible for the contents of the report. 


\section{Results}

\section{Literature Searches and Evidence Base}

The literature search identified 8,916 citations. An additional 36 references were identified through reference mining, grey literature search; and from Key Informants and Technical Experts. There were 98 original studies with a total of 13,401 patients that met inclusion criteria and were included in the systematic review (Appendix Figure A.1.). Of the 98 studies, 4 were crossover randomized controlled trials (RCTs). ${ }^{65-67} 16$ studies ${ }^{6,68-82}$ addressed Key Question (KQ)1(86, 68, 70-72, 74, 79, 82 had patients with mild exacerbation of chronic obstructive pulmonary disease (ECOPD) and 6 $6^{69,73,75-78}$, had patients with moderate to severe ECOPD, $1^{80}$ had patients with mild to severe ECOPD, and $1^{81}$ had severe ECOPD).51 $25,46,65-67,74-76,83-125$ addressed KQ2(4 $4^{74,75,89,122}$ had patients with mild ECOPD, 47 25, 46, 65-67, 76, 83-88, 90-121, 123-125 had patients with moderate to severe ECOPD, 3 studies ${ }^{126-128}$ addressed KQ 3 and 34 studies $^{75-77,129-159}$ addressed KQ4. 59 trials were in a hospital setting, 46, 65, 69, 73, 75-78, 81, 83-91, 93-103, 105-112, 114-118, 120, 122-125, 127, 129, 130, 133, 140, 142, 144, 146, 149, 151, 155, 15910 in emergency departments, 80, 82, 92, 104, 119, 121, 126, 137, 141, 15819 in an outpatient setting, 66, 68, 70-72, 74, 79, 113, 132, 136, 138, 139, 143, 145, 148, 152-154 1 was ambulance based ${ }^{25}, 2$ were in both outpatients and hospitalized patients, ${ }^{67,156}$ and in 6 trials the

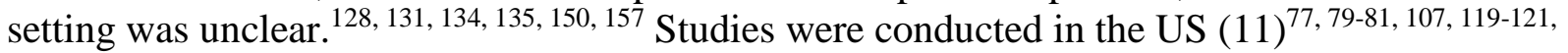
154, 156, 157, Canada (5) 6, 67, 82, 90, 118 , Europe (44) 46, 65, 66, 68, 71-74, 76, 78, 83, 84, 86, 93, 99, 102, 106, 108, 110, 113117, 124, 125, 127, 128, 130, 135, 138-144, 147, 148, 151-153, 155, 158 , Australia (7), 25, 92, 97, 104, 105, 111, 126 Africa (2), ${ }^{70,}$ 101 Asia (26), 69, 75, 85, 87-89, 91, 94-96, 98, 103, 109, 112, 122, 123, 129, 131-134, 137, 146, 149, 150, 159 Africa (2), ${ }^{70,101}$ South America (2), ${ }^{100,145}$ and 1 study $^{136}$ was conducted in 30 countries. Most studies were conducted in hospitalized patients with moderate to severe chronic obstructive pulmonary disease (COPD). The mean treatment duration was 9.9 days and there was a mean 3.7 months of reported followup. A list of the studies excluded at the full-text review stage is in Appendix C. A search of ClinicalTrials.gov identified 33 ongoing clinical trials. We also found 102 studies published as conference abstracts.

KQ1. In adult patients with exacerbation of COPD, what are the benefits and harms of systemic corticosteroids and antibiotics compared with placebo or standard care?

\section{Key Points-KQ1}

\section{Systemic Antibiotics Versus Placebo or Management Without Systemic Antibiotics}

- Antibiotics increased clinical cure of ECOPD compared with placebo or management without systemic antibiotics at the end of the intervention and at the longest followup (moderate Strength of Evidence (SOE)).

- Antibiotics reduced clinical failure rate compared with placebo at the end of the intervention (moderate SOE), but not at the longest followup (low SOE).

- Antibiotics improved dyspnea compared with placebo at the end of the intervention (low SOE) but not at the longest followup (low SOE). 
- Antibiotics did not change 30-day hospital readmission, repeat exacerbation, and quality of life, compared with placebo (low SOE).

- No statistically significant difference in adverse events was observed, including serious adverse events, between antibiotics and placebo or management without antibiotics.

\section{Systemic Corticosteroids Versus Placebo or Management Without Systemic Corticosteroids}

- Systemic corticosteroids improved dyspnea (low SOE) at the end of the intervention and reduced clinical failure rate (low SOE) at the end of the intervention, compared with placebo.

- No statistically significant difference in serious adverse events was found between systemic corticosteroids and placebo. Systemic corticosteroids were associated with statistically significantly less withdrawals but more endocrine related adverse events.

\section{KQ 1 Results}

Sixteen studies with 2,762 patients were included for KQ1. The characteristics of the studies are listed in Appendix Table D.1. Eight ${ }^{6,68-73,82}$ compared systemic antibiotics to placebo or management without systemic antibiotics and eight ${ }^{74-81}$ compared systemic corticosteroids to placebo or management without systemic corticosteroids. Eight studies has patients with mild ECOPD, ${ }^{6,68,70-72,74,79,82} 6$ studies $^{69,73,75-78}$ had patients with moderate to severe ECOPD $1^{80}$ had patients with mild to severe ECOPD, and $1^{81}$ had severe ECOPD. 7 trials were conducted in a hospital setting, ${ }^{69,73,75-78,81} 2$ in the emergency department, ${ }^{80,82}$ and 7 were in outpatients. ${ }^{6,68,70-}$ ${ }^{72,74,79}$ Studies were conducted in the US(4), ${ }^{77,79-81}$ Canada(2) ${ }^{6,82}$ Europe (7), ${ }^{68,71-74,76,78}$ Africa (1),${ }^{70}$ and Asia (2).$^{69,75}$ The average treatment length was 11.5 days and mean reported followup was 4.6 months. Details of the interventions used in each study can be found in Appendix Table F.1. Individual studies inclusion and exclusion criteria are in Appendix Table I.1.

The overall risk of bias is intermediate due to unclear allocation concealment (56.25\%), unclear and random sequence generation (43.75\%) (Appendix Table E.1.).

\section{Systemic Antibiotics Versus Placebo or Management Without Systemic Antibiotics}

Seven studies ${ }^{6,68-73,82}$ evaluated the effectiveness of antibiotics versus placebo or management without systemic antibiotics, of which 2 studies were conducted in patients with mild, ${ }^{68,70} 4$ mild to moderate ${ }^{6,71,72,82}$ ECOPD and 2 studies ${ }^{69,73}$ were conducted in patients with moderate to severe COPD. Table 4 and Table 5 provide an overview of all included studies. Tables stratified by the severity of ECOPD can be found in the Appendix (Appendix Table G.1: mild ECOPD, Appendix Table G.2: moderate to severe COPD).

\section{Mortality}

There was no statistically significant difference found between the antibiotics and placebo or management without antibiotics at the end of followup or at the end of the intervention.

When stratifying studies by severity of ECOPD, there were no statistically significant differences between groups in either mild or moderate to severe ECOPD. One study ${ }^{68}$ in patients 
with mild ECOPD did not show a statistically significant difference between antibiotics and placebo in mortality at the longest followup. In patients with moderate or severe ECOPD, there was no statistically significant difference observed between groups in mortality at the end of the intervention or at the end of followup.

\section{Symptoms}

One study showed statistically significantly less dyspnea (measured by numeric scale) at the end of the intervention in the antibiotics group compared with the placebo group ${ }^{73}$ but not at the longest followup. Another study did not show a statistically significant difference in dyspnea $^{71}$ (measured by questionnaire) at the longest followup.

In patients with mild ECOPD, there was no statistically significant difference observed in dyspnea between groups.

Cough was statistically significantly more reduced in the antibiotics groups compared with the placebo group at the end of the intervention but not at the longest followup.

Other symptoms were statistically significantly reduced in the antibiotics group compared with placebo or management without antibiotics at the end of treatment but not at the longest followup.

One study ${ }^{71}$ was conducted in patients with mild ECOPD. The study did not find a statistically significant difference in dyspnea or other symptoms between intervention and placebo. Studies conducted in patients with moderate to severe COPD showed statistically significantly less dyspnea, cough and other symptoms at the end of the intervention but not at the longest followup in the antibiotics group compared with the placebo or management without antibiotics. ${ }^{69,73}$

\section{Quality of Life}

Quality of life (QoL) was measured in one study (in patients with mild ECOPD, at the longest followup). ${ }^{71}$ There was no statistically significant difference found between antibiotics and placebo.

\section{Hospital Admission}

Thirty-day hospital admissions were measured in one study ${ }^{69}$ (including patients with moderate to severe ECOPD) with no statistically significant difference between antibiotics and management without antibiotics.

\section{Repeat Exacerbations}

Repeat exacerbations at the end of the intervention, at 30 days, 6 months, and longest followup did not show a statistically significant difference between the antibiotics and placebo or management without antibiotics. There was no statistically significant difference found in repeat exacerbations when stratifying studies by severity of ECOPD.

\section{Intubations}

The need for intubation at the end of the intervention was assessed in one study ${ }^{69}$ (in patients with moderate to severe COPD).No statistically significantly difference was found between the antibiotics and management without antibiotics. 


\section{Clinical Resolution of Exacerbation}

Statistically significantly more patients were clinically cured in the antibiotics group compared with placebo or management without antibiotics at the end of the intervention as well as at the longest followup.

In patients with mild ECOPD, statistically significantly more patients were clinically cured at the end of the intervention. Patients with mild ECOPD also had statistically significantly higher cure rates at the longest followup.

Statistically significantly less patients on antibiotics compared with patients in the placebo group had clinical failure at the end of the intervention but not at the longest followup.

In patients with mild ECOPD, statistically significantly less patients on antibiotics compared with patients in the placebo group had clinical failure at the end of the intervention but not at the longest followup.

A crossover RCT ${ }^{6}$ compared antibiotics to placebo in mild to moderate ECOPD patients. After 3 weeks, patients in the antibiotic group were found to have statistically significantly more clinical cures (68\% vs. 55\%) and less clinical failures (10\% vs. 19\%) than those in the placebo group.

Table 4. Comparison of systemic antibiotics versus control, critical outcomes

\begin{tabular}{|c|c|c|c|c|c|}
\hline Comparison & Outcome & Findings & $\begin{array}{c}\text { Study } \\
\text { Design and } \\
\text { Sample size }\end{array}$ & $\begin{array}{l}\text { Rationale for } \\
\text { Strength of } \\
\text { Evidence } \\
\text { (SOE) }\end{array}$ & $\begin{array}{c}\text { Overall } \\
\text { Strength of } \\
\text { Evidence }\end{array}$ \\
\hline $\begin{array}{l}\text { Antibiotics vs. } \\
\text { Management Without } \\
\text { Antibiotics }\end{array}$ & $\begin{array}{l}\text { Mortality } \\
\text { End of Intervention }\end{array}$ & $\begin{array}{l}\text { OR: } 2.02 ; \\
95 \% \mathrm{Cl}: 0.18 \\
\text { to } 22.66 \\
\mathrm{I}^{2}=\mathrm{N} / \mathrm{A}\end{array}$ & $\begin{array}{l}1 \mathrm{RCT}^{69} \text { with } \\
194 \text { patients }\end{array}$ & $\begin{array}{l}\text { Severe } \\
\text { imprecision }\end{array}$ & $\begin{array}{l}\text { Low SOE } \\
\text { supporting no } \\
\text { difference }\end{array}$ \\
\hline $\begin{array}{l}\text { Antibiotics vs. } \\
\text { Placebo or } \\
\text { Management without } \\
\text { Antibiotics }\end{array}$ & $\begin{array}{l}\text { Mortality } \\
\text { Longest Followup }\end{array}$ & $\begin{array}{l}\text { OR: } 1.78 ; \\
95 \% \mathrm{Cl}: 0.88 \\
\text { to } 3.59, \mathrm{I}^{2}= \\
0.00 \%\end{array}$ & $\begin{array}{l}3_{73} \mathrm{RCTs}^{68,69} \\
\text { with } 764 \\
\text { patients }\end{array}$ & $\begin{array}{l}\text { Intermediate } \\
\text { ROB and } \\
\text { severe } \\
\text { imprecision }\end{array}$ & $\begin{array}{l}\text { Insufficient } \\
\text { evidence }\end{array}$ \\
\hline $\begin{array}{l}\text { Antibiotics vs. } \\
\text { Placebo }\end{array}$ & $\begin{array}{l}\text { Dyspnea } \\
\text { (Questionnaire: } \\
\text { CRQ(dyspnea)) } \\
\text { Longest Followup }\end{array}$ & $\begin{array}{l}\text { WMD: } 0.00 ; \\
95 \% \mathrm{Cl}:-0.97 \\
\text { to } 0.97 \\
\mathrm{I}^{2}=\mathrm{N} / \mathrm{A}\end{array}$ & $\begin{array}{l}1 \mathrm{RCT}^{71} \text { with } \\
35 \text { patients }\end{array}$ & $\begin{array}{l}\text { Severe } \\
\text { imprecision }\end{array}$ & $\begin{array}{l}\text { Low SOE } \\
\text { supporting no } \\
\text { difference }\end{array}$ \\
\hline $\begin{array}{l}\text { Antibiotics vs. } \\
\text { Placebo }\end{array}$ & $\begin{array}{l}\text { Dyspnea } \\
\text { (Numeric Scale: } \\
\text { VAS) } \\
\text { End of Intervention }\end{array}$ & $\begin{array}{l}\text { WMD: }-0.80 ; \\
95 \% \mathrm{Cl}:-1.49 \\
\text { to }-0.11 \\
\mathrm{I}^{2}=\mathrm{N} / \mathrm{A}\end{array}$ & $\begin{array}{l}1 \mathrm{RCT}^{73} \text { with } \\
265 \text { patients }\end{array}$ & $\begin{array}{l}\text { High ROB and } \\
\text { imprecision }\end{array}$ & $\begin{array}{l}\text { Low SOE } \\
\text { supporting } \\
\text { improvement }\end{array}$ \\
\hline $\begin{array}{l}\text { Antibiotics vs. } \\
\text { Placebo }\end{array}$ & $\begin{array}{l}\text { Dyspnea } \\
\text { (Numeric Scale: } \\
\text { VAS) } \\
\text { Longest Followup }\end{array}$ & $\begin{array}{l}\text { WMD: }-0.06 ; \\
95 \% \mathrm{Cl}:-1.27 \\
\text { to } 0.07 \\
\mathrm{I}^{2}=\mathrm{N} / \mathrm{A}\end{array}$ & $\begin{array}{l}1 \mathrm{RCT}^{73} \text { with } \\
265 \text { patients }\end{array}$ & $\begin{array}{l}\text { High ROB and } \\
\text { imprecision }\end{array}$ & $\begin{array}{l}\text { Low SOE } \\
\text { supporting no } \\
\text { difference }\end{array}$ \\
\hline $\begin{array}{l}\text { Antibiotics vs. } \\
\text { Placebo }\end{array}$ & $\begin{array}{l}\text { Quality of Life } \\
\text { (CRQ) } \\
\text { Longest Followup }\end{array}$ & $\begin{array}{l}\text { WMD: } 0.00 ; \\
95 \% \mathrm{Cl}:-1.80 \\
\text { to } 1.79 \\
\mathrm{I}^{2}=\mathrm{N} / \mathrm{A}\end{array}$ & $\begin{array}{l}1 \mathrm{RCT}^{71} \text { with } \\
35 \text { patients }\end{array}$ & $\begin{array}{l}\text { Severe } \\
\text { imprecision }\end{array}$ & $\begin{array}{l}\text { Low SOE } \\
\text { supporting no } \\
\text { difference }\end{array}$ \\
\hline $\begin{array}{l}\text { Antibiotics vs. } \\
\text { Management Without } \\
\text { Antibiotics }\end{array}$ & $\begin{array}{l}\text { Hospital Admission } \\
30 \text { days }\end{array}$ & $\begin{array}{l}\text { OR: } 1.72 ; \\
95 \% \mathrm{Cl}: 0.68 \\
\text { to } 4.36 \\
\mathrm{I}^{2}=\mathrm{N} / \mathrm{A}\end{array}$ & $\begin{array}{l}1 \mathrm{RCT}{ }^{69} \text { with } \\
194 \text { patients }\end{array}$ & $\begin{array}{l}\text { Severe } \\
\text { imprecision }\end{array}$ & $\begin{array}{l}\text { Low SOE } \\
\text { supporting no } \\
\text { difference }\end{array}$ \\
\hline
\end{tabular}




\begin{tabular}{|c|c|c|c|c|c|}
\hline Comparison & Outcome & Findings & $\begin{array}{c}\text { Study } \\
\text { Design and } \\
\text { Sample size }\end{array}$ & $\begin{array}{l}\text { Rationale for } \\
\text { Strength of } \\
\text { Evidence } \\
\text { (SOE) }\end{array}$ & $\begin{array}{c}\text { Overall } \\
\text { Strength of } \\
\text { Evidence }\end{array}$ \\
\hline \multirow[t]{2}{*}{$\begin{array}{l}\text { Antibiotics vs. } \\
\text { Placebo or } \\
\text { Management Without } \\
\text { Antibiotics }\end{array}$} & \multirow[t]{2}{*}{$\begin{array}{l}\text { Repeat } \\
\text { Exacerbation } \\
\text { End of Intervention }\end{array}$} & $\begin{array}{l}\text { OR: } 1.80 ; \\
95 \% \mathrm{Cl}: 0.89 \\
\text { to } 3.62 \\
\mathrm{I}^{2}=0.00 \%\end{array}$ & $\begin{array}{l}2 \mathrm{RCTs}^{69,71} \\
\text { with } 229 \\
\text { patients }\end{array}$ & $\begin{array}{l}\text { Severe } \\
\text { imprecision }\end{array}$ & $\begin{array}{l}\text { Low SOE } \\
\text { supporting no } \\
\text { difference }\end{array}$ \\
\hline & & $\begin{array}{l}\text { Rate Ratio; } \\
1.01 ; 95 \% \mathrm{Cl} \text { : } \\
0.14 \text { to } 1.75 \\
\mathrm{I}^{2}=\mathrm{N} / \mathrm{A}\end{array}$ & $\begin{array}{l}1 \mathrm{RCT}^{68} \text { with } \\
305 \text { patients }\end{array}$ & $\begin{array}{l}\text { Severe } \\
\text { imprecision }\end{array}$ & $\begin{array}{l}\text { Low SOE } \\
\text { supporting no } \\
\text { difference }\end{array}$ \\
\hline \multirow[t]{2}{*}{$\begin{array}{l}\text { Antibiotics vs. } \\
\text { Placebo or } \\
\text { Management Without } \\
\text { Antibiotics }\end{array}$} & \multirow[t]{2}{*}{$\begin{array}{l}\text { Repeat } \\
\text { Exacerbation } \\
30 \text { days }\end{array}$} & $\begin{array}{l}\text { OR: } 1.69 ; \\
95 \% \mathrm{Cl}: 0.78 \\
\text { to } 3.68 \\
\mathrm{I}^{2}=0.18 \%\end{array}$ & $\begin{array}{l}2 \mathrm{RCTs}^{69,71} \\
\text { with } 229 \\
\text { patients }\end{array}$ & $\begin{array}{l}\text { Severe } \\
\text { imprecision }\end{array}$ & $\begin{array}{l}\text { Low SOE } \\
\text { supporting no } \\
\text { difference }\end{array}$ \\
\hline & & $\begin{array}{l}\text { Rate Ratio: } \\
1.57 ; 95 \% \mathrm{Cl} \text { : } \\
0.76 \text { to } 3.24 \\
\mathrm{I}^{2}=0.00 \%\end{array}$ & $\begin{array}{l}2 \text { RCTs }^{69,71} \\
\text { with } 229 \\
\text { patients }\end{array}$ & $\begin{array}{l}\text { Intermediate } \\
\text { ROB and } \\
\text { severe } \\
\text { imprecision }\end{array}$ & $\begin{array}{l}\text { Insufficient } \\
\text { evidence }\end{array}$ \\
\hline $\begin{array}{l}\text { Antibiotics vs. } \\
\text { Placebo }\end{array}$ & $\begin{array}{l}\text { Repeat } \\
\text { Exacerbation } \\
6 \text { months }\end{array}$ & $\begin{array}{l}\text { OR: } 2.24 \\
95 \% \mathrm{Cl}: 0.58 \\
\text { to } 8.69 \\
\mathrm{I}^{2}=\mathrm{N} / \mathrm{A}\end{array}$ & $\begin{array}{l}1 \mathrm{RCT}^{71} \text { with } \\
35 \text { patients }\end{array}$ & $\begin{array}{l}\text { Severe } \\
\text { imprecision }\end{array}$ & $\begin{array}{l}\text { Low SOE } \\
\text { supporting no } \\
\text { difference }\end{array}$ \\
\hline $\begin{array}{l}\text { Antibiotics vs. } \\
\text { Placebo or } \\
\text { Management Without } \\
\text { Antibiotics }\end{array}$ & $\begin{array}{l}\text { Repeat } \\
\text { Exacerbation } \\
\text { Longest Followup }\end{array}$ & $\begin{array}{l}\text { OR: } 1.80 ; \\
95 \% \mathrm{Cl}: 0.89 \\
\text { to } 3.62 \\
\mathrm{I}^{2}=\mathrm{N} / \mathrm{A}\end{array}$ & $\begin{array}{l}2 \mathrm{RCTs}^{69,71} \\
\text { with } 229 \\
\text { patients }\end{array}$ & $\begin{array}{l}\text { Severe } \\
\text { imprecision }\end{array}$ & $\begin{array}{l}\text { Low SOE } \\
\text { supporting no } \\
\text { difference }\end{array}$ \\
\hline $\begin{array}{l}\text { Antibiotics vs. } \\
\text { Management Without } \\
\text { Antibiotics }\end{array}$ & $\begin{array}{l}\text { Need for Intubation } \\
\text { End of Intervention }\end{array}$ & $\begin{array}{l}\text { OR: } 0.49 ; \\
95 \% \mathrm{Cl}: 0.04 \\
\text { to } 5.55 \\
\mathrm{I}^{2}=\mathrm{N} / \mathrm{A}\end{array}$ & $\begin{array}{l}1 \mathrm{RCT}^{69} \text { with } \\
194 \text { patients }\end{array}$ & $\begin{array}{l}\text { Severe } \\
\text { imprecision }\end{array}$ & $\begin{array}{l}\text { Low SOE } \\
\text { supporting no } \\
\text { difference }\end{array}$ \\
\hline $\begin{array}{l}\text { Antibiotics vs. } \\
\text { Placebo }\end{array}$ & $\begin{array}{l}\text { Clinical Cure } \\
\text { End of Intervention }\end{array}$ & $\begin{array}{l}\text { OR: } 2.03 ; \\
95 \% \mathrm{Cl}: 1.47 \\
\text { to } 2.80 \\
\mathrm{I}^{2}=0.00 \%\end{array}$ & $\begin{array}{l}3 \text { RCTs }^{70,72,} \\
73 \text { with } 683 \\
\text { patients }\end{array}$ & High ROB & $\begin{array}{l}\text { Moderate SOE } \\
\text { supporting } \\
\text { improvement }\end{array}$ \\
\hline $\begin{array}{l}\text { Antibiotics vs. } \\
\text { Placebo or } \\
\text { Management Without } \\
\text { Antibiotics }\end{array}$ & $\begin{array}{l}\text { Clinical Cure } \\
\text { Longest Followup }\end{array}$ & $\begin{array}{l}\text { OR: } 1.50 ; \\
95 \% \mathrm{Cl}: 1.01 \\
\text { to } 2.24 \\
\mathrm{I}^{2}=20.33 \%\end{array}$ & $\begin{array}{l}4 \text { RCTs }^{69,71-} \\
73 \text { with } 812 \\
\text { patients }\end{array}$ & $\begin{array}{l}\text { Intermediate } \\
\text { ROB }\end{array}$ & $\begin{array}{l}\text { Moderate SOE } \\
\text { supporting } \\
\text { improvement }\end{array}$ \\
\hline $\begin{array}{l}\text { Antibiotics vs. } \\
\text { Placebo }\end{array}$ & $\begin{array}{l}\text { Clinical Failure } \\
\text { End of Intervention }\end{array}$ & $\begin{array}{l}\text { OR: } 0.54 ; \\
95 \% \mathrm{Cl}: 0.34 \\
\text { to } 0.86 \\
\mathrm{I}^{2}=20.32 \%\end{array}$ & $\begin{array}{l}2 \mathrm{RCTS}^{68,70} \\
\text { with } 405 \\
\text { patients }\end{array}$ & $\begin{array}{l}\text { Intermediate } \\
\text { ROB }\end{array}$ & $\begin{array}{l}\text { Moderate SOE } \\
\text { supporting } \\
\text { improvement }\end{array}$ \\
\hline $\begin{array}{l}\text { Antibiotics vs. } \\
\text { Placebo }\end{array}$ & $\begin{array}{l}\text { Clinical Failure } \\
\text { Longest Followup }\end{array}$ & $\begin{array}{l}\text { OR: } 0.82 ; \\
95 \% \mathrm{Cl}: 0.58 \\
\text { to } 1.14 \\
\mathrm{I}^{2}=0.00 \%\end{array}$ & $\begin{array}{l}2 \mathrm{RCTs}^{68,73} \\
\text { with } 570 \\
\text { patients }\end{array}$ & $\begin{array}{l}\text { Intermediate } \\
\text { ROB and } \\
\text { imprecision }\end{array}$ & $\begin{array}{l}\text { Low SOE } \\
\text { supporting no } \\
\text { difference }\end{array}$ \\
\hline
\end{tabular}

CI = confidence interval; CRQ = Chronic Respiratory Questionnaire; N/A = not applicable; OR = odds ratio; RCT = randomized controlled trial; ROB = Risk of Bias; SOE = Strength of Evidence; VAS = visual analog scale; $\mathrm{WMD}=$ weighted mean difference

\section{Lung Function}

Forced expiratory volume in one second (FEV1) percent predicted and FEV1 absolute at the end of the intervention and at the longest followup were not statistically significantly different between the antibiotics and placebo or management without antibiotics. This finding was 
independent of whether studies were conducted in patients with mild or moderate to severe ECOPD.

Table 5. Comparison of systemic antibiotics versus control, additional outcomes

\begin{tabular}{|c|c|c|c|}
\hline Comparison & Outcome & Findings & Study Design and Sample Size \\
\hline $\begin{array}{l}\text { Antibiotics vs. } \\
\text { Placebo }\end{array}$ & $\begin{array}{l}\text { Cough (VAS) } \\
\text { End of Intervention }\end{array}$ & $\begin{array}{l}\text { WMD: }-1.1 ; 95 \% \mathrm{Cl}:-1.80 \text { to } \\
-0.40, \mathrm{I}^{2}=\mathrm{N} / \mathrm{A}\end{array}$ & $1 \mathrm{RCT}^{73}$ with 265 patients \\
\hline $\begin{array}{l}\text { Antibiotics vs. } \\
\text { Placebo }\end{array}$ & $\begin{array}{l}\text { Cough (VAS) } \\
\text { Longest Followup }\end{array}$ & $\begin{array}{l}\text { WMD: }-0.40 ; 95 \% \mathrm{Cl}:-1.33 \\
\text { to } 0.33, \mathrm{I}^{2}=\mathrm{N} / \mathrm{A}\end{array}$ & $1 \mathrm{RCT}^{73}$ with 265 patients \\
\hline $\begin{array}{l}\text { Antibiotics vs. } \\
\text { Placebo/ } \\
\text { Management } \\
\text { Without Antibiotics }\end{array}$ & $\begin{array}{l}\text { Other Symptoms(Total } \\
\text { Symptom Score } \\
\text { (Dyspnea, Fatigue, } \\
\text { Cough, Sputum); VAS; } \\
\text { CCQ Symptom Score) } \\
\text { End of Intervention }\end{array}$ & $\begin{array}{l}\text { SMD: } 0.29 ; 95 \% \mathrm{Cl}: 0.11 \text { to } \\
0.47,\left.\right|^{2}=41.0 \%\end{array}$ & 3 RCTs $^{69,71,73}$ with 494 patients \\
\hline $\begin{array}{l}\text { Antibiotics vs. } \\
\text { Placebo }\end{array}$ & $\begin{array}{l}\text { Other Symptoms(Total } \\
\text { Symptom Score } \\
\text { (Dyspnea, Fatigue, } \\
\text { Cough, Sputum)) } \\
\text { Longest Followup }\end{array}$ & $\begin{array}{l}\text { WMD: }-1.10 ; 95 \% \mathrm{Cl}:-3.31 \\
\text { to } 1.11, I^{2}=\mathrm{N} / \mathrm{A}\end{array}$ & $1 \mathrm{RCT}^{73}$ with 265 patients \\
\hline $\begin{array}{l}\text { Antibiotics vs. } \\
\text { Management } \\
\text { Without Antibiotics }\end{array}$ & $\begin{array}{l}\text { FEV1 \% Predicted } \\
\text { End of Intervention }\end{array}$ & $\begin{array}{l}\text { WMD: }-1.70 ; 95 \% \mathrm{Cl}:-7.31 \\
\text { to } 3.91, I^{2}=\mathrm{N} / \mathrm{A}\end{array}$ & $1 \mathrm{RCT}^{69}$ with 194 patients \\
\hline $\begin{array}{l}\text { Antibiotics vs. } \\
\text { Placebo }\end{array}$ & $\begin{array}{l}\text { FEV1 \% Predicted } \\
\text { Longest Followup }\end{array}$ & $\begin{array}{l}\text { WMD: }-0.80 ; 95 \% \mathrm{Cl}:-6.67 \\
\text { to } 5.07, \mathrm{I}^{2}=\mathrm{N} / \mathrm{A}\end{array}$ & $1 \mathrm{RCT}^{71}$ with 35 patients \\
\hline $\begin{array}{l}\text { Antibiotics vs. } \\
\text { Placebo }\end{array}$ & $\begin{array}{l}\text { FEV1 Absolute } \\
\text { End of Intervention }\end{array}$ & $\begin{array}{l}\text { WMD: } 0.05 ; 95 \% \mathrm{Cl}:-0.01 \\
\text { to } 0.11, \mathrm{I}^{2}=0.0 \%\end{array}$ & $2 \mathrm{RCTs}^{70,73}$ with 365 patients \\
\hline $\begin{array}{l}\text { Antibiotics vs. } \\
\text { Placebo }\end{array}$ & $\begin{array}{l}\text { FEV1 Absolute } \\
\text { Longest Followup }\end{array}$ & $\begin{array}{l}\text { WMD: } 0.05 ; 95 \% \mathrm{Cl}:-0.01 \\
\text { to } 0.12,1^{2}=44.13 \%\end{array}$ & $2 \mathrm{RCTs}^{71,73}$ with 300 patients \\
\hline
\end{tabular}

\section{Adverse Events}

Appendix Table H.1. lists the rate ratio of adverse events(AEs) between antibiotics and placebo or management without antibiotics. There was no statistical difference between antibiotics and placebo on withdrawals, withdrawals due to AEs, total number of AEs, or any specific category of AEs (cardiovascular AE, dermatological AE, gastrointestinal AE, general internal medicine AE, musculoskeletal AE, or oncological AE). 2 studies ${ }^{68,73}$ reported serious $\mathrm{AE}$ in the antibiotics group, including pneumonia (1 case), urinary tract infection (1 case), cardiovascular (22 cases), hypoglycemia (1 case), malignancy-related (6 cases), and other unspecified (36 cases). There was no statistical difference on serious AEs.

\section{Systemic Corticosteroids Versus Placebo or Management Without Systemic Corticosteroids}

Nine studies compared the effectiveness of systemic corticosteroids versus placebo or management without systemic corticosteroids, ${ }^{74-82}$ of which three studies were conducted in patients with mild ECOPD, ${ }^{74,79,82}$ four studies in patients with moderate to severe ECOPD, ${ }^{75-78,}$ 100 one study in patients with severe ECOPD ${ }^{81}$ and one in patients with mild to severe ECOPD. ${ }^{80}$ Table 6 and Table 7 provide an overview of all included studies. Tables stratified by the severity 
of ECOPD can be found in the Appendix (Appendix Table G.3: mild ECOPD, Appendix Table G.4: moderate to severe COPD).

\section{Mortality}

The pooled estimate of three studies (all conducted in patients with moderate to severe ECOPD) did not show any statistically significant difference between the systemic corticosteroids and the placebo or management without systemic corticosteroids group at the end of the intervention or at the end followup.

There were no studies measuring mortality in patients with mild ECOPD.

\section{Symptoms}

Dyspnea was statistically significantly better in the systemic corticosteroid group compared with the placebo group at the end of the intervention. There was no statistically significant difference in dyspnea at the end of the intervention when stratifying studies by severity of ECOPD.

\section{Hospital Admission}

Thirty-day hospital admissions were measured in two studies (including patients with moderate to severe ECOPD) with no statistically significant difference found between systemic corticosteroid and placebo group.

\section{Repeat Exacerbations}

Repeat exacerbations at the end of the intervention, at 1 month, 3 months, and longest followup did not show a statistically significant difference between systemic corticosteroid and placebo or management without systemic corticosteroids. There was no statistically significant difference observed in repeat exacerbations when stratifying studies by severity of ECOPD.

\section{Intubations}

The need for intubation at the end of the intervention and at the longest followup was found to be not statistically significantly different between the systemic corticosteroid and placebo group. There were no studies in mild ECOPD that assessed need for intubation as outcome.

\section{Clinical Resolution of Exacerbation}

Statistically significantly less patients on systemic corticosteroids compared with patients in the placebo group had clinical failure at the end of the intervention but not at the longest followup.

In patients with mild ECOPD, clinical failure in patients on systemic corticosteroids compared with patients in the placebo group was statistically significantly lower at the end of the intervention. 
Table 6. Comparison of systemic corticosteroids versus control, critical outcomes

\begin{tabular}{|c|c|c|c|c|c|}
\hline Comparison & Outcome & Findings & $\begin{array}{l}\text { Study Design } \\
\text { and Sample } \\
\text { Size }\end{array}$ & $\begin{array}{l}\text { Rationale for } \\
\text { Strength of } \\
\text { Evidence } \\
\text { (SOE) }\end{array}$ & $\begin{array}{c}\text { Overall } \\
\text { Strength of } \\
\text { Evidence }\end{array}$ \\
\hline $\begin{array}{l}\text { Systemic } \\
\text { Corticosteroids vs. } \\
\text { Placebo }\end{array}$ & $\begin{array}{l}\text { Mortality } \\
\text { End of } \\
\text { Intervention }\end{array}$ & $\begin{array}{l}\text { OR: } 1.61 ; 95 \% \\
\mathrm{Cl}: 0.47 \text { to } 5.47 \\
\mathrm{I}^{2}=0.00 \%\end{array}$ & $\begin{array}{l}4 \mathrm{RCTs}^{76,77,8281} \\
\text { with } 510 \\
\text { patients }\end{array}$ & $\begin{array}{l}\text { Intermediate } \\
\text { ROB and } \\
\text { severe } \\
\text { imprecision }\end{array}$ & $\begin{array}{l}\text { Insufficient } \\
\text { evidence }\end{array}$ \\
\hline $\begin{array}{l}\text { Systemic } \\
\text { Corticosteroids vs. } \\
\text { Placebo or } \\
\text { Management Aithout } \\
\text { Systemic } \\
\text { Corticosteroids }\end{array}$ & $\begin{array}{l}\text { Mortality } \\
\text { Longest } \\
\text { Followup }\end{array}$ & $\begin{array}{l}\text { OR: } 0.89 ; 95 \% \\
\mathrm{Cl}: 0.37 \text { to } 2.16 \\
\mathrm{I}^{2}=0.00 \%\end{array}$ & $\begin{array}{l}\mathrm{RCTs}^{75,77,78} \\
\text { with } 353 \\
\text { patients }\end{array}$ & $\begin{array}{l}\text { High ROB and } \\
\text { severe } \\
\text { imprecision }\end{array}$ & $\begin{array}{l}\text { Insufficient } \\
\text { evidence }\end{array}$ \\
\hline $\begin{array}{l}\text { Systemic } \\
\text { Corticosteroids vs. } \\
\text { Placebo }\end{array}$ & $\begin{array}{l}\text { Dyspnea } \\
\text { (Numeric Scale: } \\
\text { Modified Borg, } \\
\text { VAS) } \\
\text { End of } \\
\text { Intervention } \\
\end{array}$ & $\begin{array}{l}\text { SMD: } 0.40 ; \\
95 \% \mathrm{Cl}: 0.07 \text { to } \\
0.70, \mathrm{I}^{2}=0.00 \%\end{array}$ & $\begin{array}{l}2 \mathrm{RCTs}^{76,79} \\
\text { with } 154 \\
\text { patients }\end{array}$ & $\begin{array}{l}\text { High ROB and } \\
\text { imprecision }\end{array}$ & $\begin{array}{l}\text { Low SOE } \\
\text { supporting } \\
\text { improvement }\end{array}$ \\
\hline \multirow[t]{2}{*}{$\begin{array}{l}\text { Systemic } \\
\text { Corticosteroids vs. } \\
\text { Placebo }\end{array}$} & \multirow[t]{2}{*}{$\begin{array}{l}\text { Hospital } \\
\text { Admission } \\
30 \text { days }\end{array}$} & $\begin{array}{l}\text { OR: } 0.54 ; 95 \% \\
\mathrm{Cl}: 0.10 \text { to } 2.88, \\
\mathrm{I}^{2}=\mathrm{N} / \mathrm{A}\end{array}$ & $\begin{array}{l}1 \mathrm{RCT}^{77} \text { with } \\
191 \text { patients }\end{array}$ & $\begin{array}{l}\text { High ROB and } \\
\text { severe } \\
\text { imprecision }\end{array}$ & $\begin{array}{l}\text { Insufficient } \\
\text { evidence }\end{array}$ \\
\hline & & $\begin{array}{l}\text { Rate Ratio: } \\
0.50 ; 95 \% \mathrm{Cl} \text { : } \\
0.15 \text { to } 1.66 \\
\text { I}^{2}=\mathrm{N} / \mathrm{A}\end{array}$ & $\begin{array}{l}1 \mathrm{RCT}^{75} \text { with } \\
106 \text { patients }\end{array}$ & $\begin{array}{l}\text { High ROB and } \\
\text { severe } \\
\text { imprecision }\end{array}$ & $\begin{array}{l}\text { Insufficient } \\
\text { evidence }\end{array}$ \\
\hline $\begin{array}{l}\text { Systemic } \\
\text { Corticosteroids vs. } \\
\text { Placebo or } \\
\text { Management Aithout } \\
\text { Systemic } \\
\text { Corticosteroids }\end{array}$ & $\begin{array}{l}\text { Repeat } \\
\text { Exacerbation } \\
\text { End of } \\
\text { Intervention }\end{array}$ & $\begin{array}{l}\text { OR: } 0.97 ; 95 \% \\
\mathrm{Cl}: 0.52 \text { to } 1.81 \\
\mathrm{I}^{2}=45.12 \%\end{array}$ & $\begin{array}{l}\text { RCTs }^{75,78,80} \\
\text { with } 266 \\
\text { patients }\end{array}$ & $\begin{array}{l}\text { High ROB and } \\
\text { severe } \\
\text { imprecision }\end{array}$ & $\begin{array}{l}\text { Insufficient } \\
\text { evidence }\end{array}$ \\
\hline $\begin{array}{l}\text { Systemic } \\
\text { Corticosteroids vs. } \\
\text { Management Aithout } \\
\text { Systemic } \\
\text { Corticosteroids }\end{array}$ & $\begin{array}{l}\text { Repeat } \\
\text { Exacerbation } \\
1 \text { Month }\end{array}$ & $\begin{array}{l}\text { Rate Ratio: } \\
0.52 ; 95 \% \mathrm{Cl}: \\
0.24 \text { to } 1.36 \\
\mathrm{I}^{2}=\mathrm{N} / \mathrm{A}\end{array}$ & $\begin{array}{l}1 \mathrm{RCT}^{75} \text { with } \\
106 \text { patients }\end{array}$ & $\begin{array}{l}\text { High ROB and } \\
\text { severe } \\
\text { imprecision }\end{array}$ & $\begin{array}{l}\text { Insufficient } \\
\text { evidence }\end{array}$ \\
\hline $\begin{array}{l}\text { Systemic } \\
\text { Corticosteroids vs. } \\
\text { Placebo }\end{array}$ & $\begin{array}{l}\text { Repeat } \\
\text { Exacerbation } \\
3 \text { Months }\end{array}$ & $\begin{array}{l}\text { OR: } 1.29 ; 95 \% \\
\mathrm{Cl}: 0.40 \text { to } 4.13 \text {, } \\
\mathrm{I}^{2}=\mathrm{N} / \mathrm{A}\end{array}$ & $\begin{array}{l}1 \mathrm{RCT}^{78} \text { with } 56 \\
\text { patients }\end{array}$ & $\begin{array}{l}\text { Intermediate } \\
\text { ROB and } \\
\text { severe } \\
\text { imprecision }\end{array}$ & $\begin{array}{l}\text { Insufficient } \\
\text { evidence }\end{array}$ \\
\hline $\begin{array}{l}\text { Systemic } \\
\text { Corticosteroids vs. } \\
\text { Placebo }\end{array}$ & $\begin{array}{l}\text { Repeat } \\
\text { Exacerbation } \\
\text { Longest } \\
\text { Followup } \\
\end{array}$ & $\begin{array}{l}\text { OR: } 1.29 ; 95 \% \\
\mathrm{Cl}: 0.40 \text { to } 4.13, \\
\mathrm{I}^{2}=\mathrm{N} / \mathrm{A}\end{array}$ & $\begin{array}{l}1 \mathrm{RCT}^{78} \text { with } 56 \\
\text { patients }\end{array}$ & $\begin{array}{l}\text { Intermediate } \\
\text { ROB and } \\
\text { severe } \\
\text { imprecision }\end{array}$ & $\begin{array}{l}\text { Insufficient } \\
\text { evidence }\end{array}$ \\
\hline $\begin{array}{l}\text { Systemic } \\
\text { Corticosteroids vs. } \\
\text { Management without } \\
\text { Systemic } \\
\text { Corticosteroids }\end{array}$ & $\begin{array}{l}\text { Repeat } \\
\text { Exacerbation } \\
\text { Longest } \\
\text { Followup }\end{array}$ & $\begin{array}{l}\text { Rate Ratio: } \\
0.57,95 \% \mathrm{Cl}: \\
0.24 \text { to } 1.36 \\
\mathrm{I}^{2}=\mathrm{N} / \mathrm{A}\end{array}$ & $\begin{array}{l}1 \mathrm{RCT}^{75} \text { with } \\
106 \text { patients }\end{array}$ & $\begin{array}{l}\text { High ROB and } \\
\text { severe } \\
\text { imprecision }\end{array}$ & $\begin{array}{l}\text { Insufficient } \\
\text { evidence }\end{array}$ \\
\hline $\begin{array}{l}\text { Systemic } \\
\text { Corticosteroids vs. } \\
\text { Placebo }\end{array}$ & $\begin{array}{l}\text { Need for } \\
\text { Intubation } \\
\text { End of } \\
\text { Intervention }\end{array}$ & $\begin{array}{l}\text { OR: } 0.42 ; 95 \% \\
\mathrm{Cl}: 0.06 \text { to } 2.68, \\
\mathrm{I}^{2}=0.00 \%\end{array}$ & $\begin{array}{l}2 \mathrm{RCTs}^{76,77} \\
\text { with } 319 \\
\text { patients }\end{array}$ & $\begin{array}{l}\text { High ROB and } \\
\text { severe } \\
\text { imprecision }\end{array}$ & $\begin{array}{l}\text { Insufficient } \\
\text { evidence }\end{array}$ \\
\hline
\end{tabular}




\begin{tabular}{|c|c|c|c|c|c|}
\hline Comparison & Outcome & Findings & $\begin{array}{c}\text { Study Design } \\
\text { and Sample } \\
\text { Size }\end{array}$ & $\begin{array}{l}\text { Rationale for } \\
\text { Strength of } \\
\text { Evidence } \\
\text { (SOE) }\end{array}$ & $\begin{array}{c}\text { Overall } \\
\text { Strength of } \\
\text { Evidence }\end{array}$ \\
\hline $\begin{array}{l}\text { Systemic } \\
\text { Corticosteroids vs. } \\
\text { Placebo }\end{array}$ & $\begin{array}{l}\text { Need for } \\
\text { Intubation } \\
\text { Longest } \\
\text { Followup }\end{array}$ & $\begin{array}{l}\text { OR: } 0.92 ; 95 \% \\
\text { Cl: } 0.15 \text { to } 5.66 \text {, } \\
\text { I²=N/A }^{2}\end{array}$ & $\begin{array}{l}1 \mathrm{RCT}^{77} \text { with } \\
191 \text { patients }\end{array}$ & $\begin{array}{l}\text { High ROB and } \\
\text { severe } \\
\text { imprecision }\end{array}$ & $\begin{array}{l}\text { Insufficient } \\
\text { evidence }\end{array}$ \\
\hline $\begin{array}{l}\text { Systemic } \\
\text { Corticosteroids vs. } \\
\text { Placebo }\end{array}$ & $\begin{array}{l}\text { Clinical Failure } \\
\text { End of } \\
\text { Intervention }\end{array}$ & $\begin{array}{l}\text { OR: } 0.01 ; 95 \% \\
\text { Cl: } 0.00 \text { to } 0.13 \\
\mathrm{I}^{2}=0.00 \%\end{array}$ & $\begin{array}{l}2 \text { RCTs }^{77,79} \\
\text { with } 217 \\
\text { patients }\end{array}$ & $\begin{array}{l}\text { High ROB and } \\
\text { imprecision }\end{array}$ & $\begin{array}{l}\text { Low SOE } \\
\text { supporting } \\
\text { improvement }\end{array}$ \\
\hline $\begin{array}{l}\text { Systemic } \\
\text { Corticosteroids vs. } \\
\text { Placebo }\end{array}$ & $\begin{array}{l}\text { Clinical Failure } \\
\text { Longest } \\
\text { Followup }\end{array}$ & $\begin{array}{l}\text { OR: } 0.92 ; 95 \% \\
\text { Cl: } 0.58 \text { to } 1.45 \\
\mathrm{I}^{2}=81.42 \%\end{array}$ & $\begin{array}{l}2 \mathrm{RCTs}^{77,82} \\
\text { with } 338 \\
\text { patients }\end{array}$ & $\begin{array}{l}\text { Intermediate } \\
\text { ROB and } \\
\text { severe } \\
\text { imprecision }\end{array}$ & $\begin{array}{l}\text { Insufficient } \\
\text { evidence }\end{array}$ \\
\hline
\end{tabular}

$\mathrm{CI}$ = confidence interval; N/A = not applicable; OR = odds ratio; RCT = randomized controlled trial; ROB = risk of bias; SMD = standardized mean difference; $\mathrm{SOE}=$ strength of evidence

\section{Lung Function}

FEV1percent predicted was statistically significantly increased in the systemic corticosteroid group compared with placebo or management without systemic corticosteroids at the end of the intervention but not at the end of followup. FEV1percent absolute was statistically significantly increased in the systemic corticosteroid group compared with placebo or management without systemic corticosteroids at the end of the intervention.

Two stuides ${ }^{74,79}$ measured lung function in patients with mild ECOPD and found a statistically significantly increased FEV1 percent absolute in the systemic corticosteroid group compared with the placebo group at the end of the intervention.

Table 7. Comparison of systemic corticosteroids versus control, additional outcomes

\begin{tabular}{|c|c|c|c|}
\hline Comparison & Outcome & Findings & Study Design and Sample Size \\
\hline $\begin{array}{l}\text { Systemic } \\
\text { Corticosteroids } \\
\text { vs. Placebo or } \\
\text { Management } \\
\text { Without Systemic } \\
\text { Corticosteroids }\end{array}$ & $\begin{array}{l}\text { FEV1 \% Predicted } \\
\text { End of Intervention }\end{array}$ & $\begin{array}{l}\text { WMD: } 4.64 ; 95 \% \mathrm{Cl}: 1.28 \text { to } \\
7.99, \mathrm{I}^{2}=0.00 \%\end{array}$ & $4 \mathrm{RCTs}^{75,80,81}$ with 310 patients \\
\hline $\begin{array}{l}\text { Systemic } \\
\text { Corticosteroids } \\
\text { vs. Placebo }\end{array}$ & $\begin{array}{l}\text { FEV1 \% Predicted } \\
\text { Longest Followup }\end{array}$ & $\begin{array}{l}\text { WMD: } 7.75 ; 95 \% \mathrm{Cl}:-0.30 \text { to } \\
15.80,1^{2}=0.62 \%\end{array}$ & $2 \mathrm{RCTs}^{78,81}$ with 100 patients \\
\hline $\begin{array}{l}\text { Systemic } \\
\text { Corticosteroids } \\
\text { vs. Placebo }\end{array}$ & $\begin{array}{l}\text { FEV1 Absolute } \\
\text { End of Intervention }\end{array}$ & $\begin{array}{l}\text { WMD: } 0.36 ; 95 \% \mathrm{Cl}: 0.28 \text { to } \\
0.45, \mathrm{I}^{2}=87.61 \%\end{array}$ & $3 \mathrm{RCTs}^{74,78,79}$ with 112 patients \\
\hline
\end{tabular}

$\mathrm{CI}$ = confidence interval; FEV1 = forced expiratory volume in one second; N/A = not applicable; RCT = randomized controlled trial; WMD = weighted mean difference

\section{Adverse Events}

Appendix Table H.2. presents adverse events between systemic corticosteroids and placebo or management without systemic corticosteroids. The systemic corticosteroids group had statistically significant fewer withdrawals, and more endocrine AEs. One study ${ }^{76}$ reported 5 cases of serious AEs (unspecified) in the systemic corticosteroids group. There was no statistical difference on serious AEs or other AEs. 
KQ2. In adult patients with exacerbation of COPD, what are the benefits and harms of emerging and other pharmacologic and nonpharmacologic therapies compared with placebo or standard care?

\section{Key Points-KQ2}

\section{Pharmacologic Therapies Versus Placebo or Management Without Pharmacologic Therapies}

- The evidence was insufficient for the effect of aminophyllines, magnesium sulfate, mucolytics, inhaled corticosteroids, inhaled antibiotics, 5-lipoxygenase inhibitor and statins on mortality, dyspnea, need for intubation, clinical failure, or hospital admission.

- Aminophyllines were associated with statistically significantly more gastrointestinal adverse events than placebo. No other statistically significant difference was found in adverse events between the remaining pharmacologic therapies and placebo or management without pharmacologic therapies

\section{Nonpharmacologic Therapies Versus Placebo or Management Without Nonpharmacologic Therapies}

- Resistance training improved dyspnea, and quality of life compared with management without nonpharmacologic therapies (low SOE).

- Early pulmonary rehabilitation, commenced before hospital discharge during the initial most acute phase of exacerbation rather than the convalescence period, improved dyspnea compared with management without nonpharmacologic therapies (low SOE).

- Whole body vibration training improved QoL compared with management without nonpharmacologic therapies (low SOE).

- Titrated oxygen reduced mortality compared with high flow oxygen at the longest followup (low SOE).

- Vitamin D supplementation improved quality of life compared with usual diet (low SOE).

- Omega-3 fatty acid enriched diet did not change quality of life, need for intubation, or dyspnea at the end of intervention compared with usual diet (low SOE).

- Few adverse events were reported in studies of nonpharmacologic therapies. There was no statistically significant difference found in adverse events between nonpharmacologic therapies and management without nonpharmacologic therapies.

\section{Results KQ2}

There were 51studies $25,46,65-67,74-76,83-125$, with 4,280 patients included for KQ2. The characteristics of the studies are listed in Appendix Table D.2. Four ${ }^{66,74,113,122}$ studies had patients with mild ECOPD, $6^{25,46,65,75,76,83-112,114-121,123-125}$ studies had patients with moderate to severe ECOPD, and $1{ }^{67}$ study had patients with mild to severe ECOPD. Twenty $65,66,74-76,89,92,93$, 96, 98, 101, 104, 107, 112-114, 116, 119-121 evaluated pharmacologic therapies compared with management without pharmacologic therapies and $31^{25,46,67,83-88,90,91,94,95,97, ~ 99, ~ 100, ~ 102, ~ 103, ~ 105, ~ 106, ~ 108-111, ~ 115, ~} 117$, 118, 122-125 evaluated nonpharmacologic therapies to management without nonpharmacologic 
therapies. 41 were conducted in a hospital setting, 46, 65, 75, 76, 83-91, 93-103, 105-112, 114-118, 120, 123-125 4 in the Emergency Department, ${ }^{92,104,119,121} 3$ in outpatients, ${ }^{66,74,113} 1$ in both outpatients and hospital settings, ${ }^{67}$ one in an ambulance ${ }^{25}$ and in one study the setting was unclear. ${ }^{122}$ Studies were conducted in the US (4), ${ }^{107,119-121}$ Canada (3), ${ }^{67,90,118}$ Europe (21), 46, 65, 66, 74, 76, 83, 84, 86, 93, 99, 102, 106, 108, 110, 113-117, 124, 125 Africa (1), ${ }^{101}$, Asia (15), ${ }^{75,85,87-89,91,94-96,98,103,109,112,122,123}$ Australia (6) ${ }^{25,92,97,104,105,111}$ and South America (1). ${ }^{100}$ Average treatment length was 9.6 days and the mean reported followup was 3.6 months. Details of the interventions in each study can be found in Appendix Table F.2. Individual studies inclusion and exclusion criteria are in Appendix Table I.1.

The overall risk of bias is intermediate to high due to unclear sequence generation (49.02\%), unclear allocation concealment (66.67\%), and high risk or unclear risk of incomplete outcome data (56.86\%) (Appendix Table E.1.).

\section{Pharmacologic Therapies}

\section{Aminophyllines Versus Placebo}

Three studies ${ }^{114,120,121}$ evaluated the effectiveness of intravenous aminophyllines compared with placebo (Table 8 and Table 9). All studies were conducted in patients with moderate to severe ECOPD.

There were no statistically significant differences observed between groups in mortality, dyspnea, cough, other symptoms, FEV1 absolute, and need for intubation. Statistically significantly more patients in the aminophyllines group had total number of AEs and gastrointestinal AEs than those in the placebo group ${ }^{114}$ (Appendix Table H.3.). No other statistically significant difference on withdrawals, other AEs (cardiovascular AE, neurological AE, withdrawals due to AEs) were found.

Table 8. Comparison of intravenous aminophyllines versus placebo, critical outcomes

\begin{tabular}{|l|l|l|l|l|}
\hline \multicolumn{1}{|c|}{ Outcome } & \multicolumn{1}{|c|}{ Findings } & $\begin{array}{l}\text { Study Design and } \\
\text { Sample Size }\end{array}$ & \multicolumn{1}{|c|}{$\begin{array}{c}\text { Rationale for } \\
\text { Strength of } \\
\text { Evidence (SOE) }\end{array}$} & $\begin{array}{l}\text { Overall Strength of } \\
\text { Evidence }\end{array}$ \\
\hline $\begin{array}{l}\text { Mortality } \\
\text { End of intervention }\end{array}$ & $\begin{array}{l}\text { OR: } 0.81 ; 95 \% \mathrm{Cl}: \\
0.29 \text { to } 2.28, \mathrm{I}^{2}= \\
0.00 \%\end{array}$ & $\begin{array}{l}2 \mathrm{RCT}^{114,121} \text { with } \\
132 \text { patients }\end{array}$ & $\begin{array}{l}\text { Intermediate ROB } \\
\text { and severe } \\
\text { imprecision }\end{array}$ & Insufficient evidence \\
\hline $\begin{array}{l}\text { Dyspnea } \\
\text { (Numeric Scale: }\end{array}$ & $\begin{array}{l}\mathrm{SMD}:-0.01 ; 95 \% \\
\text { Borg) }-0.35 \text { to } 0.33, \\
\text { End of Intervention }\end{array}$ & $\begin{array}{l}2 \mathrm{RCT}^{114,121} \text { with } \\
132 \text { patients }\end{array}$ & $\begin{array}{l}\text { Intermediate ROB } \\
\text { and severe } \\
\text { imprecision }\end{array}$ & Insufficient evidence \\
\hline $\begin{array}{l}\text { Need for Intubation } \\
\text { End of Intervention }\end{array}$ & $\begin{array}{l}\text { OR: } 0.87 ; 95 \% \mathrm{Cl}: \\
0.05 \text { to } 15.28,\end{array}$ & $\begin{array}{l}1 \mathrm{RCT}^{114,120,121} \text { with } \\
30 \text { patients }\end{array}$ & $\begin{array}{l}\text { Intermediate ROB } \\
\text { and severe } \\
\text { imprecision }\end{array}$ & Insufficient evidence \\
\hline
\end{tabular}

$\mathrm{CI}=$ confidence interval; N/A = not applicable; $\mathrm{OR}$ = odds ratio; $\mathrm{RCT}$ = randomized controlled trial; ROB = risk of bias; SMD = standardized mean difference

Table 9. Comparison of intravenous aminophyllines versus placebo, additional outcomes

\begin{tabular}{|l|l|l|}
\hline \multicolumn{1}{|c|}{ Outcome } & \multicolumn{1}{|c|}{ Findings } & \multicolumn{1}{|c|}{ Study Design and Sample Size } \\
\hline $\begin{array}{l}\text { Cough (Scale no } \\
\text { problem to severe } \\
\text { problem) }\end{array}$ & WMD: $-0.30 ; 95 \% \mathrm{Cl}:-0.94$ to 0.34, & $1 \mathrm{RCT}^{121}$ with 52 patients \\
End of Intervention & & \\
\hline
\end{tabular}




\begin{tabular}{|l|l|l|}
\hline \multicolumn{1}{|c|}{ Outcome } & \multicolumn{1}{|c|}{ Findings } & \multicolumn{1}{c|}{ Study Design and Sample Size } \\
\hline $\begin{array}{l}\text { Other Symptoms } \\
\text { (VAS) }\end{array}$ & $\begin{array}{l}\text { WMD: }-10.8 ; 95 \% \mathrm{Cl}:-21.59 \text { to } 0.00, \\
\text { End of Intervention }\end{array}$ & $\mathrm{I}^{2}=\mathrm{N} / \mathrm{A}$ \\
\hline $\begin{array}{l}\text { FEV1 Absolute } \\
\text { End of Intervention }\end{array}$ & $\begin{array}{l}\text { WMD: }-0.02 ; 95 \% \mathrm{Cl}:-0.09 \text { to } 0.06, \\
\mathrm{I}^{2}=0.00 \%\end{array}$ & $3 \mathrm{RCT}^{114,120,121}$ with 162 patients \\
\hline
\end{tabular}

$\mathrm{CI}$ = confidence interval; FEV1 = forced expiratory volume in one second; N/A = not applicable; RCT = randomized controlled trial; VAS: visual analog scale; WMD = weighted mean difference

\section{Magnesium Sulfate Versus Placebo}

Four studies evaluated the effectiveness of intravenous magnesium sulfate, ${ }^{65,92,98,119}$ and one study ${ }^{104}$ evaluated the effectiveness of nebulized magnesium sulfate (Table 10 and Table 11). All studies were conducted in patients with moderate to severe ECOPD.

There were no statistically significant differences observed in FEV1percent predicted at the longest followup and FEV1 absolute and dyspnea scores at the end of the intervention between intravenous magnesium and placebo. The intravenous magnesium sulfate group had a statistically significantly increased FEV1absolute at the longest followup. There was no statistically significant difference found in FEV1 absolute at the end of the intervention between nebulized magnesium sulfate and placebo. No adverse events were reported in the magnesium group. ${ }^{119}$

Table 10. Comparison of intravenous magnesium sulfate versus placebo, critical outcomes

\begin{tabular}{|c|c|c|c|c|}
\hline Outcome & Findings & $\begin{array}{l}\text { Study Design } \\
\text { and Sample } \\
\text { Size }\end{array}$ & $\begin{array}{c}\text { Rationale for } \\
\text { Strength of } \\
\text { Evidence (SOE) }\end{array}$ & $\begin{array}{l}\text { Overall Strength of } \\
\text { Evidence }\end{array}$ \\
\hline $\begin{array}{l}\text { Dyspnea } \\
\text { (Numeric Scale: } \\
\text { Dyspnea 1-7) End } \\
\text { of Intervention }\end{array}$ & $\begin{array}{l}\text { No statistical } \\
\text { difference }\end{array}$ & $\begin{array}{l}1 \mathrm{RCT}^{119} \text { with } 72 \\
\text { patients }\end{array}$ & $\begin{array}{l}\text { High ROB and } \\
\text { severe } \\
\text { imprecision }\end{array}$ & Insufficient evidence \\
\hline
\end{tabular}

RCT = randomized controlled trial; ROB = risk of bias; SOE = strength of evidence

Table 11. Comparison of magnesium sulfate versus placebo, additional outcomes

\begin{tabular}{|c|c|c|c|}
\hline Comparison & Outcome & Findings & Study Design and Sample Size \\
\hline \multirow{5}{*}{$\begin{array}{l}\text { Intravenous } \\
\text { Magnesium Sulfate } \\
\text { vs. Placebo }\end{array}$} & $\begin{array}{l}\text { FEV1 Absolute } \\
\text { End of Intervention }\end{array}$ & $\begin{array}{l}\text { WMD: } 0.02 ; 95 \% \mathrm{Cl}:- \\
0.02 \text { to } 0.05, I^{2}=\mathrm{N} / \mathrm{A}\end{array}$ & $1 \mathrm{RCT}^{98}$ with 30 patients \\
\hline & $\begin{array}{l}\text { FEV1 Absolute } \\
\text { End of Intervention }\end{array}$ & $\begin{array}{l}\text { No statistical } \\
\text { difference }\end{array}$ & 1 Crossover RCT $\mathrm{R}^{65}$ with 24 patients \\
\hline & $\begin{array}{l}\text { FEV1\% Predicted } \\
\text { End of intervention }\end{array}$ & $\begin{array}{l}\text { No statistical } \\
\text { difference }\end{array}$ & 1 Crossover $\mathrm{RCT}^{65}$ with 24 patients \\
\hline & $\begin{array}{l}\text { FEV1 Absolute } \\
\text { Longest Followup }\end{array}$ & $\begin{array}{l}\text { WMD: } 0.09 ; 95 \% \mathrm{Cl}: \\
0.01 \text { to } 0.16, \mathrm{I}^{2}=\mathrm{N} / \mathrm{A}\end{array}$ & $1 \mathrm{RCT}^{92}$ with 33 patients \\
\hline & $\begin{array}{l}\text { FEV1\% predicted } \\
\text { Longest Followup }\end{array}$ & $\begin{array}{l}\text { WMD: } 0.00 ; 95 \% \mathrm{Cl}:- \\
48.08 \text { to } 48.08, \mathrm{I}^{2}= \\
\text { N/A }\end{array}$ & $1 \mathrm{RCT}^{98}$ with 30 patients \\
\hline $\begin{array}{l}\text { Nebulized } \\
\text { Magnesium Sulfate } \\
\text { vs. Placebo }\end{array}$ & $\begin{array}{l}\text { FEV1 Absolute } \\
\text { End of Intervention }\end{array}$ & $\begin{array}{l}\text { WMD: }-0.03 ; 95 \% \mathrm{Cl}: \\
-0.15 \text { to } 0.09, \mathrm{I}^{2}=\mathrm{N} / \mathrm{A}\end{array}$ & $1 \mathrm{RCT}^{104}$ with 116 patients \\
\hline
\end{tabular}

$\mathrm{CI}$ = confidence interval; FEV1 = forced expiratory volume in one second; N/A = not applicable; RCT = randomized controlled trial; $\mathrm{WMD}=$ weighted mean difference 


\section{Mucolytics Versus Placebo or Management Without Mucolytics}

The effectiveness of the oral mucolytics N-Acetylcysteine and Erdosteine compared with placebo or management without mucolytics was evaluated in $4^{96,101,113,116}$ studies and 1 study ${ }^{93,}$ ${ }^{101}$ respectively. One N-Acetylcysteine study was in patients with mild ECOPD, ${ }^{113}$ the remaining studies $^{93,96,101,116}$ were in patients with moderate to severe ECOPD. Tables stratified by the severity of ECOPD can be found in the Appendix (Appendix Table G.5: mild ECOPD, Appendix Table G.6: moderate to severe COPD).

Erdosteine statistically significantly reduced repeat exacerbations at 3-month followup but not at 1-month followup compared with management without Erdosteine (low SOE). Erdosteine was associated with significantly reduced symptoms (based on the breathlessness, cough and sputum scale) at the end of the intervention but not at the longest followup. FEV1 percent predicted was statistically significantly increased in the erdosteine group compared with the management without Erdosteine group at the end of the intervention but not at the longest followup (Table 12 and Table 13). No AEs were reported in both groups.

Dyspnea and FEV1 absolute at the end of the intervention were not found to have a statistically significantly difference between the N-Acetylcysteine and the placebo group. There was no statistically significant difference found in FEV1 absolute at the end of the intervention between the N-Acetylcysteine and the placebo group in patients with mild or moderate to severe ECOPD (Table 12 and Table 13). There was no statistically significant difference observed on gastrointestinal AEs and total number of AEs (Appendix Table H.4.). ${ }^{116}$

Table 12. Comparison of oral mucolytics versus control, critical outcomes

\begin{tabular}{|c|c|c|c|c|c|}
\hline Comparison & Outcome & Findings & $\begin{array}{c}\text { Study Design } \\
\text { and Sample Size }\end{array}$ & $\begin{array}{c}\text { Rationale } \\
\text { for Strength } \\
\text { of Evidence } \\
\text { (SOE) }\end{array}$ & $\begin{array}{c}\text { Overall } \\
\text { Strength of } \\
\text { Evidence }\end{array}$ \\
\hline $\begin{array}{l}\text { N-Acetylcysteine } \\
\text { (Mucolytic) vs. } \\
\text { Placebo }\end{array}$ & $\begin{array}{l}\text { Dyspnea } \\
\text { (Numeric Scale: } \\
\text { Dyspnea) } \\
\text { End of intervention }\end{array}$ & $\begin{array}{l}\text { SMD: }-0.16 ; 95 \% \\
\text { Cl: }-0.57 \text { to } 0.25 \text {, } \\
\mathrm{I}^{2}=50.36 \%\end{array}$ & $\begin{array}{l}2 \mathrm{RCTs}^{96,116} \text { with } \\
92 \text { patients }\end{array}$ & $\begin{array}{l}\text { High ROB } \\
\text { and severe } \\
\text { imprecision }\end{array}$ & $\begin{array}{l}\text { Insufficient } \\
\text { evidence }\end{array}$ \\
\hline $\begin{array}{l}\text { N-Acetylcysteine } \\
\text { (Mucolytic) vs. } \\
\text { Placebo }\end{array}$ & $\begin{array}{l}\text { Hospital Admission } \\
\text { Longest Followup }\end{array}$ & $\begin{array}{l}\text { Rate Ratio: } 1.70 ; \\
95 \% \mathrm{Cl}: 0.80 \text { to } \\
3.63, \mathrm{I}^{2}=\mathrm{N} / \mathrm{A}\end{array}$ & $\begin{array}{l}1 \mathrm{RCT}^{96} \text { with } 44 \\
\text { patients }\end{array}$ & $\begin{array}{l}\text { High ROB } \\
\text { and severe } \\
\text { imprecision }\end{array}$ & $\begin{array}{l}\text { Insufficient } \\
\text { evidence }\end{array}$ \\
\hline $\begin{array}{l}\text { Erdosteine } \\
\text { (Mucolytic) vs. } \\
\text { Management } \\
\text { Without } \\
\text { Erdosteine }\end{array}$ & $\begin{array}{l}\text { Repeat } \\
\text { Exacerbation } \\
1 \text { month }\end{array}$ & $\begin{array}{l}\text { OR: } 0.05 ; 95 \% \mathrm{Cl}: \\
0.00 \text { to } 1.04, \\
\mathrm{I}^{2}=\mathrm{N} / \mathrm{A}\end{array}$ & $\begin{array}{l}1 \mathrm{RCT}^{93} \text { with } 40 \\
\text { patients }\end{array}$ & $\begin{array}{l}\text { High ROB } \\
\text { and severe } \\
\text { imprecision }\end{array}$ & $\begin{array}{l}\text { Insufficient } \\
\text { evidence }\end{array}$ \\
\hline $\begin{array}{l}\text { Erdosteine } \\
\text { (Mucolytic) vs. } \\
\text { Management } \\
\text { Without } \\
\text { Erdosteine }\end{array}$ & $\begin{array}{l}\text { Repeat } \\
\text { Exacerbation } \\
3 \text { months }\end{array}$ & $\begin{array}{l}\text { OR: } 0.14 ; 95 \% \mathrm{Cl}: \\
0.03 \text { to } 0.65, \\
\mathrm{I}^{2}=\mathrm{N} / \mathrm{A}\end{array}$ & $\begin{array}{l}1 \mathrm{RCT}^{93} \text { with } 40 \\
\text { patients }\end{array}$ & $\begin{array}{l}\text { High ROB } \\
\text { and } \\
\text { imprecision }\end{array}$ & $\begin{array}{l}\text { Low SOE } \\
\text { supporting } \\
\text { reduction }\end{array}$ \\
\hline $\begin{array}{l}\text { Erdosteine } \\
\text { (Mucolytic) vs. } \\
\text { Management } \\
\text { Without } \\
\text { Erdosteine }\end{array}$ & $\begin{array}{l}\text { Repeat } \\
\text { Exacerbation } \\
\text { Longest Followup }\end{array}$ & $\begin{array}{l}\text { OR: } 0.14 ; 95 \% \mathrm{Cl}: \\
0.03 \text { to } 0.65, \\
\mathrm{I}^{2}=\mathrm{N} / \mathrm{A}\end{array}$ & $\begin{array}{l}1 \mathrm{RCT}^{93} \text { with } 40 \\
\text { patients }\end{array}$ & $\begin{array}{l}\text { High ROB } \\
\text { and } \\
\text { imprecision }\end{array}$ & $\begin{array}{l}\text { Low SOE } \\
\text { supporting } \\
\text { reduction }\end{array}$ \\
\hline
\end{tabular}

$\mathrm{CI}$ = confidence interval; $\mathrm{OR}$ = odds ratio; N/A = not applicable; $\mathrm{RCT}$ = randomized controlled trial; ROB = risk of bias; $\mathrm{SMD}=$ standardized mean difference; $\mathrm{SOE}=$ strength of evidence 
Table 13. Comparison of oral mucolytics versus control, additional outcomes

\begin{tabular}{|c|c|c|c|}
\hline Comparison & Outcome & Findings & Study Design and Sample Size \\
\hline $\begin{array}{l}\text { Erdosteine } \\
\text { (Mucolytic) vs. } \\
\text { Management } \\
\text { Without } \\
\text { Erdosteine }\end{array}$ & $\begin{array}{l}\text { Symptoms BCSS } \\
\text { End of } \\
\text { Intervention }\end{array}$ & $\begin{array}{l}\text { WMD: }-1.00 ; 95 \% \mathrm{Cl}:-1.63 \text { to - } \\
0.37, \mathrm{I}^{2}=\mathrm{N} / \mathrm{A}\end{array}$ & $1 \mathrm{RCT}^{93}$ with 40 patients \\
\hline $\begin{array}{l}\text { Erdosteine } \\
\text { (Mucolytic) vs. } \\
\text { Management } \\
\text { Without } \\
\text { Erdosteine }\end{array}$ & $\begin{array}{l}\text { Symptoms BCSS } \\
\text { Longest } \\
\text { Followup }\end{array}$ & $\begin{array}{l}\text { WMD: }-0.90 ; 95 \% \mathrm{Cl}:-1.82 \text { to } 0.02 \text {, } \\
\mathrm{I}^{2}=\mathrm{N} / \mathrm{A}\end{array}$ & $1 \mathrm{RCT}^{93}$ with 40 patients \\
\hline $\begin{array}{l}\text { Mucolytics } \\
\text { (Erdosteine, N- } \\
\text { Acetylcysteine) vs. } \\
\text { Placebo or } \\
\text { Management } \\
\text { Without } \\
\text { Erdosteine }\end{array}$ & $\begin{array}{l}\text { FEV1\% } \\
\text { Predicted End } \\
\text { of Intervention }\end{array}$ & $\begin{array}{l}\text { All mucolytics: WMD: } 1.98 ; 95 \% \\
\text { Cl: } 0.51 \text { to } 3.44, I^{2}=89.12 \% \\
\text { Erdosteine: WMD: } 9.00 ; 95 \% \mathrm{Cl} \text { : } \\
3.17 \text { to } 14.83, \mathrm{I}^{2}=\mathrm{N} / \mathrm{A} ; \\
\text { Acetylcysteine: WMD: } 1.50 ; 95 \% \\
\mathrm{Cl}:-0.02 \text { to } 3.02, \mathrm{I}^{2}=\mathrm{N} / \mathrm{A}\end{array}$ & $\begin{array}{l}\text { All mucolytics: } 2 \mathrm{RCT}^{93,101} \text { with } 70 \\
\text { patients; Erdosteine: } 1 \mathrm{RCT}^{93} \text { with } \\
40 \text { patients; Acetylcysteine: } 1 \\
\text { RCT }^{101} \text { with } 30 \text { patients }\end{array}$ \\
\hline $\begin{array}{l}\text { Erdosteine } \\
\text { (Mucolytic) vs. } \\
\text { Management } \\
\text { Without } \\
\text { Erdosteine }\end{array}$ & $\begin{array}{l}\text { FEV1\% } \\
\text { Predicted } \\
\text { Longest } \\
\text { Followup }\end{array}$ & $\begin{array}{l}\text { WMD: } 8.30 ; 95 \% \mathrm{Cl}:-0.69 \text { to } \\
17.29, \mathrm{I}^{2}=\mathrm{N} / \mathrm{A}\end{array}$ & 1 RCT $^{93}$ with 40 patients \\
\hline $\begin{array}{l}\text { N-Acetylcysteine } \\
\text { (Mucolytic) vs. } \\
\text { Placebo }\end{array}$ & $\begin{array}{l}\text { FEV1 Absolute } \\
\text { End of } \\
\text { Intervention }\end{array}$ & $\begin{array}{l}\text { WMD: } 0.07 ; 95 \% \mathrm{Cl}:-0.11 \text { to } 0.25 \text {, } \\
\mathrm{I}^{2}=0.00 \%\end{array}$ & $2 \mathrm{RCT}^{96,113}$ with 125 patients \\
\hline
\end{tabular}

BCSS = Breathlessness, Cough and Sputum Scale; CI = confidence interval; FEV1 = forced expiratory volume in one second; $\mathrm{N} / \mathrm{A}=$ not applicable; $\mathrm{RCT}=$ randomized controlled trial; $\mathrm{WMD}=$ weighted mean difference

\section{Inhaled Corticosteroids With or Without Inhaled Short- and Long- Acting Bronchodilators Versus Placebo}

Four studies $^{74-76,112}$ evaluated the effectiveness of different inhaler treatments containing an inhaled corticosteroid (ICS) versus placebo, of which 3 were conducted in patients with moderate to severe ECOPD ${ }^{75,76,112}$ and 1 in mild ECOPD ${ }^{74}$ (Table 14 and Table 15).

In patients with moderate to severe ECOPD, ICS were associated with a statistically significantly higher FEV1percent predicted at the end of the intervention compared with placebo, but there was no statistically significant difference found in dyspnea, 30-day hospital admission, and need for intubation between groups (Appendix Table G.7).

In patients with moderate to severe ECOPD, a combination of ICS+SABA (budesonide+ terbutaline) was associated with a statistically significantly higher FEV1percent predicted and FEV1 absolute at the end of the intervention compared with placebo (Appendix Table G.8.).

In patients with mild ECOPD, a combination of ICS+ long-acting beta-2-agonists (LABA) (budesonide+formoterol) was not associated with a statistically significant difference in FEV1 absolute and clinical failure at the end of the intervention compared with placebo ${ }^{74}$ (Appendix Table G.9).

No statistically significant difference was found on total number of AEs, number of withdrawals and number of withdrawals due to AEs (Appendix Table H.5). Eight unspecified serious AEs were reported in the ICS group (8 in the ICS group while 9 cases in the placebo group). 
Table 14. Comparison of inhaled corticosteroids with or without inhaled short- and long-acting bronchodilators versus placebo, critical outcomes

\begin{tabular}{|c|c|c|c|c|c|}
\hline Comparison & Outcome & Findings & $\begin{array}{c}\text { Study Design } \\
\text { and Sample } \\
\text { Size }\end{array}$ & $\begin{array}{l}\text { Rationale for } \\
\text { Strength of } \\
\text { Evidence } \\
\text { (SOE) }\end{array}$ & $\begin{array}{c}\text { Overall } \\
\text { Strength of } \\
\text { Evidence }\end{array}$ \\
\hline \multirow[t]{3}{*}{$\begin{array}{l}\text { ICS (Budesonide) vs. } \\
\text { Placebo }\end{array}$} & $\begin{array}{l}\text { Dyspnea } \\
\text { (Numeric Scale: } \\
\text { Modified Borg) } \\
\text { End of } \\
\text { Intervention }\end{array}$ & $\begin{array}{l}\text { WMD: }-0.10 ; \\
95 \% \mathrm{Cl}:-0.92 \\
\text { to } 0.22, I^{2}=\mathrm{N} / \mathrm{A}\end{array}$ & $\begin{array}{l}1 \mathrm{RCT}^{76} \text { with } \\
137 \text { patients }\end{array}$ & $\begin{array}{l}\text { High ROB and } \\
\text { severe } \\
\text { imprecision }\end{array}$ & $\begin{array}{l}\text { Insufficient } \\
\text { evidence }\end{array}$ \\
\hline & $\begin{array}{l}\text { Need for } \\
\text { Intubation } \\
\text { End of } \\
\text { Intervention }\end{array}$ & $\begin{array}{l}\text { OR: } 0.31 ; 95 \% \\
\text { Cl: } 0.01 \text { to } \\
7.63, I^{2}=\mathrm{N} / \mathrm{A}\end{array}$ & $\begin{array}{l}1 \mathrm{RCT}^{76} \text { with } \\
137 \text { patients }\end{array}$ & $\begin{array}{l}\text { High ROB and } \\
\text { severe } \\
\text { imprecision }\end{array}$ & $\begin{array}{l}\text { Insufficient } \\
\text { evidence }\end{array}$ \\
\hline & $\begin{array}{l}\text { Hospital } \\
\text { Admission } \\
30 \text { Days }\end{array}$ & $\begin{array}{l}\text { Rate Ratio: } \\
0.63 ; 95 \% \mathrm{Cl} \text { : } \\
0.20 \text { to } 1.91 \\
\mathrm{I}^{2}=\mathrm{N} / \mathrm{A}\end{array}$ & $\begin{array}{l}1 \mathrm{RCT}^{75} \text { with } \\
106 \text { patients }\end{array}$ & $\begin{array}{l}\text { High ROB and } \\
\text { severe } \\
\text { imprecision }\end{array}$ & $\begin{array}{l}\text { Insufficient } \\
\text { evidence }\end{array}$ \\
\hline $\begin{array}{l}\text { ICS+ LABA } \\
\text { (Budesonide } \\
\text { +Formoterol) vs. } \\
\text { Placebo } \\
\end{array}$ & $\begin{array}{l}\text { Clinical failure } \\
\text { End of } \\
\text { Intervention }\end{array}$ & $\begin{array}{l}\text { OR: } 1.00 ; 95 \% \\
\text { Cl: } 0.06 \text { to } \\
17.62, I^{2}=N / A\end{array}$ & $\begin{array}{l}1 \mathrm{RCT}^{74} \text { with } \\
30 \text { patients }\end{array}$ & $\begin{array}{l}\text { High ROB and } \\
\text { severe } \\
\text { imprecision }\end{array}$ & $\begin{array}{l}\text { Insufficient } \\
\text { evidence }\end{array}$ \\
\hline
\end{tabular}

CI = confidence interval; ICS = inhaled corticosteroid; LABA = Long-acting beta-agonist; N/A = not applicable; OR = odds ratio; $\mathrm{RCT}=$ randomized controlled trial; $\mathrm{ROB}=$ risk of bias; $\mathrm{SOE}=$ strength of evidence; $\mathrm{WMD}=$ weighted mean difference

Table 15. Comparison of inhaled corticosteroids with or without inhaled short- and long-acting bronchodilators versus placebo, additional outcomes

\begin{tabular}{|c|c|c|c|}
\hline Comparison & Outcome & Findings & $\begin{array}{l}\text { Study Design and Sample } \\
\text { Size }\end{array}$ \\
\hline $\begin{array}{l}\text { ICS (Budesonide) vs. } \\
\text { Placebo }\end{array}$ & $\begin{array}{l}\text { FEV1\% Predicted } \\
\text { End of Intervention }\end{array}$ & $\begin{array}{l}\text { WMD: } 10.10 ; 95 \% \mathrm{Cl}: 4.23 \\
\text { to } 15.97, \mathrm{I}^{2}=\mathrm{N} / \mathrm{A}\end{array}$ & $1 \mathrm{RCT}^{75}$ with 106 patients \\
\hline $\begin{array}{l}\text { ICS+SABA } \\
\text { (Budesonide+ } \\
\text { Terbutaline) vs. } \\
\text { Placebo }\end{array}$ & $\begin{array}{l}\text { FEV1\% Predicted } \\
\text { End of Intervention }\end{array}$ & $\begin{array}{l}\text { WMD: } 8.30 ; 95 \% \mathrm{Cl}: 2.92 \text { to } \\
13.68, \mathrm{I}^{2}=\mathrm{N} / \mathrm{A}\end{array}$ & 1 RCT ${ }^{112}$ with 40 patients \\
\hline $\begin{array}{l}\text { ICS+SABA } \\
\text { (Budesonide } \\
\text { +Terbutaline) vs. } \\
\text { Placebo }\end{array}$ & $\begin{array}{l}\text { FEV1 Absolute } \\
\text { End of Intervention }\end{array}$ & $\begin{array}{l}\text { WMD: } 0.35 ; 95 \% \mathrm{Cl}: 0.05 \text { to } \\
0.65, \mathrm{I}^{2}=\mathrm{N} / \mathrm{A}\end{array}$ & 1 RCT ${ }^{112}$ with 40 patients \\
\hline $\begin{array}{l}\text { ICS+ LABA } \\
\text { (Budesonide } \\
\text { +Formoterol) vs. } \\
\text { Placebo }\end{array}$ & $\begin{array}{l}\text { FEV1 Absolute } \\
\text { End of Intervention }\end{array}$ & $\begin{array}{l}\text { WMD: } 0.18 ; 95 \% \mathrm{Cl}:-0.17 \\
\text { to } 0.53,1^{2}=\mathrm{N} / \mathrm{A}\end{array}$ & $1 \mathrm{RCT}^{74}$ with 30 patients \\
\hline
\end{tabular}

CI = confidence interval; FEV1 = forced expiratory volume in one second; ICS = inhaled corticosteroid; LABA = Long-acting beta-agonist; N/A = not applicable; RCT = randomized controlled trial; ROB = risk of bias; SABA = Short acting beta agonists; $\mathrm{SOE}=$ strength of evidence; $\mathrm{WMD}=$ weighted mean difference

\section{Inhaled Antibiotics Versus Placebo}

One study ${ }^{89}$ evaluated the effectiveness of inhaled (nebulized) gentamicin compared with placebo in patients with moderate to severe ECOPD (Table 16). Inhaled gentamicin was associated with a statistically significantly higher FEV1 absolute at the end of the intervention compared with placebo. 
Table 16. Comparison of inhaled antibiotics versus placebo

\begin{tabular}{|l|l|l|}
\hline \multicolumn{1}{|c|}{ Outcome } & \multicolumn{1}{|c|}{ Findings } & Study Design and Sample Size \\
\hline $\begin{array}{l}\text { FEV1 Absolute } \\
\text { End of Intervention }\end{array}$ & WMD: $3.49 ; 95 \% \mathrm{Cl}: 1.49$ to $5.49, \mathrm{I}^{2}=\mathrm{N} / \mathrm{A}$ & $1 \mathrm{RCT}^{89}$ with 86 patients \\
\hline
\end{tabular}

$\mathrm{CI}$ = confidence interval; FEV1 = forced expiratory volume in one second; N/A = not applicable; RCT = randomized controlled trial; WMD = weighted mean difference

\section{Long-Acting Muscarinic Antagonists Versus Placebo}

One crossover RCT ${ }^{66}$ compared inhaled oxitropium, a long-acting muscarinic antagonist (LAMA) to placebo in 50 patients with ECOPD and heart disease (ischemic heart disease and/or arrhythmias). There was a statistically significant improvement of FEV1 absolute compared with placebo at the end of intervention $(\mathrm{p}<0.05)$ (Table 17).

Table 17. Long-acting muscarinic antagonists versus placebo

\begin{tabular}{|l|l|l|}
\hline \multicolumn{1}{|c|}{ Outcome } & \multicolumn{1}{c|}{ Findings } & Study Design and Sample Size \\
\hline $\begin{array}{l}\text { FEV1 Absolute } \\
\text { End of } \\
\text { Intervention }\end{array}$ & 0.16 vs. $0.05, \mathrm{p}<0.05$ & 1 crossover RCT $^{66}$ with 50 patients \\
\hline
\end{tabular}

FEV1 = forced expiratory volume in one second; RCT = randomized controlled trial

\section{5-Lipoxygenase Inhibitor Versus Placebo}

The effectiveness of (oral) zileuton, a 5-lipoxygenase inhibitor, was evaluated in one study of patients with moderate to severe ECOPD. ${ }^{107}$ 5-Lipoxygenase inhibitor was associated with a statistically significantly increased FEV1 absolute at the end of the intervention and at the longest followup compared with placebo. There were no statistically significant differences observed in FEV1 percent predicted, mortality, hospital admissions, clinical resolution and need for intubation between the zileuton and placebo group (Table 18 and Table 19). There was no statistically significant difference found on AEs between 5-lipoxygenase inhibitor and placebo (Appendix Table H.6.). 17 cases of unspecified serious AEs were reported in the 5-lipoxygenase inhibitor group while 21 cases in the placebo group $(\mathrm{p}=0.40)$.

Table 18. Comparison of 5-lipoxygenase inhibitor (zileuton) versus placebo, critical outcomes

\begin{tabular}{|l|l|l|l|l|}
\hline \multicolumn{1}{|c|}{ Outcome } & \multicolumn{1}{|c|}{ Findings } & $\begin{array}{l}\text { Study Design and } \\
\text { Sample Size }\end{array}$ & \multicolumn{1}{|c|}{$\begin{array}{c}\text { Rationale for } \\
\text { Strength of } \\
\text { Evidence (SOE) }\end{array}$} & $\begin{array}{l}\text { Overall Strength of } \\
\text { Evidence }\end{array}$ \\
\hline $\begin{array}{l}\text { Mortality } \\
\text { End of Intervention }\end{array}$ & $\begin{array}{l}\text { OR: } 0.48 ; 95 \% \mathrm{Cl}: \\
0.04 \text { to } 5.48, \mathrm{I}^{2}=\mathrm{N} / \mathrm{A}\end{array}$ & $\begin{array}{l}1 \mathrm{RCT}^{107} \text { with } 119 \\
\text { patients }\end{array}$ & $\begin{array}{l}\text { High ROB and } \\
\text { severe imprecision }\end{array}$ & Insufficient evidence \\
\hline $\begin{array}{l}\text { Hospital Admission } \\
\text { Longest Followup }\end{array}$ & $\begin{array}{l}\text { OR: } 0.98 ; 95 \% \mathrm{Cl}: \\
0.32 \text { to } 2.99, \mathrm{I}^{2}=\mathrm{N} / \mathrm{A}\end{array}$ & $\begin{array}{l}1 \mathrm{RCT}^{107} \text { with } 119 \\
\text { patients }\end{array}$ & $\begin{array}{l}\text { High ROB and } \\
\text { severe imprecision }\end{array}$ & Insufficient evidence \\
\hline $\begin{array}{l}\text { Clinical Failure } \\
\text { Longest Followup }\end{array}$ & $\begin{array}{l}\text { OR: } 0.82 ; 95 \% \mathrm{Cl}: \\
0.36 \text { to } 1.87, \mathrm{I}^{2}=\mathrm{N} / \mathrm{A}\end{array}$ & $\begin{array}{l}1 \mathrm{RCT}^{107} \text { with } 119 \\
\text { patients }\end{array}$ & $\begin{array}{l}\text { High ROB and } \\
\text { severe imprecision }\end{array}$ & Insufficient evidence \\
\hline $\begin{array}{l}\text { Need for Intubation } \\
\text { End of Intervention }\end{array}$ & $\begin{array}{l}\text { OR: } 0.98 ; 95 \% \mathrm{Cl}: \\
0.06 \text { to } 16.09, \mathrm{I}^{2}=\mathrm{N} / \mathrm{A}\end{array}$ & $\begin{array}{l}1 \mathrm{RCT} \mathrm{T}^{107} \text { with } 119 \\
\text { patients }\end{array}$ & $\begin{array}{l}\text { High ROB and } \\
\text { severe imprecision }\end{array}$ & Insufficient evidence \\
\hline
\end{tabular}

$\mathrm{CI}$ = confidence interval; N/A = not applicable; $\mathrm{OR}=$ odds ratio; $\mathrm{RCT}$ = randomized controlled trial; $\mathrm{ROB}=$ risk of bias; $\mathrm{SOE}=$ strength of evidence

Table 19. Comparison of 5-lipoxygenase inhibitor (zileuton) versus placebo, additional outcomes

\begin{tabular}{|l|l|l|}
\hline \multicolumn{1}{|c|}{ Outcome } & \multicolumn{1}{|c|}{ Findings } & \multicolumn{1}{c|}{ Study Design and Sample Size } \\
\hline $\begin{array}{l}\text { FEV1\% Predicted } \\
\text { Longest Followup }\end{array}$ & WMD: 4:30; $95 \%$ Cl: -2.28 to $10.88, \mathrm{I}^{2}=\mathrm{N} / \mathrm{A}$ & $1 \mathrm{RCT} \mathrm{T}^{107}$ with 119 patients \\
\hline $\begin{array}{l}\text { FEV1 Absolute } \\
\text { End of Intervention }\end{array}$ & WMD: $0.17 ; 95 \% \mathrm{Cl}: 0.13$ to $0.21, \mathrm{I}^{2}=\mathrm{N} / \mathrm{A}$ & $1 \mathrm{RCT}^{107}$ with 119 patients \\
\hline
\end{tabular}




\begin{tabular}{|l|l|c|}
\hline \multicolumn{1}{|c|}{ Outcome } & \multicolumn{1}{|c|}{ Findings } & Study Design and Sample Size \\
\hline $\begin{array}{l}\text { FEV1 Absolute } \\
\text { Longest Followup }\end{array}$ & WMD: $0.23 ; 95 \% \mathrm{Cl}: 0.02$ to $0.44, \mathrm{I}^{2}=\mathrm{N} / \mathrm{A}$ & $1 \mathrm{RCT}^{107}$ with 119 patients \\
\hline
\end{tabular}

CI = confidence interval; FEV1 = forced expiratory volume in one second; N/A = not applicable; RCT = randomized controlled trial; WMD = weighted mean difference

\section{Statin (Simvastatin) Versus Management Without Statin}

Statin therapy with simvastatin was compared with management without statin in one study, 122 which found a statistically significantly increased FEV1percent predicted at the end of the intervention in the statin group (Table 20).

Table 20. Comparison of statin versus management without statin

\begin{tabular}{|l|l|l|}
\hline \multicolumn{1}{|c|}{ Outcome } & \multicolumn{1}{|c|}{ Findings } & \multicolumn{1}{|c|}{ Study Design and Sample Size } \\
\hline FEV1\% Predicted & WMD: $0.68,95 \% \mathrm{Cl}: 0.38$ to 0.98, & $1 \mathrm{RCT}^{122}$ with 60 patients \\
End of Intervention & $\mathrm{I}^{2}=\mathrm{N} / \mathrm{A}$ & \\
\hline
\end{tabular}

$\mathrm{CI}$ = confidence interval; FEV1 = Forced expiratory volume in one second; N/A = not applicable; RCT = randomized controlled trial; WMD = weighted mean difference

\section{Nonpharmacologic Therapies}

\section{Chest Physiotherapy (Breathing Technique; Vibration/Percussion; Positive Expiratory Pressure) Versus Management Without Chest Physiotherapy}

\section{Chest Physiotherapy Using Breathing Technique}

Three studies ${ }^{88,106,110}$ evaluated the effectiveness of chest physiotherapy using a specific breathing technique compared with management without chest physiotherapy in patients with moderate to severe ECOPD (Table 21 and Table 22).

Chest physiotherapy using a specific breathing technique significantly reduced hospital admission at the longest followup compared with management without chest physiotherapy (low SOE). There were no statistically significantly differences between the intervention and management without chest physiotherapy group for other outcomes, including: mortality at end of followup, mortality at the longest followup, dyspnea based on a questionnaire, dyspnea based on a numeric scale, other symptoms, quality of life, and FEV1 predicted. No statistically significant difference was found in withdrawals, and withdrawals due to AEs ${ }^{106,110}$ (Appendix Table H.7.). The intervention group ${ }^{106}$ reported cardiovascular AEs (3 cases), respiratory AEs (7 cases), and general internal medicine AEs (5 cases).

Table 21. Comparison of chest physiotherapy using breathing technique versus management without chest physiotherapy, critical outcomes

\begin{tabular}{|l|l|l|l|l|}
\hline \multicolumn{1}{|c|}{ Outcome } & \multicolumn{1}{|c|}{ Findings } & $\begin{array}{l}\text { Study Design and } \\
\text { Sample Size }\end{array}$ & \multicolumn{1}{|c|}{$\begin{array}{c}\text { Rationale for } \\
\text { Strength of } \\
\text { Evidence (SOE) }\end{array}$} & $\begin{array}{l}\text { Overall Strength of } \\
\text { Evidence }\end{array}$ \\
\hline $\begin{array}{l}\text { Mortality } \\
\text { End of intervention }\end{array}$ & $\begin{array}{l}\text { OR: } 0.97 ; 95 \% \mathrm{Cl}: \\
0.06 \text { to } 16.20, \mathrm{I}^{2}= \\
\text { N/A }\end{array}$ & $\begin{array}{l}1 \mathrm{RCT}^{110} \text { with } 59 \\
\text { patients }\end{array}$ & $\begin{array}{l}\text { High ROB and } \\
\text { severe imprecision }\end{array}$ & Insufficient evidence \\
\hline $\begin{array}{l}\text { Mortality } \\
\text { Longest Followup }\end{array}$ & $\begin{array}{l}\text { OR: } 0.90 ; 95 \% \mathrm{Cl}: \\
0.54 \text { to } 1.49, \mathrm{I}^{2}=\mathrm{N} / \mathrm{A}\end{array}$ & $\begin{array}{l}1 \mathrm{RCT}^{106} \text { with } 522 \\
\text { patients }\end{array}$ & $\begin{array}{l}\text { Intermediate ROB } \\
\text { and imprecision }\end{array}$ & $\begin{array}{l}\text { Low SOE } \\
\text { supporting no } \\
\text { difference }\end{array}$ \\
\hline
\end{tabular}




\begin{tabular}{|c|c|c|c|c|}
\hline Outcome & Findings & $\begin{array}{l}\text { Study Design and } \\
\text { Sample Size }\end{array}$ & $\begin{array}{c}\text { Rationale for } \\
\text { Strength of } \\
\text { Evidence (SOE) }\end{array}$ & $\begin{array}{l}\text { Overall Strength of } \\
\text { Evidence }\end{array}$ \\
\hline $\begin{array}{l}\text { Dyspnea } \\
\text { (Questionnaire: } \\
\text { MRC) } \\
\text { End of Intervention }\end{array}$ & $\begin{array}{l}\text { WMD: } 0.40 ; 95 \% \mathrm{Cl}: \\
-0.24 \text { to } 1.04, \mathrm{I}^{2}= \\
\text { N/A }\end{array}$ & $\begin{array}{l}1 \mathrm{RCT}^{110} \text { with } 59 \\
\text { patients }\end{array}$ & $\begin{array}{l}\text { High } \mathrm{ROB} \text { and } \\
\text { severe imprecision }\end{array}$ & Insufficient evidence \\
\hline $\begin{array}{l}\text { Dyspnea } \\
\text { (Numeric Scale: } \\
\text { VAS, Borg) } \\
\text { End of Intervention }\end{array}$ & $\begin{array}{l}\text { SMD: }-0.42 ; 95 \% \\
\text { Cl: }-0.89 \text { to } 0.05 \text {, } \\
\mathrm{I}^{2}=98.92 \%\end{array}$ & $\begin{array}{l}2 \mathrm{RCT}^{88,110} \text { with } 119 \\
\text { patients }\end{array}$ & $\begin{array}{l}\text { High ROB, } \\
\text { inconsistency and } \\
\text { severe imprecision }\end{array}$ & Insufficient evidence \\
\hline $\begin{array}{l}\text { QoL(SGRQ) } \\
\text { End of Intervention }\end{array}$ & $\begin{array}{l}\text { SMD: }-0.02 ; 95 \% \\
\text { Cl: }-0.18 \text { to } 0.14 \text {, } \\
\mathrm{I}^{2}=0.00 \%\end{array}$ & $\begin{array}{l}2 \text { RCTs } \\
581 \text { patients }\end{array}$ & $\begin{array}{l}\text { High ROB and } \\
\text { severe imprecision }\end{array}$ & Insufficient evidence \\
\hline $\begin{array}{l}\text { Hospital Admission } \\
\text { Longest Followup }\end{array}$ & $\begin{array}{l}\text { Rate Ratio: } 0.91 ; \\
95 \% \mathrm{Cl}: 0.83 \text { to } \\
0.99, \mathrm{I}^{2}=79.5 \%\end{array}$ & $\begin{array}{l}2 \mathrm{RCTs}^{106,110} \text { with } \\
581 \text { patients }\end{array}$ & $\begin{array}{l}\text { High ROB, and } \\
\text { inconsistency }\end{array}$ & $\begin{array}{l}\text { Low SOE } \\
\text { supporting reduction }\end{array}$ \\
\hline
\end{tabular}

$\mathrm{CI}=$ confidence interval; MRC = Medical Research Council; N/A = not applicable; $\mathrm{OR}=$ odds ratio; QoL = quality of life; RCT = randomized controlled trial; ROB = risk of bias; SGRQ = St. George Respiratory Questionnaire; SMD = standardized mean difference; SOE = strength of evidence; VAS = visual analog scale; WMD = weighted mean difference

Table 22. Comparison of chest physiotherapy using breathing technique versus management without chest physiotherapy, additional outcomes

\begin{tabular}{|l|l|l|}
\hline \multicolumn{1}{|c|}{ Outcome } & \multicolumn{1}{|c|}{ Findings } & \multicolumn{1}{c|}{ Study Design and Sample Size } \\
\hline $\begin{array}{l}\text { Other Symptoms } \\
\text { (SGRQ, symptom } \\
\text { score) }\end{array}$ & $\begin{array}{l}\text { WMD: }-0.02 ; 95 \% \mathrm{Cl}:-3.99 \text { to } \\
\text { End of Intervention }\end{array}$ & $1 \mathrm{RCT}^{2}=\mathrm{N} / \mathrm{A}$ \\
\hline $\begin{array}{l}\text { Symptoms BCSS } \\
\text { End of Intervention }\end{array}$ & $\begin{array}{l}\text { WMD: }-0.06 ; 95 \% \mathrm{Cl} \text { : }-0.56 \text { to } \\
0.44, \mathrm{I}^{2}=\mathrm{N} / \mathrm{A}\end{array}$ & $1 \mathrm{RCT}^{106}$ with 522 patients \\
\hline FEV1\% Predicted & WMD: $6.50 ; 95 \% \mathrm{Cl}:-8.46$ to & $1 \mathrm{RCT}^{110}$ with 59 patients \\
End of Intervention & $21.46, \mathrm{I}^{2}=\mathrm{N} / \mathrm{A}$ & \\
\hline
\end{tabular}

BCSS = breathlessness, cough and sputum scale; CI = confidence interval; FEV1 = forced expiratory volume in one second; N/A = not applicable; RCT = randomized controlled trial; SGRQ = St. George respiratory questionnaire; SMD = standardized mean difference; WMD = weighted mean difference

\section{Chest Physiotherapy Using Vibration, Percussion, or Massage}

Three studies ${ }^{67,102,103}$ evaluated the effectiveness of chest physiotherapy using vibration, percussion, or massage compared with management without chest physiotherapy in patients with moderate to severe ECOPD (Table 23 and Table 24).

There was no difference between the intervention and management without chest physiotherapy group for all evaluated outcomes including: dyspnea at the end of the intervention and at the longest followup, FEV1percent predicted at the end of the intervention and at the longest followup, FEV1 absolute at the end of the intervention, and 6-minute walking distance at the end of the intervention. No patient withdrew during the study (Appendix Table H.8.).

Table 23. Comparison of chest physiotherapy using vibration, percussion, or massage versus management without chest physiotherapy, critical outcomes

\begin{tabular}{|c|c|c|c|c|}
\hline Outcome & Findings & $\begin{array}{l}\text { Study Design and } \\
\text { Sample Size }\end{array}$ & $\begin{array}{c}\text { Rationale for } \\
\text { Strength of } \\
\text { Evidence (SOE) }\end{array}$ & $\begin{array}{l}\text { Overall Strength } \\
\text { of Evidence }\end{array}$ \\
\hline $\begin{array}{l}\text { Dyspnea } \\
\text { (Questionnaire: MRC; } \\
\text { MMRC) } \\
\text { End of Intervention }\end{array}$ & $\begin{array}{l}\text { SMD: } 0.15 ; 95 \% \mathrm{Cl}: \\
-0.29 \text { to } 0.60, \mathrm{I}^{2}= \\
0.00 \%\end{array}$ & $\begin{array}{l}2 \text { RCTs } 102,103 \text { with } \\
80 \text { patients }\end{array}$ & $\begin{array}{l}\text { High ROB and } \\
\text { severe } \\
\text { imprecision }\end{array}$ & $\begin{array}{l}\text { Insufficient } \\
\text { evidence }\end{array}$ \\
\hline
\end{tabular}




\begin{tabular}{|l|l|l|l|l|}
\hline \multicolumn{1}{|c|}{ Outcome } & \multicolumn{1}{|c|}{ Findings } & $\begin{array}{l}\text { Study Design and } \\
\text { Sample Size }\end{array}$ & $\begin{array}{l}\text { Rationale for } \\
\text { Strength of } \\
\text { Evidence (SOE) }\end{array}$ & $\begin{array}{l}\text { Overall Strength } \\
\text { of Evidence }\end{array}$ \\
\hline $\begin{array}{l}\text { Dyspnea } \\
\text { Questionnaire: MMRC) } \\
\text { Longest Followup }\end{array}$ & $\begin{array}{l}\text { WMD: }-0.24 ; 95 \% \\
\mathrm{Cl}:-0.73 \text { to } 0.25, \mathrm{I}^{2}=\end{array}$ & $\begin{array}{l}1 \mathrm{RCT}^{103} \text { with } 50 \\
\text { patients }\end{array}$ & $\begin{array}{l}\text { High ROB and } \\
\text { severe } \\
\text { imprecision }\end{array}$ & $\begin{array}{l}\text { Insufficient } \\
\text { evidence }\end{array}$ \\
\hline
\end{tabular}

CI = confidence interval; MMRC = Modified Medical Research Council; MRC = Medical Research Council; N/A = not applicable; RCT = randomized controlled trial; ROB = risk of bias; SMD = standardized mean difference; SOE = strength of evidence; $\mathrm{WMD}$ = weighted mean difference

Table 24. Comparison of chest physiotherapy using vibration, percussion, or massage versus management without chest physiotherapy, additional outcomes

\begin{tabular}{|l|l|l|}
\hline \multicolumn{1}{|c|}{ Outcome } & \multicolumn{1}{|c|}{ Findings } & Study Design and Sample Size \\
\hline $\begin{array}{l}\text { FEV1\% Predicted } \\
\text { End of Intervention }\end{array}$ & WMD: $4.88 ; 95 \% \mathrm{Cl}:-0.37$ to $10.12, \mathrm{I}^{2}=87.37 \%$ & 2 RCTs ${ }^{102,103}$ with 80 patients \\
\hline $\begin{array}{l}\text { FEV1\% Predicted } \\
\text { Longest Followup }\end{array}$ & WMD: $0.00 ; 95 \% \mathrm{Cl}:-5.98$ to $5.98, \mathrm{I}^{2}=\mathrm{N} / \mathrm{A}$ & $1 \mathrm{RCT}^{103}$ with 50 patients \\
\hline $\begin{array}{l}\text { FEV1 Absolute } \\
\text { End of Intervention }\end{array}$ & WMD: $0.00 ; 95 \% \mathrm{Cl}:-0.45$ to $0.45, \mathrm{I}^{2}=\mathrm{N} / \mathrm{A}$ & $1 \mathrm{RCT}^{103}$ with 30 patients \\
\cline { 2 - 3 } & $\begin{array}{l}0.9(\mathrm{SD}: 0.5) \text { vS. } 0.9 \text { (SD: } 0.5), \mathrm{p}=\mathrm{non}- \\
\text { statistically significant }\end{array}$ & 1 crossover RCT ${ }^{67}$ with 24 patients \\
\hline $\begin{array}{l}\text { 6MWD } \\
\text { End of Intervention }\end{array}$ & WMD: $56.20 ; 95 \% \mathrm{Cl}:-8.18$ to $120.58, \mathrm{I}^{2}=\mathrm{N} / \mathrm{A}$ & $1 \mathrm{RCT}^{102}$ with 30 patients \\
\hline
\end{tabular}

$\mathrm{CI}$ = confidence interval; FEV1 = forced expiratory volume in one second; N/A = not applicable; RCT = randomized controlled trial; $\mathrm{SD}=$ standard deviation; $\mathrm{WMD}=$ weighted mean difference

\section{Chest Physiotherapy Using Positive Expiratory Pressure}

One study ${ }^{97}$ evaluated the effectiveness of chest physiotherapy using positive expiratory pressure compared with management without positive expiratory pressure in patients with moderate to severe ECOPD (Table 25 and Table 26).

There was no difference between the intervention and management without positive expiratory pressure group for all evaluated outcomes including: mortality at the end of the intervention and at the longest followup, dyspnea at the end of the intervention and at the longest followup, FEV1 predicted at the end of the intervention and at the longest followup, repeat exacerbations at the end of the intervention, 6-minute walking distance at the end of the intervention, and quality of life at the longest followup.

No statistically significant difference on withdrawals, withdrawals due to AEs, and total number of AEs was found (Appendix Table H.9.). Serious AEs (serious clinical deterioration) were reported in 9 patients in the chest physiotherapy compared with 6 patients in the management without positive expiratory pressure group $(\mathrm{p}=0.40)$.

Table 25. Comparison of chest physiotherapy using positive expiratory pressure versus management without positive expiratory pressure, critical outcomes

\begin{tabular}{|c|c|c|c|c|}
\hline Outcome & Findings & $\begin{array}{l}\text { Study Design and } \\
\text { Sample Size }\end{array}$ & $\begin{array}{c}\text { Rationale for } \\
\text { Strength of } \\
\text { Evidence (SOE) }\end{array}$ & $\begin{array}{l}\text { Overall Strength of } \\
\text { Evidence }\end{array}$ \\
\hline $\begin{array}{l}\text { Mortality } \\
\text { End of Intervention }\end{array}$ & $\begin{array}{l}\text { OR: } 1.00 ; 95 \% \mathrm{Cl}: \\
0.06 \text { to } 16.48, \mathrm{I}^{2}= \\
\text { N/A }\end{array}$ & $\begin{array}{l}1 \mathrm{RCT}^{97} \text { with } 92 \\
\text { patients }\end{array}$ & Severe imprecision & $\begin{array}{l}\text { Low SOE supporting } \\
\text { no difference }\end{array}$ \\
\hline $\begin{array}{l}\text { Mortality } \\
\text { Longest Followup }\end{array}$ & $\begin{array}{l}\text { OR: } 1.58 ; 95 \% \mathrm{Cl}: \\
0.41 \text { to } 6.00, \mathrm{I}^{2}=\mathrm{N} / \mathrm{A}\end{array}$ & $\begin{array}{l}1 \mathrm{RCT}^{97} \text { with } 92 \\
\text { patients }\end{array}$ & Severe imprecision & $\begin{array}{l}\text { Low SOE supporting } \\
\text { no difference }\end{array}$ \\
\hline $\begin{array}{l}\text { Dyspnea } \\
\text { (Questionnaire: } \\
\text { MMRC) } \\
\text { End of Intervention }\end{array}$ & $\begin{array}{l}\text { WMD: } 0.40 ; 95 \% \mathrm{Cl} \text { : } \\
-0.16 \text { to } 0.96, \mathrm{I}^{2}= \\
\text { N/A }\end{array}$ & $\begin{array}{l}1 \mathrm{RCT}^{97} \text { with } 92 \\
\text { patients }\end{array}$ & Severe imprecision & $\begin{array}{l}\text { Low SOE supporting } \\
\text { no difference }\end{array}$ \\
\hline
\end{tabular}




\begin{tabular}{|c|c|c|c|c|}
\hline Outcome & Findings & $\begin{array}{l}\text { Study Design and } \\
\text { Sample Size }\end{array}$ & $\begin{array}{c}\text { Rationale for } \\
\text { Strength of } \\
\text { Evidence (SOE) }\end{array}$ & $\begin{array}{l}\text { Overall Strength of } \\
\text { Evidence }\end{array}$ \\
\hline $\begin{array}{l}\text { Dyspnea } \\
\text { (Questionnaire: } \\
\text { MMRC) } \\
\text { Longest Followup }\end{array}$ & $\begin{array}{l}\text { WMD: } 0.50 ; 95 \% \mathrm{Cl}: \\
-0.06 \text { to } 1.06, \mathrm{I}^{2}= \\
\text { N/A }\end{array}$ & $\begin{array}{l}1 \mathrm{RCT}^{97} \text { with } 92 \\
\text { patients }\end{array}$ & Severe imprecision & $\begin{array}{l}\text { Low SOE supporting } \\
\text { no difference }\end{array}$ \\
\hline $\begin{array}{l}\text { Repeat } \\
\text { Exacerbation End } \\
\text { of Intervention }\end{array}$ & $\begin{array}{l}\text { OR: } 1.00 ; 95 \% \mathrm{Cl}: \\
0.06 \text { to } 16.48, \mathrm{I}^{2}= \\
\text { N/A }\end{array}$ & $\begin{array}{l}1 \mathrm{RCT}^{97} \text { with } 92 \\
\text { patients }\end{array}$ & Severe imprecision & $\begin{array}{l}\text { Low SOE supporting } \\
\text { no difference }\end{array}$ \\
\hline $\begin{array}{l}\text { Repeat } \\
\text { Exacerbation } \\
\text { Longest Followup }\end{array}$ & $\begin{array}{l}\text { Rate Ratio: } 1.05 ; \\
95 \% 0.69 \text { to } 1.59, \\
\mathrm{I}^{2}=\text { N/A }\end{array}$ & $\begin{array}{l}1 \mathrm{RCT}^{97} \text { with } 92 \\
\text { patients }\end{array}$ & Severe imprecision & $\begin{array}{l}\text { Low SOE supporting } \\
\text { no difference }\end{array}$ \\
\hline $\begin{array}{l}\text { QoL(SGRQ) } \\
\text { Longest Followup }\end{array}$ & $\begin{array}{l}\text { WMD: }-1.50 ; 95 \% \\
\text { Cl: }-5.99 \text { to } 8.99,1^{2}= \\
\text { N/A }\end{array}$ & $\begin{array}{l}1 \mathrm{RCT}^{97} \text { with } 92 \\
\text { patients }\end{array}$ & Severe imprecision & $\begin{array}{l}\text { Low SOE supporting } \\
\text { no difference }\end{array}$ \\
\hline
\end{tabular}

CI = confidence interval; MMRC: Modified Medical Research Council Scale N/A = not applicable; OR = odds ratio; RCT = randomized controlled trial; QoL = quality of life; ROB = risk of bias; SGRQ = St. George Respiratory Questionnaire; WMD = weighted mean difference

Table 26. Comparison of chest physiotherapy using positive expiratory pressure versus management without positive expiratory pressure, additional outcomes

\begin{tabular}{|l|l|l|}
\hline \multicolumn{1}{|c|}{ Outcome } & \multicolumn{1}{|c|}{ Findings } & \multicolumn{1}{|c|}{ Study Design and Sample Size } \\
\hline $\begin{array}{l}\text { FEV1\% Predicted } \\
\text { End of Intervention }\end{array}$ & WMD: $-0.30 ; 95 \% \mathrm{Cl}:-4.18$ to $3.58, \mathrm{I}^{2}=\mathrm{N} / \mathrm{A}$ & $1 \mathrm{RCT}^{97}$ with 92 patients \\
\hline $\begin{array}{l}\text { FEV1\% Predicted } \\
\text { Longest Followup }\end{array}$ & WMD: $-1.30 ; 95 \% \mathrm{Cl}:-7.30$ to $4.70, \mathrm{I}^{2}=\mathrm{N} / \mathrm{A}$ & $1 \mathrm{RCT}^{97}$ with 92 patients \\
\hline $\begin{array}{l}\text { 6MWD } \\
\text { End of Intervention }\end{array}$ & $\begin{array}{l}\text { WMD: }-26.00 ; 95 \% \mathrm{Cl}:-89.61 \text { to } 37.62, \mathrm{I}^{2}= \\
\text { N/A }\end{array}$ & $1 \mathrm{RCT}^{97}$ with 92 patients \\
\hline $\begin{array}{l}\text { 6MWD } \\
\text { Longest Followup }\end{array}$ & \begin{tabular}{l} 
WMD: $-4.00 ; 95 \% \mathrm{Cl}:-82.49$ to $74.49, \mathrm{I}^{2}=$ \\
\hline $\begin{array}{l}\text { Symptoms BCSS } \\
\text { End of Intervention }\end{array}$
\end{tabular} WMD: $0.20 ; 95 \% \mathrm{Cl}:-0.91$ to $1.31, \mathrm{I}^{2}=\mathrm{N} / \mathrm{A}$ & $1 \mathrm{RCT}^{97}$ with 92 patients \\
\hline $\begin{array}{l}\text { Symptoms BCSS } \\
\text { Longest Followup }\end{array}$ & WMD: $0.10 ; 95 \% \mathrm{Cl}:-1.01$ to $1.21, \mathrm{I}^{2}=\mathrm{N} / \mathrm{A}$ & $1 \mathrm{RCT}^{97}$ with 92 patients \\
\hline
\end{tabular}

6MWD = 6 minute walking distance; BCSS = Breathlessness, Cough and Sputum Scale; $\mathrm{CI}=$ confidence interval; FEV1 = forced expiratory volume in one second; N/A = not applicable; RCT = randomized controlled trial; WMD = weighted mean difference

\section{Exercise Versus Management Without Exercise}

\section{Exercise Using Resistance Training Versus Management Without Resistance Training}

Three studies ${ }^{84,86,100,108}$ evaluated the effectiveness of exercise using resistance training compared with management without resistance training in patients with moderate to severe ECOPD (Table 27 and Table 28).

Exercise using resistance training was associated with statistically significantly better dyspnea, better QoL, and higher 6-minute walking distance at the end of the intervention, compared with management without resistance training.

There was no difference between the intervention and management without resistance training group for all other evaluated outcomes including: mortality at the longest followup, other symptoms at the end of the intervention and at the longest followup, FEV1percent predicted at the end of intervention, and hospital admissions at the longest followup. No statistically significant difference was found on number of withdrawals (Appendix Table H.10.). 
Table 27. Comparison of exercise using resistance training versus management without resistance training, critical outcomes

\begin{tabular}{|c|c|c|c|c|}
\hline Outcome & Findings & $\begin{array}{l}\text { Study Design and } \\
\text { Sample Size }\end{array}$ & $\begin{array}{l}\text { Rationale } \\
\text { for } \\
\text { Strength of } \\
\text { Evidence } \\
\text { (SOE) }\end{array}$ & $\begin{array}{l}\text { Overall Strength of } \\
\text { Evidence }\end{array}$ \\
\hline $\begin{array}{l}\text { Mortality } \\
\text { Longest Followup }\end{array}$ & $\begin{array}{l}\text { OR: } 0.22 ; 95 \% \mathrm{Cl}: \\
0.01 \text { to } 4.81, \mathrm{I}^{2}= \\
\text { N/A }\end{array}$ & $\begin{array}{l}1 \mathrm{RCT}^{100} \text { with } 46 \\
\text { patients }\end{array}$ & $\begin{array}{l}\text { Intermediate } \\
\text { ROB and } \\
\text { severe } \\
\text { imprecision }\end{array}$ & Insufficient evidence \\
\hline $\begin{array}{l}\text { Dyspnea } \\
\text { (Numeric Scale; } \\
\text { Modified Borg) } \\
\text { End of Intervention }\end{array}$ & $\begin{array}{l}\text { WMD: }-2.11 ; 95 \% \\
\mathrm{Cl}:-3.50 \text { to }-0.72 \\
\mathrm{I}^{2}=\mathrm{N} / \mathrm{A}\end{array}$ & $\begin{array}{l}1 \mathrm{RCT}^{84} \text { with } 60 \\
\text { patients }\end{array}$ & $\begin{array}{l}\text { Intermediate } \\
\text { ROB and } \\
\text { imprecision }\end{array}$ & $\begin{array}{l}\text { Low SOE supporting } \\
\text { improvement }\end{array}$ \\
\hline $\begin{array}{l}\text { QoL(EQ-5D VAS) } \\
\text { End of Intervention }\end{array}$ & $\begin{array}{l}\text { WMD: } 18.70 ; 95 \% \\
\text { Cl: } 5.06 \text { to } 32.34, \\
\mathrm{I}^{2}=\mathrm{N} / \mathrm{A}\end{array}$ & $\begin{array}{l}1 \mathrm{RCT}^{84} \text { with } 60 \\
\text { patients }\end{array}$ & $\begin{array}{l}\text { Intermediate } \\
\text { ROB and } \\
\text { imprecision }\end{array}$ & $\begin{array}{l}\text { Low SOE supporting } \\
\text { improvement }\end{array}$ \\
\hline $\begin{array}{l}\text { Hospital Admission } \\
\text { Longest Followup }\end{array}$ & $\begin{array}{l}\text { OR: } 1.23 ; 95 \% \mathrm{Cl}: \\
0.35 \text { to } 4.31, I^{2}= \\
\text { N/A }\end{array}$ & $\begin{array}{l}1 \mathrm{RCT}^{108} \text { with } 40 \\
\text { patients }\end{array}$ & $\begin{array}{l}\text { High ROB } \\
\text { and severe } \\
\text { imprecision }\end{array}$ & Insufficient evidence \\
\hline
\end{tabular}

CI = confidence interval; EQ-5d = EuroQol, 5 Dimensions; N/A = not applicable; OR = odds ratio; QoL = quality of life; RCT = randomized controlled trial; $\mathrm{ROB}=$ risk of bias; $\mathrm{SOE}=$ strength of evidence; VAS = visual analog scale; WMD = weighted mean difference

Table 28. Comparison of exercise using resistance training versus management without resistance training, additional outcomes

\begin{tabular}{|l|l|l|}
\hline \multicolumn{1}{|c|}{ Outcome } & \multicolumn{1}{|c|}{ Findings } & \multicolumn{1}{c|}{ Study Design and Sample Size } \\
\hline $\begin{array}{l}\text { Other Symptoms } \\
\text { (Health related } \\
\text { Quality of Life, sub } \\
\text { scale) } \\
\text { End of Intervention }\end{array}$ & WMD: $5.90 ; 95 \% \mathrm{Cl}:-1.20$ to $13.00, \mathrm{I}^{2}=\mathrm{N} / \mathrm{A}$ & $1 \mathrm{RCT}^{100}$ with 46 patients \\
\hline $\begin{array}{l}\text { Other Symptoms } \\
\text { (Health related } \\
\text { Quality of Life, sub } \\
\text { scale) } \\
\text { Longest Followup }\end{array}$ & WMD: $0.80 ; 95 \% \mathrm{Cl}:-10.31$ to $11.90, \mathrm{I}^{2}=\mathrm{N} / \mathrm{A}$ & $1 \mathrm{RCT}^{100}$ with 46 patients \\
\hline $\begin{array}{l}\text { FEV1\% Predicted } \\
\text { End of Intervention }\end{array}$ & WMD: $2.37 ; 95 \% \mathrm{Cl}:-2.83$ to $7.57, \mathrm{I}^{2}=0.00 \%$ & $2 \mathrm{RCTS}^{84,100}$ with 106 patients \\
\hline $\begin{array}{l}\text { 6MWD } \\
\text { End of Intervention }\end{array}$ & WMD: $74.42 ; 95 \% \mathrm{Cl}: 46.85$ to $101.99, \mathrm{I}^{2}=$ & $2 \mathrm{RCTS}^{100,108}$ with 86 patients \\
\hline
\end{tabular}

6MWD = 6-minute walking distance; $\mathrm{CI}$ = confidence interval; FEV1 = Forced expiratory volume in one second; N/A = not applicable; RCT = randomized controlled trial; WMD = weighted mean difference

\section{Exercise Using Aerobic Training Versus Management Without Aerobic Training}

Four studies reported relevant outcomes comparing aerobic training versus management without aerobic training. ${ }^{83,86,124,125}$ Aerobic exercise was associated with significantly improved dyspnea and quality of life at the end of intervention, worse dyspnea at the longest followup, statistically significant improvement in 6 minute walking distance, number of steps walked per day, and endurance based on a 30-second sit-to-stand test, compared with management without 
aerobic exercise. There was no difference between the intervention and control group for mortality, hospital admissions and repeat exacerbations (Table 29 and Table 30).

Table 29. Comparison of exercise using aerobic training versus management without aerobic training, critical outcomes

\begin{tabular}{|c|c|c|c|c|}
\hline Outcome & Findings & $\begin{array}{l}\text { Study Design and } \\
\text { Sample Size }\end{array}$ & $\begin{array}{c}\text { Rationale } \\
\text { for Strength } \\
\text { of Evidence } \\
\text { (SOE) } \\
\end{array}$ & $\begin{array}{l}\text { Overall Strength of } \\
\text { Evidence }\end{array}$ \\
\hline $\begin{array}{l}\text { Mortality } \\
\text { End of Intervention }\end{array}$ & $\begin{array}{l}\text { OR: } 1.00 ; 95 \% \mathrm{Cl}: \\
0.06 \text { to } 17.02, \mathrm{I}^{2}= \\
\text { N/A }\end{array}$ & $\begin{array}{l}1 \mathrm{RCT}^{124} \text { with } 46 \\
\text { patients }\end{array}$ & $\begin{array}{l}\text { High ROB } \\
\text { and severe } \\
\text { imprecision }\end{array}$ & Insufficient evidence \\
\hline $\begin{array}{l}\text { Dyspnea } \\
\text { (Questionnaire: } \\
\text { Transitional } \\
\text { Dyspnea Index) } \\
\text { End of Intervention }\end{array}$ & $\begin{array}{l}\text { WMD: } 7.20 ; 95 \% \\
\text { Cl: } 4.53 \text { to } 9.87 \text {, } \\
I^{2}=\text { N/A }\end{array}$ & $\begin{array}{l}1 \mathrm{RCT}^{124} \text { with } 46 \\
\text { patients }\end{array}$ & $\begin{array}{l}\text { High ROB } \\
\text { and } \\
\text { imprecision }\end{array}$ & $\begin{array}{l}\text { Low SOE supporting better } \\
\text { outcome }\end{array}$ \\
\hline $\begin{array}{l}\text { Dyspnea } \\
\text { (Questionnaire: } \\
\text { MRC) } \\
\text { Longest Followup }\end{array}$ & $\begin{array}{l}\text { WMD: } 1.20 ; 95 \% \\
\text { Cl: } 0.33 \text { to } 2.07, \mathrm{I}^{2}= \\
\text { N/A }\end{array}$ & $\begin{array}{l}1 \mathrm{RCT}^{83} \text { with } 29 \\
\text { patients }\end{array}$ & $\begin{array}{l}\text { Intermediate } \\
\text { ROB and } \\
\text { imprecision }\end{array}$ & $\begin{array}{l}\text { Low SOE supporting worse } \\
\text { outcome }\end{array}$ \\
\hline $\begin{array}{l}\text { QoL (CRQ) } \\
\text { End of Intervention }\end{array}$ & $\begin{array}{l}\text { WMD: } 38.00 ; 95 \% \\
\text { Cl: } 24.51 \text { to } 51.49 \\
\mathrm{I}^{2}=\mathrm{N} / \mathrm{A}\end{array}$ & $\begin{array}{l}\text { 1 RCT }{ }^{124} \text { with } 46 \\
\text { patients }\end{array}$ & $\begin{array}{l}\text { High ROB } \\
\text { and } \\
\text { imprecision }\end{array}$ & $\begin{array}{l}\text { Low SOE supporting better } \\
\text { outcome }\end{array}$ \\
\hline $\begin{array}{l}\text { QoL(CAT) } \\
\text { Longest Followup }\end{array}$ & $\begin{array}{l}\text { WMD: }-5.20 ; 95 \% \\
\text { Cl: }-2.99 \text { to } 13.39 \text {, } \\
\mathrm{I}^{2}=\mathrm{N} / \mathrm{A}\end{array}$ & $\begin{array}{l}1 \mathrm{RCT}^{83} \text { with } 29 \\
\text { patients }\end{array}$ & $\begin{array}{l}\text { Intermediate } \\
\text { ROB and } \\
\text { severe } \\
\text { imprecision }\end{array}$ & Insufficient evidence \\
\hline \multirow[t]{2}{*}{$\begin{array}{l}\text { Hospital Admission } \\
\text { Longest Followup }\end{array}$} & $\begin{array}{l}\text { OR: } 1.50 ; 95 \% \mathrm{Cl} \\
0.33 \text { to } 6.77, \mathrm{I}^{2}= \\
\text { N/A }\end{array}$ & $\begin{array}{l}1 \mathrm{RCT}^{83} \text { with } 29 \\
\text { patients }\end{array}$ & $\begin{array}{l}\text { Intermediate } \\
\text { ROB and } \\
\text { severe } \\
\text { imprecision }\end{array}$ & Insufficient evidence \\
\hline & $\begin{array}{l}\text { Rate Ratio: } 0.96 ; \\
95 \% \mathrm{Cl}: 0.39 \text { to } \\
2.37, \mathrm{I}^{2}=\mathrm{N} / \mathrm{A}\end{array}$ & $\begin{array}{l}1 \mathrm{RCT}^{83} \text { with } 29 \\
\text { patients }\end{array}$ & $\begin{array}{l}\text { Intermediate } \\
\text { ROB and } \\
\text { severe } \\
\text { imprecision }\end{array}$ & Insufficient evidence \\
\hline $\begin{array}{l}\text { Repeat } \\
\text { Exacerbation } \\
\text { End of Intervention }\end{array}$ & $\begin{array}{l}\text { OR: } 0.74 ; 95 \% \mathrm{Cl}: \\
0.22 \text { to } 2.49, \mathrm{I}^{2}= \\
0.0 \%\end{array}$ & $\begin{array}{l}2 \mathrm{RCTs}^{83124} \text { with } 75 \\
\text { patients }\end{array}$ & $\begin{array}{l}\text { High ROB } \\
\text { and severe } \\
\text { imprecision }\end{array}$ & Insufficient evidence \\
\hline
\end{tabular}

CAT = COPD Assessment Test; CI = confidence interval; CRQ = Chronic Respiratory Disease Questionnaire; MRC = Medical Research Council Scale; OR = odds ratio; RCT = randomized controlled trial; ROB = risk of bias; SOE = strength of evidence; WMD = weighted mean difference

Table 30. Comparison of exercise using aerobic training versus management without aerobic training, additional outcomes

\begin{tabular}{|l|l|l|}
\hline \multicolumn{1}{|c|}{ Outcome } & \multicolumn{1}{|c|}{ Findings } & \multicolumn{1}{c|}{$\begin{array}{c}\text { Study Design and Sample } \\
\text { Size }\end{array}$} \\
\hline $\begin{array}{l}\text { FEV1\% Predicted } \\
\text { End of Intervention }\end{array}$ & $\begin{array}{l}\text { WMD: } 0.10 ; 95 \% \mathrm{Cl}:-8.36 \text { to } \\
8.56, \mathrm{I}^{2}=\mathrm{N} / \mathrm{A}\end{array}$ & 1 RCT ${ }^{25}$ with 29 patients \\
\hline $\begin{array}{l}\text { FEV1 Absolute } \\
\text { End of Intervention }\end{array}$ & $\begin{array}{l}\text { WMD: } 0.19 ; 95 \% \mathrm{Cl}:-0.08 \text { to } \\
0.46, \mathrm{I}^{2}=\mathrm{N} / \mathrm{A}\end{array}$ & $1 \mathrm{RCT}^{124}$ with 46 patients \\
\hline $\begin{array}{l}\text { 6MWD } \\
\text { End of Intervention }\end{array}$ & $\begin{array}{l}\text { WMD: } 28.71 ; 95 \% \mathrm{Cl}: 10.91 \text { to } \\
46.50, \mathrm{I}^{2}=98.4 \%\end{array}$ & $\begin{array}{l}2 \mathrm{RCTs}^{124} 125 \\
\text { patients }\end{array}$ \\
\hline $\begin{array}{l}\text { Number of steps walked } 75 \\
\text { per day } \\
\text { End of Intervention }\end{array}$ & $\begin{array}{l}\text { WMD: } 663.03 ; 95 \% \mathrm{Cl}: 496.34 \\
\text { to } 829.72, \mathrm{I}^{2}=\mathrm{N} / \mathrm{A}\end{array}$ & $1 \mathrm{RCT}^{86}$ with 58 patients \\
\hline $\begin{array}{l}\text { 30-second sit-to-stand test } \\
\text { End of Intervention }\end{array}$ & $\begin{array}{l}\text { WMD: } 4.63 ; 95 \% \mathrm{Cl}: 2.54 \text { to } \\
6.72, \mathrm{I}^{2}=\mathrm{N} / \mathrm{A}\end{array}$ & $1 \mathrm{RCT}^{86}$ with 58 patients \\
\hline
\end{tabular}


6MWD = 6 minute walking distance; $\mathrm{CI}$ = confidence interval; $\mathrm{RCT}=$ randomized controlled trial; $\mathrm{WMD}=$ weighted mean difference

\section{Exercise Using Combined Aerobic + Resistance Training Versus Management Without Exercise Training}

One study ${ }^{105}$ evaluated the effectiveness of exercise using combined aerobic and resistance training compared with management without exercise training in patients with moderate to severe ECOPD (Table 31). The study had two active treatment arms: one low-intensity exercise arm and one moderate to high-intensity exercise arm, in addition to a management without exercise training arm.

Low-intensity and moderate-to-high intensity aerobic and resistance training was not associated with a statistical difference in FEV1 percent predicted, 3-minute walking distance test, and upper limb muscle strength at the end of the intervention compared with management without exercise training. There was no statistical difference in total number of AEs (Appendix Table H.11).

Table 31. Comparison of exercise using combined aerobic + resistance training versus management without exercise training

\begin{tabular}{|c|c|c|c|}
\hline Comparison & Outcome & Findings & $\begin{array}{c}\text { Study Design and Sample } \\
\text { Size }\end{array}$ \\
\hline \multirow{3}{*}{$\begin{array}{l}\text { Low Intensity } \\
\text { Exercise Group } \\
\text { vs. Management } \\
\text { Without Exercise } \\
\text { Training }\end{array}$} & $\begin{array}{l}\text { FEV1\% Predicted } \\
\text { End of Intervention }\end{array}$ & $\begin{array}{l}\text { WMD: }-4.80 ; 95 \% \mathrm{Cl}:-13.23 \text { to } \\
3.63, \mathrm{I}^{2}=\mathrm{N} / \mathrm{A}\end{array}$ & $1 \mathrm{RCT}^{105}$ with 22 patients \\
\hline & $\begin{array}{l}\text { 3-minute Walking Distance } \\
\text { Test } \\
\text { End of Intervention }\end{array}$ & $\begin{array}{l}\text { SMD: } 0.40 ; 95 \% \mathrm{Cl}:-0.50 \text { to } \\
1.30, \mathrm{I}^{2}=\mathrm{N} / \mathrm{A}\end{array}$ & $1 \mathrm{RCT}^{105}$ with 22 patients \\
\hline & $\begin{array}{l}\text { Upper Limb Muscle } \\
\text { Strength }\end{array}$ & $\begin{array}{l}\text { SMD: } 0.20 ; 95 \% \mathrm{Cl}:-0.70 \text { to } \\
1.00, \mathrm{I}^{2}=\mathrm{N} / \mathrm{A}\end{array}$ & $1 \mathrm{RCT}^{105}$ with 22 patients \\
\hline \multirow{2}{*}{$\begin{array}{l}\text { Moderate-to- } \\
\text { High Intensity } \\
\text { Exercise Group } \\
\text { vs. Management } \\
\text { Without Exercise } \\
\text { Training }\end{array}$} & $\begin{array}{l}\text { 3-minute Walking Distance } \\
\text { Test } \\
\text { End of Intervention }\end{array}$ & $p=N S$ & $1 \mathrm{RCT}^{105}$ with 22 patients \\
\hline & $\begin{array}{l}\text { Upper Limb Muscle } \\
\text { Strength }\end{array}$ & $\mathrm{p}=\mathrm{NS}$ & $1 \mathrm{RCT}^{105}$ with 22 patients \\
\hline
\end{tabular}

\section{Chest Physiotherapy + Exercise Combined Versus Management Without Exercise Training}

One study ${ }^{84}$ evaluated the effectiveness of chest physiotherapy and exercise (breathing technique and range of motion exercises) combined compared with management without exercise training in patients with moderate to severe ECOPD (Table 32 and Table 33).

The chest physiotherapy and exercise combination group was found to have statistically significantly more improvements in quality of life at the end of the intervention. No other statistically significant difference was found. 
Table 32. Comparison of chest physiotherapy + exercise (breathing technique + range of motion exercises) combined versus management without exercise training, critical outcomes

\begin{tabular}{|l|l|l|l|l|}
\hline \multicolumn{1}{|c|}{ Outcome } & \multicolumn{1}{|c|}{ Findings } & \multicolumn{1}{c|}{$\begin{array}{c}\text { Study Design and } \\
\text { Sample Size }\end{array}$} & $\begin{array}{c}\text { Rationale for } \\
\text { Strength of } \\
\text { Evidence (SOE) }\end{array}$ & $\begin{array}{l}\text { Overall Strength of } \\
\text { Evidence }\end{array}$ \\
\hline $\begin{array}{l}\text { Dyspnea } \\
\text { (Numeric Scale: } \\
\text { Modified Borg } \\
\text { Scale) } \\
\text { End of Intervention }\end{array}$ & $\begin{array}{l}\text { WMD: } 1.15 ; 95 \% \mathrm{Cl}: \\
-0.61 \text { to } 2.91, \mathrm{I}^{2}=\end{array}$ & $\begin{array}{l}1 \mathrm{RCT}^{84} \text { with } 60 \\
\text { patients }\end{array}$ & $\begin{array}{l}\text { Intermediate ROB } \\
\text { and severe } \\
\text { imprecision }\end{array}$ & Insufficient evidence \\
\hline $\begin{array}{l}\text { QOL } \\
\text { (EQ-5D) } \\
\text { End of Intervention }\end{array}$ & $\begin{array}{l}\text { WMD: } 14.89 ; 95 \% \\
\text { Cl: } 5.30 \text { to } 24.50, \\
\mathrm{I}^{2}=\mathrm{N} / \mathrm{A}\end{array}$ & $\begin{array}{l}1 \mathrm{RCT}^{84} \text { with } 60 \\
\text { patients }\end{array}$ & $\begin{array}{l}\text { Intermediate ROB } \\
\text { and imprecision }\end{array}$ & $\begin{array}{l}\text { Low SOE supporting } \\
\text { improvement }\end{array}$ \\
\hline
\end{tabular}

CI = confidence interval; EQ-5D = EuroQol 5 Dimension Index; N/A = not applicable; RCT = randomized controlled trial; QoL

$=$ quality of life; $\mathrm{ROB}=$ risk of bias; $\mathrm{SOE}=$ strength of evidence; $\mathrm{WMD}=$ weighted mean difference

Table 33. Comparison of chest physiotherapy + exercise (breathing technique + range of motion exercises) combined versus management without exercise training, additional outcomes

\begin{tabular}{|l|l|c|}
\hline \multicolumn{1}{|c|}{ Outcome } & \multicolumn{1}{|c|}{ Findings } & Study Design and Sample Size \\
\hline $\begin{array}{l}\text { FEV1\% Predicted } \\
\text { End of Intervention }\end{array}$ & WMD: 2.48; 95\% Cl: -1.81 to $6.77, \mathrm{I}^{2}=\mathrm{N} / \mathrm{A}$ & $1 \mathrm{RCT}^{84}$ with 60 patients \\
\hline \\
CI = confidence interval; FEV1 = forced expiratory volume in one second; N/A = not applicable; RCT = randomized controlled \\
trial; WMD = weighted mean difference
\end{tabular}

\section{Early Pulmonary Rehabilitation (During ECOPD) Versus Management Without Early Pulmonary Rehabilitation}

Four studies 46, 83, 94, 95, 111 evaluated the effectiveness of early pulmonary rehabilitation during ECOPD compared with management without early pulmonary rehabilitation in patients with moderate to severe ECOPD (Table 34 and Table 35).

Early pulmonary rehabilitation was associated with a statistically significantly longer 6minute walking distance at the end of the intervention, compared with management without early pulmonary rehabilitation.

Dyspnea based on a numeric scale was statistically significantly improved at the end of the intervention, but not at the longest followup in the early pulmonary rehabilitation group compared with the management without early pulmonary rehabilitation group. Early pulmonary rehabilitation was also statistically significantly associated with decreased cough at the end of the intervention compared with management without early pulmonary rehabilitation.

There was no statistical difference between the intervention and management without early pulmonary rehabilitation for other outcomes including: dyspnea (based on questionnaire) at the end of the intervention and at the longest followup, hospital admissions at 30 days and 1 year.

Serious AEs were reported in 7 cases in the early pulmonary rehabilitation group, including worsening COPD exacerbations (5 cases), pneumonia (1 case), a not further specified respiratory, thoracic and mediastinal disorder (1 case), and vascular disorder (1 case). There was no statistically significant difference observed between the early pulmonary rehabilitation group and the management without early pulmonary rehabilitation in serious AEs, total number of AEs, withdrawals, and withdrawals due to AEs. (Appendix Table H.12.) 
Table 34. Comparison of early pulmonary rehabilitation versus management without early pulmonary rehabilitation, critical outcomes

\begin{tabular}{|c|c|c|c|c|}
\hline Outcome & Findings & $\begin{array}{l}\text { Study Design } \\
\text { and Sample Size }\end{array}$ & $\begin{array}{c}\text { Rationale for } \\
\text { Strength of } \\
\text { Evidence (SOE) }\end{array}$ & $\begin{array}{l}\text { Overall Strength } \\
\text { of Evidence }\end{array}$ \\
\hline $\begin{array}{l}\text { Mortality } \\
\text { End of Intervention }\end{array}$ & $\begin{array}{l}\text { OR: } 3.26 ; 95 \% \mathrm{Cl}: \\
0.13 \text { to } 81.98, \mathrm{I}^{2}=\mathrm{N} / \mathrm{A}\end{array}$ & $\begin{array}{l}1 \mathrm{RCT}^{111} \text { with } 97 \\
\text { patients }\end{array}$ & $\begin{array}{l}\text { High ROB and } \\
\text { severe imprecision }\end{array}$ & $\begin{array}{l}\text { Insufficient } \\
\text { evidence }\end{array}$ \\
\hline $\begin{array}{l}\text { Dyspnea } \\
\text { (Questionnaire: } \\
\text { MMRC) } \\
\text { End of Intervention }\end{array}$ & $\begin{array}{l}\text { WMD: }-0.50 ; 95 \% \mathrm{Cl}:- \\
3.01 \text { to } 2.06,1^{2}=\mathrm{N} / \mathrm{A}\end{array}$ & $\begin{array}{l}1 \mathrm{RCT}^{95} \text { with } 94 \\
\text { patients }\end{array}$ & $\begin{array}{l}\text { High ROB and } \\
\text { severe imprecision }\end{array}$ & $\begin{array}{l}\text { Insufficient } \\
\text { evidence }\end{array}$ \\
\hline $\begin{array}{l}\text { Dyspnea } \\
\text { (Questionnaire: } \\
\text { MMRC) } \\
\text { Longest Followup }\end{array}$ & $\begin{array}{l}\text { WMD: } 0.04 ; 95 \% \mathrm{Cl}:- \\
0.48 \text { to } 0.56,1^{2}=\mathrm{N} / \mathrm{A}\end{array}$ & $\begin{array}{l}1 \mathrm{RCT}^{95} \text { with } 94 \\
\text { patients }\end{array}$ & $\begin{array}{l}\text { High ROB and } \\
\text { severe imprecision }\end{array}$ & $\begin{array}{l}\text { Insufficient } \\
\text { evidence }\end{array}$ \\
\hline $\begin{array}{l}\text { Dyspnea } \\
\text { (Numeric Scale: } \\
\text { Modified Borg; Borg) } \\
\text { End of Intervention }\end{array}$ & $\begin{array}{l}\text { SMD: } 0.66 ; 95 \% \mathrm{Cl}: \\
0.31 \text { to } 1.00 \\
\mathrm{I}^{2}=89.21 \%\end{array}$ & $\begin{array}{l}2 \mathrm{RCT}^{94,95} \text { with } \\
156 \text { patients }\end{array}$ & $\begin{array}{l}\text { High ROB and } \\
\text { inconsistency }\end{array}$ & $\begin{array}{l}\text { Low SOE } \\
\text { supporting } \\
\text { improvement }\end{array}$ \\
\hline $\begin{array}{l}\text { Dyspnea } \\
\text { (Numeric Scale: Borg) } \\
\text { Longest Followup }\end{array}$ & $\begin{array}{l}\text { WMD: } 0.20 ; 95 \% \mathrm{Cl}:- \\
0.69 \text { to } 0.29,1^{2}=\mathrm{N} / \mathrm{A}\end{array}$ & $\begin{array}{l}1 \mathrm{RCT}^{111} \text { with } 97 \\
\text { patients }\end{array}$ & $\begin{array}{l}\text { High ROB and } \\
\text { severe imprecision }\end{array}$ & $\begin{array}{l}\text { Insufficient } \\
\text { evidence }\end{array}$ \\
\hline \multirow[t]{2}{*}{$\begin{array}{l}\text { Hospital Admission } \\
\text { At } 30 \text { days }\end{array}$} & $\begin{array}{l}\text { OR: } 0.65 ; 95 \% \mathrm{Cl}: \\
0.26 \text { to } 1.60, I^{2}=\mathrm{N} / \mathrm{A}\end{array}$ & $\begin{array}{l}\text { 1 } \mathrm{RCT}^{111} \text { with } 97 \\
\text { patients }\end{array}$ & $\begin{array}{l}\text { High ROB and } \\
\text { severe imprecision }\end{array}$ & $\begin{array}{l}\text { Insufficient } \\
\text { evidence }\end{array}$ \\
\hline & $\begin{array}{l}\text { Rate Ratio: } 0.60 ; 95 \% \\
\mathrm{Cl}: 0.31 \text { to } 1.15, \mathrm{I}^{2}= \\
\mathrm{N} / \mathrm{A}\end{array}$ & $\begin{array}{l}1 \mathrm{RCT}^{111} \text { with } 97 \\
\text { patients }\end{array}$ & $\begin{array}{l}\text { High ROB and } \\
\text { severe imprecision }\end{array}$ & $\begin{array}{l}\text { Insufficient } \\
\text { evidence }\end{array}$ \\
\hline $\begin{array}{l}\text { Hospital Admission } \\
\text { Longest Followup }\end{array}$ & $\begin{array}{l}\text { Hazard ratio: } 1.19, \\
95 \% \mathrm{Cl}: 0.90 \text { to } 1.60, \\
\mathrm{I}^{2}=\mathrm{N} / \mathrm{A}\end{array}$ & $\begin{array}{l}1 \mathrm{RCT}^{46,} \text { with } 320 \\
\text { patients }\end{array}$ & $\begin{array}{l}\text { High ROB and } \\
\text { severe imprecision }\end{array}$ & $\begin{array}{l}\text { Insufficient } \\
\text { evidence }\end{array}$ \\
\hline
\end{tabular}

CAT = COPD Assessment Test; $\mathrm{CI}=$ confidence interval; MMRC = Modified Medical Research Council Scale; MRC = Medical Research Council Scale; N/A = not applicable; OR = odds ratio; RCT = randomized controlled trial; QoL = quality of life; ROB

$=$ risk of bias; $\mathrm{SMD}=$ standardized mean difference; $\mathrm{SOE}=$ strength of evidence; $\mathrm{WMD}=$ weighted mean difference

Table 35. Comparison of early pulmonary rehabilitation versus management without early pulmonary rehabilitation, additional outcomes

\begin{tabular}{|l|l|l|}
\hline \multicolumn{1}{|c|}{ Outcome } & \multicolumn{1}{|c|}{ Findings } & \multicolumn{1}{c|}{ Study Design and Sample Size } \\
\hline $\begin{array}{l}\text { Cough (VAS) } \\
\text { End of Intervention }\end{array}$ & W/A : $-2.00 ; 95 \% \mathrm{Cl}:-2.98$ to $-1.02, \mathrm{I}^{2}=$ & $1 \mathrm{RCT}^{94}$ with 62 patients \\
\hline $\begin{array}{l}\text { 6MWD } \\
\text { End of Intervention }\end{array}$ & $\begin{array}{l}\text { WMD: } 20.02 ; 95 \% \mathrm{Cl}: 12.06 \text { to } 28.67, \\
\mathrm{I}^{2}=79.08 \%\end{array}$ & $3 \mathrm{RCTS}^{94,95,111}$ with 253 patients \\
\hline
\end{tabular}

6MWD = 6 minute walking distance; $\mathrm{CI}$ = confidence interval; N/A = not applicable; RCT = randomized controlled trial; SMD = standardized mean difference; WMD = weighted mean difference; VAS= visual analog score

\section{Whole Body Vibration Training Versus Management Without Whole Body Vibration}

One study ${ }^{99}$ evaluated the effectiveness of whole body vibration training during ECOPD compared with management without whole body vibration in patients with moderate to severe ECOPD (Table 36 and Table 37).

There was a statistically significantly higher quality of life and a longer 6-minute walking distance at the end of the intervention in the intervention compared with the management without whole body vibration group. Whole body vibration training during ECOPD was not associated with statistically significant difference in FEV1 percent predicted.

No AEs were reported in the whole body vibration group. There was no statistical difference on withdrawals (Appendix Table H.13.). 
Table 36. Comparison of whole body vibration training during ECOPD versus management without whole body vibration, critical outcomes

\begin{tabular}{|l|l|l|l|l|}
\hline \multicolumn{1}{|c|}{ Outcome } & \multicolumn{1}{|c|}{ Findings } & \multicolumn{1}{c|}{$\begin{array}{c}\text { Study Design and } \\
\text { Sample Size }\end{array}$} & $\begin{array}{c}\text { Rationale for } \\
\text { Strength of } \\
\text { Evidence (SOE) }\end{array}$ & $\begin{array}{c}\text { Overall Strength } \\
\text { of Evidence }\end{array}$ \\
\hline $\begin{array}{l}\text { QoL(SGRQ) } \\
\begin{array}{l}\text { End of } \\
\text { Intervention }\end{array}\end{array}$ & $\begin{array}{l}\text { WMD: }-12.02 ; 95 \% \\
\mathrm{Cl}:-21.41 \text { to }-2.63,\end{array}$ & $1 \mathrm{RCT}^{99}$ with 49 patients & $\begin{array}{l}\text { Intermediate ROB } \\
\text { and imprecision }\end{array}$ & $\begin{array}{l}\text { Low SOE } \\
\text { supporting } \\
\text { improvement }\end{array}$ \\
\hline
\end{tabular}

CI = confidence interval; N/A = not applicable; QoL = quality of life; RCT = randomized controlled trial; SGRQ = St. George

Respiratory Questionnaire; WMD = weighted mean difference

Table 37. Comparison of whole body vibration training during ECOPD versus management without whole body vibration, additional outcomes

\begin{tabular}{|l|l|l|}
\hline \multicolumn{1}{|c|}{ Outcome } & \multicolumn{1}{|c|}{ Findings } & Study Design and Sample Size \\
\hline 6MWD & WMD: 89.42; 95\% Cl: 45.18 & $1 \mathrm{RCT}^{99}$ with 49 patients \\
End of Intervention & to133.66, $\mathrm{I}^{2}=\mathrm{N} / \mathrm{A}$ & \\
\hline FEV1\% Predicted & WMD: $-6.52 ; 95 \% \mathrm{Cl}:-16.96$ to & $1 \mathrm{RCT}^{99}$ with 49 patients \\
End of Intervention & $3.92, \mathrm{I}^{2}=\mathrm{N} / \mathrm{A}$ & \\
\hline
\end{tabular}

6MWD = 6 minute walking distance; $\mathrm{CI}$ = confidence interval; FEV1 = forced expiratory volume in one second; N/A = not applicable; RCT = randomized controlled trial; WMD = weighted mean difference

\section{Transcutaneous Electrical Nerve Stimulation (TENS) Versus Management Without TENS}

One study ${ }^{87}$ evaluated the effectiveness of transcutaneous electrical nerve stimulation (TENS) during ECOPD compared with management without TENS in patients with moderate to severe ECOPD (Table 38 and Table 39).

TENS during ECOPD was associated with a statistically significantly longer 6-minute walking distance at the end of the intervention, but no difference in dyspnea, FEV1 absolute, and number of withdrawals at the end of the intervention compared with management without TENS group (Appendix Table H.14.).

Table 38. Comparison of transcutaneous electrical nerve stimulation (TENS) during ECOPD versus management without TENS, critical outcomes

\begin{tabular}{|l|l|l|l|l|}
\hline \multicolumn{1}{|c|}{ Outcome } & \multicolumn{1}{|c|}{ Findings } & \multicolumn{1}{c|}{$\begin{array}{c}\text { Study Design and } \\
\text { Sample Size }\end{array}$} & $\begin{array}{c}\text { Rationale for } \\
\text { Strength of } \\
\text { Evidence (SOE) }\end{array}$ & $\begin{array}{c}\text { Overall Strength of } \\
\text { Evidence }\end{array}$ \\
\hline $\begin{array}{l}\text { Dyspnea } \\
\text { (Questionnaire: }\end{array}$ & $\begin{array}{l}\text { WMD: }-0.23 ; 95 \% \\
\text { MRC) }\end{array}$ & $\begin{array}{l}1 \mathrm{RCT}^{87} \text { with } 82 \\
\text { patients }\end{array}$ & $\begin{array}{l}\text { High ROB and } \\
\text { severe imprecision }\end{array}$ & Insufficient evidence \\
End of Intervention & $\mathrm{I}^{2}=\mathrm{N} / \mathrm{A}$ & & & \\
\hline
\end{tabular}

$\mathrm{CI}=$ confidence interval; ECPOD = Exacerbations of Chronic Obstructive Pulmonary Disease; MRC = Medical Research

Council Scale N/A = not applicable; RCT = randomized controlled trial; ROB = risk of bias; WMD = weighted mean difference

Table 39. Comparison of transcutaneous electrical nerve stimulation (TENS) during ECOPD versus management without TENS, additional outcomes

\begin{tabular}{|c|c|c|}
\hline Outcome & Findings & Study Design and Sample Size \\
\hline $\begin{array}{l}\text { 6MWD } \\
\text { End of Intervention }\end{array}$ & $\begin{array}{l}\text { WMD: } 64.54 ; 95 \% \mathrm{Cl}: 53.76 \text { to } 75.32, \mathrm{I}^{2}= \\
\text { N/A }\end{array}$ & $1 \mathrm{RCT}^{87}$ with 82 patients \\
\hline $\begin{array}{l}\text { FEV1 Absolute } \\
\text { End of Intervention }\end{array}$ & WMD: $-0.05 ; 95 \% \mathrm{Cl}:-0.33$ to $0.23, \mathrm{I}^{2}=\mathrm{N} / \mathrm{A}$ & $1 \mathrm{RCT}^{87}$ with 82 patients \\
\hline
\end{tabular}




\section{Supplemental Oxygen Versus Supplemental Air During Mobilization With a Walking Aid}

One study ${ }^{117}$ randomized 120 patients with moderate to severe ECOPD to one of the four groups: gutter frame with supplemental oxygen; gutter frame with supplemental air; rollator with supplemental oxygen, or rollator with supplemental air (Table 40). The presence of hypoxemia at rest was not a requirement for study inclusion, and paO2 ranged from $72-80 \mathrm{mmHg}$ in the different groups at baseline. Oxygen levels were not measured during mobilization. There was no statistically significant difference observed in dyspnea, mortality, and 30-day hospital admissions, AEs, withdrawals, and withdrawals due to AEs between groups (Appendix Tables H.15. and H.16.).

Table 40. Supplemental oxygen versus supplemental air

\begin{tabular}{|c|c|c|c|c|c|}
\hline Comparison & Outcome & Findings & $\begin{array}{l}\text { Study Design } \\
\text { and Sample } \\
\text { Size }\end{array}$ & $\begin{array}{l}\text { Rationale for } \\
\text { Strength of } \\
\text { Evidence } \\
\text { (SOE) }\end{array}$ & $\begin{array}{l}\text { Overall } \\
\text { Strength of } \\
\text { Evidence }\end{array}$ \\
\hline \multirow{3}{*}{$\begin{array}{l}\text { Gutter Frame } \\
\text { with } \\
\text { Supplemental } \\
\text { Oxygen vs. } \\
\text { Gutter Frame } \\
\text { with } \\
\text { Supplemental } \\
\text { Air }\end{array}$} & $\begin{array}{l}\text { Dyspnea } \\
\text { (Numeric: Borg } \\
\text { Dyspnea Scale) } \\
\text { End of intervention }\end{array}$ & $\begin{array}{l}\text { WMD: } 0.80 ; 95 \% \\
\text { Cl: }-0.90 \text { to } 2.50 \text {, } \\
\mathrm{I}^{2}=\mathrm{N} / \mathrm{A}\end{array}$ & $\begin{array}{l}1 \mathrm{RCT}^{117} \text { with } 60 \\
\text { patients }\end{array}$ & $\begin{array}{l}\text { High ROB and } \\
\text { severe } \\
\text { imprecision }\end{array}$ & $\begin{array}{l}\text { Insufficient } \\
\text { evidence }\end{array}$ \\
\hline & $\begin{array}{l}\text { Mortality } \\
\text { Longest Followup }\end{array}$ & $\begin{array}{l}\text { OR:1.00; } 95 \% \\
\text { Cl: } 0.06 \text { to } 16.76, \\
l^{2}=N / A\end{array}$ & $\begin{array}{l}1 \mathrm{RCT}^{117} \text { with } 60 \\
\text { patients }\end{array}$ & $\begin{array}{l}\text { High ROB and } \\
\text { severe } \\
\text { imprecision }\end{array}$ & $\begin{array}{l}\text { Insufficient } \\
\text { evidence }\end{array}$ \\
\hline & $\begin{array}{l}\text { Hospital } \\
\text { Admission } \\
30 \text { days }\end{array}$ & $\begin{array}{l}\text { OR: } 1.63 ; 95 \% \\
\text { Cl: } 0.41 \text { to } 6.47 \\
I^{2}=N / A\end{array}$ & $\begin{array}{l}\text { 1 } \mathrm{RCT}^{117} \text { with } 60 \\
\text { patients }\end{array}$ & $\begin{array}{l}\text { High ROB and } \\
\text { severe } \\
\text { imprecision }\end{array}$ & $\begin{array}{l}\text { Insufficient } \\
\text { evidence }\end{array}$ \\
\hline \multirow[t]{3}{*}{$\begin{array}{l}\text { Rollator with } \\
\text { Supplemental } \\
\text { Oxygen, vs. } \\
\text { Rollator with } \\
\text { Supplemental } \\
\text { Air }\end{array}$} & $\begin{array}{l}\text { Dyspnea } \\
\text { (Numeric: Borg } \\
\text { Dyspnea Scale) } \\
\text { End of } \\
\text { Intervention } \\
\end{array}$ & $\begin{array}{l}\text { WMD: }-0.90 \\
95 \% \mathrm{Cl}:-2.35 \text { to } \\
0.58, \mathrm{I}^{2}=\mathrm{N} / \mathrm{A}\end{array}$ & $\begin{array}{l}1 \mathrm{RCT}^{117} \text { with } 60 \\
\text { patients }\end{array}$ & $\begin{array}{l}\text { High ROB and } \\
\text { severe } \\
\text { imprecision }\end{array}$ & $\begin{array}{l}\text { Insufficient } \\
\text { evidence }\end{array}$ \\
\hline & $\begin{array}{l}\text { Mortality } \\
\text { Longest Followup }\end{array}$ & $\begin{array}{l}0 \text { case in both } \\
\text { groups. }\end{array}$ & $\begin{array}{l}1 \mathrm{RCT}^{117} \text { with } 60 \\
\text { patients }\end{array}$ & $\begin{array}{l}\text { High ROB and } \\
\text { severe } \\
\text { imprecision }\end{array}$ & $\begin{array}{l}\text { Insufficient } \\
\text { evidence }\end{array}$ \\
\hline & $\begin{array}{l}\text { Hospital } \\
\text { Admission } \\
30 \text { days }\end{array}$ & $\begin{array}{l}\text { OR: } 1.63 ; 95 \% \\
\text { Cl: } 0.41 \text { to } 6.47 \\
I^{2}=\mathrm{N} / \mathrm{A}\end{array}$ & $\begin{array}{l}1 \mathrm{RCT}^{117} \text { with } 60 \\
\text { patients }\end{array}$ & $\begin{array}{l}\text { High ROB and } \\
\text { severe } \\
\text { imprecision }\end{array}$ & $\begin{array}{l}\text { Insufficient } \\
\text { evidence }\end{array}$ \\
\hline
\end{tabular}

CI = confidence interval; N/A = not applicable; OR = odds ratio; RCT = randomized controlled trial; ROB = risk of bias; WMD = weighted mean difference

\section{Titrated Oxygen Versus High Flow Oxygen}

A study ${ }^{25}$ randomized 405 patients with a presumed moderate to severe ECOPD to titrated oxygen (with a target oxygen saturation between 88 percent and 92 percent delivered by nasal prongs) or high flow oxygen (8-10 liters/minute, administered by a non-rebreather face mask). In the subgroup analysis of confirmed ECOPD patients (117 in titrated oxygen vs. 97 in high flow oxygen), titrated oxygen was associated with statistically significantly reduced mortality compared with high flow oxygen at the longest followup (Table 41). 
Table 41. Titrated oxygen versus high flow oxygen, critical outcomes

\begin{tabular}{|l|l|l|l|l|}
\hline \multicolumn{1}{|c|}{ Outcome } & \multicolumn{1}{|c|}{ Findings } & $\begin{array}{l}\text { Study Design and } \\
\text { Sample Size }\end{array}$ & \multicolumn{1}{c|}{$\begin{array}{c}\text { Rationale for } \\
\text { Strength of } \\
\text { Evidence (SOE) }\end{array}$} & $\begin{array}{l}\text { Overall Strength of } \\
\text { Evidence }\end{array}$ \\
\hline $\begin{array}{l}\text { Mortality } \\
\text { Longest Followup }\end{array}$ & $\begin{array}{l}\text { OR: } 0.36 ; 95 \% \mathrm{Cl}: \\
0.14 \text { to } 0.88, \mathrm{I}^{2}=\mathrm{N} / \mathrm{A} \\
\mathrm{I}^{2}\end{array}$ & $\begin{array}{l}1 \mathrm{RCT}^{25} \text { with } 214 \\
\text { patients }\end{array}$ & $\begin{array}{l}\text { High ROB and } \\
\text { imprecision }\end{array}$ & $\begin{array}{l}\text { Low SOE supporting } \\
\text { improvement }\end{array}$ \\
\hline $\begin{array}{l}\text { Need for Intubation } \\
\text { Longest Followup }\end{array}$ & $\begin{array}{l}\mathrm{OR}: 0.13 ; 95 \% \mathrm{Cl}: \\
0.02 \text { to } 1.00, \mathrm{I}^{2}=\mathrm{N} / \mathrm{A}\end{array}$ & $\begin{array}{l}1 \mathrm{RCT}^{25} \text { with } 214 \\
\text { patients }\end{array}$ & $\begin{array}{l}\text { High ROB and } \\
\text { severe imprecision }\end{array}$ & Insufficient evidence \\
\hline
\end{tabular}

$\mathrm{CI}$ = confidence interval; N/A = not applicable; $\mathrm{OR}=$ odds ratio; $\mathrm{RCT}$ = randomized controlled trial; $\mathrm{ROB}=$ risk of bias; $\mathrm{SOE}=$ strength of evidence

\section{Automated Oxygen Titration Versus Manual Oxygen Titration}

One RCT ${ }^{90}$ compared automated oxygen titration with manual oxygen titration and found no statistically significant difference in mortality, need for intubation, ICU admission, and hospital admissions. The target oxygen saturation was determined by the attending physician before randomization (Table 42).

Table 42. Titrated oxygen versus free flow oxygen, critical outcomes

\begin{tabular}{|c|c|c|c|c|}
\hline Outcome & Findings & $\begin{array}{l}\text { Study Design and } \\
\text { Sample Size }\end{array}$ & $\begin{array}{c}\text { Rationale for } \\
\text { Strength of } \\
\text { Evidence (SOE) }\end{array}$ & $\begin{array}{l}\text { Overall Strength of } \\
\text { Evidence }\end{array}$ \\
\hline $\begin{array}{l}\text { Mortality } \\
\text { End of Intervention }\end{array}$ & $\begin{array}{l}\text { OR: } 1.00 ; 95 \% \mathrm{Cl}: \\
0.06 \text { to } 16.93, \\
\mathrm{l}^{2}=\mathrm{N} / \mathrm{A}\end{array}$ & $\begin{array}{l}1 \mathrm{RCT}^{90} \text { with } 50 \\
\text { patients }\end{array}$ & $\begin{array}{l}\text { Intermediate ROB } \\
\text { and severe } \\
\text { imprecision }\end{array}$ & Insufficient evidence \\
\hline $\begin{array}{l}\text { Need for intubation } \\
\text { End of intervention }\end{array}$ & $\begin{array}{l}\text { OR: } 3.12 ; 95 \% \mathrm{Cl}: \\
0.12 \text { to } 80.39, \\
\mathrm{I}^{2}=\mathrm{N} / \mathrm{A}\end{array}$ & $\begin{array}{l}1 \mathrm{RCT}^{90} \text { with } 50 \\
\text { patients }\end{array}$ & $\begin{array}{l}\text { Intermediate ROB } \\
\text { and severe } \\
\text { imprecision }\end{array}$ & Insufficient evidence \\
\hline $\begin{array}{l}\text { Hospital Admissions } \\
30 \text { days }\end{array}$ & $\begin{array}{l}\text { OR: } 1.00 ; 95 \% \mathrm{Cl}: \\
0.27 \text { to } 3.66,1^{2}=\mathrm{N} / \mathrm{A}\end{array}$ & $\begin{array}{l}1 \mathrm{RCT}^{90} \text { with } 50 \\
\text { patients }\end{array}$ & $\begin{array}{l}\text { Intermediate ROB } \\
\text { and severe } \\
\text { imprecision }\end{array}$ & Insufficient evidence \\
\hline $\begin{array}{l}\text { Hospital Admissions } \\
\text { Longest Followup }\end{array}$ & $\begin{array}{l}\text { OR: } 1.63 ; 95 \% \mathrm{Cl}: \\
0.53 \text { to } 4.98,1^{2}=\mathrm{N} / \mathrm{A}\end{array}$ & $\begin{array}{l}1 \mathrm{RCT}^{90} \text { with } 50 \\
\text { patients }\end{array}$ & $\begin{array}{l}\text { Intermediate ROB } \\
\text { and severe } \\
\text { imprecision }\end{array}$ & Insufficient evidence \\
\hline $\begin{array}{l}\text { ICU Admission } \\
\text { End of Intervention }\end{array}$ & $\begin{array}{l}\text { OR: } 3.12 ; 95 \% \mathrm{Cl}: \\
0.12 \text { to } 80.39, \\
\mathrm{I}^{2}=\mathrm{N} / \mathrm{A}\end{array}$ & $\begin{array}{l}1 \mathrm{RCT}^{90} \text { with } 50 \\
\text { patients }\end{array}$ & $\begin{array}{l}\text { Intermediate ROB } \\
\text { and severe } \\
\text { imprecision }\end{array}$ & Insufficient evidence \\
\hline
\end{tabular}

$\mathrm{CI}=$ confidence interval; ICU = intensive care unit; N/A = not applicable; OR = odds ratio; RCT = randomized controlled trial; $\mathrm{ROB}=$ risk of bias; $\mathrm{SOE}=$ strength of evidence

\section{Dietary Intervention Versus Usual Diet}

\section{Dietary Intervention Using a Caloric Supplement During ECOPD Versus Usual Diet}

One study ${ }^{118}$ evaluated the effectiveness of a dietary intervention using a caloric supplement during ECOPD compared with a usual diet in patients with moderate to severe ECOPD (Table 43 and Table 44). There were no statistically significant differences found between the intervention and usual diet for mortality, dyspnea, QoL, FEV1percent predicted, and number of withdrawals (Appendix Table H.17.). 
Table 43. Comparison of a dietary intervention using a caloric supplement during ECOPD versus usual diet, critical outcomes

\begin{tabular}{|c|c|c|c|c|}
\hline Outcome & Findings & $\begin{array}{l}\text { Study Design and } \\
\text { Sample Size }\end{array}$ & $\begin{array}{c}\text { Rationale for } \\
\text { Strength of } \\
\text { Evidence (SOE) }\end{array}$ & $\begin{array}{l}\text { Overall Strength of } \\
\text { Evidence }\end{array}$ \\
\hline $\begin{array}{l}\text { Mortality } \\
\text { End of Intervention }\end{array}$ & $\begin{array}{l}\text { OR: } 0.81 ; 95 \% \mathrm{Cl}: \\
0.05 \text { to } 14.28, \mathrm{I}^{2}= \\
\text { N/A }\end{array}$ & $\begin{array}{l}1 \mathrm{RCT}^{118} \text { with } 31 \\
\text { patients }\end{array}$ & $\begin{array}{l}\text { High ROB and } \\
\text { severe imprecision }\end{array}$ & Insufficient evidence \\
\hline $\begin{array}{l}\text { Dyspnea } \\
\text { (Questionnaire: } \\
\text { Oxygen Cost } \\
\text { Diagram) } \\
\text { End of Intervention }\end{array}$ & $\begin{array}{l}\text { WMD: } 05.95 ; 95 \% \\
\text { Cl: }-5.74 \text { to } 17.64, \\
I^{2}=\text { N/A }\end{array}$ & $\begin{array}{l}1 \mathrm{RCT}^{118} \text { with } 31 \\
\text { patients }\end{array}$ & $\begin{array}{l}\text { High ROB and } \\
\text { imprecision }\end{array}$ & Insufficient evidence \\
\hline $\begin{array}{l}\text { QoL(General Well- } \\
\text { Being) } \\
\text { End of Intervention }\end{array}$ & $\begin{array}{l}\text { WMD: } 22.21 ; 95 \% \\
\mathrm{Cl}:-6.99 \text { to } 151.40 \\
\mathrm{I}^{2}=\mathrm{N} / \mathrm{A}\end{array}$ & $\begin{array}{l}1 \mathrm{RCT}^{118} \text { with } 31 \\
\text { patients }\end{array}$ & $\begin{array}{l}\text { High ROB and } \\
\text { imprecision }\end{array}$ & Insufficient evidence \\
\hline
\end{tabular}

Table 44. Comparison of a dietary intervention using a caloric supplement during ECOPD versus usual diet, additional outcomes

\begin{tabular}{|l|l|c|}
\hline \multicolumn{1}{|c|}{ Outcome } & \multicolumn{1}{|c|}{ Findings } & Study Design and Sample Size \\
\hline $\begin{array}{l}\text { FEV1\% Predicted } \\
\text { End of Intervention }\end{array}$ & WMD: $6.14 ; 95 \% \mathrm{Cl}:-0.76$ to $13.04, \mathrm{I}^{2}=\mathrm{N} / \mathrm{A}$ & $1 \mathrm{RCT}^{118}$ with 31 patients \\
\hline
\end{tabular}

CI = confidence interval; FEV1 = forced expiratory volume in one second; N/A = not applicable; RCT = randomized controlled trial; WMD = weighted mean difference

\section{Dietary Intervention Using a Caloric and a Protein Supplement During ECOPD Versus Placebo (Non-Caloric Fluid, Vanilla Flavored Water)}

One study ${ }^{115}$ evaluated the effectiveness of a dietary intervention using a caloric and a protein supplement during ECOPD compared with placebo (non-caloric fluid, vanilla flavored water) in patients with moderate to severe ECOPD (Table 45 and Table 46).

A caloric and protein supplement during ECOPD was not associated with statistically significant differences in dyspnea and FEV1 percent predicted at the end of the intervention compared with placebo (non-caloric fluid, vanilla flavored water).

There was no statistically significant difference observed in withdrawals, withdrawals due to AEs, gastrointestinal AEs, and total number of AEs (Appendix Table H.18.).

Table 45. Comparison of a dietary intervention using a caloric and a protein supplement during ECOPD versus placebo (non-caloric fluid, vanilla flavored water), critical outcomes

\begin{tabular}{|c|c|c|c|c|}
\hline Outcome & Findings & $\begin{array}{l}\text { Study Design and } \\
\text { Sample Size }\end{array}$ & $\begin{array}{l}\text { Rationale for } \\
\text { Strength of } \\
\text { Evidence (SOE) }\end{array}$ & $\begin{array}{l}\text { Overall Strength of } \\
\text { Evidence }\end{array}$ \\
\hline $\begin{array}{l}\text { Dyspnea } \\
\text { (Numeric Scale: } \\
\text { VAS, dyspnea score } \\
\text { while eating) } \\
\text { End of Intervention }\end{array}$ & $\begin{array}{l}\text { WMD: } 0.5 ; 95 \% \mathrm{Cl} \text { : } \\
-1.14 \text { to } 2.14, \mathrm{I}^{2}= \\
\text { N/A }\end{array}$ & $\begin{array}{l}1 \mathrm{RCT}^{115} \text { with } 47 \\
\text { patients }\end{array}$ & $\begin{array}{l}\text { High } \mathrm{ROB} \text { and } \\
\text { severe imprecision }\end{array}$ & Insufficient evidence \\
\hline
\end{tabular}

CI = confidence interval; N/A = not applicable; RCT = randomized controlled trial; ROB = risk of bias; SMD = standardized mean difference; VAS = visual analog scale 
Table 46. Comparison of a dietary intervention using a caloric and a protein supplement during ECOPD versus placebo (non-caloric fluid, vanilla flavored water), additional outcomes

\begin{tabular}{|l|c|c|}
\hline \multicolumn{1}{|c|}{ Outcome } & \multicolumn{1}{|c|}{ Findings } & Study Design and Sample Size \\
\hline $\begin{array}{l}\text { FEV1\% Predicted } \\
\text { End of Intervention }\end{array}$ & WMD: $0.00 ; 95 \% \mathrm{Cl}:-4.36$ to $4.36, \mathrm{I}^{2}=\mathrm{N} / \mathrm{A}$ & $1 \mathrm{RCT} \mathrm{T}^{115}$ with 47 patients \\
\hline
\end{tabular}

$\mathrm{CI}$ = confidence interval; FEV1 = forced expiratory volume in one second; N/A = not applicable; RCT = randomized controlled trial; WMD = weighted mean difference

\section{Dietary Intervention Using a High Fat Low Carbohydrate Diet During ECOPD Versus Usual Diet}

One study ${ }^{109}$ evaluated the effectiveness of a dietary intervention using a high fat low carbohydrate diet during ECOPD compared with a usual diet in patients with moderate to severe ECOPD (Table 47).

A high fat low carbohydrate diet during ECOPD was not found to have a statistically significant difference in FEV1 percent absolute at the end of the intervention compared with usual diet.

Table 47. Comparison of a dietary intervention using a high fat low carbohydrate diet during ECOPD versus usual diet

\begin{tabular}{|l|l|l|}
\hline \multicolumn{1}{|c|}{ Outcome } & \multicolumn{1}{|c|}{ Findings } & \multicolumn{1}{c|}{ Study Design and Sample Size } \\
\hline FEV1 Absolute & WMD: $-0.01 ; 95 \% \mathrm{Cl}:-0.31$ to 0.29, & $1 \mathrm{RCT}^{109}$ with 30 patients \\
End of Intervention & $\mathrm{I}^{2}=\mathrm{N} / \mathrm{A}$ & \\
\hline
\end{tabular}

$\mathrm{CI}$ = confidence interval; FEV1 = forced expiratory volume in one second; N/A = not applicable; RCT = randomized controlled trial; WMD = weighted mean difference

\section{Omega-3 Fatty Acid Enriched Diet Versus Usual Diet}

One study compared omega-3 fatty acids to a usual diet in patients with moderate to severe ECOPD (Table 48 and Appendix table H.19). ${ }^{123}$ There was no statistically significant difference observed in dyspnea, need for intubation, and quality of life between the two groups.

Table 48. Comparison of a dietary intervention using omega-3 fatty acid versus usual diet

\begin{tabular}{|l|l|l|l|l|}
\hline \multicolumn{1}{|c|}{ Outcome } & \multicolumn{1}{|c|}{ Findings } & $\begin{array}{l}\text { Study Design and } \\
\text { Sample Size }\end{array}$ & $\begin{array}{l}\text { Rationale for } \\
\text { Strength of } \\
\text { Evidence (SOE) }\end{array}$ & $\begin{array}{l}\text { Overall Strength } \\
\text { of Evidence }\end{array}$ \\
\hline $\begin{array}{l}\text { QoL (CAT) } \\
\text { End of Intervention }\end{array}$ & $\begin{array}{l}\text { WMD: } 0.00 ; 95 \% \\
\mathrm{Cl}:-3.46 \text { to } 3.46, \\
\mathrm{I}^{2}=\mathrm{N} / \mathrm{A}\end{array}$ & $\begin{array}{l}1 \mathrm{RCT}^{123} \text { with } 50 \\
\text { patients }\end{array}$ & $\begin{array}{l}\text { Severe } \\
\text { imprecision }\end{array}$ & $\begin{array}{l}\text { Low SOE } \\
\text { supporting no } \\
\text { difference }\end{array}$ \\
\hline $\begin{array}{l}\text { Need for Intubation } \\
\text { End of Intervention }\end{array}$ & $\begin{array}{l}\mathrm{OR}: 0.18 ; 95 \% \mathrm{Cl}: \\
0.01 \text { to } 4.04, \mathrm{I}^{2}= \\
\mathrm{N} / \mathrm{A}\end{array}$ & $\begin{array}{l}1 \mathrm{RCT}^{123} \text { with } 50 \\
\text { patients }\end{array}$ & $\begin{array}{l}\text { Severe } \\
\text { imprecision }\end{array}$ & $\begin{array}{l}\text { Low SOE } \\
\text { supporting no } \\
\text { difference }\end{array}$ \\
\hline $\begin{array}{l}\text { Dyspnea (Questionnaire: } \\
\text { MMRC) } \\
\text { End of intervention }\end{array}$ & $\begin{array}{l}\text { WMD: } 0.00 ; 95 \% \\
\mathrm{Cl}:-0.55 \text { to } 0.55,\end{array}$ & $\begin{array}{l}1 \mathrm{RCT}^{123} \text { with } 50 \\
\text { patients }\end{array}$ & $\begin{array}{l}\text { Severe } \\
\text { imprecision }\end{array}$ & $\begin{array}{l}\text { Low SOE } \\
\text { supporting no } \\
\text { difference }\end{array}$ \\
\hline
\end{tabular}

CAT = COPD Assessment Test; CI = confidence interval; MMRC = Modified Medical Research Ccouncil Scale; N/A = not applicable; OR = odds ratio; QoL = quality of life; RCT = randomized controlled trial; SMD = standardized mean difference

\section{Vitamin D Versus Placebo}

Two studies ${ }^{85,91}$ evaluated the effectiveness of vitamin D during ECOPD compared with placebo in patients with moderate to severe ECOPD (Table 49 and Table 50). In one study, ${ }^{91}$ vitamin $\mathrm{D}$ was given orally, in the other, ${ }^{85}$ it was given as intermuscular injection. 
Vitamin D during ECOPD was associated with a statistically significantly better quality of life and less symptoms at the end of the intervention and at the longest followup compared with placebo.

There was no statistically significant difference found in mortality at longest followup, dyspnea at the end of the intervention and at the longest followup, FEV1 percent predicted at the end of the intervention, and number of withdrawals (Appendix Table H.20.).

Table 49. Comparison of a dietary intervention using vitamin D during ECOPD versus placebo, critical outcomes

\begin{tabular}{|c|c|c|c|c|}
\hline Outcome & Findings & $\begin{array}{l}\text { Study Design and } \\
\text { Sample Size }\end{array}$ & $\begin{array}{c}\text { Rationale for } \\
\text { Strength of } \\
\text { Evidence (SOE) }\end{array}$ & $\begin{array}{l}\text { Overall Strength of } \\
\text { Evidence }\end{array}$ \\
\hline $\begin{array}{l}\text { Mortality } \\
\text { Longest Followup }\end{array}$ & $\begin{array}{l}\text { OR: } 1.55 ; 95 \% \mathrm{Cl}: \\
0.24 \text { to } 9.88,1^{2}=\mathrm{N} / \mathrm{A}\end{array}$ & $\begin{array}{l}1 \mathrm{RCT}^{85} \text { with } 70 \\
\text { patients }\end{array}$ & $\begin{array}{l}\text { High ROB and } \\
\text { severe imprecision }\end{array}$ & Insufficient evidence \\
\hline $\begin{array}{l}\text { Dyspnea } \\
\text { (Questionnaire: } \\
\text { MMRC) } \\
\text { End of Intervention }\end{array}$ & $\begin{array}{l}\text { SMD: }-0.11 ; 95 \% \\
\text { Cl: }-0.42 \text { to } 0.20, \\
I^{2}=0.00 \%\end{array}$ & $\begin{array}{l}2 \mathrm{RCTs}^{8591} \text { with } \\
160 \text { patients }\end{array}$ & $\begin{array}{l}\text { High ROB and } \\
\text { severe imprecision }\end{array}$ & Insufficient evidence \\
\hline $\begin{array}{l}\text { Dyspnea } \\
\text { (Questionnaire: } \\
\text { MMRC) } \\
\text { Longest Followup }\end{array}$ & $\begin{array}{l}\text { SMD: } 0.27 ; 95 \% \mathrm{Cl} \\
-0.09 \text { to } 0.63, \mathrm{I}^{2}= \\
\text { N/A }\end{array}$ & $\begin{array}{l}1 \mathrm{RCT}^{85} \text { with } 70 \\
\text { patients }\end{array}$ & $\begin{array}{l}\text { High ROB and } \\
\text { severe imprecision }\end{array}$ & Insufficient evidence \\
\hline $\begin{array}{l}\text { QoL(SGRQ) } \\
\text { End of Intervention }\end{array}$ & $\begin{array}{l}\text { WMD: }-1.96 ; 95 \% \\
\text { Cl: }-2.89 \text { to }-1.03 \text {, } \\
\mathrm{I}^{2}=\mathrm{N} / \mathrm{A}\end{array}$ & $\begin{array}{l}1 \mathrm{RCT}^{85} \text { with } 70 \\
\text { patients }\end{array}$ & $\begin{array}{l}\text { High ROB and } \\
\text { imprecision }\end{array}$ & $\begin{array}{l}\text { Low SOE supporting } \\
\text { improvement }\end{array}$ \\
\hline $\begin{array}{l}\text { QoL(SGRQ) } \\
\text { Longest Followup }\end{array}$ & $\begin{array}{l}\text { WMD: }-4.67 ; 95 \% \\
\text { Cl: }-6.00 \text { to }-3.35 \text {, } \\
\mathrm{I}^{2}=\mathrm{N} / \mathrm{A}\end{array}$ & $\begin{array}{l}1 \mathrm{RCT}^{85} \text { with } 70 \\
\text { patients }\end{array}$ & $\begin{array}{l}\text { High ROB and } \\
\text { imprecision }\end{array}$ & $\begin{array}{l}\text { Low SOE supporting } \\
\text { improvement }\end{array}$ \\
\hline
\end{tabular}

CI = confidence interval; MMRC = Modified Medical Research Council Scale; N/A = not applicable; OR = odds ratio; QoL = quality of life; RCT = randomized controlled trial; ROB = risk of bias; SMD = standardized mean difference; SGRQ = St.

George Respiratory Questionnaire; SOE = strength of evidence; WMD = weighted mean difference

Table 50. Comparison of a dietary intervention using vitamin D during ECOPD versus placebo, additional outcomes

\begin{tabular}{|l|l|l|}
\hline \multicolumn{1}{|c|}{ Outcome } & \multicolumn{1}{c|}{ Findings } & \multicolumn{1}{c|}{$\begin{array}{c}\text { Study Design and Sample } \\
\text { Size }\end{array}$} \\
\hline $\begin{array}{l}\text { Other Symptoms(SGRQ } \\
\text { symptom score) } \\
\text { End of Intervention }\end{array}$ & WMD: $-4.47 ; 95 \% \mathrm{Cl}:-8.10$ to $-0.84, \mathrm{I}^{2}=\mathrm{N} / \mathrm{A}$ & $1 \mathrm{RCT}^{85}$ with 70 patients \\
\hline $\begin{array}{l}\text { Other Symptoms(SGRQ } \\
\text { symptom score) } \\
\text { Longest Followup }\end{array}$ & WMD: $-7.19 ; 95 \% \mathrm{Cl}:-11.12$ to $-3.26, \mathrm{I}^{2}=\mathrm{N} / \mathrm{A}$ & $1 \mathrm{RCT}^{85}$ with 70 patients \\
\hline $\begin{array}{l}\text { FEV1\% Predicted } \\
\text { End of Intervention }\end{array}$ & WMD: $2.20 ; 95 \% \mathrm{Cl}:-7.24$ to $11.64, \mathrm{I}^{2}=\mathrm{N} / \mathrm{A}$ & $1 \mathrm{RCT}^{91}$ with 90 patients \\
\hline
\end{tabular}

$\mathrm{CI}$ = confidence interval; FEV1 = forced expiratory volume in one second; N/A = not applicable; RCT = randomized controlled trial; SGRQ = St. George Respiratory Questionnaire; WMD = weighted mean difference 
KQ3. In adult patients with exacerbation of COPD, what are the benefits and harms of combinations of treatments that are individually effective (based on empirical evidence in stable COPD)?

\section{Key Point-KQ3}

- No statistically significant difference in adverse events was found between any of the combined treatments and individual treatments.

\section{KQ3 Results}

There were 3 studies ${ }^{126-128}$ with 149 patients included for KQ3. The characteristics of the studies are listed in Appendix Table D.3. 1 trial was conducted in a hospital setting, ${ }^{127} 1$ in the emergency department, ${ }^{126}$ and 1 in an unclear setting. ${ }^{128}$ Studies were conducted in Europe (2), ${ }^{127,128}$ and in Australia (1). ${ }^{126}$ The average length of treatment was 14.5 days. Followup was not reported in any study. Details of the interventions used in each study can be found in Appendix Table F.3. Individual studies inclusion and exclusion criteria are in Appendix Table I.1.

The overall risk of bias is moderate to high due to unclear risk of bias for most items, including sequence generation, allocation concealment, blinding of outcome assessors, and unknown risk of other bias (Appendix Table E.1.).

Three studies ${ }^{126-128}$ assessed the comparative effectiveness of different inhalation treatments for ECOPD (Table 51 and Table 52).

Direct comparison of Ipratropium (short-acting muscarinic antagonists (SAMA) versus Salbutamol (SABA) ${ }^{126}$ did not show any statistically significant difference in FEV1 absolute at the end of the intervention. Combination therapy with ipratropium + salbutamol

$(\mathrm{SAMA}+\mathrm{SABA})^{126,127}$ did not result in a statistically significant change in FEV1 absolute at the end of the intervention compared with salbutamol (SABA) alone $\mathrm{a}^{126,127}$ or ipratropium $(\text { SAMA })^{126}$ alone.

Combination therapy of beclomethasone + salbutamol (ICS+SABA) $)^{128}$ was not found to have a statistically significant difference in clinical cure, clinical failure and FEV1 percent predicted at the end of the intervention compared with fenoterol (SABA) only.

There was no statistically significant difference found on withdrawals, general internal medicine AEs, neurological AEs, and total number of AEs (Appendix Table H.21.).

Table 51. Comparative effectiveness of inhalation treatments, critical outcomes

\begin{tabular}{|c|c|c|c|c|c|}
\hline Comparison & Outcome & Findings & $\begin{array}{l}\text { Study Design and } \\
\text { Sample Size }\end{array}$ & $\begin{array}{l}\text { Rationale for } \\
\text { Strength of } \\
\text { Evidence } \\
\text { (SOE) }\end{array}$ & $\begin{array}{c}\text { Overall } \\
\text { Strength of } \\
\text { Evidence }\end{array}$ \\
\hline $\begin{array}{l}\text { ICS+ SABA } \\
\text { (Beclomethasone+ } \\
\text { Salbutamol) vs. } \\
\text { SABA (Fenoterol) }\end{array}$ & $\begin{array}{l}\text { Clinical Cure } \\
\text { End of } \\
\text { Intervention }\end{array}$ & $\begin{array}{l}\text { OR: } 1.00 ; \\
95 \% \mathrm{Cl} \text {; } \\
0.17 \text { to } \\
5.98, \mathrm{I}^{2}= \\
\mathrm{N} / \mathrm{A}\end{array}$ & $\begin{array}{l}1 \mathrm{RCT}^{128} \text { with } 30 \\
\text { patients }\end{array}$ & $\begin{array}{l}\text { High ROB } \\
\text { and severe } \\
\text { imprecision }\end{array}$ & $\begin{array}{l}\text { Insufficient } \\
\text { evidence }\end{array}$ \\
\hline
\end{tabular}




\begin{tabular}{|c|c|c|c|c|c|}
\hline Comparison & Outcome & Findings & $\begin{array}{l}\text { Study Design and } \\
\text { Sample Size }\end{array}$ & $\begin{array}{l}\text { Rationale for } \\
\text { Strength of } \\
\text { Evidence } \\
\text { (SOE) }\end{array}$ & $\begin{array}{c}\text { Overall } \\
\text { Strength of } \\
\text { Evidence }\end{array}$ \\
\hline $\begin{array}{l}\text { ICS+ SABA } \\
\text { (Beclomethasone+ } \\
\text { Salbutamol) vs. } \\
\text { SABA (Fenoterol) }\end{array}$ & $\begin{array}{l}\text { Clinical } \\
\text { Failure } \\
\text { End of } \\
\text { Intervention }\end{array}$ & $\begin{array}{l}\text { OR: } 0.31 ; \\
95 \% \mathrm{Cl} \text {; } \\
0.01 \text { to } \\
8.28, \mathrm{I}^{2}= \\
\mathrm{N} / \mathrm{A}\end{array}$ & $\begin{array}{l}1 \mathrm{RCT}^{128} \text { with } 30 \\
\text { patients }\end{array}$ & $\begin{array}{l}\text { High ROB } \\
\text { and severe } \\
\text { imprecision }\end{array}$ & $\begin{array}{l}\text { Insufficient } \\
\text { evidence }\end{array}$ \\
\hline
\end{tabular}

CI = confidence interval; ICS = inhaled corticosteroid; N/A = not applicable; OR = odds ratio; RCT = randomized controlled trial; SABA = short-acting beta adrenergic agonists; SOE = strength of evidence

Table 52. Comparative effectiveness of inhalation treatments, additional outcomes

\begin{tabular}{|c|c|c|c|}
\hline Comparison & Outcome & Findings & Study Design and Sample Size \\
\hline $\begin{array}{l}\text { ICS + SABA } \\
\text { (Beclomethasone } \\
\text { + Salbutamol) vs. } \\
\text { SABA (Fenoterol) }\end{array}$ & $\begin{array}{l}\text { FEV1\% } \\
\text { Predicted } \\
\text { End of } \\
\text { Intervention }\end{array}$ & $\begin{array}{l}\text { WMD: } 7.60 ; \\
95 \% \mathrm{Cl}:-2.53 \\
\text { to } 17.73, \mathrm{I}^{2}=\mathrm{N} / \mathrm{A}\end{array}$ & $1 \mathrm{RCT}^{128}$ with 30 patients \\
\hline $\begin{array}{l}\text { SABA + SAMA } \\
\text { (Salbutamol + } \\
\text { Ipratropium) vs. } \\
\text { SABA } \\
\text { (Salbutamol) }\end{array}$ & $\begin{array}{l}\text { FEV1 Absolute } \\
\text { End of } \\
\text { Intervention }\end{array}$ & $\begin{array}{l}\text { WMD: }-0.07 ; \\
95 \% \mathrm{Cl}:-0.17 \\
\text { to } 0.02 \text {, } \\
\mathrm{I}^{2}=0.00 \%\end{array}$ & 2 RCTs $^{126,127}$ with 104 patients \\
\hline $\begin{array}{l}\text { SAMA } \\
\text { (Ipratropium) vs. } \\
\text { SABA } \\
\text { (Salbutamol) }\end{array}$ & $\begin{array}{l}\text { FEV1 Absolute } \\
\text { End of } \\
\text { Intervention }\end{array}$ & $\begin{array}{l}\text { WMD: }-0.11 ; \\
95 \% \mathrm{Cl}:-0.22 \\
\text { to } 0.00, \mathrm{I}^{2}=\mathrm{N} / \mathrm{A}\end{array}$ & $1 \mathrm{RCT}^{126}$ with 31 patients \\
\hline $\begin{array}{l}\text { SABA + SAMA } \\
\text { (Ipratropium vs. } \\
\text { Salbutamol) + } \\
\text { SAMA } \\
\text { (Ipratropium) }\end{array}$ & $\begin{array}{l}\text { FEV1 Absolute } \\
\text { End of } \\
\text { Intervention }\end{array}$ & $\begin{array}{l}\text { WMD: }-0.04 ; \\
95 \% \mathrm{Cl}:-0.13 \\
\text { to } 0.05, \mathrm{I}^{2}=\mathrm{N} / \mathrm{A}\end{array}$ & $1 \mathrm{RCT}^{126}$ with 33 patients \\
\hline
\end{tabular}

CI = confidence interval; FEV1 = forced expiratory volume in one second; ICS = inhaled corticosteroid; N/A = not applicable; RCT = randomized controlled trial; SABA = short-acting beta adrenergic agonists; SAMA = short-acting muscarinic antagonists; $\mathrm{WMD}=$ weighted mean difference

KQ4. In adult patients with exacerbation of COPD, what is the comparative effectiveness of different regimens of antibiotics and systemic corticosteroids based on type of agents (e.g.. broad-spectrum vs. narrowspectrum antibiotics), delivery modes (e.g., intravenous, oral), and durations of treatment?

\section{Key Points-KQ4}

\section{Comparative Effectiveness of Different Antibiotics}

- Numerous antibiotics, given as empirical initial therapy for ECOPD (in the absence of pneumonia), were compared against each other but the evidence was insufficient to estimate an effect on final health outcomes; except that levofloxacin reduced repeat exacerbations at 3 months of followup, compared with prulifloxacin (;ow SOE). 
- Amoxicillin plus clavulanic acid was associated with statistically significantly more adverse events than telithromycin. Imipenem plus cilastatin was associated with statistically significantly more adverse events than meropenem.

\section{Comparative Effectiveness of Different Dosages of the Same Antibiotic}

- The evidence comparing different dosages of the same antibiotic was insufficient for mortality, clinical cure, and clinical failure.

- No difference in adverse events was found between trovafloxacin $200 \mathrm{mg}$ and trovafloxacin $100 \mathrm{mg}$.

\section{Comparative Effectiveness of Different Application Routes for Antibiotics}

- No studies were found.

\section{Comparative Effectiveness of Different Durations of Treatment With Antibiotics}

- The evidence was insufficient comparing 3 day versus 10 day regimens of amoxicillin plus clavulanic acid.

- No statistically significant difference of AEs was found between 3 day and 10 day regimens of amoxicillin plus clavulanic acid.

\section{Comparative Effectiveness of Different Corticosteroids}

- The evidence was insufficient comparing the different corticosteroids for mortality, need for intubation, clinical failures, and dyspnea.

- There was no statistically significant difference in AEs found between the different systemic corticosteroids.

\section{Comparative Effectiveness of Different Routes of Administration for Corticosteroids}

- $\quad$ No difference between intravenous methylprednisolone and inhaled budesonide $40 \mathrm{mg}$ was found in quality of life and repeat exacerbations (low SOE).

- Inhaled budesonide $40 \mathrm{mg}$ was associated with statistically significantly less endocrinerelated AEs than intravenous methylprednisolone.

\section{Comparative Effectiveness of Different Durations of Treatment With Corticosteroids}

- The evidence was insufficient comparing the different durations of corticosteroid treatment for mortality, hospital admission, need for intubation, clinical failure, quality of life, repeat exacerbation, and dyspnea.

- There was no statistically significant difference in AEs found between the different systemic corticosteroid durations. 


\section{KQ 4 Results}

There were 34 studies $^{75-77,129-159}$ with 7,311 patients included for KQ4. The characteristics of the studies are listed in Appendix Table D.4. 14 trials were conducted in a hospital setting, ${ }^{75-77,}$ 129, 130, 133, 140, 142, 144, 146, 149, 151, 155, 1593 in the Emergency Department, ${ }^{137,}{ }^{141,} 15810$ in an outpatient setting, ${ }^{132,136,138,139,143,145,148,152-154}$ one in both outpatients and hospitalized patients, ${ }^{156}$ and in 5 the setting was unclear. ${ }^{131}$, 134, 135, 150, 157 Studies were conducted in the US (4), ${ }^{77,154,156,157}$ Europe (17), ${ }^{76,130,135,138-144,147,148,151-153,155,158}$ Asia (11), ${ }^{75}, 129,131-134,137,146,149,150,159$ South America (1), ${ }^{145}$ and 1 study was conducted in 30 countries. ${ }^{136}$ Mean treatment length was 10.6 days and mean reported followup was 3 months. Details of the interventions used in each study can be found in Appendix Table F.4. Individual studies inclusion and exclusion criteria are in Appendix Table I.1.

The overall risk of bias is high due to unclear sequence generation (50.00\%), unclear allocation concealment (79.41\%), high risk or unclear blinding of patients or care providers (58.82\%), high risk or unclear blinding of outcome assessors (88.24\%) and high risk or unclear risk of other bias (94.12\%) (Appendix Table E.1.).

Figure 2 gives an overview of the different comparisons made in different studies.

\section{Figure 2. Summary of comparisons between different antibiotic agents}

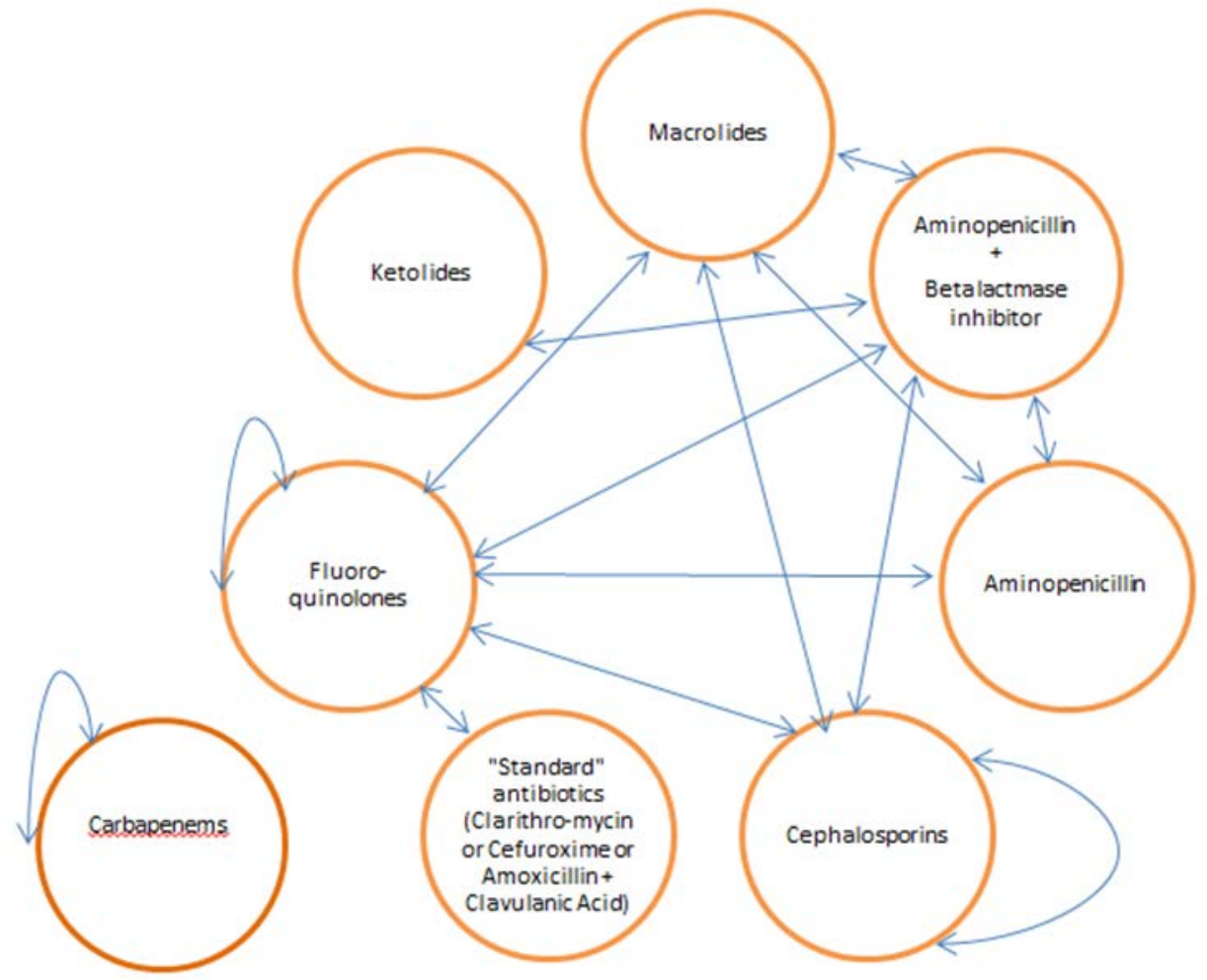

Note: Each arrowed line represents a comparison between two treatments reported by the literature. 


\section{Fluoroquinolone Versus Aminopenicillin Plus Beta-Lactamase Inhibitor}

Three studies $^{136,150,152}$ evaluated the effectiveness of a fluoroquinolone (either moxifloxacin, trovafloxacin, or ciprofloxacin) compared with an aminopenicillin (amoxicillin or ampicillin) plus a beta-lactamase inhibitor (clavulanic acid or sulbactam). There were no statistically significant differences observed between the groups in any of the outcomes (Table 53 and Table 54) and adverse events (Appendix Table H.22.). One study ${ }^{136}$ compared moxifloxacin to amoxicillin + clavulanic acid and reported 97 cases of serious unspecified AEs (46 in the moxifloxacin group vs. 51 in the amoxicillin + clavulanic acid group). Four cases in the moxifloxacin group were considered as treatment related, including anaphylactic reaction, bronchitis, gastroenteritis, and tachyarrhythmia); while 2 cases (allergic dermatitis and radial nerve palsy) were reported in the amoxicillin + clavulanic acid group.

Table 53. Comparison of fluoroquinolone versus aminopenicillin plus beta-lactamase inhibitor, critical outcomes

\begin{tabular}{|c|c|c|c|c|c|}
\hline Comparison & Outcome & Findings & $\begin{array}{l}\text { Study Design } \\
\text { and Sample } \\
\text { Size }\end{array}$ & $\begin{array}{l}\text { Rationale for } \\
\text { Strength of } \\
\text { Evidence } \\
\text { (SOE) }\end{array}$ & $\begin{array}{c}\text { Overall } \\
\text { Strength of } \\
\text { Evidence }\end{array}$ \\
\hline $\begin{array}{l}\text { Fluoroquinolone vs. } \\
\text { Amoxicillin + } \\
\text { Clavulanic Acid } \\
\text { Moxifloxacin vs. } \\
\text { Amoxicillin + } \\
\text { Clavulanic Acid } \\
\\
\text { Trovafloxacin vs. } \\
\text { Amoxicillin + } \\
\text { Clavulanic Acid }\end{array}$ & $\begin{array}{l}\text { Mortality } \\
\text { End of } \\
\text { Intervention }\end{array}$ & 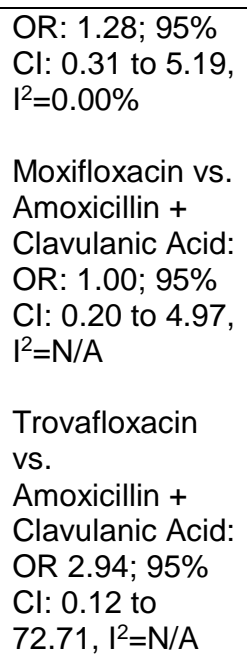 & $\begin{array}{l}\text { 2 RCTs }{ }^{136,152} \\
\text { with } 1656 \\
\text { patients } \\
\text { Moxifloxacin } \\
\text { vs. Amoxicillin } \\
\text { + Clavulanic } \\
\text { Acid: } 1 \text { RCT }{ }^{136} \\
\text { with } 1372 \\
\text { patients } \\
\text { Trovafloxacin } \\
\text { vs. } \\
\text { Amoxicillin + } \\
\text { Clavulanic } \\
\text { Acid:1 RCT }{ }^{152} \\
\text { with } 284 \\
\text { patients }\end{array}$ & $\begin{array}{l}\text { High ROB and } \\
\text { severe } \\
\text { imprecision }\end{array}$ & $\begin{array}{l}\text { Insufficient } \\
\text { evidence }\end{array}$ \\
\hline \multirow[t]{3}{*}{$\begin{array}{l}\text { Moxifloxacin vs. } \\
\text { Amoxicillin }+ \\
\text { Clavulanic Acid }\end{array}$} & $\begin{array}{l}\text { QoL(SGRQ) } \\
\text { End of } \\
\text { Intervention }\end{array}$ & $\begin{array}{l}\text { WMD: }-0.73 ; \\
95 \% \mathrm{Cl}:-2.04 \text { to } \\
0.58, \mathrm{I}^{2}=\mathrm{N} / \mathrm{A}\end{array}$ & $\begin{array}{l}\text { 1 RCT }{ }^{136} \text { with } \\
1372 \text { patients }\end{array}$ & $\begin{array}{l}\text { High ROB and } \\
\text { severe } \\
\text { imprecision }\end{array}$ & $\begin{array}{l}\text { Insufficient } \\
\text { evidence }\end{array}$ \\
\hline & $\begin{array}{l}\text { QOL(SGRQ) } \\
\text { Longest } \\
\text { Followup }\end{array}$ & $\begin{array}{l}\text { WMD: }-0.05 ; \\
95 \% \mathrm{Cl}:-2.31 \text { to } \\
2.21, \mathrm{I}^{2}=\mathrm{N} / \mathrm{A}\end{array}$ & $\begin{array}{l}1 \mathrm{RCT}^{136} \text { with } \\
1372 \text { patients }\end{array}$ & $\begin{array}{l}\text { High ROB and } \\
\text { severe } \\
\text { imprecision }\end{array}$ & $\begin{array}{l}\text { Insufficient } \\
\text { evidence }\end{array}$ \\
\hline & $\begin{array}{l}\text { Hospital } \\
\text { Admission } \\
\text { Longest } \\
\text { Followup }\end{array}$ & $\begin{array}{l}\text { OR: } 0.86 ; 95 \% \\
\text { Cl: } 0.56 \text { to } 1.33 \text {, } \\
\mathrm{I}^{2}=\mathrm{N} / \mathrm{A}\end{array}$ & $\begin{array}{l}1 \text { RCT }{ }^{136} \text { with } \\
1372 \text { patients }\end{array}$ & $\begin{array}{l}\text { High ROB and } \\
\text { severe } \\
\text { imprecision }\end{array}$ & $\begin{array}{l}\text { Insufficient } \\
\text { evidence }\end{array}$ \\
\hline
\end{tabular}




\begin{tabular}{|c|c|c|c|c|c|}
\hline Comparison & Outcome & Findings & $\begin{array}{l}\text { Study Design } \\
\text { and Sample } \\
\text { Size }\end{array}$ & $\begin{array}{l}\text { Rationale for } \\
\text { Strength of } \\
\text { Evidence } \\
\text { (SOE) }\end{array}$ & $\begin{array}{c}\text { Overall } \\
\text { Strength of } \\
\text { Evidence }\end{array}$ \\
\hline $\begin{array}{l}\text { Fluoroquinolone vs. } \\
\text { Aminopenicillin plus } \\
\text { Beta-lactamase } \\
\text { inhibitor }\end{array}$ & $\begin{array}{l}\text { Clinical Cure } \\
\text { End of } \\
\text { Intervention }\end{array}$ & $\begin{array}{l}\text { OR: } 1.05 ; 95 \% \\
\mathrm{Cl}: 0.61 \text { to } 1.81 \\
\mathrm{I}^{2}=64.80 \%\end{array}$ & $\begin{array}{l}2 \mathrm{RCTs}^{150,152} \\
\text { with } 337 \\
\text { patients }\end{array}$ & $\begin{array}{l}\text { High ROB and } \\
\text { severe } \\
\text { imprecision }\end{array}$ & $\begin{array}{l}\text { Insufficient } \\
\text { evidence }\end{array}$ \\
\hline $\begin{array}{l}\text { Ciprofloxacin vs. } \\
\text { Ampicillin + } \\
\text { Sulbactam }\end{array}$ & & $\begin{array}{l}\text { OR: } 11 ; 95 \% \\
\mathrm{Cl}: 0.58 \text { to } \\
209.90,1^{2}=\mathrm{N} / \mathrm{A}\end{array}$ & $\begin{array}{l}1 \mathrm{RCT}^{150} \text { with } \\
53 \text { patients }\end{array}$ & & \\
\hline $\begin{array}{l}\text { Trovafloxacin vs. } \\
\text { Amoxicillin }+ \\
\text { Clavulanic Acid }\end{array}$ & & $\begin{array}{l}\text { OR: } 0.87 ; 95 \% \\
\mathrm{Cl}: 0.49 \text { to } 1.55 \\
\mathrm{I}^{2}=\mathrm{N} / \mathrm{A}\end{array}$ & $\begin{array}{l}1 \mathrm{RCT} \text { with } \\
284 \text { patients }\end{array}$ & & \\
\hline $\begin{array}{l}\text { Moxifloxacin vs. } \\
\text { Amoxicillin + } \\
\text { Clavulanic Acid }\end{array}$ & $\begin{array}{l}\text { Clinical Failure } \\
\text { Longest } \\
\text { Followup }\end{array}$ & $\begin{array}{l}\text { OR: } 0.93 ; 95 \% \\
\mathrm{Cl}: 0.72 \text { to } 1.21 \\
\mathrm{I}^{2}=\mathrm{N} / \mathrm{A}\end{array}$ & $\begin{array}{l}1 \mathrm{RCT}^{136} \text { with } \\
1372 \text { patients }\end{array}$ & $\begin{array}{l}\text { High ROB and } \\
\text { severe } \\
\text { imprecision }\end{array}$ & $\begin{array}{l}\text { Insufficient } \\
\text { evidence }\end{array}$ \\
\hline
\end{tabular}

$\mathrm{CI}$ = confidence interval; N/A = not applicable; $\mathrm{OR}$ = odds ratio; QoL = quality of life; RCT = randomized controlled trial; ROB = risk of bias; SGRQ $=$ St. George respiratory questionnaire; SOE = strength of the body of evidence; WMD = weighted mean difference

Table 54. Comparison of fluoroquinolone versus aminopenicillin plus beta-lactamase inhibitor, additional outcomes

\begin{tabular}{|c|c|c|c|}
\hline Comparison & Outcome & Findings & Study Design and Sample Size \\
\hline $\begin{array}{l}\text { Moxifloxacin vs. } \\
\text { Amoxicillin }+ \\
\text { Clavulanic Acid }\end{array}$ & $\begin{array}{l}\text { FEV1\% } \\
\text { Predicted } \\
\text { End of } \\
\text { Intervention }\end{array}$ & $\begin{array}{l}\text { WMD: } 0.76 ; \\
95 \% \mathrm{Cl}:-0.94 \text { to } \\
2.46, \mathrm{I}^{2}=\mathrm{N} / \mathrm{A}\end{array}$ & $1 \mathrm{RCT}^{136}$ with 1372 patients \\
\hline $\begin{array}{l}\text { Moxifloxacin vs. } \\
\text { Amoxicillin }+ \\
\text { Clavulanic Acid }\end{array}$ & $\begin{array}{l}\text { FEV1\% } \\
\text { Predicted } \\
\text { Longest } \\
\text { Followup }\end{array}$ & $\begin{array}{l}\text { WMD:1.10; } 95 \% \\
\mathrm{Cl}:-0.61 \text { to } \\
2.81, \mathrm{I}^{2}=\mathrm{N} / \mathrm{A}\end{array}$ & $1 \mathrm{RCT}^{136}$ with 1372 patients \\
\hline $\begin{array}{l}\text { Fluoroquinolone vs. } \\
\text { Aminopenicillin + } \\
\text { Beta-lactamase } \\
\text { inhibitor } \\
\text { Moxifloxacin vs. } \\
\text { Amoxicillin + } \\
\text { Clavulanic Acid } \\
\text { Ciprofloxacin vs. } \\
\text { Ampicillin + } \\
\text { Sulbactam }\end{array}$ & $\begin{array}{l}\text { FEV1 Absolute } \\
\text { End of } \\
\text { Intervention }\end{array}$ & $\begin{array}{l}\text { WMD: } 0.03 ; \\
95 \% \mathrm{Cl}:-0.02 \text { to } \\
\text { 0.08, } \mathrm{I}^{2}=0.56 \% \\
\text { WMD: } 0.03 ; \\
95 \% \mathrm{Cl}:-0.02 \text { to } \\
\text { 0.08, I²=N/A } \\
\text { WMD: } 0.13 ; \\
95 \% \mathrm{Cl}:-0.20 \text { to } \\
0.46, \mathrm{I}^{2}=\mathrm{N} / \mathrm{A}\end{array}$ & $\begin{array}{l}2 \mathrm{RCTs}^{136,150} \text { with } 1425 \text { patients } \\
1 \mathrm{RCT}^{136} \text { with } 1372 \text { patients } \\
1 \mathrm{RCT}^{150} \text { with } 53 \text { patients }\end{array}$ \\
\hline $\begin{array}{l}\text { Moxifloxacin vs. } \\
\text { Amoxicillin + } \\
\text { Clavulanic Acid }\end{array}$ & $\begin{array}{l}\text { FEV1 Absolute } \\
\text { Longest } \\
\text { Followup }\end{array}$ & $\begin{array}{l}\text { WMD: } 0.04 ; \\
95 \% \mathrm{Cl}:-0.01 \text { to } \\
0.09, \mathrm{I}^{2}=\mathrm{N} / \mathrm{A}\end{array}$ & $1 \mathrm{RCT}^{136}$ with 1372 patients \\
\hline
\end{tabular}

$\mathrm{CI}$ = confidence interval; FEV1 = forced expiratory volume in one second; N/A = not applicable; RCT = randomized controlled trial; $\mathrm{WMD}=$ weighted mean difference 


\section{Ciprofloxacin (Fluoroquinolone) Versus Amoxicillin (Aminopenicillin)}

One study ${ }^{153}$ evaluated the effectiveness of ciprofloxacin, a fluoroquinolone, compared with amoxicillin, an aminopenicillin, and did not find a statistically significant difference between groups for FEV1 percent predicted at the end of the intervention (Table 55). Five patients in the ciprofloxacin group withdrew compared with 1 patient in the amoxicillin group (Appendix Table H.23.).

Table 55. Comparison of ciprofloxacin versus amoxicillin

\begin{tabular}{|l|l|l|}
\hline \multicolumn{1}{|c|}{ Outcome } & \multicolumn{1}{|c|}{ Findings } & \multicolumn{1}{c|}{ Study Design and Sample Size } \\
\hline FEV1\% & WMD: $5.00 ; 95 \% \mathrm{Cl}:-$ & $1 \mathrm{RCT}^{153}$ with 12 patients \\
$\begin{array}{l}\text { Predicted } \\
\text { End of } \\
\text { Intervention }\end{array}$ & 7.52 to $17.52, \mathrm{I}^{2}=\mathrm{N} / \mathrm{A}$ & \\
\hline
\end{tabular}

$\mathrm{CI}=$ confidence interval; FEV1 = forced expiratory volume in one second; N/A = not applicable; RCT = randomized controlled trial; WMD = weighted mean difference

\section{Levofloxacin (Fluoroquinolone) Versus “Standard” Antibiotic Therapy (Clarithromycin or Cefuroxime or Amoxicillin + Clavulanic Acid)}

One study ${ }^{141}$ evaluated the effectiveness of levofloxacin, a fluoroquinolone, compared with "standard" antibiotic therapy (defined as clarithromycin or cefuroxime or amoxicillin + clavulanic acid). Levofloxacin was associated with significantly lower risk of 30-day hospital admission than "standard" antibiotic therapy (low SOE). There were no statistically significant differences found in mortality, quality of life, repeat exacerbation, and FEV1 predicted between the two groups (Table 56 and Table 57).

There was no statistically significant difference observed in AEs (Appendix Table H.24.). Three patients in the levofloxacin group withdrew due to serious AEs (2 dizziness and 1 diarrhea), compared with 4 patients in the "standard" antibiotic therapy group (3 diarrhea cases and 1 urticaria). 
Table 56. Comparison of levofloxacin versus "standard" antibiotic therapy (clarithromycin or cefuroxime or amoxicillin + clavulanic acid), critical outcomes

\begin{tabular}{|c|c|c|c|c|}
\hline Outcome & Findings & $\begin{array}{l}\text { Study Design and } \\
\text { Sample Size }\end{array}$ & $\begin{array}{c}\text { Rationale for } \\
\text { Strength of } \\
\text { Evidence (SOE) }\end{array}$ & $\begin{array}{c}\text { Overall } \\
\text { Strength of } \\
\text { Evidence }\end{array}$ \\
\hline $\begin{array}{l}\text { Mortality } \\
\text { Longest } \\
\text { Followup }\end{array}$ & $\begin{array}{l}\text { OR : } 0.71 ; 95 \% \\
\mathrm{Cl}: 0.26 \text { to } 1.96 \text {, } \\
\left.\right|^{2}=\mathrm{N} / \mathrm{A}\end{array}$ & $\begin{array}{l}1 \mathrm{RCT}^{141} \text { with } 102 \\
\text { patients }\end{array}$ & $\begin{array}{l}\text { High ROB and } \\
\text { severe } \\
\text { imprecision }\end{array}$ & $\begin{array}{l}\text { Insufficient } \\
\text { evidence }\end{array}$ \\
\hline $\begin{array}{l}\text { QoL(HRQOL) } \\
\text { End of } \\
\text { Intervention }\end{array}$ & $\begin{array}{l}\text { WMD : }-0.73 ; \\
95 \% \mathrm{Cl}:-2.57 \text { to } \\
0.44, \mathrm{I}^{2}=\mathrm{N} / \mathrm{A}\end{array}$ & $\begin{array}{l}1 \mathrm{RCT}^{141} \text { with } 102 \\
\text { patients }\end{array}$ & $\begin{array}{l}\text { High ROB and } \\
\text { severe } \\
\text { imprecision }\end{array}$ & $\begin{array}{l}\text { Insufficient } \\
\text { evidence }\end{array}$ \\
\hline $\begin{array}{l}\text { QoL(HRQOL) } \\
\text { Longest } \\
\text { Followup }\end{array}$ & $\begin{array}{l}\text { WMD : }-2.12 ; \\
95 \% \mathrm{Cl}:-1.97 \text { to } \\
6.21, \mathrm{I}^{2}=\mathrm{N} / \mathrm{A}\end{array}$ & $\begin{array}{l}1 \mathrm{RCT}^{141} \text { with } 102 \\
\text { patients }\end{array}$ & $\begin{array}{l}\text { High ROB and } \\
\text { severe } \\
\text { imprecision }\end{array}$ & $\begin{array}{l}\text { Insufficient } \\
\text { evidence }\end{array}$ \\
\hline $\begin{array}{l}\text { Hospital } \\
\text { Admission } \\
30 \text { day }\end{array}$ & $\begin{array}{l}\text { Rate Ratio: 0.46; } \\
95 \% \mathrm{Cl}: 0.23 \text { to } \\
0.91, \mathrm{I}^{2}=\mathrm{N} / \mathrm{A}\end{array}$ & $\begin{array}{l}1 \mathrm{RCT}^{141} \text { with } 102 \\
\text { patients }\end{array}$ & $\begin{array}{l}\text { High ROB and } \\
\text { imprecision }\end{array}$ & $\begin{array}{l}\text { Low SOE } \\
\text { supporting } \\
\text { reduction }\end{array}$ \\
\hline $\begin{array}{l}\text { Repeat } \\
\text { Exacerbation } \\
6 \text { Month } \\
\text { Followup }\end{array}$ & $\begin{array}{l}\text { Rate Ratio: 0.84: } \\
95 \% \mathrm{Cl}: 0.53 \text { to } \\
1.32, \mathrm{I}^{2}=\mathrm{N} / \mathrm{A}\end{array}$ & $\begin{array}{l}1 \mathrm{RCT}^{141} \text { with } 102 \\
\text { patients }\end{array}$ & $\begin{array}{l}\text { High ROB and } \\
\text { severe } \\
\text { imprecision }\end{array}$ & $\begin{array}{l}\text { Insufficient } \\
\text { evidence }\end{array}$ \\
\hline $\begin{array}{l}\text { Repeat } \\
\text { Exacerbation } \\
\text { Longest } \\
\text { Followup }\end{array}$ & $\begin{array}{l}\text { Rate Ratio: 0.84: } \\
95 \% \mathrm{Cl}: 0.53 \text { to } \\
1.32, \mathrm{I}^{2}=\mathrm{N} / \mathrm{A}\end{array}$ & $\begin{array}{l}1 \mathrm{RCT}^{141} \text { with } 102 \\
\text { patients }\end{array}$ & $\begin{array}{l}\text { High ROB and } \\
\text { severe } \\
\text { imprecision }\end{array}$ & $\begin{array}{l}\text { Insufficient } \\
\text { evidence }\end{array}$ \\
\hline
\end{tabular}

$\mathrm{CI}=$ confidence interval; HRQL = health related quality of life; N/A = not applicable; OR = odds ratio; QoL = quality of life; $\mathrm{RCT}$ = randomized controlled trial; $\mathrm{ROB}$ = risk of bias; $\mathrm{SOE}=$ strength of evidence; $\mathrm{WMD}=$ weighted mean difference

Table 57. Comparison of levofloxacin versus "standard" antibiotic therapy (clarithromycin or cefuroxime or amoxicillin + clavulanic acid), additional outcomes

\begin{tabular}{|l|l|l|}
\hline \multicolumn{1}{|c|}{ Outcome } & \multicolumn{1}{|c|}{ Findings } & \multicolumn{1}{c|}{ Study Design and Sample Size } \\
\hline FEV1\% & WMD: $-0.95 ; 95 \% \mathrm{Cl}:-8.11$ to & $1 \mathrm{RCT}^{141}$ with 102 patients \\
Predicted & $6.21, \mathrm{I}^{2}=\mathrm{N} / \mathrm{A}$ & \\
$\begin{array}{l}\text { End of } \\
\text { Intervention }\end{array}$ & & \\
\hline FEV1\% & WMD: 4.17; 95\% Cl:- 6.00 to & $1 \mathrm{RCT}^{141}$ with 102 patients \\
$\begin{array}{l}\text { Predicted } \\
\text { Longest } \\
\text { Followup }\end{array}$ & $14.34, \mathrm{I}^{2}=\mathrm{N} / \mathrm{A}$ & \\
\hline
\end{tabular}

$\mathrm{CI}$ = confidence interval; FEV1 = forced expiratory volume in one second; N/A = not applicable; RCT = randomized controlled trial; WMD = weighted mean difference

\section{Azithromycin (Macrolide) Versus Amoxicillin (Aminopenicillin)}

Azithromycin was compared with amoxicillin in one study, ${ }^{145}$ which showed that azithromycin was associated with a statistically significantly higher FEV1percent predicted at the end of the intervention but statistically significantly lower FEV1 percent predicted at the longest followup compared with amoxicillin. There were no statistically significant differences found in other outcomes (Table 58 and Table 59 ) and AEs (Appendix Table H.25.) between groups. 5 patients treated with azithromycin and 2 patients with amoxicillin t reported serious AEs, including respiratory conditions with bronchoconstriction (4 cases), convulsive seizure (1 case), and rib fracture (1 case). 
Table 58. Comparison of azithromycin versus amoxicillin, critical outcomes

\begin{tabular}{|c|c|c|c|c|}
\hline Outcome & Findings & $\begin{array}{c}\text { Study Design } \\
\text { and Sample } \\
\text { Size }\end{array}$ & $\begin{array}{c}\text { Rationale for } \\
\text { Strength of } \\
\text { Evidence (SOE) }\end{array}$ & $\begin{array}{c}\text { Overall } \\
\text { Strength of } \\
\text { Evidence }\end{array}$ \\
\hline $\begin{array}{l}\text { Clinical Cure } \\
\text { End of } \\
\text { Intervention }\end{array}$ & $\begin{array}{l}\text { OR: } 1.60 ; 95 \% \\
\text { Cl: } 0.62 \text { to } 4.11 \text {, } \\
\text { I }^{2}=N / A\end{array}$ & $\begin{array}{l}1 \mathrm{RCT}^{145} \text { with } \\
102 \text { patients }\end{array}$ & $\begin{array}{l}\text { High ROB and } \\
\text { severe } \\
\text { imprecision }\end{array}$ & $\begin{array}{l}\text { Insufficient } \\
\text { evidence }\end{array}$ \\
\hline $\begin{array}{l}\text { Clinical Cure } \\
\text { Longest } \\
\text { Followup }\end{array}$ & $\begin{array}{l}\text { OR: } 1.24 ; 95 \% \\
\text { Cl: } 0.50 \text { to } 3.07 \text {, } \\
\text { I }^{2}=N / A\end{array}$ & $\begin{array}{l}1 \mathrm{RCT}^{145} \text { with } \\
102 \text { patients }\end{array}$ & $\begin{array}{l}\text { High ROB and } \\
\text { severe } \\
\text { imprecision }\end{array}$ & $\begin{array}{l}\text { Insufficient } \\
\text { evidence }\end{array}$ \\
\hline $\begin{array}{l}\text { Clinical Failure } \\
\text { End of } \\
\text { Intervention }\end{array}$ & $\begin{array}{l}\text { OR: } 0.64 ; 95 \% \\
\text { CI: } 0.22 \text { to } 1.80 \text {, } \\
\left.\right|^{2}=N / A\end{array}$ & $\begin{array}{l}1 \mathrm{RCT}^{145} \text { with } \\
102 \text { patients }\end{array}$ & $\begin{array}{l}\text { High ROB and } \\
\text { severe } \\
\text { imprecision }\end{array}$ & $\begin{array}{l}\text { Insufficient } \\
\text { evidence }\end{array}$ \\
\hline $\begin{array}{l}\text { Clinical Failure } \\
\text { Longest } \\
\text { Followup }\end{array}$ & $\begin{array}{l}\text { OR: } 0.75 ; 95 \% \\
\text { Cl: } 0.27 \text { to } 2.04 \text {, } \\
\text { I}^{2}=N / A\end{array}$ & $\begin{array}{l}1 \mathrm{RCT}^{145} \text { with } \\
102 \text { patients }\end{array}$ & $\begin{array}{l}\text { High ROB and } \\
\text { severe } \\
\text { imprecision }\end{array}$ & $\begin{array}{l}\text { Insufficient } \\
\text { evidence }\end{array}$ \\
\hline
\end{tabular}

$\mathrm{CI}=$ confidence interval; N/A = not applicable; $\mathrm{OR}=$ odds ratio; $\mathrm{RCT}=$ randomized controlled trial; $\mathrm{ROB}=$ risk of bias; $\mathrm{SOE}=$ strength of evidence

Table 59. Comparison of azithromycin versus amoxicillin, additional outcomes

\begin{tabular}{|l|l|l|}
\hline \multicolumn{1}{|c|}{ Outcome } & \multicolumn{1}{|c|}{ Findings } & Study Design and Sample Size \\
\hline $\begin{array}{l}\text { FEV1\% Predicted } \\
\text { End of Intervention }\end{array}$ & $\begin{array}{l}\text { WMD: } 3.35 ; 95 \% \mathrm{Cl}: 3.23 \text { to } 3.47, \\
\mathrm{I}^{2}=\mathrm{N} / \mathrm{A}\end{array}$ & $1 \mathrm{RCT}^{145}$ with 102 patients \\
\hline $\begin{array}{l}\text { FEV1\% Predicted } \\
\text { Longest Followup }\end{array}$ & $\begin{array}{l}\text { WMD: }-5.10 ; 95 \% \mathrm{Cl}:-5.24 \text { to }-4.96, \\
\mathrm{I}^{2}=\mathrm{N} / \mathrm{A}\end{array}$ & $1 \mathrm{RCT}^{145}$ with 102 patients \\
\hline $\begin{array}{l}\text { FEV1 Absolute } \\
\text { End of Intervention }\end{array}$ & $\begin{array}{l}\text { WMD: } 0.10 ; 95 \% \mathrm{Cl}:-0.21 \text { to } 0.41, \\
\mathrm{I}^{2}=\mathrm{N} / \mathrm{A}\end{array}$ & $1 \mathrm{RCT}^{145}$ with 102 patients \\
\hline $\begin{array}{l}\text { FEV1 Absolute } \\
\text { Longest Followup }\end{array}$ & $\begin{array}{l}\text { WMD: }-0.10 ; 95 \% \mathrm{Cl}:-0.43 \text { to } 0.23, \\
\mathrm{I}^{2}=\mathrm{N} / \mathrm{A}\end{array}$ & $1 \mathrm{RCT}^{145}$ with 102 patients \\
\hline
\end{tabular}

CI = confidence interval; FEV1 = forced expiratory volume in one second; N/A = not applicable; RCT = randomized controlled trial; WMD = weighted mean difference

\section{Cefaclor (Cephalosporin) Versus Ampicillin + Sulbactam (Aminopenicillin Plus Beta-Lactamase Inhibitor)}

Cefaclor, a cephalosporin, was compared with ampicillin + sulbactam (aminopenicillin plus beta-lactamase inhibitor) in one study. ${ }^{150}$ There was no statistically significant difference found in FEV1 absolute and clinical cure at the end of the intervention between the two groups (Table 60 and Table 61).

Table 60. Comparison of cefaclor versus ampicillin + sulbactam, critical outcomes

\begin{tabular}{|l|l|l|l|l|}
\hline \multicolumn{1}{|c|}{ Outcome } & \multicolumn{1}{|c|}{ Findings } & \multicolumn{1}{|c|}{$\begin{array}{c}\text { Study Design and Sample } \\
\text { Size }\end{array}$} & $\begin{array}{l}\text { Rationale for } \\
\text { Strength of } \\
\text { Evidence (SOE) }\end{array}$ & $\begin{array}{c}\text { Overall Strength of } \\
\text { Evidence }\end{array}$ \\
\hline $\begin{array}{l}\text { Clinical Cure } \\
\text { End of } \\
\text { Intervention }\end{array}$ & $\begin{array}{l}\text { OR: } 0.96 ; 95 \% \mathrm{Cl}: \\
0.24 \text { to } 3.75,\end{array}$ & $1 \mathrm{RCT}^{150}$ with 57 patients & $\begin{array}{l}\text { High ROB and } \\
\text { severe imprecision }\end{array}$ & Insufficient evidence \\
\hline
\end{tabular}

CI = confidence interval; N/A = not applicable; OR = odds ratio; RCT = randomized controlled trial; SOE = strength of evidence

Table 61. Comparison of cefaclor versus ampicillin + sulbactam, additional outcomes

\begin{tabular}{|l|l|l|}
\hline \multicolumn{1}{|c|}{ Outcome } & \multicolumn{1}{|c|}{ Findings } & \multicolumn{1}{c|}{ Study Design and Sample Size } \\
\hline $\begin{array}{l}\text { FEV1 Absolute } \\
\text { End of } \\
\text { Intervention }\end{array}$ & WMD: $0.05 ; 95 \% \mathrm{Cl}:-0.23$ to & $1 \mathrm{RCT}^{150}$ with 57 patients \\
\hline
\end{tabular}

$\mathrm{CI}$ = confidence interval; FEV1 = forced expiratory volume in one second; N/A = not applicable; RCT = randomized controlled trial; WMD = weighted mean difference 


\section{Fluoroquinolone Versus Cephalosporin}

Three studies ${ }^{134,143,150}$ evaluated the effectiveness of a fluoroquinolone (ciprofloxacin or levofloxacin) compared with a cephalosporin (cefaclor or cefuroxime). There were no statistically significant differences found between the groups in any of the outcomes (Table 62 and Table 63). Two serious AEs were reported, including 1 case of hypersensitivity reaction in the levofloxacin group, and 1 case of bronchitis in the cefuroxime group. There was no statistically significant difference observed in AEs, serious AEs, withdrawals, and withdrawals due to AEs (Appendix Table H.26.).

Table 62. Comparison of fluoroquinolone versus cephalosporin, critical outcomes

\begin{tabular}{|c|c|c|c|c|c|}
\hline Comparison & Outcome & Findings & $\begin{array}{c}\text { Study Design } \\
\text { and Sample } \\
\text { Size }\end{array}$ & $\begin{array}{l}\text { Rationale for } \\
\text { Strength of } \\
\text { Evidence } \\
\text { (SOE) }\end{array}$ & $\begin{array}{c}\text { Overall } \\
\text { Strength of } \\
\text { Evidence }\end{array}$ \\
\hline $\begin{array}{l}\text { Fluoroquinolones, vs. } \\
\text { Cephalosporin } \\
\text { Ciprofloxacin vs. } \\
\text { Cefaclor } \\
\text { Levofloxacin vs. } \\
\text { Cefuroxime }\end{array}$ & $\begin{array}{l}\text { Clinical Cure } \\
\text { End of } \\
\text { Intervention }\end{array}$ & $\begin{array}{l}\text { OR: } 1.22 ; 95 \% \\
\text { Cl: } 0.85 \text { to } \\
\text { 1.75, } \\
\mathrm{I}^{2}=57.69 \% \\
\text { OR: } 11.47 ; \\
95 \% \mathrm{Cl}: 0.60 \text { to } \\
219.08, \mathrm{I}^{2}=\mathrm{N} / \mathrm{A} \\
\text { OR: } 1.13 ; 95 \% \\
\mathrm{Cl}: 0.78 \text { to } \\
\text { 1.64, } \mathrm{I}^{2}=\mathrm{N} / \mathrm{A}\end{array}$ & $\begin{array}{l}2 \mathrm{RCTs}^{143,150} \\
\text { with } 741 \\
\text { patients } \\
1 \mathrm{RCT}^{150} \text { with } \\
52 \text { patients } \\
1 \mathrm{RCT}^{143} \text { with } \\
689 \text { patients }\end{array}$ & $\begin{array}{l}\text { High ROB and } \\
\text { severe } \\
\text { imprecision }\end{array}$ & $\begin{array}{l}\text { Insufficient } \\
\text { evidence }\end{array}$ \\
\hline $\begin{array}{l}\text { Levofloxacin vs. } \\
\text { Cefuroxime }\end{array}$ & $\begin{array}{l}\text { Clinical Cure } \\
\text { Longest } \\
\text { Followup }\end{array}$ & $\begin{array}{l}\text { OR: } 1.01 ; 95 \% \\
\mathrm{Cl}: 0.49 \text { to } \\
2.09, \mathrm{I}^{2}=\mathrm{N} / \mathrm{A}\end{array}$ & $\begin{array}{l}1 \mathrm{RCT}^{134} \text { with } \\
137 \text { patients }\end{array}$ & $\begin{array}{l}\text { Intermediate } \\
\text { ROB and } \\
\text { severe } \\
\text { imprecision }\end{array}$ & $\begin{array}{l}\text { Insufficient } \\
\text { evidence }\end{array}$ \\
\hline $\begin{array}{l}\text { Levofloxacin vs. } \\
\text { Cefuroxime }\end{array}$ & $\begin{array}{l}\text { Clinical Failure } \\
\text { Longest } \\
\text { Followup }\end{array}$ & $\begin{array}{l}\text { OR: } 0.92 ; 95 \% \\
\mathrm{Cl}: 0.27 \text { to } \\
3.16, \mathrm{I}^{2}=\mathrm{N} / \mathrm{A}\end{array}$ & $\begin{array}{l}1 \mathrm{RCT}^{134} \text { with } \\
137 \text { patients }\end{array}$ & $\begin{array}{l}\text { Intermediate } \\
\text { ROB and } \\
\text { severe } \\
\text { imprecision }\end{array}$ & $\begin{array}{l}\text { Insufficient } \\
\text { evidence }\end{array}$ \\
\hline
\end{tabular}

Table 63. Comparison of fluoroquinolone versus cephalosporin, additional outcomes

\begin{tabular}{|l|l|l|l|}
\hline \multicolumn{1}{|c|}{ Comparison } & \multicolumn{1}{c|}{ Outcome } & \multicolumn{1}{c|}{ Findings } & \multicolumn{1}{c|}{ Study Design and Sample Size } \\
\hline Ciprofloxacin vs. & FEV1 Absolute & WMD: $0.08 ;$ & $1 \mathrm{RCT}^{150}$ with 52 patients \\
Cefaclor & End of & $95 \% \mathrm{Cl}:-0.23$ & \\
& Intervention & to $0.39, \mathrm{I}^{2}=\mathrm{N} / \mathrm{A}$ & \\
\hline
\end{tabular}

CI = confidence interval; FEV1 = forced expiratory volume in one second; N/A = not applicable; RCT = randomized controlled trial; $\mathrm{WMD}=$ weighted mean difference

\section{Azithromycin (Macrolide) Versus Cefaclor (Cephalosporin)}

Two studies ${ }^{150,157}$ compared azithromycin, a macrolide, with cefaclor, a cephalosporin. There were no statistically significant differences found between groups in FEV1 absolute, clinical cure and clinical failure at the end of the intervention (Table 64 and Table 65). No statistical difference was found in AEs, withdrawals and withdrawals due to AEs (Appendix Table H.27). 
Table 64. Comparison of azithromycin versus cefaclor, critical outcomes

\begin{tabular}{|l|l|l|l|l|}
\hline \multicolumn{1}{|c|}{ Outcome } & \multicolumn{1}{|c|}{ Findings } & \multicolumn{1}{|c|}{$\begin{array}{c}\text { Study Design and } \\
\text { Sample Size }\end{array}$} & $\begin{array}{l}\text { Rationale for } \\
\text { Strength of } \\
\text { Evidence (SOE) }\end{array}$ & $\begin{array}{c}\text { Overall Strength of } \\
\text { Evidence }\end{array}$ \\
\hline $\begin{array}{l}\text { Clinical Cure } \\
\text { End of Intervention }\end{array}$ & $\begin{array}{l}\text { OR: } 2.26 ; 95 \% \mathrm{Cl}: \\
0.94 \text { to } 5.48, \mathrm{I}^{2}=0.00 \%\end{array}$ & $\begin{array}{l}2 \mathrm{RCTS}^{150,157} \text { with } 254 \\
\text { patients }\end{array}$ & $\begin{array}{l}\text { High ROB and } \\
\text { severe } \\
\text { imprecision }\end{array}$ & Insufficient evidence \\
\hline $\begin{array}{l}\text { Clinical Failure } \\
\text { End of Intervention }\end{array}$ & $\begin{array}{l}\text { OR: } 0.09 ; 95 \% \mathrm{Cl}: \\
0.00 \text { to } 1.97, \mathrm{I}^{2}=\mathrm{N} / \mathrm{A}\end{array}$ & $\begin{array}{l}1 \mathrm{RCT}^{157} \text { with } 201 \\
\text { patients }\end{array}$ & $\begin{array}{l}\text { High ROB and } \\
\text { severe } \\
\text { imprecision }\end{array}$ & Insufficient evidence \\
\hline
\end{tabular}

$\mathrm{CI}$ = confidence interval; $\mathrm{OR}$ = odds ratio; N/A = not applicable; $\mathrm{RCT}$ = randomized controlled trial; $\mathrm{ROB}$ = risk of bias; $\mathrm{SOE}$ = strength of evidence

Table 65. Comparison of azithromycin versus cefaclor, additional outcomes

\begin{tabular}{|l|l|l|}
\hline \multicolumn{1}{|c|}{ Outcome } & \multicolumn{1}{|c|}{ Findings } & \multicolumn{1}{|c|}{ Study Design and Sample Size } \\
\hline FEV1 Absolute & WMD: $-0.03 ; 95 \% \mathrm{Cl}:-0.30$ to & $1 \mathrm{RCT}^{150}$ with 53 patients \\
End of Intervention & $0.23, \mathrm{I}^{2}=\mathrm{N} / \mathrm{A}$ & \\
\hline
\end{tabular}

CI = confidence interval; FEV1 = forced expiratory volume in one second; N/A = not applicable; RCT = randomized controlled trial; WMD = weighted mean difference

\section{Azithromycin (Macrolide) Versus Ciprofloxacin (Fluoroquinolone)}

One study ${ }^{150}$ compared azithromycin, a macrolide, with ciprofloxacin, a fluoroquinolone. There were no statistically significant differences found between groups in FEV1 absolute and clinical cure at the end of the intervention (Table 66 and Table 67).

Table 66. Comparison of azithromycin versus ciprofloxacin, critical outcomes

\begin{tabular}{|c|c|c|c|c|}
\hline Outcome & Findings & $\begin{array}{l}\text { Study Design and Sample } \\
\text { Size }\end{array}$ & $\begin{array}{c}\text { Rationale for } \\
\text { Strength of } \\
\text { Evidence (SOE) }\end{array}$ & $\begin{array}{l}\text { Overall Strength of } \\
\text { Evidence }\end{array}$ \\
\hline $\begin{array}{l}\text { Clinical Cure } \\
\text { End of } \\
\text { Intervention }\end{array}$ & $\begin{array}{l}\text { OR: } 0.19 ; 95 \% \mathrm{Cl} \\
0.01 \text { to } 4.21 \\
\mathrm{I}^{2}=\mathrm{N} / \mathrm{A}\end{array}$ & $1 \mathrm{RCT}^{150}$ with 49 patients & $\begin{array}{l}\text { High ROB and } \\
\text { severe imprecision }\end{array}$ & Insufficient evidence \\
\hline
\end{tabular}

Table 67. Comparison of azithromycin versus ciprofloxacin, additional outcomes

\begin{tabular}{|l|l|l|}
\hline \multicolumn{1}{|c|}{ Outcome } & \multicolumn{1}{|c|}{ Findings } & \multicolumn{1}{c|}{ Study Design and Sample Size } \\
\hline $\begin{array}{l}\text { FEV1 Absolute } \\
\begin{array}{l}\text { End of } \\
\text { Intervention }\end{array}\end{array}$ & WMD: $-0.05 ; 95 \% \mathrm{Cl}:-0.37$ to 0.28, & $1 \mathrm{RCT}^{150}$ with 49 patients \\
\hline
\end{tabular}

CI = confidence interval; FEV1 = forced expiratory volume in one second; N/A = not applicable; RCT = randomized controlled trial; WMD = weighted mean difference

\section{Amoxicillin (Aminopenicillin) Versus Amoxicillin Plus Clavulanic Acid}

One study ${ }^{139}$ compared amoxicillin with amoxicillin plus clavulanic acid. There were no statistically significant differences observed between groups in clinical cure and clinical failure at the end of the intervention and clinical cure at the longest followup (Table 68). No statistical difference was found in AEs and withdrawals (Appendix Table H.28.). 
Table 68. Comparison of amoxicillin versus amoxicillin plus clavulanic acid

\begin{tabular}{|c|c|c|c|c|}
\hline Outcome & Findings & $\begin{array}{l}\text { Study Design and } \\
\text { Sample Size }\end{array}$ & $\begin{array}{c}\text { Rationale for } \\
\text { Strength of } \\
\text { Evidence (SOE) }\end{array}$ & $\begin{array}{l}\text { Overall Strength } \\
\text { of Evidence }\end{array}$ \\
\hline $\begin{array}{l}\text { Clinical Cure } \\
\text { End of Intervention }\end{array}$ & $\begin{array}{l}\text { OR: } 0.68 ; 95 \% \mathrm{Cl} \\
0.21 \text { to } 2.26 \\
\mathrm{I}^{2}=\mathrm{N} / \mathrm{A}\end{array}$ & $\begin{array}{l}1 \mathrm{RCT}^{139} \text { with } 137 \\
\text { patients }\end{array}$ & $\begin{array}{l}\text { High ROB and } \\
\text { severe } \\
\text { imprecision }\end{array}$ & $\begin{array}{l}\text { Insufficient } \\
\text { evidence }\end{array}$ \\
\hline $\begin{array}{l}\text { Clinical Cure } \\
\text { Longest Followup }\end{array}$ & $\begin{array}{l}\text { OR: } 0.59 ; 95 \% \mathrm{Cl} \\
0.21 \text { to } 1.61, \\
\mathrm{I}^{2}=\mathrm{N} / \mathrm{A}\end{array}$ & $\begin{array}{l}1 \mathrm{RCT}^{139} \text { with } 137 \\
\text { patients }\end{array}$ & $\begin{array}{l}\text { High ROB and } \\
\text { severe } \\
\text { imprecision }\end{array}$ & $\begin{array}{l}\text { Insufficient } \\
\text { evidence }\end{array}$ \\
\hline $\begin{array}{l}\text { Clinical Failure } \\
\text { End of Intervention }\end{array}$ & $\begin{array}{l}\text { OR: } 2.13 ; 95 \% \mathrm{Cl} \\
0.51 \text { to } 8.88, \\
\mathrm{I}^{2}=\mathrm{N} / \mathrm{A}\end{array}$ & $\begin{array}{l}1 \mathrm{RCT}^{139} \text { with } 137 \\
\text { patients }\end{array}$ & $\begin{array}{l}\text { High ROB and } \\
\text { severe } \\
\text { imprecision }\end{array}$ & $\begin{array}{l}\text { Insufficient } \\
\text { evidence }\end{array}$ \\
\hline
\end{tabular}

$\mathrm{CI}$ = confidence interval; OR = odds ratio; N/A = not applicable; RCT = randomized controlled trial; ROB = risk of bias; $\mathrm{SOE}=$ strength of evidence

\section{Telithromycin (Ketolide) Versus Amoxicillin Plus Clavulanic Acid (Aminopenicillin Plus Beta-Lactamase Inhibitor)}

One study ${ }^{148}$ compared telithromycin, a ketolide, with amoxicillin plus clavulanic acid. There were no statistically significant differences found between groups in clinical cure at the end of the intervention and at the longest followup (Table 69). Statistically significantly more patients in the amoxicillin plus clavulanic acid group reported AEs and withdrew due to AEs than those in the telithromycin group (Appendix Table H.29.). 13 patients reported unspecified serious AEs: 7 in the telithromycin group and 6 in the amoxicillin plus clavulanic acid group.

Table 69. Comparison of telithromycin versus amoxicillin plus clavulanic acid

\begin{tabular}{|c|c|c|c|c|}
\hline Outcome & Findings & $\begin{array}{c}\text { Study Design } \\
\text { and Sample } \\
\text { Size }\end{array}$ & $\begin{array}{c}\text { Rationale for } \\
\text { Strength of } \\
\text { Evidence (SOE) }\end{array}$ & $\begin{array}{l}\text { Overall } \\
\text { Strength of } \\
\text { Evidence }\end{array}$ \\
\hline $\begin{array}{l}\text { Clinical Cure } \\
\text { End of } \\
\text { Intervention }\end{array}$ & $\begin{array}{l}\text { OR: } 1.13 ; 95 \% \\
\mathrm{Cl}: 0.67 \text { to } 1.93 \\
\mathrm{I}^{2}=\mathrm{N} / \mathrm{A}\end{array}$ & $\begin{array}{l}1 \mathrm{RCT}^{148} \text { with } \\
324 \text { patients }\end{array}$ & $\begin{array}{l}\text { High ROB and } \\
\text { severe } \\
\text { imprecision }\end{array}$ & $\begin{array}{l}\text { Insufficient } \\
\text { evidence }\end{array}$ \\
\hline $\begin{array}{l}\text { Clinical Cure } \\
\text { Longest } \\
\text { Followup }\end{array}$ & $\begin{array}{l}\text { OR: } 1.08 ; 95 \% \\
\mathrm{Cl}: 0.67 \text { to } 1.75 \\
\mathrm{l}^{2}=\mathrm{N} / \mathrm{A}\end{array}$ & $\begin{array}{l}1 \mathrm{RCT}^{148} \text { with } \\
324 \text { patients }\end{array}$ & $\begin{array}{l}\text { High ROB and } \\
\text { severe } \\
\text { imprecision }\end{array}$ & $\begin{array}{l}\text { Insufficient } \\
\text { evidence }\end{array}$ \\
\hline
\end{tabular}

$\mathrm{CI}$ = confidence interval; OR = odds ratio; N/A = not applicable; RCT = randomized controlled trial; ROB = risk of bias; $\mathrm{SOE}$ = strength of evidence

\section{Azithromycin (Macrolide) Versus Aminopenicillin Plus Beta- Lactamase Inhibitor}

Two studies ${ }^{150,154}$ evaluated the effectiveness of azithromycin, a macrolide, compared with an aminopenicillin plus beta-lactamase inhibitor (amoxicillin or ampicillin). There were no statistically significant differences observed between the groups in clinical cure, clinical failure, and FEV1 absolute at the end of the intervention (Table 70 and Table 71). No statistically significant difference in AEs and withdrawals was found (Appendix Tables H.30. and H.31). 
Table 70. Comparison of macrolide versus aminopenicillin plus beta-lactamase inhibitor, critical outcomes

\begin{tabular}{|c|c|c|c|c|c|}
\hline Comparison & Outcome & Findings & $\begin{array}{c}\text { Study Design } \\
\text { and Sample } \\
\text { Size }\end{array}$ & $\begin{array}{c}\text { Rationale for } \\
\text { Strength of } \\
\text { Evidence (SOE) }\end{array}$ & $\begin{array}{c}\text { Overall } \\
\text { Strength of } \\
\text { Evidence }\end{array}$ \\
\hline $\begin{array}{l}\text { Macrolides vs. } \\
\text { Aminopenicillin } \\
\text { plus Beta- } \\
\text { lactamase } \\
\text { inhibitor }\end{array}$ & $\begin{array}{l}\text { Clinical Cure } \\
\text { End of } \\
\text { Intervention }\end{array}$ & $\begin{array}{l}\text { OR: } 1.46 ; 95 \% \\
\text { Cl: } 0.64 \text { to } 3.32 \text {, } \\
I^{2}=0.00 \%\end{array}$ & $\begin{array}{l}2 \text { RCTs }{ }^{150,154} \\
\text { with 124 } \\
\text { patients }\end{array}$ & $\begin{array}{l}\text { High ROB and } \\
\text { severe } \\
\text { imprecision }\end{array}$ & $\begin{array}{l}\text { Insufficient } \\
\text { evidence }\end{array}$ \\
\hline $\begin{array}{l}\text { Azithromycin vs. } \\
\text { Ampicillin }+ \\
\text { Sulbactam }\end{array}$ & & $\begin{array}{l}\text { OR: } 2.40 ; 95 \% \\
\text { Cl: } 0.42 \text { to } 13.60, \\
I^{2}=N / A\end{array}$ & $\begin{array}{l}1 \mathrm{RCT}^{150} \text { with } 54 \\
\text { patients }\end{array}$ & & \\
\hline $\begin{array}{l}\text { Azithromycin vs. } \\
\text { Amoxicillin }+ \\
\text { Clavulanic Acid }\end{array}$ & & $\begin{array}{l}\text { OR: } 1.24 ; 95 \% \\
\text { Cl: } 0.48 \text { to } 3.20 \text {, } \\
\mathrm{I}^{2}=\mathrm{N} / \mathrm{A}\end{array}$ & $\begin{array}{l}1 \mathrm{RCT}^{154} \text { with } 70 \\
\text { patients }\end{array}$ & & \\
\hline $\begin{array}{l}\text { Azithromycin vs. } \\
\text { Amoxicillin + } \\
\text { Clavulanic Acid }\end{array}$ & $\begin{array}{l}\text { Clinical Failure } \\
\text { End of } \\
\text { Intervention }\end{array}$ & $\begin{array}{l}\text { OR: } 0.15 ; 95 \% \\
\text { Cl: } 0.01 \text { to } 3.23 \text {, } \\
\mathrm{I}^{2}=\mathrm{N} / \mathrm{A}\end{array}$ & $\begin{array}{l}1 \mathrm{RCT}^{154} \text { with } 70 \\
\text { patients }\end{array}$ & $\begin{array}{l}\text { High ROB and } \\
\text { severe } \\
\text { imprecision }\end{array}$ & $\begin{array}{l}\text { Insufficient } \\
\text { evidence }\end{array}$ \\
\hline
\end{tabular}

$\mathrm{CI}$ = confidence interval; FEV1 = Forced expiratory volume in one second; OR = odds ratio; N/A = not applicable; RCT = randomized controlled trial; $\mathrm{ROB}=$ risk of bias; $\mathrm{SOE}=$ strength of evidence; $\mathrm{WMD}=$ weighted mean difference

Table 71. Comparison of macrolide versus aminopenicillin plus beta-lactamase inhibitor, additional outcomes

\begin{tabular}{|l|l|l|l|}
\hline \multicolumn{1}{|c|}{ Comparison } & \multicolumn{1}{|c|}{ Outcome } & \multicolumn{1}{c|}{ Findings } & \multicolumn{1}{c|}{$\begin{array}{c}\text { Study Design and } \\
\text { Sample Size }\end{array}$} \\
\hline $\begin{array}{l}\text { Macrolides vs. } \\
\text { Aminopenicillin plus Beta- } \\
\text { lactamase inhibitor }\end{array}$ & $\begin{array}{l}\text { FEV1 Absolute } \\
\text { End of Intervention }\end{array}$ & $\begin{array}{l}\text { WMD: 0.08; 95\% Cl: }-0.21 \\
\text { to 0.38, I2=N/A }\end{array}$ & 1 RCT150 with 54 patients \\
& & & \\
\hline
\end{tabular}

CI = confidence interval; FEV1 = Forced expiratory volume in one second; N/A = not applicable; RCT = randomized controlled trial; WMD = weighted mean difference

\section{Prulifloxacin ( $4^{\text {th }}$ Generation Fluoroquinolone) Versus Levofloxacin ( ${ }^{\text {nd }}$ Generation Fluoroquinolone)}

Two studies ${ }^{130,135}$ evaluated the effectiveness of prulifloxacin, a $4^{\text {th }}$ generation fluoroquinolone, compared with levofloxacin, a $2^{\text {nd }}$ generation fluoroquinolone. The only statistically significant difference in outcomes between groups was repeat exacerbations at 3 months, which were statistically significantly higher with prulifloxacin compared with levofloxacin (Table 72 and Table 73). Unspecified serious AEs were reported in 5 patients in the prulifloxacin group and 6 patients in the levofloxacin group. There was no statistically significant difference found in AEs, withdrawals, and withdrawals due to AEs (Appendix Table H.32.).

Table 72. Comparison of prulifloxacin versus levofloxacin, critical outcomes

\begin{tabular}{|l|l|l|l|l|}
\hline \multicolumn{1}{|c|}{ Outcome } & \multicolumn{1}{|c|}{ Findings } & $\begin{array}{c}\text { Study Design and } \\
\text { Sample Size }\end{array}$ & $\begin{array}{l}\text { Rationale for } \\
\text { Strength of } \\
\text { Evidence (SOE) }\end{array}$ & $\begin{array}{l}\text { Overall Strength } \\
\text { of Evidence }\end{array}$ \\
\hline $\begin{array}{l}\text { Mortality } \\
\text { Longest Followup }\end{array}$ & $\begin{array}{l}\text { OR: } 0.43 ; 95 \% \mathrm{Cl}: \\
0.14 \text { to } 1.23, \mathrm{I}^{2}=\mathrm{N} / \mathrm{A}\end{array}$ & $\begin{array}{l}1 \mathrm{RCT}^{130} \text { with } 258 \\
\text { patients }\end{array}$ & $\begin{array}{l}\text { High ROB and } \\
\text { severe } \\
\text { imprecision }\end{array}$ & $\begin{array}{l}\text { Insufficient } \\
\text { evidence }\end{array}$ \\
\hline
\end{tabular}




\begin{tabular}{|c|c|c|c|c|}
\hline Outcome & Findings & $\begin{array}{l}\text { Study Design and } \\
\text { Sample Size }\end{array}$ & $\begin{array}{c}\text { Rationale for } \\
\text { Strength of } \\
\text { Evidence (SOE) }\end{array}$ & $\begin{array}{l}\text { Overall Strength } \\
\text { of Evidence }\end{array}$ \\
\hline $\begin{array}{l}\text { Dyspnea } \\
\text { (Numeric Scale: } \\
\text { Dyspnea Score) } \\
\text { End of Intervention }\end{array}$ & $\begin{array}{l}\text { WMD: }-0.05 ; 95 \% \\
\text { Cl: }-0.21 \text { to } \\
0.11, I^{2}=N / A\end{array}$ & $\begin{array}{l}1 \mathrm{RCT}^{135} \text { with } 357 \\
\text { patients }\end{array}$ & $\begin{array}{l}\text { Intermediate ROB } \\
\text { and severe } \\
\text { imprecision }\end{array}$ & $\begin{array}{l}\text { Insufficient } \\
\text { evidence }\end{array}$ \\
\hline $\begin{array}{l}\text { Repeat Exacerbation } \\
\text { End of Intervention }\end{array}$ & $\begin{array}{l}\text { OR: } 1.37 ; 95 \% \mathrm{Cl}: \\
0.84 \text { to } 2.24, \\
\mathrm{I}^{2}=7.10 \%\end{array}$ & $\begin{array}{l}2 \mathrm{RCTS}^{130,135} \text { with } \\
\text { patients }\end{array}$ & $\begin{array}{l}\text { High ROB and } \\
\text { severe } \\
\text { imprecision }\end{array}$ & $\begin{array}{l}\text { Insufficient } \\
\text { evidence }\end{array}$ \\
\hline \multirow[t]{2}{*}{$\begin{array}{l}\text { Repeat Exacerbation } \\
3 \text { Months }\end{array}$} & $\begin{array}{l}\text { OR: } 2.26 ; 95 \% \mathrm{Cl} \\
1.15 \text { to } 4.45, \mathrm{I}^{2}=\mathrm{N} / \mathrm{A}\end{array}$ & $\begin{array}{l}1 \mathrm{RCT}^{135} \text { with } 357 \\
\text { patients }\end{array}$ & $\begin{array}{l}\text { Intermediate ROB } \\
\text { and imprecision }\end{array}$ & $\begin{array}{l}\text { Low SOE } \\
\text { supporting } \\
\text { worsening }\end{array}$ \\
\hline & $\begin{array}{l}\text { Rate Ratio: } 0.77 ; \\
95 \% \mathrm{Cl}: 0.42 \text { to } \\
1.43, \mathrm{I}^{2}=\mathrm{N} / \mathrm{A}\end{array}$ & $\begin{array}{l}1 \mathrm{RCT}^{130} \text { with } 258 \\
\text { patients }\end{array}$ & $\begin{array}{l}\text { High ROB and } \\
\text { severe } \\
\text { imprecision }\end{array}$ & $\begin{array}{l}\text { Insufficient } \\
\text { evidence }\end{array}$ \\
\hline $\begin{array}{l}\text { Repeat Exacerbation } \\
6 \text { Months }\end{array}$ & $\begin{array}{l}\text { OR: } 1.29 ; 95 \% \mathrm{Cl}: \\
0.72 \text { to } 2.31, \mathrm{I}^{2}=\mathrm{N} / \mathrm{A}\end{array}$ & $\begin{array}{l}1 \mathrm{RCT}^{135} \text { with } 357 \\
\text { patients }\end{array}$ & $\begin{array}{l}\text { Intermediate ROB } \\
\text { and severe } \\
\text { imprecision }\end{array}$ & $\begin{array}{l}\text { Insufficient } \\
\text { evidence }\end{array}$ \\
\hline $\begin{array}{l}\text { Repeat Exacerbation } \\
12 \text { Months }\end{array}$ & $\begin{array}{l}\text { Rate Ratio: } 1.16 ; \\
95 \% \mathrm{Cl}: 0.76 \text { to } \\
1.76, \mathrm{I}^{2}=\mathrm{N} / \mathrm{A}\end{array}$ & $\begin{array}{l}1 \mathrm{RCT}^{130} \text { with } 258 \\
\text { patients }\end{array}$ & $\begin{array}{l}\text { High ROB and } \\
\text { severe } \\
\text { imprecision }\end{array}$ & $\begin{array}{l}\text { Insufficient } \\
\text { evidence }\end{array}$ \\
\hline \multirow[t]{2}{*}{$\begin{array}{l}\text { Repeat Exacerbation } \\
\text { Longest Followup }\end{array}$} & $\begin{array}{l}\text { OR: } 1.29 ; 95 \% \mathrm{Cl}: \\
0.72 \text { to } 2.31, \mathrm{I}^{2}=\mathrm{N} / \mathrm{A}\end{array}$ & $\begin{array}{l}1 \mathrm{RCT}^{135} \text { with } 357 \\
\text { patients }\end{array}$ & $\begin{array}{l}\text { Intermediate ROB } \\
\text { and severe } \\
\text { imprecision }\end{array}$ & $\begin{array}{l}\text { Insufficient } \\
\text { evidence }\end{array}$ \\
\hline & $\begin{array}{l}\text { Rate Ratio: } 1.16 ; \\
95 \% \mathrm{Cl}: 0.76 \text { to } \\
1.76, \mathrm{I}^{2}=\mathrm{N} / \mathrm{A}\end{array}$ & $\begin{array}{l}1 \mathrm{RCT}^{130} \text { with } 258 \\
\text { patients }\end{array}$ & $\begin{array}{l}\text { High ROB and } \\
\text { severe } \\
\text { imprecision }\end{array}$ & $\begin{array}{l}\text { Insufficient } \\
\text { evidence }\end{array}$ \\
\hline $\begin{array}{l}\text { Clinical Cure } \\
\text { End of Intervention }\end{array}$ & $\begin{array}{l}\text { OR: } 0.96 ; 95 \% \mathrm{Cl}: \\
0.52 \text { to } 1.78, \\
\mathrm{l}^{2}=68.97 \%\end{array}$ & $\begin{array}{l}2 \mathrm{RCTs}^{130,135} \text { with } 615 \\
\text { patients }\end{array}$ & $\begin{array}{l}\text { High ROB and } \\
\text { severe } \\
\text { imprecision }\end{array}$ & $\begin{array}{l}\text { Insufficient } \\
\text { evidence }\end{array}$ \\
\hline $\begin{array}{l}\text { Clinical Cure } \\
\text { Longest Followup }\end{array}$ & $\begin{array}{l}\text { OR: } 0.83 ; 95 \% \mathrm{Cl}: \\
0.51 \text { to } 1.37, \mathrm{I}^{2}=\mathrm{N} / \mathrm{A}\end{array}$ & $\begin{array}{l}\text { 1 RCT }{ }^{135} \text { with } 357 \\
\text { Patients }\end{array}$ & $\begin{array}{l}\text { Intermediate ROB } \\
\text { and severe } \\
\text { imprecision }\end{array}$ & $\begin{array}{l}\text { Insufficient } \\
\text { evidence }\end{array}$ \\
\hline $\begin{array}{l}\text { Clinical Failure } \\
\text { End of Intervention }\end{array}$ & $\begin{array}{l}\text { OR: } 2.24 ; 95 \% \mathrm{Cl}: \\
0.83 \text { to } 6.05, \mathrm{I}^{2}=\mathrm{N} / \mathrm{A}\end{array}$ & $\begin{array}{l}1 \mathrm{RCT}^{135} \text { with } 357 \\
\text { patients }\end{array}$ & $\begin{array}{l}\text { Intermediate ROB } \\
\text { and severe } \\
\text { imprecision }\end{array}$ & $\begin{array}{l}\text { Insufficient } \\
\text { evidence }\end{array}$ \\
\hline $\begin{array}{l}\text { Clinical Failure } \\
\text { Longest Followup }\end{array}$ & $\begin{array}{l}\text { OR: } 3.05 ; 95 \% \mathrm{Cl}: \\
0.61 \text { to } 15.33, \\
\mathrm{I}^{2}=\mathrm{N} / \mathrm{A}\end{array}$ & $\begin{array}{l}1 \mathrm{RCT}^{135} \text { with } 357 \\
\text { patients }\end{array}$ & $\begin{array}{l}\text { Intermediate ROB } \\
\text { and severe } \\
\text { imprecision }\end{array}$ & $\begin{array}{l}\text { Insufficient } \\
\text { evidence }\end{array}$ \\
\hline
\end{tabular}

CI = confidence interval; OR = odds ratio; N/A = not applicable; RCT = randomized controlled trial; ROB = risk of bias; $\mathrm{SOE}$ = strength of the body of vidence; WMD = weighted mean difference

Table 73. Comparison of prulifloxacin versus levofloxacin, additional outcomes

\begin{tabular}{|l|l|l|}
\hline \multicolumn{1}{|c|}{ Outcome } & \multicolumn{1}{|c|}{ Findings } & \multicolumn{1}{c|}{ Study Design and Sample Size } \\
\hline $\begin{array}{l}\text { Cough (Scale 0-4 from absent } \\
\text { to severe) }\end{array}$ & $\begin{array}{l}\text { WMD: }-0.07 ; 95 \% \mathrm{Cl}:-0.19 \\
\text { Longest Followup }\end{array}$ & $1 \mathrm{RCT}^{135}$ with 357 patients \\
\hline $\begin{array}{l}\text { Other Symptoms (Total } \\
\text { Symptom Score) } \\
\text { End of Intervention }\end{array}$ & $\begin{array}{l}\text { SMD: } 0.05 ; 95 \% \mathrm{Cl}:-0.11 \text { to } \\
0.21, \mathrm{I}^{2}=0.00 \%\end{array}$ & $2 \mathrm{RCTs}^{130,135}$ with 615 patients \\
\hline $\begin{array}{l}\text { FEV1\% Predicted } \\
\text { End of Intervention }\end{array}$ & $\begin{array}{l}\text { WMD: }-1.50 ; 95 \% \mathrm{Cl}:-4.72 \\
\text { to } 1.72, \mathrm{I}^{2}=\mathrm{N} / \mathrm{A}\end{array}$ & $1 \mathrm{RCT}^{135}$ with 357 patients \\
\hline
\end{tabular}

$\mathrm{CI}=$ confidence interval; FEV1 = forced expiratory volume in one second; N/A = not applicable; OR = odds ratio; RCT = randomized controlled trial; SMD = standardized mean difference; $\mathrm{WMD}=$ weighted mean difference 


\section{Zabofloxacin (Next Generation Fluoroquinolone) Versus Moxifloxacin ( $4^{\text {th }}$ Generation Fluoroquinolone)}

One study ${ }^{132}$ evaluated the effectiveness of zabofloxacin, a next generation fluoroquinolone, compared with moxifloxacin, a $4^{\text {th }}$ generation fluoroquinolone. There were no statistically significant differences observed in outcomes (Table 74), and AEs (Appendix Table H.33.). Serious AEs were reported in 15 cases: 7 cases in the zabofloxacin group (4 COPD exacerbations, 1 influenza like illness, 1 pneumonia, and 1 acute pyelonephritis); and 8 cases in the fluoroquinolone group (4 COPD exacerbations, 2 pneumonia, 1 variant angina, and 1 urethral stenosis).

Table 74. Comparison of zabofloxacin versus moxifloxacin

\begin{tabular}{|c|c|c|c|c|}
\hline Outcome & Findings & $\begin{array}{c}\text { Study Design } \\
\text { and Sample Size }\end{array}$ & $\begin{array}{c}\text { Rationale for } \\
\text { Strength of } \\
\text { Evidence (SOE) }\end{array}$ & $\begin{array}{l}\text { Overall Strength } \\
\text { of Evidence }\end{array}$ \\
\hline $\begin{array}{l}\text { Repeat } \\
\text { Exacerbation } \\
30 \text { Days }\end{array}$ & $\begin{array}{l}\text { OR: } 0.82 ; 95 \% \mathrm{Cl} \\
0.39 \text { to } 1.74, \\
\left.\right|^{2}=\mathrm{N} / \mathrm{A}\end{array}$ & $\begin{array}{l}1 \mathrm{RCT}^{132} \text { with } 342 \\
\text { patients }\end{array}$ & $\begin{array}{l}\text { High ROB and } \\
\text { severe } \\
\text { imprecision }\end{array}$ & $\begin{array}{l}\text { Insufficient } \\
\text { evidence }\end{array}$ \\
\hline $\begin{array}{l}\text { Repeat } \\
\text { Exacerbation } \\
\text { Longest Followup }\end{array}$ & $\begin{array}{l}\text { OR: } 0.82 ; 95 \% \mathrm{Cl} \\
0.39 \text { to } 1.74, \\
\left.\right|^{2}=\mathrm{N} / \mathrm{A}\end{array}$ & $\begin{array}{l}1 \mathrm{RCT}^{132} \text { with } 342 \\
\text { patients }\end{array}$ & $\begin{array}{l}\text { High ROB and } \\
\text { severe } \\
\text { imprecision }\end{array}$ & $\begin{array}{l}\text { Insufficient } \\
\text { evidence }\end{array}$ \\
\hline $\begin{array}{l}\text { Clinical Cure } \\
\text { End of Intervention }\end{array}$ & $\begin{array}{l}\text { OR: } 0.99 ; 95 \% \mathrm{Cl} \\
0.60 \text { to } 1.65, \\
\mathrm{I}^{2}=\mathrm{N} / \mathrm{A}\end{array}$ & $\begin{array}{l}1 \mathrm{RCT}^{132} \text { with } 342 \\
\text { patients }\end{array}$ & $\begin{array}{l}\text { High ROB and } \\
\text { severe } \\
\text { imprecision }\end{array}$ & $\begin{array}{l}\text { Insufficient } \\
\text { evidence }\end{array}$ \\
\hline $\begin{array}{l}\text { Clinical Cure } \\
\text { Longest Followup }\end{array}$ & $\begin{array}{l}\text { OR: } 1.19 ; 95 \% \mathrm{Cl} \\
0.76 \text { to } 1.87 \\
\mathrm{I}^{2}=\mathrm{N} / \mathrm{A}\end{array}$ & $\begin{array}{l}1 \mathrm{RCT}^{132} \text { with } 342 \\
\text { patients }\end{array}$ & $\begin{array}{l}\text { High ROB and } \\
\text { severe } \\
\text { imprecision }\end{array}$ & $\begin{array}{l}\text { Insufficient } \\
\text { evidence }\end{array}$ \\
\hline $\begin{array}{l}\text { Clinical Failure } \\
\text { End of Intervention }\end{array}$ & $\begin{array}{l}\text { OR: } 1.07 ; 95 \% \mathrm{Cl} \\
0.54 \text { to } 2.10, \\
\mathrm{I}^{2}=\mathrm{N} / \mathrm{A}\end{array}$ & $\begin{array}{l}1 \mathrm{RCT}^{132} \text { with } 342 \\
\text { patients }\end{array}$ & $\begin{array}{l}\text { High ROB and } \\
\text { severe } \\
\text { imprecision }\end{array}$ & $\begin{array}{l}\text { Insufficient } \\
\text { evidence }\end{array}$ \\
\hline $\begin{array}{l}\text { Clinical Failure } \\
\text { Longest Followup }\end{array}$ & $\begin{array}{l}\text { OR: } 0.94 ; 95 \% \mathrm{Cl} \\
0.55 \text { to } 1.60, \\
\text { I}^{2}=\mathrm{N} / \mathrm{A}\end{array}$ & $\begin{array}{l}1 \mathrm{RCT}^{132} \text { with } 342 \\
\text { patients }\end{array}$ & $\begin{array}{l}\text { High ROB and } \\
\text { severe } \\
\text { imprecision }\end{array}$ & $\begin{array}{l}\text { Insufficient } \\
\text { evidence }\end{array}$ \\
\hline
\end{tabular}

\section{Cefpodoxime ( $3^{\text {rd }}$ Generation Cephalosporin) Versus Cefaclor ( $2^{\text {nd }}$ Generation Cephalosporin)}

One study ${ }^{156}$ evaluated the effectiveness of cefpodoxime, a 3rd generation cephalosporin, compared with cefaclor, a 2nd generation cephalosporin. There were no statistically significant differences in outcomes found (Table 75), AEs, and withdrawals (Appendix Table H.34.). 
Table 75. Comparison of cefpodoxime versus cefaclor

\begin{tabular}{|c|c|c|c|c|}
\hline Outcome & Findings & $\begin{array}{l}\text { Study Design and } \\
\text { Sample Size }\end{array}$ & $\begin{array}{c}\text { Rationale for } \\
\text { Strength of } \\
\text { Evidence (SOE) }\end{array}$ & $\begin{array}{l}\text { Overall Strength } \\
\text { of Evidence }\end{array}$ \\
\hline $\begin{array}{l}\text { Repeat } \\
\text { Exacerbation } \\
\text { End of Intervention }\end{array}$ & $\begin{array}{l}\text { OR: } 1.51 ; 95 \% \mathrm{Cl}: \\
0.57 \text { to } 3.99 \\
\mathrm{I}^{2}=\mathrm{N} / \mathrm{A}\end{array}$ & $\begin{array}{l}1 \mathrm{RCT}^{156} \text { with } 301 \\
\text { patients }\end{array}$ & $\begin{array}{l}\text { High ROB and } \\
\text { severe imprecision }\end{array}$ & $\begin{array}{l}\text { Insufficient } \\
\text { evidence }\end{array}$ \\
\hline $\begin{array}{l}\text { Repeat } \\
\text { Exacerbation } \\
30 \text { Days } \\
\end{array}$ & $\begin{array}{l}\text { OR: } 1.51 ; 95 \% \mathrm{Cl}: \\
0.57 \text { to } 3.99, \\
\text { I}^{2}=\mathrm{N} / \mathrm{A}\end{array}$ & $\begin{array}{l}1 \mathrm{RCT}^{156} \text { with } 301 \\
\text { patients }\end{array}$ & $\begin{array}{l}\text { High ROB and } \\
\text { severe imprecision }\end{array}$ & $\begin{array}{l}\text { Insufficient } \\
\text { evidence }\end{array}$ \\
\hline $\begin{array}{l}\text { Repeat } \\
\text { Exacerbation } \\
\text { Longest Followup }\end{array}$ & $\begin{array}{l}\text { OR: } 1.51 ; 95 \% \mathrm{Cl}: \\
0.57 \text { to } 3.99, \\
\mathrm{I}^{2}=\mathrm{N} / \mathrm{A}\end{array}$ & $\begin{array}{l}1 \mathrm{RCT}^{156} \text { with } 301 \\
\text { patients }\end{array}$ & $\begin{array}{l}\text { High ROB and } \\
\text { severe imprecision }\end{array}$ & $\begin{array}{l}\text { Insufficient } \\
\text { evidence }\end{array}$ \\
\hline $\begin{array}{l}\text { Clinical Cure } \\
\text { End of Intervention }\end{array}$ & $\begin{array}{l}\text { OR: } 1.42 ; 95 \% \mathrm{Cl}: \\
0.84 \text { to } 2.40, \\
\text { I}^{2}=\mathrm{N} / \mathrm{A}\end{array}$ & $\begin{array}{l}1 \mathrm{RCT}^{156} \text { with } 301 \\
\text { patients }\end{array}$ & $\begin{array}{l}\text { High ROB and } \\
\text { severe imprecision }\end{array}$ & $\begin{array}{l}\text { Insufficient } \\
\text { evidence }\end{array}$ \\
\hline $\begin{array}{l}\text { Clinical Failure } \\
\text { End of Intervention }\end{array}$ & $\begin{array}{l}\text { OR: } 0.13 ; 95 \% \mathrm{Cl}: \\
0.01 \text { to } 1.21 \\
\text { I}^{2}=\mathrm{N} / \mathrm{A}\end{array}$ & $\begin{array}{l}1 \mathrm{RCT}^{156} \text { with } 301 \\
\text { patients }\end{array}$ & $\begin{array}{l}\text { High ROB and } \\
\text { severe imprecision }\end{array}$ & $\begin{array}{l}\text { Insufficient } \\
\text { evidence }\end{array}$ \\
\hline
\end{tabular}

CI = confidence interval; OR = odds ratio; N/A = not applicable; RCT = randomized controlled trial; ROB = risk of bias

\section{Meropenem (Carbapenem) Versus Imipenem (Carbapenem)}

One study ${ }^{155}$ evaluated the effectiveness of meropenem, a carbapenem, compared with imipenem, a carbapenem (combined with cilastatin, which is an inhibitor of the enzyme dehydropeptidase and prolongs the antibacterial effect of imipenem). There were no statistically significant differences observed in outcomes between groups (Table 76). Statistically significantly more AEs cases were reported in the imipenem group than those in the meropenem group, though there is no statistically significant difference in specific AEs, withdrawals, and withdrawals due to AEs (Appendix Table H.35.).

Table 76. Comparison of meropenem versus imipenem

\begin{tabular}{|l|l|l|l|l|}
\hline \multicolumn{1}{|c|}{ Outcome } & \multicolumn{1}{|c|}{ Findings } & \multicolumn{1}{c|}{$\begin{array}{c}\text { Study Design and } \\
\text { Sample Size }\end{array}$} & $\begin{array}{c}\text { Rationale for } \\
\text { Strength of } \\
\text { Evidence (SOE) }\end{array}$ & $\begin{array}{c}\text { Overall Strength of } \\
\text { Evidence }\end{array}$ \\
\hline $\begin{array}{l}\text { Repeat Exacerbation } \\
30 \text { Days }\end{array}$ & $\begin{array}{l}\text { OR: } 1.01 ; 95 \% \mathrm{Cl} \text { : } \\
0.28 \text { to } 3.63, \mathrm{I}^{2}=\mathrm{N} / \mathrm{A}\end{array}$ & $1 \mathrm{RCT}^{155}$ with 173 patients & $\begin{array}{l}\text { High ROB and } \\
\text { severe imprecision }\end{array}$ & Insufficient evidence \\
\hline $\begin{array}{l}\text { Repeat Exacerbation } \\
\text { Longest Followup }\end{array}$ & $\begin{array}{l}\text { OR: } 1.01 ; 95 \% \mathrm{Cl}: \\
0.28 \text { to } 3.63, \mathrm{I}^{2}=\mathrm{N} / \mathrm{A}\end{array}$ & $1 \mathrm{RCT}^{155}$ with 173 patients & $\begin{array}{l}\text { High ROB and } \\
\text { severe imprecision }\end{array}$ & Insufficient evidence \\
\hline $\begin{array}{l}\text { Clinical Cure } \\
\text { End of Intervention }\end{array}$ & $\begin{array}{l}\text { OR: } 0.93 ; 95 \% \mathrm{Cl}: \\
0.48 \text { to } 1.81, \mathrm{I}^{2}=\mathrm{N} / \mathrm{A}\end{array}$ & $1 \mathrm{RCT}^{155}$ with 173 patients & $\begin{array}{l}\text { High ROB and } \\
\text { severe imprecision }\end{array}$ & Insufficient evidence \\
\hline $\begin{array}{l}\text { Clinical Cure } \\
\text { Longest Followup }\end{array}$ & $\begin{array}{l}\text { OR: } 0.85 ; 95 \% \mathrm{Cl}: \\
0.47 \text { to } 1.55, \mathrm{I}^{2}=\mathrm{N} / \mathrm{A}\end{array}$ & $1 \mathrm{RCT}^{155}$ with 173 patients & $\begin{array}{l}\text { High ROB and } \\
\text { severe imprecision }\end{array}$ & Insufficient evidence \\
\hline $\begin{array}{l}\text { Clinical Failure } \\
\text { End of Intervention }\end{array}$ & $\begin{array}{l}\text { OR: } 0.67 ; 95 \% \mathrm{Cl}: \\
0.11 \text { to } 4.09, \mathrm{I}^{2}=\mathrm{N} / \mathrm{A}\end{array}$ & $1 \mathrm{RCT}^{155}$ with 173 patients & $\begin{array}{l}\text { High ROB and } \\
\text { severe imprecision }\end{array}$ & Insufficient evidence \\
\hline $\begin{array}{l}\text { Clinical Failure } \\
\text { Longest Followup }\end{array}$ & $\begin{array}{l}\text { OR: } 1.01 ; 95 \% \mathrm{Cl}: \\
0.06 \text { to } 16.44, \mathrm{I}^{2}=\mathrm{N} / \mathrm{A}\end{array}$ & $1 \mathrm{RCT}^{155}$ with 173 patients & $\begin{array}{l}\text { High ROB and } \\
\text { severe imprecision }\end{array}$ & Insufficient evidence \\
\hline
\end{tabular}

CI = confidence interval; OR = odds ratio; N/A = not applicable; RCT = randomized controlled trial; ROB = risk of bias 


\section{Comparative Effectiveness of Different Dosages of the Same Antibiotic}

\section{Trovafloxacin $200 \mathrm{Mg}$ Versus Trovafloxacin $100 \mathrm{Mg}$}

One study ${ }^{152}$ evaluated the effectiveness of trovafloxacin 200 mg compared with trovafloxacin $100 \mathrm{mg}$. There were no statistically significant differences found in outcomes between groups (Table 77). Unspecified serious AEs were reported in 13 patients treated by trovafloxacin $200 \mathrm{mg}$ and 8 patients in trovafloxacin $100 \mathrm{mg}$. No statistically significant difference was found in AEs, and withdrawals (Appendix Table H.36.).

Table 77. Comparison of trovafloxacin $200 \mathrm{mg}$ versus trovafloxacin $100 \mathrm{mg}$

\begin{tabular}{|c|c|c|c|c|}
\hline Outcome & Findings & $\begin{array}{l}\text { Study Design and } \\
\text { Sample Size }\end{array}$ & $\begin{array}{c}\text { Rationale for } \\
\text { Strength of } \\
\text { Evidence (SOE) }\end{array}$ & $\begin{array}{l}\text { Overall Strength } \\
\text { of Evidence }\end{array}$ \\
\hline $\begin{array}{l}\text { Mortality } \\
\text { End of } \\
\text { Intervention }\end{array}$ & $\begin{array}{l}\text { OR: } 3.02 ; 95 \% \mathrm{Cl}: \\
0.12 \text { to } 74.78, \\
\mathrm{I}^{2}=\mathrm{N} / \mathrm{A}\end{array}$ & $1 \mathrm{RCT}^{152}$ with 288 patients & $\begin{array}{l}\text { High ROB and } \\
\text { severe imprecision }\end{array}$ & $\begin{array}{l}\text { Insufficient } \\
\text { evidence }\end{array}$ \\
\hline $\begin{array}{l}\text { Clinical Cure } \\
\text { End of } \\
\text { Intervention }\end{array}$ & $\begin{array}{l}\text { OR: } 1 ; 95 \% \mathrm{Cl}: \\
0.57 \text { to } 1.75, \\
\mathrm{I}^{2}=\mathrm{N} / \mathrm{A}\end{array}$ & $1 \mathrm{RCT}^{152}$ with 288 patients & $\begin{array}{l}\text { High ROB and } \\
\text { severe imprecision }\end{array}$ & $\begin{array}{l}\text { Insufficient } \\
\text { evidence }\end{array}$ \\
\hline $\begin{array}{l}\text { Clinical Cure } \\
\text { Longest Followup }\end{array}$ & $\begin{array}{l}\text { OR:1.34; } 95 \% \mathrm{Cl}: \\
0.81 \text { to } 2.20, \\
\mathrm{I}^{2}=\mathrm{N} / \mathrm{A}\end{array}$ & $1 \mathrm{RCT}^{152}$ with 288 patients & $\begin{array}{l}\text { High ROB and } \\
\text { severe imprecision }\end{array}$ & $\begin{array}{l}\text { Insufficient } \\
\text { evidence }\end{array}$ \\
\hline
\end{tabular}

$\mathrm{CI}=$ confidence interval; $\mathrm{OR}$ = odds ratio; N/A = not applicable; RCT = randomized controlled trial; ROB = risk of bias

\section{Intermittent Cefotaxime Versus Continuous Cefotaxime}

One study ${ }^{140}$ evaluated the effectiveness of intermittent compared with continuous intravenous dosing of cefotaxime, a $3^{\text {rd }}$ generation cephalosporin.

There were no statistically significant differences observed in clinical cure, clinical failure, and number of withdrawals at the end of the intervention between groups (Table 78 and Appendix Table H.37.).

Table 78. Comparison of intermittent intravenous cefotaxime versus continuous intravenous cefotaxime

\begin{tabular}{|l|l|l|l|l|}
\hline \multicolumn{1}{|c|}{ Outcome } & \multicolumn{1}{|c|}{ Findings } & $\begin{array}{l}\text { Study Design and } \\
\text { Sample Size }\end{array}$ & $\begin{array}{c}\text { Rationale for } \\
\text { Strength of } \\
\text { Evidence (SOE) }\end{array}$ & $\begin{array}{l}\text { Overall Strength } \\
\text { of Evidence }\end{array}$ \\
\hline $\begin{array}{l}\text { Clinical Cure } \\
\text { End of Intervention }\end{array}$ & $\begin{array}{l}\text { OR: } 1.80 ; 95 \% \mathrm{Cl}: \\
0.60 \text { to } 5.45, \\
\mathrm{I}^{2}=\mathrm{N} / \mathrm{A}\end{array}$ & $\begin{array}{l}1 \mathrm{RCT}^{140} \text { with } 93 \\
\text { patients }\end{array}$ & $\begin{array}{l}\text { High ROB and } \\
\text { severe imprecision }\end{array}$ & $\begin{array}{l}\text { Insufficient } \\
\text { evidence }\end{array}$ \\
\hline $\begin{array}{l}\text { Clinical Failure } \\
\text { End of Intervention }\end{array}$ & $\begin{array}{l}\text { OR: } 1.02 ; 95 \% \mathrm{Cl}: \\
0.20 \text { to } 5.35, \\
\mathrm{I}^{2}=\mathrm{N} / \mathrm{A}\end{array}$ & $\begin{array}{l}1 \mathrm{RCT}^{140} \text { with } 93 \\
\text { patients }\end{array}$ & $\begin{array}{l}\text { High ROB and } \\
\text { severe imprecision }\end{array}$ & $\begin{array}{l}\text { Insufficient } \\
\text { evidence }\end{array}$ \\
\hline
\end{tabular}

$\mathrm{CI}=$ confidence interval; $\mathrm{OR}$ = odds ratio; $\mathrm{N} / \mathrm{A}=$ not applicable; $\mathrm{RCT}$ = randomized controlled trial; $\mathrm{ROB}=$ risk of bias 


\section{Comparative Effectiveness of Different Application Routes for}

Antibiotics

No eligible studies were identified that compared different routes of application (for example oral, intravenous) for the same antibiotic (class).

\section{Comparative Effectiveness of Different Durations of Treatment With Antibiotics}

\section{Amoxicillin + Clavulanic for 3 Days Versus Amoxicillin + Clavulanic for 10 Days}

One study ${ }^{142}$ compared amoxicillin + clavulanic for 3 days with amoxicillin + clavulanic for 10 days. There was no statistically significant difference found in outcomes and adverse events between the two groups (Table 79). Only mild gastrointestinal AEs were reported: 1 patient in the 3-day group and 5 patients in the 10-day group ( $\mathrm{p}=0.11$ ) (Appendix Table H. 38.).

Table 79. Comparison of amoxicillin + clavulanic for 3 days versus 10 days

\begin{tabular}{|c|c|c|c|c|}
\hline Outcome & Findings & $\begin{array}{l}\text { Study Design and } \\
\text { Sample Size }\end{array}$ & $\begin{array}{c}\text { Rationale for } \\
\text { Strength of } \\
\text { Evidence (SOE) }\end{array}$ & $\begin{array}{l}\text { Overall Strength } \\
\text { of Evidence }\end{array}$ \\
\hline $\begin{array}{l}\text { Mortality } \\
\text { Longest Followup }\end{array}$ & $\begin{array}{l}\text { OR: } 0.35 ; 95 \% \mathrm{Cl}: \\
0.01 \text { to } 8.96, \\
\mathrm{I}^{2}=\mathrm{N} / \mathrm{A}\end{array}$ & $\begin{array}{l}1 \mathrm{RCT}^{142} \text { with } 48 \\
\text { patients }\end{array}$ & $\begin{array}{l}\text { Intermediate ROB } \\
\text { and severe } \\
\text { imprecision }\end{array}$ & $\begin{array}{l}\text { Insufficient } \\
\text { evidence }\end{array}$ \\
\hline $\begin{array}{l}\text { Hospital Admission } \\
30 \text { Days }\end{array}$ & $\begin{array}{l}\text { OR: } 0.14 ; 95 \% \mathrm{Cl}: \\
0.01 \text { to } 2.80, \\
\mathrm{I}^{2}=\mathrm{N} / \mathrm{A}\end{array}$ & $\begin{array}{l}1 \mathrm{RCT}^{142} \text { with } 48 \\
\text { patients }\end{array}$ & $\begin{array}{l}\text { Intermediate ROB } \\
\text { and severe } \\
\text { imprecision }\end{array}$ & $\begin{array}{l}\text { Insufficient } \\
\text { evidence }\end{array}$ \\
\hline $\begin{array}{l}\text { Repeat Exacerbation } \\
\text { End of Intervention }\end{array}$ & $\begin{array}{l}\text { OR: } 0.38 ; 95 \% \mathrm{Cl}: \\
0.07 \text { to } 2.19, \\
\mathrm{I}^{2}=\mathrm{N} / \mathrm{A}\end{array}$ & $\begin{array}{l}1 \mathrm{RCT}^{142} \text { with } 48 \\
\text { patients }\end{array}$ & $\begin{array}{l}\text { Intermediate ROB } \\
\text { and severe } \\
\text { imprecision }\end{array}$ & $\begin{array}{l}\text { Insufficient } \\
\text { evidence }\end{array}$ \\
\hline $\begin{array}{l}\text { Repeat Exacerbation } \\
30 \text { Days }\end{array}$ & $\begin{array}{l}\text { OR: } 0.71 ; 95 \% \mathrm{Cl}: \\
0.19 \text { to } 2.68, \\
\mathrm{I}^{2}=\mathrm{N} / \mathrm{A}\end{array}$ & $\begin{array}{l}1 \mathrm{RCT}^{142} \text { with } 48 \\
\text { patients }\end{array}$ & $\begin{array}{l}\text { Intermediate ROB } \\
\text { and severe } \\
\text { imprecision }\end{array}$ & $\begin{array}{l}\text { Insufficient } \\
\text { evidence }\end{array}$ \\
\hline $\begin{array}{l}\text { Repeat Exacerbation } \\
\text { Longest Followup }\end{array}$ & $\begin{array}{l}\text { OR: } 0.71 ; 95 \% \mathrm{Cl}: \\
0.19 \text { to } 2.68, \\
\mathrm{I}^{2}=\mathrm{N} / \mathrm{A}\end{array}$ & $\begin{array}{l}1 \mathrm{RCT}^{142} \text { with } 48 \\
\text { patients }\end{array}$ & $\begin{array}{l}\text { Intermediate ROB } \\
\text { and severe } \\
\text { imprecision }\end{array}$ & $\begin{array}{l}\text { Insufficient } \\
\text { evidence }\end{array}$ \\
\hline $\begin{array}{l}\text { Clinical Cure } \\
\text { End of Intervention }\end{array}$ & $\begin{array}{l}\text { OR: } 0.57 ; 95 \% \mathrm{Cl}: \\
0.15 \text { to } 2.14 \\
\mathrm{I}^{2}=\mathrm{N} / \mathrm{A}\end{array}$ & $\begin{array}{l}1 \mathrm{RCT}^{142} \text { with } 48 \\
\text { patients }\end{array}$ & $\begin{array}{l}\text { Intermediate ROB } \\
\text { and severe } \\
\text { imprecision }\end{array}$ & $\begin{array}{l}\text { Insufficient } \\
\text { evidence }\end{array}$ \\
\hline $\begin{array}{l}\text { Clinical Cure } \\
\text { Longest Followup }\end{array}$ & $\begin{array}{l}\text { OR: } 1.02 ; 95 \% \mathrm{Cl}: \\
0.33 \text { to } 3.20 \\
\mathrm{I}^{2}=\mathrm{N} / \mathrm{A}\end{array}$ & $\begin{array}{l}1 \mathrm{RCT}^{142} \text { with } 48 \\
\text { patients }\end{array}$ & $\begin{array}{l}\text { Intermediate ROB } \\
\text { and severe } \\
\text { imprecision }\end{array}$ & $\begin{array}{l}\text { Insufficient } \\
\text { evidence }\end{array}$ \\
\hline $\begin{array}{l}\text { Resolution } \\
\text { Clinical Failure } \\
\text { End of Intervention }\end{array}$ & $\begin{array}{l}\text { OR: } 1.10 ; 95 \% \mathrm{Cl}: \\
0.20 \text { to } 6.09 \\
\mathrm{I}^{2}=\mathrm{N} / \mathrm{A}\end{array}$ & $\begin{array}{l}1 \mathrm{RCT}^{142} \text { with } 48 \\
\text { patients }\end{array}$ & $\begin{array}{l}\text { Intermediate ROB } \\
\text { and severe } \\
\text { imprecision }\end{array}$ & $\begin{array}{l}\text { Insufficient } \\
\text { evidence }\end{array}$ \\
\hline
\end{tabular}

CI = confidence interval; OR = odds ratio; N/A = not applicable; $\mathrm{RCT}$ = randomized controlled trial; ROB = risk of bias 


\section{Corticosteroids-Comparative Effectiveness Studies}

\section{Comparative Effectiveness of Different Systemic Corticosteroid Agents}

One study ${ }^{151}$ compared intravenous methylprednisolone with intravenous deflazacort hemisuccinate for 7 days. There were no statistically significant differences found in mortality and FEV1 absolute at the end of the intervention between groups (Table 80 and Table 81). No statistically significant difference was observed in AEs, withdrawals and withdrawals due to AEs (Appendix Table H.39.).

One study ${ }^{137}$ compared intravenous methylprednisolone, followed by oral methylprednisone with intravenous hydrocortisone, followed by oral prednisolone. The total treatment duration was 14 days. FEV1 absolute at the end of treatment was statistically significantly higher in the methylprednisolone group compared with the hydrocortisone/prednisolone group, but there were no statistically significant differences observed in the other evaluated outcomes between groups (Table 80 and Table 81). No statistically significant difference in AEs, withdrawals and withdrawals due to AEs was found (Appendix Table H.40).

Table 80. Comparison of different systemic corticosteroid agents, critical outcomes

\begin{tabular}{|c|c|c|c|c|c|}
\hline Comparison & Outcome & Findings & $\begin{array}{l}\text { Study } \\
\text { Design } \\
\text { and } \\
\text { Sample } \\
\text { Size }\end{array}$ & $\begin{array}{l}\text { Rationale } \\
\text { for Strength } \\
\text { of Evidence } \\
\text { (SOE) }\end{array}$ & $\begin{array}{c}\text { Overall } \\
\text { Strength of } \\
\text { Evidence }\end{array}$ \\
\hline $\begin{array}{l}\text { iv Methyl- } \\
\text { prednisolone vs. } \\
\text { iv Deflazacort } \\
\text { Hemisuccinate }\end{array}$ & $\begin{array}{l}\text { Mortality } \\
\text { End of } \\
\text { Interventio } \\
\mathrm{n}\end{array}$ & $\begin{array}{l}\text { OR : } 0.32 ; \\
95 \% \mathrm{Cl}: 0.01 \\
\text { to } 8.24 \\
\text { I2=N/A }\end{array}$ & $\begin{array}{l}1 \mathrm{RCT}^{151} \\
\text { with } 60 \\
\text { patients }\end{array}$ & $\begin{array}{l}\text { High ROB } \\
\text { and severe } \\
\text { imprecision }\end{array}$ & $\begin{array}{l}\text { Insufficient } \\
\text { evidence }\end{array}$ \\
\hline $\begin{array}{l}\text { iv Methyl- } \\
\text { prednisolone, } \\
\text { followed by oral } \\
\text { Methylprednison } \\
\text { e vs. iv Hydro- } \\
\text { cortisone, } \\
\text { followed by oral } \\
\text { Prednisolone }\end{array}$ & $\begin{array}{l}\text { Mortality } \\
\text { Longest } \\
\text { Followup }\end{array}$ & $\begin{array}{l}\text { OR: } 1.07 ; \\
95 \% \mathrm{Cl}: 0.06 \\
\text { to } 17.53 \\
\text { I2=N/A }\end{array}$ & $\begin{array}{l}1 \mathrm{RCT}^{137} \\
\text { with } 97 \\
\text { patients }\end{array}$ & $\begin{array}{l}\text { Intermediate } \\
\text { ROB and } \\
\text { severe } \\
\text { imprecision }\end{array}$ & $\begin{array}{l}\text { Insufficient } \\
\text { evidence }\end{array}$ \\
\hline $\begin{array}{l}\text { iv Methyl- } \\
\text { prednisolone, } \\
\text { followed by oral } \\
\text { Methylprednison } \\
\text { e vs. iv Hydro- } \\
\text { cortisone, } \\
\text { followed by oral } \\
\text { Prednisolone }\end{array}$ & $\begin{array}{l}\text { Need for } \\
\text { Intubation } \\
\text { Longest } \\
\text { Followup }\end{array}$ & $\begin{array}{l}\text { OR: } 1.07 ; \\
95 \% \mathrm{Cl}: 0.06 \\
\text { to } 17.53 \\
\text { I2=N/A }\end{array}$ & $\begin{array}{l}1 \mathrm{RCT}^{137} \\
\text { with } 97 \\
\text { patients }\end{array}$ & $\begin{array}{l}\text { Intermediate } \\
\text { ROB and } \\
\text { severe } \\
\text { imprecision }\end{array}$ & $\begin{array}{l}\text { Insufficient } \\
\text { evidence }\end{array}$ \\
\hline $\begin{array}{l}\text { iv Methyl- } \\
\text { prednisolone, } \\
\text { followed by oral } \\
\text { Methylprednison } \\
\text { e vs. iv Hydro- } \\
\text { cortisone, } \\
\text { followed by oral } \\
\text { Prednisolone }\end{array}$ & $\begin{array}{l}\text { Clinical } \\
\text { Failure } \\
\text { End of } \\
\text { Interventio } \\
n\end{array}$ & $\begin{array}{l}\text { OR: } 1.37 ; \\
95 \% \mathrm{Cl}: 0.34 \\
\text { to } 5.44 \\
\text { I2=N/A }\end{array}$ & $\begin{array}{l}1 \mathrm{RCT}^{137} \\
\text { with } 97 \\
\text { patients }\end{array}$ & $\begin{array}{l}\text { Intermediate } \\
\text { ROB and } \\
\text { severe } \\
\text { imprecision }\end{array}$ & $\begin{array}{l}\text { Insufficient } \\
\text { evidence }\end{array}$ \\
\hline
\end{tabular}




\begin{tabular}{|c|c|c|c|c|c|}
\hline Comparison & Outcome & Findings & $\begin{array}{l}\text { Study } \\
\text { Design } \\
\text { and } \\
\text { Sample } \\
\text { Size }\end{array}$ & $\begin{array}{l}\text { Rationale } \\
\text { for Strength } \\
\text { of Evidence } \\
\text { (SOE) }\end{array}$ & $\begin{array}{c}\text { Overall } \\
\text { Strength of } \\
\text { Evidence }\end{array}$ \\
\hline $\begin{array}{l}\text { iv Methyl- } \\
\text { prednisolone, } \\
\text { followed by oral } \\
\text { Methylprednison } \\
\text { e vs. iv Hydro- } \\
\text { cortisone, } \\
\text { followed by oral } \\
\text { Prednisolone }\end{array}$ & $\begin{array}{l}\text { Dyspnea } \\
\text { (Questionn } \\
\text { aire: MRC) } \\
\text { Longest } \\
\text { Followup }\end{array}$ & $\begin{array}{l}\text { WMD: }-01 ; 95 \% \\
\text { Cl: }-0.29 \text { to } \\
0.09, I^{2}=\mathrm{N} / \mathrm{A}\end{array}$ & $\begin{array}{l}\mathrm{RCT}^{137} \\
\text { with } 97 \\
\text { patients }\end{array}$ & $\begin{array}{l}\text { Intermediate } \\
\text { ROB and } \\
\text { severe } \\
\text { imprecision }\end{array}$ & $\begin{array}{l}\text { Insufficient } \\
\text { evidence }\end{array}$ \\
\hline
\end{tabular}

CI = confidence interval; N/A = not applicable; MRC = Medical Research Council Scale; OR = odds ratio; RCT = randomized controlled trial; $\mathrm{ROB}=$ risk of bias; $\mathrm{WMD}$ = weighted mean difference

Table 81. Comparison of different systemic corticosteroid agents, additional outcomes

\begin{tabular}{|c|c|c|c|}
\hline Comparison & Outcome & Findings & Study Design and Sample Size \\
\hline $\begin{array}{l}\text { iv Methyl- } \\
\text { prednisolone vs. } \\
\text { iv Deflazacort } \\
\text { Hemisuccinate }\end{array}$ & $\begin{array}{l}\text { FEV1 } \\
\text { Absolute } \\
\text { End of } \\
\text { Intervention }\end{array}$ & $\begin{array}{l}\text { WMD: } 0.12 ; 95 \% \mathrm{Cl}:-0.06 \text { to } 0.30 \text {, } \\
\mathrm{I}^{2}=\mathrm{N} / \mathrm{A}\end{array}$ & $1 \mathrm{RCT}^{151}$ with 60 patients \\
\hline $\begin{array}{l}\text { iv Methyl- } \\
\text { prednisolone, } \\
\text { followed by oral } \\
\text { Methylprednisone } \\
\text { vs iv Hydro- } \\
\text { cortisone, } \\
\text { followed by oral } \\
\text { Prednisolone }\end{array}$ & $\begin{array}{l}\text { FEV1 } \\
\text { Absolute } \\
\text { Longest } \\
\text { Followup }\end{array}$ & $\begin{array}{l}\text { WMD: } 0.20 ; 95 \% \mathrm{Cl}: 0.06 \text { to } 0.34 \text {, } \\
\mathrm{I}^{2}=\mathrm{N} / \mathrm{A}\end{array}$ & $1 \mathrm{RCT}^{137}$ with 97 patients \\
\hline
\end{tabular}

$\mathrm{CI}=$ confidence interval; FEV1 = forced expiratory volume in one second; I.V. = intravenous; OR = odds ratio; N/A = not applicable; RCT = randomized controlled trial; WMD = weighted mean difference

\section{Comparative Effectiveness of Different Routes of Application for Corticosteroids}

\section{Systemic Corticosteroids Versus Inhaled Corticosteroids}

One study ${ }^{75}$ compared subcutaneous prednisolone with nebulized budesonide given for at least 10 days. FEV1 percent predicted at the end of treatment was statistically significantly lower with subcutaneous prednisolone compared with nebulized budesonide (Table 82 and Table 83).

Oral prednisolone versus nebulized budesonide given for 10 days was compared in one study. ${ }^{76}$ There were no statistically significant differences found between groups in mortality and dyspnea at the end of the intervention (Table 82 and Table 83). Unspecified serious AEs were reported in 8 patients treated by nebulized budesonide and 5 treated by oral prednisolone. No statistical difference in AEs, withdrawals, and withdrawals due to AEs were found (Appendix Table H.41.).

Intravenous prednisolone versus nebulized budesonide ${ }^{146}$ given for 10 days was compared in one study. No AEs were reported in any of the two groups.

Another study ${ }^{138}$ evaluated the effectiveness of oral prednisolone + inhaled formoterol (in a metered dose inhaler) compared with inhaled budesonide + formoterol (in a single metered dose inhaler) for 14 days. There were no statistically significant differences observed between groups in any of the evaluated outcomes (Table 82 and Table 83) and AEs (Appendix Table H.42.). 
One study ${ }^{131}$ compared methylprednisolone (initially intravenous, then oral) versus inhaled budesonide. Patients in the inhaled budesonide group had significantly lower incidence of adverse events than those in the systemic methylprednisolone group. There was no statistically significant difference in FEV1 percent predicted. Another study ${ }^{159}$ compared intravenous methylprednisolone versus inhaled budesonide. There was no statistically significantly difference found in quality of life, FEV1 absolute values, and repeat exacerbation between the groups (Table 82 and Table 83). However, budesonide was associated with statistically significantly less endocrine-related AEs (Appendix Tables H.43 and H.44). Another study ${ }^{133}$ compared methylprednisolone (intravenous) to inhaled budesonide $4 \mathrm{mg}$ or inhaled budesonide $8 \mathrm{mg}$. There was no statistically significant difference found in FEV1 percent predicted, FEV1 absolute, dyspnea (Table 82 and Table 83) and AEs (Appendix Tables H. 45 and H.46.) between groups.

Table 82. Systemic corticosteroids versus inhaled corticosteroids, critical outcomes

\begin{tabular}{|c|c|c|c|c|c|}
\hline Comparison & Outcome & Findings & $\begin{array}{l}\text { Study Design and } \\
\text { Sample Size }\end{array}$ & $\begin{array}{l}\text { Rationale for } \\
\text { Strength of } \\
\text { Evidence } \\
\text { (SOE) }\end{array}$ & $\begin{array}{c}\text { Overall } \\
\text { Strength of } \\
\text { Evidence }\end{array}$ \\
\hline \multirow[t]{2}{*}{$\begin{array}{l}\text { Oral Prednisolone vs. } \\
\text { Nebulized } \\
\text { Budesonide }\end{array}$} & $\begin{array}{l}\text { Mortality } \\
\text { End of Intervention }\end{array}$ & $\begin{array}{l}\text { OR: } 3.49 ; \\
95 \% \mathrm{Cl}: 0.14 \\
\text { to } 87.18 \\
\mathrm{I}^{2}=\mathrm{N} / \mathrm{A}\end{array}$ & $\begin{array}{l}1 \mathrm{RCT}^{76} \text { with } 133 \\
\text { patients }\end{array}$ & $\begin{array}{l}\text { High ROB and } \\
\text { severe } \\
\text { imprecision }\end{array}$ & $\begin{array}{l}\text { Insufficient } \\
\text { evidence }\end{array}$ \\
\hline & $\begin{array}{l}\text { Dyspnea } \\
\text { (Numeric Scale: } \\
\text { Modified Borg } \\
\text { Scale)End of } \\
\text { Intervention }\end{array}$ & $\begin{array}{l}\text { WMD: }-0.70 \\
95 \% \mathrm{Cl}:- \\
1.48 \text { to } 0.08 \\
\mathrm{I}^{2}=\mathrm{N} / \mathrm{A}\end{array}$ & $\begin{array}{l}1 \mathrm{RCT}^{76} \text { with } 133 \\
\text { patients }\end{array}$ & $\begin{array}{l}\text { High ROB and } \\
\text { severe } \\
\text { imprecision }\end{array}$ & $\begin{array}{l}\text { Insufficient } \\
\text { evidence }\end{array}$ \\
\hline \multirow[t]{2}{*}{$\begin{array}{l}\text { Subcutaneous } \\
\text { Prednisolone vs. } \\
\text { Nebulized } \\
\text { Budesonide }\end{array}$} & $\begin{array}{l}\text { Hospital Admission } \\
30 \text { days }\end{array}$ & $\begin{array}{l}\text { Rate Ratio: } \\
0.80 ; 95 \% \mathrm{Cl}: \\
0.21 \text { to } 2.98 \\
\mathrm{I}^{2}=\mathrm{N} / \mathrm{A}\end{array}$ & $\begin{array}{l}1 \mathrm{RCT}^{75} \text { with } 106 \\
\text { patients }\end{array}$ & $\begin{array}{l}\text { High ROB and } \\
\text { severe } \\
\text { imprecision }\end{array}$ & $\begin{array}{l}\text { Insufficient } \\
\text { evidence }\end{array}$ \\
\hline & $\begin{array}{l}\text { Repeat Exacerbation } \\
1 \text { Month Followup }\end{array}$ & $\begin{array}{l}\text { Rate Ratio: } \\
0.88 ; 95 \% \mathrm{Cl}: \\
0.34 \text { to } 2.33 \text {, } \\
\mathrm{I}^{2}=\mathrm{N} / \mathrm{A}\end{array}$ & $\begin{array}{l}1 \mathrm{RCT}^{75} \text { with } 106 \\
\text { patients }\end{array}$ & $\begin{array}{l}\text { High ROB and } \\
\text { severe } \\
\text { imprecision }\end{array}$ & $\begin{array}{l}\text { Insufficient } \\
\text { evidence }\end{array}$ \\
\hline \multirow{5}{*}{$\begin{array}{l}\text { Oral Prednisolone (+ } \\
\text { Inhaled Formoterol) } \\
\text { vs Inhaled } \\
\text { Budesonide (+Inhaled } \\
\text { Formoterol) }\end{array}$} & $\begin{array}{l}\text { QoL(CCQ) } \\
\text { End of Intervention }\end{array}$ & $\begin{array}{l}\text { WMD: }-0.09 ; \\
95 \% \mathrm{Cl}:- \\
0.53 \text { to } 0.35 \\
\text { I }^{2}=\mathrm{N} / \mathrm{A}\end{array}$ & $\begin{array}{l}1 \mathrm{RCT}^{138} \text { with } 109 \\
\text { patients }\end{array}$ & $\begin{array}{l}\text { Intermediate } \\
\text { ROB and } \\
\text { severe } \\
\text { imprecision }\end{array}$ & $\begin{array}{l}\text { Insufficient } \\
\text { evidence }\end{array}$ \\
\hline & $\begin{array}{l}\text { QoL(CCQ) } \\
\text { Longest Followup }\end{array}$ & $\begin{array}{l}\text { WMD: }-0.20 \\
95 \% \mathrm{Cl}:- \\
0.63 \text { to } 0.23 \text {, } \\
\mathrm{I}^{2}=\mathrm{N} / \mathrm{A}\end{array}$ & $\begin{array}{l}1 \mathrm{RCT}^{138} \text { with } 109 \\
\text { patients }\end{array}$ & $\begin{array}{l}\text { Intermediate } \\
\text { ROB and } \\
\text { severe } \\
\text { imprecision }\end{array}$ & $\begin{array}{l}\text { Insufficient } \\
\text { evidence }\end{array}$ \\
\hline & $\begin{array}{l}\text { Repeat Exacerbation } \\
\text { End of Intervention }\end{array}$ & $\begin{array}{l}\text { Rate Ratio: } \\
1.02 ; 95 \% \mathrm{Cl} ; \\
0.49 \text { to } 2.14 \text {, } \\
\mathrm{I}^{2}=\mathrm{N} / \mathrm{A}\end{array}$ & $\begin{array}{l}1 \mathrm{RCT}^{138} \text { with } 109 \\
\text { patients }\end{array}$ & $\begin{array}{l}\text { Intermediate } \\
\text { ROB and } \\
\text { severe } \\
\text { imprecision }\end{array}$ & $\begin{array}{l}\text { Insufficient } \\
\text { evidence }\end{array}$ \\
\hline & $\begin{array}{l}\text { Repeat Exacerbation } \\
\text { End of Intervention }\end{array}$ & $\begin{array}{l}\text { OR: } 0.91 ; \\
95 \% \mathrm{Cl}: 0.35 \\
\text { to } 2.36 \\
\mathrm{I}^{2}=\mathrm{N} / \mathrm{A}\end{array}$ & $\begin{array}{l}1 \mathrm{RCT}^{138} \text { with } 109 \\
\text { patients }\end{array}$ & $\begin{array}{l}\text { Intermediate } \\
\text { ROB and } \\
\text { severe } \\
\text { imprecision }\end{array}$ & $\begin{array}{l}\text { Insufficient } \\
\text { evidence }\end{array}$ \\
\hline & $\begin{array}{l}\text { Clinical Failure } \\
\text { End of Intervention }\end{array}$ & $\begin{array}{l}\text { OR: } 0.2 ; 95 \% \\
\text { Cl: } 0.01 \text { to } \\
4.19, I^{2}=N / A\end{array}$ & $\begin{array}{l}1 \mathrm{RCT}^{138} \text { with } 109 \\
\text { patients }\end{array}$ & $\begin{array}{l}\text { Intermediate } \\
\text { ROB and } \\
\text { severe } \\
\text { imprecision }\end{array}$ & $\begin{array}{l}\text { Insufficient } \\
\text { evidence }\end{array}$ \\
\hline
\end{tabular}




\begin{tabular}{|c|c|c|c|c|c|}
\hline Comparison & Outcome & Findings & $\begin{array}{l}\text { Study Design and } \\
\text { Sample Size }\end{array}$ & $\begin{array}{l}\text { Rationale for } \\
\text { Strength of } \\
\text { Evidence } \\
\text { (SOE) }\end{array}$ & $\begin{array}{c}\text { Overall } \\
\text { Strength of } \\
\text { Evidence }\end{array}$ \\
\hline \multirow[t]{2}{*}{$\begin{array}{l}\text { Intravenous } \\
\text { Methylprednisolone } \\
\text { vs. inhaled } \\
\text { Budesonide }\end{array}$} & $\begin{array}{l}\text { QoL(CAT) } \\
\text { End of Intervention }\end{array}$ & $\begin{array}{l}\text { WMD: }-0.27, \\
95 \% \mathrm{Cl}:- \\
1.28 \text { to } 1.26 \\
\mathrm{I}^{2}=\mathrm{N} / \mathrm{A}\end{array}$ & $\begin{array}{l}\text { 1 } \text { RCT }^{159} \text { with } 471 \\
\text { patients }\end{array}$ & $\begin{array}{l}\text { Intermediate } \\
\text { ROB and } \\
\text { imprecision }\end{array}$ & $\begin{array}{l}\text { Low SOE } \\
\text { supporting no } \\
\text { difference }\end{array}$ \\
\hline & $\begin{array}{l}\text { Repeat } \\
\text { Exacerbations } \\
\text { End of Intervention }\end{array}$ & $\begin{array}{l}\text { Rate Ratio: } \\
0.90 ; 95 \% \mathrm{Cl} \text { : } \\
0.76 \text { to } 1.06 \text {, } \\
\mathrm{I}^{2}=\mathrm{N} / \mathrm{A}\end{array}$ & $\begin{array}{l}\text { 1 } \mathrm{RCT}^{159} \text { with } 471 \\
\text { patients }\end{array}$ & $\begin{array}{l}\text { Intermediate } \\
\text { ROB and } \\
\text { imprecision }\end{array}$ & $\begin{array}{l}\text { Low SOE } \\
\text { supporting no } \\
\text { difference }\end{array}$ \\
\hline $\begin{array}{l}\text { Intravenous } \\
\text { Methylprednisolone } \\
\text { vs. inhaled } \\
\text { Budesonide } 4 \mathrm{mg} \\
\end{array}$ & $\begin{array}{l}\text { Dyspnea } \\
\text { (Numeric Scale) } \\
\text { End of Intervention }\end{array}$ & $\mathrm{P}=\mathrm{NS}$ & $\begin{array}{l}1 \mathrm{RCT}^{133} \text { with } 60 \\
\text { patients }\end{array}$ & $\begin{array}{l}\text { High ROB and } \\
\text { severe } \\
\text { imprecision }\end{array}$ & $\begin{array}{l}\text { Insufficient } \\
\text { evidence }\end{array}$ \\
\hline $\begin{array}{l}\text { Intravenous } \\
\text { Methylprednisolone } \\
\text { vs. inhaled } \\
\text { Budesonide } 8 \mathrm{mg}\end{array}$ & $\begin{array}{l}\text { Dyspnea } \\
\text { (Numeric Scale) } \\
\text { End of Intervention }\end{array}$ & $\mathrm{P}=\mathrm{NS}$ & $\begin{array}{l}1 \mathrm{RCT}^{133} \text { with } 59 \\
\text { patients }\end{array}$ & $\begin{array}{l}\text { High ROB and } \\
\text { severe } \\
\text { imprecision }\end{array}$ & $\begin{array}{l}\text { Insufficient } \\
\text { evidence }\end{array}$ \\
\hline
\end{tabular}

CAT = COPD Assessment Test; CCQ = Clinical COPD Questionnaire; CI = confidence interval; FEV1 = forced expiratory volume in one second; OR = odds ratio; N/A = not applicable; NS = not statistically significant; QoL = quality of life; RCT = randomized controlled trial; $\mathrm{ROB}=$ risk of bias; $\mathrm{WMD}=$ weighted mean difference

Table 83. Systemic corticosteroids versus inhaled corticosteroids, additional outcomes

\begin{tabular}{|c|c|c|c|}
\hline Comparison & Outcome & Findings & Study Design and Sample Size \\
\hline $\begin{array}{l}\text { Subcutaneous } \\
\text { Prednisolone vs. } \\
\text { Nebulized } \\
\text { Budesonide }\end{array}$ & $\begin{array}{l}\text { FEV1\% Predicted } \\
\text { End of Intervention }\end{array}$ & $\begin{array}{l}\text { WMD: }-6.60 ; 95 \% \mathrm{Cl}:-12.39 \text { to }- \\
0.81, I^{2}=\mathrm{N} / \mathrm{A}\end{array}$ & $1 \mathrm{RCT}^{75}$ with 106 patients \\
\hline \multirow{4}{*}{$\begin{array}{l}\text { Oral Prednisolone (+ } \\
\text { inhaled Formoterol) } \\
\text { vs. inhaled } \\
\text { Budesonide (+inhaled } \\
\text { Formoterol) }\end{array}$} & $\begin{array}{l}\text { FEV1\% Predicted } \\
\text { End of Intervention }\end{array}$ & $\begin{array}{l}\text { WMD: }-1.40 ; 95 \% \mathrm{Cl}:-5.79 \text { to } 2.99 \text {, } \\
\mathrm{I}^{2}=\mathrm{N} / \mathrm{A}\end{array}$ & $1 \mathrm{RCT}^{138}$ with 109 patients \\
\hline & $\begin{array}{l}\text { FEV1\% Predicted } \\
\text { Longest Followup }\end{array}$ & $\begin{array}{l}\text { WMD: }-0.40 ; 95 \% \mathrm{Cl}:-4.98 \text { to } 4.18 \text {, } \\
\mathrm{I}^{2}=\mathrm{N} / \mathrm{A}\end{array}$ & $1 \mathrm{RCT}^{138}$ with 109 patients \\
\hline & $\begin{array}{l}\text { FEV1 Absolute } \\
\text { End of Intervention }\end{array}$ & $\begin{array}{l}\text { WMD: }-0.02 ; 95 \% \mathrm{Cl}:-0.17 \text { to } 0.13 \text {, } \\
\mathrm{I}^{2}=\mathrm{N} / \mathrm{A}\end{array}$ & 1 RCT ${ }^{138}$ with 109 patients \\
\hline & $\begin{array}{l}\text { FEV1 Absolute } \\
\text { Longest Followup }\end{array}$ & $\begin{array}{l}\text { WMD: } 0.02 ; 95 \% \mathrm{Cl}:-0.14 \text { to } 0.18 \text {, } \\
\mathrm{l}^{2}=\mathrm{N} / \mathrm{A}\end{array}$ & $1 \mathrm{RCT}^{138}$ with 109 patients \\
\hline \multirow{2}{*}{$\begin{array}{l}\text { Intravenous } \\
\text { Methylprednisolone } \\
\text { vs. inhaled } \\
\text { Budesonide }\end{array}$} & $\begin{array}{l}\text { FEV1\% Predicted } \\
\text { End of Intervention }\end{array}$ & $\begin{array}{l}\text { WMD: }-0.48 ; 95 \% \mathrm{Cl}:-10.18 \text { to } \\
9.22, I^{2}=\mathrm{N} / \mathrm{A}\end{array}$ & $1 \mathrm{RCT}^{131}$ with 30 patients \\
\hline & $\begin{array}{l}\text { FEV1 Absolute } \\
\text { End of Intervention }\end{array}$ & $\begin{array}{l}\text { WMD: }-0.04,95 \% \mathrm{Cl}:-0.12 \text { to } 0.04 \text {, } \\
\mathrm{I}^{2}=\mathrm{N} / \mathrm{A}\end{array}$ & $1 \mathrm{RCT}^{159}$ with 471 patients \\
\hline $\begin{array}{l}\text { Intravenous } \\
\text { Methylprednisolone } \\
\text { vs. Inhaled } \\
\text { Budesonide } 4 \mathrm{mg} \\
\end{array}$ & $\begin{array}{l}\text { FEV1 \% Predicted } \\
\text { End of Intervention }\end{array}$ & 50.7 vs. $44.5 ; p=N S$ & $1 \mathrm{RCT}^{133}$ with 60 patients \\
\hline $\begin{array}{l}\text { Intravenous } \\
\text { Methylprednisolone } \\
\text { vs. Inhaled } \\
\text { Budesonide } 8 \mathrm{mg} \\
\end{array}$ & $\begin{array}{l}\text { FEV1 \% Predicted } \\
\text { End of Intervention }\end{array}$ & 54.8 vs. $44.5 ; p=N S$ & $1 \mathrm{RCT}^{133}$ with 59 patients \\
\hline
\end{tabular}

$\mathrm{CI}=$ confidence interval; FEV1 = forced expiratory volume in one second; $\mathrm{mg}$ = milligram; N/A = not applicable; NS = not statistically significant; QoL = quality of life; RCT = randomized controlled trial; ROB = risk of bias; WMD = weighted mean difference 


\section{Oral Corticosteroids Versus Intravenous Corticosteroids}

One study ${ }^{144}$ compared oral prednisolone with intravenous prednisolone for 5 days. There were no statistically significant differences observed between groups in any of the evaluated outcomes (Table 84 and Table 85) and number of withdrawals (Appendix Table H.47).

Intravenous methylprednisolone, (+nebulized salbutamol and ipratropium bromide) was compared with oral methylprednisolone (+fenoterol and ipratropium bromide via metered dose inhaler) in one study. ${ }^{147}$ There were no statistically significant differences observed in any of the evaluated outcomes and withdrawals between groups (Table 84 and Table 85 and Appendix Table H.48.).

Table 84. Comparison of oral corticosteroids versus intravenous corticosteroids, critical outcomes

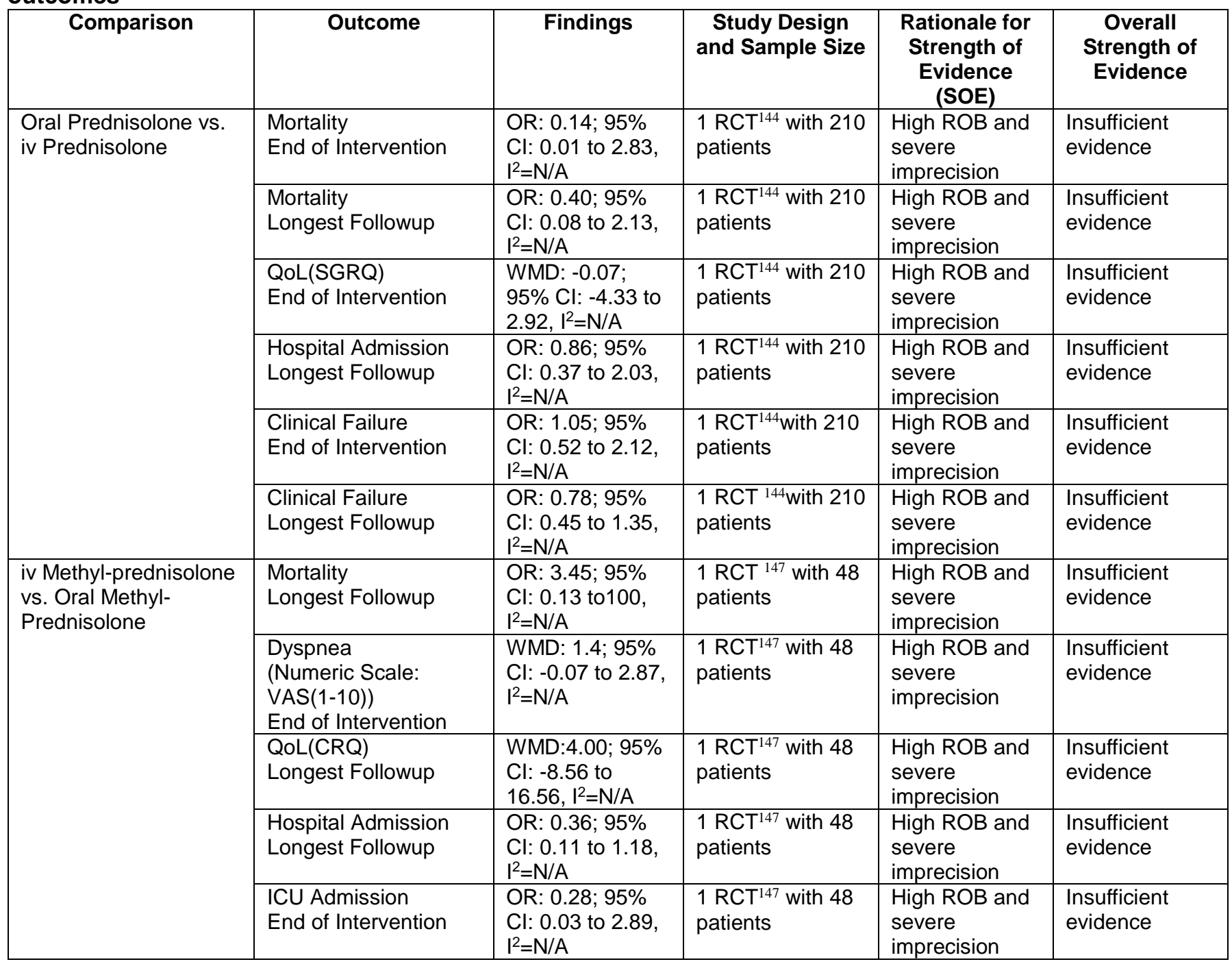

CI = confidence interval; I.V. = intravenous; N/A = not applicable; OR = odds ratio; QoL = quality of life; RCT = randomized controlled trial; ROB = risk of bias; SGRQ = St. George Respiratory Questionnaire; WMD = weighted mean difference 
Table 85. Comparison of oral corticosteroids versus intravenous corticosteroids, additional outcomes

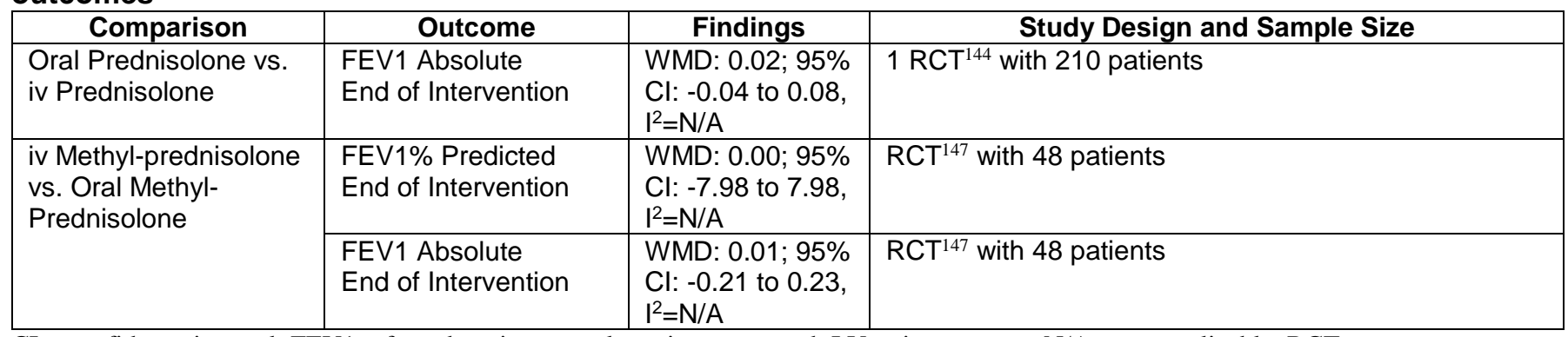

$\mathrm{CI}$ = confidence interval; FEV1 = forced expiratory volume in one second; I.V. = intravenous; N/A = not applicable; RCT = randomized controlled trial; $\mathrm{WMD}=$ weighted mean difference

\section{Comparative Effectiveness of Different Durations of Treatment With Corticosteroids}

One study ${ }^{77}$ compared systemic corticosteroid treatment for 8 weeks versus 2 weeks. Treatment in both groups started with intravenous methylprednisolone, followed by oral prednisone. There were no statistically significant differences found in any of the outcomes (Table 86 and Table 87) and AEs (Appendix Table H.49.) between groups.

Another study ${ }^{158}$ compared systemic corticosteroid treatment for 5 days versus 14 days. Intravenous methylprednisolone was given on day 1 in both treatment groups, followed by prednisone for days 2-5 and placebo on days 6-14 in the shorter active treatment group, and prednisone for days 2-14 in the longer active treatment group. There were no statistically significant differences observed in any of the outcomes (Table 86 and Table 87), AEs, and number of withdrawals (Appendix Table H.50.) between groups.

Intravenous methylprednisolone for 3 days versus 10 days was assessed in one study. ${ }^{149}$ FEV1 absolute at the end of intervention was statistically significantly lower in the group treated with methylprednisolone for 3 days compared with the group treated for 10 days, but all other outcomes, AEs, and withdrawals were not found to be statistically significantly different between groups (Table 86, Table 87 and Appendix Table H.51.).

Table 86. Comparison of different durations of treatment with corticosteroids, critical outcomes

\begin{tabular}{|c|c|c|c|c|c|}
\hline Comparison & Outcome & Findings & $\begin{array}{c}\text { Study Design } \\
\text { and Sample Size }\end{array}$ & $\begin{array}{c}\text { Rationale } \\
\text { for } \\
\text { Strength of } \\
\text { Evidence } \\
\text { (SOE) }\end{array}$ & $\begin{array}{c}\text { Overall } \\
\text { Strength of } \\
\text { Evidence }\end{array}$ \\
\hline \multirow[t]{2}{*}{$\begin{array}{l}\text { Systemic } \\
\text { Corticosteroids } \\
\text { for } 8 \text { Weeks vs. } \\
2 \text { Weeks }\end{array}$} & $\begin{array}{l}\text { Mortality } \\
\text { End of Intervention }\end{array}$ & $\begin{array}{l}\text { OR: 5.13; } \\
95 \% \mathrm{Cl} \text { : } \\
0.24 \text { to } \\
108.51 \\
\left.\right|^{2}=\mathrm{N} / \mathrm{A}\end{array}$ & $\begin{array}{l}1 \mathrm{RCT}^{77} \text { with } 160 \\
\text { patients }\end{array}$ & $\begin{array}{l}\text { High ROB } \\
\text { and severe } \\
\text { imprecision }\end{array}$ & $\begin{array}{l}\text { Insufficient } \\
\text { evidence }\end{array}$ \\
\hline & $\begin{array}{l}\text { Mortality } \\
\text { Longest Followup }\end{array}$ & $\begin{array}{l}\text { OR: } 1.82 ; \\
95 \% \mathrm{Cl} \text {; } \\
0.51 \text { to } \\
6.49 \\
\left.\right|^{2}=\mathrm{N} / \mathrm{A}\end{array}$ & $\begin{array}{l}1 \mathrm{RCT}^{77} \text { with } 160 \\
\text { patients }\end{array}$ & $\begin{array}{l}\text { High ROB } \\
\text { and severe } \\
\text { imprecision }\end{array}$ & $\begin{array}{l}\text { Insufficient } \\
\text { evidence }\end{array}$ \\
\hline
\end{tabular}




\begin{tabular}{|c|c|c|c|c|c|}
\hline Comparison & Outcome & Findings & $\begin{array}{c}\text { Study Design } \\
\text { and Sample Size }\end{array}$ & $\begin{array}{c}\text { Rationale } \\
\text { for } \\
\text { Strength of } \\
\text { Evidence } \\
\text { (SOE) }\end{array}$ & $\begin{array}{c}\text { Overall } \\
\text { Strength of } \\
\text { Evidence }\end{array}$ \\
\hline & $\begin{array}{l}\text { Hospital Admission } \\
30 \text { Days }\end{array}$ & $\begin{array}{l}\text { OR: } 0.49 ; \\
95 \% \mathrm{Cl} \text {; } \\
0.09 \text { to } \\
2.74 \text {, } \\
\mathrm{I}^{2}=\mathrm{N} / \mathrm{A}\end{array}$ & $\begin{array}{l}1 \mathrm{RCT}^{77} \text { with } 160 \\
\text { patients }\end{array}$ & $\begin{array}{l}\text { High ROB } \\
\text { and severe } \\
\text { imprecision }\end{array}$ & $\begin{array}{l}\text { Insufficient } \\
\text { evidence }\end{array}$ \\
\hline & $\begin{array}{l}\text { Hospital Admission } \\
\text { Longest Followup }\end{array}$ & $\begin{array}{l}\text { OR: } 1.10 ; \\
95 \% \mathrm{Cl} \text {; } \\
0.47 \text { to } \\
2.58 \\
\mathrm{I}^{2}=\mathrm{N} / \mathrm{A}\end{array}$ & $\begin{array}{l}1 \mathrm{RCT}^{77} \text { with } 160 \\
\text { patients }\end{array}$ & $\begin{array}{l}\text { High ROB } \\
\text { and severe } \\
\text { imprecision }\end{array}$ & $\begin{array}{l}\text { Insufficient } \\
\text { evidence }\end{array}$ \\
\hline & $\begin{array}{l}\text { Need for Intubation } \\
\text { End of Intervention }\end{array}$ & $\begin{array}{l}\text { OR: } 0.49 ; \\
95 \% \mathrm{Cl} \text {; } \\
0.04 \text { to } \\
5.59 \\
\text { I }^{2}=\mathrm{N} / \mathrm{A}\end{array}$ & $\begin{array}{l}1 \mathrm{RCT}^{77} \text { with } 160 \\
\text { patients }\end{array}$ & $\begin{array}{l}\text { High ROB } \\
\text { and severe } \\
\text { imprecision }\end{array}$ & $\begin{array}{l}\text { Insufficient } \\
\text { evidence }\end{array}$ \\
\hline & $\begin{array}{l}\text { Need for Intubation } \\
\text { Longest Followup }\end{array}$ & $\begin{array}{l}\text { OR: } 0.66 ; \\
95 \% \mathrm{Cl} \text { : } \\
0.11 \text { to } \\
4.05 \\
\mathrm{I}^{2}=\mathrm{N} / \mathrm{A}\end{array}$ & $\begin{array}{l}1 \mathrm{RCT}^{77} \text { with } 160 \\
\text { patients }\end{array}$ & $\begin{array}{l}\text { High ROB } \\
\text { and severe } \\
\text { imprecision }\end{array}$ & $\begin{array}{l}\text { Insufficient } \\
\text { evidence }\end{array}$ \\
\hline & $\begin{array}{l}\text { Clinical Failure } \\
\text { Longest Followup }\end{array}$ & $\begin{array}{l}\text { OR: } 0.94 ; \\
95 \% \mathrm{Cl} \text {; } \\
0.46 \text { to } \\
1.90 \\
\text { I}^{2}=\mathrm{N} / \mathrm{A}\end{array}$ & $\begin{array}{l}1 \mathrm{RCT}^{77} \text { with } 160 \\
\text { patients }\end{array}$ & $\begin{array}{l}\text { High ROB } \\
\text { and severe } \\
\text { imprecision }\end{array}$ & $\begin{array}{l}\text { Insufficient } \\
\text { evidence }\end{array}$ \\
\hline \multirow[t]{6}{*}{$\begin{array}{l}\text { Systemic } \\
\text { Corticosteroids } \\
\text { for } 5 \text { Days vs. } 14 \\
\text { Days }\end{array}$} & $\begin{array}{l}\text { Dyspnea } \\
\text { (Questionnaire: MRC) } \\
\text { Longest Followup }\end{array}$ & $\begin{array}{l}\text { WMD: - } \\
0.08 ; 95 \% \\
\mathrm{Cl}:-0.33 \text { to } \\
0.17 \\
\mathrm{I}^{2}=\mathrm{N} / \mathrm{A}\end{array}$ & $\begin{array}{l}1 \mathrm{RCT}^{158} \text { with } 313 \\
\text { patients }\end{array}$ & $\begin{array}{l}\text { Severe } \\
\text { imprecision }\end{array}$ & $\begin{array}{l}\text { Low SOE } \\
\text { supporting } \\
\text { no } \\
\text { difference }\end{array}$ \\
\hline & $\begin{array}{l}\text { Mortality } \\
\text { Longest Followup }\end{array}$ & $\begin{array}{l}\text { OR: } 0.91 ; \\
95 \% \mathrm{Cl} \text {; } \\
0.40 \text { to } \\
2.06 \text {, } \\
\mathrm{I}^{2}=\mathrm{N} / \mathrm{A}\end{array}$ & $\begin{array}{l}1 \mathrm{RCT}^{158} \text { with } 313 \\
\text { patients }\end{array}$ & $\begin{array}{l}\text { Severe } \\
\text { imprecision }\end{array}$ & $\begin{array}{l}\text { Low SOE } \\
\text { supporting } \\
\text { no } \\
\text { difference }\end{array}$ \\
\hline & $\begin{array}{l}\text { QoL(Bronchitis-Associated } \\
\text { Quality of Life) } \\
\text { End of Intervention }\end{array}$ & $\begin{array}{l}\text { WMD: } 0.05 ; \\
95 \% \mathrm{Cl}:- \\
0.12 \text { to } \\
0.22 \\
\mathrm{I}^{2}=\mathrm{N} / \mathrm{A}\end{array}$ & $\begin{array}{l}1 \mathrm{RCT}^{158} \text { with } 313 \\
\text { patients }\end{array}$ & $\begin{array}{l}\text { Severe } \\
\text { imprecision }\end{array}$ & $\begin{array}{l}\text { Low SOE } \\
\text { supporting } \\
\text { no } \\
\text { difference }\end{array}$ \\
\hline & $\begin{array}{l}\text { QoL(Bronchitis-Associated } \\
\text { Quality of Life) } \\
\text { Longest Followup }\end{array}$ & $\begin{array}{l}\text { WMD: - } \\
0.02 ; 95 \% \\
\mathrm{Cl}:-0.18 \text { to } \\
0.14 \\
\mathrm{I}^{2}=\mathrm{N} / \mathrm{A}\end{array}$ & $\begin{array}{l}1 \mathrm{RCT}^{158} \text { with } 313 \\
\text { patients }\end{array}$ & $\begin{array}{l}\text { Severe } \\
\text { imprecision }\end{array}$ & $\begin{array}{l}\text { Low SOE } \\
\text { supporting } \\
\text { no } \\
\text { difference }\end{array}$ \\
\hline & $\begin{array}{l}\text { Repeat Exacerbation } \\
\text { End of Intervention }\end{array}$ & $\begin{array}{l}\text { OR: } 0.96 \text {; } \\
95 \% \mathrm{Cl} \text { : } \\
0.61 \text { to } \\
1.53 \text {, } \\
\mathrm{I}^{2}=\mathrm{N} / \mathrm{A}\end{array}$ & $\begin{array}{l}1 \mathrm{RCT}^{158} \text { with } 313 \\
\text { patients }\end{array}$ & $\begin{array}{l}\text { Severe } \\
\text { imprecision }\end{array}$ & $\begin{array}{l}\text { Low SOE } \\
\text { supporting } \\
\text { no } \\
\text { difference }\end{array}$ \\
\hline & $\begin{array}{l}\text { Need for Intubation } \\
\text { Longest } \\
\text { Followup }\end{array}$ & $\begin{array}{l}\text { OR: } 0.78 ; \\
95 \% \mathrm{Cl} \text { : } \\
0.39 \text { to } \\
1.54 \\
\mathrm{I}^{2}=\mathrm{N} / \mathrm{A}\end{array}$ & $\begin{array}{l}1 \mathrm{RCT}^{158} \text { with } 313 \\
\text { patients }\end{array}$ & $\begin{array}{l}\text { Severe } \\
\text { imprecision }\end{array}$ & $\begin{array}{l}\text { Low SOE } \\
\text { supporting } \\
\text { no } \\
\text { difference }\end{array}$ \\
\hline
\end{tabular}




\begin{tabular}{|c|c|c|c|c|c|}
\hline Comparison & Outcome & Findings & $\begin{array}{c}\text { Study Design } \\
\text { and Sample Size }\end{array}$ & $\begin{array}{c}\text { Rationale } \\
\text { for } \\
\text { Strength of } \\
\text { Evidence } \\
\text { (SOE) }\end{array}$ & $\begin{array}{c}\text { Overall } \\
\text { Strength of } \\
\text { Evidence }\end{array}$ \\
\hline \multirow[t]{4}{*}{$\begin{array}{l}\text { iv Methyl- } \\
\text { prednisolone for } \\
3 \text { Days vs. } 10 \\
\text { Days }\end{array}$} & $\begin{array}{l}\text { Dyspnea } \\
\text { (Numeric Scale: Dyspnea } \\
\text { Score) } \\
\text { End of Intervention }\end{array}$ & $\begin{array}{l}\text { WMD: } 0.20 ; \\
95 \% \mathrm{Cl}:- \\
0.35 \text { to } \\
0.75 \\
\mathrm{I}^{2}=\mathrm{N} / \mathrm{A}\end{array}$ & $\begin{array}{l}1 \mathrm{RCT}^{149} \text { with } 34 \\
\text { patients }\end{array}$ & $\begin{array}{l}\text { Intermediate } \\
\text { ROB and } \\
\text { severe } \\
\text { imprecision }\end{array}$ & $\begin{array}{l}\text { Insufficient } \\
\text { evidence }\end{array}$ \\
\hline & $\begin{array}{l}\text { Dyspnea (Numeric Scale: } \\
\text { Dyspnea Score) } \\
\text { Longest Followup }\end{array}$ & $\begin{array}{l}\text { WMD: - } \\
0.10 ; 95 \% \\
\mathrm{Cl}:-0.81 \text { to } \\
0.61 \\
\mathrm{I}^{2}=\mathrm{N} / \mathrm{A}\end{array}$ & $\begin{array}{l}1 \mathrm{RCT}^{149} \text { with } 34 \\
\text { patients }\end{array}$ & $\begin{array}{l}\text { Intermediate } \\
\text { ROB and } \\
\text { severe } \\
\text { imprecision }\end{array}$ & $\begin{array}{l}\text { Insufficient } \\
\text { evidence }\end{array}$ \\
\hline & \multirow[t]{2}{*}{$\begin{array}{l}\text { Repeat Exacerbation } \\
\text { End of Intervention }\end{array}$} & $\begin{array}{l}\text { OR: } 1.31 ; \\
95 \% \mathrm{Cl} \text { : } \\
0.31 \text { to } \\
5.53 \\
\mathrm{I}^{2}=\mathrm{N} / \mathrm{A}\end{array}$ & $\begin{array}{l}1 \mathrm{RCT}^{149} \text { with } 34 \\
\text { patients }\end{array}$ & $\begin{array}{l}\text { Intermediate } \\
\text { ROB and } \\
\text { severe } \\
\text { imprecision }\end{array}$ & $\begin{array}{l}\text { Insufficient } \\
\text { evidence }\end{array}$ \\
\hline & & $\begin{array}{l}\text { Rate Ratio: } \\
1.60 ; 95 \% \\
\mathrm{Cl}: 0.52 \text { to } \\
4.89 \\
\mathrm{I}^{2}=\mathrm{N} / \mathrm{A}\end{array}$ & $\begin{array}{l}1 \mathrm{RCT}^{149} \text { with } 34 \\
\text { patients }\end{array}$ & $\begin{array}{l}\text { Intermediate } \\
\text { ROB and } \\
\text { severe } \\
\text { imprecision }\end{array}$ & $\begin{array}{l}\text { Insufficient } \\
\text { evidence }\end{array}$ \\
\hline
\end{tabular}

CI = confidence interval; I.V. = intravenous; MRQ = Medical Research Council Scale; N/A = not applicable; OR = odds ratio; $\mathrm{QoL}=$ quality of life; RCT $=$ randomized controlled trial; $\mathrm{ROB}=$ risk of bias; $\mathrm{WMD}=$ weighted mean difference

Table 87. Comparison of different durations of treatment with corticosteroids, additional outcomes

\begin{tabular}{|c|c|c|c|}
\hline Comparison & Outcome & Findings & $\begin{array}{c}\text { Study Design and Sample } \\
\text { Size }\end{array}$ \\
\hline \multirow{2}{*}{$\begin{array}{l}\text { Systemic } \\
\text { Corticosteroids for } \\
5 \text { Days vs. } 14 \text { Days }\end{array}$} & $\begin{array}{l}\text { FEV1\% Predicted } \\
\text { End of Intervention }\end{array}$ & $\begin{array}{l}\text { WMD: }-1.16 ; 95 \% \mathrm{Cl}:- \\
4.63 \text { to } 2.31, \mathrm{I}^{2}=\mathrm{N} / \mathrm{A}\end{array}$ & $1 \mathrm{RCT}^{158}$ with 313 patients \\
\hline & $\begin{array}{l}\text { FEV1\% Predicted } \\
\text { Longest Followup }\end{array}$ & $\begin{array}{l}\text { WMD: } 0.80 ; 95 \% \mathrm{Cl}:- \\
2.91 \text { to } 4.51,1^{2}=\mathrm{N} / \mathrm{A}\end{array}$ & $1 \mathrm{RCT}^{158}$ with 313 patients \\
\hline \multirow{3}{*}{$\begin{array}{l}\text { iv Methyl- } \\
\text { prednisolone for } 3 \\
\text { Days vs. } 10 \text { Days }\end{array}$} & $\begin{array}{l}\text { Cough(Cough Scale) } \\
\text { End of Intervention }\end{array}$ & $\begin{array}{l}\text { WMD: } 0.00 ; 95 \% \mathrm{Cl}:- \\
0.83 \text { to } 0.83,1^{2}=\mathrm{N} / \mathrm{A}\end{array}$ & $1 \mathrm{RCT}^{149}$ with 34 patients \\
\hline & $\begin{array}{l}\text { Cough(Cough Scale) } \\
\text { Longest Followup }\end{array}$ & $\begin{array}{l}\text { WMD: } 0.30 ; 95 \% \mathrm{Cl}:- \\
0.41 \text { to } 1.00, \mathrm{I}^{2}=\mathrm{N} / \mathrm{A}\end{array}$ & $1 \mathrm{RCT}^{149}$ with 34 patients \\
\hline & $\begin{array}{l}\text { FEV1 Absolute } \\
\text { End of Intervention }\end{array}$ & $\begin{array}{l}\text { WMD: }-0.23 ; 95 \% \mathrm{Cl}:- \\
0.41 \text { to }-0.04,1^{2}=\mathrm{N} / \mathrm{A}\end{array}$ & $1 \mathrm{RCT}^{149}$ with 34 patients \\
\hline
\end{tabular}

$\mathrm{CI}=$ confidence interval; FEV1 = forced expiratory volume in one second; I.V. = intravenous; N/A = not applicable; RCT = randomized controlled trial; $\mathrm{WMD}=$ weighted mean difference 


\section{Discussion}

\section{Overview}

We conducted a systematic review to assess the effectiveness of pharmacologic and nonpharmacologic therapies in adults with exacerbations of chronic obstructive pulmonary disease (ECOPD). We assessed the effectiveness of systemic antibiotics, systemic corticosteroids and potentially emerging pharmacologic and nonpharmacologic therapies stratified by severity of ECOPD. Further, we assessed the effectiveness of combinations of treatments, and we compared different regimens (different agents, routes of administration, and duration of treatment) of antibiotics and corticosteroids.

The majority of studies were conducted in hospitalized patients with moderate or severe ECOPD with only a small number of studies conducted in outpatients with mild or mild to moderate ECOPD. Figures 3 and 4 are evidence maps that summarize the distribution of evidence for the effectiveness of interventions compared with placebo or management without intervention treatment. Lung function (forced expiratory volume in one second (FEV1) absolute or FEV \% predicted) was the most commonly evaluated outcome. For most interventions there was only one randomized controlled trial (RCT) per outcome available.

Figure 3. Evidence map showing distribution of trials in mild (or mild/moderate) ECOPD (Key Questions 1 and 2)

\begin{tabular}{|c|c|c|c|c|c|c|c|c|}
\hline & Mortality & Dyspnea & QoL & $\begin{array}{l}\text { FEV1 } \\
\text { (absolute or } \\
\% \\
\text { predicted) }\end{array}$ & $\begin{array}{l}\text { 6MWD } \\
\text { or } \\
\text { shuttle } \\
\text { walk }\end{array}$ & $\begin{array}{l}\text { Need for } \\
\text { intubation }\end{array}$ & $\begin{array}{l}\text { Repeat } \\
\text { exacerbation } \\
\text { and/or } \\
\text { hospital } \\
\text { admissions }\end{array}$ & $\begin{array}{l}\text { ECOPD } \\
\text { resolution } \\
\text { (clinical } \\
\text { cure, } \\
\text { failure) }\end{array}$ \\
\hline \multicolumn{9}{|c|}{ Key Question 1} \\
\hline $\begin{array}{l}\text { Systemic } \\
\text { Antibiotics }\end{array}$ & $1 \mathrm{RCT}$ & $1 \mathrm{RCT}$ & $1 \mathrm{RCT}$ & 2 RCTs & & & $1 \mathrm{RCT}$ & 4 RCTs \\
\hline $\begin{array}{l}\text { Systemic } \\
\text { Corticosteroids }\end{array}$ & $1 \mathrm{RCT}$ & $1 \mathrm{RCT}$ & 1RCT & 2 RCTs & & & $1 \mathrm{RCT}$ & 2 RCTs \\
\hline \multicolumn{9}{|c|}{ Key Question 2} \\
\hline Oral Mucolytics & & & & $1 \mathrm{RCT}$ & & & & \\
\hline ICS+LABA & & & & $1 \mathrm{RCT}$ & & & & $1 \mathrm{RCT}$ \\
\hline Statin & & & & $1 \mathrm{RCT}$ & & & & \\
\hline
\end{tabular}

6MWD = 6 minute walking distance; ECPOD = exacerbations of chronic obstructive pulmonary disease; FEV1 = forced expiratory volume in one second; ICS = inhaled corticosteroids; LABA = long-acting beta-agonist; RCT = randomized controlled trial

White cells = no RCTS; Grey cells = 1 RCT; Black cells = 2 or more RCTs 
Figure 4. Evidence map showing distribution of trials in moderate to severe ECOPD (Key Questions 1 and 2)

\section{Key Question 1}

\begin{tabular}{|l|l|l|l|l|l|l|l|l|}
\hline & Mortality & Dyspnea & QoL & $\begin{array}{l}\text { FEV1 } \\
\text { (absolute } \\
\text { or \% } \\
\text { predicted) }\end{array}$ & $\begin{array}{l}\text { 6MWD } \\
\text { or } \\
\text { shuttle } \\
\text { walk }\end{array}$ & $\begin{array}{l}\text { Need for } \\
\text { intubation }\end{array}$ & $\begin{array}{l}\text { Repeat } \\
\text { exacerbation } \\
\text { and/or } \\
\text { hospital } \\
\text { admissions }\end{array}$ & $\begin{array}{l}\text { Resolution } \\
\text { of ECOPD } \\
\text { (clinical } \\
\text { cure, } \\
\text { failure) }\end{array}$ \\
\hline $\begin{array}{l}\text { Key Question 1 } \\
\text { Systemic } \\
\text { Antibiotics }\end{array}$ & 2 RCTs & 1 RCT & & 2 RCTs & & 1 RCT & 1 RCT & 2 RCTs \\
\hline $\begin{array}{l}\text { Systemic } \\
\text { Corticosteroids }\end{array}$ & 4 RCTs & 1 RCT & & 3 RCTs & & 2 RCTs & 3 RCTs & 1 RCT \\
\hline
\end{tabular}

$6 \mathrm{MWD}=6$ minute walking distance; ECPOD = exacerbations of chronic obstructive pulmonary disease; FEV1 = forced expiratory volume in one second; QoL = quality of life; RCT = randomized controlled trial

White cells = no RCTS; grey cells = 1 RCT; black cells = 2 or more RCTs

\section{Key Question 2 - pharmacologic interventions}

\begin{tabular}{|c|c|c|c|c|c|c|c|c|}
\hline & Mortality & Dyspnea & QoL & $\begin{array}{l}\text { FEV1 } \\
\text { (absolute } \\
\text { or \% } \\
\text { predicted) }\end{array}$ & $\begin{array}{l}\text { 6MWD } \\
\text { or } \\
\text { shuttle } \\
\text { walk }\end{array}$ & $\begin{array}{l}\text { Need for } \\
\text { intubation }\end{array}$ & $\begin{array}{l}\text { Repeat } \\
\text { exacerbation } \\
\text { and/or } \\
\text { hospital } \\
\text { admissions } \\
\end{array}$ & $\begin{array}{l}\text { Resolution } \\
\text { of ECOPD } \\
\text { (clinical } \\
\text { cure, } \\
\text { failure) }\end{array}$ \\
\hline \multicolumn{9}{|c|}{ Key Question 2} \\
\hline \multicolumn{9}{|c|}{ Pharmacologic interventions } \\
\hline $\begin{array}{l}\text { i.v. } \\
\text { Aminophyllines }\end{array}$ & & 2 RCTs & & 3 RCTs & & $1 \mathrm{RCT}$ & & \\
\hline $\begin{array}{l}\text { i.v. Magnesium } \\
\text { Sulfate }\end{array}$ & & $1 \mathrm{RCT}$ & & 3 RCTs & & & & \\
\hline $\begin{array}{l}\text { Nebulized } \\
\text { Magnesium } \\
\text { Sulfate }\end{array}$ & & & & $1 \mathrm{RCT}$ & & & & \\
\hline Oral Mucolytics & & 2 RCTs & & 3 RCTs & & & 2 RCTs & \\
\hline ICS & & $1 \mathrm{RCT}$ & & $1 \mathrm{RCT}$ & & 1RCT & $1 \mathrm{RCT}$ & \\
\hline ICS+SABA & & & & $1 \mathrm{RCT}$ & & & & \\
\hline $\begin{array}{l}\text { Inhaled } \\
\text { Antibiotics }\end{array}$ & & & & $1 \mathrm{RCT}$ & & & & \\
\hline $\begin{array}{l}\text { 5-lipogygenase } \\
\text { inhibitor }\end{array}$ & $1 \mathrm{RCT}$ & & & $1 \mathrm{RCT}$ & & $1 \mathrm{RCT}$ & $1 \mathrm{RCT}$ & $1 \mathrm{RCT}$ \\
\hline
\end{tabular}

6MWD = 6 minute walking distance; ECPOD = exacerbations of chronic obstructive pulmonary disease; FEV1 = forced expiratory volume in one second; ICS = inhaled corticosteroids; i.v. = intravenous; SABA = short-acting beta-agonist; QoL = quality of life; RCT = randomized controlled trial

White cells = no RCTS; grey cells = 1 RCT; black cells = 2 or more RCTs 
Key Question 2 - nonpharmacologic interventions

\begin{tabular}{|c|c|c|c|c|c|c|c|c|}
\hline & Mortality & Dyspnea & QoL & $\begin{array}{l}\text { FEV1 } \\
\text { (absolute } \\
\text { or \% } \\
\text { predicted) }\end{array}$ & $\begin{array}{l}\text { 6MWD } \\
\text { or } \\
\text { shuttle } \\
\text { walk }\end{array}$ & $\begin{array}{l}\text { Need for } \\
\text { intubation }\end{array}$ & $\begin{array}{l}\text { Repeat } \\
\text { exacerbation } \\
\text { and/or } \\
\text { hospital } \\
\text { admissions }\end{array}$ & $\begin{array}{l}\text { Resolution } \\
\text { of ECOPD } \\
\text { (clinical } \\
\text { cure, } \\
\text { failure) }\end{array}$ \\
\hline \multicolumn{9}{|l|}{ Key Question 2} \\
\hline \multicolumn{9}{|c|}{ Nonpharmacologic interventions } \\
\hline $\begin{array}{l}\text { Chest Physio, } \\
\text { Breathing } \\
\text { Technique }\end{array}$ & & 2 RCTs & $2 \mathrm{RCTs}$ & $1 \mathrm{RCT}$ & & & 2 RCTs & \\
\hline $\begin{array}{l}\text { Chest Physio, } \\
\text { Vibration/Percu } \\
\text { ssion }\end{array}$ & & 2 RCTs & & 3 RCTs & $1 \mathrm{RCT}$ & & & \\
\hline $\begin{array}{l}\text { Chest Physio, } \\
\text { Positive } \\
\text { Expiratory } \\
\text { Pressure }\end{array}$ & $1 \mathrm{RCT}$ & $1 \mathrm{RCT}$ & $1 \mathrm{RCT}$ & $1 \mathrm{RCT}$ & $1 \mathrm{RCT}$ & & $1 \mathrm{RCT}$ & \\
\hline $\begin{array}{l}\text { Resistance } \\
\text { Training }\end{array}$ & $1 \mathrm{RCT}$ & $1 \mathrm{RCT}$ & $1 \mathrm{RCT}$ & 2 RCTs & 2 RCTs & & $1 \mathrm{RCT}$ & \\
\hline Aerobic Training & & $1 \mathrm{RCT}$ & $1 \mathrm{RCT}$ & & & & $1 \mathrm{RCT}$ & \\
\hline $\begin{array}{l}\text { Aerobic + } \\
\text { Resistance } \\
\text { Training }\end{array}$ & & & & $1 \mathrm{RCT}$ & & & & \\
\hline $\begin{array}{l}\text { Chest Physio + } \\
\text { Exercise }\end{array}$ & & $1 \mathrm{RCT}$ & $1 \mathrm{RCT}$ & 1 RCT & & & & \\
\hline $\begin{array}{l}\text { Early Pulmonary } \\
\text { Rehab }\end{array}$ & & 3 RCTs & 1RCT & & 3 RCTs & & $1 \mathrm{RCT}$ & \\
\hline $\begin{array}{l}\text { Whole Body } \\
\text { Vibration } \\
\text { Training }\end{array}$ & & & 1RCT & 1RCT & $1 \mathrm{RCT}$ & & & \\
\hline $\begin{array}{l}\text { Transcutaneous } \\
\text { Electrical Nerve } \\
\text { Stimulation }\end{array}$ & & $1 \mathrm{RCT}$ & & 1 RCT & 1 RCT & & & \\
\hline $\begin{array}{l}\text { Dietary } \\
\text { Intervention, } \\
\text { Caloric } \\
\text { Supplement }\end{array}$ & $1 \mathrm{RCT}$ & $1 \mathrm{RCT}$ & $1 \mathrm{RCT}$ & $1 \mathrm{RCT}$ & & & & \\
\hline $\begin{array}{l}\text { Dietary } \\
\text { Intervention, } \\
\text { Caloric and } \\
\text { Protein } \\
\text { supplement }\end{array}$ & & $1 \mathrm{RCT}$ & & $1 \mathrm{RCT}$ & & & & \\
\hline $\begin{array}{l}\text { Dietary } \\
\text { Intervention, } \\
\text { High Fat Low } \\
\text { Carbohydrate }\end{array}$ & & & & $1 \mathrm{RCT}$ & & & & \\
\hline $\begin{array}{l}\text { Dietary } \\
\text { Intervention, } \\
\text { Vitamin D }\end{array}$ & $1 \mathrm{RCT}$ & 2 RCTs & 1RCT & $1 \mathrm{RCT}$ & & & & \\
\hline $\begin{array}{l}\text { Dietary } \\
\text { Intervention, } \\
\text { Omega-3 Fatty } \\
\text { Acid }\end{array}$ & & 1 RCT & $1 \mathrm{RCT}$ & & & $1 \mathrm{RCT}$ & & \\
\hline
\end{tabular}

$6 \mathrm{MWD}=6$ minute walking distance; ECPOD = exacerbations of chronic obstructive pulmonary disease; FEV1 = forced expiratory volume in one second; RCT = randomized controlled trial

White cells = no RCTS; grey cells = 1 RCT; black cells = 2 or more RCTs 
We found moderate strength of evidence for antibiotics to improve clinical cure and reduce clinical failure in ECOPD compared with placebo. The finding was independent of the severity of an exacerbation episode including in patients with only mild ECOPD treated as outpatients. The review did not identify any antibiotic (group) that was clearly outperforming another.

There was low strength of evidence for the effectiveness of systemic corticosteroids (oral and intravenous) to reduce clinical failure at the end of the intervention. No significant differences were found between different systemic corticosteroid agents or routes of administration. A 5-day course of systemic corticosteroids was not statistically different to a 14-day course based on the final health outcomes mortality, quality of life, repeat exacerbation, and need for intubation.

Additional nonpharmacologic treatments showed in general more promising results than pharmacologic treatments. Some pharmacologic interventions had only been assessed for intermediate outcomes (lung function, symptoms) including magnesium sulfate, mucolytics, inhaled corticosteroid (ICS) and ICS+ short-acting beta adrenergic agonists (SABA), and inhaled antibiotics. None of the pharmacological interventions that were assessed for final health outcomes (aminophyllines, ICS+ long-acting beta-agonist (LABA) and the 5-lipoxygenase inhibitor zileuton) showed a significant improvement in any final health outcomes compared with placebo. In addition to intermediate outcomes, many studies on nonpharmacologic interventions assessed exercise capacity (e.g. 6-minute walking distance) and/or quality of life. For nonpharmacologic interventions, a significant improvement in exercise capacity compared with management without nonpharmacologic was found for resistance training, early pulmonary rehabilitation, whole body vibration training, and transcutaneous electrical nerve stimulation. This suggests that these interventions can potentially successfully address ECOPD-associated deconditioning. An improvement in quality of life was observed for combined chest physiotherapy (using breathing technique) and motion exercises, whole body vibration training, caloric supplements and vitamin D. Evidence from one study indicated that oxygen titration in ECOPD compared with high flow oxygen may reduce mortality.

Chest physiotherapy is commonly prescribed in patients hospitalized for ECOPD; however, the evidence-base for chest physiotherapy, as shown in our review, is weak. There was no evidence of improved final health outcomes, other than reduced hospital admission, from relatively small, low quality trials in our review.

Inhaled salbutamol (SABA) and inhaled ipratropium (short-acting muscarinic antagonists (SAMA)) had comparable effects on lung function and combining the SABA and SAMA did not result in better lung function than either medication alone.

\section{Findings in Relation to What Is Known}

This review provides a comprehensive overview of pharmacologic and nonpharmacologic interventions in ECOPD. The literature on interventions for chronic obstructive pulmonary disease (COPD) and ECOPD has proliferated substantially in recent years with numerous published systematic reviews on different interventions for the management of ECOPD. For clinicians, health policy makers and other end users of the evidence, it has become an almost impossible task to keep up with the ever increasing body of evidence on the management of ECOPD. This review therefore addresses an urgent need to provide an up-to-date summary of the current state of evidence for the management of ECOPD. 
One of the main findings of this systematic review is that despite a proliferation of the COPD literature, the evidence base for most interventions in ECOPD remains low. While significant progress has been made in recent years in assessing interventions to prevent ECOPD (during stable COPD), the same cannot be said for acute interventions used during ECOPD.

For the standard therapy of ECOPD with systemic antibiotics, corticosteroids and bronchodilators, many questions remain unanswered. While the discussion of COPD phenotypes (and ECOPD phenotypes) has taken center stage on the COPD research agenda, very limited information on ECOPD phenotypes (e.g. infective versus noninfective, high versus low eosinophil count) has been included in trials of intervention for ECOPD. In particular, whether a response to systemic corticosteroid treatment of ECOPD depends on the blood eosinophil level remains unexplored. Studies on ICS for prevention of ECOPD in stable COPD suggest that patients with higher blood eosinophil levels might be more likely to benefit from ICS treatment in terms of reducing the risk of ECOPD. ${ }^{44}$

Despite the ubiquitous use of SABAs and SAMAs in ECOPD, we found only two studies (Key Question [KQ]3) that studied their effectiveness. The role of LABAs and long-acting muscarinic antagonists (LAMAs) in ECOPD remains largely unexplored with only one crossover trial identified in our review that assessed a LAMA versus placebo.

Another important insight from our systemic review is that some nonpharmacologic interventions (resistance training, early pulmonary rehabilitation, whole body vibration training transcutaneous electrical nerve stimulation, caloric supplementation, and vitamin D) show promise, but the current evidence is largely based on single, relatively small RCTs. In stable COPD, pulmonary rehabilitation is one of the most effective (though underused) interventions. In recent years, there has been a significant interest in exploring the effects of pulmonary rehabilitation in patients who have recently experienced an ECOPD or even in patients who are in the acute phase of ECOPD (e.g. before hospital discharge).

Our review indicated that pulmonary rehabilitation during ECOPD may increase functional capacity (based on 6-minute walking distance). A potential risk for increased mortality associated with pulmonary rehabilitation commenced during hospitalization for ECOPD has previously been flagged in the guidelines on management of COPD exacerbations by the European Respiratory Society and the American Thoracic Society, published in $2017 .{ }^{45}$ We did not find a significant association with increased mortality for pulmonary rehabilitation or any form of exercise commenced during hospitalization. Our review did not include studies conducted in an intensive care unit (ICU), chronic ventilator unit, or respiratory care unit, which might have contributed to the discrepancy in the findings. Also, a trial of rehabilitation commenced within 48 hours of hospital admission in 389 patients with exacerbations of different chronic respiratory conditions found an increase in mortality in the intervention group at one year (odds ratio 1.74 , 95\% confidence interval: 1.05 to 2.88 ).$^{46}$ Mortality was, however, not reported in the subgroup of patients with COPD and is therefore not included in our review. Given the potential of exercise programs during hospitalization for ECOP to ameliorate deconditioning and improve functional status, further research in this area is urgently needed. Other nonpharmacologic interventions during ECOPD that may improve functional capacity included resistance training, whole body vibration training and transcutaneous electrical nerve stimulation. As these findings were based on single, relatively small studies, evidence from wellconducted large RCTs will be required to confirm these findings. Similarly, caloric 
supplementation and vitamin D may improve quality of life in patients with ECOPD, but confirmation from well-conducted large RCTs is required before any definite conclusions can be drawn.

\section{Limitations}

For most interventions, only one RCT was available per outcome (KQ1-4), which limits inferences from the quantitative synthesis. Failure to detect statistical significance for most of the outcomes may have resulted from type II error. There was some heterogeneity in the definition of the severity of ECOPD, although in general mild ECOPD referred to patients that could be treated in an outpatient setting, whereas moderate to severe ECOPD was used for hospitalized patients. A number of studies included patients assessed in an emergency department with a broad range of severity of ECOPD. We used the definition of serious AEs listed by the original studies, which could have varied between studies.

Defining resolution of ECOPD and differentiating poor resolution from re-exacerbation can be challenging. We used outcomes as described in the original studies, which might have resulted in heterogeneity of definitions of ECOPD resolution and overlap between clinical failure and re-exacerbation between studies.

Very limited information on ECOPD phenotypes (e.g. infective versus noninfective, high versus low eosinophil count) has been included in trials of intervention. We could therefore not draw any conclusions about interventions for different ECOPD phenotypes. In particular, whether a response to systemic corticosteroids depends on the blood eosinophil levels remains unexplored.

Studies were overall at high risk of bias. This, together with the low number of studies per intervention/outcome, makes interpretation of the body of evidence challenging. We were unable to statistically evaluate publication bias and only included studies published in English. An evaluation of completed clinical trials registered in clinicaltrials.gov showed that 62 percent (24 out of 39) studies were not published.

\section{Applicability}

Most studies were conducted in hospitalized patients with moderate to severe ECOPD, and the results of these studies may not be applicable to patients with milder forms of ECOPD treated in an outpatient setting. KQ1 and KQ2 were stratified by severity of ECOPD, which allows determination of the generalizability of the results based on the severity of ECOPD. For KQ2, almost all studies were conducted in hospitalized patients. As we excluded studies conducted in an ICU setting, some of our findings may not be extrapolated to the most severely sick patients who require ICU admission for ECOPD.

The results of comparisons of different antibiotic agents/classes are context-specific, as the optimal antibiotic choice depends on local antimicrobial resistance patterns, which can change over time. The results of these comparisons (KQ4) are therefore not necessarily applicable to patients in different geographic locations and at different points in time.

COPD terminology has not been used consistently in the past with some older studies referring to chronic bronchitis without airflow obstruction as COPD. We excluded studies with patients who had chronic bronchitis but no evidence of chronic airflow obstruction to increase applicability of the results to patients with chronic airflow obstruction. 
Not all studies explicitly excluded patients with potential asthma or asthma-COPD overlap syndrome (ACOS), and there is therefore a potential for misclassification.

Pulmonary rehabilitation is a complex (multi-component) intervention, which consists of exercise training, patient education, and behavior change. The detailed interventions for pulmonary rehabilitation were reported in the included studies, which should facilitate reproducibility and applicability. While there are published standards for pulmonary rehabilitation programs, ${ }^{47}$ these have been developed in the context of pulmonary rehabilitation in patients with stable COPD (as opposed to patients with ECOPD).

\section{Suggestions for Future Research}

Lung function (FEV1) was the most commonly assessed outcome in studies of interventions to manage ECOPD, while final health outcomes, such as resolution of ECOPD (clinical cure, clinical failure), and repeat exacerbation (with or without hospital admission) were rarely assessed. Future studies in ECOPD should focus on final health outcomes and include clinical resolution of ECOPD and risk of repeat exacerbation in addition to other final health outcomes such as dyspnea and quality of life.

The response to antibiotic therapy as well as corticosteroid therapy in ECOPD likely differs based on the phenotype of the exacerbation episode. A number of studies that used procalcitonin-guided treatment algorithms have been conducted on antibiotic therapy versus placebo in ECOPD, ${ }^{48}$ but identification of responders to systemic corticosteroid treatment of ECOPD based on blood eosinophils remains unexplored. This contrasts with the increasing recognition of eosinophilic phenotypes in stable COPD which appear to be more likely to benefit from longterm inhaled corticosteroids. ${ }^{44}$ Future studies on systemic corticosteroids in ECOPD should assess the treatment effect stratified by blood eosinophil count.

Chest physiotherapy using breathing technique and/or vibration/percussions and/or positive expiratory pressure (PEP) is commonly prescribed in patients hospitalized for ECOPD, but there was insufficient evidence that these interventions improve outcomes. As these are resourceintensive interventions, large well-designed trials with final health outcomes including clinical resolution of ECOPD and repeat exacerbations should be conducted to assess the role of chest physiotherapy for airway clearance in ECOPD and inform clinical practice.

It is currently unclear whether pulmonary rehabilitation commenced during hospitalization for ECOPD is associated with increased mortality. An increased mortality was found in the review conducted for the guidelines on management of COPD exacerbations by the European Respiratory Society and the American Thoracic Society but was not found in our systematic review. Given the potential benefit of pulmonary rehabilitation to counteract the deconditioning associated with ECOPD, we believe that conducting high-quality RCTs to answer this question should be a priority.

The relatively new treatment options of whole body vibration and transcutaneous electrical nerve stimulation (TENS) and dietary interventions with caloric supplements and vitamin D need to be assessed in large high quality RCTs to inform recommendations about these treatments. Such literature (e.g., on vitamin D) is notorious for contradictory findings over time.

Further research is required to determine the optimal route of administration for systemic corticosteroids, i.e. to determine whether oral corticosteroids are generally not inferior to intravenous corticosteroids and to determine a potential role of inhaled corticosteroids (possibly as alternative to systemic corticosteroids) in ECOPD. 
Patients hospitalized with COPD exacerbations are at high risk for hospital readmissions and death after hospital discharge, which emphasizes the importance of improving the hospital-tohome continuum of care. Our systematic review only focused on the acute episode of an exacerbation and did not include health service interventions, but there is an urgent need for research that assesses interventions to reduce the risk of adverse outcomes following hospital discharge. Much of the recent debate on adverse outcomes following hospital discharge has focused on reducing 30-day hospital readmissions in ECOPD, as the Medicare's Hospital Readmissions Reduction Program (HRRP) lowered payments to Inpatient Prospective Payment System hospitals with too many readmissions within 30 days. Recent evidence, however, showed that implementation of the HRRP was associated with a significant increase in trends in 30-day post-discharge mortality among patients hospitalized for heart failure and pneumonia. ${ }^{49}$ It is therefore evident that future research that aims to improve outcomes post-hospital discharge for any disease with frequent hospital readmissions including ECOPD should not focus on reducing 30-day hospital admissions in isolation but only in conjunction with final health outcomes such as QoL and mortality.

\section{Conclusion}

Despite a proliferation of the COPD literature, the evidence base for most interventions in ECOPD remains limited. Systemic antibiotics and corticosteroids are associated with improved outcomes in mild and moderate to severe ECOPD. Titrated oxygen reduces mortality. Future research is required to assess the effectiveness of several emerging nonpharmacological and dietary treatments. 


\section{References}

1. Adeloye D, Chua S, Lee C, et al. Global and regional estimates of COPD prevalence: Systematic review and meta-analysis. J Glob Health. 2015 Dec;5(2):020415. doi: 10.7189/jogh.05-020415. PMID: 26755942.

2. Murphy SL, Xu J, Kochanek KD, et al. Mortality in the United States, 2017. NCHS Data Brief. 2018 Nov(328):1-8. PMID: 30500322.

3. Kochanek KD, Murphy SL, Xu J, et al. Deaths: Final Data for 2014. Natl Vital Stat Rep. 2016 Jun;65(4):1-122. PMID: 27378572.

4. Ford ES, Murphy LB, Khavjou O, et al. Total and state-specific medical and absenteeism costs of COPD among adults aged $>/=18$ years in the United States for 2010 and projections through 2020. Chest. 2015 Jan;147(1):31-45. doi:

10.1378/chest.14-0972. PMID: 25058738.

5. Disease GIfCOL. Global Strategy for the Diagnosis, Management, and Prevention of Chronic Obstructive Lung Disease. 2019.

6. Anthonisen NR, Manfreda J, Warren CP, et al. Antibiotic therapy in exacerbations of chronic obstructive pulmonary disease. Annals of Internal Medicine. 1987 Feb;106(2):196-204. PMID: 3492164.

7. Connors AF, Jr., Dawson NV, Thomas C, et al. Outcomes following acute exacerbation of severe chronic obstructive lung disease. The SUPPORT investigators (Study to Understand Prognoses and Preferences for Outcomes and Risks of Treatments). Am J Respir Crit Care Med. 1996 Oct;154(4 Pt 1):959-67. doi:

10.1164/ajrccm.154.4.8887592. PMID: 8887592.

8. Seemungal TA, Donaldson GC, Paul EA, et al. Effect of exacerbation on quality of life in patients with chronic obstructive pulmonary disease. Am J Respir Crit Care Med. 1998 May;157(5 Pt 1):1418-22. doi: 10.1164/ajrccm.157.5.9709032. PMID: 9603117.
9.

Miravitlles M, Murio C, Guerrero T, et al. Pharmacoeconomic evaluation of acute exacerbations of chronic bronchitis and COPD. Chest. 2002 May;121(5):1449-55. PMID: 12006427.

10. Miravitlles M, Garcia-Polo C, Domenech A, et al. Clinical outcomes and cost analysis of exacerbations in chronic obstructive pulmonary disease. Lung. 2013 Oct;191(5):523-30. doi: 10.1007/s00408013-9487-z. PMID: 23842641.

11. Seemungal TA, Donaldson GC, Bhowmik A, et al. Time course and recovery of exacerbations in patients with chronic obstructive pulmonary disease. Am J Respir Crit Care Med. 2000 May;161(5):1608-13. doi: 10.1164/ajrccm.161.5.9908022. PMID: 10806163.

12. Donaldson GC, Seemungal TA, Bhowmik A, et al. Relationship between exacerbation frequency and lung function decline in chronic obstructive pulmonary disease. Thorax. 2002 Oct;57(10):847-52. PMID: 12324669.

13. Doll H, Miravitlles M. Health-related QOL in acute exacerbations of chronic bronchitis and chronic obstructive pulmonary disease: a review of the literature. Pharmacoeconomics. 2005;23(4):345-63. PMID: 15853435.

14. Patil SP, Krishnan JA, Lechtzin N, et al. Inhospital mortality following acute exacerbations of chronic obstructive pulmonary disease. Arch Intern Med. 2003 May 26;163(10):1180-6. doi: 10.1001/archinte.163.10.1180. PMID: 12767954 .

15. Wouters EF. Economic analysis of the Confronting COPD survey: an overview of results. Respir Med. 2003 Mar;97 Suppl C:S3-14. PMID: 12647938

16. Aaron SD. Management and prevention of exacerbations of COPD. Bmj. 2014 Sep 22;349:g5237. doi: 10.1136/bmj.g5237. PMID: 25245156. 
17. Welte T. Acute exacerbation in COPD: we must do more. Eur Respir J. 2016

Jan;47(1):14-5. doi:

10.1183/13993003.01981-2015. PMID: 26721956.

18. Mackay AJ, Hurst JR. COPD exacerbations: causes, prevention, and treatment. Immunol Allergy Clin North Am. 2013 Feb;33(1):95115. doi: 10.1016/j.iac.2012.10.006. PMID: 23337067.

19. Calverley P. Current drug treatment, chronic and acute. Clin Chest Med. 2014

Mar;35(1):177-89. doi:

10.1016/j.ccm.2013.09.009. PMID: 24507845.

20. Cabrini L, Landoni G, Oriani A, et al. Noninvasive ventilation and survival in acute care settings: a comprehensive systematic review and metaanalysis of randomized controlled trials. Crit Care Med. 2015 Apr;43(4):880-8. doi: 10.1097/CCM.0000000000000819. PMID: 25565461.

21. Barjaktarevic IZ, Arredondo AF, Cooper CB. Positioning new pharmacotherapies for COPD. Int J Chron Obstruct Pulmon Dis. 2015;10:1427-42. doi: 10.2147/COPD.S83758. PMID: 26244017.

22. Poole PJ. Role of mucolytics in the management of COPD. Int J Chron Obstruct Pulmon Dis. 2006;1(2):123-8. PMID: 18046889.

23. Poole P, Black PN, Cates CJ. Mucolytic agents for chronic bronchitis or chronic obstructive pulmonary disease. Cochrane Database of Systematic Reviews. 2012 Aug 15(8):CD001287. doi: https://dx.doi.org/10.1002/14651858.CD001 287.pub4. PMID: 22895919.

24. Wijesinghe M, Perrin K, Healy B, et al. Prehospital oxygen therapy in acute exacerbations of chronic obstructive pulmonary disease. Intern Med J. 2011 Aug;41(8):618-22. doi: 10.1111/j.14455994.2010.02207.x. PMID: 20214690.
25. Austin MA, Wills KE, Blizzard L, et al. Effect of high flow oxygen on mortality in chronic obstructive pulmonary disease patients in prehospital setting: randomised controlled trial. BMJ. 2010 Oct 18;341:c5462. PMID: 20959284.

26. O'Driscoll BR, Howard LS, Earis J, et al. British Thoracic Society Guideline for oxygen use in adults in healthcare and emergency settings. BMJ Open Respir Res. 2017;4(1):e000170. doi: 10.1136/bmjresp2016-000170. PMID: 28883921.

27. Holland AE. Physiotherapy management of acute exacerbations of chronic obstructive pulmonary disease. J Physiother. 2014 Dec;60(4):181-8. doi: 10.1016/j.jphys.2014.08.018. PMID: 25450482

28. Spencer L. Pulmonary rehabilitation for patients with acute chronic obstructive pulmonary disease exacerbations: is the evidence strengthening? Curr Opin Pulm Med. 2018 Mar;24(2):147-51. doi: 10.1097/mcp.0000000000000453. PMID: 29210749.

29. Osadnik CR, McDonald CF, Jones AP, et al. Airway clearance techniques for chronic obstructive pulmonary disease. Cochrane Database Syst Rev. 2012 Mar 14(3):CD008328. doi: 10.1002/14651858.CD008328.pub2. PMID: 22419331.

30. Creutzberg EC, Schols AM, BothmerQuaedvlieg FC, et al. Prevalence of an elevated resting energy expenditure in patients with chronic obstructive pulmonary disease in relation to body composition and lung function. Eur J Clin Nutr. 1998 Jun;52(6):396-401. PMID: 9683390.

31. Law S, Kumar P, Woods S, et al. Malnutrition screening in patients admitted to hospital with an exacerbation of chronic obstructive pulmonary disease and its association with patient outcomes. Hosp Pract (1995). 2016 Oct;44(4):207-12. doi: 10.1080/21548331.2016.1224007. PMID: 27560297. 
32. Calder PC, Laviano A, Lonnqvist F, et al. Targeted medical nutrition for cachexia in chronic obstructive pulmonary disease: a randomized, controlled trial. J Cachexia Sarcopenia Muscle. 2018 Feb;9(1):28-40. doi: 10.1002/jcsm.12228. PMID: 28891198.

33. Manalan K, Rashid T, Singanayagam A. Antibiotic treatment in exacerbations of chronic obstructive pulmonary disease: Recent trial results. Clinical Investigation. 201501 Feb;5(2):189-204. doi: http://dx.doi.org/10.4155/cli.14.113. PMID: 602447476.

34. Laue J, Reierth E, Melbye H. When should acute exacerbations of COPD be treated with systemic corticosteroids and antibiotics in primary care: a systematic review of current COPD guidelines. NPJ Prim Care Respir Med. 2015 Feb 19;25:15002. doi: 10.1038/npjpcrm.2015.2. PMID: 25695630.

35. Baker EH, Bell D. Blood glucose: of emerging importance in COPD

exacerbations. Thorax. 2009

Oct;64(10):830-2. doi:

10.1136/thx.2009.118638. PMID: 19786710.

36. Qureshi H, Sharafkhaneh A, Hanania NA. Chronic obstructive pulmonary disease exacerbations: latest evidence and clinical implications. Ther Adv Chronic Dis. 2014 Sep;5(5):212-27. doi: 10.1177/2040622314532862. PMID: 25177479 .

37. Bourbeau J, Sedeno MF, Metz K, et al. Early COPD Exacerbation Treatment with Combination of ICS and LABA for Patients Presenting with Mild-to-Moderate Worsening of Dyspnea. Copd. 2016 Aug;13(4):439-47. doi: 10.3109/15412555.2015.1101435. PMID: 26752024 .

38. Woods JA, Wheeler JS, Finch CK, et al. Corticosteroids in the treatment of acute exacerbations of chronic obstructive pulmonary disease. Int J Chron Obstruct Pulmon Dis. 2014;9:421-30. doi: 10.2147/copd.s51012. PMID: 24833897.
39. Bonilla Arcos D, Krishnan JA, Vandivier RW, et al. High-Dose Versus Low-Dose Systemic Steroids in the Treatment of Acute Exacerbations of Chronic Obstructive Pulmonary Disease: Systematic Review. Chronic Obstructive Pulmonary Diseases. 2016 Feb 17;3(2):580-8. doi: https://dx.doi.org/10.15326/jcopdf.3.2.2015. 0178. PMID: 28848882.

40. Walters JA, Tan DJ, White CJ, et al. Different durations of corticosteroid therapy for exacerbations of chronic obstructive pulmonary disease. Cochrane Database Syst Rev. 2018 Mar 19;3:Cd006897. doi: 10.1002/14651858.CD006897.pub4. PMID: 29553157.

41. Zhang HL, Tan M, Qiu AM, et al. Antibiotics for treatment of acute exacerbation of chronic obstructive pulmonary disease: a network meta-analysis. BMC Pulm Med. 2017 Dec 12;17(1):196. doi: 10.1186/s12890-017-0541-0. PMID: 29233130.

42. Methods Guide for Effectiveness and Comparative Effectiveness Reviews. AHRQ Publication No. 10(14)-EHC063-EF. Rockville, MD: Agency for Healthcare Research and Quality; January 2014. Chapters available at: www.effectivehealthcare.ahrq.gov.

43. Moher D, Liberati A, Tetzlaff J, et al. Preferred reporting items for systematic reviews and meta-analyses: the PRISMA statement. PLoS Med. 2009 Jul 21;6(7):e1000097. doi: 10.1371/journal.pmed.1000097. PMID: 19621072.

44. Barnes NC, Sharma R, Lettis S, et al. Blood eosinophils as a marker of response to inhaled corticosteroids in COPD. Eur Respir J. 2016 May;47(5):1374-82. doi: 10.1183/13993003.01370-2015. PMID: 26917606.

45. Wedzicha JA, Miravitlles M, Hurst JR, et al. Management of COPD exacerbations: A European respiratory society/American thoracic society guideline. European Respiratory Journal. 2017;49(3):1600791. 
46. Greening NJ, Williams JEA, Hussain SF, et al. An early rehabilitation intervention to enhance recovery during hospital admission for an exacerbation of chronic respiratory disease: randomised controlled trial. BMJ : British Medical Journal. 2014;349:g4315. doi: 10.1136/bmj.g4315.

47. Spruit MA, Singh SJ, Garvey C, et al. An official American Thoracic Society/European Respiratory Society statement: key concepts and advances in pulmonary rehabilitation. Am J Respir Crit Care Med. 2013 Oct 15;188(8):e13-64. doi: 10.1164/rccm.201309-1634ST. PMID: 24127811.

48. Mathioudakis AG, ChatzimavridouGrigoriadou V, Corlateanu A, et al. Procalcitonin to guide antibiotic administration in COPD exacerbations: a meta-analysis. Eur Respir Rev. 2017 Jan;26(143). doi: 10.1183/16000617.00732016. PMID: 28143877.

49. Wadhera RK, Joynt Maddox KE, Wasfy JH, et al. Association of the Hospital Readmissions Reduction Program With Mortality Among Medicare Beneficiaries Hospitalized for Heart Failure, Acute Myocardial Infarction, and Pneumonia. JAMA. 2018 Dec 25;320(24):2542-52. doi: 10.1001/jama.2018.19232. PMID: 30575880 .

50. Niewoehner DE, Collins D, Erbland ML. Relation of FEV(1) to clinical outcomes during exacerbations of chronic obstructive pulmonary disease. Department of Veterans Affairs Cooperative Study Group. Am J Respir Crit Care Med. 2000 Apr;161(4 Pt 1):1201-5. doi:

10.1164/ajrccm.161.4.9907143. PMID: 10764312.

51. Higgins JPT, Green S, eds. Cochrane handbook for systematic reviews of interventions Version 5.1.0 [updated March 2011]: The Cochrane Collaboration; 2011.

52. Li T, Yu T, Hawkins BS, et al. Design, analysis, and reporting of crossover trials for inclusion in a meta-analysis. PLoS One. 2015;10(8):e0133023.

53. Services CfMM. Hospital Readmissions Reduction Program (HRRP). 2018.
54. DerSimonian R, Laird N. Meta-analysis in clinical trials. Controlled clinical trials. 1986 Sep;7(3):177-88. PMID: 3802833.

55. Sidik K, Jonkman JN. A simple confidence interval for meta-analysis. Stat Med. 2002 Nov 15;21(21):3153-9. doi: 10.1002/sim.1262. PMID: 12375296.

56. Hartung J, Knapp G. On tests of the overall treatment effect in meta-analysis with normally distributed responses. Stat Med. 2001 Jun 30;20(12):1771-82. doi: 10.1002/sim.791. PMID: 11406840.

57. Hartung J, Knapp G. A refined method for the meta-analysis of controlled clinical trials with binary outcome. Stat Med. 2001 Dec 30;20(24):3875-89. PMID: 11782040.

58. Hardy RJ, Thompson SG. A likelihood approach to meta-analysis with random effects. Stat Med. 1996 Mar 30;15(6):61929. doi: 10.1002/(sici)10970258(19960330)15:6<619::aidsim188>3.0.co;2-a. PMID: 8731004.

59. Morton SC MM, O'Connor E, Lee CS, Booth M, Vandermeer BW, Snowden JM, D'Anci KE, Fu R, Gartlehner G, Wang Z, Steele DW. . Quantitative Synthesis-An Update. Methods Guide for Comparative Effectiveness Reviews. (Prepared by the Scientific Resource Center under Contract No. 290-2012-0004-C). Agency for Healthcare Research and Quality. 2018;AHRQ Publication No. 18-EHC007EF.

60. Wiksten A, Rücker G, Schwarzer G. Hartung-Knapp method is not always conservative compared with fixed-effect meta-analysis. Statistics in medicine. 2016;35(15):2503-15.

61. Copas J. A simple confidence interval for meta-analysis. K. Sidik and JN Jonkman, Statistics in Medicine 2002; 21: 3153-3159. Statistics in medicine. 2003;22(16):2667-8.

62. Jackson D, Law M, Rücker G, et al. The Hartung-Knapp modification for randomeffects meta-analysis: A useful refinement but are there any residual concerns? Statistics in medicine. 2017;36(25):3923-34. 
63. Guyatt G, Rennie D, Meade M, et al. Users' guides to the medical literature. A manual for evidence-based clinical practice. Third edition. ed. New York: McGraw-Hill Education Medical; 2015.

64. Ioannidis JP, Trikalinos TA. The appropriateness of asymmetry tests for publication bias in meta-analyses: a large survey. Canadian Medical Association Journal. 2007;176(8):1091-6.

65. Abreu Gonzalez J, Hernandez Garcia C, Abreu Gonzalez P, et al. [Effect of intravenous magnesium sulfate on chronic obstructive pulmonary disease exacerbations requiring hospitalization: a randomized placebo-controlled trial]. Archivos de Bronconeumologia. 2006 Aug;42(8):384-7. PMID: 16948990.

66. Centanni S, Santus P, Casanova F, et al. Bronchodilating effect of oxitropium bromide in heart disease patients with exacerbations of COPD: double-blind, randomized, controlled study. Respiratory Medicine. 2002 Mar;96(3):137-41. PMID: 11905547.

67. Brown PM, Manfreda J, McCarthy DS, et al. The effect of mechanical vibration in patients with acute exacerbations of chronic obstructive pulmonary disease. Physiother Can. 1987;39(6):371-4.

68. van Velzen P, ter Riet G, Bresser P, et al. Doxycycline for outpatient-treated acute exacerbations of COPD: a randomised double-blind placebo-controlled trial. The lancet respiratory medicine. 2017(pagination). PMID: CN-01374427.

69. Wang J-X, Zhang S-M, Li X-H, et al. Acute exacerbations of chronic obstructive pulmonary disease with low serum procalcitonin values do not benefit from antibiotic treatment: a prospective randomized controlled trial. International Journal of Infectious Diseases. 2016 Jul;48:40-5. PMID: 27155210.

70. Hassan WA, Shalan I, Elsobhy M. Impact of antibiotics on acute exacerbations of COPD. Egyptian Journal of Chest Diseases and Tuberculosis. 201501 Jul;64(3):579-85. PMID: 603624004.
71. Brusse-Keizer M, VanderValk P, Hendrix $\mathrm{R}$, et al. Necessity of amoxicillin clavulanic acid in addition to prednisolone in mild-tomoderate COPD exacerbations. BMJ open respiratory research. 2014;1(1):e000052. PMID: 25562034.

72. Llor C, Moragas A, Hernandez S, et al. Efficacy of antibiotic therapy for acute exacerbations of mild to moderate chronic obstructive pulmonary disease. American Journal of Respiratory \& Critical Care Medicine. 2012 Oct 15;186(8):716-23. PMID: 22923662.

73. Daniels JMA, Snijders D, e Graaff CS, et al. Antibiotics in addition to systemic corticosteroids for acute exacerbations of chronic obstructive pulmonary disease. American Journal of Respiratory \& Critical Care Medicine. 2010 Jan 15;181(2):150-7. PMID: 19875685.

74. Bathoorn E, Liesker JJW, Postma DS, et al. Anti-inflammatory effects of combined budesonide/formoterol in COPD exacerbations. Copd: Journal of Chronic Obstructive Pulmonary Disease. 2008 Oct;5(5):282-90. PMID: 18972276.

75. Gunen H, Hacievliyagil SS, Yetkin O, et al. The role of nebulised budesonide in the treatment of exacerbations of COPD.[Erratum appears in Eur Respir J. 2014 Jul;44(1):272]. European Respiratory Journal. 2007 Apr;29(4):660-7. PMID: 17251232.

76. Maltais F, Ostinelli J, Bourbeau J, et al. Comparison of nebulized budesonide and oral prednisolone with placebo in the treatment of acute exacerbations of chronic obstructive pulmonary disease: a randomized controlled trial. American Journal of Respiratory \& Critical Care Medicine. 2002 Mar 01;165(5):698-703. PMID: 11874817.

77. Niewoehner DE, Erbland ML, Deupree RH, et al. Effect of systemic glucocorticoids on exacerbations of chronic obstructive pulmonary disease. Department of Veterans Affairs Cooperative Study Group. New England Journal of Medicine. 1999 Jun 24;340(25):1941-7. PMID: 10379017. 
78. Davies L, Angus RM, Calverley PM. Oral corticosteroids in patients admitted to hospital with exacerbations of chronic obstructive pulmonary disease: a prospective randomised controlled trial. Lancet. 1999 Aug 07;354(9177):456-60. PMID: 10465169.

79. Thompson WH, Nielson CP, Carvalho P, et al. Controlled trial of oral prednisone in outpatients with acute COPD exacerbation. American Journal of Respiratory \& Critical Care Medicine. 1996 Aug;154(2 Pt 1):40712. PMID: 8756814.

80. Emerman CL, Connors AF, Lukens TW, et al. A randomized controlled trial of methylprednisolone in the emergency treatment of acute exacerbations of COPD. Chest. 1989 Mar;95(3):563-7. PMID: 2920584.

81. Albert RK, Martin TR, Lewis SW. Controlled clinical trial of methylprednisolone in patients with chronic bronchitis and acute respiratory insufficiency. Annals of Internal Medicine. 1980 Jun;92(6):753-8. PMID: 6770731.

82. Aaron SD, Vandemheen KL, Hebert P, et al. Outpatient oral prednisone after emergency treatment of chronic obstructive pulmonary disease. N Engl J Med. 2003 Jun 26;348(26):2618-25. doi: 10.1056/NEJMoa023161. PMID: 12826636.

83. Cox M, Connor C, Biggs K, et al. The feasibility of early pulmonary rehabilitation and activity after COPD exacerbations: External pilot randomised controlled trial, qualitative case study and exploratory economic evaluation. Health Technology Assessment. 2018;22(11).

84. Torres-Sánchez I, Valenza MC, Cebriá i Iranzo MDÀ, et al. Effects of different physical therapy programs on perceived health status in acute exacerbation of chronic obstructive pulmonary disease patients: a randomized clinical trial. Disability and Rehabilitation. 2017:1-7.
85. Pourrashid MH, Dastan F, Salamzadeh J, et al. Role of vitamin D replacement on health related quality of life in hospitalized patients with "acute exacerbation of chronic obstructive pulmonary disease”. Iranian Journal of Pharmaceutical Research. 2018;17(2):801-10.

86. Torres-Sanchez I, Valenza MC, CabreraMartos I, et al. Effects of an Exercise Intervention in Frail Older Patients with Chronic Obstructive Pulmonary Disease Hospitalized due to an Exacerbation: A Randomized Controlled Trial. Copd: Journal of Chronic Obstructive Pulmonary Disease. 2017 Feb;14(1):37-42. PMID: 27715322.

87. Oncu E, Zincir H. The effect of transcutaneous electrical nerve stimulation in patients with acute exacerbation of chronic obstructive pulmonary disease: randomised controlled trial. Journal of Clinical Nursing. 2017 Jul;26(13-14):183444. PMID: 27325551.

88. Basri R, Tahir M, Naseem M. Short-term effects of chest physiotherapy in acute exacerbation of chronic obstructive pulmonary disease. Journal of Medical Sciences (Peshawar). 2017 July;25(3):323-7. PMID: 620855828.

89. Soltaninejad F, Kheiri S, Habibian R, et al. Evaluation effects of nebulized gentamicin in exacerbation of chronic obstructive lung disease. Journal of Research in Medical Sciences. 2016;21 (4) (no pagination)(56). PMID: 612021136.

90. Lellouche F, Bouchard PA, Roberge M, et al. Automated oxygen titration and weaning with FreeO $<$ inf $>2<$ inf $>$ in patients with acute exacerbation of COPD: A pilot randomized trial. International Journal of COPD. 201624 Aug;11(1):1983-90. PMID: 611880937.

91. Sanjari M, Soltani A, Habibi Khorasani A, et al. The effect of vitamin D on COPD exacerbation: a double blind randomized placebo-controlled parallel clinical trial. Journal of Diabetes \& Matabolic Disorders. 2015;15(1):33. PMID: 27570748. 
92. Mukerji S, Shahpuri B, Clayton-Smith B, et al. Intravenous magnesium sulphate as an adjuvant therapy in acute exacerbations of chronic obstructive pulmonary disease: a single centre, randomised, double-blinded, parallel group, placebo-controlled trial: a pilot study. New Zealand Medical Journal. 2015 Nov 20;128(1425):34-42. PMID: 26905985.

93. Moretti M, Fagnani S. Erdosteine reduces inflammation and time to first exacerbation postdischarge in hospitalized patients with AECOPD. International Journal of Copd. 2015;10:2319-25. PMID: 26604731.

94. Liao L-Y, Chen K-M, Chung W-S, et al. Efficacy of a respiratory rehabilitation exercise training package in hospitalized elderly patients with acute exacerbation of COPD: a randomized control trial. International Journal of Copd. 2015;10:1703-9. PMID: 26345529.

95. He M, Yu S, Wang L, et al. Efficiency and safety of pulmonary rehabilitation in acute exacerbation of chronic obstructive pulmonary disease. Medical Science Monitor. 2015 Mar 18;21:806-12. PMID: 25783889.

96. Ayfer Aytemur Z, Baysak A, Ozdemir O, et al. N-acetylcysteine in patients with COPD exacerbations associated with increased sputum. Wiener Klinische Wochenschrift. 2015 Apr;127(7-8):256-61. PMID: 25595117.

97. Osadnik CR, McDonald CF, Miller BR, et al. The effect of positive expiratory pressure (PEP) therapy on symptoms, quality of life and incidence of re-exacerbation in patients with acute exacerbations of chronic obstructive pulmonary disease: a multicentre, randomised controlled trial. Thorax. 2014 Feb;69(2):137-43. PMID: 24005444.

98. Solooki M, Miri M, Mokhtari M, et al. Magnesium sulfate in exacerbations of COPD in patients admitted to internal medicine ward. Iranian Journal of Pharmaceutical Research. 201401 Sep;13(4):1235-9. PMID: 600071239.
99. Greulich T, Nell C, Koepke J, et al. Benefits of whole body vibration training in patients hospitalised for COPD exacerbations - a randomized clinical trial. BMC Pulmonary Medicine. 2014 Apr 11;14:60. PMID: 24725369.

100. Borges RC, Carvalho CR. Impact of resistance training in chronic obstructive pulmonary disease patients during periods of acute exacerbation. Archives of Physical Medicine \& Rehabilitation. 2014 Sep;95(9):1638-45. PMID: 24879965.

101. Mahmoud Abd El Hafiz A, Mohammed El Wakeel L, Mohammed El Hady H, et al. High dose $\mathrm{N}$-acetyl cysteine improves inflammatory response and outcome in patients with COPD exacerbations. Egyptian Journal of Chest Diseases and Tuberculosis. 2013 January;62(1):51-7. PMID: 369315238.

102. Kurzaj M, Wierzejski W, Dor A, et al. The impact of specialized physiotherapy methods on BODE index in COPD patients during hospitalization. Advances in Clinical \& Experimental Medicine. 2013 SepOct;22(5):721-30. PMID: 24285458.

103. Goktalay T, Akdemir SE, Alpaydin AO, et al. Does high-frequency chest wall oscillation therapy have any impact on the infective exacerbations of chronic obstructive pulmonary disease? A randomized controlled single-blind study. Clinical Rehabilitation. 2013 Aug;27(8):710-8. PMID: 23503735.

104. Edwards L, Shirtcliffe P, Wadsworth K, et al. Use of nebulised magnesium sulphate as an adjuvant in the treatment of acute exacerbations of COPD in adults: a randomised double-blind placebo-controlled trial. Thorax. 2013 Apr;68(4):338-43. PMID: 23299960.

105. Tang CY, Blackstock FC, Clarence M, et al. Early rehabilitation exercise program for inpatients during an acute exacerbation of chronic obstructive pulmonary disease: a randomized controlled trial. Journal of Cardiopulmonary Rehabilitation \& Prevention. 2012 May-Jun;32(3):163-9. PMID: 22561417. 
106. Cross JL, Elender F, Barton G, et al. Evaluation of the effectiveness of manual chest physiotherapy techniques on quality of life at six months post exacerbation of COPD (MATREX): a randomised controlled equivalence trial. BMC Pulmonary Medicine. 2012 Jul 02;12:33. PMID: 22748085.

107. Woodruff PG, Albert RK, Bailey WC, et al. Randomized trial of zileuton for treatment of COPD exacerbations requiring hospitalization. Copd: Journal of Chronic Obstructive Pulmonary Disease. 2011 Feb;8(1):21-9. PMID: 21299475.

108. Troosters T, Probst VS, Crul T, et al. Resistance training prevents deterioration in quadriceps muscle function during acute exacerbations of chronic obstructive pulmonary disease. American Journal of Respiratory \& Critical Care Medicine. 2010 May 15;181(10):1072-7. PMID: 20133927.

109. Tumer G, Mercanligil SM, Uzun O, et al. The effects of a high-fat, low-carbohydrate diet on the prognosis of patients with an acute attack of chronic obstructive pulmonary disease. Turkiye Klinikleri Journal of Medical Sciences. 2009;29(4):895-904. PMID: 358101090.

110. Kodric M, Garuti G, Colomban M, et al. The effectiveness of a bronchial drainage technique (ELTGOL) in COPD exacerbations. Respirology. 2009 Apr;14(3):424-8. PMID: 19192230.

111. Eaton T, Young P, Fergusson W, et al. Does early pulmonary rehabilitation reduce acute health-care utilization in COPD patients admitted with an exacerbation? A randomized controlled study. Respirology. 2009 Mar;14(2):230-8. PMID: 19272084.

112. Xiong G, Xu L, Wei L, et al. Atomization inhalation of terbutaline and budesonide efficiently improved immunity and lung function of AECOPD patients. Cellular \& Molecular Immunology. 2008 Aug;5(4):287-91. PMID: 18761816.

113. Zuin R, Palamidese A, Negrin R, et al. High-dose $\mathrm{N}$-acetylcysteine in patients with exacerbations of chronic obstructive pulmonary disease. Clinical Drug Investigation. 2005;25(6):401-8. PMID: 41019987.
114. Duffy N, Walker P, Diamantea F, et al. Intravenous aminophylline in patients admitted to hospital with non-acidotic exacerbations of chronic obstructive pulmonary disease: a prospective randomised controlled trial. Thorax. 2005 Sep;60(9):713-7. PMID: 15939732.

115. Vermeeren MAP, Wouters EFM, GeraertsKeeris AJW, et al. Nutritional support in patients with chronic obstructive pulmonary disease during hospitalization for an acute exacerbation; a randomized controlled feasibility trial. Clinical Nutrition. 2004 Oct;23(5):1184-92. PMID: 15380912.

116. Black PN, Morgan-Day A, McMillan TE, et al. Randomised, controlled trial of $\mathrm{N}$ acetylcysteine for treatment of acute exacerbations of chronic obstructive pulmonary disease [ISRCTN21676344]. BMC Pulmonary Medicine. 2004 Dec 06;4:13. PMID: 15581425.

117. Yohannes AM, Connolly MJ. Early mobilization with walking aids following hospital admission with acute exacerbation of chronic obstructive pulmonary disease. Clinical Rehabilitation. 2003

Aug;17(5):465-71. PMID: 12952150.

118. Saudny-Unterberger H, Martin JG, GrayDonald K. Impact of nutritional support on functional status during an acute exacerbation of chronic obstructive pulmonary disease. American Journal of Respiratory \& Critical Care Medicine. 1997 Sep;156(3 Pt 1):794-9. PMID: 9309995.

119. Skorodin MS, Tenholder MF, Yetter B, et al. Magnesium sulfate in exacerbations of chronic obstructive pulmonary disease. Archives of Internal Medicine. 1995 Mar 13;155(5):496-500. PMID: 7864705.

120. Rice KL, Leatherman JW, Duane PG, et al. Aminophylline for acute exacerbations of chronic obstructive pulmonary disease. A controlled trial. Annals of Internal Medicine. 1987 Sep;107(3):305-9. PMID: 3619219.

121. Seidenfeld JJ, Jones WN, Moss RE, et al. Intravenous aminophylline in the treatment of acute bronchospastic exacerbations of chronic obstructive pulmonary disease. Annals of Emergency Medicine. 1984 Apr;13(4):248-52. PMID: 6367554. 
122. Du F, Liu D, He G, et al. Effect of simvastatin on serum gammaglutamyltransferase and C-reactive protein in patient with acute exacerbation chronic obstructive pulmonary disease. Acta Medica Mediterranea. 2018;34(5):1221-6. PMID: 623957543.

123. Ogasawara T, Marui S, Miura E, et al. Effect of eicosapentaenoic acid on prevention of lean body mass depletion in patients with exacerbation of chronic obstructive pulmonary disease: A prospective randomized controlled trial. Clinical Nutrition ESPEN. 2018 Dec;28:67-73. PMID: 30390895.

124. Behnke M, Taube C, Kirsten D, et al. Homebased exercise is capable of preserving hospital-based improvements in severe chronic obstructive pulmonary disease. Respir Med. 2000 Dec;94(12):1184-91. doi: 10.1053/rmed.2000.0949. PMID: 11192954.

125. Kirsten DK, Taube C, Lehnigk B, et al. Exercise training improves recovery in patients with COPD after an acute exacerbation. Respir Med. 1998 Oct;92(10):1191-8. PMID: 9926148.

126. Koutsogiannis Z, Kelly AM. Does high dose ipratropium bromide added to salbutamol improve pulmonary function for patients with chronic obstructive airways disease in the emergency department? Australian \& New Zealand Journal of Medicine. 2000 Feb;30(1):38-40. PMID: 10800876.

127. Moayyedi P, Congleton J, Page RL, et al. Comparison of nebulised salbutamol and ipratropium bromide with salbutamol alone in the treatment of chronic obstructive pulmonary disease. Thorax. 1995 Aug;50(8):834-7. PMID: 7570433.

128. Perri G, Giovannini M, Spada E. Salbutamol plus beclomethasone dipropionate (Ventolin Flogo) vs. fenoterol (Dosberotec) in chronic obstructive lung disease therapeutic strategy: a 4-week clinical trial. International Journal of Clinical Pharmacology, Therapy, \& Toxicology. 1985 May;23(5):274-8. PMID: 3891642.
129. Ardestani ME, Kalantary E, Samaiy V, et al. Methyl prednisolone vs dexamethasone in management of COPD exacerbation; a randomized clinical trial. Emergency. 2017;5(1):195-200. PMID: 614121265.

130. Giusti M, Blasi F, Iori I, et al. Prulifloxacin vs Levofloxacin for Exacerbation of COPD after Failure of Other Antibiotics. Copd: Journal of Chronic Obstructive Pulmonary Disease. 2016 Oct;13(5):555-60. PMID: 27027547.

131. Sun X, He Z, Zhang J, et al. Compare the efficacy of inhaled budesonide and systemic methylprednisolone on systemic inflammation of AECOPD. Pulmonary Pharmacology \& Therapeutics. 2015 Apr;31:111-6. PMID: 25281894.

132. Rhee CK, Chang JH, Choi EG, et al. Zabofloxacin versus moxifloxacin in patients with COPD exacerbation: a multicenter, double-blind, double-dummy, randomized, controlled, Phase III, noninferiority trial. International Journal of Copd. 2015;10:2265-75. PMID: 26543359.

133. Yilmazel Ucar E, Araz O, Meral M, et al. Two different dosages of nebulized steroid versus parenteral steroid in the management of COPD exacerbations: a randomized control trial. Medical Science Monitor. 2014 Mar 28;20:513-20. PMID: 24675102.

134. Yoon HI, Lee C-H, Kim DK, et al. Efficacy of levofloxacin versus cefuroxime in treating acute exacerbations of chronic obstructive pulmonary disease. International Journal of Copd. 2013;8:329-34. PMID: 23874094.

135. Blasi F, Schaberg T, Centanni S, et al. Prulifloxacin versus levofloxacin in the treatment of severe COPD patients with acute exacerbations of chronic bronchitis. Pulmonary Pharmacology \& Therapeutics. 2013 Oct;26(5):609-16. PMID: 23538168.

136. Wilson R, Anzueto A, Miravitlles M, et al. Moxifloxacin versus amoxicillin/clavulanic acid in outpatient acute exacerbations of COPD: MAESTRAL results.[Erratum appears in Eur Respir J. 2012 Sep;40(3):800]. European Respiratory Journal. 2012 Jul;40(1):17-27. PMID: 22135277. 
137. Aggarwal P, Wig N, Bhoi S. Efficacy of two corticosteroid regimens in acute exacerbation of chronic obstructive pulmonary disease. International Journal of Tuberculosis \& Lung Disease. 2011 May;15(5):687-92. PMID: 21756523.

138. Stallberg B, Selroos O, Vogelmeier C, et al. Budesonide/formoterol as effective as prednisolone plus formoterol in acute exacerbations of COPD. A double-blind, randomised, non-inferiority, parallel-group, multicentre study. Respiratory Research. 2009 Feb 19;10:11. PMID: 19228428.

139. Llor C, Hernandez S, Ribas A, et al. Efficacy of amoxycillin versus amoxycillin/clavulanate in acute exacerbations of chronic pulmonary obstructive disease in primary care. International Journal of Copd. 2009;4:45-53. PMID: 19436696.

140. van Zanten ARH, Oudijk M, NohlmansPaulssen MKE, et al. Continuous vs. intermittent cefotaxime administration in patients with chronic obstructive pulmonary disease and respiratory tract infections: pharmacokinetics/pharmacodynamics, bacterial susceptibility and clinical efficacy. British Journal of Clinical Pharmacology. 2007 Jan;63(1):100-9. PMID: 16869814.

141. Ruiz-Gonzalez A, Gimenez A, GomezArbones X, et al. Open-label, randomized comparison trial of long-term outcomes of levofloxacin versus standard antibiotic therapy in acute exacerbations of chronic obstructive pulmonary disease. Respirology. 2007 Jan;12(1):117-21. PMID: 17207036.

142. Roede BM, Bresser P, El Moussaoui R, et al. Three vs. 10 days of amoxycillinclavulanic acid for type 1 acute exacerbations of chronic obstructive pulmonary disease: a randomised, doubleblind study. Clinical Microbiology \& Infection. 2007 Mar;13(3):284-90. PMID: 17391383.
143. Petitpretz P, Chone C, Tremolieres F, et al. Levofloxacin $500 \mathrm{mg}$ once daily versus cefuroxime $250 \mathrm{mg}$ twice daily in patients with acute exacerbations of chronic obstructive bronchitis: clinical efficacy and exacerbation-free interval. International Journal of Antimicrobial Agents. 2007 Jul;30(1):52-9. PMID: 17512704.

144. de Jong YP, Uil SM, Grotjohan HP, et al. Oral or IV prednisolone in the treatment of COPD exacerbations: a randomized, controlled, double-blind study. Chest. 2007 Dec;132(6):1741-7. PMID: 17646228.

145. Andre-Alves MR, Jardim JR, Frare e Silva $\mathrm{R}$, et al. Comparison between azithromycin and amoxicillin in the treatment of infectious exacerbation of chronic obstructive pulmonary disease. Jornal Brasileiro De Pneumologia: Publicacao Oficial Da Sociedade Brasileira De Pneumologia E Tisilogia. 2007 JanFeb;33(1):43-50. PMID: 17568867.

146. Mirici A, Meral M, Akgun M. Comparison of the efficacy of nebulised budesonide with parenteral corticosteroids in the treatment of acute exacerbations of chronic obstructive pulmonary disease. Clinical Drug Investigation. 2003;23(1):55-62. PMID: 23319094.

147. Willaert W, Daenen M, Bomans P, et al. What is the optimal treatment strategy for chronic obstructive pulmonary disease exacerbations? European Respiratory Journal. 2002 May;19(5):928-35. PMID: 12030735.

148. Aubier M, Aldons PM, Leak A, et al. Telithromycin is as effective as amoxicillin/clavulanate in acute exacerbations of chronic bronchitis. Respiratory Medicine. 2002 Nov;96(11):862-71. PMID: 12418583.

149. Sayiner A, Aytemur ZA, Cirit M, et al. Systemic glucocorticoids in severe exacerbations of COPD. Chest. 2001 Mar;119(3):726-30. PMID: 11243949. 
150. Umut S, Tutluoglu B, Aydin Tosun G, et al. Determination of the etiological organism during acute exacerbations of COPD and efficacy of azithromycin, ampicillinsulbactam, ciprofloxacin and cefaclor. Turkish Thoracic Society COPD Working Group. Journal of Chemotherapy. 1999 Jun;11(3):211-4. PMID: 10435684.

151. Rizzato G. Effects of deflazacort hemisuccinate vs. methylprednisolone succinate in COPD exacerbations. Internista. 1998;6(1):35-40. PMID: 28122103.

152. Leophonte P, Baldwin RJ, Pluck N. Trovafloxacin versus amoxicillin/clavulanic acid in the treatment of acute exacerbations of chronic obstructive bronchitis. European Journal of Clinical Microbiology \& Infectious Diseases. 1998 Jun;17(6):434-40. PMID: 9758289.

153. Hasani A, Pavia D, Rotondetto S, et al. Effect of oral antibiotics on lung mucociliary clearance during exacerbation of chronic obstructive pulmonary disease. Respiratory Medicine. 1998 Mar;92(3):4427. PMID: 9692103.

154. Whitlock W. Multicenter comparison of azithromycin and amoxicillin/clavulanate in the treatment of patients with acute exacerbations of chronic obstructive pulmonary disease. Current Therapeutic Research - Clinical and Experimental. 1995;56(10):985-95. PMID: 25335828.
155. Hamacher J, Vogel F, Lichey J, et al. Treatment of acute bacterial exacerbations of chronic obstructive pulmonary disease in hospitalised patients--a comparison of meropenem and imipenem/cilastatin. COPD Study Group. Journal of Antimicrobial Chemotherapy. 1995 Jul;36 Suppl A:121-33. PMID: 8543488.

156. Phillips H, Van Hook CJ, Butler T, et al. A comparison of cefpodoxime proxetil and cefaclor in the treatment of acute exacerbation of COPD in adults. Chest. 1993 Nov;104(5):1387-92. PMID: 8222793.

157. Dark D. Azithromycin versus cefaclor in the treatment of acute exacerbations of chronic obstructive pulmonary disease. Current Therapeutic Research - Clinical and Experimental. 1993;53(2):203-11. PMID: 23119468.

158. Leuppi JD, Schuetz P, Bingisser R, et al. Short-term vs conventional glucocorticoid therapy in acute exacerbations of chronic obstructive pulmonary disease: the REDUCE randomized clinical trial. Jama. 2013;309(21):2223-31.

159. Ding Z, Li X, Lu Y, et al. A randomized, controlled multicentric study of inhaled budesonide and intravenous methylprednisolone in the treatment on acute exacerbation of chronic obstructive pulmonary disease. Respir Med. 2016 Dec;121:39-47. doi: 10.1016/j.rmed.2016.10.013. PMID: 27888990. 


\section{Abbreviations and Acronyms}

ACOS

AE

AHRQ

ATS

CI

COPD

$\mathrm{D}-\mathrm{L}$

ECOPD

ED

EPC

ERS

FDA

FEV1

GOLD

HKSJ

ICS

ICU

KQ

LABA

LAMA

Mg

OR

PICOTS

PRISMA

QoL

RCTs

RCU

SABAs

SAMAs

SMD

SOE

TENS

US

WMD
Asthma-COPD overlap syndrome

Adverse Event

Agency for Healthcare Research and Quality

American Thoracic Society

Confidence Interval

Chronic Obstructive Pulmonary Disease

DerSimonian and Laird

Exacerbations of Chronic Obstructive Pulmonary Disease

Emergency Department

Evidence-based Practice Center

European Respiratory Society

Food and Drug Administration

Forced Expiratory Volume in One Second

Global Initiative for Chronic Obstructive Lung Disease

Hartung-Knapp-Sidik-Jonkman

Inhaled Corticosteroid

Intensive Care Unit

Key Question

Long-acting beta-agonist

Long-acting muscarinic antagonists

Milligram

Odds Ratio

Population, Interventions, Comparisons, Outcomes, Timing, and Setting

Preferred Reporting Items for Systematic Reviews and Meta-Analyses

Quality of Life

Randomized Controlled Trials

Respiratory Care Unit

Short-acting Beta Adrenergic Agonists

Short-acting Muscarinic Antagonists

Standardized Mean Difference

Strength of Evidence

Transcutaneous Electrical Nerve Stimulation

United States of America

Weighted Mean Difference 


\section{Appendixes}

\section{Tables}

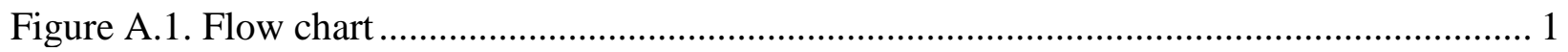

Table D.1. KQ1: Characteristics of included studies................................................................... 1

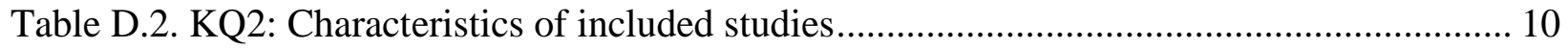

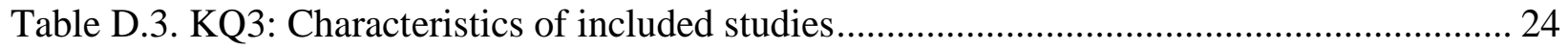

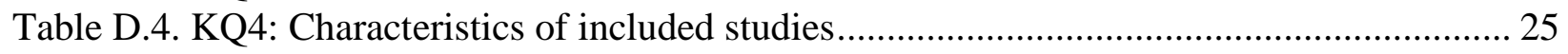

Table E.1. Risk of Bias (Cochrane ROB tool) for included studies .......................................... 1

Table F.1. KQ1: Intervention description and conclusions .................................................... 1

Table F.2. KQ2: Intervention description and conclusions ........................................................ 8

Table F.3. KQ3: Intervention description and conclusions ........................................................... 26

Table F.4. KQ4: Intervention description and conclusions .......................................................... 27

Table G.1. KQ1 results: mild ECOPD severity- antibiotics vs. placebo .......................................... 1

Table G.2. KQ1 results: moderate-severe ECOPD severity-antibiotics vs. control ......................... 2

Table G.3. KQ1 results: Mild Severity-systemic corticosteroids vs. placebo ................................ 3

Table G.4. KQ1 results: Moderate-severe severity- systemic corticosteroids vs. control................ 3

Table G.5. KQ2 results: Mild severity- mucolytics vs management without mucolytics............... 4

Table G.6. KQ2 results: Moderate-severe severity - mucolytics vs placebo.................................... 5

Table G.7. KQ2 results: Moderate-severe severity-inhaled corticosteroids vs control ................... 5

Table G.8. KQ2 results: Moderate-severe severity-inhaled corticosteroids with inhaled shortacting bronchodilators vs management without inhaled corticosteroids ......................................... 6

Table G.9. KQ2 results: Mild severity-inhaled corticosteroids with inhaled long acting

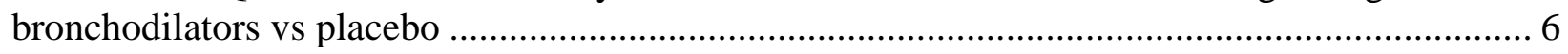

Table H.1. KQ1: Adverse events. Antibiotics compared with control ........................................... 1

Table H.2. KQ1: Adverse events. Systemic corticosteroids compared with control....................... 1

Table H.3. KQ2: Adverse events. Intravenous aminophyllines compared with placebo ................. 2

Table H.4. KQ2: Adverse events. Oral mucolytics compared with placebo .................................. 2

Table H.5. KQ2: Adverse events. Inhaled corticosteroids with or without inhaled short- and long acting bronchodilators compared with placebo ......................................................................... 2

Table H.6. KQ2: Adverse events. 5-lipoxygenase inhibitor (zileuton) compared with placebo .... 2 Table H.7. KQ2: Adverse events. Chest physiotherapy using breathing technique compared with management without chest physiotherapy ...................................................................................... 3

Table H.8. KQ2: Adverse events. Chest physiotherapy using vibration/percussion/massage compared with management without chest physiotherapy ………………………............................. 3

Table H.9. KQ2: Adverse events. Chest physiotherapy using positive expiratory pressure compared with management without positive expiratory pressure................................................... 3

Table H.10. KQ2: Adverse events. Exercise using resistance training compared with management without resistance training..................................................................................... 3

Table H.11. KQ2: Adverse events. Exercise using combined aerobic + resistance training compared with management without exercise training. ................................................................. 3

Table H.12. KQ2: Adverse events. Early pulmonary rehabilitation compared with management without early pulmonary rehabilitation.......................................................................................... 4 Table H.13. KQ2: Adverse events. Whole body vibration training during ECOPD compared with management without whole body vibration. 
Table H.14. KQ2: Adverse events. Transcutaneous electrical nerve stimulation (TENS) during ECOPD compared with vs management without Transcutaneous Electrical Nerve Stimulation .. 4 Table H.15. KQ2: Adverse events. Gutter frame with supplemental oxygen compared with gutter frame supplemental air. 4 Table H.16. KQ2: Adverse events. Rollator with supplemental oxygen compared with gutter frame supplemental air 4

Table H.17. KQ2: Adverse events. Dietary intervention using a caloric supplement during ECOPD compared with usual diet

Table H.18. KQ2: Adverse events. Dietary intervention using a caloric and a protein supplement during ECOPD compared with Placebo (non-caloric fluid, vanilla flavored water) ..................... 5 Table. H.19. KQ. Adverse events. Dietary intervention using omega-3 fatty acid compared with usual diet

Table H.20. KQ2: Adverse events. Dietary intervention using vitamin D during ECOPD compared with placebo 5

Table H.21. KQ3: Adverse events. ICS+ SABA (beclomethasone+ salbutamol compared with SABA (Fenoterol). 5

Table H.22. KQ4: Adverse events. Aminopenicillin plus beta-lactamase compared with fluoroquinolone. 5

Table H.23. KQ4: Adverse events. Ciprofloxacin compared with amoxicillin. 6 Table H.24. KQ4: Adverse events. "Standard" antibiotic therapy (clarithromycin or cefuroxime or amoxicillin + clavulanic acid) compared with levofloxacin ............................................... 6

Table H.25. KQ4: Adverse events. Amoxicillin compared with azithromycin........................... 6 Table H.26. KQ4: Adverse events. Cephalosporin compared with fluoroquinolone ..................... 6 Table H.27. KQ4: Adverse events. Azithromycin compared with cefaclor ................................ 7 Table H.28. KQ4: Adverse events. Amoxicillin compared with amoxicillin plus clavulanic acid 7 Table H.29. KQ4: Adverse events. Amoxicillin plus clavulanic acid compared with telithromycin

Table H.30. KQ4: Adverse events. Aminopenicillin plus beta-lactamases inhibitor plus clavulanic acid compared with macrolides ....................................................................... 7

Table H.31. KQ4: Adverse events. Amoxicillin + Clavulanic acid compared azithromycin........ 8

Table H.32. KQ4: Adverse events. Levofloxacin compared with prulifloxacin ......................... 8

Table H.33. KQ4: Adverse events. Moxifloxacin compared with zabofloxacin .......................... 8

Table H.34. KQ4: Adverse events. Cefaclor compared with cefpodoxime.................................. 8

Table H.35. KQ4: Adverse events. Imipenem+cilastatin compared with meropenem ................. 9

Table H.36. KQ4: Adverse events. Trovafloxacin $200 \mathrm{mg}$ compared with trovafloxacin $100 \mathrm{mg} 9$ Table H.37. KQ4: Adverse events. Intermittent intravenous cefotaxime compared with continuous intravenous cefotaxime.

Table H.38. KQ4: Adverse events. Amoxicillin + clavulanic for 10 days compared with amoxicillin + Clavulanic for 3 days. 9 Table H.39. KQ4: Adverse events. Deflazacort hemisuccinate compared with methylprednisolone. 9 Table H.40. KQ4: Adverse events. Hydrocortisone i.v. followed by prednisolone oral compared with methylprednisolone i.v. followed by methylprednisone oral ......................................... 10 Table H.41. KQ4: Adverse events. Nebulized budesonide compared with oral prednisolone..... 10 Table H.42. KQ4: Adverse events. Oral prednisolone + inhaled formoterol compared with inhaled budesonide + formoterol 
Table H.43. KQ4: Adverse events. Inhaled budesonide + formoterol compared with systemic methylprednisolone

Table H.44. KQ4: Adverse events. Inhaled Budesonide 40mg compared with Intravenous Methylprednisolone (initially intravenous, then oral) ...................................................... 10

Table H.45. KQ4: Adverse events. Inhaled Budesonide 4mg compared with Intravenous Methylprednisolone 11

Table H.46. KQ4: Adverse events. Inhaled Budesonide 8mg compared with intravenous methylprednisolone 11

Table H.47. KQ4: Adverse events. Oral prednisolone compared with intravenous prednisolone 11 Table H.48. KQ4: Adverse events. Oral methyl-prednisolone compared with intravenous methylprednisolone.... 11 Table H.49. KQ4: Adverse events. Glucocorticoid for 2 weeks compared with glucocorticoid for 8 weeks. 11

Table H.50. KQ4: Adverse events. Methylprednisolone for 5 days compared with methylprednisolone for 14 days

Table H.51. KQ4: Adverse events. Methylprednisolone for 3 days compared with

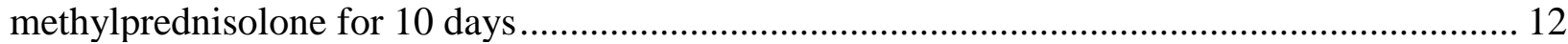

Table I.1. Inclusion and Exclusion Criteria of Included Studies .............................................. 1

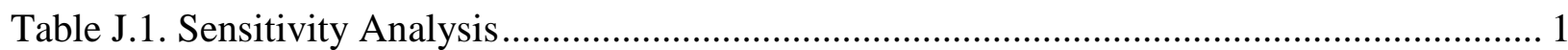

\section{Figures}

Figure A.1. Flow chart 


\section{Appendix A. Flow Chart}

Figure A.1. Flow chart

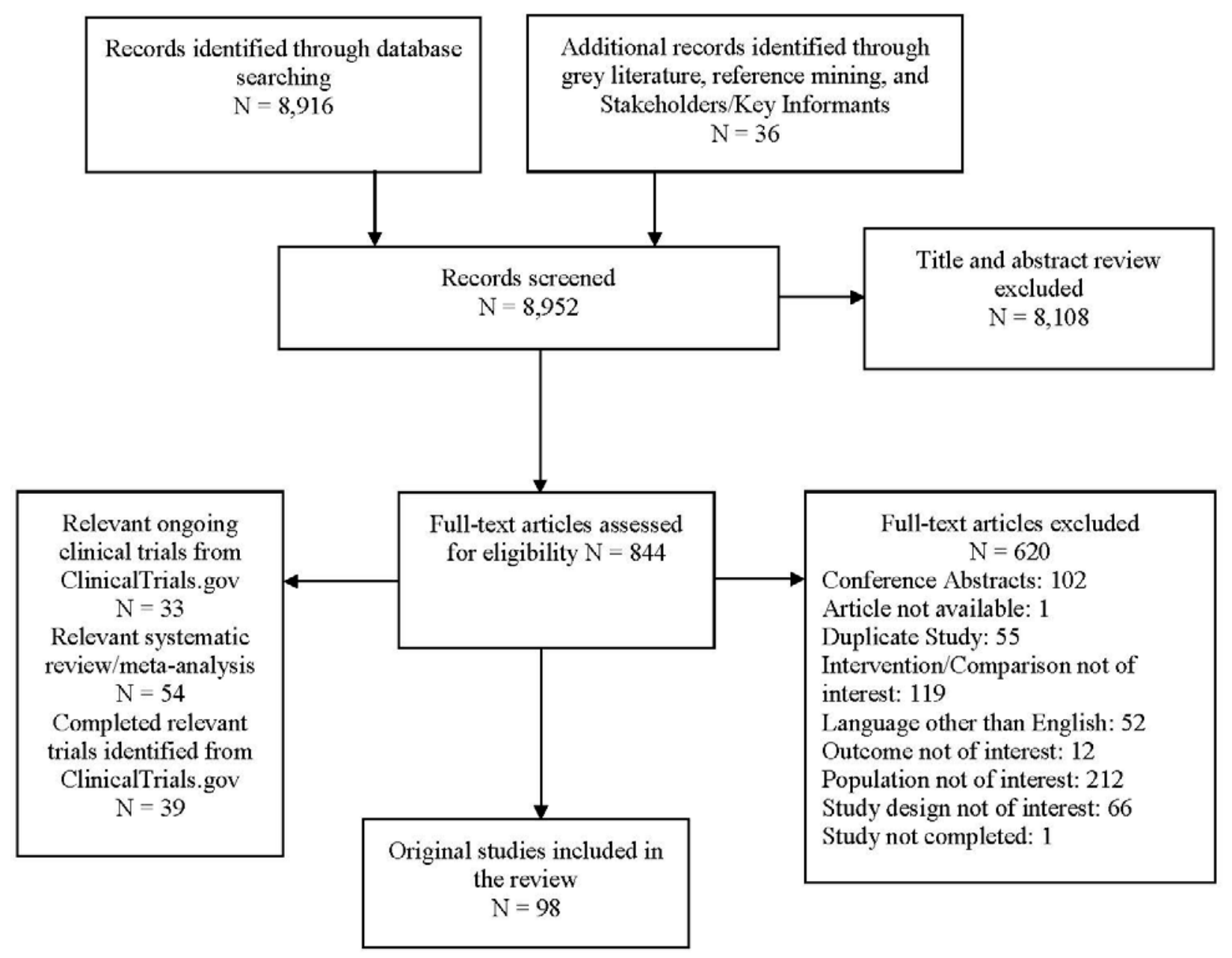




\section{Appendix B. Search Strategy}

\section{Ovid}

Database(s): EBM Reviews - Cochrane Central Register of Controlled Trials November 2018, EBM Reviews - Cochrane Database of Systematic Reviews 2005 to December 28, 2018, Embase 1974 to 2018 January 02, Ovid MEDLINE(R) and Epub Ahead of Print, In-Process \& Other Non-Indexed Citations and Daily 1946 to January 02, 2019

Search Strategy:

\# $\quad$ Searches

1 exp Pulmonary Disease, Chronic Obstructive/dh, dt, px, rh, th or exp Lung Diseases, Obstructive/dh, dt, px, rh, th

2 exp chronic obstructive lung disease/dm, dt, rh, th

3 ((chronic* adj3 bronchiti*) or (obstruct* adj3 (pulmonary or lung* or airway* or airflow* or bronch* or respirat*)) or aecb or "chronic airflow disease*" or "chronic airflow disorder*" or "chronic airflow limitation*" or "chronic airflow obstruction*" or "chronic airway disease*" or "chronic airway disorder*" or "chronic airway limitation*" or "chronic airway obstruction*" or "chronic bronchitis" or "chronic obstructive airflow disease*" or "chronic obstructive airflow disorder*" or "chronic obstructive airway disease*" or "chronic obstructive airway disorder*" or "chronic obstructive bronchitis" or "chronic obstructive bronchopulmonary disease*" or "chronic obstructive broncho-pulmonary disease*" or "chronic obstructive bronchopulmonary disorder*" or "chronic obstructive broncho-pulmonary disorder*" or "chronic obstructive lung disease*" or "chronic obstructive lung disorder*" or "chronic obstructive pulmonary disease*" or "chronic obstructive pulmonary disorder*" or "chronic obstructive respiratory disease*" or "chronic obstructive respiratory disorder*" or coad or cobd or copd or emphysema* or "obstructive lung disease" or "obstructive lung disorder*" or "obstructive pulmonary disease*" or "obstructive pulmonary disorder*" or "obstructive pulmonary tract disease*" or "obstructive pulmonary tract disorder*" or "obstructive respiratory disease*" or "obstructive respiratory disorder*" or "obstructive respiratory tract disease*" or "obstructive respiratory tract disorder*").ti,ab,hw,kw. 4 ((increas* adj3 (severity or seriousness)) or exacerbation* or worsen*).ti,ab,hw,kw.

$5 \quad$ (1 or 2 or 3$)$ and 4

6 exp Bronchodilator Agents/ or exp Adrenergic beta-2 Receptor Agonists/ or exp Cholinergic Antagonists/ or exp Phosphodiesterase 4 Inhibitors/ or exp Antibiotic Prophylaxis/ or exp Anti-Bacterial Agents/ or exp antibiotic agent/ or exp Benzodiazepines/ or exp Respiration, Artificial/ or exp Adrenal Cortex Hormones/ or exp corticosteroid/ or exp corticosteroid therapy/ or exp Expectorants/ or exp narcotic analgesic agent/ or exp Analgesics, Opioid/ or exp Smoking Cessation/ or exp Respiratory Therapy/ or exp exercise/ or exp Exercise Therapy/ or exp Breathing Exercises/ or exp Exercise Movement Techniques/ or exp Nutrition Therapy/ or exp Influenza Vaccines/ or exp Pneumococcal Vaccines/ or exp vaccination/ or exp Psychotherapy/ or exp Cognitive Therapy/ or exp Cognitive Behavior Therapy/ or exp Mindfulness/ or exp Mind-Body Therapies/ or exp Self Care/ or exp Acupuncture exp Complementary Therapies/ or exp Electric Stimulation Therapy/

$7 \quad$ ((action adj3 plan*) or (disease adj2 manag*) or (management adj1 program*) or Acupuncture or "Adrenal Cortex Hormone*" or "Adrenergic beta-2 Receptor Agonist*" or "Adrenergic beta-2 Receptor Antagonist*" or "alternative medicine*" or antibacterial* or "AntiBacterial*" or antibiotic* or Anticholinergic* or "artificial respiration" or behavior* or behaviour* or Benzodiazepine* or "Beta adrenergic agonist*" or "Beta adrenergic Antagonist*" 
or "Breathing Exercise*" or Bronchodilator* or chemotherap* or "Chest physiotherap*" or "Cholinergic agonist*" or "Cholinergic Antagonist*" or "Cognitive Behavior Therap*" or "Cognitive Therap*" or "Complementary Therap*" or corticosteroid* or diet or drug* or educat* or "Electric Stimulation*" or empower* or exercise* or Expectorant* or Glucocorticoid* or instruct* or "management plan*" or "Mind-Body" or Mindfulnes* or narcotic* or Nutrition* or opioid* or "Oxygen therap*" or "patient cent*" or "patient educat*" or "patient focus*" or pharmacotherap* or "Phosphodiesterase 4 Inhibitor*" or Psychotherap* or respirator* or "Respiratory Therap*" or "Self Car*" or "self-efficac*" or "self-manag*" or "Smoking Cessation" or steroid* or train* or Vaccin* or ventilation or ventilator*).ti,ab,hw,kw.

86 or 7

95 and 8

10 limit 9 to ("all adult (19 plus years)" or "young adult (19 to 24 years)" or "adult (19 to 44 years)" or "young adult and adult (19-24 and 19-44)" or "middle age (45 to 64 years)" or "middle aged (45 plus years)" or "all aged (65 and over)" or "aged (80 and over)") [Limit not valid in CCTR,CDSR,Embase; records were retained]

11 limit 10 to (adult $<18$ to 64 years $>$ or aged $<65+$ years $>$ ) [Limit not valid in CCTR,CDSR,Ovid MEDLINE(R),Ovid MEDLINE(R) Daily Update,Ovid MEDLINE(R) InProcess,Ovid MEDLINE(R) Publisher; records were retained]

12 limit 9 to ("all infant (birth to 23 months)" or "all child (0 to 18 years)" or "newborn infant (birth to 1 month)" or "infant (1 to 23 months)" or "preschool child (2 to 5 years)" or "child (6 to 12 years)" or "adolescent (13 to 18 years)") [Limit not valid in CCTR,CDSR,Embase; records were retained]

13 limit 12 to (embryo or infant or child or preschool child $<1$ to 6 years $>$ or school child $<7$ to 12 years $>$ or adolescent $<13$ to 17 years $>$ ) [Limit not valid in CCTR,CDSR,Ovid MEDLINE(R),Ovid MEDLINE(R) Daily Update,Ovid MEDLINE(R) In-Process, Ovid MEDLINE(R) Publisher; records were retained]

$14 \quad 13$ not 11

159 not 14

16 limit 15 to (editorial or erratum or note or addresses or autobiography or bibliography or biography or blogs or comment or dictionary or directory or interactive tutorial or interview or lectures or legal cases or legislation or news or newspaper article or overall or patient education handout or periodical index or portraits or published erratum or video-audio media or webcasts) [Limit not valid in CCTR,CDSR,Embase,Ovid MEDLINE(R),Ovid MEDLINE(R) Daily Update,Ovid MEDLINE(R) In-Process,Ovid MEDLINE(R) Publisher; records were retained]

17 from 16 keep 8-93

$18 \quad$ (15 not 16$)$ or 17

19 limit 18 to yr="2017 -Current"

20 limit 19 to yr="2018 -Current"

21 remove duplicates from 20

2219 not 20

23 remove duplicates from 22

$24 \quad 21$ or 23

25 exp meta analysis/

26 exp Meta-Analysis as Topic/

27 exp "systematic review"/

28 ((meta adj analys*) or (systematic* adj3 review*)).mp,pt. 
25 or 26 or 27 or 28

$30 \quad 24$ and 29

31 exp controlled study/

32 exp Randomized Controlled Trial/

33 exp triple blind procedure/

34 exp Double-Blind Method/

35 exp Single-Blind Method/

36 exp latin square design/

37 ((control* adj3 study) or (control* adj3 trial) or (randomized adj3 study) or (randomized adj3 trial) or (randomised adj3 study) or (randomised adj3 trial) or "pragmatic clinical trial" or (doubl* adj blind*) or (doubl* adj mask*) or (singl* adj blind*) or (singl* adj mask*) or (tripl* adj blind*) or (tripl* adj mask*) or (trebl* adj blind*) or (trebl* adj mask*) or "latin

square").mp,pt.

38 or $/ 31-37$

$39 \quad$ (24 not 30) and 38

\section{Scopus}

1 TITLE-ABS-KEY((chronic* W/3 bronchiti*) or (obstruct* W/3 (pulmonary or lung* or airway* or airflow* or bronch* or respirat*)) or aecb or "chronic airflow disease*" or "chronic airflow disorder*" or "chronic airflow limitation*" or "chronic airflow obstruction*" or "chronic airway disease*" or "chronic airway disorder*" or "chronic airway limitation*" or "chronic airway obstruction*" or "chronic bronchitis" or "chronic obstructive airflow disease*" or "chronic obstructive airflow disorder*" or "chronic obstructive airway disease*" or "chronic obstructive airway disorder*" or "chronic obstructive bronchitis" or "chronic obstructive bronchopulmonary disease*" or "chronic obstructive broncho-pulmonary disease*" or "chronic obstructive bronchopulmonary disorder*" or "chronic obstructive broncho-pulmonary disorder*" or "chronic obstructive lung disease*" or "chronic obstructive lung disorder*" or "chronic obstructive pulmonary disease*" or "chronic obstructive pulmonary disorder*" or "chronic obstructive respiratory disease*" or "chronic obstructive respiratory disorder*" or coad or cobd or copd or emphysema* or "obstructive lung disease" or "obstructive lung disorder*" or "obstructive pulmonary disease*" or "obstructive pulmonary disorder*" or "obstructive pulmonary tract disease*" or "obstructive pulmonary tract disorder*" or "obstructive respiratory disease*" or "obstructive respiratory disorder*" or "obstructive respiratory tract disease*" or "obstructive respiratory tract disorder*")

2 TITLE-ABS-KEY((increas* W/3 (severity or seriousness)) or exacerbation* or worsen*)

3 TITLE-ABS-KEY((action W/3 plan*) or (disease W/2 manag*) or (management W/1 program*) or Acupuncture or "Adrenal Cortex Hormone*" or "Adrenergic beta-2 Receptor Agonist*" or "Adrenergic beta-2 Receptor Antagonist*" or "alternative medicine*" or antibacterial* or "Anti-Bacterial*" or antibiotic* or Anticholinergic* or "artificial respiration" or behavior* or behaviour* or Benzodiazepine* or "Beta adrenergic agonist*" or "Beta adrenergic Antagonist*" or "Breathing Exercise*" or Bronchodilator* or chemotherap* or "Chest physiotherap*" or "Cholinergic agonist*" or "Cholinergic Antagonist*" or "Cognitive Behavior Therap*" or "Cognitive Therap*" or "Complementary Therap*" or corticosteroid* or diet or drug* or educat* or "Electric Stimulation*" or empower* or exercise* or Expectorant* or Glucocorticoid* or instruct* or "management plan*" or "Mind-Body" or Mindfulnes* or narcotic* or Nutrition* or opioid* or "Oxygen therap*" or "patient cent*" or "patient educat*" or 
"patient focus*" or pharmacotherap* or "Phosphodiesterase 4 Inhibitor*" or Psychotherap* or respirator* or "Respiratory Therap*" or "Self Car*" or "self-efficac*" or "self-manag*" or "Smoking Cessation" or steroid* or train* or Vaccin* or ventilation or ventilator*)

$4 \quad 1$ and 2 and 3

5 TITLE-ABS-KEY(newborn* or neonat* or infant* or toddler* or child* or adolescent* or paediatric* or pediatric* or girl or girls or boy or boys or teen or teens or teenager* or preschooler* or "pre-schooler*" or preteen or preteens or "pre-teen" or "pre-teens" or youth or youths) AND NOT TITLE-ABS-KEY(adult or adults or "middle age" or "middle aged" OR elderly OR geriatric* OR "old people" OR "old person*" OR "older people" OR "older person*" OR "very old")

64 and not 5

7 DOCTYPE(ed) OR DOCTYPE(bk) OR DOCTYPE(er) OR DOCTYPE(no) OR DOCTYPE(sh)

$8 \quad 6$ and not 7

$9 \quad \mathrm{PMID}\left(0^{*}\right)$ OR PMID(1*) OR PMID(2*) OR PMID(3*) OR PMID(4*) OR PMID(5*) OR PMID(6*) OR PMID(7*) OR PMID(8*) OR PMID(9*)

$10 \quad 8$ and not 9

11 TITLE-ABS-KEY("consensus development" or guideline* or "position statement*")

$12 \quad 10$ and not 11

13 TITLE-ABS-KEY((meta W/1 analys*) or (systematic* W/3 review*))

$14 \quad 12$ and 13

$15 \quad 12$ and not 14

16 TITLE-ABS-KEY((control* W/3 study) or (control* W/3 trial) or (randomized W/3 study) or (randomized W/3 trial) or (randomised W/3 study) or (randomised W/3 trial) or "pragmatic clinical trial" or (doubl* W/1 blind*) or (doubl* W/1 mask*) or (singl* W/1 blind*) or (singl* W/1 mask*) or (tripl* W/1 blind*) or (tripl* W/1 mask*) or (trebl* W/1 blind*) or (trebl* W/1 mask*) or "latin square")

$17 \quad 15$ and 16

\section{CInicalTrials.Gov}

(aecb OR "airflow obstruction" OR "airway obstruction" OR "bronchial obstruction" OR "bronchus obstruction" OR "chronic airflow disease") AND ("increased severity" OR "increasing severity" OR "increased seriousness" OR "increasing seriousness" OR exacerbation OR worsening)

("chronic airflow disorder" OR "chronic airflow limitation" OR "chronic airflow obstruction" OR "chronic airway disease" OR "chronic airway disorder" OR "chronic airway limitation") AND ("increased severity" OR "increasing severity" OR "increased seriousness" OR "increasing seriousness" OR exacerbation OR worsening)

("chronic airway obstruction" OR "chronic bronchitis" OR "chronic obstructive airflow disease" OR "chronic obstructive airflow disorder" OR "chronic obstructive airway disease" OR "chronic obstructive airway disorder") AND ("increased severity" OR "increasing severity" OR "increased seriousness" OR "increasing seriousness" OR exacerbation OR worsening) 
("chronic obstructive bronchitis" OR "chronic obstructive bronchopulmonary disease" OR "chronic obstructive broncho-pulmonary disease" OR "chronic obstructive bronchopulmonary disorder") AND ("increased severity" OR "increasing severity" OR "increased seriousness" OR "increasing seriousness" OR exacerbation OR worsening)

("chronic obstructive broncho-pulmonary disorder" OR "chronic obstructive lung disease" OR "chronic obstructive lung disorder" OR "chronic obstructive pulmonary disease" OR "chronic obstructive pulmonary disorder") AND ("increased severity" OR "increasing severity" OR "increased seriousness" OR "increasing seriousness" OR exacerbation OR worsening)

("chronic obstructive respiratory disease" OR "chronic obstructive respiratory disorder" OR coad OR cobd OR copd OR emphysema OR "lung obstruction" OR "obstructive lung disease" OR "obstructive lung disorder") AND ("increased severity" OR "increasing severity" OR "increased seriousness" OR "increasing seriousness" OR exacerbation OR worsening)

("obstructive pulmonary disease" OR "obstructive pulmonary disorder" OR "obstructive pulmonary tract disease" OR "obstructive pulmonary tract disorder" OR "obstructive respiratory disease") AND ("increased severity" OR "increasing severity" OR "increased seriousness" OR "increasing seriousness" OR exacerbation OR worsening)

("obstructive respiratory disorder" OR "obstructive respiratory tract disease" OR "obstructive respiratory tract disorder" OR "pulmonary obstruction" OR "respiratory obstruction") AND ("increased severity" OR "increasing severity" OR "increased seriousness" OR "increasing seriousness" OR exacerbation OR worsening)

All limited to adults. 


\section{Appendix C. Excluded Studies}

1. 1-year Study to Assess the Efficacy, Safety, and Tolerability of Glycopyrronium Bromide (NVA237) in Chronic Obstructive Pulmonary Disease (COPD). 2009 June. PMID: NCT00929110. [Population not of interest].

2. A 24-week Study to Compare Umeclidinium/Vilanterol (UMEC/VI), UMEC and Salmeterol in Subjects With Chronic Obstructive Pulmonary Disease (COPD). Https://clinicaltrials.gov/show/nct03034915. 2017. PMID: CN-01561596. [Duplicate Study].

3. A 52-Week, Multicenter, Randomized, Double-Blind, Parallel-Group, PlaceboControlled Study to Evaluate the Safety and Tolerability of GSK573719/GW642444 and GSK573719 in Subjects With Chronic Obstructive Pulmonary Disease (COPD). 2011 January 1. PMID: NCT01316887. [Population not of interest].

4. A Comparison Study Between the Fixed Dose Triple Combination of Fluticasone Furoate/ Umeclidinium/ Vilanterol Trifenatate (FF/UMEC/VI) With Budesonide/Formoterol in Subjects With Chronic Obstructive Pulmonary Disease (COPD). 2015 January. PMID: NCT02345161. [Population not of interest].

5. A Dose-Finding Study Evaluating Safety and Efficacy in Patients With Chronic Obstructive Pulmonary Disease. 2006 November. PMID: NCT00403286. [Population not of interest].

6. A Multicenter, Randomized, Double-Blind, Double-Dummy Trial of Azithromycin SR Compared With Levofloxacin for the Treatment of Acute Symptoms of Chronic Bronchitis. 2003 January. PMID: NCT00644449. [Population not of interest].

7. A Multicentre, Randomised, Open-Label Study To Compare The Efficacy And Safety Of Azithromycin For 5 Days With Those Of Amoxicillin-Clavulanic Acid In Patients With Chronic Bronchitis. 2002 October. PMID: NCT00649831. [Clinical Trial].

8. A Pilot Safety Study of Inhaled Dry Powder Mannitol in Acute Exacerbations of COPD. 2006 October. PMID: NCT00446667. [Study design not of interest].

9. A Pivotal Study of the Safety and Effectiveness of Arformoterol in Subjects With Chronic Obstructive Pulmonary Disease (COPD). 2002 February. PMID: NCT00685841. [Population not of interest].

10. A Randomised Effectiveness Study Comparing Fluticasone Furoate (FF, GW685698)/Vilanterol (VI, GW642444) With Standard Treatment in Chronic Obstructive Pulmonary Disease (COPD). 2012 March 13. PMID: NCT01551758. [Population not of interest].

11. A Study Comparing the Efficacy, Safety and Tolerability of Fixed Dose Combination (FDC) of FF/UMEC/VI With the FDC of FF/VI and UMEC/VI; Administered Oncedaily Via a Dry Powder Inhaler (DPI) in Subjects With Chronic Obstructive Pulmonary Disease (COPD). 2014 June 30. PMID: NCT02164513. [Population not of interest].

12. A Study of Andrographolide Sulfonate in Patients With Acute Exacerbation of Chronic Bronchitis. Https://clinicaltrials.gov/show/nct03132610. 2017. PMID: CN-01493692. [Duplicate Study].

13. A Study of Arformoterol Tartrate Inhalation Solution and Tiotropium Bromide on Rehospitalization in Chronic Obstructive Pulmonary Disease (COPD) Subjects. 2014 November. PMID: NCT02275481. [Population not of interest].

14. A Study to Evaluate the Efficacy and Safety of Fluticasone Furoate (FF)/GW642444 Inhalation Powder in Subjects With Chronic Obstructive Pulmonary Disease (COPD). 2009 September 25. PMID: NCT01009463. [Population not of interest]. 
15. A Study to Evaluate the Safety, Efficacy and Changes in Induced Sputum and Blood Biomarkers Following Daily Repeat Doses of Inhaled GSK2269557 in Chronic Obstructive Pulmonary Disease (COPD)

Subjects With Acute Exacerbation. 2015 November 4. PMID: NCT02522299. [Clinical Trial].

16. A Study To Investigate The Effects Of GW856553 On Patients With COPD (Chronic Obstructive Pulmonary Disease). 2006 August. PMID: NCT00392587. [Population not of interest].

17. A Trial To Evaluate Two Antibiotics For The Treatment Of Acute Exacerbation Of Chronic Bronchitis (AECB). 2006 February. PMID: NCT00254566. [Population not of interest].

18. Aaron SD, Vandemheen K, Maltais F, et al. Randomized, double-blind, placebocontrolled trial of TNF-alpha antagonists for acute exacerbations of copd. American Journal of Respiratory and Critical Care Medicine. Conference: American Thoracic Society International Conference, ATS. 2012;185(MeetingAbstracts). PMID: 71987621. [Duplicate Study].

19. Aaron SD, Vandemheen KL, Maltais F, et al. TNFalpha antagonists for acute exacerbations of COPD: a randomised double-blind controlled trial. Thorax. 2013 Feb;68(2):142-8. PMID: 23161645. [Duplicate Study].

20. Aaron SD, Vandemheen KL, Maltais F, et al. TNF $\alpha$ antagonists for acute exacerbations of COPD: A randomised double-blind controlled trial. Thorax. 2013;68(2):142-8. [Intervention/Comparison not of interest].

21. Aaron SD. Controlled trial of oral glucocorticoids in outpatients with acute COPD exacerbations who present to the emergency department: a randomized, double-blind, placebo-controlled pilot study [Dissertation]. University of Ottawa. 1999. PMID: CN-00462253 UPDATE. [Study design not of interest].

22. Abdullah Al Mamun SM, Rahman S. Role of 7-day and 14-day courses of oral prednisolone treatment in acute exacerbation of COPD. Thorax. 2011 December;4):A172A3. PMID: 70627568. [Abstract].
23. Active for Life: chronic Obstructive Pulmonary Disease. Https://clinicaltrials.gov/show/nct03201198. 2017. PMID: CN-01495280. [Population not of interest].

24. ADVAIR ${ }^{\circledR}$ DISKUS ${ }^{\circledR}$ Inhaler (Fluticasone Propionate/Salmeterol) Versus SEREVENT ${ }^{\circledR}$ DISKUS ${ }^{\circledR}$ Inhaler (Salmeterol) For The Treatment Of Chronic Obstructive Pulmonary Disease Exacerbations. ADVAIR ${ }^{\circledR}$ DISKUS ${ }^{\circledR}$ Inhaler and SEREVENT ${ }^{\circledR}$ DISKUS ${ }^{\circledR}$ Inhaler Are Trademarks of the GSK Group of Companies. 2004 October. PMID: NCT00144911. [Population not of interest].

25. Advair ${ }^{\circledR}$ DISKUS ${ }^{\circledR}$ Versus Serevent ${ }^{\circledR}$ DISKUS ${ }^{\circledR}$ For Chronic Obstructive Pulmonary Disease Exacerbations. 2004 December. PMID: NCT00115492. [Population not of interest].

26. Aggarwal R, Shaphe MA, George C, et al. A comparison of flutter device and active cycle of breathing techniques in acute exacerbation of chronic obstructive pulmonary disease patients. Indian Journal of Physiotherapy and Occupational Therapy. 2010;4(3):60-4. [Duplicate Study].

27. Agusti AG, Carrera M, Barbe F, et al. Oxygen therapy during exacerbations of chronic obstructive pulmonary disease. European Respiratory Journal. 1999 Oct;14(4):934-9. PMID: 10573245. [Outcome not of interest].

28. Aimonino Ricauda N, Tibaldi V, Leff B, et al. Substitutive "hospital at home" versus inpatient care for elderly patients with exacerbations of chronic obstructive pulmonary disease: a prospective randomized, controlled trial. Journal of the American Geriatrics Society. 2008 Mar;56(3):493-500. PMID: 18179503. [Intervention/Comparison not of interest].

29. Ain Shams U, Trudell Medical I. Oscillating Positive Expiratory Pressure Devices and Acute Exacerbation of Chronic Obstructive Pulmonary Disease. 2018 January 22. PMID: NCT03299231. [Study design not of interest]. 
30. Air Liquide Santé I, Services I, Lincoln M, et al. The COPD Patient Management European Trial (COMET). 2010 September. PMID: NCT01241526. [Population not of interest].

31. Air Liquide Santé I. Efficacy of Helium/Oxygen Compared to Air/Oxygen in Severe Exacerbation of Chronic Obstructive Pulmonary Disease (COPD). 2010 May. PMID: NCT01155310. [Population not of interest].

32. Alderman CP. Cefpodoxime proxetil for infective exacerbations of chronic obstructive airway disease. Annals of Pharmacotherapy. 1996 Feb;30(2):196. PMID: 8835062. [Abstract].

33. Alexa I, Alexa-Stratulat T, Antoniu S, et al. Roflumilast in patients with advanced chronic obstructive pulmonary disease: towards a better-targeted use. Expert Opinion on Pharmacotherapy. 2018. PMID: 625222748. [Duplicate Study].

34. Ali MS, Talwar D, Jain SK. The effect of a short-term pulmonary rehabilitation on exercise capacity and quality of life in patients hospitalised with acute exacerbation of chronic obstructive pulmonary disease. Indian J Chest Dis Allied Sci. 2014;56(1):13-9. [Duplicate Study].

35. Ali MS, Talwar D, Singh RK, et al. Controlled trial of short term (3 WEEKS) pulmonary rehabilitation in copd following acute exacerbation. American Journal of Respiratory and Critical Care Medicine. Conference: American Thoracic Society International Conference, ATS. 2012;185(MeetingAbstracts). PMID: 71987623. [Abstract].

36. Aljassem A, Fodor E, LaBan MM, et al. Pulmonary rehabilitation: Its role in decreasing length of stay, cost of hospitalization, and 30-day readmission rates for chronic obstructive pulmonary disease. PM and R. 2012 October;1):S215S6. PMID: 70909843. [Abstract].
37. Allegra L, Konietzko N, Leophonte P, et al. Comparative safety and efficacy of sparfloxacin in the treatment of acute exacerbations of chronic obstructive pulmonary disease: a double-blind, randomised, parallel, multicentre study. Journal of Antimicrobial Chemotherapy. 1996 May;37 Suppl A:93-104. PMID: 8737129. [Population not of interest].

38. Allegra L, Konietzko N, Leophonte P, et al. Comparative safety and efficacy of sparfloxacin in the treatment of acute exacerbations of chronic obstructive pulmonary disease: a double-blind, randomised, parallel, multicentre study. Journal of Antimicrobial Chemotherapy. 1996;37(suppl_A):93-104. [Duplicate Study].

39. Alvarez Gutierrez FJ, Soto Campos G, el Castillo Otero D, et al. [A randomized comparative study of 3 days of azithromycin treatment and 10 days of cefuroxime treatment in exacerbations in patients with chronic obstructive pulmonary disease]. Medicina Clinica. 1999 Jul 03;113(4):124-8. PMID: 10472596. [Language other than English].

40. Amaducci S, Colombo B, Iuliano A, et al. COPD exacerbations and long term oxygen therapy (LTOT): comparative trial of three empirical antibiotic treatments. European Respiratory Journal - Supplement. 1995;8(Suppl 19):311S. PMID: CN00392893 UPDATE. [Abstract].

41. American Thoracic Society 2018 International Conference. American Journal of Respiratory and Critical Care Medicine. Conference: American Thoracic Society International Conference, ATS. 2018;197(MeetingAbstracts). PMID: 622967216. [Abstract].

42. An Efficacy Study of GSK2269557 Added to Standard Care in Subjects With an Acute Exacerbation of Chronic Obstructive Pulmonary Disease. 2015 March 31. PMID: NCT02294734. [Clinical Trial].

43. Anon Elizalde JM, Garcia de Lorenzo y Mateos A, Alvarez-Sala Walther R, et al. Treatment and prognosis of the severe exacerbation in the chronic obstructive pulmonary disease. Revista Clinica Espanola. 2001;201(11):658-66. PMID: 34026536. [Language other than English]. 
44. Aquinox Pharmaceuticals I. Efficacy and Safety of AQX-1125 in Unstable COPD. 2013 October. PMID: NCT01954628. [Population not of interest].

45. Ardestani ME, Klantar E, Azimian M. Comparison of the efficacy of budesonide nebulizer suspension and budesonide inhalation suspension in the treatment of acute exacerbation of chronic obstructive pulmonary disease. Tanaffos. 2018;17(2):96-102. PMID: 625362133. [Intervention/Comparison not of interest].

46. Assistance Publique - Hôpitaux de P. Efficacy of Corticosteroids to Treat Outpatients With Acute Exacerbations of COPD. 2015 February 10. PMID: NCT02330952. [Clinical Trial].

47. AstraZeneca MTPC. Efficacy and Safety of Roflumilast in Japanese Patients Older Than 40 Years With Chronic Obstructive Pulmonary Disease (APTA-2217-06). 2004 November. PMID: NCT00242294. [Population not of interest].

48. AstraZeneca Q. A Study to Assess Safety, Tolerability and Pharmacokinetics of of AZD6553 in Healthy Volunteers and Patients With Chronic Obstructive Pulmonary Disease (COPD). 2010 February. PMID: NCT01068184. [Population not of interest].

49. Austin MA, Wills KE, Walters EH, et al. Effect of high flow oxygen on mortality in chronic obstructive pulmonary disease patients in prehospital setting: Randomized controlled trial. Academic Emergency Medicine. 2012 April;1):S255. PMID: 70745644. [Population not of interest].

50. AVANTI - Avelox ${ }^{\circledR}$ in Acute Exacerbations of chroNic bronchiTIs. 2008 April. PMID: NCT00846911. [Population not of interest].

51. Avdeev SN, Guseva NA, Nuralieva GS. Efficacy of high frequency chest wall oscillation method in acute exacerbation of chronic obstructive pulmonary disease. [Russian]. Pulmonologiya. 2016;26(4):46672. PMID: 614678609. [Language other than English].

52. Aveiro U. Pulmonary Rehabilitation Innovation and Microbiota in Exacerbations of COPD. 2018 September 1. PMID: NCT03701945. [Intervention/Comparison not of interest].
53. AZD2423 Safety and Tolerability Study in Patients With Moderate and Severe Chronic Obstructive Pulmonary Disease(COPD). 2010 October. PMID: NCT01215279. [Population not of interest].

54. Azevedo P, Costa J, Vaz-Carneiro A. Analysis of the cochrane review: Use of systemic corticosteroids for acute exacerbations of chronic obstructive pulmonary disease. cochrane database syst rev. 2014, 9:Cd001288. [Portuguese]. Acta Medica Portuguesa. 201401 Sep;27(5):53740. PMID: 600320802. [Language other than English].

55. Azienda Ospedaliera San Giovanni B. Hospitalization at Home of Elderly Patients With Exacerbated COPD. 2004 April. PMID: NCT00369083. [Intervention/Comparison not of interest].

56. Babu AS, Noone MS, Haneef M, et al. The effects of 'on-call/out of hours' physical therapy in acute exacerbations of chronic obstructive pulmonary disease: a randomized controlled trial. Clinical Rehabilitation. 2010 Sep;24(9):802-9. PMID: 20543018. [Intervention/Comparison not of interest].

57. Bafadhel M, Davies L, Calverley PMA, et al. Blood eosinophil guided prednisolone therapy for exacerbations of COPD: a further analysis. European Respiratory Journal. 2014 Sep;44(3):789-91. PMID: 24925917. [Study design not of interest].

58. Bafadhel M, Greening NJ, Harvey-Dunston $\mathrm{T}$, et al. Severe hospitalised exacerbations of COPD with an eosinophilic phenotype have favourable outcomes with prednisolone therapy: Sub-analysis from a prospective multi-centre randomised control trial. Thorax. 2013 December;3):A16. PMID: 71367049. [Abstract].

59. Bafadhel M, McKenna S, Terry S, et al. A double-blind randomised control trial of peripheral blood eosinophils to direct prednisolone use in COPD exacerbations. European Respiratory Journal. Conference: European Respiratory Society Annual Congress. 2011;38(SUPPL. 55). PMID: 72122584. [Abstract]. 
60. Bafadhel M, McKenna S, Terry S, et al. Blood eosinophils to direct corticosteroid treatment of exacerbations of chronic obstructive pulmonary disease: a randomized placebo-controlled trial. American Journal of Respiratory \& Critical Care Medicine. 2012 Jul 01;186(1):48-55. PMID: 22447964. [Intervention/Comparison not of interest].

61. Bangabandhu Sheikh Mujib Medical University D. Effect of Vitamin D3 on Lung Function and Exercise Tolerance in COPD Patients. 2017 March 1. PMID: NCT03781895. [Population not of interest].

62. Barber CM, Bradshaw LM, Buttery P, et al. Assisted discharge for patients with exacerbations of COPD [4] (multiple letters). Thorax. 2001;56(5):417-8. PMID: 32398139. [Study design not of interest].

63. Bardsley G, Pilcher J, McKinstry S, et al. Oxygen versus air-driven nebulisers for exacerbations of chronic obstructive pulmonary disease: A randomised controlled trial 11 Medical and Health Sciences 1102 Cardiorespiratory Medicine and Haematology. BMC Pulmonary Medicine. 201803 Oct;18 (1) (no pagination)(157). PMID: 624140780. [Duplicate Study].

64. Bardsley G, Pilcher J, McKinstry S, et al. Oxygen versus air-driven nebulisers for exacerbations of chronic obstructive pulmonary disease: a randomised controlled trial. BMC Pulmonary Medicine. 2018 Oct 03;18(1):157. PMID: 30285695. [Outcome not of interest].

65. Bardsley PA, Tweney J, Morgan N, et al. Oral almitrine in treatment of acute respiratory failure and cor pulmonale in patients with an exacerbation of chronic obstructive airways disease. Thorax. 1991 Jul;46(7):493-8. PMID: 1908604. [Intervention/Comparison not of interest].

66. Baroi S, McNamara RJ, McKenzie DK, et al. Advances in Remote Respiratory Assessments for People with Chronic Obstructive Pulmonary Disease: A Systematic Review. Telemedicine Journal \& E-Health. 2018 06;24(6):415-24. PMID: 29083268. [Population not of interest].
67. Barry HC. Amoxicillin/clavulanate during COPD exacerbations decreases symptoms and delays subsequent exacerbations. American Family Physician. 201301 Mar;87(7):512. PMID: 368623481. [Study design not of interest].

68. Basran GS, Joseph J, Abbas AM, et al. Treatment of acute exacerbations of chronic obstructive airways disease--a comparison of amoxycillin and ciprofloxacin. Journal of Antimicrobial Chemotherapy. 1990 Dec;26 Suppl F:19-24. PMID: 2292540. [Population not of interest].

69. Baveja S, Akpa B, Ferris J, et al. A randomized, double dummy, crossover study comparing the bronchodilator effect of formoterol administered by nebulizer versus dry powder inhaler in patients recovering from severe exacerbations of chronic obstructive pulmonary disease. American Journal of Respiratory and Critical Care Medicine. Conference: American Thoracic Society International Conference, ATS. 2018;197(MeetingAbstracts). PMID: 622969450. [Abstract].

70. Beijing Chao Yang H. Treatment in Patients Hospitalized With Acute Exacerbation of Chronic Obstructive Pulmonary Disease. 2014 August. PMID: NCT02219360. [Study design not of interest].

71. Benzo R, Vickers K, Novotny PJ, et al. Health Coaching and Chronic Obstructive Pulmonary Disease Rehospitalization. A Randomized Study. American Journal of Respiratory \& Critical Care Medicine. 2016 Sep 15;194(6):672-80. PMID: 26953637. [Intervention/Comparison not of interest].

72. Berry RB, Shinto RA, Wong FH, et al. Nebulizer vs spacer for bronchodilator delivery in patients hospitalized for acute exacerbations of COPD. Chest. 1989 Dec;96(6):1241-6. PMID: 2582828. [Intervention/Comparison not of interest].

73. Bhatt SP, Sanders JG, Anderson EM, et al. Telehealth exercise intervention reduces COPD readmissions. American Journal of Respiratory and Critical Care Medicine. Conference: American Thoracic Society International Conference, ATS. 2018;197(MeetingAbstracts). PMID: 622965773. [Abstract]. 
74. Bilancia R, Sebastio G, Mastrandrea L, et al. Ceftriaxone+amikacin versus piperacillin+amikacin in the treatment of COPD infectious exacerbations. Clinical and economical evaluation. [Italian]. Minerva Pneumologica. 1998 June;37(2):47-53. PMID: 28535662. [Language other than English].

75. Boehringer I. Combivent Respimat 1-year Safety Study in Patients With Chronic Obstructive Pulmonary Disease. 2009 November. PMID: NCT01019694. [Population not of interest].

76. Boehringer I. Comparative Effectiveness of COPD Treatments. 2017 December 1. PMID: NCT03376295. [Study design not of interest].

77. Boehringer I. Comparison of Safety and Efficacy of COMBIVENT HFA to COMBIVENT (CFC) in Patients With Chronic Obstructive Pulmonary Disease (COPD). 2000 October. PMID: NCT02194205. [Population not of interest].

78. Boehringer I. Effectiveness of Triple Therapy in COPD. 2018 November 10. PMID: NCT03724877. [Population not of interest].

79. Boehringer I. Efficacy and Safety of Tiotropium Compared to Salmeterol and Placebo in Patients With Chronic Obstructive Bronchitis (COPD). 1999 February. PMID: NCT02173691. [Article not available ].

80. Boehringer I. Investigate the Impact of Early Treatment Initiation With Tiotropium in Patients Recovering From Hospitalization for an Acute COPD Exacerbation 1. 2012 August. PMID: NCT01663987. [Population not of interest].

81. Boehringer I. Investigate the Impact of Early Treatment Initiation With Tiotropium in Patients Recovering From Hospitalization for an Acute COPD Exacerbation 2. 2012 August. PMID: NCT01662986. [Population not of interest].
82. Boehringer I. Ipratropium

Bromide/Salbutamol Delivered by the Respimat ${ }^{\circledR}$ Inhaler Compared to Ipratropium Bromide Respimat ${ }^{\circledR}$, COMBIVENT ${ }^{\circledR}$ Inhalation Aerosol and Placebo in Adults With Chronic Obstructive Pulmonary Disease. 2002 October. PMID: NCT02177253. [Population not of interest].

83. Boehringer I. Multiple Dose Comparison of Tiotropium Inhalation Capsules, Salmeterol Inhalation Aerosol and Placebo in Patients With Chronic Obstructive Pulmonary Disease (COPD). 1999 February. PMID: NCT02172287. [Population not of interest].

84. Boehringer I. Respimat ${ }^{\circledR}$ Combivent Trial in Chronic Obstructive Pulmonary Disease (COPD). 2006 November. PMID: NCT00400153. [Population not of interest].

85. Boehringer I. Safety and Efficacy of BI 1744 CL in Patients With Chronic Obstructive Pulmonary Disease I. 2009 February. PMID: NCT00793624. [Population not of interest].

86. Boehringer I. Safety and Efficacy of BI 1744 CL in Patients With Chronic Obstructive Pulmonary Disease II. 2009 January. PMID: NCT00796653. [Population not of interest].

87. Boehringer I. Tiotropium (18 Mcg) Once Daily Via the HandiHaler ${ }^{\circledR}$ in Patients With Chronic Obstructive Pulmonary Disease (COPD). 2001 September. PMID: NCT00274547. [Population not of interest].

88. Borglykke A, Pisinger C, Jorgensen T, et al. The effectiveness of smoking cessation groups offered to hospitalised patients with symptoms of exacerbations of chronic obstructive pulmonary disease (COPD). The clinical respiratory journal. 2008 Jul;2(3):158-65. PMID: 20298324. [Intervention/Comparison not of interest].

89. Borodulina EA, Chernogayeva GY, Gladunova EP, et al. Noninvasive ventilation and inhalational nebulized therapy in the intensive care of acute exacerbation of chronic obstructive pulmonary disease. [Russian]. Pulmonologiya. 2018;28(3):313-7. PMID: 623324697. [Language other than English]. 
90. Bourbeau J, Kessler R, Casan P, et al. An international randomised study of a homebased self-management program for severe COPD: The COPD patient management European trial. Canadian Journal of Respiratory Critical Care and Sleep Medicine. 2017;1 (2):98. PMID: 624482909. [Population not of interest].

91. Boyd M, Blakely T, Atkinson J. Ethnic counts on mortality, New Zealand Cancer Registry and census data: 2006-2011. New Zealand Medical Journal. 2016 Jan 29;129(1429):22-39. PMID: 26914296. [Population not of interest].

92. Boye NP, Gaustad P. Double-blind comparative study of ofloxacin (Hoe 280) and trimethoprim-sulfamethoxazole in the treatment of patients with acute exacerbations of chronic bronchitis and chronic obstructive lung disease. Infection. 1991;19 Suppl 7:S388-90. PMID: 1804789. [Population not of interest].

93. Breo Ellipta: An inhaled fluticasone/ vilanterol combination for COPD. Medical Letter on Drugs and Therapeutics. 201302 Sep;55(1424):69-71. PMID: 369736488. [Study design not of interest].

94. Brightling CE, Monteiro W, Ward R, et al. Sputum eosinophilia and short-term response to prednisolone in chronic obstructive pulmonary disease: a randomised controlled trial. The Lancet. 2000;356(9240):1480-5. [Duplicate Study].

95. Budesonide Formoterol in Treatment of Exacerbations of COPD. 2001 January. PMID: NCT00239278. [Clinical Trial].

96. Buess M, Schilter D, Schneider T, et al. Treatment of COPD Exacerbation in Switzerland: results and Recommendations of the European COPD Audit. Respiration. 2017(pagination). PMID: CN-01395049 NEW. [Abstract].

97. Buess M, Schneider T, Maurer M, et al. Treatment of COPD exacerbation in Switzerland - Results and recommendations of the European COPD audit. Respiration. 2017;94 (1):76. PMID: 623934064. [Duplicate Study].
98. Bullard MJ, Liaw SJ, Tsai YH, et al. Early corticosteroid use in acute exacerbations of chronic airflow obstruction. American Journal of Emergency Medicine. 1996 Mar;14(2):139-43. PMID: 8924134. [Population not of interest].

99. Campus Bio-Medico U, National Institute of Environmental Health S. Hyaluronic Acid in Acute Exacerbation of COPD - Duration Of Noninvasive Assistance. 2016 March. PMID: NCT02674880. [Intervention/Comparison not of interest].

100.Cantex P. A Study Designed to Evaluate ODSH in Subjects With Exacerbations of COPD. 2007 April. PMID: NCT00457951. [Clinical Trial].

101.Catalan Society of Family M, Fondo de Investigacion S. Effectiveness of Antibiotic Therapy for Exacerbations of Chronic Obstructive Pulmonary Disease. 2007 October. PMID: NCT00495586. [Clinical Trial].

102.Cazzola M, Califano C, Di Perna F, et al. Acute effects of higher than customary doses of salmeterol and salbutamol in patients with acute exacerbation of COPD. Respiratory Medicine. 2002 Oct;96(10):7905. PMID: 12412978. [Intervention/Comparison not of interest].

103.Cazzola M, D'Amato M, Califano C, et al. Formoterol as dry powder oral inhalation compared with salbutamol metered-dose inhaler in acute exacerbations of chronic obstructive pulmonary disease. Clinical Therapeutics. 2002 Apr;24(4):595-604. PMID: 12017404. [Intervention/Comparison not of interest].

104.Cazzola M, Matera MG, Amato M, et al. Long-acting $\beta 2$-agonists in the treatment of acute exacerbations of COPD. Clinical Drug Investigation. 2002;22(6):369-76. [Duplicate Study].

105.Cazzola M, Matera MG, D'Amato M, et al. Long-acting beta2-agonists in the treatment of acute exacerbations of COPD. Clinical drug investigation. 2002;22(6):369-76. PMID: CN-00443342 UPDATE. [Intervention/Comparison not of interest]. 
106.Cazzola M, Rogliani P, Calzetta L, et al. Impact of Mucolytic Agents on COPD Exacerbations: A Pair-wise and Network Meta-analysis. Copd: Journal of Chronic Obstructive Pulmonary Disease. 2017 Oct;14(5):552-63. PMID: 28753070. [Intervention/Comparison not of interest].

107.Cazzola M, Salzillo A, De Giglio C, et al. Formoterol/budesonide (FBC) in the treatment of acute exacerbation of COPD. Eur Respir J. 2003;22(suppl 45):395s. [Duplicate Study].

108.Cazzola M, Santus P, Matera MG, et al. A single high dose of formoterol is as effective as the same dose administered in a cumulative manner in patients with acute exacerbation of COPD. Respiratory Medicine. 2003 May;97(5):458-62. PMID: 12735660. [Intervention/Comparison not of interest].

109. Center for Integrated Rehabilitation OFH, Centocor I. Effects of Infliximab (Remicade) on Fat Free Mass in Patients With Moderate to Severe COPD Suffering From Cachexia. 2003 October. PMID: NCT00244192. [Population not of interest].

110.Centre de Recherche de l'Institut Universitaire de Cardiologie et de Pneumologie de Q. Using a Closed-loop System for Oxygen Delivery (FreeO2) to Optimize Oxygentherapy in Patients With COPD Exacerbation. 2011 July. PMID: NCT01393015. [Outcome not of interest].

111. Chang CL, Wong C, Beckert L, et al. betablockers in exacerbations of COPD: Feasibility of a randomised controlled trial. ERJ Open Research. 201701 Jan;3 (1) (no pagination)(00090-2016). PMID:

614172090. [Study design not of interest].

112. Chang CL, Wong C, Beckert L, et al. $\beta$ blockers in exacerbations of COPD: Feasibility of a randomised controlled trial. ERJ Open Research. 2017;3(1). [Study design not of interest].
113.Chen G, Xie C-M, Luo Y-F. [The effects and therapeutic duration of oral corticosteroids in patients with acute exacerbation of chronic obstructive pulmonary diseases]. Chung-Hua Chieh Ho Ho Hu Hsi Tsa Chih Chinese Journal of Tuberculosis \& Respiratory Diseases. 2008 Aug;31(8):577-80. PMID: 19080399. [Language other than English].

114. Chen HY, Ma CH, Cao K-J, et al. A systematic review and meta-analysis of herbal medicine on chronic obstructive pulmonary diseases. Evidence-Based Complementary \& Alternative Medicine: eCAM. 2014;2014:925069. PMID: 24795773. [Intervention/Comparison not of interest].

115. Chest Hospital MoH, Welfare T. Respiratory Rehabilitation Exercises in Older Adults With Acute Exacerbations of Chronic Obstructive Pulmonary Disease. 2011 November. PMID: NCT02329873. [Clinical Trial].

116. Chiesi Farmaceutici SpA. 2-arm Parallel Group Study of Fixed Combination of CHF 5993 vs Ultibro ${ }^{\circledR}$ in COPD Patients. 2015 May 29. PMID: NCT02579850. [Population not of interest].

117. Chiesi Farmaceutici SpA. A 24-wk Dose Ranging Study to Evaluate the Efficacy and Safety of 4 Doses of a New PDE4 Inhibitor in Patients With COPD. 2016 December 15. PMID: NCT02986321. [Population not of interest].

118. Chiesi Farmaceutici SpA. Active Controlled Trial of CHF5993 Pressurized Metered-dose Inhaler ( pMDI) vs Symbicort ${ }^{\circledR}$ Turbuhaler ${ }^{\circledR}$ in Patients With Chronic Obstructive Pulmonary Disease ( COPD). 2016 December. PMID: NCT03197818. [Population not of interest].

119. Chiesi Farmaceutici SpA. Efficacy of Fixed Combination of Beclometasone + Formoterol + Glycopyrrolate in Chronic Obstructive Pulmonary Disease. 2014 January. PMID: NCT01911364. [Population not of interest].

120.Chiesi Farmaceutici SpA. Efficacy of Fixed Combination of Beclometasone + Formoterol + Glycopyrrolate Versus Foster® in COPD. 2014 March. PMID: NCT01917331. [Population not of interest]. 
121.China-Japan Friendship H, Peking U, China Soong Ching Ling F. Acute Exacerbation of Chronic Obstructive Pulmonary Disease Inpatient Registry Study. 2016 January. PMID: NCT02657525. [Study design not of interest].

122.China-Japan Friendship H. Extracorporeal Carbon Dioxide Removal in Severe Chronic Obstructive Pulmonary Disease Exacerbation. 2017 January. PMID: NCT03692117. [Study design not of interest].

123. Chinese Pla General Hospital FHattCPLAGH, Chinese People's Liberation Army RFGH, Chinese People's Liberation Army HoPLA. Evaluate the Efficacy of Xuebijing Injection in Acute Exacerbations of COPD. 2016 October. PMID: NCT02937974. [Intervention/Comparison not of interest].

124. Chinese University of Hong K. Early Pulmonary Rehabilitation for Hospitalized Patients With Exacerbations of Chronic Obstructive Pulmonary Disease (COPD). 2006 January. PMID: NCT00287625. [Clinical Trial].

125. Chinese University of Hong K. Short-course Out-patient Pulmonary Rehabilitation and COPD Exacerbations. 2015 July. PMID: NCT02471235. [Population not of interest].

126. Chow JWY, Khullar K, Katechia K, et al. Do ambulance paramedics administer too much oxygen to patients with acute exacerbations of chronic obstructive airways disease? EMA - Emergency Medicine Australasia. 201501 Jun;27(3):275-6. PMID: 603966118. [Study design not of interest].

127.Chronic Obstructive Pulmonary Disease (COPD)-Related Outcomes and Costs for Patients on Combination Fluticasone Propionate-Salmeterol Xinafoate 250/50mcg Versus Anticholinergics in a COPDComorbid Depression/Anxiety Population. 2010 October. PMID: NCT01337336. [Population not of interest].
128. Cimera HSE, Hospital Doce De Octubre HCoB, Germans Trias i Pujol Hospital HFJD, et al. Study of Chronic Obstructive Pulmonary Disease (COPD) Exacerbations In Spain. 2006 September. PMID: NCT01750658. [Intervention/Comparison not of interest].

129. City UoL. Fan Therapy in COPD Patients. 2017 May 24. PMID: NCT03137524. [Population not of interest].

130.Clini EM, Crisafulli E, Costi S, et al. Effects of early inpatient rehabilitation after acute exacerbation of COPD. Respiratory medicine. 2009;103(10):1526-31. [Duplicate Study].

131. Clinical Outcome of Corticosteroids in the Treatment of COPD Exacerbations in China. 2017 February 28. PMID: NCT03633838. [Study design not of interest].

132. Colebourn CL, Barber V, Young JD. Use of helium-oxygen mixture in adult patients presenting with exacerbations of asthma and chronic obstructive pulmonary disease: a systematic review. Anaesthesia. 2007 Jan;62(1):34-42. PMID: 17156225. [Population not of interest].

133. Comert S, Kiyan E, Okumus G, et al. [Efficiency of nebulised magnesium sulphate in infective exacerbations of chronic obstructive pulmonary disease]. Tuberkuloz ve Toraks. 2016 Mar;64(1):1726. PMID: 27266281. [Language other than English].

134. Comparative study of adaptive support ventilation vs synchronized intermittent mandatory ventilation in acute exacerbation of COPD patients. Chest. 2017;Conference: CHEST 2017 Annual Meeting. Canada. 152(4 Supplement 1):A228. PMID: CN01622619. [Abstract].

135. Comparative Study of Fluticasone Furoate(FF)/Umeclidinium Bromide (UMEC)/ Vilanterol (VI) Closed Therapy Versus FF/VI Plus UMEC Open Therapy in Subjects With Chronic Obstructive Pulmonary Disease (COPD). 2016 June 29. PMID: NCT02729051. [Population not of interest].

136.Comparison Between Symbicort ${ }^{\circledR}$ and Prednisolone in COPD. 2005 September. PMID: NCT00259779. [Clinical Trial]. 
137. Cordero PJ, Benlloch E, Sol AA, et al. Corticosteroid therapy for COPD exacerbations at inpatient settings: a controlled-randomized study. Archivos de bronconeumologa-a. 1996;32(Suppl 2):17. PMID: CN-00689747. [Language other than English].

138. Cordero PJ, Sole A, Benlloch E, et al. A randomized controlled study of prednisone in outpatients with acute exacerbation of COPD. European Respiratory Journal Supplement. 1996;9(Suppl 23):110s-1s. PMID: CN-00382559 UPDATE. [Abstract].

139. Cotton MM, Bucknall CE, Dagg KD, et al. Early discharge for patients with exacerbations of chronic obstructive pulmonary disease: a randomized controlled trial. Thorax. 2000 Nov;55(11):902-6. PMID: 11050257. [Intervention/Comparison not of interest].

140.Cox M, O'Connor C, Biggs K, et al. The feasibility of early pulmonary rehabilitation and activity after COPD exacerbations: external pilot randomised controlled trial, qualitative case study and exploratory economic evaluation. Health Technology Assessment (Winchester, England). 2018 Mar;22(11):1-204. PMID: 29516853. [Duplicate Study].

141.Criner GJ, Dransfield MT, Arora S, et al. A randomized, double-blind, placebo controlled, multicenter phase 2a study to assess safety, daily respiratory symptoms, pharmacokinetics, and biomarker variations after administration of either YPL-001, or placebo in patients with moderate to severe chronic obstructive pulmonary disease. American Journal of Respiratory and Critical Care Medicine. Conference: American Thoracic Society International Conference, ATS. 2018;197(MeetingAbstracts). PMID: 622970903. [Abstract].

142. Cross J, Elender F, Barton G, et al. A randomised controlled equivalence trial to determine the effectiveness and cost-utility of manual chest physiotherapy techniques in the management of exacerbations of chronic obstructive pulmonary disease (MATREX). Health Technology Assessment (Winchester, England). 2010 May;14(23):1147, iii-iv. PMID: 20487638.

[Intervention/Comparison not of interest].
143. Cross J, Elender F, Barton G, et al. A randomised controlled equivalence trial to determine the effectiveness and cost-utility of manual chest physiotherapy techniques in the management of exacerbations of chronic obstructive pulmonary disease (MATREX). Health Technology Assessment. 2010;14(23):i-175. [Duplicate Study].

144. Cushen B, Alsaid A, Abdulkareem A, et al. Pilot study to assess bronchodilator response during an acute exacerbation of COPD using a vibrating mesh nebuliser versus jet nebuliser for bronchodilator delivery. Thorax. 2016 December;71 (Supplement 3):A250. PMID: 615030777. [Abstract].

145.Cydulka RK, Emerman CL. Effects of combined treatment with glycopyrrolate and albuterol in acute exacerbation of chronic obstructive pulmonary disease. Annals of Emergency Medicine. 1995 Apr;25(4):4703. PMID: 7710150. [Study design not of interest].

146.Daitx RB, Dos Santos K, Dohnert MB, et al. Limited utility of Kinesio Taping in the physiotherapy treatment for patients with chronic obstructive pulmonary disease exacerbation. Physiotherapy Theory \& Practice. 2018 Jan 08:1-6. PMID: 29308939. [Intervention/Comparison not of interest].

147.Daitx RB, Dos Santos K, Dohnert MB, et al. Limited utility of Kinesio Taping(R) in the physiotherapy treatment for patients with chronic obstructive pulmonary disease exacerbation. Physiotherapy theory and practice. 2018. PMID: CN-01456787 NEW. [Duplicate Study].

148.Dal Negro R, Caputo M, Conti A, et al. Procalcitonin guided assessment of bacterial etiological role in patients admitted for severe COPD exacerbations a double blind study [Abstract]. European respiratory journal. 2007;30(Suppl 51):224s [E1371]. PMID: CN-00642828 UPDATE. [Abstract].

149.Danish Procalcitonin Study Group CUH. Procalcitonin To Reduce Antibiotics in Chronic Obstructive Lung Disease (ProToCOLD). 2012 November. PMID: NCT01950936. [Intervention/Comparison not of interest]. 
150.Dasgupta A, Kjarsgaard M, Capaldi D, et al. A pilot randomised clinical trial of mepolizumab in COPD with eosinophilic bronchitis. European Respiratory Journal. 2017;49(3). [Population not of interest].

151.Davies L, Angus RM, Calverley PMA. Oral corticosteroids in patients admitted to hospital with exacerbations of chronic obstructive pulmonary disease: a prospective randomised controlled trial. The Lancet. 1999;354(9177):456-60. [Duplicate Study].

152.Davies L, Angus RMCPMA. A prospective, randomised, double-blind, placebo controlled study of oral corticosteroids in patients admitted with normocapnic acute exacerbations of chronic obstructive pulmonary disease (COPD). Thorax. Issue. 1997;52(6). PMID: CN-00267032 UPDATE. [Study design not of interest].

153.Davies L, Wilkinson M, Bonner S, et al. "Hospital at home" versus hospital care in patients with exacerbations of chronic obstructive pulmonary disease: prospective randomised controlled trial. BMJ. $2000 \mathrm{Nov}$ 18;321(7271):1265-8. PMID: 11082090. [Intervention/Comparison not of interest].

154. Davis L, Wilkinson M, Bonner S, et al. "Hospital at Home" versus hospital care in patients with exacerbations of chronic obstructive pulmonary disease: Prospective randomised controlled trial. Revista Portuguesa de Pneumologia. 2001;7(2):1635. PMID: 32452045.

[Intervention/Comparison not of interest].

155.De Graaf P, Doggen CJM, De Jongh FH, et al. Evaluation of the effect of the alphacoreTM on breathlessness and time to discharge in hospitalized patient with chronic obstuctive pulmonary disease (COPD). American Journal of Respiratory and Critical Care Medicine. Conference: American Thoracic Society International Conference, ATS. 2013;187(MeetingAbstracts). PMID: 71980821. [Population not of interest].

156.Denysova O, Sukhin R. Efficacy of administration of $0.02 \%$ solution of decamethoxine via nebulizer in patients with infectious exacerbations of COPD. European Respiratory Journal. Conference: European Respiratory Society Annual Congress. 2013;42(SUPPL. 57). PMID: 71840611. [Abstract].
157.Devereux G, Cotton S, Fielding S, et al. Effect of Theophylline as Adjunct to Inhaled Corticosteroids on Exacerbations in Patients With COPD: A Randomized Clinical Trial. JAMA. 201810 16;320(15):1548-59. PMID: 30326124. [Population not of interest].

158.Di Marco F, Verga M, Santus P, et al. Effect of formoterol, tiotropium, and their combination in patients with acute exacerbation of chronic obstructive pulmonary disease: a pilot study. Respiratory Medicine. 2006 Nov;100(11):1925-32. PMID: 16626956. [Intervention/Comparison not of interest].

159.Diez-Manglano J, Barquero-Romero J, Mena PA, et al. Polypharmacy in patients hospitalised for acute exacerbation of COPD. European Respiratory Journal. 2014 01 Sep;44(3):791-4. PMID: 373874384. [Study design not of interest].

160.Dixit D, Bridgeman MB, Madduri RP, et al. Pharmacological management and prevention of exacerbations of chronic obstructive pulmonary disease in hospitalized patients. $\mathrm{P}$ and $\mathrm{T}$. 2016;41(11):703-12. [Study design not of interest].

161.Dong L, Xia JW, Gong Y, et al. Effect of lianhuaqingwen capsules on airway inflammation in patients with acute exacerbation of chronic obstructive pulmonary disease. Evidence-based Complementary and Alternative Medicine. 2014;2014 (no pagination)(637969). PMID: 373279335. [Intervention/Comparison not of interest].

162. Dose Finding Study of Nemiralisib (GSK2269557) in Subjects With an Acute Moderate or Severe Exacerbation of Chronic Obstructive Pulmonary Disease (COPD). Https://clinicaltrials.gov/show/nct03345407. 2017. PMID: CN-01565900. [Duplicate Study].

163.Dr. López V, Puerta de Hierro University H. Early Assisted Discharge for COPD Exacerbations With Telemonitoring. 2012 March. PMID: NCT01951261. [Population not of interest]. 
164.Dransfield MT, Rowe SM, Johnson JE, et al. Use of $\beta$ blockers and the risk of death in hospitalised patients with acute exacerbations of COPD. Thorax. 2008;63(4):301-5. [Intervention/Comparison not of interest].

165.Duenk RG, Verhagen C, Bronkhorst EM, et al. Proactive palliative care for patients with COPD (PROLONG): A pragmatic cluster controlled trial. International Journal of COPD. 2017;12:2795-806. [Study design not of interest].

166.Duran CA, Winnicka L, Bista A, et al. Reducing hospital readmission among high risk patients admitted with COPD exacerbation: Randomized trial of a respiratory therapist conducted home-based structured intervention. American Journal of Respiratory and Critical Care Medicine. Conference: American Thoracic Society International Conference, ATS. 2017;195(no pagination). PMID: 617713443. [Abstract].

167.Durzo A, Bader G, Shen S, et al. Comparison of glycopyrronium versus tiotropium on the time to clinically important deteriorations in patients with COPD: A post-hoc analysis of randomized trials. npj Primary Care Respiratory Medicine. 2018;28(1). [Population not of interest].

168.e la Poza Abad M, Mas Dalmau G, Moreno Bakedano M, et al. Rationale, design and organization of the delayed antibiotic prescription (DAP) trial: a randomized controlled trial of the efficacy and safety of delayed antibiotic prescribing strategies in the non-complicated acute respiratory tract infections in general practice. BMC Family Practice. 2013 May 19;14:63. PMID: 23682979. [Population not of interest].

169.Early Extubation by ECCO2R Compared to IMV in Patients With Severe Acute Exacerbation of COPD. Https://clinicaltrials.gov/show/nct03584295. 2018. PMID: CN-01625629. [Population not of interest].
170.eBoisblanc BP, DeBleiux P, Resweber S, et al. Randomized trial of the use of heliox as a driving gas for updraft nebulization of bronchodilators in the emergent treatment of acute exacerbations of chronic obstructive pulmonary disease. Critical Care Medicine. 2000 Sep;28(9):3177-80. PMID: 11008978. [Intervention/Comparison not of interest].

171.Echevarria C, Brewin K, Horobin H, et al. Early supported discharge/hospital at home for exacerbation of chronic obstructive pulmonary disease, A review and metaanalysis. European Respiratory Journal. Conference: European Respiratory Society Annual Congress. 2016;48(Supplement 60). PMID: 614780019.

[Intervention/Comparison not of interest].

172.Edmond P. The Efficacy and Safety of Erdosteine in Chronic Obstructive Pulmonary Disease (COPD). 2009 August. PMID: NCT01032304. [Population not of interest].

173.Effect of Acupuncture on Patients With Chronic Obstructive Pulmonary Disease. Https:/clinicaltrials.gov/show/nct03169504. 2017. PMID: CN-01494541. [Duplicate Study].

174.Effect of Roflumilast on Lung Function in Chronic Obstructive Pulmonary Disease (COPD) Patients Treated With Salmeterol: The EOS Study (BY217/M2-127). 2006 April. PMID: NCT00313209. [Population not of interest].

175.Effectiveness of Vibrating Mesh Versus Small Volume Nebuliser in Chronic Obstructive Pulmonary Disease (COPD). Https://clinicaltrials.gov/show/nct03286855. 2017. PMID: CN-01564180. [Duplicate Study].

176. Efficacy and Safety of Aclidinium Bromide at Two Dose Levels Versus Placebo Administered in Chronic Obstructive Pulmonary Disease (COPD) Patients. 2009 October. PMID: NCT01001494. [Population not of interest].

177.Efficacy and Safety of Levalbuterol in Chronic Obstructive Pulmonary Disease (COPD). 2002 February. PMID: NCT00665600. [Population not of interest]. 
178.Efficacy and Safety of Mepolizumab as an Add-on Treatment in Chronic Obstructive Pulmonary Disease (COPD). 2014 April 24. PMID: NCT02105961. [Population not of interest].

179.Efficacy and Safety of Oral Roflumilast Taken Once Daily in Patients Older Than 40 Years With Chronic Obstructive Pulmonary Disease (BY217/M2-119). 2005 August. PMID: NCT00242320. [Population not of interest].

180.Efficacy and Tolerability of Symbicort as an add-on Treatment to Spiriva Compare With Spiriva Alone in Patients With Severe Chronic Obstructive Pulmonary Disease (COPD). 2011 July. PMID: NCT01397890. [Population not of interest].

181.Efficiency of Budesonide Combined With Formoterol and Tiotropium in the Treatment of Acute Exacerbation of ACO. Https://clinicaltrials.gov/show/nct03504527. 2018. PMID: CN-01568258. [Study design not of interest].

182.Effing T, Kerstjens H, van der Valk P, et al. (Cost)-effectiveness of self-treatment of exacerbations on the severity of exacerbations in patients with COPD: the COPE II study. Thorax. 2009 Nov;64(11):956-62. PMID: 19736179. [Intervention/Comparison not of interest].

183. Eisai Co L. Effects of Lysozyme on Acute Exacerbation of Chronic Obstructive Pulmonary Disease : A Randomised Placebo-Controlled Study. 2012 August. PMID: NCT01645800. [Population not of interest].

184.ElectroCore LLC. Vagus Nerve Stimulation for Management of Bronchoconstriction in Patients Hospitalized With COPD Exacerbations. 2012 October. PMID: NCT01612793. [Clinical Trial].

185.Elpen Pharmaceutical Co I. EvAluation of Clinical Effectiveness Of RoLenium Administered With Elpenhaler in Chronic Obstructive Pulmonary Disease (COPD) patientS in Daily Clinical Practice, in Greece. 2017 January. PMID: NCT02978703. [Population not of interest].
186.Emami Ardestani M, Kalantary E, Samaiy $\mathrm{V}$, et al. Methyl prednisolone vs Dexamethasone in Management of COPD Exacerbation; a Randomized Clinical Trial. Emergency (Tehran, Iran). 2017;5(1):e35. PMID: 28286842. [Duplicate Study].

187.Emerman CL, Cydulka RK. Effect of different albuterol dosing regimens in the treatment of acute exacerbation of chronic obstructive pulmonary disease. Annals of Emergency Medicine. 1997 Apr;29(4):4748. PMID: 9095007. [Intervention/Comparison not of interest].

188. Erbland ML, Deupree RH, Niewoehner DE. Systemic Corticosteroids in Chronic Obstructive Pulmonary Disease Exacerbations (SCCOPE): rationale and design of an equivalence trial. Veterans Administration Cooperative Trials SCCOPE Study Group. Controlled Clinical Trials. 1998 Aug;19(4):404-17. PMID: 9683314. [Study design not of interest].

189.Erratum: a multicenter randomized trial assessing the efficacy of helium/oxygen in severe exacerbations of chronic obstructive pulmonary disease (American Journal of Respiratory and Critical Care Medicine (2017) 195 (871-880) DOI: 10.1164/rccm.201601-00830C). American journal of respiratory and critical care medicine. 2018;197(6):839-40. PMID: CN01571373. [Study design not of interest].

190.Exacerbation recovery patterns in newly diagnosed or maintenance treatment-naive patients with COPD: secondary analyses of TICARI 1 trial data. International journal of COPD. 2018;13(pp 1515-1525). PMID: CN01628506 NEW. [Duplicate Study].

191.Extracorporeal CO2 Removal With the Hemolung RAS for Mechanical Ventilation Avoidance During Acute Exacerbation of COPD.

Https:/clinicaltrials.gov/show/nct03255057. 2017. PMID: CN-01495891. [Population not of interest].

192.Fabbri LM, Luppi F, Beghe B, et al. Update in chronic obstructive pulmonary disease 2005. American Journal of Respiratory and Critical Care Medicine. 200615 May;173(10):1056-65. PMID: 43726885. [Study design not of interest]. 
193.Faber M, Jahnz-Rozyk K, Targowski T, et al. [Comparative analysis of the effectiveness and costs of azithromycin and cefoperazone treatment of patients during COPD exacerbation]. Polski Merkuriusz Lekarski. 2003 Jan;14(79):36-8. PMID: 12712826. [Language other than English].

194.Facius A, Bagul N, Gardiner P, et al. Pharmacokinetics and pharmacodynamics of a 4-week up-titration regimen of roflumilast in the optimize study. Pneumologie. Conference. 2018;59. PMID: 622904793. [Abstract].

195.Fadoi Foundation I. Comparison Between Levofloxacin and Prulifloxacin, in Internal Medicine Patients With Acute Exacerbation of COPD. 2009 May. PMID: NCT01710488. [Clinical Trial].

196. Falagas ME, Avgeri SG, Matthaiou DK, et al. Short- versus long-duration antimicrobial treatment for exacerbations of chronic bronchitis: a meta-analysis. Journal of Antimicrobial Chemotherapy. 2008 Sep;62(3):442-50. PMID: 18467303. [Population not of interest].

197.Fally M, Corti C, Fabricius-Bjerre A, et al. Point-of-care procalcitonin test to reduce antibiotics in COPD exacerbation: A quasirandomised control trial. European Respiratory Journal. 2015;46. PMID: CN01135066 NEW. [Abstract].

198.Far Eastern Memorial H. The Doses of Systemic Steroid in Acute Exacerbation of Chronic Obstructive Pulmonary Disease (AECOPD). 2010 January. PMID: NCT01215825. [Study design not of interest].

199.Farquhar D. Inhaled corticosteroids and COPD. Cmaj. 200008 Aug;163(3):326-7. PMID: 30670695. [Population not of interest].

200.Fartoukh M, Similowski T, Brun-Buisson C. ANTEAB: A study of early antibiotherapy in the ICU management of acute exacerbations of COPD. [French]. Revue des Maladies Respiratoires. 2004 April;21(2 PART 1):381-9. PMID: 38858506. [Intervention/Comparison not of interest].
201.Fekri A, Hôpital Universitaire Fattouma B. Oral Prednisolone in Severe Acute Exacerbation of Chronic Obstructive Pulmonary Disease (COPD). 2010 April. PMID: NCT01353235. [Population not of interest].

202.Ferguson G, Barnes C, Pendyala S, et al. Efficacy and safety of revefenacin, a longacting muscarinic antagonist for nebulization from phase 3 trials in patients with moderate to very severe chronic obstructive pulmonary disease. Consultant Pharmacist. 2018 October;33 (10):600. PMID: 625245305. [Abstract].

203.Ferguson GT, Buhl R, Bothner U, et al. Safety of tiotropium/olodaterol in chronic obstructive pulmonary disease: pooled analysis of three large, 52-week, randomized clinical trials. Respiratory Medicine. 2018 October;143:67-73. PMID: 2001076207. [Population not of interest].

204.Fernandez Blanco R, Diez Rodriguez C, Garcia Pozo AM, et al. [Evaluation of the Efficiency of Cleaning the Air between Two Methods of Making in Patients with Aerosol Chronic Obstructive Pulmonary Disease]. Revista de Enfermeria. 2015 Jan;38(1):32-7. PMID: 26540907. [Language other than English].

205.Finch AP, van Velzen P, Ter Riet G, et al. Doxycycline Added to Prednisolone in Outpatient-Treated Acute Exacerbations of COPD: A Cost-Effectiveness Analysis Alongside a Randomised Controlled Trial. Pharmacoeconomics. 2018 Dec 19;19:19. PMID: 30565022. [Intervention/Comparison not of interest].

206.Folch-Ayora A, Orts-Cortes MI, MaciaSoler L, et al. Patient education during hospital admission due to exacerbation of chronic obstructive pulmonary disease: Effects on quality of life-Controlled and randomized experimental study. Patient Education \& Counseling. 2018 Sep 16;16:16. PMID: 30279028. [Intervention/Comparison not of interest]. 
207.Folch-Ayora A, Orts-Cortés MI, MaciaSoler L, et al. Patient education during hospital admission due to exacerbation of chronic obstructive pulmonary disease: Effects on quality of life-Controlled and randomized experimental study. Patient Education and Counseling. 2018. [Duplicate Study].

208.Fondazione Don Carlo Gnocchi O. Continuous Chest Wall Vibration in COPD Rehabilitation. 2018 September 12. PMID: NCT03644888. [Population not of interest].

209.Frederikssunds Hospital D. Progressive Resistance Training of the Extensor Muscle of the Thigh in COPD-patients Hospitalized With an Exacerbation. 2012 December. PMID: NCT01812460. [Clinical Trial].

210.Fundació Institut de Recerca de l'Hospital de la Santa Creu i Sant P, Spanish Society of P, Thoracic S. Impact of Early Respiratory Rehabilitation in the Exacerbations of Readmitted COPD Patients. 2013 April. PMID: NCT02190461. [Population not of interest].

211. Fundació Sant Joan de D. Acupoint Transcutaneous Electrical Nerve Stimulation in Hospitalized COPD Patients With Severe Dyspnoea. 2016 November. PMID: NCT02998957. [Population not of interest].

212.Fundacion Clinic per a la Recerca B, Instituto de Salud C, III. Antibiotic or Not in Non-purulent Exacerbations of COPD: a Trial of Security and Efficacy. 2010 July. PMID: NCT01091493. [Clinical Trial].

213.Garre M, Garo B, Ansart S, et al. Acute exacerbation of chronic obstructif pulmonary disease and antibiotics: analysis of comparative trials. [French]. Medecine et Maladies Infectieuses. 2006 November/December;36(11-12):690-6. PMID: 44856165. [Language other than English].

214.Gendron LM, Nyberg A, Saey D, et al. Active mind-body movement therapies as an adjunct to or in comparison with pulmonary rehabilitation for people with chronic obstructive pulmonary disease. Cochrane Database of Systematic Reviews. 2018 Oct 10;10:CD012290. PMID: 30306545. [Population not of interest].
215.Gerards MC, Venema GE, Patberg KW, et al. Dapagliflozin for prednisone-induced hyperglycaemia in acute exacerbation of chronic obstructive pulmonary disease. Diabetes, Obesity and Metabolism. 2018 May;20(5):1306-10. PMID: 620566672. [Population not of interest].

216.Gerards MC, Vriesendorp TM, Hoekstra JBL, et al. Dapagliflozin for treatment of prednisone induced hyperglycaemia: A double-blind randomised clinical trial. Diabetologia. 2017 September;60 (1 Supplement 1):S414-S5. PMID: 618053136. [Abstract].

217. Giusti M, Valerio A, Sgambato F, et al. Fluoroquinolones in the treatment of resistant exacerbations of COPD: Preliminary results from the FADOI-FLOR study. Italian Journal of Medicine. 2013 May;2):60. PMID: 71295825. [Abstract].

218.Gloeckl R, Heinzelmann I, Baeuerle S, et al. Effects of whole body vibration in patients with chronic obstructive pulmonary diseasea randomized controlled trial. Respiratory medicine. 2012;106(1):75-83. [Duplicate Study].

219.Glostrup University Hospital C. Outpatient Management of Patients With Exacerbation of Chronic Obstructive Pulmonary Disease. 2006 April. PMID: NCT00317057. [Population not of interest].

220.Goktalay T, Akdemir S, Coskun A, et al. Evaluation of contribution of high frequency chest wall oscillation treatment to medical treatment in patients with acute exacerbations of COPD. European Respiratory Journal. Conference: European Respiratory Society Annual Congress. 2011;38(SUPPL. 55). PMID: 72116799. [Abstract].

221.Gomersall CD, Joynt GM, Freebairn RC, et al. Oxygen therapy for hypercapnic patients with chronic obstructive pulmonary disease and acute respiratory failure: a randomized, controlled pilot study. Critical Care Medicine. 2002 Jan;30(1):113-6. PMID: 11905405. [Population not of interest].

222. Gomersall CD, Joynt GM, Freebairn RC, et al. Randomized controlled pilot study of oxygen therapy for hypercapnic patients with an acute exacerbation of COPD. Chest. 1998;114(4 SUPPL.). [Abstract]. 
223.Gonzalez P, Sepulveda R. [Treatment of exacerbations of chronic obstructive bronchial disease with purulent sputum. Double-blind study with ampicillin and in association with sulfamethopyrazine and trimethoprim]. Revista Medica de Chile. 1982 Nov;110(11):1075-80. PMID: 6764012. [Language other than English].

224.Gotfried M, Notario G, Spiller J, et al. Comparative efficacy of once daily, 5-day short-course therapy with clarithromycin extended-release versus twice daily, 7-day therapy with clarithromycin immediaterelease in acute bacterial exacerbation of chronic bronchitis. Current Medical Research \& Opinion. 2005 Feb;21(2):24554. PMID: 15801995. [Population not of interest].

225. Gow CH, Hsu HJ, Zhang JH, et al. Observation of the effect of metered dose inhalers and electrical impedance tomography patterns on mechanically ventilated patients with acute exacerbation of obstructive pulmonary diseases. Respirology. 2018 November;23 (Supplement 2):12. PMID: 625398456. [Intervention/Comparison not of interest].

226. Gravil JH. Home treatment of acute exacerbations of COPD. CME Bulletin Respiratory Medicine. 2001;3(1):7-9. PMID: 32619055. [Intervention/Comparison not of interest].

227.Greening N, Willams J, Harvey-Dunstan T, et al. Effects on health care utilisation of early pulmonary rehabilitation on hospitalisation for an acute exacerbation of chronic respiratory disease. European Respiratory Journal. Conference: European Respiratory Society Annual Congress. 2013;42(SUPPL. 57). PMID: 71840775. [Abstract].

228. Greenstone M, Lasserson TJ. Doxapram for ventilatory failure due to exacerbations of chronic obstructive pulmonary disease. Cochrane Database of Systematic Reviews. 2009(4). PMID: 00075320-10000000000878. [Population not of interest].
229.Greulich T, Kostikas K, Gaga M, et al. Indacaterol/glycopyrronium (IND/GLY) reduces the risk of clinically important deterioration (CID) in patients with moderate COPD: Results from the CRYSTAL study. Pneumologie. Conference. 2018;59. PMID: 622904776. [Population not of interest].

230.Guangzhou Institute of Respiratory D. Quality Control for Chronic Obstructive Pulmonary Disease. 2018 March 20. PMID: NCT03604146. [Abstract].

231.Guarnieri R, Pappacoda A, Solitro S, et al. Supplementation with high dose antioxidants improves clinical response in acute exacerbation of chronic obstructive pulmonary disease (COPD) treated with antibiotic therapy. Trends in Medicine. 2009;9(3):167-74. [Language other than English].

232. Guce EP. The role of tetracycline in COPD among adult Filipinos. Scient Proc. 1991;2(1):22-5. PMID: CN-00776695 UPDATE. [Study design not of interest].

233.Gulea C, Zakeri R, Quint JK. Effect of betablocker therapy on clinical outcomes, safety, health-related quality of life and functional capacity in patients with chronic obstructive pulmonary disease (COPD): a protocol for a systematic literature review and metaanalysis with multiple treatment comparison. BMJ Open. 2018 Nov 13;8(11):e024736. PMID: 30429149. [Population not of interest].

234.GW815SF For Chronic Obstructive Pulmonary Disease (Chronic Bronchitis, Emphysema). 2005 January. PMID: NCT00269087. [Population not of interest].

235.Halpin DMG, Birk R, Brealey N, et al. Single-inhaler triple therapy in symptomatic COPD patients: FULFIL subgroup analyses. ERJ open research. 2018;4(2). PMID: CN01606772. [Population not of interest].

236. Hanlim Pharm. Co L. A Multicenter, Randomized, Double-blind, Parallel Group Study to Determine the Optimal Dose of HL301 After 7 Days Oral Administration in Acute Bronchitis or Acute Exacerbations of Chronic Bronchitis Patients (HL301: Mixed Extract of Rehmannia Glutinosa, Schisandra and so on). 2014 March. PMID:

NCT02250027. [Population not of interest]. 
237. Haynes JM. Randomized controlled trial of a breath-activated nebulizer in patients with exacerbation of COPD. Respiratory Care. 2012 Sep;57(9):1385-90. PMID: 22348309. [Intervention/Comparison not of interest].

238.He W, Xu G, Zhang C, et al. Clinical efficacy and safety of cardio-selective betareceptor blocker in management of AECOPD complicated with right heart failure. Biomedical Research (India). 2017;28(10):4507-11. PMID: 616782986. [Duplicate Study].

239.He W, Xu G, Zhang C, et al. Clinical efficacy and safety of cardio-selective $\beta$ receptor blocker in management of AECOPD complicated with right heart failure. Biomedical Research (India). 2017;28(10):4507-11. [Intervention/Comparison not of interest].

240.Henan University of Traditional Chinese M. A Randomized Comparative Effectiveness Research Trial of Three Treatments for COPD Patients. 2013 May. PMID: NCT01836016. [Population not of interest].

241.Henan University of Traditional Chinese M. Effect of Acupuncture on Patients With Chronic Obstructive Pulmonary Disease. 2017 May. PMID: NCT03169504. [Population not of interest].

242.Henan University of Traditional Chinese M. Effect of Traditional Chinese Medicine on Outcomes in COPD Patients. 2016 December. PMID: NCT03007914. [Population not of interest].

243.Henan University of Traditional Chinese M. Effects of Traditional Chinese Medicine on AECOPD Patients. 2018 March 1. PMID: NCT03428412. [Intervention/Comparison not of interest].

244.Henry Ford Health S. Pre-Discharge Bundle for Patients With Acute Exacerbations of Chronic Obstructive Pulmonary Disease to Reduce Readmissions. 2010 February. PMID: NCT02135744. [Outcome not of interest].

245.Hernandez C, Casas A, Escarrabill J, et al. Home hospitalisation of exacerbated chronic obstructive pulmonary disease patients. European Respiratory Journal. 2003 Jan;21(1):58-67. PMID: 12570110. [Intervention/Comparison not of interest].
246.Hessel E, Hamblin J, Robertson J, et al. Inhaled PI3Kdelta inhibitor nemiralisib improves lung function and reduces reexacerbations in exacerbating chronic obstructive pulmonary disease (COPD) patients (PII116678). American Journal of Respiratory and Critical Care Medicine. Conference: American Thoracic Society International Conference, ATS. 2018;197(MeetingAbstracts). PMID: 622964633. [Abstract].

247.High Flow Nasal Cannula vs NPPV in Moderate Chronic Obstructive Pulmonary Disease Exacerbation.

Https:/clinicaltrials.gov/show/nct03014869. 2017. PMID: CN-01561123. [Duplicate Study].

248.Hitchings AW, Lai D, Jones PW, et al. Antihyperglycaemic effects of metformin in acute exacerbations of chronic obstructive pulmonary disease (COPD): A multi-centre, randomised, double-blind, placebocontrolled trial. American Journal of Respiratory and Critical Care Medicine. Conference: American Thoracic Society International Conference, ATS. 2015;191(MeetingAbstracts). PMID: 72051844. [Abstract].

249. Hitchings AW, Lai D, Jones PW, et al. Effects of metformin on serum C-reactive protein and interleukin-6 concentration in acute exacerbations of chronic obstructive pulmonary disease: A multi-centre, randomised, double-blind, placebocontrolled trial. American Journal of Respiratory and Critical Care Medicine. Conference: American Thoracic Society International Conference, ATS. 2015;191(MeetingAbstracts). PMID: 72051843. [Abstract].

250.Hitchings AW, Lai D, Jones PW, et al. Metformin in severe exacerbations of chronic obstructive pulmonary disease: A randomised controlled trial. Thorax. 201601 Jul;71(7):587-93. PMID: 608796119. [Intervention/Comparison not of interest].

251.Hoang BX, Han B, Nguyen HX, et al. Nutritional Supplement "Bao Khi Khang" as an Adjuvant Therapy in Acute Exacerbations of Chronic Obstructive Pulmonary Disease. Journal of Medicinal Food. 2018 Apr 23;23:23. PMID: 29683370. [Intervention/Comparison not of interest]. 
252.Hoffmann-La R. A Study to Evaluate Safety and Efficacy of Lebrikizumab in Participants With Chronic Obstructive Pulmonary Disease (COPD). 2015 September 30. PMID: NCT02546700. [Population not of interest].

253. Horizon Pharma Usa I. A Phase 1b Study to Evaluate the Safety and PK of MP-376 in Chronic Obstructive Pulmonary Disease (COPD) Patients. 2008 July. PMID: NCT00752414. [Population not of interest].

254.Hospital Universitari Son D, Fondo de Investigación S, Sociedad Española de Neumología y Cirugía T. Effects of LowDose Theophylline During Chronic Obstructive Pulmonary Disease (COPD) Exacerbations. 2005 June. PMID: NCT00671151. [Clinical Trial].

255.Hospital Universitario G, Grant Ip041233 From Fondo De Investigacion S. Evaluation Of The Efficacy Of Corticosteroids In Patients With An Acute Exacerbation Of Chronic Obstructive Pulmonary Disease Receiving Ventilator Support. 2005 July. PMID: NCT01281748. [Population not of interest].

256. Hospital Universitario La P. Long-term Effect of an Health Education Program on Daily Physical Activity in Patients With Moderate to Very Severe Chronic Obstructive Pulmonary Disease. 2016 October. PMID: NCT02924870. [Population not of interest].

257. Howcroft M, Walters EH, Wood-Baker R, et al. Action plans with brief patient education for exacerbations in chronic obstructive pulmonary disease. Cochrane Database of Systematic Reviews. 201612 19;12:CD005074. PMID: 27990628. [Intervention/Comparison not of interest].

258.Hu J, Hong QY, Bai CX. Efficacy of beta $<$ inf $>2<$ inf $>$-agonist and impratropium bromide in chronic obstructive pulmonary disease. [Chinese]. Fudan University Journal of Medical Sciences. 2002;29(4):314-6. PMID: 37081253. [Language other than English].
259.Hua Z-Q, Chang Y-J. [Clinical study of decoction of invigorating Qi and clearing lung combined standardized myrtol on acute exacerbation of chronic obstructive pulmonary disease (AECOPD)]. Zhongguo Zhong Yao Za Zhi/Zhongguo Zhongyao Zazhi/China Journal of Chinese Materia Medica. 2013 Feb;38(3):440-2. PMID: 23668026. [Language other than English].

260.Huang XY, Zhang R, Zhao JY, et al. Clinical efficacy of kuanxionglifei decoction combined with Sanziyangqin decoction in treatment of acute exacerbation of chronic obstructive pulmonary disease. [Chinese]. Academic Journal of Second Military Medical University. 2015 December;36(12):1374-8. PMID: 607409792. [Intervention/Comparison not of interest].

261.Huashan H. Efficiency of Budesonide Combined With Formoterol and Tiotropium in the Treatment of Acute Exacerbation of ACO. 2018 May 1. PMID: NCT03504527. [Population not of interest].

262.Huashan H. Study of Prophylactic Effect of Rivaroxaban on Venous Thromboembolism in AECOPD. 2017 October 1. PMID: NCT03277001. [Intervention/Comparison not of interest].

263.Hugues FC, Le JC, Bernard Y, et al. A controlled, multicentre study of cefixime versus amoxicillin in the treatment of lower respiratory tract infections in adults.

$<$ ORIGINAL $>$ ETUDE MULTICENTRIQUE CONTROLEE COMPARANT LE CEFIXIME DE L'AMOXICILLINE DANS LE TRAITEMENT DES INFECTIONS RESPIRATOIRES BASSES DE L'ADULTE. Presse medicale (Paris, France .. 1989;1983) 18(32):1600-4. PMID: CN00194845 UPDATE. [Language other than English].

264.Hull EYHNHST. Erdosteine (Erdotin) Versus Standard Care Plus Placebo on Erdosteine for Treatment of Cough in Acute Exacerbations of Chronic Obstructive Pulmonary Disease (COPD). PMID: NCT01176318. [Clinical Trial]. 
265.Hvidovre University H, Innovation Fund D. Automated Oxygen Delivery by O2matic to Patients Admitted With an Exacerbation in COPD. 2018 April. PMID: NCT03464695. [Clinical Trial].

266. Ichinose M, Nishimura M, Akimoto M, et al. Tiotropium/olodaterol versus tiotropium in Japanese patients with COPD: results from the DYNAGITO study. International journal of chronic obstructive pulmonary disease. 2018;13:2147-56. PMID: 625118172. [Population not of interest].

267.Ilvan A, Gur A, Musellim B, et al. Acute infectious exacerbations in chronic obstructive lung disease and effects of azithromycine, sultamycilline, ciprofloxacin and cefaclor monohydrate. [Turkish]. Cerrahpasa Tip Dergisi. 1997;28(2):73-7. PMID: 27361375. [Language other than English].

268.Ilvan A, Gur A, Musellim B, et al. Acute infectious exacerbations in chronic obstructive lung disease and effects of azithromycine, sultamycilline, ciprofloxacin and cefaclor monohydrate. <ORIGINAL> KRONIK OBSTRUKTIF AKCIGER HASTALIGI OLGULARINDA AKUT INFEKSIYON DONEMINDE PATOJEN AJANIN SAPTANMASI VE AZITROMISIN, SULTAMISILIN, SIPROFLOKSASIN VE SEFAKLOR MONOHIDRATIN ETKINLIGI. Cerrahpasa Tip Dergisi. 1997;28(2):73-7. PMID: CN-00198467 UPDATE. [Language other than English].

269.Impact of Initiating Tiotropium Alone Versus Initiating Tiotropium in Combination With Fluticasone Propionate/Salmeterol Xinafoate Combination (FSC) on Chronic Obstructive Pulmonary Disease-related Outcomes in Patients With Pre-existing Exacerbations. 2008 July. PMID: NCT01381406. [Population not of interest].

270.Imperial College L. Metformin to Reduce Airway Glucose in COPD Patients. 2018 September 1. PMID: NCT03651895. [Population not of interest].

271.Imperial College L. Targeted Retreatment of COPD Exacerbations. 2014 June. PMID: NCT02300220. [Population not of interest].
272.Influence Of Salmeterol Xinafoate/Fluticasone Propionate (50/500 $\mu \mathrm{g}$ BID) On The Course Of The Disease And Exacerbation Frequency In COPD Patients Gold Stage III And IV. 2007 November. PMID: NCT00527826. [Population not of interest].

273.Inmunotek SL. Evaluation of the Efficacy and Safety of MV130 in Chronic Obstructive Pulmonary Disease (COPD). 2013 April. PMID: NCT01842360. [Population not of interest].

274. Inonu U. The Role of Nebulized Budesonide in the Treatment of Acute Exacerbations of COPD. 2003 January. PMID:

NCT00274222. [Study design not of interest].

275. Intravenous Versus Oral Administration of Prednisolone in Exacerbations of Chronic Obstructive Pulmonary Disease (COPD). 2001 June. PMID: NCT00311961. [Clinical Trial].

276.INvestigating COPD Outcomes, Genomics and Neutrophilic Inflammation With Tiotropium and Olodaterol. Https://clinicaltrials.gov/show/nct03152149. 2017. PMID: CN-01575080. [Duplicate Study].

277. Isbaniah F, Wiyono WH, Yunus F, et al. Echinacea purpurea along with zinc, selenium and vitamin $\mathrm{C}$ to alleviate exacerbations of chronic obstructive pulmonary disease: results from a randomized controlled trial. Journal of Clinical Pharmacy \& Therapeutics. 2011 Oct;36(5):568-76. PMID: 21062330. [Intervention/Comparison not of interest].

278.Jafarinejad H, Moghoofei M, Mostafaei S, et al. Worldwide prevalence of viral infection in AECOPD patients: A meta-analysis. Microbial Pathogenesis. 2017 Dec;113:1906. PMID: 29038056.

[Intervention/Comparison not of interest].

279.Jahnz-Rozyk K, Kucharczyk A, Chcialowski A, et al. [The effect of inhaled ambroxol treatment on clinical symptoms and chosen parameters of ventilation in patients with exacerbation of chronic obstructive pulmonary disease patients]. Polski Merkuriusz Lekarski. 2001 Sep;11(63):23943. PMID: 11761819. [Language other than English]. 
280.Jakobsen AS, Laursen LC, Ostergaard B, et al. Hospital admitted COPD patients treated at home using telemedicine technology A randomized, multicentre trial. European Respiratory Journal. Conference: European Respiratory Society Annual Congress. 2012;40(SUPPL. 56). PMID: 71923683. [Intervention/Comparison not of interest].

281.Jamaly S, Leidag M, Schneider HW, et al. The effect of singing therapy compared to standard physiotherapeutic lung sport in COPD. Pneumologie. Conference. 2017;58. PMID: 623446922. [Population not of interest].

282.Janaudis-Ferreira T, Carr SJ, Harrison SL, et al. Can Patients With COPD Assimilate Disease-Specific Information During an Acute Exacerbation?: Results of a Pilot Randomized Controlled Trial. Chest. 2018 Sep;154(3):588-96. PMID: 29879395. [Intervention/Comparison not of interest].

283.Janaudis-Ferreira T, Harrison S, Carr J, et al. Can patients with COPD assimilate disease information at a time of being acutely unwell due to an exacerbation of their disease? Results of a randomized controlled trial. Canadian Journal of Respiratory Critical Care and Sleep Medicine. 2017;1 (3):160. PMID: 624483066. [Abstract].

284.Janjua S, Dias S, Threapleton JDC, et al. Prophylactic antibiotics for adults with chronic obstructive pulmonary disease: a network meta-analysis. Cochrane Database of Systematic Reviews. 2018(11). PMID: 00075320-100000000-11608. [Population not of interest].

285.Jennings JH, Thavarajah K, Mendez MP, et al. Predischarge bundle for patients with acute exacerbations of COPD to reduce readmissions and ED visits: a randomized controlled trial. Chest. 2015 May;147(5):1227-34. PMID: 25940250. [Intervention/Comparison not of interest].

286.Jens Bräunlich MD. NHF in Acute Hypercapnic Acute Exacerbation of COPD. 2018 May 15. PMID: NCT03523481. [Population not of interest].
287.Jeppesen E, Brurberg KG, Vist GE, et al. Hospital at home for acute exacerbations of chronic obstructive pulmonary disease. Cochrane Database of Systematic Reviews. 2012 May 16(5):CD003573. PMID: 22592692. [Intervention/Comparison not of interest].

288.Jia Z, Feng Z, Tian R, et al. Thymosin alpha1 plus routine treatment inhibit inflammatory reaction and improve the quality of life in AECOPD patients. Immunopharmacology \& Immunotoxicology. 2015;37(4):388-92. PMID: 26250523. [Intervention/Comparison not of interest].

289.Jiang D, Wang X, Su Q, et al. Milkvetch root improves immune function in patients with acute exacerbation of COPD. BioMedical Materials \& Engineering. 2015;26 Suppl 1:S2113-21. PMID: 26405990. [Intervention/Comparison not of interest].

290.Jiang DH, Wang X, Liu LS, et al. The effect of ventilator mask atomization inhalation of ipratropium bromide and budesonide suspension liquid in the treatment of COPD in acute exacerbation period on circulating levels of inflammation and prognosis. European Review for Medical \& Pharmacological Sciences. 2017 Nov;21(22):5211-6. PMID: 29228436. [Duplicate Study].

291.Jiang GY, Li Q, Lv YX. Short-term treatment of irbesartan and hydrochlorothiazide decreases plasma Nterminal pro-brain natriuretic peptide levels in subjects with acute exacerbations of COPD. International journal of chronic obstructive pulmonary disease. 2019;14:7380. PMID: 625705532. [Outcome not of interest].

292.Jiang HL, Mao B. Evidence-based treatment of a patient with acute exacerbation of chronic obstructive pulmonary disease with nebulized budesonide. Chinese Journal of Evidence-Based Medicine. 2010;10(6):7568. [Language other than English].

293.Johns Hopkins University H-R. Chest Wall Oscillation for Asthma and COPD Exacerbations Trial (COAT). 2003 October. PMID: NCT00181285. [Clinical Trial]. 
294.Johnson CL, Rogers DF. Why is erdosteine recommended as a treatment for acute exacerbations of chronic bronchitis? A systematic review of clinical trials. Thorax. 2016 December;71 (Supplement 3):A157. PMID: 615030720. [Abstract].

295.Jones SE, Barker RE, Nolan CM, et al. Pulmonary rehabilitation in patients with an acute exacerbation of chronic obstructive pulmonary disease. Journal of Thoracic Disease. 2018 May;10(Suppl 12):S1390-S9. PMID: 29928521. [Study design not of interest].

296.Jones SM, Albert P, Warburton CJ, et al. Effect of a case management study on primary care use and prescribing for AECOPD. Thorax. 2009 December;4):A119. PMID: 70254645. [Abstract].

297.Juan Ortega J, Abu-Omar Peris L, López Matoses MJ. Effect of withdrawal and/or replacement of inhaled corticosteroids in favor of double bronchodilation in symptomatic COPD patients. RETICORTEPOC study. Revista de Patologia Respiratoria. 2018;21(2):71-3. [Duplicate Study].

298.Juanes A, Garin N, Mangues MA, et al. Impact of a pharmaceutical care programme for patients with chronic disease initiated at the emergency department on drug-related negative outcomes: A randomised controlled trial. European Journal of Hospital Pharmacy. 201801 Sep;25(5):274-80. PMID: 617562826. [Duplicate Study].

299.Kaplan AG, Prefontaine D, Belle-Isle J, et al. Power: prospective cohort study for the real-life effectiveness of glycopyrronium with indacaterol combination in the management of COPD in Canada. Canadian Journal of Respiratory Critical Care and Sleep Medicine. 2017;1 (3):161-2. PMID: 624483102. [Abstract].

300. Karaoren G, Tomruk SG, Esquinas AM. Automated versus manual oxygen titration in COPD exacerbation: Machine or hands, this is the question. International Journal of COPD. 201703 Apr;12:1057-8. PMID: 615237679. [Study design not of interest].
301.Karpel JP, Pesin J, Greenberg D, et al. A comparison of the effects of ipratropium bromide and metaproterenol sulfate in acute exacerbations of COPD. Chest. 1990 Oct;98(4):835-9. PMID: 2145136. [Intervention/Comparison not of interest].

302. Katholieke Universiteit L. Effects of Physical Activity Counseling After an Exacerbation in COPD. 2013 March. PMID: NCT02223962. [Population not of interest].

303.Katholieke Universiteit L. Exacerbation and Training in Patients With Chronic Obstructive Pulmonary Disease. 2004 January. PMID: NCT00877084. [Clinical Trial].

304.Katsaras T, Milsis A, Rizikari M, et al. The use of the "healthwear" wearable system in chronic patients' early hospital discharge: Control randomized clinical trial. 2011 5th International Symposium on Medical Information and Communication Technology, ISMICT 2011. 2011:143-6. [Intervention/Comparison not of interest].

305.Kessler R, Casan-Clara P, Koehler D, et al. COMET: a multicomponent home-based disease-management programme versus routine care in severe COPD. The European respiratory journal. 2018;51(1). PMID: 625302790. [Abstract].

306.Khon Kaen U, Thailand Research F. Effect of Home-base Exercise With Conical-PEP Device on Physical Performance and Quality of Life in COPD. 2016 October. PMID: NCT02790047. [Population not of interest].

307.Kim H, Lee Y, Oh YM, et al. Zabofloxacin $367 \mathrm{mg}$ for five days versus moxifloxacin $400 \mathrm{mg}$ for seven days in patients with COPD exacerbation: A multicenter, randomized, double-blind, phase 3, noninferiority trial. American Journal of Respiratory and Critical Care Medicine. Conference: American Thoracic Society International Conference, ATS. 2015;191(MeetingAbstracts). PMID: 72050649. [Abstract]. 
308.King J, Tessier S, Charrette MJ, et al. Patient education interventions provided by physiotherapists to patients with chronic obstructive pulmonary disease: The result of a scoping review. Respirology. 2017 March;22 (Supplement 2):135. PMID: 617841424. [Abstract].

309.King's College Hospital NHST, British Lung F. Early Pulmonary Rehabilitation Following Acute COPD Exacerbation. 2006 January. PMID: NCT00557115. [Population not of interest].

310.Kiszka-Kanowitz M, Matzen P. Doxapram treatment in acute exacerbation of chronic obstructive lung disease. [Danish]. Ugeskrift for Laeger. 1997;159(41):6055-6. PMID: 27437489. [Language other than English].

311.Kniajeskaya N. The treatment of acute respiratory viral infections in a patients with a chronic obstructive pulmonary disease. European Respiratory Journal. Conference: European Respiratory Society Annual Congress. 2012;40(SUPPL. 56). PMID: 71926044. [Population not of interest].

312.Ko FWS, Chan PKS, Ip A, et al. Multiplex molecular detection of respiratory pathogens of adult patients admitted to hospital for acute exacerbation chronic obstructive pulmonary disease and their clinical outcomes. American Journal of Respiratory and Critical Care Medicine. Conference: American Thoracic Society International Conference, ATS. 2018;197(MeetingAbstracts). PMID: 622965383. [Abstract].

313.Ko FWS, Dai DLK, Ngai J, et al. Effect of early pulmonary rehabilitation on health care utilization and health status in patients hospitalized with acute exacerbations of COPD. Respirology. 2011 May;16(4):61724. PMID: 21199163.

[Intervention/Comparison not of interest].

314.Kocks JWH, Tuinenga MG, Uil SM, et al. Health status measurement in COPD: the minimal clinically important difference of the clinical COPD questionnaire.

Respiratory Research. 2006 Apr 07;7:62. PMID: 16603063. [Intervention/Comparison not of interest].
315.Kreis SR, Herrera N, Golzar N, et al. A comparison of moxifloxacin and azithromycin in the treatment of acute exacerbations of chronic bronchitis. JCOMWAYNE PA-. 2000;7(12):33-41.

[Population not of interest].

316.Kunafina TV, Chuchalin AG, Belevsky AS, et al. Neuromuscular electrical stimulation as an alternative to physical exercise in patients with COPD. Bulletin of Russian State Medical University. 2018;7(3):59-64. [Duplicate Study].

317.Kunter E, Ilvan A, Ozmen N, et al. Effect of corticosteroids on hemostasis and pulmonary arterial pressure during chronic obstructive pulmonary disease exacerbation. Respiration. 2008;75(2):145-54. PMID: 17143000. [Study design not of interest].

318.La Trobe U, The A, Austin H, et al. Does Positive Expiratory Pressure Mask Therapy Improve Recovery From Acute Exacerbations of Chronic Obstructive Pulmonary Disease? 2010 July. PMID: NCT01101282. [Clinical Trial].

319.Lallemand Pharma I, Sprim Advanced Life S. Advanced Immunological Approach in COPD Exacerbation. 2009 September. PMID: NCT02417649. [Population not of interest].

320.Landau Z, Schlaffer F, Pitlik S. Cefuroxime axetil vs. augmentin for the treatment of acute bronchitis and exacerbation of chronic obstructive pulmonary disease. Israel Journal of Medical Sciences. 1992 Nov;28(11):797-9. PMID: 1468894. [Intervention/Comparison not of interest].

321.Lange P. [Antibiotics for exacerbations of chronic obstructive pulmonary disease]. Ugeskrift for Laeger. 2007 May 07;169(19):1780-2. PMID: 17537351. [Language other than English].

322.Laval U, Canadian Institutes of Health R. Substudy : Patients With an Acute Exacerbation of Chronic Obstructive Pulmonary Disease. 2012 May. PMID: NCT02282436. [Study design not of interest].

323.Laval U. Clinical Evaluation of FreeO2 (Version 4) in All Patients Receiving Oxygen. 2017 January 30. PMID: NCT03119727. [Population not of interest]. 
324.Laval University G. Effects of Advair $®$ in Outpatients With Chronic Obstructive Pulmonary Disease (COPD) Acute Exacerbation. 2007 November. PMID: NCT00531791. [Clinical Trial].

325.Lawson Health Research Institute P. COPE With COPD Trial. 2011 October. PMID: NCT02343055. [Population not of interest].

326.Lazaar AL, Miller BE, Tabberer M, et al. Effect of the CXCR2 antagonist danirixin on symptoms and health status in COPD. European Respiratory Journal. 201801 Oct;52 (4) (no pagination)(1801020). PMID: 624259157. [Study design not of interest].

327. Leaver S, Higton A, MacIntyre J, et al. A randomised controlled trial to assess the effect of Heliox in patients with exacerbations of asthma and chronic obstructive pulmonary disease [Abstract]. Thorax. 2005;2(Suppl II):ii9. PMID: CN00592096 UPDATE. [Abstract].

328.Lee JS, Liu AI, Pounds D, et al. Characteristics of COPD patients who agree to participate in a pragmatic trial of physical activity coaching compared to nonparticipants. American Journal of Respiratory and Critical Care Medicine. Conference: American Thoracic Society International Conference, ATS. 2018;197(MeetingAbstracts). PMID: 622968923. [Population not of interest].

329.Lellouche F, Bouchard PA, Roberge M, et al. Automated oxygen titration and weaning with FreeO2 in patients with acute exacerbation of COPD: a pilot randomized trial. International journal of chronic obstructive pulmonary disease. 1983;11:1983-90. PMID: CN-01180214. [Duplicate Study].

330. Lellouche F, Bouchard PA, Roberge M, et al. Oxygen titration and weaning with FREEO2 in COPD patients hospitalized for exacerbation. A randomized controlled pilot study. American Journal of Respiratory and Critical Care Medicine. Conference: American Thoracic Society International Conference, ATS. 2015;191(MeetingAbstracts). PMID: 72051722. [Abstract].
331.Lellouche F, Bouchard PA, Roberge M, et al. Utilization of a closed loop device to titrate oxygen flow (FREEO2) in COPD exacerbation. A randomized controlled pilot study. Intensive Care Medicine. 2012 October;1):S68. PMID: 71013456. [Abstract].

332.Lenferink A, Brusse-Keizer M, Van Der Valk P, et al. Self-management interventions including COPD exacerbation action plans improve hospitalisation rate and healthrelated quality of life-A cochrane review. Respirology. 2017 March;22 (Supplement 2):73. PMID: 617841223.

[Intervention/Comparison not of interest].

333.Lenferink A, Brusse-Keizer M, Van Der Valk P, et al. Self-management interventions that include COPD exacerbation action plans improve healthrelated quality of life-a cochrane review. European Respiratory Journal. Conference: European Respiratory Society Annual Congress. 2016;48(Supplement 60). PMID: 614780110. [Intervention/Comparison not of interest].

334.Lenferink A, Brusse-Keizer M, van der Valk PD, et al. Self-management interventions including action plans for exacerbations versus usual care in patients with chronic obstructive pulmonary disease. Cochrane Database of Systematic Reviews. 2017 Aug 04;8:CD011682. PMID: 28777450. [Intervention/Comparison not of interest].

335.Lenferink A, Van Der Palen J, Van Der Valk P, et al. Self-management action plans for COPD patients with comorbidities reduce exacerbation duration and respiratory-related hospitalizations - The cope-iii study. American Journal of Respiratory and Critical Care Medicine. Conference: American Thoracic Society International Conference, ATS. 2017;195(no pagination). PMID: 617713413. [Abstract].

336.Leophonte P, Chidiac C, Drugeon HB, et al. [Treatment of exacerbations of chronic obstructive pulmonary disease with pristinamycin]. Revue des Maladies Respiratoires. 2004 Apr;21(2 Pt 1):261-71. PMID: 15211234. [Language other than English]. 
337.Leuppi JD, Schutz P, Bingisser R, et al. Short term glucocorticoid therapy in acute exacerbations of chronic obstructive pulmonary disease: "REDUCE[Study design not of interest].

338.Li H, He G, Chu H, et al. A step-wise application of methylprednisolone versus dexamethasone in the treatment of acute exacerbations of COPD. Respirology. 2003 Jun;8(2):199-204. PMID: 12753536. [Intervention/Comparison not of interest].

339.Li H, He G, Chu H, et al. A step-wise application of methylprednisolone versus dexamethasone in the treatment of acute exacerbations of COPD. Respirology. 2003;8(2):199-204. [Duplicate Study].

340.Li W, Mao B, Wang G, et al. [A study of the mechanism of Qingre Huatan therapy in treatment of acute exacerbation of chronic obstructive pulmonary disease by improving airway inflammation and mucus hypersecretion]. Zhong Xi Yi Jie He Xue Bao/Journal of Chinese Integrative Medicine. 2008 Aug;6(8):799-805. PMID: 18664347. [Language other than English].

341.Li W, Mao B, Wang G, et al. Effect of Tanreqing Injection on treatment of acute exacerbation of chronic obstructive pulmonary disease with Chinese medicine syndrome of retention of phlegm and heat in Fei. Chinese Journal of Integrative Medicine. 2010 Apr;16(2):131-7. PMID: 20473738. [Intervention/Comparison not of interest].

342.Lin C, Pang Q. Meta-analysis and systematic review of procalcitonin-guided treatment in acute exacerbation of chronic obstructive pulmonary disease. The clinical respiratory journal. 2018 Jan;12(1):10-5. PMID: 27328801. [Intervention/Comparison not of interest].

343.Lin Q, Liu K, Liu S, et al. [The efficacy and safety of moxifloxacin in the treatment of patients with acute exacerbation of chronic obstructive pulmonary disease]. Chung-Hua Chieh Ho Ho Hu Hsi Tsa Chih Chinese Journal of Tuberculosis \& Respiratory Diseases. 2015 May;38(5):366-9. PMID: 26463489. [Language other than English].
344.Lipson DA, Barnhart F, Brealey N, et al. Reduction in all-cause mortality with single inhaler triple therapy (FF/UMEC/VI) versus dual therapy (FF/VI and UMEC/VI) in symptomatic patients with COPD: prespecified analysis of the phase III impact trial. American Journal of Respiratory and Critical Care Medicine. Conference: American Thoracic Society International Conference, ATS.

2018;197(MeetingAbstracts). PMID: 622967744. [Population not of interest].

345.Lipson DA, Barnhart F, Brealey N, et al. Single inhaler triple-therapy (ICS/LAMA/LABA) versus dual-therapy (ICS/LABA or LAMA/LABA) in patients with COPD at risk of exacerbations: Efficacy and safety results of the phase iii impact trial. American Journal of Respiratory and Critical Care Medicine. Conference: American Thoracic Society International Conference, ATS. 2018;197(MeetingAbstracts). PMID: 622967683. [Abstract].

346.Lirsac B, Benezet O, Dansin E, et al. [Evaluation and symptomatic treatment of surinfectious exacerbations of COPD: preliminary study of antibiotic treatment combined with fenspiride (Pneumorel 80mg) versus placebo]. Revue de Pneumologie Clinique. 2000 Feb;56(1):17-24. PMID: 10740110. [Language other than English].

347.Liu M, Zhong X, Li Y, et al. Xuan Bai Cheng Qi formula as an adjuvant treatment of acute exacerbation of chronic obstructive pulmonary disease of the syndrome type phlegm-heat obstructing the lungs: a multicenter, randomized, double-blind, placebo-controlled clinical trial. BMC Complementary \& Alternative Medicine. 2014 Jul 11;14:239. PMID: 25014996. [Intervention/Comparison not of interest].

348.Liu S, Shergis J, Chen X, et al. Chinese herbal medicine (weijing decoction) combined with pharmacotherapy for the treatment of acute exacerbations of chronic obstructive pulmonary disease. Evidencebased Complementary and Alternative Medicine. 2014;2014. [Intervention/Comparison not of interest]. 
349.Lloberes P, Ramis L, Montserrat JM, et al. Effect of three different bronchodilators during an exacerbation of chronic obstructive pulmonary disease. European Respiratory Journal. 1988 Jun;1(6):536-9. PMID: 2971565. [Intervention/Comparison not of interest].

350.Llor C, Moragas A, Hernandez S, et al. Amoxicillin/clavulanate vs placebo: More exacerbation cures, fewer recurrences in mild-to-moderate COPD. Annals of internal medicine. 2013;158(6):JC3. PMID: CN00920635 UPDATE. [Study design not of interest].

351.Long-acting beta-2-agonists in COPD safe. Australian Journal of Pharmacy. 2008 July;89(1059):110. PMID: 352017370. [Study design not of interest].

352.Lopez Lopez L, Granados Santiago M, Donaire Galindo M, et al. Efficacy of combined electrostimulation in patients with acute exacerbation of COPD: randomised clinical trial. Medicina Clinica. 2018 Apr 25;25:25. PMID: 29705158. [Language other than English].

353.Lopez Lopez L, Granados Santiago M, Donaire Galindo M, et al. Eficacia de la electroestimulacion combinada en pacientes con exacerbacion aguda de enfermedad pulmonar obstructiva cronica: ensayo clinico aleatorizado, Efficacy of combined electrostimulation in patients with acute exacerbation of COPD: randomised clinical trial. Medicina clinica. 2018;151(8):323-8. PMID: CN-01651256 NEW. [Language other than English].

354.LudwLudwig Boltzmann Institute for C, Respiratory E. Endothelial Dysfunction in Acute Exacerbations of Chronic Obstructive Pulmonary Disease (COPD). 2008 August. PMID: NCT01460082. [Study design not of interest].

355.Luo J, Wang K, Liu D, et al. Can roflumilast, a phosphodiesterase-4 inhibitor, improve clinical outcomes in patients with moderate-to-severe chronic obstructive pulmonary disease? A meta-analysis. Respiratory Research. 2016 Feb 17;17:18. PMID: 26887407. [Population not of interest].
356.Luo Q, Zheng Z, Cen H, et al. A modified nebulization modality versus classical ultrasonic nebulization and oxygen-driven nebulization in facilitating airway clearance in patients with acute exacerbation of chronic obstructive pulmonary disease: A randomized controlled trial. Journal of Thoracic Disease. 2015;7(7):1130-41. PMID: 605719499.

[Intervention/Comparison not of interest].

357. Maastricht University Medical Center Z, The Netherlands Organisation for Health Research D. Morphine for Treatment of Dyspnea in Patients With COPD. 2016 November 1. PMID: NCT02429050. [Population not of interest].

358. Mackay AJ, Patel ARC, Singh R, et al. Randomized Double-Blind Controlled Trial of Roflumilast at Acute Exacerbations of Chronic Obstructive Pulmonary Disease. American Journal of Respiratory \& Critical Care Medicine. 201709 01;196(5):656-9. PMID: 28146642. [Study design not of interest].

359.Mahajan AK, Diette GB, Hatipoglu U, et al. High frequency chest wall oscillation for asthma and chronic obstructive pulmonary disease exacerbations: a randomized shamcontrolled clinical trial. Respiratory Research. 2011 Sep 10;12:120. PMID: 21906390. [Population not of interest].

360. Makarova EV, Varvarina GN, Menkov NV, et al. [Nebulized budesonide in the treatment of exacerbations of chronic obstructive pulmonary disease: Efficacy, safety, and effects on the serum levels of soluble differentiation molecules]. Terapevticheskii Arkhiv. 2016;88(3):24-31. PMID: 27030325. [Language other than English].

361.Maltais F, Ostinelli J, Bourbeau J, et al. Comparison of nebulized budesonide and oral prednisolone with placebo in the treatment of acute exacerbations of chronic obstructive pulmonary disease: a randomized controlled trial. American journal of respiratory and critical care medicine. 2002;165(5):698-703. [Duplicate Study]. 
362.Mannino DM, Clerisme-Beaty EM, Franceschina J, et al. Exacerbation recovery patterns in newly diagnosed or maintenance treatment-naive patients with COPD: secondary analyses of TICARI 1 trial data. International Journal of Copd. 2018;13:1515-25. PMID: 29785101. [Study design not of interest].

363.Mansour G, Salih M, Kukreja R, et al. COPD exacerbation, does procalcitonin help? A metaanalysis. Chest. Conference: CHEST. 2015;148(4 MEETING ABSTRACT). PMID: 72132618. [Intervention/Comparison not of interest].

364. Mao M. A clinical study of AECOPD in Southern China. American Journal of Respiratory and Critical Care Medicine. Conference: American Thoracic Society International Conference, ATS. 2018;197(MeetingAbstracts). PMID: 622964634. [Abstract].

365. Maquet Cardiopulmonary AG. The PALP ${ }^{\mathrm{TM}}$-COPD Trial (Low-Flow CO2Removal (ECCO2-R) in Exacerbated COPD). 2012. PMID: NCT02107222. [Population not of interest].

366. Marchetti N, Jacobs MR, Zhao H, et al. Effects of roflumilast on re-hospitalization and mortality in patients hospitalized with a COPD exacerbation. American Journal of Respiratory and Critical Care Medicine. Conference: American Thoracic Society International Conference, ATS. 2018;197(MeetingAbstracts). PMID: 622970526. [Abstract].

367. Martínez JA, Guillén MDLG. Evidence for the use of methylxantines in exacerbations of COPD. Revista del Instituto Nacional de Enfermedades Respiratorias. 2006;19(4):309-15. [Language other than English].

368.Martin-Salvador A, Colodro-Amores G, Torres-Sanchez I, et al. [Physical therapy intervention during hospitalization in patients with acute exacerbation of chronic obstructive pulmonary disease and pneumonia: A randomized clinical trial]. Medicina Clinica. 2016 Apr 01;146(7):3014. PMID: 26726117. [Language other than English].
369. Mathioudakis AG, ChatzimavridouGrigoriadou V, Corlateanu A, et al. Clinical effectiveness of procalcitonin based protocols to guide the administration of antibiotics in patients presenting with COPD exacerbations: Systematic review and metaanalysis. Thorax. 2016 December;71 (Supplement 3):A110-A1. PMID: 615030853. [Intervention/Comparison not of interest].

370. Mathioudakis AG, ChatzimavridouGrigoriadou V, Corlateanu A, et al. Procalcitonin to guide antibiotic administration in COPD exacerbations: a meta-analysis. European Respiratory Review. 2017 Jan;26(143). PMID: 28143877. [Intervention/Comparison not of interest].

371. Maugeri F. Randomized Trial of the FreeAspire Versus PEP-bottle After Acute Exacerbation in COPD Patients. 2016 October. PMID: NCT02640430. [Population not of interest].

372. McCrory DC, Hasselblad V. The results of a randomized controlled trial of hydrocortisone in acute exacerbation of COPD [10]. American Journal of Emergency Medicine. 2000;18(1):122. PMID: 30073220. [Abstract].

373.McCurdy BR. hospital-at-home programs for patients with acute exacerbations of chronic obstructive pulmonary disease (COPD): An evidence-based analysis. Ontario Health Technology Assessment Series. 2012;12(10):1-65. PMID: 369081372. [Intervention/Comparison not of interest].

374.McGill University Health Center G. Innovations in Treating COPD Exacerbations: Pilot Project on Action Plans Using New Technology. 2013 October. PMID: NCT02275078. [Population not of interest].

375.McGill University Health Center G. Strategy for Early Treatment of Exacerbations in COPD: Standing Prescriptions of Advair With a Written Action Plan in the Event of an Exacerbation. 2008 July. PMID: NCT02136875. [Intervention/Comparison not of interest]. 
376. McKinstry S, Pilcher J, Bardsley G, et al. Nasal high flow therapy and PtCO < inf $>2<$ inf $>$ in stable COPD: a randomized controlled cross-over trial. Respirology. 2017(pagination). PMID: CN01423208. [Duplicate Study].

377. McMaster University F. Heated High Humidity After COPD Exacerbation. 2019 January 16. PMID: NCT03772626. [Intervention/Comparison not of interest].

378. Medical Center A. CRP-guided Antibiotic Treatment in COPD Exacerbations Admitted to the Hospital. 2011 July. PMID: NCT01232140. [Intervention/Comparison not of interest].

379. Medical Center A. Placebo Versus Antibiotics in Acute Exacerbations of Chronic Obstructive Pulmonary Disease (COPD). 2002 July. PMID: NCT00170222. [Clinical Trial].

380.MedImmune Llc A. A Study to Evaluate the Effectiveness of MEDI-563 in Subjects With Chronic Obstructive Pulmonary Disease (COPD). 2010 November. PMID: NCT01227278. [Population not of interest].

381. MedImmune LLC. A Study to Evaluate the Efficacy of MEDI8968 in Chronic Obstructive Pulmonary Disease. 2011 November. PMID: NCT01448850. [Population not of interest].

382. Mehta R, Farrell C, Kilbride S, et al. Population pharmacokinetic analysis of single inhaler triple therapy (ICS/LAMA/LABA) versus dual therapy (LAMA/LABA AND ICS/LABA) in patients with symptomatic COPD: Combined results from three phase iii trials. American Journal of Respiratory and Critical Care Medicine. Conference: American Thoracic Society International Conference, ATS. 2018;197(MeetingAbstracts). PMID: 622969129. [Abstract].

383. Merck S, Dohme C. An Investigational Drug Study In Patients With COPD (Chronic Obstructive Pulmonary Disease) (MK-0873005). 2004 June 1. PMID: NCT00132730. [Population not of interest].
384. Merck S, Dohme C. Long-Term Study of the Effects of Navarixin (SCH 527123, MK7123) in Participants With Moderate to Severe COPD (MK-7123-019). 2009 October. PMID: NCT01006616. [Population not of interest].

385. Mereo B. Efficacy and Safety of BCT197 in Subjects With Acute Respiratory Exacerbations of Chronic Obstructive Pulmonary Disease. 2016 August 1. PMID: NCT02700919. [Clinical Trial].

386. Miao R, Yang B, Huang X. Effect of inhaling penehyclidine hydyochloride in treatment of patients with acute exacerbation of chronic obstrnctive pulmonary disease. [Chinese]. Chinese Journal of Emergency Medicine. 200610 Oct;15(10):909-12. PMID: 44596687. [Language other than English].

387. Mokry M, Joppa P, Slaba E, et al. Beta $<$ inf $>2<$ inf $>$-adrenergic receptor haplotype and bronchodilator response to salbutamol in patients with acute exacerbations of COPD. Medical Science Monitor. 2008 August;14(8):CR392-CR8. PMID: 352234056. [Study design not of interest].

388. Moretti M, Montagna A. Positive outcomes of $900 \mathrm{mg}$ daily erdosteine in acute exacerbation of COPD. European respiratory journal. 2013;42(7). PMID: CN01099938 NEW. [Study design not of interest].

389. Moretti M. Erdosteine improves airflow in patients with severe exacerbation of COPD (AECOPD). European Respiratory Journal. Conference: European Respiratory Society Annual Congress. 2016;48(Supplement 60). PMID: 614778264. [Abstract].

390.Multi-centre Study to Assess the Efficacy and Safety of AZD5423 in COPD Patients on a Background Therapy of Formoterol. 2012 April. PMID: NCT01555099. [Population not of interest].

391. Mundipharma Research L. A Study to Show That Flutiform is Well Tolerated and Effective in the Treatment of COPD. 2013 October. PMID: NCT01946620. [Population not of interest]. 
392.Musil J, Hirsch V, Vondra V, et al. [What dosage is sufficient in combined inhalation therapy (fenoterol + ipratropium bromide) in patients with exacerbation of chronic obstructive lung disease?]. Vnitrni Lekarstvi. 1998 Jul;44(7):415-7. PMID: 9748878. [Language other than English].

393. Musil J, Hirsch V, Vondra V, et al. What is the adequate dose in combined inhalation treatment of patients with exacerbations of chronic obstructive pulmonary disease?: $<$ ORIGINAL $>$ JAKA DAVKA JE DOSTATECNA PRI KOMBINOVANE INHALACNI LECBE (FENOTEROL + IPRATROPIUM BROMID) U NEMOCNYCH S EXACERBACI CHRONICKE OBSTRUKCNI PLICNI NEMOCI (CHOPN)? Vnitrni lekarstvi. 1998;44(7):415-7. PMID: CN-00200534 UPDATE. [Language other than English].

394.Musil J. Cefuroxime in acute exacerbation of chronic obstructive lung disease. [Czech]. Prakticky Lekar. 1997;77(2):84-5. PMID: 27083651. [Language other than English].

395. my mhealth L, Portsmouth Hospitals NHST. Comparing Online Pulmonary Rehabilitation 'myPR' Versus Conventional Pulmonary Rehabilitation for Patients With COPD. 2015 July. PMID: NCT02706613. [Population not of interest].

396.my mhealth L, Portsmouth Hospitals NHST. Trial of E-Health Platform Supported Care vs Usual Care After Exacerbation of COPD. 2015 June. PMID: NCT02706600. [Population not of interest].

397.Nair S, Thomas E, Pearson SB, et al. A randomized controlled trial to assess the optimal dose and effect of nebulized albuterol in acute exacerbations of COPD. Chest. 2005 Jul;128(1):48-54. PMID: 16002915. [Intervention/Comparison not of interest].

398. Nannini LJ. Hospitalization due to COPD exacerbation. Chest. 2012 December;142(6):1697. PMID: 366204582. [Study design not of interest].
399.Naya I, Lipson DA, Boucot I, et al. Impact of prior and concurrent medication on exacerbation risk with long-acting bronchodilators in chronic obstructive pulmonary disease: A post hoc analysis. American Journal of Respiratory and Critical Care Medicine. Conference: American Thoracic Society International Conference, ATS.

2018;197(MeetingAbstracts). PMID: 622969661. [Abstract].

400.Nefedova MN, Shmelev EI. [Inhalational antibacterial therapy for an exacerbation of chronic obstructive pulmonary disease]. Problemy Tuberkuleza I Boleznej Legkih. 2008(8):8-11. PMID: 18822473. [Language other than English].

401.New York Methodist H. Vibratory PEP Device and Hospital Length of Stay for Acute Exacerbation of COPD. 2013 October. PMID: NCT03094806. [Clinical Trial].

402. Niewoehner DE, Collins D, Erbland ML. Relation of FEV(1) to clinical outcomes during exacerbations of chronic obstructive pulmonary disease. Department of Veterans Affairs Cooperative Study Group. American Journal of Respiratory \& Critical Care Medicine. 2000 Apr;161(4 Pt 1):1201-5. PMID: 10764312. [Study design not of interest].

403.Nissen I, Jensen MS. [Nurse-supported discharge of patients with exacerbation of chronic obstructive pulmonary disease]. Ugeskrift for Laeger. 2007 Jun 04;169(23):2220-3. PMID: 17592691. [Language other than English].

404.Northumbria Healthcare NHSFT. Mortality and Symptom Burden Post Hospitalisation With COPD. 2018 September. PMID: NCT03657121. [Intervention/Comparison not of interest].

405. Northwell H, Patient-Centered Outcomes Research I. A Comprehensive Disease Management Program to Improve Quality of Life in Disparity Hispanic and AfricanAmerican Patients Admitted With Exacerbation of Chronic Pulmonary Diseases. 2017 April 1. PMID: NCT03007485. [Population not of interest]. 
406. Nouira S, Bouida W, Grissa MH, et al. Magnesium sulfate versus ipratropium bromide in chronic obstructive pulmonary disease exacerbation: a randomized trial. American Journal of Therapeutics. 2014 May-Jun;21(3):152-8. PMID: 22407196. [Intervention/Comparison not of interest].

407. Novartis Pharmaceuticals N. A Study to Assess the Efficacy, Safety and Tolerability of Once-daily (q.d.) QVA149 in Patients With Moderate to Severe Chronic Obstructive Pulmonary Disease (COPD). 2010 September. PMID: NCT01202188. [Population not of interest].

408. Novartis Pharmaceuticals N. Effect of QVA149 Versus NVA237 and Tiotropium on Chronic Obstructive Pulmonary Disorder (COPD) Exacerbations. 2010 April. PMID: NCT01120691. [Population not of interest].

409. Novartis Pharmaceuticals N. Efficacy and Safety of QMF149 vs. Salmeterol Xinafoate/Fluticasone Propionate in Patients With Chronic Obstructive Pulmonary Disease (COPD). 2012 November. PMID: NCT01636076. [Population not of interest].

410. Novartis Pharmaceuticals N. Efficacy, Safety and Tolerability of NVA237 in Patients With Chronic Obstructive Pulmonary Disease. 2012 March. PMID: NCT01566604. [Population not of interest].

411.Novartis Pharmaceuticals N. Exacerbation Study. 2009 March. PMID: NCT00845728. [Population not of interest].

412.Novartis Pharmaceuticals N. Long Term Safety Study of NVA237 vs QAB149 in COPD Patients. 2012 October. PMID: NCT01697696. [Population not of interest].

413. Novartis Pharmaceuticals N. QVA vs. Salmeterol/Fluticasone, 52-week Exacerbation Study, FLAME (EFfect of Indacaterol Glycopyronium Vs Fluticasone Salmeterol on COPD Exacerbations). 2013 July. PMID: NCT01782326. [Population not of interest].

414. Novartis Pharmaceuticals N. Safety \& Efficacy of BCT197A2201 in Chronic Obstructive Pulmonary Disease (COPD) Patients Presenting With an Exacerbation. 2011 March. PMID: NCT01332097. [Clinical Trial].
415.Ntoumenopoulos G. Using titrated oxygen instead of high flow oxygen during an acute exacerbation of chronic obstructive pulmonary disease (COPD) saves lives. Journal of physiotherapy. 2011;57(1):55. PMID: CN-00797082 UPDATE. [Study design not of interest].

416. Oba Y, Keeney E, Ghatehorde N, et al. Dual combination therapy versus long-acting bronchodilators alone for chronic obstructive pulmonary disease (COPD): a systematic review and network metaanalysis. Cochrane Database of Systematic Reviews. 2018 Dec 03;12:CD012620. PMID: 30521694. [Population not of interest].

417. Odense University Hospital KS, Svendborg Hospital FH, Naestved Hospital HH, et al. Antibiotics in Chronic Obstructive Pulmonary Disease (COPD). 2009 October. PMID: NCT00952861. [Clinical Trial].

418. Odonchimeg P, Ichinnorov D, Sarantuyaa Z, et al. Efficacy of different regimens of steroid therapy in patients with exacerbation of chronic obstructive pulmonary disease. [Russian]. Pulmonologiya. 2015;25(1):5863. PMID: 613728823. [Language other than English].

419. Ohar JA, Crater G, Emmett A, et al. Effects of fluticasone propionate/salmeterol combination 250/50mcg bid (advair diskusTM) Vs. Salmeterol 50mcg bid (serevent diskusTM) on chronic obstructive pulmonary disease (COPD) exacerbation rate, following acute exacerbation or hospitalization. American Journal of Respiratory and Critical Care Medicine. Conference: American Thoracic Society International Conference, ATS. 2013;187(MeetingAbstracts). PMID: 71981900. [Intervention/Comparison not of interest].

420.Ole Frobert MD, PhD RÖC. Beta-blockers to Patients With Chronic Obstructive Pulmonary Disease. 2018 June 12. PMID: NCT03566667. [Population not of interest].

421. Oral prednisolone as effective as IV dor COPD exacerbation. Australian Journal of Pharmacy. 2009 March;90(1066):79. PMID: 354326988. [Study design not of interest]. 
422. Osadnik C, McDonald CF, Miller B, et al. Positive expiratory pressure therapy does not improve symptoms, quality of life or incidence of exacerbations in individuals with acute exacerbations of COPD-a multicentre randomized controlled trial. Respirology. 2013 April;2):26. PMID: 71011003. [Abstract].

423. Osadnik C, McDonald CF, Miller BR, et al. A multi-centre, randomised controlled trial of positive expiratory pressure mask therapy for inpatients with acute exacerbations of COPD and sputum expectoration. European Respiratory Journal. Conference: European Respiratory Society Annual Congress. 2013;42(SUPPL. 57). PMID: 71843394. [Abstract].

424. Ottawa Hospital Research I, Behring CSL, Grifols Biologicals I. Ig PRx in AECOPD: Pilot Study. 2016 September. PMID: NCT02690038. [Population not of interest].

425. Ottawa Hospital Research I. Feasibility and Safety of Immunoglobulin (Ig) Treatment in COPD Outpatients With Frequent Exacerbations: Pilot Study 1. 2018 January. PMID: NCT03018652. [Population not of interest].

426. Ottawa Hospital Research I. TNF-alpha Antagonists for AECOPD: A Randomized, Double-Blind, Placebo-Controlled Pilot Trial. 2008 November. PMID: NCT00789997. [Duplicate Study].

427. Outcome Study to Evaluate Klaricid XL ${ }^{\circledR}$ (Clarithromycin Modified Release) in Patients With Acute Exacerbation of Chronic Bronchitis. 2011 May. PMID: NCT01354938. [Population not of interest].

428. Outcomes for Chronic Obstructive Pulmonary Disease Moderate Exacerbators Initiating Treatment. 2011 March. PMID: NCT01395875. [Population not of interest].

429. Outcomes From Initial Maintenance Therapy With Fluticasone Propionate 250/Salmeterol 50 (FSC) or Tiotropium in Chronic Obstructive Pulmonary Disease. 2008 July. PMID: NCT01387178. [Population not of interest].
430. Owusuaa C, Dijkland S, Van Der Rijt K, et al. Prognostic factors and tools of mortality in patients with chronic lung disease-A systematic review and meta-analysis. Palliative Medicine. 2018 May;32 (1 Supplement 1):105. PMID: 622331141. [Population not of interest].

431.Parc de Salut M. Respiratory Therapy in COPD Exacerbations. 2011 December. PMID: NCT02125747. [Outcome not of interest].

432.Parkin JM, Vaz M, Orford C, et al. Designing an acute intervention trial in patients hospitalised for AeCOPD: Experience from the $\mathrm{PH} 2$ aether study of acumapimod (BCT197) P38 map kinase inhibitor. American Journal of Respiratory and Critical Care Medicine. Conference: American Thoracic Society International Conference, ATS. 2017;195(no pagination). PMID: 617704496. [Abstract].

433.Patel K, McNeilly R, Collins C, et al. Nebulized hypertonic saline for inpatient use in COPD. Chest. 2017 October;152 (4 Supplement 1):A786. PMID: 619297259. [Abstract].

434.Pearl Therapeutics I. A Study to Assess the Efficacy and Safety of PT009 Compared to PT005 on COPD Exacerbations Over a 52Week Period in Subjects With Moderate to Very Severe COPD (Sophos). 2016 April 29. PMID: NCT02727660. [Population not of interest].

435.Pearl Therapeutics I. Study to Assess the Efficacy and Safety of PT010 Relative to PT003 and PT009 in Subjects With Moderate to Very Severe COPD (Ethos). 2015 June 30. PMID: NCT02465567. [Population not of interest].

436.Peking Union Medical College Hospital XARPsH, Cangzhou Central Hospital CsPsH, Chengde Central Hospital BFH, et al. Effects of Particulate Matter on the Pulmonary Function and Acute Exacerbation of COPD and Asthma. 2015 December. PMID: NCT03193879. [Population not of interest]. 
437.Perkins-Porras L, Riaz M, Okekunle A, et al. Feasibility study to assess the effect of a brief mindfulness intervention for patients with chronic obstructive pulmonary disease: A randomized controlled trial. Chronic Respiratory Disease. 2018 Jan 01:1479972318766140. PMID: 29690786. [Intervention/Comparison not of interest].

438.Person-centred Care at Distance. Https://clinicaltrials.gov/show/nct03183817. 2017. PMID: CN-01494890. [Population not of interest].

439.PERSPECTIVE: Telithromycin - Acute Exacerbation of Chronic Bronchitis. 2004 January. PMID: NCT00132938. [Population not of interest].

440.Pilcher J, Eastlake L, Richards M, et al. Physiological effects of titrated oxygen via nasal high-flow cannulae in COPD exacerbations: A randomized controlled cross-over trial. Respirology. 2017 Aug;22(6):1149-55. PMID: 28470831. [Outcome not of interest].

441.Pishbin E, Moradi EV. Intravenous magnesium sulfate in the treatment of acute exacerbations of COPD: A randomized controlled trial. Critical Care. Conference: 38th International Symposium on Intensive Care and Emergency Medicine, ISICEM. 2018;22(Supplement 1). PMID: 621461220. [Abstract].

442.Pishbin E. Intravenous magnesium sulfate in the treatment of acute exacerbations of COPD. Journal of Emergency Medicine. 2017 September;53 (3):442-3. PMID: 618810768. [Abstract].

443.Plant PK, Watson JP, Muers MF, et al. Randomised controlled trial of oral corticosteroids in acute exacerbations of COPD. European respiratory society annual congress, madrid, spain. 1999. PMID: CN01343140 NEW. [Study design not of interest].

444.PneumRx I. COPD Co-Pilot AIR Substudy of CLN0014. 2017 March 7. PMID: NCT03267992. [Population not of interest].
445.Poder TG, Kouakou CRC, Bouchard PA, et al. Cost-effectiveness of FreeO $<$ inf $>2<$ inf $>$ in patients with chronic obstructive pulmonary disease hospitalised for acute exacerbations: Analysis of a pilot study in Quebec. BMJ Open. 201801 Jan;8 (1) (no pagination)(e018835). PMID: 623794356. [Outcome not of interest].

446.Poitiers University H. High-flow Nasal Cannula Nebulization of Beta 2 Adrenergic Agonist During Acute Exacerbation of Chronic Obstructive Pulmonary Disease. 2018 May. PMID: NCT03449056. [Population not of interest].

447.Portsmouth Hospitals Nhs Trust P. MISSION COPD - Modern Innovative SolutionS in Improving Outcomes iN COPD. 2015 September. PMID: NCT02534766. [Population not of interest].

448.Powrie DJ, Wilkinson TMA, Donaldson GC, et al. Effect of tiotropium on sputum and serum inflammatory markers and exacerbations in COPD. [Portuguese]. Revista Portuguesa de Pneumologia. 2008 July/August;14(4):573-6. PMID: 352088306. [Population not of interest].

449.Price DB, Cotton S, Fielding S, et al. Use of 'low-dose' theophylline to reduce exacerbations of COPD: A pragmatic multicentre randomised placebo controlled trial. American Journal of Respiratory and Critical Care Medicine. Conference: American Thoracic Society International Conference, ATS. 2018;197(MeetingAbstracts). PMID: 622969217. [Abstract].

450.Prinsv HJ, Snijders D, Daniels JMA, et al. Eosinophilia as marker of outcome in hospitalised patients with AECOPD. European Respiratory Journal. Conference: European Respiratory Society Annual Congress. 2013;42(SUPPL. 57). PMID: 71843553. [Abstract].

451.Pristinamycin use in the treatment of acute exacerbations of COPD (COPD = chronic obstructive pulmonary disease). [French]. Medecine et Maladies Infectieuses. 2008;38(2 PART 2):5-7. PMID: CN01194574 NEW. [Language other than English]. 
452.Puig-Junoy J, Casas A, Font-Planells J, et al. The impact of home hospitalization on healthcare costs of exacerbations in COPD patients. European Journal of Health Economics. 2007 Dec;8(4):325-32. PMID: 17221178. [Intervention/Comparison not of interest].

453. Pulmonary Rehabilitation for Chronic Obstructive Pulmonary Disease (COPD) Exacerbation. 2006 October. PMID: NCT00413543. [Clinical Trial].

454.Punekar YS, Sharma S, Pahwa A, et al. Rescue medication use as a patient-reported outcome in COPD: a systematic review and regression analysis. Respiratory Research. 201705 08;18(1):86. PMID: 28482883. [Population not of interest].

455. Rabe KF, Halpin D, Martinez F, et al. Relative timing of clinically important deterioration and related long-term outcomes in COPD: A post hoc analysis of the UPLIFT study. Pneumologie. Conference. 2018;59. PMID: 622904795. [Abstract].

456. Rabe KF, Hanauer G, Strigun A, et al. Effect of roflumilast on the serum metabolome of COPD patients. American Journal of Respiratory and Critical Care Medicine. Conference: American Thoracic Society International Conference, ATS. 2018;197(MeetingAbstracts). PMID: 622968067. [Abstract].

457. Radboud U. Exacerbation Self-management in COPD: The ACCESS Study. 2015 June. PMID: NCT02553096. [Population not of interest].

458. Rahlfs VW, Macciocchi A, Monti T. Clinical efficacy of brodimoprim in the treatment of acute exacerbation of chronic bronchitis. A meta-analysis. Clinical Drug Investigation. 1996;11(3):121-30. PMID: 26097447. [Population not of interest].

459. Ram FS, Rodriguez-Roisin R, GranadosNavarrete A, et al. WITHDRAWN: Antibiotics for exacerbations of chronic obstructive pulmonary disease. Cochrane Database of Systematic Reviews. 2011 Jan 19(1):CD004403. PMID: 21249661. [Abstract].
460.Ram FSF, Barnes NC, Granados-Navarrete A, et al. Comparing antibiotics for acute exacerbations of chronic obstructive pulmonary disease. Cochrane Database of Systematic Reviews. 2008;(2) (no pagination)(CD007093). PMID: 351807258. [Study design not of interest].

461. Ram FSF, Poole PJ, Bagg W, et al. Randomized, controlled trial of oral theophylline for the treatment of acute exacerbations of chronic obstructive pulmonary disease. Am J Respir Crit Care Med. 2000;161(suppl):A489. [Duplicate Study].

462.Ram FSF, Rodriguez-Roisin R, GranadosNavarrete A, et al. Antibiotics for exacerbations of chronic obstructive pulmonary disease. Cochrane Database of Systematic Reviews. 2006 Apr 19(2):CD004403. PMID: 16625602. [Study not completed].

463. Ram FSF, Wedzicha JA, Wright J, et al. Hospital at home for patients with acute exacerbations of chronic obstructive pulmonary disease: Systematic review of evidence. British Medical Journal. 200407 Aug;329(7461):315-8. PMID: 39062294. [Intervention/Comparison not of interest].

464. Ram FSF, Wedzicha JA, Wright JJ, et al. Hospital at home for acute exacerbations of chronic obstructive pulmonary disease. Cochrane Database of Systematic Reviews. 2009;(4) (no pagination)(CD003573). PMID: 358528499.

[Intervention/Comparison not of interest].

465. Randomised, Double-Blind, PlaceboControlled, Parallel-Group, Multi-centre, Dose Ranging Study to Evaluate the Efficacy and Safety of Losmapimod Tablets Administered Twice Daily Compared With Placebo for 24 Weeks in Adult Subjects With Chronic Obstructive Pulmonary Disease (COPD). 2010 November 4. PMID: NCT01218126. [Population not of interest].

466. Rassouli F, Baty F, Stolz D, et al. Telehealth care vs. standard care in COPD - An international randomised controlled trial (interim analysis). Respiration. 2018;95 (6):508. PMID: 623934020. [Abstract]. 
467. Reed M. Short-term versus conventional glucocorticoid therapy in acute exacerbations of COPD. Pharmacy Times. 2013;79(8). PMID: 369613852. [Study design not of interest].

468. Revitt O, Sewell L, Singh S. Early versus delayed pulmonary rehabilitation: A randomized controlled trial - Can we do it? Chronic Respiratory Disease. 201801 Aug;15(3):323-6. PMID: 623392453. [Population not of interest].

469. Reyes Martin E, Fernandez Almendros C, Alvarez-Sala JL, et al. [Effect of immunomodulator AM3 on the exacerbations in patients with chronic bronchitis: a systematic review of controlled trials]. Revista Clinica Espanola. 2004 Sep;204(9):466-71. PMID: 15388020. [Language other than English].

470. Riancho JA, Cubian I, Portero I. [Effectiveness of inhaled corticosteroids in chronic obstructive lung disease: systematic review]. Medicina Clinica. $2002 \mathrm{Apr}$ 06;118(12):446-51. PMID: 11958761. [Language other than English].

471. Rittayamai N, Tscheikuna J, Praphruetkit N, et al. Use of High-Flow Nasal Cannula for Acute Dyspnea and Hypoxemia in the Emergency Department. Respiratory Care. 2015 Oct;60(10):1377-82. PMID: 26060321. [Population not of interest].

472. Roche N, Wedzicha JA, Donohue JF, et al. Indacaterol/glycopyrronium improves lung function and health status versus salmeterol/fluticasone in moderate-to-very severe COPD patients irrespective of prior ICS/LABA/LAMA therapy: The flame study. Respirology. 2017 November;22 (Supplement 3):177-8. PMID: 619968137. [Abstract].

473. Rodrigo GJ, Neffen H. A systematic review with meta-analysis of fluticasone furoate/vilanterol combination for the treatment of stable COPD. Pulmonary Pharmacology \& Therapeutics. 2017 02;42:1-6. PMID: 27864038. [Population not of interest].
474. Rodriguez DA, Rodo-Pin A, Guerrero M, et al. Impact of step exercises as an early intervention during COPD exacerbation. European Respiratory Journal. Conference: European Respiratory Society Annual Congress. 2016;48(Supplement 60). PMID: 614780709. [Abstract].

475. Rodriguez-Roisin R. COPD exacerbations . 5: Management. Thorax. 2006 June;61(6):535-44. PMID: 43901525. [Study design not of interest].

476. Roede I, Bresser P, Bindels P, et al. Antibiotics for exacerbation of chronic obstructive pulmonary disease seems to reduce relapses. [Dutch]. Huisarts en Wetenschap. 2009 May;52(5):218-24. PMID: 354813664. [Language other than English].

477. Roflumilast in Chronic Obstructive Pulmonary Disease (COPD) Patients Treated With Fixed Combinations of Longacting $\beta 2$-agonists (LABA) and Inhaled Glucocorticosteroid (ICS). 2011 May. PMID: NCT01329029. [Population not of interest].

478. Roflumilast in Chronic Obstructive Pulmonary Disease (COPD) Patients Treated With Fixed Dose Combinations of Long-acting $\beta 2$-agonist (LABA) and Inhaled Corticosteroid (ICS). 2011 September 30. PMID: NCT01443845. [Population not of interest].

479. Rogliani P, Calzetta L, Braido F, et al. LABA/LAMA fixed-dose combinations in patients with COPD: a systematic review. International Journal of Copd. 2018;13:3115-30. PMID: 30323582. [Population not of interest].

480.Rogliani P, Ora J, Ciaprini C, et al. Effect of indacaterol on arterial blood gases in patients suffering from acute exacerbation of COPD. Respiratory Medicine. 2014 Feb;108(2):307-13. PMID: 24238769. [Intervention/Comparison not of interest].

481. Romanovskikh A, Sinopalinkov A, Ratchina S. Open label randomized, comparative trial of the efficacy of levofloxacin versus clarithromycin SR therapy in complicated infectious exacerbation of COPD [Abstract]. European respiratory journal. 2007;30(Suppl 51):674s [E3919]. PMID: CN-00642856 UPDATE. [Abstract]. 
482. Royal F, University College Medical S. Effects of Long Term Macrolide Antibiotic Therapy in Patients With COPD. 2004 April. PMID: NCT00147667. [Population not of interest].

483. Rutishauser J. 5 days of prednisone was noninferior to 14 days in patients with acute COPD exacerbation. Annals of internal medicine. 2013;159(6):JC5. PMID: CN00916328 UPDATE. [Study design not of interest].

484.Saey D, Ribeiro F. Resistance training preserves skeletal muscle function in patients with COPD who are hospitalised with an acute exacerbation. Journal of Physiotherapy. 2011 September;57(3):194. PMID: 362505468. [Study design not of interest].

485. Safety and Efficacy of Arformoterol Tartrate Inhalation Solution in Subjects With Chronic Obstructive Pulmonary Disease. 2005 October. PMID: NCT00250679. [Population not of interest].

486. Safety and Efficacy Study of Losmapimod (GW856553) in Frequently Exacerbating Participants With Chronic Obstructive Pulmonary Disease (COPD). 2014 December. PMID: NCT02299375. [Population not of interest].

487. Safety Study of Arformoterol Tartrate Inhalation Solution in Chronic Obstructive Pulmonary Disease (COPD) Subjects. 2009 June. PMID: NCT00909779. [Population not of interest].

488. Safety Study to Evaluate MN-221 in Chronic Obstructive Pulmonary Disease (COPD) Patients. 2012 March. PMID: NCT01551316. [Population not of interest].

489.Salazar A, Juan A, Ballbe R, et al. Emergency short-stay unit as an effective alternative to in-hospital admission for acute chronic obstructive pulmonary disease exacerbation. American Journal of Emergency Medicine. 2007 May;25(4):4867. PMID: 46722667.

[Intervention/Comparison not of interest].

490.Samsung Medical C. Physical Activity in COPD Acute Exacerbation Patients. 2017 November 6. PMID: NCT03437252. [Population not of interest].
491.Sanchez IT, Valenza MC, Del Corral Nunez-Flores T, et al. Repercussion of different physical therapy interventions on the perceived health status of AECOPD patients. European Respiratory Journal. Conference: European Respiratory Society Annual Congress. 2016;48(Supplement 60). PMID: 614780393. [Abstract].

492.Saudny-Unterberger H, Martin JG, GrayDonald K. Impact of nutritional support on functional status during an acute exacerbation of chronic obstructive pulmonary disease. American journal of respiratory and critical care medicine. 1997;156(3):794-9. [Duplicate Study].

493. Sayiner A, Aytemur ZA, Baysak A, et al. Nacetylcysteine in exacerbations of chronic obstrutive pulmonary disease associated with increased sputum. American Journal of Respiratory and Critical Care Medicine. Conference: American Thoracic Society International Conference, ATS. 2011;183(1 MeetingAbstracts). PMID: 70847477. [Abstract].

494.Sayiner A, Aytemur ZA, Cirit M, et al. Systemic glucocorticoids in severe exacerbations of COPD. Chest. 2001;119(3):726-30. [Duplicate Study].

495. Schou L, Ostergaard B, Rydahl-Hansen S, et al. A randomised trial of telemedicine-based treatment versus conventional hospitalisation in patients with severe COPD and exacerbation - effect on self-reported outcome. Journal of Telemedicine \& Telecare. 2013 Apr;19(3):160-5. PMID: 23612519. [Intervention/Comparison not of interest].

496. Schroff P, Dransfield MT, Woodruff P, et al. Plasma acetyl-proline-glycine-proline (ACPGP) and COPD exacerbation risk in chronic azithromycin use. American Journal of Respiratory and Critical Care Medicine. Conference: American Thoracic Society International Conference, ATS. 2018;197(MeetingAbstracts). PMID: 622969889. [Abstract]. 
497.Schuetz P, Leuppi JD, Bingisser R, et al. Prospective analysis of adrenal function in patients with acute exacerbations of COPD: the Reduction in the Use of Corticosteroids in Exacerbated COPD (REDUCE) trial. European Journal of Endocrinology. 2015 Jul;173(1):19-27. PMID: 25855628. [Study design not of interest].

498.Schumaker GL, Epstein SK. Managing acute respiratory failure during exacerbation of chronic obstructive pulmonary disease. Respiratory Care. 2004 Jul;49(7):766-82. PMID: 15222909. [Study design not of interest].

499. Scuri M, Fabbri LM, Singh D, et al. Reduction in fatal events with ICScontaining medications: results of safety pooled analysis from the trilogy, trinity and tribute studies. American Journal of Respiratory and Critical Care Medicine. Conference: American Thoracic Society International Conference, ATS. 2018;197(MeetingAbstracts). PMID: 622969648. [Population not of interest].

500.Scuri M, Singh D, Fabbri LM, et al. Single inhaler extrafine triple therapy improves clinical outcomes in gold b COPD patients: Post-HOC analysis of the tribute study. American Journal of Respiratory and Critical Care Medicine. Conference: American Thoracic Society International Conference, ATS. 2018;197(MeetingAbstracts). PMID: 622969478. [Abstract].

501. Scuri M, Singh D, Fabbri LM, et al. Single inhaler extrafine triple therapy reduces clinically important deterioration (CID) in COPD compared to indacaterol/glycopyrronium: Post-hoc analysis of the tribute study. American Journal of Respiratory and Critical Care Medicine. Conference: American Thoracic Society International Conference, ATS. 2018;197(MeetingAbstracts). PMID: 622967653. [Abstract].
502.Scuri M, Singh D, Fabbri LM, et al. Single inhaler extrafine triple therapy vs indacaterol/glycopyrronium in copd patients previously treated with laba/lama: PostHOC analysis of the tribute study. American Journal of Respiratory and Critical Care Medicine. Conference: American Thoracic Society International Conference, ATS. 2018;197(MeetingAbstracts). PMID: 622969555. [Abstract].

503.Scuri M, Spinola M, Vezzoli S, et al. Efficacy of CHF5993, a novel triple extrafine combination treatment in COPD patients at high risk of exacerbations: A sub group analysis of the TRINITY study. Pneumologie. Conference. 2018;59. PMID: 622904829. [Duplicate Study].

504.Segreti A, Fiori E, Calzetta L, et al. The effect of indacaterol during an acute exacerbation of COPD. Pulmonary Pharmacology \& Therapeutics. 2013 Dec;26(6):630-4. PMID: 23578980. [Intervention/Comparison not of interest].

505.Sergey A, Galiya N, Sanjita B, et al. Effects of combined therapy with nebulized b2agonist and anticholinergic in severe exacerbation of chronic obstructive pulmonary disease. Randomised controlled trial [Abstract]. European respiratory journal. 2007;30(Suppl 51):611s [P3594]. PMID: CN-00642795 UPDATE. [Abstract].

506. Sethi S, Anzueto A, Miravitlles M, et al. Determinants of bacteriological outcomes in exacerbations of chronic obstructive pulmonary disease. Infection. 2016 Feb;44(1):65-76. PMID: 26370552. [Study design not of interest].

507. Sethi S, Kerwin EM, Watz H, et al. Amplify: A randomized, phase iii study evaluating the efficacy and safety of aclidinium/formoterol versus monotherapy in patients with copd. American Journal of Respiratory and Critical Care Medicine. Conference: American Thoracic Society International Conference, ATS. 2018;197(MeetingAbstracts). PMID: 622971059. [Abstract]. 
508. Sheikh Motahar Vahedi H, Mahshidfar B, Rabiee $\mathrm{H}$, et al. The adjunctive effect of nebulized furosemide in COPD exacerbation: a randomized controlled clinical trial. Respiratory Care. 2013 Nov;58(11):1873-7. PMID: 23650431. [Intervention/Comparison not of interest].

509. Shestovitskiy VA, Grinshteyn YI, Maksimova AV. Cytological and oxidant characteristics of local cellular immunity in acute severe exacerbation of chronic obstructive pulmonary disease. Pulmonologiya. 2017;27(4):478-83. [Duplicate Study].

510.Shi X, Li H. Anticoagulation therapy in patients with chronic obstructive pulmonary disease in the acute exacerbation stage. Experimental and Therapeutic Medicine. 2013;5(5):1367-70.

[Intervention/Comparison not of interest].

511.Shmelev EI, Kunicina YL. Comparison of fenspiride with beclomethasone as adjunctive anti-inflammatory treatment in patients with chronic obstructive pulmonary disease. Clinical Drug Investigation. 2006;26(3):151-9. PMID: 17163246. [Population not of interest].

512. Shortall SP, Blum J, Oldenburg FA, et al. Treatment of patients hospitalized for exacerbations of chronic obstructive pulmonary disease: comparison of an oral/metered-dose inhaler regimen and an intravenous/nebulizer regimen. Respiratory Care. 2002 Feb;47(2):154-8. PMID: 11812271. [Intervention/Comparison not of interest].

513. Shorter courses of corticosteroids for COPD exacerbations? Drug and Therapeutics Bulletin. 2015;53(3):28. PMID: 604440834. [Abstract].

514. Shrestha M, O'Brien T, Haddox R, et al. Decreased duration of emergency department treatment of chronic obstructive pulmonary disease exacerbations with the addition of ipratropium bromide to betaagonist therapy. Annals of Emergency Medicine. 1991 Nov;20(11):1206-9. PMID: 1835324. [Intervention/Comparison not of interest].
515. Shrestha M, O'Brien T, Haddox R, et al. Decreased duration of emergency department treatment of chronic obstructive pulmonary disease exacerbations with the addition of ipratropium bromide to $\beta$-agonist therapy. Annals of emergency medicine. 1991;20(11):1206-9. [Duplicate Study].

516.Silver PC, Kollef MH, Clinkscale D, et al. A Respiratory Therapist Disease Management Program for Subjects Hospitalized With COPD. Respiratory Care. 2017 Jan;62(1):19. PMID: 27899531.

[Intervention/Comparison not of interest].

517.Similowski T, Suissa S. Systemic steroids in severe forms of COPD exacerbations: A question of balance? European Respiratory Journal. 201401 Mar;43(3):668-70. PMID: 372532423. [Study design not of interest].

518.Singh VP, Rao V, V P, et al. Comparison of the effectiveness of music and progressive muscle relaxation for anxiety in COPD--A randomized controlled pilot study. Chronic Respiratory Disease. 2009;6(4):209-16. PMID: 19858350. [Population not of interest].

519.Sivapalan P, Moberg M, Eklof J, et al. A multi-center randomized, controlled, openlabel trial evaluating the effects of eosinophil-guided corticosteroid-sparing therapy in hospitalised patients with COPD exacerbations - The CORTICO steroid reduction in COPD (CORTICO-COP) study protocol. BMC Pulmonary Medicine. 2017 Aug 15;17(1):114. PMID: 28810909. [Intervention/Comparison not of interest].

520.Skwarska E, Cohen G, Skwarski KM, et al. Randomized controlled trial of supported discharge in patients with exacerbations of chronic obstructive pulmonary disease. Thorax. 2000 Nov;55(11):907-12. PMID: 11050258. [Intervention/Comparison not of interest].

521. Slawson D. Five-day steroid treatment effective for acute COPD exacerbation. American family physician. 2013;88(11):744A. PMID: CN-00911065 UPDATE. [Abstract]. 
522. Sliwka A, Jankowski M, Gross-Sondej I, et al. Once-daily long-acting beta2agonists/inhaled corticosteroids combined inhalers versus inhaled long-acting muscarinic antagonists for people with chronic obstructive pulmonary disease. Cochrane Database of Systematic Reviews. 201808 24;8:CD012355. PMID: 30141826. [Population not of interest].

523. Slotervaart Hospital A, Isala SG. Glucose Control During Glucocorticoid Therapy in Acute Exacerbation of Chronic Obstructive Pulmonary Disease. 2015 February. PMID: NCT02253121. [Outcome not of interest].

524.Song YJ, Zhou ZH, Liu YK, et al. Prothrombotic state in senile patients with acute exacerbations of chronic obstructive pulmonary disease combined with respiratory failure. Experimental and Therapeutic Medicine. 2013;5(4):1184-8. [Outcome not of interest].

525. Soroka University Medical C. Tight Glycemic Control in Acute Exacerbations of COPD. 2007 April. PMID: NCT00452296. [Study design not of interest].

526. Southeast University C. Effects of NAVA and PSV to AECOPD Patients' Air Distribution and Dead Space. 2014 November. PMID: NCT02289573. [Population not of interest].

527. Soutotchnikova OA, Avdeev SN, Belevsky AS, et al. Randomized controlled trial of nebulized budesonide suspension in acute severe exacerbations of COPD. European respiratory journal. 2002;20(Suppl 38):244s. PMID: CN-00461774 UPDATE. [Abstract].

528. Ställberg B, Selroos O, Vogelmeier C, et al. Budesonide/formoterol as effective as prednisolone plus formoterol in acute exacerbations of COPD A double-blind, randomised, non-inferiority, parallel-group, multicentre study. Respiratory research. 2009;10(1):11. [Duplicate Study].

529. Steurer J. [5 days of systemic steroids in COPD exacerbation are adequate]. Praxis. 2013 Aug 21;102(17):1075. PMID: 23965722. [Study design not of interest].
530.Stolbrink M, Amiry J, Blakey JD. Does antibiotic treatment duration affect the outcomes of exacerbations of asthma and COPD? A systematic review. Chronic Respiratory Disease. 2018 Aug;15(3):22540. PMID: 29232988. [Duplicate Study].

531.Stolz D, Christ-Crain M, Bingisser R, et al. Antibiotic treatment of exacerbations of COPD: a randomized, controlled trial comparing procalcitonin-guidance with standard therapy. Chest. 2007 Jan;131(1):919. PMID: 17218551.

[Intervention/Comparison not of interest].

532.Stolz D, Christ-Crain M, Bingisser R, et al. Procalcitonin-guided antibiotic therapy in acute exacerbations of COPD (AECOPD): a randomised trial - the ProCOLD study [Abstract]. European respiratory journal. 2005;26(Suppl 49):Abstract No. 3044. PMID: CN-00592471 UPDATE. [Intervention/Comparison not of interest].

533.Strambu I, Parkin JM, Magnusson B, et al. Treatment of acute exacerbations in COPD: An exploratory phase ii study of single and repeated doses of acumapimod (BCT197), an oral p38 inhibitor. American Journal of Respiratory and Critical Care Medicine. Conference: American Thoracic Society International Conference, ATS. 2017;195(no pagination). PMID: 617707134. [Abstract].

534.Study Evaluating the Efficacy and Safety of Fluticasone Furoate/Vilanterol Inhalation Powder (FF/VI) Compared With Vilanterol Inhalation Powder (VI) in Subjects With Chronic Obstructive Pulmonary Disease (COPD). 2014 April 7. PMID: NCT02105974. [Population not of interest].

535.Study In Subjects With COPD (Chronic Obstructive Pulmonary Disease). 2004 November. PMID: NCT00103922. [Population not of interest].

536. Study to Evaluate Efficacy and Safety of Mepolizumab for Frequently Exacerbating Chronic Obstructive Pulmonary Disease (COPD) Patients. 2014 April 15. PMID: NCT02105948. [Population not of interest].

537. Study to Evaluate the Safety and Efficacy of Formoterol in a Daily Dose of $18 \mu \mathrm{g}(9 \mu \mathrm{g}$ Twice Daily) in Japanese Chronic Obstructive Pulmonary Disease (COPD) Patients. 2009 December. PMID: NCT01047553. [Population not of interest]. 
538.System VAPSHC, System VAECHC, System VAPAHC. Understanding Patient Management of COPD Exacerbations. 2016 April. PMID: NCT02725294. [Study design not of interest].

539.Tabak M, Brusse-Keizer M, van der Valk P, et al. A telehealth program for selfmanagement of COPD exacerbations and promotion of an active lifestyle: a pilot randomized controlled trial. International Journal of Copd. 2014;9:935-44. PMID: 25246781. [Intervention/Comparison not of interest].

540.Tahirah F, Jenkins S, Othman SK, et al. A randomised controlled trial of individualised, progressed early exercise in patients hospitalised with an acute exacerbation of chronic obstructive pulmonary disease (AECOPD). European Respiratory Journal. Conference: European Respiratory Society Annual Congress. 2015;46(SUPPL. 59). PMID: 72105113. [Abstract].

541.Tang C, Southcott AM, Barker K, et al. Commencement of pulmonary rehabilitation during hospital admission increases rates of attendance among patients with chronic obstructive pulmonary disease (COPD): Randomised controlled trial. Respirology. 2018 March;23 (Supplement 1):122. PMID: 622091528. [Abstract].

542.Tao Y, Chen J, Zhu J, et al. The clinical applications of penehyclidine hydyochloride in patients with AECOPD. [Chinese]. Chinese Journal of Emergency Medicine. 2006 March;15(3):255-9. PMID: 43421654. [Language other than English].

543.Targeted AntiBiotics for Chronic Obstructive Pulmonary Disease. Https://clinicaltrials.gov/show/nct03262142. 2017. PMID: CN-01496082. [Population not of interest].

544.Taylor J, Martinez C, Sjoding M, et al. Transitional care management for COPD: The University of Michigan experience. American Journal of Respiratory and Critical Care Medicine. Conference: American Thoracic Society International Conference, ATS. 2018;197(MeetingAbstracts). PMID: 622970044. [Abstract].
545.Telithromycin in Acute Exacerbation of Chronic Bronchitis. 2006 January. PMID: NCT00288223. [Population not of interest].

546.Telithromycin: in the Treatment of Acute Exacerbation of Chronic Bronchitis in Adult Outpatients With COPD. 2002 November. PMID: NCT00538148. [Clinical Trial].

547.Temple U, Forest L. Effects of Roflumilast in Hospitalized Chronic Obstructive Pulmonary Disease( COPD) on Mortality and Re-hospitalization. 2013 November. PMID: NCT01973998. [Clinical Trial].

548. Temple U, Pennsylvania Department of $\mathrm{H}$. Pennsylvania Study Of Chronic Obstructive Pulmonary Exacerbations. 2004 June. PMID: NCT00774176. [Study design not of interest].

549.Temple University F. Effects of Nasal Highflow Oxygen in Patients With an Exacerbation of Chronic Obstructive Pulmonary Disease (COPD). 2017 July 20. PMID: NCT03214458. [Study design not of interest].

550. Teva Branded Pharmaceutical Products R, D Inc TPI. A Study of 3 Doses of Tiotropium Hydrofluoralkane (HFA) Breath Actuated Inhaler (BAI), in Patients With Chronic Obstructive Pulmonary Disease. 2014 August. PMID: NCT02203474. [Population not of interest].

551.Teva Branded Pharmaceutical Products R, D Inc TPI. Evaluate the Relationship Between Use of Albuterol Multidose Dry Powder Inhaler With an eModule (eMDPI) and Exacerbations in Patients With Chronic Obstructive Pulmonary Disease (COPD). 2017 September 28. PMID: NCT03256695. [Clinical Trial].

552.The Effect of Azithromycin in Patients With Chronic Obstructive Pulmonary Disease (COPD) and Chronic Productive Cough. 2009 September. PMID: NCT01071161. [Population not of interest].

553.The Effectiveness of Roflumilast in Improving Mucociliary Clearance in Patients With COPD and Chronic Bronchitis. Https://clinicaltrials.gov/show/nct03073798. 2017. PMID: CN-01562453. [Duplicate Study]. 
554.The Effects of Singing Training for Patients With Chronic Obstructive Pulmonary Disease (COPD). Https://clinicaltrials.gov/show/nct03280355. 2017. PMID: CN-01564011. [Duplicate Study].

555. The George Institute NH, Medical Research Council A. Theophylline and Steroids in Chronic Obstructive Pulmonary Disease (COPD) Study. 2014 May. PMID: NCT02261727. [Population not of interest].

556. The Korean Academy of Tuberculosis RD. Clinical Outcomes of Tiotropium Plus Fluticasone Propionate/Salmeterol Compared With Tiotropium for Chronic Obstructive Pulmonary Disease (COPD) in Korea. 2009 March. PMID: NCT00864812. [Population not of interest].

557.The Royal Bournemouth Hospital S. Insulin Therapy in Chronic Obstructive Pulmonary Disease (COPD) Exacerbations. 2007 July. PMID: NCT00467636. [Clinical Trial].

558. The Study Will Evaluate the Effect of AZD9164 in Patients With Chronic Obstructive Pulmonary Disease. 2009 June. PMID: NCT00939211. [Population not of interest].

559.Thompson WH, Nielson CP, Carvalho P, et al. Controlled trial of oral prednisone in outpatients with acute COPD exacerbation. American journal of respiratory and critical care medicine. 1996;154(2):407-12. [Duplicate Study].

560.Tongji Hospital AhoGmu, China UhoHufn, China Th, et al. Quality Control and Evaluation in Standard COPD Management. 2017 November 1. PMID: NCT03314077. [Population not of interest].

561.Torres A. Chronic obstructive lung disease: Antibiotic therapy of acute exacerbations. [Spanish]. Archivos de Bronconeumologia. 2003;39(SUPPL. 3):27-31. PMID: 37023004. [Language other than English].

562. Torres-Sanchez I, Cabrera-Martos I, Jose Cruz-Rueda J, et al. Multimodal therapeutic program during hospitalization in obese COPD patients. European Respiratory Journal. Conference: European Respiratory Society Annual Congress. 2015;46(SUPPL. 59). PMID: 72105220. [Abstract].
563.Torres-Sanchez I, Valenza MC, CabreraMartos I, et al. Physical therapy effects on psychological functions in patients with acute COPD exacerbation. European Respiratory Journal. Conference: European Respiratory Society Annual Congress. 2013;42(SUPPL. 57). PMID: 71843404. [Abstract].

564.Torres-Sanchez I, Valenza MC, Cebria I Iranzo MDA, et al. Effects of different physical therapy programs on perceived health status in acute exacerbation of chronic obstructive pulmonary disease patients: a randomized clinical trial. Disability \& Rehabilitation. 2017 May 08:17. PMID: 28478693. [Duplicate Study].

565.Torres-Sanchez I, Valenza MC, Cebria I Iranzo MDA, et al. Effects of different physical therapy programs on perceived health status in acute exacerbation of chronic obstructive pulmonary disease patients: a randomized clinical trial. Disability \& Rehabilitation. 2018 08;40(17):2025-31. PMID: 28478693. [Duplicate Study].

566.Torres-Sanchez I, Valenza MC, Saez-Roca G, et al. Results of a Multimodal Program During Hospitalization in Obese COPD Exacerbated Patients. Copd: Journal of Chronic Obstructive Pulmonary Disease. 2016;13(1):19-25. PMID: 26418629. [Duplicate Study].Torres-Sanchez I, Valenza MC, Valenza-Demet G, et al. Effects of a physical therapy program in hospitalized COPD patients due to an acute exacerbation. Chest. Conference: CHEST World Congress. 2014;145(3 MEETING ABSTRACT). PMID: 71428952. [Abstract].

567.Torres-Sanchez I, Valenza MC, ValenzaDemet G, et al. Quality of life in hospitalized patients for exacerbation of COPD included in a physical therapy program. Chest. Conference: CHEST World Congress. 2014;145(3 MEETING ABSTRACT). PMID: 71428955. [Abstract].

568.Touaty E, Viau F, Pariente R. A therapeutic trial of intravenous almitrine in acute exacerbations of chronic obstructive respiratory failure. Revue francaise des maladies respiratoires. 1980;8(SYMP.):2115. PMID: CN-00187210 UPDATE. [Language other than English]. 
569.Translational Research Center for Medical Innovation K, Hyogo J. Study of Long-term HFNC for COPD Patients With HOT. 2017 September 6. PMID: NCT03282019. [Population not of interest].

570.Treatment and Exacerbation in COPD Subjects. 2017 August 25. PMID: NCT03375450. [Population not of interest].

571.Tricco AC, Strifler L, Veroniki A-A, et al. Comparative safety and effectiveness of long-acting inhaled agents for treating chronic obstructive pulmonary disease: a systematic review and network metaanalysis. BMJ Open. 2015 Oct 26;5(10):e009183. PMID: 26503392. [Population not of interest].

572. Troosters T, Probst VS, Crul T, et al. Resistance training prevents deterioration in quadriceps muscle function during acute exacerbations of chronic obstructive pulmonary disease. American journal of respiratory and critical care medicine. 2010;181(10):1072-7. [Duplicate Study].

573. Turato G, Semenzato U, Bazzan E, et al. Blood eosinophilia neither reflects tissue eosinophils nor worsens clinical outcomes in chronic obstructive pulmonary disease. American Journal of Respiratory and Critical Care Medicine. 201801 May;197(9):1216-9. PMID: 622058301. [Study design not of interest].

574.Ucar EY, Araz O, Ozturk D, et al. Comparison of different dosage nebulised budesonide in COPD exacerbation. European Respiratory Journal. Conference: European Respiratory Society Annual Congress. 2012;40(SUPPL. 56). PMID: 71923645. [Abstract].

575.Universidad de G. Effect of Physiotherapy on Perceived Health Status in Hospitalized COPD Patients. 2015 September. PMID: NCT02509299. [Clinical Trial].

576.Universidad de G. Physiotherapy in Exacerbation Chronic Obstructive Pulmonary Disease. 2013 March. PMID: NCT01826682. [Clinical Trial].

577.Università degli Studi di Ferrara G. Effects of Inhaled Corticosteroids on Sputum Bacterial Load in COPD. 2009 May. PMID: NCT01213693. [Population not of interest].
578.University College L. Pulmonary Rehabilitation and Cardiovascular Risk in COPD. 2017 January. PMID:

NCT03003208. [Population not of interest].

579.University Hospital A. High Flow Oxygen Therapy in Patients Suffering From Chronic Obstructive Pulmonary Disease Exacerbation: Effects and Mechanisms of Action. 2015 February. PMID: NCT02371564. [Intervention/Comparison not of interest].

580.University Hospital B. Efficacy of Varenicline on Smoking Cessation at the Acute Phase of an Exacerbation of Chronic Obstructive Pulmonary Disease. 2012 August. PMID: NCT01694732. [Population not of interest].

581. University Hospital B. Predictor for an Additional Benefit of Inhaled Corticosteroid in Patients Treated With Tiotropium for COPD. 2007 April. PMID: NCT00860938. [Population not of interest].

582. University Hospital G, University Ghent G. Pharmaceutical Care for Chronic Obstructive Pulmonary Disease (COPD) Study. 2010 December. PMID: NCT01260389. [Population not of interest].

583.University Hospital G. COPD-EXAREHAB. Early Pulmonary Rehabilitation of Patients With Acute Exacerbation of COPD. 2013 November. PMID: NCT02987439. [Clinical Trial].

584.University Hospital G. Early Supported Discharge and Enhanced Homecare After Emergency Department Admission for Acute Exacerbation of COPD. 2016 April. PMID: NCT03474575.

[Intervention/Comparison not of interest].

585. University Hospital M. Procalcitonin-guided Antibiotic Therapy During Severe Acute Exacerbation of COPD. 2017 October 5. PMID: NCT03440060.

[Intervention/Comparison not of interest].

586. University Hospital M. Sevoflurane in Chronic Obstructive Pulmonary Disease Exacerbation. 2018 February 27. PMID: NCT03460015. [Population not of interest]. 
587.University of C, San F, Kaiser P, et al. Rehabilitation in Safety-net Settings for Patients With COPD. 2017 September 1. PMID: NCT03287440. [Population not of interest].

588. University of E, Philips H. Physical Activity Enhancing Programme in COPD. 2015 February. PMID: NCT02347566. [Population not of interest].

589.University of M. Acute Exacerbation of COPD and Physical Activity - Preliminary Study. 2013 February. PMID: NCT01871246. [Study design not of interest].

590.University of M. Ceftidoren Versus Levofloxacin in the Treatment of Patients With Acute Exacerbations of Chronic Bronchitis (AECB). 2012 January. PMID: NCT01467297. [Population not of interest].

591.University of M. Magnesium Sulfate Versus Ipratropuim Bromide in Acute Exacerbation of Chronic Obstructive Pulmonary Disease. 2005 January. PMID: NCT01136421. [Clinical Trial].

592.University of M. TrimethoprimSulfamethoxazole Versus Ciprofloxacin in Acute Exacerbation of Chronic Obstructive Pulmonary Disease (COPD) Requiring Mechanical Ventilation. 2002 July. PMID: NCT00791505. [Population not of interest].

593.University of M. $\beta 2$-agonist Versus Ipratropium Bromide Associated With $\beta 2$ agonists in Chronic Obstructive Pulmonary Disease Exacerbation. 2013 July. PMID: NCT01944033. [Clinical Trial].

594.University of Milan P. Azithromycin Treatment of Patients With Chronic Obstructive Pulmonary Disease (COPD) and Tracheostomy. 2004 October. PMID: NCT00323986. [Population not of interest].

595.University of Minnesota - Clinical TSI, National Heart L, Blood Institute CIoHR. Simvastatin Therapy for Moderate and Severe COPD. 2010 March. PMID: NCT01061671. [Population not of interest].

596. University of North C, Chapel H, University of P, et al. Validation of PROMIS Banks With COPD Exacerbations. 2008 July. PMID: NCT00784342. [Study design not of interest].
597.University of Sao P, Fundação de Amparo à Pesquisa do Estado de São P. The Role of Resistance Exercise in Chronic Obstructive Pulmonary Disease Exacerbation. 2009 April. PMID: NCT01786928. [Clinical Trial].

598. University of T. Exacerbations and Health Related Quality of Life in Chronic Obstructive Pulmonary Disease. 2002 May. PMID: NCT00884975. [Study design not of interest].

599.University of T. Granzymes and Perforin at the Onset of Chronic Obstructive Pulmonary Disease (COPD) Exacerbations. 2002 December. PMID: NCT00883701. [Intervention/Comparison not of interest].

600.University of Z. Home-based Exercise Training for COPD Patients (HOMEX-2). 2018 October 19. PMID: NCT03654092. [Population not of interest].

601.University RA. COPD Exacerbations. 2016 June. PMID: NCT02798575. [Population not of interest].

602.Use of Respiratory Therapists (RTs) to Improve Outcomes and Quality of Life in Patients With COPD. 2014 March. PMID: NCT02078622. [Population not of interest].

603.Utens CMA, Goossens LMA, Smeenk FWJM, et al. Early assisted discharge with generic community nursing for chronic obstructive pulmonary disease exacerbations: results of a randomised controlled trial. BMJ Open. 2012;2(5). PMID: 23075570. [Intervention/Comparison not of interest].

604.Utens CMA, Goossens LMA, Smeenk FWJM, et al. Effectiveness and costeffectiveness of early assisted discharge for chronic obstructive pulmonary disease exacerbations: the design of a randomised controlled trial. BMC Public Health. 2010 Oct 18;10:618. PMID: 20955582. [Intervention/Comparison not of interest].

605.Va Office of Research D. Increasing Physical Activity in COPD Through Rhythmically Enhanced Music. 2018 October 1. PMID: NCT03655028. [Population not of interest]. 
606. Va Office of Research D. Randomized Controlled Trial of Exercise Training in Patients With COPD. PMID: NCT00012792. [Population not of interest].

607.Valenza MC, Torres-Sanchez I, Del Corral Nunez-Flores T, et al. Results of an exercise intervention in frail older patients with acute exacerbation of COPD. European Respiratory Journal. Conference: European Respiratory Society Annual Congress. 2016;48(Supplement 60). PMID: 614780460. [Abstract].

608. Valenza MC, Valenza-Pena G, TorresSanchez I, et al. Effectiveness of controlled breathing techniques on anxiety and depression in hospitalized patients with COPD: a randomized clinical Trial. [Erratum appears in Respir Care. 2016 Nov;61(11):e3; PMID: 27794090]. Respiratory Care. 2014 Feb;59(2):209-15. PMID: 23882107. [Duplicate Study].

609.van den Broek KM, Wielders PLML, Creemers JPHM, et al. Efficacy of formoterol Turbuhaler in the emergency treatment of patients with obstructive airway diseases. Respiratory Medicine. 2008 Apr;102(4):579-85. PMID: 18222683. [Intervention/Comparison not of interest].

610.van Velzen P, Ter Riet G, Bresser P, et al. Doxycycline for outpatient-treated acute exacerbations of COPD: a randomised double-blind placebo-controlled trial. The Lancet Respiratory Medicine. 2017 Jun;5(6):492-9. PMID: 28483402. [Duplicate Study].

611. Van Velzen P, Ter Riet G, Bresser P, et al. Long-Term Effects of Antibiotics in COPD Exacerbations: a Randomized Clinical Trial. American journal of respiratory and critical care medicine. 2016;193(Meeting Abstracts):A1021. PMID: CN-01262042 NEW. [Abstract].

612. Vandenbos F, Caisso C, Harb E, et al. [Is it possible to shorten the antibiotic therapy in patients hospitalized for acute COPD exacerbation?]. Revue des Maladies Respiratoires. 2017 03;34(3):275-7. PMID: 28189433. [Language other than English].
613. Varol E. Role of mean platelet volume in acute exacerbation of chronic obstructive pulmonary disease. Polskie Archiwum Medycyny Wewnetrznej. 2012;122(78):380. PMID: 365615741. [Intervention/Comparison not of interest].

614. Vastra Gotaland R. Remote Monitoring of Patients With COPD. 2018 June 7. PMID: NCT03558763. [Intervention/Comparison not of interest].

615. Verduri A, Luppi F, D'Amico R, et al. Antibiotic treatment of severe exacerbations of chronic obstructive pulmonary disease with procalcitonin: a randomized noninferiority trial. PLoS ONE [Electronic Resource]. 2015;10(3):e0118241. PMID: 25760346. [Intervention/Comparison not of interest].

616. Vermeersch K, Gabrovska M, Deslypere G, et al. The Belgian trial with azithromycin for acute COPD exacerbations requiring hospitalization: an investigator-initiated study protocol for a multicenter, randomized, double-blind, placebocontrolled trial. International Journal of Copd. 2016;11:687-96. PMID: 27099485. [Study design not of interest].

617.Vianello A, Fusello M, Gubian L, et al. Home telemonitoring for patients with acute exacerbation of chronic obstructive pulmonary disease: a randomized controlled trial. BMC Pulmonary Medicine. 201611 22;16(1):157. PMID: 27876029. [Intervention/Comparison not of interest].

618. VICTOR - Avelox ${ }^{\circledR}$ Intravenous (i.v.) in Acute Exacerbations of Chronic Bronchitis. 2009 May. PMID: NCT00876577. [Population not of interest].

619. Vivodtzev I, L'Her E, Vottero G, et al. Automated $\mathrm{O}<\mathrm{inf}>2</$ inf $>$ titration improves exercise capacity in patients with hypercapnic chronic obstructive pulmonary disease: a randomised controlled cross-over trial. Thorax. 2018(pagination). PMID: CN01645054 NEW. [Population not of interest].

620.Vivodtzev I, L'Her E, Vottero G, et al. Automated $\mathrm{O} 2$ titration improves exercise capacity in patients with hypercapnic chronic obstructive pulmonary disease: A randomised controlled cross-over trial. Thorax. 2018. [Population not of interest]. 
621. Vogelmeier C, Fabbri LM, Bell S, et al. Identification of factors associated with exacerbation risk in severe COPD: Multivariate analysis of the WISDOM study. Pneumologie. Conference. 2017;58. PMID: 623446524. [Duplicate Study].

622. Vogelmeier C, Wedzicha J, Donohue J, et al. Once-daily indacaterol/glycopyrronium reduces the rate and risk of moderate or severe exacerbations compared with twicedaily salmeterol/fluticasone in a subset of gold froup D COPD patients with a history of $>=2$ exacerbations or 1 hospitalization: The FLAME study. Pneumologie. Conference. 2018;59. PMID: 622904789. [Abstract].

623. Vollenweider DJ, Frei A, Steurer-Stey CA, et al. Antibiotics for exacerbations of chronic obstructive pulmonary disease. Cochrane Database of Systematic Reviews. 201810 29;10:CD010257. PMID: 30371937. [Abstract].

624.Wang FX, Zhang TM, Ding CL. Qingfei Huazhuo treating 50 chronic obstructive pulmonary disease patients during acute exacerbation period. Chinese journal of experimental traditional medical formulae [zhong guo shi yan fang ji xue za zhi]. 2015;21(3):187-90. PMID: CN-01435696 NEW. [Abstract].

625. Wang JY, Yu WJ. The effect of Peitu Shengjin Acupuncture Therapy on patients with acute exacerbation type of COPD. Journal of emergency in traditional chinese medicine [zhong guo zhong yi ji zheng]. 2015;24(4):728-30. PMID: CN-01435686 NEW. [Abstract].

626. Wang X, Liu X. Moxifloxacin versus levofloxacin for acute exacerbation of chronic obstructive pulmonary diseases: A systematic review. [Chinese]. Chinese Journal of Evidence-Based Medicine. 2012;12(6):694-9. PMID: 373959757. [Language other than English].

627. Wang Z. Analysis of influential factors of medication adherence of inhalation devices in patients with chronic obstructive pulmonary disease. Acta Medica Mediterranea. 2018;34(6):1703-9. PMID: 624676382. [Intervention/Comparison not of interest].
628. Weber C, Stirnemann J, Herrmann F, et al. Can early introduction of specialized palliative care limit intensive care, emergency and hospital admissions in patients with severe and very severe COPD? A pilot randomized study. Respiration. 2017;94 (1):80. PMID: 623934145.

[Abstract].

629. Wedzicha JA, MacKinnon A, Parkin JM. Effectiveness of acumapimod oral p38 inhibitor in the treatment of acute severe exacerbations of COPD: Results of the aether phase ii trial. American Journal of Respiratory and Critical Care Medicine. Conference: American Thoracic Society International Conference, ATS. 2018;197(MeetingAbstracts). PMID: 622969272. [Abstract].

630. Wedzicha JA, Singh D, Kostikas K, et al. Efficacy of indacaterol/glycopyrronium versus salmeterol/fluticasone by gender in patients with moderate-to-very severe COPD: Analyses from the flame study. American Journal of Respiratory and Critical Care Medicine. Conference: American Thoracic Society International Conference, ATS.

2018;197(MeetingAbstracts). PMID: 622969225. [Abstract].

631. Wei Z, Tang Z, Wu Y. Comparison of tiotropium and salmeterol for treating COPD: A systematic review and metaanalysis. International Journal of Clinical and Experimental Medicine. 201830 May;11(5):4397-404. PMID: 622367791. [Population not of interest].

632. Weill Medical College of Cornell University NH, Blood Institute UoM, National Jewish Health UoMC, et al. The CAPTURE Study: Validating a Unique COPD Case Finding Tool in Primary Care (Aim 1). 2018 September 17. PMID: NCT03581227. [Intervention/Comparison not of interest].

633. West Park Healthcare C. Effectiveness and Feasibility of Delivering an Education Program to Patients With an Acute Exacerbation of COPD. 2015 January. PMID: NCT02321215. [Outcome not of interest]. 
634. Whale CA, MacLaran SJA, Whale CI, et al. Pilot study to assess the credibility of acupuncture in acute exacerbations of chronic obstructive pulmonary disease. Acupuncture in Medicine. 2009 Mar;27(1):13-5. PMID: 19369188. [Intervention/Comparison not of interest].

635. Wiest GH, Ferguson GT, Watz H, et al. The impact of grouping patients by the 2017 GOLD COPD strategy on response to therapy: Post hoc results from the TONADO tiotropium+olodaterol trials. Pneumologie. Conference. 2018;59. PMID: 622904798. [Abstract].

636. Wilkinson M, Calverley PMA, Angus RM. A randomised placebo controlled trial of doxapram in acute hypercapnic respiratory failure complicating exacerbation of COPD [Abstract]. European respiratory journal. 2003;22(Suppl 45):Abstract P2828. PMID: CN-00486452 UPDATE. [Abstract].

637.Wilson R, Anzueto A, Miravitlles M, et al. A novel study design for antibiotic trials in acute exacerbations of COPD: MAESTRAL methodology. International Journal of Copd. 2011;6:373-83. PMID: 21760724. [Study design not of interest].

638. Wilson R, Anzueto A, Miravitlles M, et al. Moxifloxacin (MXF) vs amoxicillin/clavulanic acid (AMC) in acute exacerbations of COPD (AECOPD): Results of a large clinical trial with a novel endpoint. European Respiratory Journal. Conference: European Respiratory Society Annual Congress. 2011;38(SUPPL. 55). PMID: 72116359. [Abstract].

639. Wilson R, Sethi S, Anzueto A, et al. Maestral (moxifloxacin in aecb superiority trial) study protocol: Use of a novel 8-week post-therapy endpoint. American Journal of Respiratory and Critical Care Medicine. Conference: American Thoracic Society International Conference, ATS. 2011;183(1 MeetingAbstracts). PMID: 70847454. [Abstract].

640.Wim J, Agentschap voor Innovatie door Wetenschap en T, Katholieke Universiteit L. Azithromycin for Acute Exacerbations Requiring Hospitalization. 2014 August 1. PMID: NCT02135354. [Clinical Trial].
641. Wise RA, Scirica BM, Schoenfeld DA, et al. Effects of aclidinium bromide on major adverse cardiovascular events and COPD exacerbations in patients with COPD and cardiovascular risk factors. American Journal of Respiratory and Critical Care Medicine. Conference: American Thoracic Society International Conference, ATS. 2018;197(MeetingAbstracts). PMID: 622969296. [Abstract].

642.Women's College H, Markham Stouffville H. Evaluating the Cloud DX Platform as a Tool for Self-management and Asynchronous Remote-monitoring of COPD. 2018 January 1. PMID:

NCT03741855. [Population not of interest].

643. Wood-Baker R, Wilkinson J, Pearce M, et al. A double-blind, randomised, placebocontrolled trial of corticosteroids for acute exacerbations of chronic obstructive pulmonary disease. [abstract]. Australian and New Zealand journal of medicine. 1998;28(262). PMID: CN-00320763 UPDATE. [Study design not of interest].

644.Wood-Baker R, Wilkinson J, Pearce M, et al. A double-blind, randomised, placebocontrolled trial of corticosteroids for acute exacerbations of chronic obstructive pulmonary disease. Thoracic Society of Australia \& New Zealand. 199p. 1997. PMID: CN-00285737 UPDATE. [Abstract].

645. Wortmann-Schmid B. Exacerbation of chronic obstructive bronchitis. [German]. Therapiewoche Schweiz. 1999;15(2):74-5. PMID: 29129883. [Language other than English].

646.Wu R, Fengjie Z, Li Y, et al. Modified dachengqi decoction combined with conventional treatment for treating acute exacerbation of chronic obstructive pulmonary disease: a systematic review based on randomized controlled trials. Evidence-Based Complementary \& Alternative Medicine: eCAM. 2013;2013:323715. PMID: 23662130. [Intervention/Comparison not of interest].

647.Wu W-w, Hong H-h, Shao X-p, et al. Effect of oxygen-driven nebulization at different oxygen flows in acute exacerbation of chronic obstructive pulmonary disease patients. American Journal of the Medical Sciences. 2014 May;347(5):343-6. PMID: 23689047. [Outcome not of interest]. 
648.Xie Y, Li X, Wang J, et al. Acupuncture therapy for acute exacerbation in chronic obstructive pulmonary disease: A literature analysis of clinical study. Respirology. 2018 November;23 (Supplement 2):125. PMID: 625398358. [Abstract].

649. Yuan G, Fu L, Sun W, et al. Effect of lowdose glucocorticoid on corticosteroid insufficient patients with acute exacerbation of chronic obstructive pulmonary disease. Chinese Journal of Emergency Medicine. 2014;23(5):543-8. [Intervention/Comparison not of interest].

650. Yuan L, Feng J, Luo FM. Low molecular weight heparin in treating patients with acute exacerbation of COPD: A metaanalysis. [Chinese]. Chinese Journal of Evidence-Based Medicine. 2014;14(7):8216. PMID: 373859422. [Language other than English].

651.Zhang X, Wang L, Liu CH, et al. Effect of compound ephedra and white peony decoction on pulmonary function and serum cytokine level in patients with chronic obstructive pulmonary diseases at acute exacerbation phase. [Chinese]. Chinese Journal of Clinical Rehabilitation. 200620 Jul;10(27):73-5. PMID: 44236597. [Language other than English].

652.Zheng F, Sun Y, Zhong X, et al. A multicenter randomized, double-blind, placebo-controlled trial to evaluate the safety and efficacy of rhubarb in treating acute exacerbation of chronic obstructive pulmonary disease of the syndrome type phlegm-heat obstructing the lungs. Journal of Traditional Chinese Medical Sciences. 201601 Apr;3(2):71-80. PMID: 612608845. [Intervention/Comparison not of interest].
653.Zheng J, Lin J, Zhou X, et al. Nebulized budesonide in the treatment of acute exacerbations of chronic obstructive pulmonary disease (AECOPD): A randomized, double blind, double dummy, parallel controlled, multicenter trial. Chest. Conference: CHEST. 2011;140(4 MEETING ABSTRACT). PMID: 70635108. [Abstract].

654.Zhong LJ, Zhang LS, Gu CF, et al. Procalcitonin impact analysis, respiratory function and blood gas analysis on Xiaoqinglong decoction combined with noninvasive ventilation in treatment of AECOPD patients. Zhongguo Zhongyao Zazhi. 2018;43(14):3026-30. [Language other than English].

655.Zhong Y, Mao B, Wang G, et al. Tanreqing injection combined with conventional Western medicine for acute exacerbations of chronic obstructive pulmonary disease: a systematic review. Journal of Alternative \& Complementary Medicine. 2010 Dec;16(12):1309-19. PMID: 21091297. [Intervention/Comparison not of interest].

656.Zhong Y, Wang X, Dong S, et al. Modified Weijing formula for the treatment of acute exacerbations of chronic obstructive pulmonary disease: A systematic review of randomized controlled trials. European Journal of Integrative Medicine. 2014 June;6(3):342-54. PMID: 52946504. [Intervention/Comparison not of interest].

657.Zujovic D, Zugic V. Efficacy and safety of formoterol fumarate in acute exacerbation of chronic obstructive pulmonary disease. American journal of respiratory and critical care medicine. Conference: american thoracic society international conference, ATS. 2017;195(no pagination). PMID: CN01409353 NEW. [Abstract].

658.Zwerink M, Kerstjens HA, van der Palen J, et al. (Cost-)effectiveness of self-treatment of exacerbations in patients with COPD: 2 years follow-up of a RCT. Respirology. 2016 Apr;21(3):497-503. PMID: 26647766. [Intervention/Comparison not of interest]. 


\section{Appendix D. Characteristics of Included Studies}

Table D.1. KQ1: Characteristics of included studies

\begin{tabular}{|c|c|c|c|c|c|c|c|c|}
\hline $\begin{array}{l}\text { Author, } \\
\text { Year }\end{array}$ & $\begin{array}{l}\text { Country, } \\
\text { Study } \\
\text { Period }\end{array}$ & $\begin{array}{l}\text { Risk of } \\
\text { Bias }\end{array}$ & $\begin{array}{c}\text { COPD } \\
\text { Definition }\end{array}$ & $\begin{array}{c}\text { COPD } \\
\text { Exacerbation }\end{array}$ & $\begin{array}{l}\text { Severity of } \\
\text { ECOPD }\end{array}$ & $\begin{array}{l}\text { Intervention(s) and } \\
\text { comparison }\end{array}$ & $\begin{array}{c}\text { COPD } \\
\text { Severity } \\
(\% \\
\text { predicted } \\
\text { unless } \\
\text { specified) } \\
\end{array}$ & $\begin{array}{c}\text { Patient } \\
\text { Characteristics }\end{array}$ \\
\hline \multirow[t]{2}{*}{$\begin{array}{l}\text { Aaron, } \\
2003^{1}\end{array}$} & \multirow[t]{2}{*}{ Canada } & \multirow[t]{2}{*}{ Low risk } & \multirow{2}{*}{$\begin{array}{l}\text { Diagnosis by a } \\
\text { physician or } \\
\text { had at least a } \\
\text { one-year } \\
\text { history of } \\
\text { chronic } \\
\text { dyspnea or } \\
\text { cough with } \\
\text { sputum } \\
\text { production }\end{array}$} & \multirow{2}{*}{$\begin{array}{l}\text { The presence } \\
\text { of at least two } \\
\text { of the following } \\
\text { three clinical } \\
\text { criteria: a } \\
\text { recent increase } \\
\text { in } \\
\text { breathlessness, } \\
\text { sputum } \\
\text { volume, or } \\
\text { sputum } \\
\text { purulence }\end{array}$} & \multirow[t]{2}{*}{ Mild/Moderate } & Prednisone & NR & $\begin{array}{l}74 \text { patients } \\
\text { aged } 68.9 \pm 11.2 \\
\text { years, } 43 \% \\
\text { female }\end{array}$ \\
\hline & & & & & & Placebo & NR & $\begin{array}{l}73 \text { patients } \\
\text { aged } 69.9 \pm 10.4 \\
\text { years, } 42 \% \\
\text { female }\end{array}$ \\
\hline \multirow[t]{2}{*}{$\begin{array}{l}\text { Albert, } \\
1980^{2}\end{array}$} & \multirow{2}{*}{$\begin{array}{l}\text { United } \\
\text { States of } \\
\text { America } \\
\text { 10/25/1976 } \\
\text { to } \\
\text { 03/27/1978 }\end{array}$} & \multirow[t]{2}{*}{ Low risk } & \multirow{2}{*}{$\begin{array}{l}\text { Chronic } \\
\text { bronchitis } \\
\text { (FEV1 } 60 \% \\
\text { predicted or } \\
\text { FEV1 } 1 \leq 60 \% \\
\text { than FVC) }\end{array}$} & \multirow{2}{*}{$\begin{array}{l}\text { Acute } \\
\text { bronchitis } \\
\text { (increase in } \\
\text { cough, sputum } \\
\text { production, and } \\
\text { sputum color } \\
\text { within prior } 5 \\
\text { days) + acute } \\
\text { respiratory } \\
\text { failure (PaO2 } \\
<65 \mathrm{mmHg} \text { on } \\
\text { room air, or } \\
\mathrm{PaCO} 2 \geq 50 \text { or } \\
\text { pH<7.35) }\end{array}$} & \multirow[t]{2}{*}{ Severe } & Methylprednisolone & $\begin{array}{l}\text { FEV1 (L): } \\
\text { Mean } \\
0.673 \pm 0.239\end{array}$ & $\begin{array}{l}22 \text { patients } \\
\text { aged } 61 \pm 9 \\
\text { years }\end{array}$ \\
\hline & & & & & & Placebo & $\begin{array}{l}\text { FEV1 (L): } \\
\text { Mean } \\
0.719 \pm 0.313\end{array}$ & $\begin{array}{l}22 \text { patients } \\
\text { aged } 62 \pm 10 \\
\text { years }\end{array}$ \\
\hline
\end{tabular}




\begin{tabular}{|c|c|c|c|c|c|c|c|c|}
\hline $\begin{array}{l}\text { Author, } \\
\text { Year }\end{array}$ & $\begin{array}{c}\text { Country, } \\
\text { Study } \\
\text { Period }\end{array}$ & $\begin{array}{c}\text { Risk of } \\
\text { Bias }\end{array}$ & $\begin{array}{c}\text { COPD } \\
\text { Definition }\end{array}$ & $\begin{array}{c}\text { COPD } \\
\text { Exacerbation }\end{array}$ & $\begin{array}{l}\text { Severity of } \\
\text { ECOPD }\end{array}$ & $\begin{array}{l}\text { Intervention(s) and } \\
\text { comparison }\end{array}$ & $\begin{array}{c}\text { COPD } \\
\text { Severity } \\
(\% \\
\text { predicted } \\
\text { unless } \\
\text { specified) }\end{array}$ & $\begin{array}{c}\text { Patient } \\
\text { Characteristics }\end{array}$ \\
\hline $\begin{array}{l}\text { Anthonisen, } \\
1987^{3}\end{array}$ & $\begin{array}{l}\text { Canada } \\
11 / 01 / 1981 \\
\text { to } 09 / 1984\end{array}$ & High risk & $\begin{array}{l}\text { FEV1/FVC }<0.7, \\
\text { FEV1<70\% } \\
\text { predicted, } \\
\text { clinical } \\
\text { diagnosis }\end{array}$ & $\begin{array}{l}\text { Type I } \\
\text { exacerbation: } \\
\text { increased } \\
\text { dyspnea, } \\
\text { sputum } \\
\text { volume, and } \\
\text { sputum } \\
\text { purulence } \\
\text { Type II } \\
\text { exacerbation: } \\
\text { two of these } \\
\text { three } \\
\text { symptoms } \\
\text { Type III: one of } \\
\text { these three } \\
\text { symptoms plus } \\
\text { at least one of } \\
\text { the following } \\
\text { findings: upper } \\
\text { respiratory } \\
\text { infection (sore } \\
\text { throat, nasal } \\
\text { discharge) } \\
\text { within the past } \\
5 \text { days, fever } \\
\text { without other } \\
\text { cause, } \\
\text { increased } \\
\text { wheezing, } \\
\text { increased } \\
\text { cough, or } \\
\text { increase in } \\
\text { respiratory rate } \\
\text { or heart rate by } \\
20 \% \text { as } \\
\text { compared with } \\
\text { baseline. }\end{array}$ & Mild/Moderate & Antibiotics & $\begin{array}{l}\text { FEV1: } \\
\text { Mean } \\
33.9 \pm 13.7\end{array}$ & $\begin{array}{l}173 \text { total } \\
\text { patients aged } \\
67.3 \pm 9.0 \text { years, } \\
20 \% \text { female }\end{array}$ \\
\hline
\end{tabular}




\begin{tabular}{|c|c|c|c|c|c|c|c|c|}
\hline $\begin{array}{l}\text { Author, } \\
\text { Year }\end{array}$ & $\begin{array}{l}\text { Country, } \\
\text { Study } \\
\text { Period }\end{array}$ & $\begin{array}{l}\text { Risk of } \\
\text { Bias }\end{array}$ & $\begin{array}{c}\text { COPD } \\
\text { Definition }\end{array}$ & $\begin{array}{c}\text { COPD } \\
\text { Exacerbation }\end{array}$ & $\begin{array}{l}\text { Severity of } \\
\text { ECOPD }\end{array}$ & $\begin{array}{l}\text { Intervention(s) and } \\
\text { comparison }\end{array}$ & $\begin{array}{c}\text { COPD } \\
\text { Severity } \\
(\% \\
\text { predicted } \\
\text { unless } \\
\text { specified) }\end{array}$ & $\begin{array}{c}\text { Patient } \\
\text { Characteristics }\end{array}$ \\
\hline \multirow[t]{3}{*}{$\begin{array}{l}\text { Bathoorn, } \\
2008^{4}\end{array}$} & \multirow[t]{3}{*}{ Netherlands } & \multirow[t]{3}{*}{ High risk } & \multirow{3}{*}{$\begin{array}{l}\text { Post } \\
\text { bronchodilator } \\
\text { FEV1 <85\% } \\
\text { predicted but } \\
>0.7 \text { liters, and } \\
\text { an Abnormal } \\
\text { post } \\
\text { bronchodilator } \\
\text { FEV1/slow } \\
\text { inspiratory vital } \\
\text { capacity (VC) } \\
\text { (<88\% } \\
\text { predicted in } \\
\text { men and <89\% } \\
\text { predicted in } \\
\text { women) }\end{array}$} & \multirow{3}{*}{$\begin{array}{l}\text { History of } \\
\text { increased } \\
\text { breathlessness } \\
\text { and at least two } \\
\text { of the following } \\
\text { symptoms for } \\
\geq 24 \text { hours: } \\
\text { increased } \\
\text { cough } \\
\text { frequency or } \\
\text { severity, } \\
\text { sputum volume } \\
\text { or purulence, } \\
\text { and wheeze. }\end{array}$} & \multirow[t]{3}{*}{ Mild } & $\begin{array}{l}\text { Budesonide + } \\
\text { Formoterol }\end{array}$ & NR & $\begin{array}{l}15 \text { patients } \\
\text { aged } 61.4 \pm 8 \\
\text { years, } 33 \% \\
\text { female }\end{array}$ \\
\hline & & & & & & Prednisolone & NR & $\begin{array}{l}15 \text { patients } \\
\text { aged } 64.8 \pm 7 \\
\text { years, } 15 \% \\
\text { female }\end{array}$ \\
\hline & & & & & & Placebo & NR & $\begin{array}{l}15 \text { patients } \\
\text { aged } 64.6 \pm 9.1 \\
7 \% \text { female }\end{array}$ \\
\hline \multirow[t]{2}{*}{$\begin{array}{l}\text { Brusse- } \\
\text { Keizer, } \\
2014^{5}\end{array}$} & \multirow[t]{2}{*}{$\begin{array}{l}\text { Netherlands } \\
05 / 2005 \text { to } \\
01 / 2007\end{array}$} & \multirow[t]{2}{*}{ Low risk } & \multirow[t]{2}{*}{ GOLD criteria } & \multirow{2}{*}{$\begin{array}{l}\text { An acute } \\
\text { negative } \\
\text { change in } \\
\text { dyspnea and/or } \\
\text { sputum volume } \\
\text { and/or color of } \\
\text { sputum } \\
\text { (yellowish or } \\
\text { greenish) } \\
\text { and/or cough, } \\
\text { which warrants } \\
\text { additional } \\
\text { treatment with } \\
\text { prednisolone } \\
\text { with or without } \\
\text { antibiotics by a } \\
\text { physician, } \\
\text { outpatient } \\
\text { evaluation }\end{array}$} & \multirow[t]{2}{*}{ Mild/Moderate } & Antibiotics & $\begin{array}{l}\text { FEV1: } \\
\text { Mean } \\
44.7 \pm 15.4\end{array}$ & $\begin{array}{l}18 \text { patients } \\
\text { aged } 68 \text { years, } \\
50 \% \text { female }\end{array}$ \\
\hline & & & & & & Placebo & $\begin{array}{l}\text { FEV1: } \\
\text { Mean } \\
52.2 \pm 15.1\end{array}$ & $\begin{array}{l}17 \text { patients } \\
\text { aged } 65 \text { years, } \\
29 \% \text { female }\end{array}$ \\
\hline
\end{tabular}




\begin{tabular}{|c|c|c|c|c|c|c|c|c|}
\hline $\begin{array}{l}\text { Author, } \\
\text { Year }\end{array}$ & $\begin{array}{c}\text { Country, } \\
\text { Study } \\
\text { Period }\end{array}$ & $\begin{array}{l}\text { Risk of } \\
\text { Bias }\end{array}$ & $\begin{array}{c}\text { COPD } \\
\text { Definition }\end{array}$ & $\begin{array}{c}\text { COPD } \\
\text { Exacerbation }\end{array}$ & $\begin{array}{l}\text { Severity of } \\
\text { ECOPD }\end{array}$ & $\begin{array}{l}\text { Intervention(s) and } \\
\text { comparison }\end{array}$ & $\begin{array}{c}\text { COPD } \\
\text { Severity } \\
(\% \\
\text { predicted } \\
\text { unless } \\
\text { specified) }\end{array}$ & $\begin{array}{c}\text { Patient } \\
\text { Characteristics }\end{array}$ \\
\hline \multirow[t]{2}{*}{$\begin{array}{l}\text { Daniels, } \\
2010^{6}\end{array}$} & \multirow[t]{2}{*}{ Netherlands } & \multirow[t]{2}{*}{ High risk } & \multirow[t]{2}{*}{$\begin{array}{l}\text { GOLD criteria, } \\
\text { stages I-IV }\end{array}$} & \multirow{2}{*}{$\begin{array}{l}\text { An acute (onset } \\
<14 \text { d) } \\
\text { exacerbation } \\
\text { Anthonisen } \\
\text { type } 1 \\
\text { [increased } \\
\text { dyspnea, } \\
\text { sputum } \\
\text { volume, and } \\
\text { sputum } \\
\text { purulence] or } \\
\text { type } 2 \text { [two of } \\
\text { three } \\
\text { symptoms]) } \\
\text { that required } \\
\text { hospitalization }\end{array}$} & \multirow[t]{2}{*}{$\begin{array}{l}\text { Moderate to } \\
\text { Severe }\end{array}$} & Doxycycline & $\begin{array}{l}\text { FEV1: } \\
\text { Mean } \\
\text { 43.9 } \pm 17.2 \\
\text { FVC: } \\
71.1 \pm 17.7\end{array}$ & $\begin{array}{l}128 \text { patients } \\
\text { aged } 71 \pm 10.2 \\
\text { years, } 43 \% \\
\text { female }\end{array}$ \\
\hline & & & & & & Placebo & $\begin{array}{l}\text { FEV1: } \\
46.9 \pm 18.5 \\
\text { FVC: } \\
72.7 \pm 18.6\end{array}$ & $\begin{array}{l}137 \text { patients } \\
\text { aged } 72.8 \pm 9.2 \\
\text { years, } 38 \% \\
\text { female }\end{array}$ \\
\hline \multirow[t]{2}{*}{$\begin{array}{l}\text { Davies, } \\
1999^{7}\end{array}$} & \multirow[t]{2}{*}{$\begin{array}{l}\text { United } \\
\text { Kingdom }\end{array}$} & \multirow[t]{2}{*}{$\begin{array}{l}\text { Intermediate } \\
\text { risk }\end{array}$} & \multirow{2}{*}{$\begin{array}{l}\text { History of at } \\
\text { least } 20 \text { pack- } \\
\text { years of } \\
\text { cigarette } \\
\text { smoking, and } \\
\text { had } \\
\text { physiological } \\
\text { evidence of } \\
\text { airflow } \\
\text { limitation with } \\
\text { initial FEV1 } \\
\text { less than } 70 \% \\
\text { predicted and } \\
\text { FEV1/forced } \\
\text { vital capacity } \\
\text { ratio less than } \\
75 \%\end{array}$} & \multirow{2}{*}{$\begin{array}{l}\text { Two of the } \\
\text { following } \\
\text { symptoms for } \\
24 \text { h or more: } \\
\text { increased } \\
\text { cough } \\
\text { frequency or } \\
\text { severity, } \\
\text { increased } \\
\text { sputum volume } \\
\text { or purulence, } \\
\text { and increased } \\
\text { wheeze }\end{array}$} & \multirow[t]{2}{*}{$\begin{array}{l}\text { Moderate to } \\
\text { Severe }\end{array}$} & Prednisolone & $\begin{array}{l}\text { FEV1: } \\
\text { Mean } \\
27.4 \pm 2.4\end{array}$ & $\begin{array}{l}29 \text { patients } \\
\text { aged } 66 \pm 1.3 \\
\text { years, } 39 \% \\
\text { female }\end{array}$ \\
\hline & & & & & & Placebo & $\begin{array}{l}\text { FEV1: } \\
\text { Mean } \\
21.4 \pm 2.5\end{array}$ & $\begin{array}{l}27 \text { patients } \\
\text { aged } 69 \pm 2.1 \\
\text { years, } 23 \% \\
\text { female }\end{array}$ \\
\hline
\end{tabular}




\begin{tabular}{|c|c|c|c|c|c|c|c|c|}
\hline $\begin{array}{l}\text { Author, } \\
\text { Year }\end{array}$ & $\begin{array}{l}\text { Country, } \\
\text { Study } \\
\text { Period }\end{array}$ & $\begin{array}{l}\text { Risk of } \\
\text { Bias }\end{array}$ & $\begin{array}{c}\text { COPD } \\
\text { Definition }\end{array}$ & $\begin{array}{c}\text { COPD } \\
\text { Exacerbation }\end{array}$ & $\begin{array}{l}\text { Severity of } \\
\text { ECOPD }\end{array}$ & $\begin{array}{l}\text { Intervention(s) and } \\
\text { comparison }\end{array}$ & $\begin{array}{c}\text { COPD } \\
\text { Severity } \\
(\% \\
\text { predicted } \\
\text { unless } \\
\text { specified) }\end{array}$ & $\begin{array}{c}\text { Patient } \\
\text { Characteristics }\end{array}$ \\
\hline \multirow[t]{2}{*}{$\begin{array}{l}\text { Emerman, } \\
1989^{8}\end{array}$} & \multirow[t]{2}{*}{$\begin{array}{l}\text { United } \\
\text { States of } \\
\text { America } \\
\text { 10/1986 to } \\
11 / 1987\end{array}$} & \multirow[t]{2}{*}{$\begin{array}{l}\text { Intermediate } \\
\text { risk }\end{array}$} & \multirow[t]{2}{*}{$\begin{array}{l}\text { Patients, with a } \\
\text { clinical history } \\
\text { of emphysema } \\
\text { or chronic } \\
\text { bronchitis with } \\
\text { an FEV1 less } \\
\text { than } 70 \% \text { of } \\
\text { predicted or a } \\
\text { FEV /FVC\% } \\
\text { less than } 60 \% \text {. }\end{array}$} & \multirow{2}{*}{$\begin{array}{l}\text { Patients, with a } \\
\text { clinical history } \\
\text { of emphysema } \\
\text { or chronic } \\
\text { bronchitis } \\
\text { presenting to } \\
\text { the Emergency } \\
\text { Department } \\
\text { with acute } \\
\text { respiratory } \\
\text { distress and } \\
\text { had initial } \\
\text { spirometry in } \\
\text { the Emergency } \\
\text { Department } \\
\text { with an FEV1 } \\
\text { less than 70\% } \\
\text { of predicted or } \\
\text { a FEV /FVC\% } \\
\text { less than } 60 \% \text {. }\end{array}$} & \multirow[t]{2}{*}{$\begin{array}{l}\text { Mild to Moderate } \\
\text { to Severe }\end{array}$} & Methylprednisolone & $\begin{array}{l}\text { FEV1: } \\
\text { Mean } \\
29 \pm 14.4\end{array}$ & $\begin{array}{l}52 \text { patients } \\
\text { aged } 63.7 \pm 8.7 \\
\text { years, } 47.91 \% \\
\text { female }\end{array}$ \\
\hline & & & & & & Placebo & $\begin{array}{l}\text { FEV1: } \\
\text { Mean } \\
26 \pm 14.4\end{array}$ & $\begin{array}{l}52 \text { patients } \\
\text { aged } 64.3 \pm 8.6 \\
\text { years, } 47.91 \% \\
\text { female }\end{array}$ \\
\hline \multirow[t]{3}{*}{$\begin{array}{l}\text { Gunen, } \\
2007^{9}\end{array}$} & \multirow[t]{3}{*}{ Turkey } & \multirow[t]{3}{*}{ High risk } & \multirow[t]{3}{*}{$\begin{array}{l}\text { American } \\
\text { Thoracic } \\
\text { Society criteria }\end{array}$} & \multirow{3}{*}{$\begin{array}{l}\text { Worsening in at } \\
\text { least two of the } \\
\text { following } \\
\text { symptoms: } \\
\text { cough, purulent } \\
\text { sputum and } \\
\text { dyspnea }\end{array}$} & \multirow[t]{3}{*}{$\begin{array}{l}\text { Moderate to } \\
\text { Severe }\end{array}$} & $\begin{array}{l}\text { Management } \\
\text { without Systemic } \\
\text { corticosteroids }\end{array}$ & $\begin{array}{l}\text { FEV1: } \\
\text { Mean } \\
36.7 \pm 11.9 \\
\end{array}$ & $\begin{array}{l}53 \text { patients } \\
\text { aged } 63.5 \pm 10.1 \text {, } \\
10 \% \text { female }\end{array}$ \\
\hline & & & & & & $\begin{array}{l}\text { Prednisolone, } 40 \mathrm{mg} \\
\text { total dose }\end{array}$ & 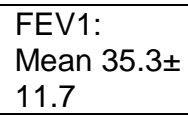 & $\begin{array}{l}53 \text { patients } \\
\text { aged } 64.9 \pm 7.1 \\
18 \% \text { female }\end{array}$ \\
\hline & & & & & & $\begin{array}{l}\text { Nebulized } \\
\text { Budesonide }\end{array}$ & $\begin{array}{l}\text { FEV1: } \\
\text { Mean } \\
39.6 \pm 12.9\end{array}$ & $\begin{array}{l}53 \text { patients } \\
\text { aged } 63.9 \pm 9.7 \\
16.7 \% \text { female }\end{array}$ \\
\hline \multirow[t]{2}{*}{$\begin{array}{l}\text { Hassan, } \\
2015^{10}\end{array}$} & \multirow[t]{2}{*}{$\begin{array}{l}\text { Egypt } \\
04 / 2013- \\
10 / 2014\end{array}$} & \multirow[t]{2}{*}{ High risk } & \multirow[t]{2}{*}{ NR } & \multirow{2}{*}{$\begin{array}{l}\text { Increase in } \\
\text { dyspnea, } \\
\text { sputum } \\
\text { purulence and } \\
\text { increased } \\
\text { sputum } \\
\text { volume, } \\
\text { outpatient } \\
\text { evaluation }\end{array}$} & \multirow[t]{2}{*}{ Mild } & Placebo & NR & $\begin{array}{l}50 \text { patients } \\
\text { aged } 63 \pm 6.1 \text {, } \\
16 \% \text { female } \\
\end{array}$ \\
\hline & & & & & & Antibiotic & NR & $\begin{array}{l}50 \text { patients } \\
\text { aged } 60.6 \pm 6.8 \\
18 \% \text { female }\end{array}$ \\
\hline
\end{tabular}




\begin{tabular}{|c|c|c|c|c|c|c|c|c|}
\hline $\begin{array}{l}\text { Author, } \\
\text { Year }\end{array}$ & $\begin{array}{l}\text { Country, } \\
\text { Study } \\
\text { Period }\end{array}$ & $\begin{array}{l}\text { Risk of } \\
\text { Bias }\end{array}$ & $\begin{array}{c}\text { COPD } \\
\text { Definition }\end{array}$ & $\begin{array}{c}\text { COPD } \\
\text { Exacerbation }\end{array}$ & $\begin{array}{l}\text { Severity of } \\
\text { ECOPD }\end{array}$ & $\begin{array}{l}\text { Intervention(s) and } \\
\text { comparison }\end{array}$ & $\begin{array}{c}\text { COPD } \\
\text { Severity } \\
(\% \\
\text { predicted } \\
\text { unless } \\
\text { specified) }\end{array}$ & $\begin{array}{c}\text { Patient } \\
\text { Characteristics }\end{array}$ \\
\hline \multirow[t]{2}{*}{ Llor, $2012^{11}$} & \multirow[t]{2}{*}{$\begin{array}{l}\text { Spain } \\
\text { 10/2007- } \\
07 / 2010\end{array}$} & \multirow[t]{2}{*}{ Low risk } & \multirow[t]{2}{*}{$\begin{array}{l}\text { FEV1/FVC } \\
<70 \% \text {, post } \\
\text { bronchodilator } \\
\text { FEV1>50\%, } \\
\text { smoking history } \\
\geq 10 \text { pack years }\end{array}$} & \multirow[t]{2}{*}{$\begin{array}{l}\text { Increase of } \\
\text { dyspnea, } \\
\text { sputum volume } \\
\text { or sputum } \\
\text { purulence }\end{array}$} & \multirow[t]{2}{*}{ Mild to Moderate } & $\begin{array}{l}\text { Amoxicillin }+ \\
\text { Clavulanate, } \\
\text { 1500mg }+375 \mathrm{mg}\end{array}$ & $\begin{array}{l}\text { Mild: } 9.5 \% \\
\text { Moderate: } \\
90.5 \% \\
\text { FEV1: } \\
\text { Mean } \\
64.2 \pm 11.8\end{array}$ & $\begin{array}{l}162 \text { patients } \\
\text { aged } 68.4 \pm 9.9 \\
16.5 \% \text { female }\end{array}$ \\
\hline & & & & & & Placebo & $\begin{array}{l}\text { Mild:13.2\% } \\
\text { Moderate: } \\
86.8 \% \\
\text { FEV1: } \\
\text { Mean } \\
65.9 \pm 12.1\end{array}$ & $\begin{array}{l}156 \text { patients } \\
\text { aged } 67.8 \pm 11 \\
21.7 \% \text { female }\end{array}$ \\
\hline \multirow[t]{2}{*}{$\begin{array}{l}\text { Maltais, } \\
2002^{12}\end{array}$} & \multirow[t]{2}{*}{$\begin{array}{l}\text { Belgium, } \\
\text { Canada, } \\
\text { and France }\end{array}$} & \multirow[t]{2}{*}{ High risk } & \multirow{2}{*}{$\begin{array}{l}\text { American } \\
\text { Thoracic } \\
\text { Society criteria, } \\
\text { chronic } \\
\text { bronchitis or } \\
\text { emphysema }\end{array}$} & \multirow{2}{*}{$\begin{array}{l}\text { Increased } \\
\text { breathlessness } \\
\text { in the past } 14 \\
\text { days, causing } \\
\text { hospital } \\
\text { admission }\end{array}$} & \multirow[t]{2}{*}{$\begin{array}{l}\text { Moderate to } \\
\text { Severe }\end{array}$} & Budesonide & NR & $\begin{array}{l}71 \text { patients } \\
\text { aged } 69.1 \pm 8.7 \\
20 \% \text { female }\end{array}$ \\
\hline & & & & & & Prednisolone & NR & $\begin{array}{l}62 \text { patients } \\
\text { aged } 70.4 \pm 7.7 \\
16 \% \text { female }\end{array}$ \\
\hline
\end{tabular}




\begin{tabular}{|c|c|c|c|c|c|c|c|c|}
\hline $\begin{array}{l}\text { Author, } \\
\text { Year }\end{array}$ & $\begin{array}{l}\text { Country, } \\
\text { Study } \\
\text { Period }\end{array}$ & $\begin{array}{l}\text { Risk of } \\
\text { Bias }\end{array}$ & $\begin{array}{c}\text { COPD } \\
\text { Definition }\end{array}$ & $\begin{array}{c}\text { COPD } \\
\text { Exacerbation }\end{array}$ & $\begin{array}{l}\text { Severity of } \\
\text { ECOPD }\end{array}$ & $\begin{array}{l}\text { Intervention(s) and } \\
\text { comparison }\end{array}$ & $\begin{array}{c}\text { COPD } \\
\text { Severity } \\
(\% \\
\text { predicted } \\
\text { unless } \\
\text { specified) }\end{array}$ & $\begin{array}{c}\text { Patient } \\
\text { Characteristics }\end{array}$ \\
\hline & & & $\begin{array}{l}14 \text { day history } \\
\text { of acute COPD } \\
\text { exacerbation } \\
\text { defined } \\
\text { as increased } \\
\text { breathlessness. } \\
\text { Patients were } \\
\text { included in the } \\
\text { study if they } \\
\text { were more than } \\
50 \text { years old, } \\
\text { had a smoking } \\
\text { history of at } \\
\text { least } 20 \text { pack } \\
\text { years, and } \\
\text { according to } \\
\text { the attending } \\
\text { physician had } \\
\text { to be treated in } \\
\text { hospital. }\end{array}$ & & & Placebo & NR & $\begin{array}{l}66 \text { patients } \\
\text { aged } 70.4 \pm 8.9 \\
20 \% \text { female }\end{array}$ \\
\hline $\begin{array}{l}\text { Niewoehner, } \\
1999^{13}\end{array}$ & $\begin{array}{l}\text { United } \\
\text { States of } \\
\text { America }\end{array}$ & High risk & $\begin{array}{l}\text { Clinical } \\
\text { diagnosis of } \\
\text { COPD plus a } \\
\text { history of } 30 \\
\text { pack years or } \\
\text { more of } \\
\text { cigarette } \\
\text { smoking, and } \\
\text { either an FEV1 } \\
\text { of } 1.50 \text { liters or } \\
\text { less or an } \\
\text { inability to } \\
\text { undergo } \\
\text { spirometry } \\
\text { because of } \\
\text { dyspnea. }\end{array}$ & $\begin{array}{l}\text { Clinical } \\
\text { diagnosis }\end{array}$ & $\begin{array}{l}\text { Moderate to } \\
\text { Severe }\end{array}$ & $\begin{array}{l}\text { Glucocorticoid for } 8 \\
\text { weeks }\end{array}$ & $\begin{array}{l}\text { FEV1 (L): } \\
\text { Mean } \\
0.785 \pm 0.288\end{array}$ & $\begin{array}{l}80 \text { patients } \\
\text { aged } 68.1 \pm 6.8 \text {, } \\
3.75 \% \text { female }\end{array}$ \\
\hline
\end{tabular}




\begin{tabular}{|c|c|c|c|c|c|c|c|c|}
\hline $\begin{array}{l}\text { Author, } \\
\text { Year }\end{array}$ & $\begin{array}{l}\text { Country, } \\
\text { Study } \\
\text { Period }\end{array}$ & $\begin{array}{l}\text { Risk of } \\
\text { Bias }\end{array}$ & $\begin{array}{l}\text { COPD } \\
\text { Definition }\end{array}$ & $\begin{array}{c}\text { COPD } \\
\text { Exacerbation }\end{array}$ & $\begin{array}{l}\text { Severity of } \\
\text { ECOPD }\end{array}$ & $\begin{array}{l}\text { Intervention(s) and } \\
\text { comparison }\end{array}$ & $\begin{array}{c}\text { COPD } \\
\text { Severity } \\
(\% \\
\text { predicted } \\
\text { unless } \\
\text { specified) }\end{array}$ & $\begin{array}{c}\text { Patient } \\
\text { Characteristics }\end{array}$ \\
\hline & & & & & & $\begin{array}{l}\text { Glucocorticoid for } 2 \\
\text { weeks }\end{array}$ & $\begin{array}{l}\text { FEV1 (L): } \\
\text { Mean } \\
0.772 \pm 0.286\end{array}$ & $\begin{array}{l}80 \text { patients } \\
\text { aged } 67.1 \pm 10.6 \text {, } \\
0 \% \text { female }\end{array}$ \\
\hline & & & & & & Placebo & $\begin{array}{l}\text { FEV1 }(L): \\
\text { Mean } \\
0.750 \pm 0.271\end{array}$ & $\begin{array}{l}\text { 111Patients, } \\
\text { aged } 67.8 \pm 10 \\
0 \% \text { female }\end{array}$ \\
\hline \multirow[t]{2}{*}{$\begin{array}{l}\text { Thompson, } \\
1996^{14}\end{array}$} & \multirow[t]{2}{*}{ USA } & \multirow[t]{2}{*}{ High risk } & \multirow{2}{*}{$\begin{array}{l}\text { Airflow } \\
\text { obstruction, } \\
\text { and a clinical } \\
\text { diagnosis of } \\
\text { chronic } \\
\text { bronchitis or } \\
\text { emphysema as } \\
\text { defined by the } \\
\text { American } \\
\text { Thoracic } \\
\text { Society }\end{array}$} & \multirow[b]{2}{*}{$\begin{array}{l}\text { Subjective } \\
\text { worsening of } \\
\text { chronic } \\
\text { baseline } \\
\text { dyspnea or } \\
\text { cough for more } \\
\text { than } 24 \mathrm{~h} \\
\text { duration and } \\
\text { necessitating a } \\
\text { hospital visit, } \\
\text { required to } \\
\text { have least a } \\
250 \% \text { increase } \\
\text { in inhaled B- } \\
\text { adrenoceptor } \\
\text { agonist use for } \\
\text { more than } 24 \text { h, } \\
\text { or an increase } \\
\text { in sputum } \\
\text { production. }\end{array}$} & \multirow[t]{2}{*}{ Mild } & Prednisone & NR & $\begin{array}{l}13 \text { Patients } \\
\text { aged } 65(9) \\
\text { female } 7 \%\end{array}$ \\
\hline & & & & & & Placebo & NR & $\begin{array}{l}13 \text { Patients } \\
\text { aged } 70(7), \\
\text { female } 0 \%\end{array}$ \\
\hline $\begin{array}{l}\text { Van Velzen, } \\
2017^{15}\end{array}$ & $\begin{array}{l}\text { The } \\
\text { Netherlands }\end{array}$ & Low Risk & $\begin{array}{l}\text { Post- } \\
\text { bronchodilator } \\
\text { FEV1/ FVC }\end{array}$ & $\begin{array}{l}\text { Increased } \\
\text { dyspnea, } \\
\text { cough, or }\end{array}$ & Mild & Doxycycline & $\begin{array}{l}\text { FEV1 }(L) \\
\text { Mean: } 61.2 \\
(18.0)\end{array}$ & $\begin{array}{l}152 \text { Patients } \\
\text { aged } 65.8(9.3) \text {, } \\
\text { female } 35 \%\end{array}$ \\
\hline
\end{tabular}




\begin{tabular}{|c|c|c|c|c|c|c|c|c|}
\hline $\begin{array}{l}\text { Author, } \\
\text { Year }\end{array}$ & $\begin{array}{c}\text { Country, } \\
\text { Study } \\
\text { Period }\end{array}$ & $\begin{array}{c}\text { Risk of } \\
\text { Bias }\end{array}$ & $\begin{array}{c}\text { COPD } \\
\text { Definition }\end{array}$ & $\begin{array}{c}\text { COPD } \\
\text { Exacerbation }\end{array}$ & $\begin{array}{l}\text { Severity of } \\
\text { ECOPD }\end{array}$ & $\begin{array}{l}\text { Intervention(s) and } \\
\text { comparison }\end{array}$ & $\begin{array}{c}\text { COPD } \\
\text { Severity } \\
(\% \\
\text { predicted } \\
\text { unless } \\
\text { specified) }\end{array}$ & $\begin{array}{c}\text { Patient } \\
\text { Characteristics }\end{array}$ \\
\hline & $\begin{array}{l}12 / 2010- \\
8 / 2013\end{array}$ & & $\begin{array}{l}<0.7, \text { post- } \\
\text { bronchodilator } \\
\text { FEV1 } \geq 30 \% \\
\text { (GOLD stage } \\
1-3), \geq 10 \text { pack } \\
\text { year smoking } \\
\text { history, } \geq 1 \\
\text { exacerbation in } \\
\text { prior } 3 \text { years, } \\
\text { no } \\
\text { exacerbations } \\
\text { in prior } 4 \text { weeks }\end{array}$ & $\begin{array}{l}\text { sputum } \\
\text { sufficient to } \\
\text { warrant a } \\
\text { change in } \\
\text { management } \\
\text { other than } \\
\text { optimization of } \\
\text { bronchodilator } \\
\text { therapy. } \\
\text { Hospital } \\
\text { admission and } \\
\text { fever excluded. }\end{array}$ & & Placebo & $\begin{array}{l}\text { FEV1 (L) } \\
\text { Mean: : } \\
60.5(17.7)\end{array}$ & $\begin{array}{l}153 \text { Patients } \\
\text { aged } 66.4(9.5) \text {, } \\
\text { female } 46 \%\end{array}$ \\
\hline \multirow[t]{2}{*}{$\begin{array}{l}\text { Wang, } \\
2016^{16}\end{array}$} & \multirow[t]{2}{*}{$\begin{array}{l}\text { China } \\
6 / 2014 \\
9 / 2015\end{array}$} & \multirow[t]{2}{*}{ Low risk } & \multirow[t]{2}{*}{$\begin{array}{l}\text { GOLD } 2014 \\
\text { criteria }\end{array}$} & \multirow{2}{*}{$\begin{array}{l}\text { Acute change } \\
\text { in symptoms } \\
\text { that were } \\
\text { beyond normal } \\
\text { day-to-day } \\
\text { variation and } \\
\text { that required a } \\
\text { change in daily } \\
\text { therapeutic } \\
\text { drug regimen, } \\
\text { hospital } \\
\text { admission }\end{array}$} & \multirow[t]{2}{*}{$\begin{array}{l}\text { Moderate to } \\
\text { Severe }\end{array}$} & Antibiotic & NR & $\begin{array}{l}97 \text { patients } \\
\text { aged } 73.4 \pm 10.1 \\
29.5 \% \text { female }\end{array}$ \\
\hline & & & & & & $\begin{array}{l}\text { Management } \\
\text { without Antibiotic }\end{array}$ & NR & $\begin{array}{l}97 \text { patients } \\
\text { aged } 72.5 \pm 9.2 \\
27.1 \% \text { female }\end{array}$ \\
\hline
\end{tabular}

Note: \pm denotes standard deviationd = day; FEV1 = forced expiration volume in 1 second; FVC = forced vital capacity; GOLD = global initiative for chronic obstructive lung disease; $\mathrm{h}$ = hour; $\mathrm{L}=$ liter; $\mathrm{NR}=$ not reported; $\mathrm{PaCO} 2$ = partial pressure of carbon dioxide in arterial blood; $\mathrm{PaO} 2$ = partial pressure of oxygen in arterial blood; $\mathrm{pH}$ = potential of hydrogen; VC = vital capacity 
Table D.2. KQ2: Characteristics of included studies

\begin{tabular}{|c|c|c|c|c|c|c|c|c|}
\hline $\begin{array}{l}\text { Author, } \\
\text { Year }\end{array}$ & $\begin{array}{l}\text { Country, } \\
\text { Study } \\
\text { Period }\end{array}$ & $\begin{array}{l}\text { Risk of } \\
\text { Bias }\end{array}$ & $\begin{array}{c}\text { COPD } \\
\text { Definition }\end{array}$ & $\begin{array}{c}\text { COPD } \\
\text { Exacerbation }\end{array}$ & $\begin{array}{l}\text { Severity of } \\
\text { ECOPD }\end{array}$ & $\begin{array}{l}\text { Intervention(s) and } \\
\text { comparison }\end{array}$ & $\begin{array}{c}\text { COPD } \\
\text { Severity } \\
(\% \\
\text { predicted } \\
\text { unless } \\
\text { specified) }\end{array}$ & $\begin{array}{c}\text { Patient } \\
\text { Characteristics }\end{array}$ \\
\hline $\begin{array}{l}\text { Abreu } \\
\text { Gonzalez, } \\
2006^{17}\end{array}$ & $\begin{array}{l}\text { Spain } \\
10 / 2003 \text { to } \\
09 / 2004\end{array}$ & High risk & NR & NR & $\begin{array}{l}\text { Moderate to } \\
\text { Severe }\end{array}$ & Magnesium Sulfate & $\begin{array}{l}\text { FEV1: } \\
\text { Mean } \\
32.5 \pm 3.2\end{array}$ & $\begin{array}{l}24 \text { total patients } \\
\text { aged } 64 \text { years, } \\
0 \% \text { female }\end{array}$ \\
\hline \multirow[t]{2}{*}{$\begin{array}{l}\text { Austin, } \\
2010^{18}\end{array}$} & \multirow[t]{2}{*}{ Australia } & \multirow[t]{2}{*}{ High risk } & \multirow{2}{*}{$\begin{array}{l}\text { Patient reported } \\
\text { history of COPD } \\
\text { or emphysema } \\
\text { or greater than } \\
10 \text { pack year } \\
\text { history of } \\
\text { smoking. }\end{array}$} & \multirow{2}{*}{$\begin{array}{l}\text { Paramedics at the } \\
\text { site of the } \\
\text { emergency } \\
\text { Determined the } \\
\text { diagnosis on the } \\
\text { basis of } \\
\text { appropriate acute } \\
\text { symptoms, a } \\
\text { history of chronic } \\
\text { obstructive } \\
\text { pulmonary disease } \\
\text { (or emphysema) } \\
\text { from the patient, or } \\
\text { a greater than } 10 \\
\text { pack year history } \\
\text { of smoking. }\end{array}$} & \multirow[t]{2}{*}{$\begin{array}{l}\text { Moderate to } \\
\text { Severe }\end{array}$} & $\begin{array}{l}\text { High Flow/Free Flow } \\
\text { Oxygen }\end{array}$ & $\begin{array}{l}\text { FEV1: } \\
\text { Mean } \\
42.1 \pm 16.4\end{array}$ & $\begin{array}{l}117 \text { patients } \\
\text { aged } 68 \pm 10.2 \\
\text { years, } 51 \% \\
\text { female }\end{array}$ \\
\hline & & & & & & Titrated Oxygen & $\begin{array}{l}\text { FEV1: } \\
\text { Mean } \\
43.3 \pm 16.5\end{array}$ & $\begin{array}{l}\text { 97 patients } \\
\text { aged } 67.9 \pm 10.3 \\
\text { years, } 54 \% \\
\text { female }\end{array}$ \\
\hline \multirow[t]{2}{*}{$\begin{array}{l}\text { Ayfer } \\
\text { Aytemur, } \\
2015^{19}\end{array}$} & \multirow[t]{2}{*}{ Turkey } & \multirow[t]{2}{*}{ High risk } & \multirow{2}{*}{$\begin{array}{l}\text { Based on } \\
\text { spirometry not } \\
\text { otherwise } \\
\text { specified, at } \\
\text { least } 20 \text { pack } \\
\text { year smoking } \\
\text { history }\end{array}$} & \multirow[t]{2}{*}{$\begin{array}{l}\text { Clinical diagnosis, } \\
\text { sputum volume of } \\
50 \text { ml or greater, } \\
\text { hospital admission }\end{array}$} & \multirow[t]{2}{*}{$\begin{array}{l}\text { Moderate to } \\
\text { Severe }\end{array}$} & N-Acetylcysteine & NR & $\begin{array}{l}20 \text { patients } \\
\text { aged } 68.6 \pm 7.5 \\
\text { years, } 11 \% \\
\text { female }\end{array}$ \\
\hline & & & & & & Placebo & NR & $\begin{array}{l}22 \text { patients } \\
\text { aged } 69.4 \pm 9.9 \\
\text { years, } 5 \% \\
\text { female }\end{array}$ \\
\hline \multirow[t]{2}{*}{$\begin{array}{l}\text { Basri, } \\
2017^{20}\end{array}$} & \multirow[t]{2}{*}{ Pakistan } & \multirow[t]{2}{*}{$\begin{array}{l}\text { Intermed } \\
\text { iate risk }\end{array}$} & \multirow[t]{2}{*}{$\begin{array}{l}\text { Clinical } \\
\text { diagnosis }\end{array}$} & \multirow[t]{2}{*}{$\begin{array}{l}\text { Clinical diagnosis, } \\
\text { hospital admission }\end{array}$} & \multirow[t]{2}{*}{$\begin{array}{l}\text { Moderate to } \\
\text { Severe }\end{array}$} & $\begin{array}{l}\text { Management without } \\
\text { Chest Physiotherapy }\end{array}$ & NR & $\begin{array}{l}30 \text { patients } \\
\text { aged } 53 \pm 3.7 \\
\text { years, } 40 \% \\
\text { female }\end{array}$ \\
\hline & & & & & & Chest Physiotherapy & NR & $\begin{array}{l}30 \text { patients } \\
\text { aged } 55 \pm 3.8 \\
\text { years, } 56 \% \\
\text { female }\end{array}$ \\
\hline
\end{tabular}




\begin{tabular}{|c|c|c|c|c|c|c|c|c|}
\hline $\begin{array}{l}\text { Author, } \\
\text { Year }\end{array}$ & $\begin{array}{l}\text { Country, } \\
\text { Study } \\
\text { Period }\end{array}$ & $\begin{array}{l}\text { Risk of } \\
\text { Bias }\end{array}$ & $\begin{array}{c}\text { COPD } \\
\text { Definition }\end{array}$ & $\begin{array}{c}\text { COPD } \\
\text { Exacerbation }\end{array}$ & $\begin{array}{c}\text { Severity of } \\
\text { ECOPD }\end{array}$ & $\begin{array}{l}\text { Intervention(s) and } \\
\text { comparison }\end{array}$ & $\begin{array}{c}\text { COPD } \\
\text { Severity } \\
(\% \\
\text { predicted } \\
\text { unless } \\
\text { specified) }\end{array}$ & $\begin{array}{c}\text { Patient } \\
\text { Characteristics }\end{array}$ \\
\hline \multirow[t]{3}{*}{$\begin{array}{l}\text { Bathoorn, } \\
2008^{4}\end{array}$} & \multirow[t]{3}{*}{ Netherlands } & \multirow[t]{3}{*}{ High risk } & \multirow{3}{*}{$\begin{array}{l}\text { Post } \\
\text { bronchodilator } \\
\text { FEV1 <85\% } \\
\text { predicted but } \\
>0.7 \text { liters, and } \\
\text { an Abnormal } \\
\text { post } \\
\text { bronchodilator } \\
\text { FEV1/slow } \\
\text { inspiratory vital } \\
\text { capacity (VC) } \\
\text { (<88\% predicted } \\
\text { in men and } \\
<89 \% \text { predicted } \\
\text { in women) }\end{array}$} & \multirow{3}{*}{$\begin{array}{l}\text { History of } \\
\text { increased } \\
\text { breathlessness } \\
\text { and at least two of } \\
\text { the following } \\
\text { symptoms for } \geq 24 \\
\text { hours: increased } \\
\text { cough frequency or } \\
\text { severity, sputum } \\
\text { volume or } \\
\text { purulence, and } \\
\text { wheeze. }\end{array}$} & \multirow[t]{3}{*}{ Mild } & $\begin{array}{l}\text { Budesonide + } \\
\text { Formoterol }\end{array}$ & NR & $\begin{array}{l}15 \text { patients } \\
\text { aged } 61.4 \pm 8 \\
\text { years, } 33 \% \\
\text { female }\end{array}$ \\
\hline & & & & & & Prednisolone & NR & $\begin{array}{l}15 \text { patients } \\
\text { aged } 64.8 \pm 7 \\
\text { years, } 15 \% \\
\text { female }\end{array}$ \\
\hline & & & & & & Placebo & NR & $\begin{array}{l}15 \text { patients } \\
\text { aged } 64.6 \pm 9.1 \\
7 \% \text { female }\end{array}$ \\
\hline \multirow[t]{2}{*}{$\begin{array}{l}\text { Behnke, } \\
2000^{21}\end{array}$} & \multirow[t]{2}{*}{ Germany } & \multirow[t]{2}{*}{ High risk } & \multirow[t]{2}{*}{$\begin{array}{l}\text { Severe COPD } \\
\text { according to } \\
\text { international } \\
\text { guidelines }\end{array}$} & \multirow[t]{2}{*}{ Hospital admission } & \multirow[t]{2}{*}{$\begin{array}{l}\text { Moderate to } \\
\text { Severe }\end{array}$} & Aerobic Exercise & $\begin{array}{l}\text { FEV1: } \\
\text { Mean } \\
34.1 \pm 7.4\end{array}$ & $\begin{array}{l}23 \text { patients } \\
\text { aged } 64.0 \pm 1.9 \\
\text { years, } 20 \% \\
\text { female }\end{array}$ \\
\hline & & & & & & $\begin{array}{l}\text { Management without } \\
\text { Aerobic Exercise }\end{array}$ & $\begin{array}{l}\text { FEV1: } \\
\text { Mean } \\
37.5 \pm 6.6\end{array}$ & $\begin{array}{l}23 \text { patients } \\
\text { aged } 68.0 \pm 2.2 \\
\text { years, } 27 \% \\
\text { female }\end{array}$ \\
\hline \multirow[t]{2}{*}{$\begin{array}{l}\text { Black, } \\
2004^{22}\end{array}$} & \multirow[t]{2}{*}{$\begin{array}{l}\text { New } \\
\text { Zealand } \\
01 / 2001- \\
10 / 2001\end{array}$} & \multirow[t]{2}{*}{$\begin{array}{l}\text { Intermed } \\
\text { iate risk }\end{array}$} & \multirow[t]{2}{*}{$\begin{array}{l}\text { FEV1 } \leq 60 \% \\
\text { predicted } \\
\text { FEV1/NC } \leq 70 \%\end{array}$} & \multirow[t]{2}{*}{$\begin{array}{l}\text { An increased } \\
\text { volume of sputum } \\
\text { as well as } \\
\text { breathlessness. }\end{array}$} & \multirow[t]{2}{*}{$\begin{array}{l}\text { Moderate to } \\
\text { Severe }\end{array}$} & $\mathrm{N}$-acetylcysteine & $\begin{array}{l}\text { FEV1: } \\
\text { Mean } \\
22 \pm 10\end{array}$ & $\begin{array}{l}25 \text { patients } \\
\text { aged } 73.6 \pm 7.8 \\
\text { years, } 56 \% \\
\text { female }\end{array}$ \\
\hline & & & & & & Placebo & $\begin{array}{l}\text { FEV1: } \\
\text { Mean } \\
24 \pm 12\end{array}$ & $\begin{array}{l}25 \text { patients } \\
\text { aged } 73 \pm 8.2 \\
\text { years, } 24 \% \\
\text { female }\end{array}$ \\
\hline $\begin{array}{l}\text { Borges, } \\
2014^{23}\end{array}$ & Brazil & $\begin{array}{l}\text { Intermed } \\
\text { iate risk }\end{array}$ & FEV1/FVC $\leq 0.7$ & $\begin{array}{l}\text { Increase in sputum } \\
\text { or cough or } \\
\text { worsening of }\end{array}$ & $\begin{array}{l}\text { Moderate to } \\
\text { Severe }\end{array}$ & Resistance Training & $\begin{array}{l}\text { FEV1: } \\
\text { Mean } \\
41.7 \pm 13.6\end{array}$ & $\begin{array}{l}21 \text { patients } \\
\text { aged } 64.1 \pm 12.5 \\
\text { years, } 29 \% \\
\text { female }\end{array}$ \\
\hline
\end{tabular}




\begin{tabular}{|c|c|c|c|c|c|c|c|c|}
\hline $\begin{array}{l}\text { Author, } \\
\text { Year }\end{array}$ & $\begin{array}{l}\text { Country, } \\
\text { Study } \\
\text { Period }\end{array}$ & $\begin{array}{l}\text { Risk of } \\
\text { Bias }\end{array}$ & $\begin{array}{c}\text { COPD } \\
\text { Definition }\end{array}$ & $\begin{array}{c}\text { COPD } \\
\text { Exacerbation }\end{array}$ & $\begin{array}{l}\text { Severity of } \\
\text { ECOPD }\end{array}$ & $\begin{array}{l}\text { Intervention(s) and } \\
\text { comparison }\end{array}$ & $\begin{array}{c}\text { COPD } \\
\text { Severity } \\
(\% \\
\text { predicted } \\
\text { unless } \\
\text { specified) }\end{array}$ & $\begin{array}{c}\text { Patient } \\
\text { Characteristics }\end{array}$ \\
\hline & & & & $\begin{array}{l}\text { dyspnea, hospital } \\
\text { admission }\end{array}$ & & $\begin{array}{l}\text { Management without } \\
\text { Resistance Training }\end{array}$ & $\begin{array}{l}\text { FEV1: } \\
\text { Mean } \\
39.1 \pm 15.5\end{array}$ & $\begin{array}{l}25 \text { patients } \\
\text { aged } 67.8 \pm 9 \\
\text { years, } 47 \% \\
\text { female }\end{array}$ \\
\hline \multirow[t]{2}{*}{$\begin{array}{l}\text { Brown, } \\
1987^{24}\end{array}$} & \multirow[t]{2}{*}{ Canada } & \multirow[t]{2}{*}{ High risk } & \multirow{2}{*}{$\begin{array}{l}\text { Chronic } \\
\text { productive cough } \\
\text { with at least } 30 \\
\text { ml of sputum } \\
\text { production daily }\end{array}$} & \multirow{2}{*}{$\begin{array}{l}\text { Acute episode of } \\
\text { pneumonia or } \\
\text { increase in sputum } \\
\text { production to at } \\
\text { least } 30 \mathrm{ml} \text { of } \\
\text { sputum production } \\
\text { daily }\end{array}$} & \multirow[t]{2}{*}{$\begin{array}{l}\text { Mild to } \\
\text { Moderate to } \\
\text { Severe }\end{array}$} & Chest Wall Vibration & \multirow[t]{2}{*}{$\begin{array}{l}\text { FEV1: } \\
\text { Mean } \\
33.4 \pm 17.5\end{array}$} & \multirow[t]{2}{*}{$\begin{array}{l}12 \text { total patients } \\
\text { aged } 66.5 \pm 11.5 \\
\text { years, } 29 \% \\
\text { female }\end{array}$} \\
\hline & & & & & & Positioning & & \\
\hline $\begin{array}{l}\text { Centanni, } \\
2002^{25}\end{array}$ & Italy & High risk & $\begin{array}{l}\text { European } \\
\text { Respiratory } \\
\text { Society (ERS) } \\
\text { guidelines, }\end{array}$ & $\begin{array}{l}\text { A sustained } \\
\text { worsening of the } \\
\text { patient's condition, } \\
\text { from the stable }\end{array}$ & Mild & Oxitropium & $\begin{array}{l}\text { FEV1: } \\
\text { Mean } \\
58.6 \pm 17.7\end{array}$ & $\begin{array}{l}50 \text { patients } \\
\text { aged } 68 \pm 0.8 \\
\text { years, } 34 \% \\
\text { female }\end{array}$ \\
\hline
\end{tabular}




\begin{tabular}{|c|c|c|c|c|c|c|c|c|}
\hline $\begin{array}{l}\text { Author, } \\
\text { Year }\end{array}$ & $\begin{array}{c}\text { Country, } \\
\text { Study } \\
\text { Period }\end{array}$ & $\begin{array}{l}\text { Risk of } \\
\text { Bias }\end{array}$ & $\begin{array}{c}\text { COPD } \\
\text { Definition }\end{array}$ & $\begin{array}{c}\text { COPD } \\
\text { Exacerbation }\end{array}$ & $\begin{array}{l}\text { Severity of } \\
\text { ECOPD }\end{array}$ & $\begin{array}{l}\text { Intervention(s) and } \\
\text { comparison }\end{array}$ & $\begin{array}{c}\text { COPD } \\
\text { Severity } \\
(\% \\
\text { predicted } \\
\text { unless } \\
\text { specified) }\end{array}$ & $\begin{array}{c}\text { Patient } \\
\text { Characteristics }\end{array}$ \\
\hline & & & $\begin{array}{l}\text { clinical history, } \\
\text { physical findings } \\
\text { and spirometry, } \\
\text { current or } \\
\text { previous } \\
\text { smokers (>10 } \\
\text { pack-years) } \\
\text { reporting chronic } \\
\text { cough with } \\
\text { sputum } \\
\text { production on } \\
\text { most days during } \\
\text { at least } 3 \\
\text { consecutive } \\
\text { months in } 2 \\
\text { consecutive } \\
\text { years, had a } \\
\text { FEV1 < } 65 \% \text { and } \\
\text { a forced vital } \\
\text { capacity (FVC) }< \\
70 \% \text { of predicted } \\
\text { normal after } \\
\text { bronchodilators } \\
\text { had been } \\
\text { withheld for } 24 \mathrm{~h} \\
\text { and a best post- } \\
\text { bronchodilator } \\
\text { FEV1/FVC of } \\
\text { less than } 0.7 \text {. }\end{array}$ & $\begin{array}{l}\text { state and beyond } \\
\text { normal day-to-day } \\
\text { variations, that is } \\
\text { acute in onset and } \\
\text { necessitates a } \\
\text { change in regular } \\
\text { medication in a } \\
\text { patient with } \\
\text { underlying COPD. }\end{array}$ & & Placebo & & \\
\hline $\begin{array}{l}\text { Cox } \\
2018^{26}\end{array}$ & $\begin{array}{l}\text { United } \\
\text { Kingdom } \\
09 / 2015 \text { to } \\
04 / 2016\end{array}$ & $\begin{array}{l}\text { Intermed } \\
\text { iate risk }\end{array}$ & $\begin{array}{l}\text { Admission to } \\
\text { hospital with } \\
\text { primary } \\
\text { diagnosis of } \\
\text { ECOPD }\end{array}$ & $\begin{array}{l}\text { Clinical diagnosis, } \\
\text { hospital admission }\end{array}$ & $\begin{array}{l}\text { Moderate to } \\
\text { Severe }\end{array}$ & $\begin{array}{l}\text { Management without } \\
\text { Aerobic Exercise }\end{array}$ & NR & $\begin{array}{l}15 \text { patients } \\
\text { aged } \\
67.8 \pm 11.12 \\
\text { years, } 67 \% \\
\text { female }\end{array}$ \\
\hline
\end{tabular}




\begin{tabular}{|c|c|c|c|c|c|c|c|c|}
\hline $\begin{array}{l}\text { Author, } \\
\text { Year }\end{array}$ & $\begin{array}{l}\text { Country, } \\
\text { Study } \\
\text { Period }\end{array}$ & $\begin{array}{l}\text { Risk of } \\
\text { Bias }\end{array}$ & $\begin{array}{c}\text { COPD } \\
\text { Definition }\end{array}$ & $\begin{array}{c}\text { COPD } \\
\text { Exacerbation }\end{array}$ & $\begin{array}{l}\text { Severity of } \\
\text { ECOPD }\end{array}$ & $\begin{array}{l}\text { Intervention(s) and } \\
\text { comparison }\end{array}$ & $\begin{array}{c}\text { COPD } \\
\text { Severity } \\
(\% \\
\text { predicted } \\
\text { unless } \\
\text { specified) }\end{array}$ & $\begin{array}{c}\text { Patient } \\
\text { Characteristics }\end{array}$ \\
\hline & & & $\begin{array}{l}\text { (clinically } \\
\text { determined by } \\
\text { treating } \\
\text { physician, pH } \\
>7.35 \text {, unstable } \\
\text { hypoxemia } \\
\text { excluded }\end{array}$ & & & Aerobic Exercise & NR & $\begin{array}{l}15 \text { patients } \\
\text { aged } \\
67.8 \pm 11.12 \\
\text { years, } 60 \% \\
\text { female }\end{array}$ \\
\hline \multirow[t]{2}{*}{$\begin{array}{l}\text { Cross, } \\
2012^{27}\end{array}$} & \multirow[t]{2}{*}{$\begin{array}{l}\text { United } \\
\text { Kingdom } \\
11 / 2005 \text { to } \\
4 / 2008\end{array}$} & \multirow[t]{2}{*}{$\begin{array}{l}\text { Intermed } \\
\text { iate risk }\end{array}$} & \multirow[t]{2}{*}{$\begin{array}{l}\text { Clinical } \\
\text { diagnosis }\end{array}$} & \multirow[t]{2}{*}{$\begin{array}{l}\text { Clinical diagnosis, } \\
\text { hospital admission }\end{array}$} & \multirow[t]{2}{*}{$\begin{array}{l}\text { Moderate to } \\
\text { Severe }\end{array}$} & $\begin{array}{l}\text { Manual Chest } \\
\text { Physiotherapy }\end{array}$ & NR & $\begin{array}{l}258 \text { patients } \\
\text { aged } \\
69.08 \pm 9.85 \\
\text { years, } 44.57 \% \\
\text { female }\end{array}$ \\
\hline & & & & & & $\begin{array}{l}\text { Management without } \\
\text { Manual Chest } \\
\text { Physiotherapy }\end{array}$ & NR & $\begin{array}{l}264 \text { patients } \\
\text { aged } \\
69.58 \pm 9.51 \\
\text { years, } 41.29 \% \\
\text { female }\end{array}$ \\
\hline \multirow[t]{2}{*}{$\begin{array}{l}\text { Du, } \\
2018^{28}\end{array}$} & \multirow[t]{2}{*}{ China } & \multirow[t]{2}{*}{ High risk } & \multirow{2}{*}{$\begin{array}{l}\text { COPD according } \\
\text { to the criteria } \\
\text { established by } \\
\text { the Global } \\
\text { initiative for } \\
\text { Chronic } \\
\text { Obstructive Lung } \\
\text { Disease }\end{array}$} & \multirow[t]{2}{*}{ NR } & \multirow[t]{2}{*}{$\begin{array}{l}\text { Mild to } \\
\text { Moderate }\end{array}$} & Simvastatin & NR & $\begin{array}{l}30 \text { patients } \\
\text { aged } \\
62.44 \pm 8.27 \\
\text { years, } 13 \% \\
\text { female }\end{array}$ \\
\hline & & & & & & Placebo & NR & $\begin{array}{l}30 \text { patients } \\
\text { aged } 64.2 \pm 9.35 \\
\text { years, } 10 \% \\
\text { female }\end{array}$ \\
\hline \multirow[t]{2}{*}{$\begin{array}{l}\text { Duffy, } \\
2005^{29}\end{array}$} & \multirow[t]{2}{*}{$\begin{array}{l}\text { United } \\
\text { Kingdom }\end{array}$} & \multirow[t]{2}{*}{ Low risk } & \multirow{2}{*}{$\begin{array}{l}\text { FEV1 of } 70 \% \\
\text { predicted, } \\
\text { FEV1/FVC of } \\
70 \% \text { predicted, } \\
\text { at least } 20 \text { pack } \\
\text { year smoking } \\
\text { history }\end{array}$} & \multirow{2}{*}{$\begin{array}{l}\text { Breathlessness } \\
\text { and two or more of } \\
\text { the following } \\
\text { symptoms for at } \\
\text { least } 24 \text { hours: } \\
\text { increased cough } \\
\text { frequency or } \\
\text { severity, increased } \\
\text { sputum volume or } \\
\text { purulence, } \\
\text { increased wheeze. }\end{array}$} & \multirow[t]{2}{*}{$\begin{array}{l}\text { Moderate to } \\
\text { Severe }\end{array}$} & Placebo & NR & $\begin{array}{l}1 \text { patients } \\
\text { aged } 67.4 \pm 7.5 \\
\text { years, } 46 \% \\
\text { female }\end{array}$ \\
\hline & & & & & & Aminophylline & NR & $\begin{array}{l}\text { 39 patients } \\
\text { aged } 69.6 \pm 8.0 \\
\text { years, } 67 \% \\
\text { female }\end{array}$ \\
\hline
\end{tabular}




\begin{tabular}{|c|c|c|c|c|c|c|c|c|}
\hline $\begin{array}{l}\text { Author, } \\
\text { Year }\end{array}$ & $\begin{array}{l}\text { Country, } \\
\text { Study } \\
\text { Period }\end{array}$ & $\begin{array}{l}\text { Risk of } \\
\text { Bias }\end{array}$ & $\begin{array}{c}\text { COPD } \\
\text { Definition }\end{array}$ & $\begin{array}{c}\text { COPD } \\
\text { Exacerbation }\end{array}$ & $\begin{array}{l}\text { Severity of } \\
\text { ECOPD }\end{array}$ & $\begin{array}{l}\text { Intervention(s) and } \\
\text { comparison }\end{array}$ & $\begin{array}{c}\text { COPD } \\
\text { Severity } \\
(\% \\
\text { predicted } \\
\text { unless } \\
\text { specified) }\end{array}$ & $\begin{array}{c}\text { Patient } \\
\text { Characteristics }\end{array}$ \\
\hline \multirow[t]{2}{*}{$\begin{array}{l}\text { Eaton, } \\
2009^{30}\end{array}$} & \multirow[t]{2}{*}{$\begin{array}{l}\text { New } \\
\text { Zealand } \\
06 / 2005 \text { to } \\
10 / 2006\end{array}$} & \multirow[t]{2}{*}{ High risk } & \multirow[t]{2}{*}{ ATS/ERS criteria } & \multirow[t]{2}{*}{$\begin{array}{l}\text { Exertional dyspnea } \\
\text { interfering with } \\
\text { daily activity, } \\
\text { hospital admission }\end{array}$} & \multirow[t]{2}{*}{$\begin{array}{l}\text { Moderate to } \\
\text { Severe }\end{array}$} & $\begin{array}{l}\text { Early Pulmonary } \\
\text { Rehabilitation }\end{array}$ & $\begin{array}{l}\text { FEV1: } \\
\text { Mean } \\
36 \pm 16 \\
0.8 \pm 0.4 \mathrm{~L}\end{array}$ & $\begin{array}{l}47 \text { patients } \\
\text { aged } 70.1 \pm 10.3 \\
\text { years, } 55 \% \\
\text { female }\end{array}$ \\
\hline & & & & & & $\begin{array}{l}\text { Management without } \\
\text { Early Rehabilitation }\end{array}$ & $\begin{array}{l}\text { FEV1: } \\
\text { Mean } \\
35 \pm 16 \\
0.8 \pm 0.4 \mathrm{~L}\end{array}$ & $\begin{array}{l}50 \text { patients } \\
\text { aged } 69.7 \pm 9.4 \\
\text { years, } 58 \% \\
\text { female }\end{array}$ \\
\hline \multirow[t]{2}{*}{$\begin{array}{l}\text { Edwards, } \\
2013^{31}\end{array}$} & \multirow{2}{*}{$\begin{array}{l}\text { New } \\
\text { Zealand } \\
06 / 2008 \text { to } \\
11 / 2011\end{array}$} & \multirow[t]{2}{*}{ Low risk } & \multirow{2}{*}{$\begin{array}{l}\text { FEV1/FVC }<0.7 \text {, } \\
\text { post- } \\
\text { bronchodilator } \\
\text { FEV1 } \leq 50 \% \text {, } \\
\text { clinical diagnosis }\end{array}$} & \multirow[t]{2}{*}{$\begin{array}{l}\text { Clinical diagnosis, } \\
\text { ED evaluation }\end{array}$} & \multirow[t]{2}{*}{$\begin{array}{l}\text { Moderate to } \\
\text { Severe }\end{array}$} & Placebo & NR & $\begin{array}{l}64 \text { patients } \\
\text { aged } 69.5 \pm 11.9 \\
\text { years, } 49.2 \% \\
\text { female }\end{array}$ \\
\hline & & & & & & Magnesium Sulfate & NR & $\begin{array}{l}52 \text { patients } \\
\text { aged } 73.2 \pm 9.8 \\
\text { years, } 43.8 \% \\
\text { female }\end{array}$ \\
\hline \multirow[t]{2}{*}{$\begin{array}{l}\text { Goktalay, } \\
2013^{32}\end{array}$} & \multirow[t]{2}{*}{$\begin{array}{l}\text { Turkey } \\
04 / 2009 \text { to } \\
07 / 2011\end{array}$} & \multirow[t]{2}{*}{ High risk } & \multirow[t]{2}{*}{ GOLD stage 3-4 } & \multirow{2}{*}{$\begin{array}{l}\text { Increased } \\
\text { dyspnea, } \\
\text { increased cough } \\
\text { and sputum } \\
\text { production, altered } \\
\text { sputum color } \\
\text { and/or viscosity, } \\
\text { fever and } \\
\text { radiologic } \\
\text { consolidation, } \\
\text { hospital admission }\end{array}$} & \multirow[t]{2}{*}{$\begin{array}{l}\text { Moderate to } \\
\text { Severe }\end{array}$} & $\begin{array}{l}\text { Management without } \\
\text { High-frequency Chest } \\
\text { Wall Oscillation } \\
\text { Therapy }\end{array}$ & $\begin{array}{l}\text { FEV1: } \\
\text { Mean } \\
30 \pm 8.93\end{array}$ & $\begin{array}{l}25 \text { patients } \\
\text { aged } \\
66.52 \pm 6.59 \\
2 \% \text { female }\end{array}$ \\
\hline & & & & & & $\begin{array}{l}\text { High-frequency Chest } \\
\text { Wall Oscillation } \\
\text { Therapy }\end{array}$ & $\begin{array}{l}\text { FEV1: } \\
\text { Mean } \\
28 \pm 8.95\end{array}$ & $\begin{array}{l}25 \text { patients } \\
\text { aged } \\
63.6 \pm 7.99,2 \% \\
\text { female }\end{array}$ \\
\hline \multirow[t]{2}{*}{$\begin{array}{l}\text { Greening, } \\
2014^{33}\end{array}$} & \multirow[t]{2}{*}{$\begin{array}{l}\text { United } \\
\text { Kingdom, }\end{array}$} & \multirow[t]{2}{*}{ High risk } & \multirow{2}{*}{$\begin{array}{l}\text { COPD } \\
\text { diagnosis, MRC } \\
\text { dyspnea } 3 \text { or } \\
\text { greater } \\
\end{array}$} & \multirow[t]{2}{*}{ NR } & \multirow[t]{2}{*}{$\begin{array}{l}\text { Moderate to } \\
\text { Severe }\end{array}$} & $\begin{array}{l}\text { Early Pulmonary } \\
\text { Rehabilitation }\end{array}$ & N/A & 169 patients \\
\hline & & & & & & $\begin{array}{l}\text { Management without } \\
\text { Early Rehabilitation }\end{array}$ & $\mathrm{N} / \mathrm{A}$ & 151 patients \\
\hline $\begin{array}{l}\text { Greulich, } \\
2014^{34}\end{array}$ & $\begin{array}{l}\text { Germany } \\
10 / 2010 \text { to } \\
07 / 2012 \\
\end{array}$ & $\begin{array}{l}\text { Intermed } \\
\text { iate risk }\end{array}$ & NR & $\begin{array}{l}\text { NR, hospital } \\
\text { admission }\end{array}$ & $\begin{array}{l}\text { Moderate to } \\
\text { Severe }\end{array}$ & $\begin{array}{l}\text { Management without } \\
\text { Whole Body Vibration }\end{array}$ & $\begin{array}{l}\text { FEV1: } \\
\text { Mean } \\
38.4 \pm 17.8 \\
2 \\
\end{array}$ & $\begin{array}{l}26 \text { patients } \\
\text { aged } 70.4 \pm 10.1 \\
\text { years, } 40 \% \\
\text { female }\end{array}$ \\
\hline
\end{tabular}




\begin{tabular}{|c|c|c|c|c|c|c|c|c|}
\hline $\begin{array}{c}\text { Author, } \\
\text { Year }\end{array}$ & $\begin{array}{l}\text { Country, } \\
\text { Study } \\
\text { Period }\end{array}$ & $\begin{array}{l}\text { Risk of } \\
\text { Bias }\end{array}$ & $\begin{array}{c}\text { COPD } \\
\text { Definition }\end{array}$ & $\begin{array}{c}\text { COPD } \\
\text { Exacerbation }\end{array}$ & $\begin{array}{l}\text { Severity of } \\
\text { ECOPD }\end{array}$ & $\begin{array}{l}\text { Intervention(s) and } \\
\text { comparison }\end{array}$ & $\begin{array}{c}\text { COPD } \\
\text { Severity } \\
(\% \\
\text { predicted } \\
\text { unless } \\
\text { specified) }\end{array}$ & $\begin{array}{c}\text { Patient } \\
\text { Characteristics }\end{array}$ \\
\hline & & & & & & Whole Body Vibration & $\begin{array}{l}\text { FEV1: } \\
\text { Mean } \\
32.71 \pm 13 \\
18\end{array}$ & $\begin{array}{l}23 \text { patients } \\
\text { aged } 66.4 \pm 9.93 \\
\text { years, } 30 \% \\
\text { female }\end{array}$ \\
\hline \multirow[t]{3}{*}{$\begin{array}{l}\text { Gunen, } \\
2007^{9}\end{array}$} & \multirow[t]{3}{*}{ Turkey } & \multirow[t]{3}{*}{ High risk } & \multirow[t]{3}{*}{$\begin{array}{l}\text { American } \\
\text { Thoracic Society } \\
\text { criteria }\end{array}$} & \multirow{3}{*}{$\begin{array}{l}\text { Worsening in at } \\
\text { least two of the } \\
\text { following } \\
\text { symptoms: cough, } \\
\text { purulent sputum } \\
\text { and dyspnea }\end{array}$} & \multirow[t]{3}{*}{$\begin{array}{l}\text { Moderate to } \\
\text { Severe }\end{array}$} & $\begin{array}{l}\text { Management without } \\
\text { Systemic } \\
\text { Corticosteroids }\end{array}$ & $\begin{array}{l}\text { FEV1: } \\
\text { Mean } \\
36.7 \pm 11.9 \\
\end{array}$ & $\begin{array}{l}53 \text { patients } \\
\text { aged } 63.5 \pm 10.1 \text {, } \\
10 \% \text { female }\end{array}$ \\
\hline & & & & & & $\begin{array}{l}\text { Prednisolone, } 40 \mathrm{mg} \\
\text { total dose }\end{array}$ & $\begin{array}{l}\text { FEV1: } \\
\text { Mean } \\
35.3 \pm 11.7\end{array}$ & $\begin{array}{l}53 \text { patients } \\
\text { aged } 64.9 \pm 7.1 \\
18 \% \text { female }\end{array}$ \\
\hline & & & & & & $\begin{array}{l}\text { Nebulized } \\
\text { Budesonide }\end{array}$ & $\begin{array}{l}\text { FEV1: } \\
\text { Mean } \\
39.6 \pm 12.9 \\
\end{array}$ & $\begin{array}{l}53 \text { patients } \\
\text { aged } 63.9 \pm 9.7 \\
16.7 \% \text { female } \\
\end{array}$ \\
\hline \multirow[t]{2}{*}{$\begin{array}{l}\mathrm{He} \\
2015^{35}\end{array}$} & \multirow[t]{2}{*}{$\begin{array}{l}\text { China } \\
12 / 2011- \\
11 / 2013\end{array}$} & \multirow[t]{2}{*}{ High risk } & \multirow[t]{2}{*}{ GOLD criteria } & \multirow{2}{*}{$\begin{array}{l}\text { The worsening of } \\
\text { respiratory } \\
\text { symptoms beyond } \\
\text { normal day-to-day } \\
\text { variation and } \\
\text { leading to a } \\
\text { change in } \\
\text { medication, MMRC } \\
>0, \text { and hospital } \\
\text { admission }\end{array}$} & \multirow[t]{2}{*}{$\begin{array}{l}\text { Moderate to } \\
\text { Severe }\end{array}$} & $\begin{array}{l}\text { Early Pulmonary } \\
\text { Rehabilitation }\end{array}$ & $\begin{array}{l}\text { FEV1: } \\
\text { Mean: } \\
38 \pm 16.7\end{array}$ & $\begin{array}{l}66 \text { patients } \\
\text { aged } 69.2 \pm 12.4 \\
9.1 \% \text { female }\end{array}$ \\
\hline & & & & & & $\begin{array}{l}\text { Management without } \\
\text { Early Rehabilitation }\end{array}$ & $\begin{array}{l}\text { FEV1: } \\
\text { Mean: } \\
39 \pm 27.8\end{array}$ & $\begin{array}{l}28 \text { patients } \\
\text { aged } 73.9 \pm 9.7 \\
17.6 \% \text { female }\end{array}$ \\
\hline \multirow[t]{2}{*}{$\begin{array}{l}\text { Kirsten, } \\
1998^{37}\end{array}$} & \multirow[t]{2}{*}{ Germany } & \multirow[t]{2}{*}{ High risk } & \multirow[t]{2}{*}{$\begin{array}{l}\text { International } \\
\text { guidelines }\end{array}$} & \multirow[t]{2}{*}{ Hospitalization } & \multirow[t]{2}{*}{$\begin{array}{l}\text { Moderate to } \\
\text { Severe }\end{array}$} & Aerobic Exercise & NR & $\begin{array}{l}15 \text { patients } \\
\text { aged } 65.5 \pm 11.8\end{array}$ \\
\hline & & & & & & $\begin{array}{l}\text { Management without } \\
\text { Aerobic Exercise }\end{array}$ & NR & $\begin{array}{l}\text { 14 patients } \\
\text { aged } 62.3 \pm 9.1\end{array}$ \\
\hline \multirow[t]{2}{*}{$\begin{array}{l}\text { Kodric, } \\
2009^{36}\end{array}$} & \multirow[t]{2}{*}{$\begin{array}{l}\text { Italy } \\
03 / 2002- \\
09 / 2002\end{array}$} & \multirow[t]{2}{*}{ High risk } & \multirow[t]{2}{*}{ GOLD criteria } & \multirow{2}{*}{$\begin{array}{l}\text { Clinical history, } \\
\text { physical } \\
\text { examination, chest } \\
\text { x-ray, severity } \\
\text { score according to } \\
\text { the Anthonisen } \\
\text { Criteria }\end{array}$} & \multirow[t]{2}{*}{$\begin{array}{l}\text { Moderate to } \\
\text { Severe }\end{array}$} & $\begin{array}{l}\text { Management without } \\
\text { Chest physiotherapy } \\
\text { technique }\end{array}$ & $\begin{array}{l}\text { FEV1: } \\
\text { Mean } \\
52.3 \pm 18.7\end{array}$ & $\begin{array}{l}29 \text { patients } \\
\text { aged } 69.1 \pm 8.3 \text {, } \\
33 \% \text { female }\end{array}$ \\
\hline & & & & & & $\begin{array}{l}\text { Chest physiotherapy } \\
\text { technique ELTGOL } \\
\text { (expiration with the } \\
\text { glottis open in the } \\
\text { lateral posture) }\end{array}$ & $\begin{array}{l}\text { FEV1: } \\
\text { Mean } \\
55.6 \pm 27.6\end{array}$ & $\begin{array}{l}30 \text { patients } \\
\text { aged } 71.3 \pm 8.4 \\
28 \% \text { female }\end{array}$ \\
\hline $\begin{array}{l}\text { Kurzaj, } \\
2013^{38}\end{array}$ & Poland & High risk & NR & $\begin{array}{l}\text { Worsening COPD } \\
\text { symptoms, hospital } \\
\text { admission }\end{array}$ & $\begin{array}{l}\text { Moderate to } \\
\text { Severe }\end{array}$ & $\begin{array}{l}\text { Specialized } \\
\text { Physiotherapy }\end{array}$ & NR & $\begin{array}{l}20 \text { patients } \\
\text { aged } 57 \pm 5.7 \\
45 \% \text { female }\end{array}$ \\
\hline
\end{tabular}




\begin{tabular}{|c|c|c|c|c|c|c|c|c|}
\hline $\begin{array}{l}\text { Author, } \\
\text { Year }\end{array}$ & $\begin{array}{l}\text { Country, } \\
\text { Study } \\
\text { Period }\end{array}$ & $\begin{array}{l}\text { Risk of } \\
\text { Bias }\end{array}$ & $\begin{array}{c}\text { COPD } \\
\text { Definition }\end{array}$ & $\begin{array}{c}\text { COPD } \\
\text { Exacerbation }\end{array}$ & $\begin{array}{l}\text { Severity of } \\
\text { ECOPD }\end{array}$ & $\begin{array}{l}\text { Intervention(s) and } \\
\text { comparison }\end{array}$ & $\begin{array}{c}\text { COPD } \\
\text { Severity } \\
(\% \\
\text { predicted } \\
\text { unless } \\
\text { specified) }\end{array}$ & $\begin{array}{c}\text { Patient } \\
\text { Characteristics }\end{array}$ \\
\hline & & & & & & $\begin{array}{l}\text { Management without } \\
\text { Specialized } \\
\text { Physiotherapy }\end{array}$ & NR & $\begin{array}{l}10 \text { patients } \\
\text { aged } 55 \pm 4.2 \text {, } \\
30 \% \text { female }\end{array}$ \\
\hline \multirow[t]{2}{*}{$\begin{array}{l}\text { Lellouche, } \\
2016^{39}\end{array}$} & \multirow[t]{2}{*}{$\begin{array}{l}\text { Canada } \\
08 / 2011- \\
02 / 2015\end{array}$} & \multirow[t]{2}{*}{$\begin{array}{l}\text { Intermed } \\
\text { iate risk }\end{array}$} & \multirow[t]{2}{*}{$\begin{array}{l}\text { Clinical } \\
\text { diagnosis, at } \\
\text { least } 10 \text { pack } \\
\text { year smoking } \\
\text { history }\end{array}$} & \multirow[t]{2}{*}{$\begin{array}{l}\text { Clinical diagnosis, } \\
\text { hospital admission }\end{array}$} & \multirow[t]{2}{*}{$\begin{array}{l}\text { Moderate to } \\
\text { Severe }\end{array}$} & $\begin{array}{l}\text { Free02 device oxygen } \\
\text { titration, } 0-20 \mathrm{~L} / \mathrm{min}\end{array}$ & $\begin{array}{l}\text { FEV1 in a } \\
\text { stable } \\
\text { state: } \\
\text { Mean } \\
40 \pm 11\end{array}$ & $\begin{array}{l}25 \text { patients } \\
\text { aged } 71 \pm 8, \\
46 \% \text { female }\end{array}$ \\
\hline & & & & & & $\begin{array}{l}\text { Manual Oxygen } \\
\text { Titration }\end{array}$ & $\begin{array}{l}\text { FEV1 in a } \\
\text { stable } \\
\text { state: } \\
\text { Mean } \\
51 \pm 20\end{array}$ & $\begin{array}{l}25 \text { patients } \\
\text { aged } 73 \pm 8, \\
46 \% \text { female }\end{array}$ \\
\hline \multirow[t]{2}{*}{$\begin{array}{l}\text { Liao, } \\
2015^{40}\end{array}$} & \multirow[t]{2}{*}{ Taiwan } & \multirow[t]{2}{*}{ High risk } & \multirow[t]{2}{*}{ NR } & \multirow{2}{*}{$\begin{array}{l}\text { An increased need } \\
\text { for medication and } \\
\text { feel the need to } \\
\text { seek additional } \\
\text { medical } \\
\text { assistance, } \\
\text { hospital admission }\end{array}$} & \multirow[t]{2}{*}{$\begin{array}{l}\text { Moderate to } \\
\text { Severe }\end{array}$} & $\begin{array}{l}\text { Early Pulmonary } \\
\text { Rehabilitation }\end{array}$ & NR & $\begin{array}{l}31 \text { patients } \\
\text { aged } 68(44- \\
89), 46.7 \% \\
\text { female }\end{array}$ \\
\hline & & & & & & $\begin{array}{l}\text { Management without } \\
\text { Early Rehabilitation }\end{array}$ & NR & $\begin{array}{l}31 \text { patients } \\
\text { aged } \pm, 32.3 \% \\
\text { female }\end{array}$ \\
\hline \multirow{3}{*}{$\begin{array}{l}\text { Mahmoud } \\
\text { Abd El } \\
\text { Hafiz, } \\
2013^{41}\end{array}$} & \multirow[t]{3}{*}{ Egypt } & \multirow[t]{3}{*}{ High risk } & \multirow{3}{*}{$\begin{array}{l}\text { FEV1/FVC } \\
<70 \% \text {, post } \\
\text { bronchodilator } \\
\text { reversibility } \\
\text { FEV1 <12\%, } \\
\text { clinical } \\
\text { diagnosis, } \\
\text { history of } 2 \text { or } \\
\text { more } \\
\text { exacerbations in } \\
2 \text { years prior }\end{array}$} & \multirow[t]{3}{*}{$\begin{array}{l}\text { Clinical diagnosis, } \\
\text { hospital admission }\end{array}$} & \multirow[t]{3}{*}{$\begin{array}{l}\text { Moderate to } \\
\text { Severe }\end{array}$} & $\begin{array}{l}\text { Management without } \\
\mathrm{N} \text {-acetylcysteine }\end{array}$ & NR & $\begin{array}{l}15 \text { patients } \\
\text { aged } 55.5 \pm 9.2 \\
0 \% \text { female }\end{array}$ \\
\hline & & & & & & $\begin{array}{l}\text { Low Dose N- } \\
\text { acetylcysteine, } 600 \mathrm{mg} \\
\text { total dose }\end{array}$ & NR & $\begin{array}{l}15 \text { patients } \\
\text { aged } 59.6 \pm 6.6 \\
0 \% \text { female }\end{array}$ \\
\hline & & & & & & $\begin{array}{l}\text { High Dose N- } \\
\text { acetylcysteine }\end{array}$ & NR & $\begin{array}{l}15 \text { patients } \\
\text { aged } 62 \pm 4.1 \\
0 \% \text { female }\end{array}$ \\
\hline \multirow[t]{2}{*}{$\begin{array}{l}\text { Maltais, } \\
2002^{12}\end{array}$} & \multirow[t]{2}{*}{$\begin{array}{l}\text { Belgium, } \\
\text { Canada, } \\
\text { and France }\end{array}$} & \multirow[t]{2}{*}{ High risk } & \multirow{2}{*}{$\begin{array}{l}\text { American } \\
\text { Thoracic Society } \\
\text { criteria, chronic } \\
\text { bronchitis or } \\
\text { emphysema }\end{array}$} & \multirow{2}{*}{$\begin{array}{l}\text { Increased } \\
\text { breathlessness in } \\
\text { the past } 14 \text { days, } \\
\text { causing hospital } \\
\text { admission }\end{array}$} & \multirow[t]{2}{*}{$\begin{array}{l}\text { Moderate to } \\
\text { Severe }\end{array}$} & Budesonide & NR & $\begin{array}{l}71 \text { patients } \\
\text { aged } 69.1 \pm 8.7 \\
20 \% \text { female }\end{array}$ \\
\hline & & & & & & Prednisolone & NR & $\begin{array}{l}62 \text { patients } \\
\text { aged } 70.4 \pm 7.7 \\
16 \% \text { female }\end{array}$ \\
\hline
\end{tabular}




\begin{tabular}{|c|c|c|c|c|c|c|c|c|}
\hline $\begin{array}{l}\text { Author, } \\
\text { Year }\end{array}$ & $\begin{array}{l}\text { Country, } \\
\text { Study } \\
\text { Period }\end{array}$ & $\begin{array}{l}\text { Risk of } \\
\text { Bias }\end{array}$ & $\begin{array}{c}\text { COPD } \\
\text { Definition }\end{array}$ & $\begin{array}{c}\text { COPD } \\
\text { Exacerbation }\end{array}$ & $\begin{array}{l}\text { Severity of } \\
\text { ECOPD }\end{array}$ & $\begin{array}{l}\text { Intervention(s) and } \\
\text { comparison }\end{array}$ & $\begin{array}{c}\text { COPD } \\
\text { Severity } \\
(\% \\
\text { predicted } \\
\text { unless } \\
\text { specified) }\end{array}$ & $\begin{array}{c}\text { Patient } \\
\text { Characteristics }\end{array}$ \\
\hline & & & $\begin{array}{l}14 \text { day history of } \\
\text { acute COPD } \\
\text { exacerbation } \\
\text { defined } \\
\text { as increased } \\
\text { breathlessness. } \\
\text { Patients were } \\
\text { included in the } \\
\text { study if they } \\
\text { were more than } \\
50 \text { year old, had } \\
\text { a smoking } \\
\text { history of at least } \\
20 \text { pack years, } \\
\text { and according to } \\
\text { the attending } \\
\text { physician had to } \\
\text { be treated in } \\
\text { hospital. }\end{array}$ & & & Placebo & NR & $\begin{array}{l}66 \text { patients } \\
\text { aged } 70.4 \pm 8.9 \\
20 \% \text { female }\end{array}$ \\
\hline \multirow[t]{2}{*}{$\begin{array}{l}\text { Moretti, } \\
2015^{42}\end{array}$} & \multirow[t]{2}{*}{$\begin{array}{l}\text { Italy } \\
\text { 10/2012- } \\
05 / 2013\end{array}$} & \multirow[t]{2}{*}{ High risk } & \multirow[t]{2}{*}{$\begin{array}{l}\text { Clinical } \\
\text { diagnosis }\end{array}$} & \multirow{2}{*}{$\begin{array}{l}\text { Clinical diagnosis, } \\
\text { fever, cough, and } \\
\text { purulent sputum in } \\
\text { the previous } 24 \\
\text { hours, hospital } \\
\text { admission }\end{array}$} & \multirow[t]{2}{*}{$\begin{array}{l}\text { Moderate to } \\
\text { Severe }\end{array}$} & $\begin{array}{l}\text { Erdosteine, } 900 \mathrm{mg} \\
\text { total dose }\end{array}$ & $\begin{array}{l}\text { FEV1: } \\
\text { Mean } \\
47.5 \pm 12.6 \\
\end{array}$ & $\begin{array}{l}20 \text { patients } \\
\text { aged } 71.4 \pm 5.4 \\
20 \% \text { female }\end{array}$ \\
\hline & & & & & & $\begin{array}{l}\text { Management without } \\
\text { Erdosteine }\end{array}$ & $\begin{array}{l}\text { FEV1: } \\
\text { Mean } \\
46.4 \pm 14.1\end{array}$ & $\begin{array}{l}20 \text { patients } \\
\text { aged } 69.7 \pm 6 \\
15 \% \text { female }\end{array}$ \\
\hline \multirow[t]{2}{*}{$\begin{array}{l}\text { Mukerji, } \\
2015^{43}\end{array}$} & \multirow[t]{2}{*}{$\begin{array}{l}\text { New } \\
\text { Zealand } \\
07 / 2013- \\
10 / 2013\end{array}$} & \multirow[t]{2}{*}{$\begin{array}{l}\text { Intermed } \\
\text { iate risk }\end{array}$} & \multirow[t]{2}{*}{$\begin{array}{l}\text { Documented } \\
\text { COPD diagnosis }\end{array}$} & \multirow[t]{2}{*}{$\begin{array}{l}\text { Clinical diagnosis, } \\
\text { ED evaluation }\end{array}$} & \multirow[t]{2}{*}{$\begin{array}{l}\text { Moderate to } \\
\text { Severe }\end{array}$} & Magnesium Sulfate & $\begin{array}{l}100 \% \text { had } \\
\text { FEV1< } \\
50 \%, \\
\text { Mean }(\mathrm{L}): \\
0.637 \pm 0.2 \\
93\end{array}$ & $\begin{array}{l}\text { 14 patients } \\
\text { aged } \\
76.1 \pm 12.47 \text {, } \\
15 \% \text { female }\end{array}$ \\
\hline & & & & & & Placebo & $\begin{array}{l}100 \% \text { had } \\
\text { FEV1< } \\
50 \% \text {, } \\
\text { Mean }(\mathrm{L}): \\
0.691 \pm 0.2 \\
88\end{array}$ & $\begin{array}{l}19 \text { patients } \\
\text { aged } 72.9 \pm 9.39 \\
41 \% \text { female }\end{array}$ \\
\hline
\end{tabular}




\begin{tabular}{|c|c|c|c|c|c|c|c|c|}
\hline $\begin{array}{l}\text { Author, } \\
\text { Year }\end{array}$ & $\begin{array}{l}\text { Country, } \\
\text { Study } \\
\text { Period }\end{array}$ & $\begin{array}{l}\text { Risk of } \\
\text { Bias }\end{array}$ & $\begin{array}{c}\text { COPD } \\
\text { Definition }\end{array}$ & $\begin{array}{c}\text { COPD } \\
\text { Exacerbation }\end{array}$ & $\begin{array}{l}\text { Severity of } \\
\text { ECOPD }\end{array}$ & $\begin{array}{l}\text { Intervention(s) and } \\
\text { comparison }\end{array}$ & $\begin{array}{c}\text { COPD } \\
\text { Severity } \\
(\% \\
\text { predicted } \\
\text { unless } \\
\text { specified) }\end{array}$ & $\begin{array}{c}\text { Patient } \\
\text { Characteristics }\end{array}$ \\
\hline \multirow[t]{2}{*}{$\begin{array}{l}\text { Ogasawar } \\
a, 2018^{44}\end{array}$} & \multirow[t]{2}{*}{ Japan } & \multirow[t]{2}{*}{ Low risk } & \multirow[t]{2}{*}{$\begin{array}{l}\text { According to the } \\
\text { GOLD criteria, } \\
\text { not otherwise } \\
\text { specified }\end{array}$} & \multirow{2}{*}{$\begin{array}{l}\text { The acute } \\
\text { worsening of } \\
\text { respiratory } \\
\text { symptoms, which } \\
\text { leads to the } \\
\text { requirement of } \\
\text { additional } \\
\text { therapy }\end{array}$} & \multirow[t]{2}{*}{$\begin{array}{l}\text { Moderate to } \\
\text { Severe }\end{array}$} & $\begin{array}{l}\text { Omega-3 fatty acid } \\
\text { enriched diet } \\
\text { (Eicosapentaenoic } \\
\text { acid) }\end{array}$ & $\begin{array}{l}\text { FEV1: } \\
\text { mean: } \\
64.2 \pm 24.7\end{array}$ & $\begin{array}{l}25 \text { patients } \\
\text { aged } 77.4 \pm 9.7 \\
\text { years, } 12 \% \\
\text { female }\end{array}$ \\
\hline & & & & & & Usual Diet & $\begin{array}{l}\text { FEV1: } \\
\text { mean: } \\
68.2 \pm 34.8\end{array}$ & $\begin{array}{l}25 \text { patients } \\
\text { aged } 79.1 \pm 7 \\
\text { years, } 5 \% \\
\text { female }\end{array}$ \\
\hline \multirow[t]{2}{*}{$\begin{array}{l}\text { Oncu, } \\
2017^{45}\end{array}$} & \multirow[t]{2}{*}{$\begin{array}{l}\text { Turkey } \\
8 / 2013- \\
5 / 2014\end{array}$} & \multirow[t]{2}{*}{ High risk } & \multirow[t]{2}{*}{$\begin{array}{l}\text { Clinical } \\
\text { diagnosis }\end{array}$} & \multirow[t]{2}{*}{$\begin{array}{l}\text { Worsening } \\
\text { pulmonary function } \\
\text { testing, hospital } \\
\text { admission }\end{array}$} & \multirow[t]{2}{*}{$\begin{array}{l}\text { Moderate to } \\
\text { Severe }\end{array}$} & $\begin{array}{l}\text { Transcutaneous } \\
\text { Electrical Nerve } \\
\text { Stimulation, } \\
45 \text { min/day }\end{array}$ & NR & $\begin{array}{l}41 \text { patients, } \\
20 \% \text { female }\end{array}$ \\
\hline & & & & & & $\begin{array}{l}\text { Management without } \\
\text { Transcutaneous } \\
\text { Electrical Nerve } \\
\text { Stimulation }\end{array}$ & NR & $\begin{array}{l}41 \text { patients, } \\
25.7 \% \text { female }\end{array}$ \\
\hline \multirow[t]{2}{*}{$\begin{array}{l}\text { Osadnik, } \\
2014^{46}\end{array}$} & \multirow[t]{2}{*}{$\begin{array}{l}\text { Australia } \\
08 / 2010- \\
01 / 2013\end{array}$} & \multirow[t]{2}{*}{ Low risk } & \multirow[t]{2}{*}{ NR } & \multirow[t]{2}{*}{$\begin{array}{l}\text { NR, hospital } \\
\text { admission }\end{array}$} & \multirow[t]{2}{*}{$\begin{array}{l}\text { Moderate to } \\
\text { Severe }\end{array}$} & $\begin{array}{l}\text { Management without } \\
\text { Positive Expiratory } \\
\text { Pressure }\end{array}$ & $\begin{array}{l}\text { FEV1: } \\
\text { Mean } \\
44.4 \pm 20.2\end{array}$ & $\begin{array}{l}46 \text { patients } \\
\text { aged } 69.5 \pm 9.8 \text {, } \\
37.8 \% \text { female }\end{array}$ \\
\hline & & & & & & $\begin{array}{l}\text { Positive Expiratory } \\
\text { Pressure }\end{array}$ & $\begin{array}{l}\text { FEV1: } \\
\text { Mean } \\
37.3 \pm 19.7\end{array}$ & $\begin{array}{l}46 \text { patients } \\
\text { aged } 67.8 \pm 11.6 \text {, } \\
33.3 \% \text { female }\end{array}$ \\
\hline \multirow[t]{2}{*}{$\begin{array}{l}\text { Pourrashi } \\
\text { d, } 2018^{47}\end{array}$} & \multirow[t]{2}{*}{$\begin{array}{l}\text { Iran } \\
12 / 2015- \\
10 / 2016\end{array}$} & \multirow[t]{2}{*}{ High risk } & \multirow{2}{*}{$\begin{array}{l}\text { Post- } \\
\text { bronchodilator } \\
\text { FEV1/FVC <0.7, } \\
\text { post- } \\
\text { bronchodilator } \\
\text { FEV1 <80\% } \\
\text { predicted }\end{array}$} & \multirow[t]{2}{*}{$\begin{array}{l}\text { Clinical diagnosis, } \\
\text { hospital admission }\end{array}$} & \multirow[t]{2}{*}{$\begin{array}{l}\text { Moderate to } \\
\text { Severe }\end{array}$} & Placebo & $\begin{array}{l}\text { Moderate: } \\
31.3 \% \\
\text { Severe: } \\
68.8 \%\end{array}$ & $\begin{array}{l}35 \text { patients } \\
\text { aged } \\
64.06 \pm 8.77 \\
15.6 \% \text { female }\end{array}$ \\
\hline & & & & & & Vitamin D, $300000 \mathrm{IU}$ & $\begin{array}{l}\text { Moderate: } \\
33.3 \% \\
\text { Severe: } \\
66.7 \%\end{array}$ & $\begin{array}{l}35 \text { patients } \\
\text { aged } \\
62.73 \pm 8.26 \text {, } \\
16.67 \% \text { female }\end{array}$ \\
\hline $\begin{array}{l}\text { Rice, } \\
1987^{48}\end{array}$ & $\begin{array}{l}\text { United } \\
\text { States of } \\
\text { America }\end{array}$ & Low risk & $\begin{array}{l}\text { FEV1/FVC ratio } \\
<60 \%, \text { FEV1 < } 2\end{array}$ & $\begin{array}{l}\text { Worsening of } \\
\text { dyspnea within } \\
\text { previous several }\end{array}$ & $\begin{array}{l}\text { Moderate to } \\
\text { Severe }\end{array}$ & Aminophylline & $\begin{array}{l}\text { FEV1 }(\mathrm{L}): \\
\text { Mean } \\
0.597 \pm 0.2\end{array}$ & $\begin{array}{l}16 \text { patients } \\
\text { aged } 66 \pm 27.9, \\
0 \% \text { female }\end{array}$ \\
\hline
\end{tabular}




\begin{tabular}{|c|c|c|c|c|c|c|c|c|}
\hline $\begin{array}{l}\text { Author, } \\
\text { Year }\end{array}$ & $\begin{array}{l}\text { Country, } \\
\text { Study } \\
\text { Period }\end{array}$ & $\begin{array}{l}\text { Risk of } \\
\text { Bias }\end{array}$ & $\begin{array}{c}\text { COPD } \\
\text { Definition }\end{array}$ & $\begin{array}{c}\text { COPD } \\
\text { Exacerbation }\end{array}$ & $\begin{array}{l}\text { Severity of } \\
\text { ECOPD }\end{array}$ & $\begin{array}{l}\text { Intervention(s) and } \\
\text { comparison }\end{array}$ & $\begin{array}{c}\text { COPD } \\
\text { Severity } \\
(\% \\
\text { predicted } \\
\text { unless } \\
\text { specified) }\end{array}$ & $\begin{array}{c}\text { Patient } \\
\text { Characteristics }\end{array}$ \\
\hline & & & $\begin{array}{l}\text { SD below } \\
\text { predicted value }\end{array}$ & $\begin{array}{l}\text { days, associated } \\
\text { with increased } \\
\text { cough and sputum, } \\
\text { sufficiently severe } \\
\text { to require hospital } \\
\text { admission }\end{array}$ & & Placebo & $\begin{array}{l}\text { FEV1 (L): } \\
\text { Mean } \\
0.561 \pm 0.3 \\
6\end{array}$ & $\begin{array}{l}14 \text { patients } \\
\text { aged } 64 \pm 22, \\
6.6 \% \text { female }\end{array}$ \\
\hline \multirow[t]{3}{*}{$\begin{array}{l}\text { Sanjari, } \\
2015^{49}\end{array}$} & \multirow[t]{3}{*}{ Iran } & \multirow[t]{3}{*}{$\begin{array}{l}\text { Intermed } \\
\text { iate risk }\end{array}$} & \multirow{3}{*}{$\begin{array}{l}\text { ERS criteria } \\
\text { (FEV1 <88\% for } \\
\text { men, <89\% for } \\
\text { women) }\end{array}$} & \multirow{3}{*}{$\begin{array}{l}\text { A dramatic } \\
\text { degradation of } \\
\text { COPD symptoms } \\
\text { (for example, the } \\
\text { quantity and the } \\
\text { color of phlegm or } \\
\text { shortness of } \\
\text { breath) that last for } \\
\text { a couple of days, } \\
\text { ED evaluation } \\
\end{array}$} & \multirow[t]{3}{*}{$\begin{array}{l}\text { Moderate to } \\
\text { Severe }\end{array}$} & Placebo & NR & $\begin{array}{l}45 \text { patients } \\
\text { aged } 58.4 \pm 9.5 \\
30.8 \% \text { female }\end{array}$ \\
\hline & & & & & & Vitamin D & NR & $\begin{array}{l}45 \text { patients } \\
\text { aged } 55.8 \pm 9.5 \\
28.6 \% \text { female }\end{array}$ \\
\hline & & & & & & Calcitriol & NR & $\begin{array}{l}45 \text { patients } \\
\text { aged } 55.6 \pm 8.9 \\
12.8 \% \text { female }\end{array}$ \\
\hline \multirow[t]{2}{*}{$\begin{array}{l}\text { Saudny- } \\
\text { Unterberg } \\
\text { er, } 1997^{50}\end{array}$} & \multirow[t]{2}{*}{$\begin{array}{l}\text { Canada } \\
11 / 1993 \\
05 / 1996\end{array}$} & \multirow[t]{2}{*}{ High risk } & \multirow{2}{*}{$\begin{array}{l}\text { Clinical } \\
\text { diagnosis of, and } \\
\text { a FEV1 that was } \\
\text { equal or less } \\
\text { than } 60 \% \\
\text { predicted }\end{array}$} & \multirow[t]{2}{*}{$\mathrm{NR}$} & \multirow[t]{2}{*}{$\begin{array}{l}\text { Moderate to } \\
\text { Severe }\end{array}$} & Nutritional Support & $\begin{array}{l}\text { FEV1: } \\
\text { Mean } \\
33.21 \pm 3.5 \\
7 \\
\end{array}$ & $\begin{array}{l}17 \text { patients } \\
\text { aged } \\
69.21 \pm 8.30 \\
43 \% \text { female }\end{array}$ \\
\hline & & & & & & Usual Diet & $\begin{array}{l}\text { FEV1: } \\
\text { Mean } \\
34.70 \pm 4.4 \\
2\end{array}$ & $\begin{array}{l}14 \text { patients } \\
\text { aged } \\
69.40 \pm 12.4 \\
30 \% \text { female }\end{array}$ \\
\hline \multirow[t]{2}{*}{$\begin{array}{l}\text { Seidenfel } \\
\text { d, } 1984^{51}\end{array}$} & \multirow[t]{2}{*}{$\begin{array}{l}\text { United } \\
\text { States of } \\
\text { America }\end{array}$} & \multirow[t]{2}{*}{$\begin{array}{l}\text { Intermed } \\
\text { iate risk }\end{array}$} & \multirow{2}{*}{$\begin{array}{l}\text { American } \\
\text { Thoracic Society } \\
\text { criteria for } \\
\text { chronic } \\
\text { bronchitis }\end{array}$} & \multirow[t]{2}{*}{ NR } & \multirow[t]{2}{*}{ Unclear } & Aminophylline & $\begin{array}{l}\text { FEV1 }(\mathrm{L}): \\
\text { Mean } \\
0.7 \pm 0.3 \\
\end{array}$ & $\begin{array}{l}22 \text { patients } \\
\text { aged } 62 \pm 8,0 \% \\
\text { female }\end{array}$ \\
\hline & & & & & & Placebo & $\begin{array}{l}\text { FEV1 (L): } \\
\text { Mean } \\
0.9 \pm 0.5\end{array}$ & $\begin{array}{l}30 \text { patients } \\
\text { aged } 63 \pm 7,0 \% \\
\text { female }\end{array}$ \\
\hline \multirow[t]{2}{*}{$\begin{array}{l}\text { Skorodin, } \\
1995^{52}\end{array}$} & \multirow[t]{2}{*}{$\begin{array}{l}\text { United } \\
\text { States of } \\
\text { America }\end{array}$} & \multirow[t]{2}{*}{ High risk } & \multirow[t]{2}{*}{$\begin{array}{l}\text { American } \\
\text { Thoracic Society } \\
\text { criteria }\end{array}$} & \multirow[t]{2}{*}{ NR } & \multirow[t]{2}{*}{$\begin{array}{l}\text { Moderate to } \\
\text { Severe }\end{array}$} & Magnesium Sulfate & NR & $\begin{array}{l}6 \text { patients } \\
\text { aged } 62.8 \pm 9, \\
3 \% \text { female }\end{array}$ \\
\hline & & & & & & Placebo & NR & $\begin{array}{l}36 \text { patients } \\
\text { aged } 66.5 \pm 7.3 \\
3 \% \text { female }\end{array}$ \\
\hline
\end{tabular}




\begin{tabular}{|c|c|c|c|c|c|c|c|c|}
\hline $\begin{array}{l}\text { Author, } \\
\text { Year }\end{array}$ & $\begin{array}{l}\text { Country, } \\
\text { Study } \\
\text { Period }\end{array}$ & $\begin{array}{l}\text { Risk of } \\
\text { Bias }\end{array}$ & $\begin{array}{c}\text { COPD } \\
\text { Definition }\end{array}$ & $\begin{array}{c}\text { COPD } \\
\text { Exacerbation }\end{array}$ & $\begin{array}{l}\text { Severity of } \\
\text { ECOPD }\end{array}$ & $\begin{array}{l}\text { Intervention(s) and } \\
\text { comparison }\end{array}$ & $\begin{array}{c}\text { COPD } \\
\text { Severity } \\
(\% \\
\text { predicted } \\
\text { unless } \\
\text { specified) }\end{array}$ & $\begin{array}{c}\text { Patient } \\
\text { Characteristics }\end{array}$ \\
\hline \multirow[t]{2}{*}{$\begin{array}{l}\text { Solooki, } \\
2014^{53}\end{array}$} & \multirow[t]{2}{*}{ Iran } & \multirow[t]{2}{*}{ High risk } & \multirow[t]{2}{*}{ NR } & \multirow[t]{2}{*}{ NR, ED evaluation } & \multirow[t]{2}{*}{$\begin{array}{l}\text { Moderate to } \\
\text { Severe }\end{array}$} & Magnesium Sulfate & $\begin{array}{l}\text { FEV1: } \\
\text { Mean } \\
26 \pm 12\end{array}$ & $\begin{array}{l}15 \text { patients } \\
\text { aged } 67 \pm 10 \text {, } \\
27 \% \text { female }\end{array}$ \\
\hline & & & & & & Placebo & $\begin{array}{l}\text { FEV1: } \\
\text { Mean } \\
35 \pm 18\end{array}$ & $\begin{array}{l}15 \text { patients } \\
\text { aged } 70 \pm 8, \\
33 \% \text { female }\end{array}$ \\
\hline \multirow[t]{2}{*}{$\begin{array}{l}\text { Soltaninej } \\
\text { ad, } 2016^{54}\end{array}$} & \multirow[t]{2}{*}{ Iran } & \multirow[t]{2}{*}{ High risk } & \multirow[t]{2}{*}{$\begin{array}{l}\text { Clinical } \\
\text { diagnosis }\end{array}$} & \multirow[t]{2}{*}{$\begin{array}{l}\text { Clinical diagnosis, } \\
\text { hospital admission }\end{array}$} & \multirow[t]{2}{*}{$\begin{array}{l}\text { Moderate to } \\
\text { Severe }\end{array}$} & Gentamicin & NR & $\begin{array}{l}43 \text { patients } \\
\text { aged } 65.5 \pm 9.7 \\
62.8 \% \text { female }\end{array}$ \\
\hline & & & & & & Placebo & NR & $\begin{array}{l}43 \text { patients } \\
\text { aged } 66 \pm 12, \\
55.8 \% \text { female }\end{array}$ \\
\hline \multirow[t]{3}{*}{$\begin{array}{l}\text { Tang, } \\
2012^{55}\end{array}$} & \multirow[t]{3}{*}{$\begin{array}{l}\text { Australia } \\
7 / 2009 \\
8 / 2010\end{array}$} & \multirow[t]{3}{*}{ Low risk } & \multirow{3}{*}{$\begin{array}{l}\text { Admitted to } \\
\text { hospital with } \\
\text { primary } \\
\text { diagnosis of } \\
\text { ECOPD }\end{array}$} & \multirow[t]{3}{*}{ NR } & \multirow[t]{3}{*}{$\begin{array}{l}\text { Moderate to } \\
\text { Severe }\end{array}$} & $\begin{array}{l}\text { Low-intensity Exercise } \\
\text { Group }\end{array}$ & $\begin{array}{l}\text { FEV1: } \\
\text { Mean } \\
45.1 \pm 18.6 \\
\end{array}$ & $\begin{array}{l}11 \text { patients } \\
\text { aged } 68 \pm 10.1 \\
55 \% \text { female }\end{array}$ \\
\hline & & & & & & $\begin{array}{l}\text { Moderate to high- } \\
\text { intensity exercise } \\
\text { group }\end{array}$ & $\begin{array}{l}\text { FEV1: } \\
\text { Mean } \\
46.1 \pm 18.3\end{array}$ & $\begin{array}{l}10 \text { patients } \\
\text { aged } 73.6 \pm 10 \\
80 \% \text { female } \\
\end{array}$ \\
\hline & & & & & & $\begin{array}{l}\text { Management without } \\
\text { Exercise Training }\end{array}$ & $\begin{array}{l}\text { FEV1: } \\
\text { Mean } \\
46.8 \pm 20.4\end{array}$ & $\begin{array}{l}11 \text { patients } \\
\text { aged } 78 \pm 8.8 \\
45 \% \text { female }\end{array}$ \\
\hline \multirow[t]{2}{*}{$\begin{array}{l}\text { Torres- } \\
\text { Sanchez, } \\
2017^{56}\end{array}$} & \multirow[t]{2}{*}{$\begin{array}{l}\text { Spain } \\
12 / 2013 \\
07 / 2014\end{array}$} & \multirow[t]{2}{*}{ Low risk } & \multirow[t]{2}{*}{$\begin{array}{l}\text { Clinical } \\
\text { diagnosis }\end{array}$} & \multirow[t]{2}{*}{$\begin{array}{l}\text { American Thoracic } \\
\text { Society criteria, } \\
\text { hospital admission }\end{array}$} & \multirow[t]{2}{*}{$\begin{array}{l}\text { Moderate to } \\
\text { Severe }\end{array}$} & Resistance Training & $\begin{array}{l}\text { FEV1: } \\
\text { Mean } \\
42.35 \pm 10 \\
62\end{array}$ & $\begin{array}{l}29 \text { patients } \\
\text { aged } \\
75.65 \pm 6.25 \\
24.13 \% \text { female }\end{array}$ \\
\hline & & & & & & $\begin{array}{l}\text { Management without } \\
\text { Resistance Training }\end{array}$ & $\begin{array}{l}\text { FEV1: } \\
\text { Mean } \\
39.12 \pm 12 \\
06\end{array}$ & $\begin{array}{l}29 \text { patients } \\
\text { aged } \\
72.12 \pm 8.19 \\
31 \% \text { female }\end{array}$ \\
\hline \multirow[t]{2}{*}{$\begin{array}{l}\text { Torres- } \\
\text { Sanchez, } \\
2017^{57}\end{array}$} & \multirow[t]{2}{*}{$\begin{array}{l}\text { Spain } \\
9 / 2015 \\
6 / 2016\end{array}$} & \multirow[t]{2}{*}{$\begin{array}{l}\text { Intermed } \\
\text { iate risk }\end{array}$} & \multirow[t]{2}{*}{$\begin{array}{l}\text { GOLD criteria } \\
\text { not otherwise } \\
\text { specified }\end{array}$} & \multirow[t]{2}{*}{$\begin{array}{l}\text { Clinical diagnosis, } \\
\text { hospital admission }\end{array}$} & \multirow[t]{2}{*}{$\begin{array}{l}\text { Moderate to } \\
\text { Severe }\end{array}$} & $\begin{array}{l}\text { Management without } \\
\text { Exercise Training }\end{array}$ & $\begin{array}{l}\text { FEV1: } \\
\text { Mean } \\
30.39 \pm 10 \\
76\end{array}$ & $\begin{array}{l}30 \text { patients } \\
\text { aged } \\
71.13 \pm 9.39 \\
20 \% \text { female }\end{array}$ \\
\hline & & & & & & $\begin{array}{l}\text { Controlled breathing + } \\
\text { Range of motion } \\
\text { exercises }\end{array}$ & $\begin{array}{l}\text { FEV1: } \\
\text { Mean } \\
31.26 \pm 5.3 \\
3\end{array}$ & $\begin{array}{l}30 \text { patients } \\
\text { aged } \\
75.07 \pm 8.71 \\
6.7 \% \text { female }\end{array}$ \\
\hline
\end{tabular}




\begin{tabular}{|c|c|c|c|c|c|c|c|c|}
\hline $\begin{array}{l}\text { Author, } \\
\text { Year }\end{array}$ & $\begin{array}{l}\text { Country, } \\
\text { Study } \\
\text { Period }\end{array}$ & $\begin{array}{l}\text { Risk of } \\
\text { Bias }\end{array}$ & $\begin{array}{c}\text { COPD } \\
\text { Definition }\end{array}$ & $\begin{array}{c}\text { COPD } \\
\text { Exacerbation }\end{array}$ & $\begin{array}{l}\text { Severity of } \\
\text { ECOPD }\end{array}$ & $\begin{array}{l}\text { Intervention(s) and } \\
\text { comparison }\end{array}$ & $\begin{array}{c}\text { COPD } \\
\text { Severity } \\
(\% \\
\text { predicted } \\
\text { unless } \\
\text { specified) }\end{array}$ & $\begin{array}{c}\text { Patient } \\
\text { Characteristics }\end{array}$ \\
\hline & & & & & & Resistance Training & $\begin{array}{l}\text { FEV1: } \\
\text { Mean } \\
30.13 \pm 8.2 \\
6 \\
\end{array}$ & $\begin{array}{l}30 \text { patients } \\
\text { aged } \\
70.12 \pm 10.6 \\
13.3 \% \text { female }\end{array}$ \\
\hline \multirow[t]{2}{*}{$\begin{array}{l}\text { Troosters, } \\
2010^{58}\end{array}$} & \multirow[t]{2}{*}{$\begin{array}{l}\text { Belgium } \\
01 / 2004 \\
03 / 2005\end{array}$} & \multirow[t]{2}{*}{ High risk } & \multirow[t]{2}{*}{$\begin{array}{l}\text { FEV1/FVC } \\
<70 \%\end{array}$} & \multirow[t]{2}{*}{$\begin{array}{l}\text { Clinical diagnosis, } \\
\text { hospital admission }\end{array}$} & \multirow[t]{2}{*}{ Severe } & $\begin{array}{l}\text { Management without } \\
\text { Resistance Training }\end{array}$ & $\begin{array}{l}\text { FEV1: } \\
\text { Mean } \\
50 \pm 18\end{array}$ & $\begin{array}{l}20 \text { patients } \\
\text { aged } 69 \pm 7, \\
26 \% \text { female }\end{array}$ \\
\hline & & & & & & Resistance Training & $\begin{array}{l}\text { FEV1: } \\
\text { Mean } \\
40 \pm 12\end{array}$ & $\begin{array}{l}20 \text { patients } \\
\text { aged } 67 \pm 8, \\
24 \% \text { female }\end{array}$ \\
\hline \multirow[t]{2}{*}{$\begin{array}{l}\text { Tumer, } \\
2009^{59}\end{array}$} & \multirow[t]{2}{*}{ Turkey } & \multirow[t]{2}{*}{ High risk } & \multirow[t]{2}{*}{$\begin{array}{l}\text { American } \\
\text { Thoracic Society } \\
\text { criteria }\end{array}$} & \multirow{2}{*}{$\begin{array}{l}\text { Increase, in at } \\
\text { least two of the } \\
\text { three following } \\
\text { symptoms: } \\
\text { dyspnea, cough } \\
\text { and sputum } \\
\text { production, } \\
\text { hospital evaluation }\end{array}$} & \multirow[t]{2}{*}{$\begin{array}{l}\text { Moderate to } \\
\text { Severe }\end{array}$} & Usual Diet & NR & $\begin{array}{l}15 \text { patients } \\
\text { aged } 63.6 \pm 4.3 \\
0 \% \text { female }\end{array}$ \\
\hline & & & & & & $\begin{array}{l}\text { High-fat Low- } \\
\text { carbohydrate Diet }\end{array}$ & NR & $\begin{array}{l}15 \text { patients } \\
\text { aged } 60.9 \pm 7 \\
0 \% \text { female }\end{array}$ \\
\hline \multirow[t]{2}{*}{$\begin{array}{l}\text { Vermeere } \\
\mathrm{n}, 2004^{60}\end{array}$} & \multirow[t]{2}{*}{ Netherlands } & \multirow[t]{2}{*}{ High risk } & \multirow[t]{2}{*}{ GOLD criteria } & \multirow{2}{*}{$\begin{array}{l}\text { Recent increase in } \\
\text { breathlessness, } \\
\text { cough and sputum } \\
\text { production of } \\
\text { sufficient severity } \\
\text { to warrant hospital } \\
\text { admission }\end{array}$} & \multirow[t]{2}{*}{$\begin{array}{l}\text { Moderate to } \\
\text { Severe }\end{array}$} & $\begin{array}{l}\text { Nutritional } \\
\text { Intervention }\end{array}$ & NR & $\begin{array}{l}23 \text { patients } \\
\text { aged } 66 \pm 8, \\
39 \% \text { female } \\
\end{array}$ \\
\hline & & & & & & $\begin{array}{l}\text { Placebo(non-caloric } \\
\text { fluid, vanilla flavored } \\
\text { water) }\end{array}$ & NR & $\begin{array}{l}24 \text { patients } \\
\text { aged } 65 \pm 10 \\
25 \% \text { female }\end{array}$ \\
\hline \multirow[t]{2}{*}{$\begin{array}{l}\text { Woodruff, } \\
2011^{61}\end{array}$} & \multirow[t]{2}{*}{$\begin{array}{l}\text { United } \\
\text { States of } \\
\text { America }\end{array}$} & \multirow[t]{2}{*}{ High risk } & \multirow{2}{*}{$\begin{array}{l}\text { Clinical } \\
\text { diagnosis of } \\
\text { AECOPD, } \geq 10 \\
\text { pack-years } \\
\text { smoking history, } \\
\text { FEV1<60\% } \\
\text { predicted at time } \\
\text { of inclusion or an } \\
\text { inability to } \\
\text { perform } \\
\text { spirometry due } \\
\text { to dyspnea. }\end{array}$} & \multirow{2}{*}{$\begin{array}{l}\text { Acute increase in } \\
\text { dyspnea, sputum } \\
\text { volume, and/or } \\
\text { sputum purulence } \\
\text { without other } \\
\text { attributable cause), } \\
\text { hospital admission }\end{array}$} & \multirow[t]{2}{*}{$\begin{array}{l}\text { Moderate to } \\
\text { Severe }\end{array}$} & Zileuton & $\begin{array}{l}\text { FEV1: } \\
\text { Mean } 32 \\
\pm 13\end{array}$ & $\begin{array}{l}60 \text { patients } \\
\text { aged } 62 \pm 8, \\
37 \% \text { female }\end{array}$ \\
\hline & & & & & & Placebo & $\begin{array}{l}\text { FEV1: } \\
\text { Mean } \\
32 \pm 11\end{array}$ & $\begin{array}{l}59 \text { patients } \\
\text { aged } 64 \pm 10 \\
30 \% \text { female }\end{array}$ \\
\hline
\end{tabular}




\begin{tabular}{|c|c|c|c|c|c|c|c|c|}
\hline $\begin{array}{l}\text { Author, } \\
\text { Year }\end{array}$ & $\begin{array}{l}\text { Country, } \\
\text { Study } \\
\text { Period }\end{array}$ & $\begin{array}{l}\text { Risk of } \\
\text { Bias }\end{array}$ & $\begin{array}{c}\text { COPD } \\
\text { Definition }\end{array}$ & $\begin{array}{c}\text { COPD } \\
\text { Exacerbation }\end{array}$ & $\begin{array}{l}\text { Severity of } \\
\text { ECOPD }\end{array}$ & $\begin{array}{l}\text { Intervention(s) and } \\
\text { comparison }\end{array}$ & $\begin{array}{c}\text { COPD } \\
\text { Severity } \\
(\% \\
\text { predicted } \\
\text { unless } \\
\text { specified) }\end{array}$ & $\begin{array}{c}\text { Patient } \\
\text { Characteristics }\end{array}$ \\
\hline \multirow[t]{2}{*}{$\begin{array}{l}\text { Xiong, } \\
2008^{62}\end{array}$} & \multirow[t]{2}{*}{ China } & \multirow[t]{2}{*}{ High risk } & \multirow[t]{2}{*}{ GOLD criteria } & \multirow[t]{2}{*}{ NR } & \multirow[t]{2}{*}{$\begin{array}{l}\text { Moderate to } \\
\text { Severe }\end{array}$} & Atomization Inhalation & NR & $\begin{array}{l}20 \text { patients } \\
\text { aged } 65.1 \pm 5.83 \text {, } \\
45 \% \text { female }\end{array}$ \\
\hline & & & & & & $\begin{array}{l}\text { Management without } \\
\text { Atomization Inhalation }\end{array}$ & NR & $\begin{array}{l}20 \text { patients } \\
\text { aged } 66.4 \pm 6.48 \text {, } \\
55 \% \text { female }\end{array}$ \\
\hline \multirow[t]{4}{*}{$\begin{array}{l}\text { Yohannes } \\
\text {, 200363 }\end{array}$} & \multirow[t]{4}{*}{$\begin{array}{l}\text { United } \\
\text { Kingdom }\end{array}$} & \multirow[t]{4}{*}{ High risk } & \multirow{4}{*}{$\begin{array}{l}\text { FEV1 <70\% } \\
\text { predicted } \\
\text { together with a } \\
<20 \% \\
\text { improvement in } \\
\text { FEV1 following } \\
\text { standard doses } \\
\text { of beta agonist } \\
\text { by inhalation or } \\
\text { nebulization. }\end{array}$} & \multirow[t]{4}{*}{ Clinical diagnosis } & \multirow[t]{4}{*}{$\begin{array}{l}\text { Moderate to } \\
\text { Severe }\end{array}$} & $\begin{array}{l}\text { Gutter Frame + } \\
\text { Supplemental Oxygen }\end{array}$ & $\begin{array}{l}\text { FEV1: } \\
\text { Mean } \\
38 \pm 11\end{array}$ & $\begin{array}{l}30 \text { patients } \\
\text { aged } 75 \pm 7 \\
54 \% \text { female }\end{array}$ \\
\hline & & & & & & $\begin{array}{l}\text { Gutter Frame + } \\
\text { Supplemental Air }\end{array}$ & $\begin{array}{l}\text { FEV1: } \\
\text { Mean } \\
38 \pm 15\end{array}$ & $\begin{array}{l}30 \text { patients } \\
\text { aged } 75 \pm 7, \\
42 \% \text { female }\end{array}$ \\
\hline & & & & & & Rollator + Oxygen & $\begin{array}{l}\text { FEV1: } \\
\text { Mean } \\
35 \pm 11\end{array}$ & $\begin{array}{l}30 \text { patients } \\
\text { aged } 74 \pm 8, \\
57 \% \text { female }\end{array}$ \\
\hline & & & & & & $\begin{array}{l}\text { Rollator + } \\
\text { Supplemental Air }\end{array}$ & $\begin{array}{l}\text { FEV1: } \\
\text { Mean } \\
39 \pm 10\end{array}$ & $\begin{array}{l}30 \text { patients } \\
\text { aged } 74 \pm 7 \text {, } \\
32 \% \text { female }\end{array}$ \\
\hline \multirow[t]{3}{*}{$\begin{array}{l}\text { Zuin, } \\
2005^{64}\end{array}$} & \multirow[t]{3}{*}{ Italy } & \multirow[t]{3}{*}{$\begin{array}{l}\text { Intermed } \\
\text { iate risk }\end{array}$} & \multirow{3}{*}{$\begin{array}{l}\text { Clinical } \\
\text { diagnosis of } \\
\text { COPD, FEV1 } \\
40-70 \% \\
\text { predicted }\end{array}$} & \multirow{3}{*}{$\begin{array}{l}1 \text { or more of } \\
\text { following: dyspnea, } \\
\text { wheezing, chest } \\
\text { tightness, mucus } \\
\text { production and } \\
\text { fever }\end{array}$} & \multirow[t]{3}{*}{ Mild } & $\begin{array}{l}\text { N-acetylcysteine } \\
\text { 1200mg }\end{array}$ & $\begin{array}{l}\text { FEV1 }(\mathrm{L}): \\
\text { Mean } \\
1.44 \pm 0.45\end{array}$ & $\begin{array}{l}39 \text { patients } \\
\text { aged } 67 \pm 12, \\
35.9 \% \text { female }\end{array}$ \\
\hline & & & & & & $\begin{array}{l}\text { N-acetylcysteine } \\
600 \mathrm{mg}\end{array}$ & $\begin{array}{l}\text { FEV1 (L): } \\
\text { Mean } \\
1.46 \pm 0.46\end{array}$ & $\begin{array}{l}41 \text { patients } \\
\text { aged } 68 \pm 13 \text {, } \\
41.5 \% \text { female }\end{array}$ \\
\hline & & & & & & Placebo & $\begin{array}{l}\text { FEV1 (L): } \\
\text { Mean } \\
1.44 \pm 0.51\end{array}$ & $\begin{array}{l}42 \text { patients } \\
\text { aged } 65 \pm 12, \\
50 \% \text { female }\end{array}$ \\
\hline
\end{tabular}

Note: \pm denotes standard deviation ECOPD = exacerbation of chronic obstructive pulmonary disease; ATS = American Thoracic Society; COPD = chronic obstructive pulmonary disease; ED = emergency department; EPA = eicosapentaenoic acid; ERS = European Respiratory Society; FEV1 = forced expiration volume in 1 second; FVC = forced vital capacity; GOLD = global initiative for chronic obstructive lung disease; $\mathrm{h}=$ hour; $\mathrm{IU}=$ international unit; $\mathrm{L}=$ liter; $\mathrm{mg}=\mathrm{milligram}$; $\mathrm{min}=$ minute; $\mathrm{ml}=$ milliliter; $\mathrm{MMRC}=$ modified medical research council; NR = not reported; ONS = oral nutrition supplementation; $\mathrm{pH}=$ potential of hydrogen; $\mathrm{SD}=$ standard deviation; VC = vital capacity 
Table D.3. KQ3: Characteristics of included studies

\begin{tabular}{|c|c|c|c|c|c|c|c|c|}
\hline $\begin{array}{l}\text { Author, } \\
\text { Year }\end{array}$ & $\begin{array}{c}\text { Country, } \\
\text { Study } \\
\text { Period }\end{array}$ & $\begin{array}{l}\text { Risk of } \\
\text { Bias }\end{array}$ & $\begin{array}{c}\text { COPD } \\
\text { Definition }\end{array}$ & $\begin{array}{c}\text { COPD } \\
\text { Exacerbation }\end{array}$ & $\begin{array}{l}\text { Severity of } \\
\text { ECOPD }\end{array}$ & $\begin{array}{l}\text { Intervention(s) and } \\
\text { comparison }\end{array}$ & $\begin{array}{c}\text { COPD } \\
\text { Severity } \\
(\% \\
\text { predicted } \\
\text { unless } \\
\text { specified) }\end{array}$ & $\begin{array}{c}\text { Patient } \\
\text { Characteristics }\end{array}$ \\
\hline \multirow[t]{3}{*}{$\begin{array}{l}\text { Koutsogia } \\
\text { nnis, } \\
2000^{65}\end{array}$} & \multirow[t]{3}{*}{ Australia } & \multirow[t]{3}{*}{$\begin{array}{l}\text { Intermed } \\
\text { iate risk }\end{array}$} & \multirow{3}{*}{$\begin{array}{l}\text { Clinical } \\
\text { diagnosis based } \\
\text { on spirometry or } \\
\text { physician } \\
\text { opinion }\end{array}$} & \multirow[t]{3}{*}{ NR } & \multirow[t]{3}{*}{ Unclear } & $\begin{array}{l}\text { Salbutamol, } 15 \mathrm{mg} \\
\text { Salbutamol, } 500 \mu \mathrm{g} \\
\text { Ipratropium Bromide }\end{array}$ & NR & 16 patients \\
\hline & & & & & & $\begin{array}{l}\text { Ipratropium Bromide, } \\
5 \mathrm{mg} \text { Salbutamol, } \\
1500 \mu \mathrm{g} \\
\text { Ipratropium Bromide }\end{array}$ & NR & 15 patients \\
\hline & & & & & & Combined & NR & 18 Patients \\
\hline \multirow[t]{2}{*}{$\begin{array}{l}\text { Moayyedi, } \\
1995^{66}\end{array}$} & \multirow[t]{2}{*}{$\begin{array}{l}\text { United } \\
\text { Kingdom }\end{array}$} & \multirow[t]{2}{*}{ High risk } & \multirow{2}{*}{$\begin{array}{l}\text { Clinical } \\
\text { diagnosis, at } \\
\text { least } 10 \text { pack } \\
\text { year smoking } \\
\text { history, } \\
\text { FEV1<65\% }\end{array}$} & \multirow[t]{2}{*}{ NR } & \multirow[t]{2}{*}{$\begin{array}{l}\text { Moderate to } \\
\text { Severe }\end{array}$} & $\begin{array}{l}\text { Salbutamol, } 20 \mathrm{mg} \\
\text { total dose }\end{array}$ & $\begin{array}{l}\text { FEV1 }(\mathrm{L}): \\
\text { Mean } \\
0.77 \pm 0.34\end{array}$ & $\begin{array}{l}37 \text { patients } \\
\text { aged } 70.4 \pm 9.1\end{array}$ \\
\hline & & & & & & $\begin{array}{l}\text { Salbutamol + } \\
\text { Ipratropium Bromide, } \\
20 \mathrm{mg}+2000 \mu \mathrm{g}\end{array}$ & $\begin{array}{l}\text { FEV1 }(L): \\
\text { Mean } \\
0.78 \pm 0.41 \\
\end{array}$ & $\begin{array}{l}33 \text { patients } \\
\text { aged } 67.8 \pm 6.7\end{array}$ \\
\hline \multirow[t]{2}{*}{$\begin{array}{l}\text { Perri, } \\
1985^{67}\end{array}$} & \multirow[t]{2}{*}{ Italy } & \multirow[t]{2}{*}{ High risk } & & \multirow[t]{2}{*}{ NR } & \multirow[t]{2}{*}{ Unclear } & $\begin{array}{l}\text { Salbutamol + } \\
\text { Beclomethasone } \\
\text { Dipropionate, } 450 \mu \mathrm{g}+ \\
300 \mu \mathrm{g}\end{array}$ & NR & 15 patients \\
\hline & & & & & & $\begin{array}{l}\text { Fenoterol, } 1200 \mu \mathrm{g} \\
\text { overall }\end{array}$ & NR & 15 patients \\
\hline
\end{tabular}

Note: \pm denotes standard deviation

FEV1 = forced expiration volume in 1 second; $\mathrm{IB}=$ ipratropium; $\mathrm{L}=$ liter; $\mathrm{mg}=$ milligram; $\mathrm{NR}=$ not reported; $\mu \mathrm{g}=$ microgram 
Table D.4. KQ4: Characteristics of included studies

\begin{tabular}{|c|c|c|c|c|c|c|c|c|}
\hline $\begin{array}{l}\text { Author, } \\
\text { Year }\end{array}$ & $\begin{array}{l}\text { Country, } \\
\text { Study } \\
\text { Period }\end{array}$ & $\begin{array}{l}\text { Risk of } \\
\text { Bias }\end{array}$ & $\begin{array}{c}\text { COPD } \\
\text { Definition }\end{array}$ & $\begin{array}{c}\text { COPD } \\
\text { Exacerbation }\end{array}$ & $\begin{array}{l}\text { Severity of } \\
\text { ECOPD }\end{array}$ & $\begin{array}{l}\text { Intervention(s) and } \\
\text { comparison }\end{array}$ & $\begin{array}{c}\text { COPD } \\
\text { Severity } \\
(\% \\
\text { predicted } \\
\text { unless } \\
\text { specified) }\end{array}$ & $\begin{array}{c}\text { Patient } \\
\text { Characteristics }\end{array}$ \\
\hline \multirow[t]{2}{*}{$\begin{array}{l}\text { Aggarwal, } \\
2011^{68}\end{array}$} & \multirow[t]{2}{*}{ India } & \multirow[t]{2}{*}{$\begin{array}{l}\text { Intermed } \\
\text { iate risk }\end{array}$} & \multirow[t]{2}{*}{ GOLD criteria } & \multirow{2}{*}{$\begin{array}{l}\text { An acute change } \\
\text { in a patient's } \\
\text { baseline } \\
\text { dyspnea, cough } \\
\text { and/or sputum } \\
\text { beyond day-to- } \\
\text { day variability, } \\
\text { sufficient to } \\
\text { warrant a change } \\
\text { in treatment, } \\
\text { hospital } \\
\text { evaluation }\end{array}$} & \multirow[t]{2}{*}{$\begin{array}{l}\text { Mild to } \\
\text { Moderate }\end{array}$} & $\begin{array}{l}\text { Hydrocortisone } \\
\text { Followed by } \\
\text { Prednisolone }\end{array}$ & $\begin{array}{l}\text { FEV1 (L): } \\
\text { Mean } \\
0.6 \pm 0.23\end{array}$ & $\begin{array}{l}50 \text { patients } \\
\text { aged } 65 \pm 9.96 \\
\text { years, } 34 \% \\
\text { female }\end{array}$ \\
\hline & & & & & & Methylprednisolone & $\begin{array}{l}\text { FEV1 (L): } \\
\text { Mean } \\
0.5 \pm 0.18\end{array}$ & $\begin{array}{l}47 \text { patients } \\
\text { aged } 64 \pm 8.19 \\
\text { years, } 25.5 \% \\
\text { female }\end{array}$ \\
\hline \multirow[t]{2}{*}{$\begin{array}{l}\text { Andre- } \\
\text { Alves, } \\
2007^{69}\end{array}$} & \multirow[t]{2}{*}{ Brazil } & \multirow[t]{2}{*}{ High risk } & \multirow[t]{2}{*}{ NR } & \multirow{2}{*}{$\begin{array}{l}\text { Increased cough, } \\
\text { increased } \\
\text { expectoration or } \\
\text { worsening of } \\
\text { dyspnea. }\end{array}$} & \multirow[t]{2}{*}{ Mild } & Azithromycin & $\begin{array}{l}\text { FEV1: } \\
\text { Mean } \\
66.1 \pm 0.3\end{array}$ & $\begin{array}{l}49 \text { patients } \\
\text { aged } 60.4 \\
\text { years, } 34.7 \% \\
\text { female }\end{array}$ \\
\hline & & & & & & Amoxicillin & $\begin{array}{l}\text { FEV1: } \\
\text { Mean } \\
66.9 \pm 0.4\end{array}$ & $\begin{array}{l}53 \text { patients } \\
\text { aged } 59.8 \\
\text { years, } 49.1 \% \\
\text { female }\end{array}$ \\
\hline \multirow[t]{2}{*}{$\begin{array}{l}\text { Aubier, } \\
2002^{70}\end{array}$} & \multirow{2}{*}{$\begin{array}{l}\text { Argentina, } \\
\text { Australia, } \\
\text { Belgium, } \\
\text { France, } \\
\text { Germany, } \\
\text { Republic of } \\
\text { Ireland, } \\
\text { South } \\
\text { Africa, and } \\
\text { United } \\
\text { Kingdom } \\
\text { 03/1998 - } \\
\text { 05/1999 }\end{array}$} & \multirow[t]{2}{*}{ High risk } & \multirow[t]{2}{*}{$\begin{array}{l}\text { FEV1/FVC } \\
<70 \% \text {, history of } \\
\text { chronic } \\
\text { bronchitis, } \\
\text { clinical } \\
\text { diagnosis, } \\
\text { bronchodilator } \\
\text { response }<12 \%\end{array}$} & \multirow{2}{*}{$\begin{array}{l}\text { Presumed } \\
\text { bacterial infection } \\
\text { (increased cough } \\
\text { and/or dyspnea, } \\
\text { increased } \\
\text { production of } \\
\text { sputum, and } \\
\text { increased } \\
\text { purulence of } \\
\text { sputum), } \\
\text { outpatient } \\
\text { evaluation }\end{array}$} & \multirow[t]{2}{*}{ Mild } & Telithromycin & $\begin{array}{l}\text { FEV/FVC } \\
<60 \%: \\
43.1 \% \\
\text { FEV/FVC } \\
60-75: \\
44.4 \% \\
\text { FEV/FVC } \\
>75: 6.3 \% \\
\end{array}$ & $\begin{array}{l}163 \text { patients } \\
\text { aged } 61.5 \\
\text { years, } 41.9 \% \\
\text { female }\end{array}$ \\
\hline & & & & & & $\begin{array}{l}\text { Amoxicillin + } \\
\text { Clavulanate }\end{array}$ & $\begin{array}{l}\text { FEV/FVC } \\
<60 \%: \\
43.8 \% \\
\text { FEV/FVC } \\
60-75: \\
40.6 \% \\
\text { FEV/FVC } \\
>75 \\
10.6 \%\end{array}$ & $\begin{array}{l}161 \text { patients } \\
\text { aged } 66 \text { years, } \\
35 \% \text { female }\end{array}$ \\
\hline
\end{tabular}




\begin{tabular}{|c|c|c|c|c|c|c|c|c|}
\hline $\begin{array}{l}\text { Author, } \\
\text { Year }\end{array}$ & $\begin{array}{l}\text { Country, } \\
\text { Study } \\
\text { Period }\end{array}$ & $\begin{array}{l}\text { Risk of } \\
\text { Bias }\end{array}$ & $\begin{array}{c}\text { COPD } \\
\text { Definition }\end{array}$ & $\begin{array}{c}\text { COPD } \\
\text { Exacerbation }\end{array}$ & $\begin{array}{l}\text { Severity of } \\
\text { ECOPD }\end{array}$ & $\begin{array}{l}\text { Intervention(s) and } \\
\text { comparison }\end{array}$ & $\begin{array}{c}\text { COPD } \\
\text { Severity } \\
(\% \\
\text { predicted } \\
\text { unless } \\
\text { specified) }\end{array}$ & $\begin{array}{c}\text { Patient } \\
\text { Characteristics }\end{array}$ \\
\hline \multirow[t]{2}{*}{$\begin{array}{l}\text { Blasi, } \\
2013^{71}\end{array}$} & \multirow{2}{*}{$\begin{array}{l}\text { Austria, } \\
\text { Germany, } \\
\text { Italy, } \\
\text { Poland, } \\
\text { Portugal, } \\
\text { and Ukraine } \\
\\
04 / 22 / 2008- \\
11 / 12 / 2010\end{array}$} & \multirow[t]{2}{*}{$\begin{array}{l}\text { Intermed } \\
\text { iate risk }\end{array}$} & \multirow{2}{*}{$\begin{array}{l}\text { FEV1/FVC < } 0.7 \text {, } \\
\text { post- } \\
\text { bronchodilator } \\
\text { FEV1 < 50\%, at } \\
\text { least } 10 \text { pack } \\
\text { year smoking } \\
\text { history, chronic } \\
\text { bronchitis, based } \\
\text { on American } \\
\text { Thoracic Society } \\
\text { criteria }\end{array}$} & \multirow[b]{2}{*}{$\begin{array}{l}\text { The presence of } \\
\text { the following } \\
\text { three symptoms } \\
\text { (or at least two } \\
\text { including sputum } \\
\text { purulence): } \\
\text { increased } \\
\text { dyspnea, } \\
\text { increased of } \\
\text { sputum volume } \\
\text { and sputum } \\
\text { purulence that } \\
\text { had to be } \\
\text { macroscopically } \\
\text { confirmed by the } \\
\text { investigator. } \\
\text { Patients, } \\
\text { requiring } \\
\text { concomitant } \\
\text { systemic } \\
\text { corticosteroids } \\
\text { administration } \\
\text { (20-40 mg/day for } \\
7 \text { days), or } \\
\text { Patients, in whom } \\
\text { an increase of the } \\
\text { daily dosage of } \\
\text { their chronically } \\
\text { corticosteroid- } \\
\text { treatment was } \\
\text { required. }\end{array}$} & \multirow[t]{2}{*}{ Unclear } & Prulifloxacin & $\begin{array}{l}\text { FEV1: } \\
\text { Mean } \\
40.2 \pm 9.0\end{array}$ & $\begin{array}{l}179 \text { patients } \\
\text { aged } \\
60.1 \pm 10.01 \\
\text { years, } 36.3 \%\end{array}$ \\
\hline & & & & & & Levofloxacin & $\begin{array}{l}\text { FEV1: } \\
\text { Mean } \\
40.7 \pm 7.7\end{array}$ & $\begin{array}{l}178 \text { patients } \\
\text { aged } 60.6 \pm 9.72 \\
\text { years, } 39.3 \% \\
\text { female }\end{array}$ \\
\hline $\begin{array}{l}\text { Dark, } \\
1993^{72}\end{array}$ & $\begin{array}{l}\text { United } \\
\text { States of } \\
\text { America }\end{array}$ & High risk & NR & NR & Unclear & Azithromycin & NR & $\begin{array}{l}136 \text { patients } \\
\text { aged } \\
56.3 \pm 12.75 \\
\text { years, } 39 \% \\
\text { female }\end{array}$ \\
\hline
\end{tabular}




\begin{tabular}{|c|c|c|c|c|c|c|c|c|}
\hline $\begin{array}{l}\text { Author, } \\
\text { Year }\end{array}$ & $\begin{array}{l}\text { Country, } \\
\text { Study } \\
\text { Period }\end{array}$ & $\begin{array}{c}\text { Risk of } \\
\text { Bias }\end{array}$ & $\begin{array}{c}\text { COPD } \\
\text { Definition }\end{array}$ & $\begin{array}{c}\text { COPD } \\
\text { Exacerbation }\end{array}$ & $\begin{array}{l}\text { Severity of } \\
\text { ECOPD }\end{array}$ & $\begin{array}{l}\text { Intervention(s) and } \\
\text { comparison }\end{array}$ & $\begin{array}{c}\text { COPD } \\
\text { Severity } \\
(\% \\
\text { predicted } \\
\text { unless } \\
\text { specified) }\end{array}$ & $\begin{array}{c}\text { Patient } \\
\text { Characteristics }\end{array}$ \\
\hline & & & & & & Cefaclor & NR & $\begin{array}{l}65 \text { patients } \\
\text { aged } 55.4 \pm 14 \\
\text { years, } 52 \% \\
\text { female }\end{array}$ \\
\hline \multirow[t]{2}{*}{$\begin{array}{l}\text { de Jong, } \\
2007^{9}\end{array}$} & \multirow[t]{2}{*}{$\begin{array}{l}\text { Netherlands } \\
\text { 05/2001 to } \\
05 / 2003\end{array}$} & \multirow[t]{2}{*}{ High risk } & \multirow{2}{*}{$\begin{array}{l}\text { History of at } \\
\text { least } 10 \text { pack- } \\
\text { years of } \\
\text { cigarette } \\
\text { smoking, and } \\
\text { evidence of } \\
\text { airflow limitation. } \\
\text { Airflow limitation } \\
\text { was defined as } \\
\text { an FEV1/FVC } \\
\text { ratio of < 70\% } \\
\text { and an FEV1 of } \\
<80 \% \text { predicted } \\
\text { (at least Global } \\
\text { Initiative for } \\
\text { Chronic } \\
\text { obstructive Lung } \\
\text { Disease [GOLD] } \\
\text { severity stage II) }\end{array}$} & \multirow{2}{*}{$\begin{array}{l}\text { An exacerbation } \\
\text { of COPD was } \\
\text { defined as a } \\
\text { history of } \\
\text { increased } \\
\text { breathlessness } \\
\text { and the presence } \\
\text { of at least two of } \\
\text { the following } \\
\text { symptoms for at } \\
\text { least } 24 \text { h: } \\
\text { increased cough } \\
\text { frequency or } \\
\text { severity; } \\
\text { increased sputum } \\
\text { volume or } \\
\text { purulence; and } \\
\text { increased } \\
\text { wheeze. }\end{array}$} & \multirow[t]{2}{*}{$\begin{array}{l}\text { Moderate to } \\
\text { Severe }\end{array}$} & $\begin{array}{l}\text { Intravenous } \\
\text { Prednisolone }\end{array}$ & $\begin{array}{l}\text { FEV1: } \\
\text { Mean } \\
36 \pm 14\end{array}$ & $\begin{array}{l}107 \text { patients } \\
\text { aged } 69.8 \pm 8.8 \\
\text { years, } 23.4 \% \\
\text { female }\end{array}$ \\
\hline & & & & & & Oral Prednisolone & $\begin{array}{l}\text { FEV1: } \\
\text { Mean } \\
39 \pm 17\end{array}$ & $\begin{array}{l}103 \text { patients } \\
\text { aged } 71.6 \pm 8.1 \\
\text { years, } 27.2 \% \\
\text { female }\end{array}$ \\
\hline $\begin{array}{l}\text { Ding, } \\
2016^{73}\end{array}$ & China & $\begin{array}{l}\text { Intermed } \\
\text { iate risk }\end{array}$ & $\begin{array}{l}\text { Symptoms of } \\
\text { chronic cough, } \\
\text { expectoration, } \\
\text { and/or dyspnea, }\end{array}$ & $\begin{array}{l}\text { Unusual } \\
\text { continuous } \\
\text { exacerbation that } \\
\text { required a }\end{array}$ & $\begin{array}{l}\text { Moderate to } \\
\text { Severe }\end{array}$ & Budesonide & NR & $\begin{array}{l}233 \text { patients } \\
\text { aged } 73.49 \pm \\
8.61 \text { years, } \\
19.1 \% \text { female }\end{array}$ \\
\hline
\end{tabular}




\begin{tabular}{|c|c|c|c|c|c|c|c|c|}
\hline $\begin{array}{l}\text { Author, } \\
\text { Year }\end{array}$ & $\begin{array}{c}\text { Country, } \\
\text { Study } \\
\text { Period }\end{array}$ & $\begin{array}{l}\text { Risk of } \\
\text { Bias }\end{array}$ & $\begin{array}{c}\text { COPD } \\
\text { Definition }\end{array}$ & $\begin{array}{c}\text { COPD } \\
\text { Exacerbation }\end{array}$ & $\begin{array}{l}\text { Severity of } \\
\text { ECOPD }\end{array}$ & $\begin{array}{l}\text { Intervention(s) and } \\
\text { comparison }\end{array}$ & $\begin{array}{c}\text { COPD } \\
\text { Severity } \\
(\% \\
\text { predicted } \\
\text { unless } \\
\text { specified) }\end{array}$ & $\begin{array}{c}\text { Patient } \\
\text { Characteristics }\end{array}$ \\
\hline & & & $\begin{array}{l}\text { incomplete } \\
\text { reversible airflow } \\
\text { (FEV1/FVC is } \\
<70 \% \text { after } \\
\text { application of a } \\
\text { bronchodilator) }\end{array}$ & $\begin{array}{l}\text { change in the } \\
\text { routine } \\
\text { medication, } \\
\text { cough, } \\
\text { expectoration, } \\
\text { dyspnea, and/or } \\
\text { wheeze } \\
\text { exacerbated, and } \\
\text { to have an } \\
\text { increased amount } \\
\text { of expectoration, } \\
\text { or to have a } \\
\text { short-term } \\
\text { change in the } \\
\text { sputum, which } \\
\text { can be } \\
\text { accompanied with } \\
\text { evident } \\
\text { aggravation of } \\
\text { inflammatory } \\
\text { (infection) } \\
\text { symptoms (such } \\
\text { as fever ) }\end{array}$ & & Methylprednisolone & NR & $\begin{array}{l}238 \text { patients } \\
\text { aged } 73.18 \pm 8.5 \\
\text { years, } 18.9 \% \\
\text { female }\end{array}$ \\
\hline \multirow[t]{2}{*}{$\begin{array}{l}\text { Emami } \\
\text { Ardestani, } \\
2017^{74}\end{array}$} & \multirow[t]{2}{*}{$\begin{array}{l}\text { Iran } \\
2013 \text { to } \\
2014\end{array}$} & \multirow[t]{2}{*}{ High risk } & \multirow[t]{2}{*}{$\begin{array}{l}\text { Post- } \\
\text { bronchodilator } \\
\text { FEV1/FVC <0.7, } \\
\text { GOLD not } \\
\text { otherwise } \\
\text { specified }\end{array}$} & \multirow{2}{*}{$\begin{array}{l}\text { Increased cough, } \\
\text { sputum, } \\
\text { wheezing, or } \\
\text { acute respiratory } \\
\text { distress for } 24 \\
\text { hours or more, } \\
\text { ED evaluation }\end{array}$} & \multirow[t]{2}{*}{$\begin{array}{l}\text { Moderate to } \\
\text { Severe }\end{array}$} & Dexamethasone & NR & $\begin{array}{l}34 \text { patients } \\
\text { aged } \\
74.67 \pm 1.79 \\
\text { years, } 17.7 \% \\
\text { female }\end{array}$ \\
\hline & & & & & & Methylprednisolone & NR & $\begin{array}{l}34 \text { patients } \\
\text { aged } \\
73.35 \pm 2.25 \\
\text { years, } 17.6 \% \\
\text { female }\end{array}$ \\
\hline $\begin{array}{l}\text { Giusti, } \\
2016^{75}\end{array}$ & Italy & High risk & $\begin{array}{l}\text { FEV1/FVC } \leq 0.7, \\
\text { FEV } 1 \leq 80 \% \text { and } \\
\geq 30 \%\end{array}$ & $\begin{array}{l}\text { Purulent sputum, } \\
\text { plus at least two } \\
\text { of the following } \\
\text { signs/symptoms } \\
\text { of at least 3-day }\end{array}$ & $\begin{array}{l}\text { Moderate to } \\
\text { Severe }\end{array}$ & Levofloxacin & NR & $\begin{array}{l}128 \text { patients } \\
\text { aged } 76.5 \pm 7.6 \\
\text { years, } 28.9 \% \\
\text { female }\end{array}$ \\
\hline
\end{tabular}




\begin{tabular}{|c|c|c|c|c|c|c|c|c|}
\hline $\begin{array}{l}\text { Author, } \\
\text { Year }\end{array}$ & $\begin{array}{l}\text { Country, } \\
\text { Study } \\
\text { Period }\end{array}$ & $\begin{array}{l}\text { Risk of } \\
\text { Bias }\end{array}$ & $\begin{array}{c}\text { COPD } \\
\text { Definition }\end{array}$ & $\begin{array}{c}\text { COPD } \\
\text { Exacerbation }\end{array}$ & $\begin{array}{l}\text { Severity of } \\
\text { ECOPD }\end{array}$ & $\begin{array}{l}\text { Intervention(s) and } \\
\text { comparison }\end{array}$ & $\begin{array}{c}\text { COPD } \\
\text { Severity } \\
(\% \\
\text { predicted } \\
\text { unless } \\
\text { specified) }\end{array}$ & $\begin{array}{c}\text { Patient } \\
\text { Characteristics }\end{array}$ \\
\hline & & & & $\begin{array}{l}\text { duration: } \\
\text { increased cough, } \\
\text { dyspnea, sputum } \\
\text { volume, hospital } \\
\text { admission }\end{array}$ & & Prulifloxacin & NR & $\begin{array}{l}130 \text { patients } \\
\text { aged } 75.1 \pm 7.5 \\
\text { years, } 30 \% \\
\text { female }\end{array}$ \\
\hline \multirow[t]{3}{*}{$\begin{array}{l}\text { Gunen, } \\
2007^{9}\end{array}$} & \multirow[t]{3}{*}{ Turkey } & \multirow[t]{3}{*}{ High risk } & \multirow[t]{3}{*}{$\begin{array}{l}\text { American } \\
\text { Thoracic Society } \\
\text { criteria }\end{array}$} & \multirow{3}{*}{$\begin{array}{l}\text { Worsening in at } \\
\text { least two of the } \\
\text { following } \\
\text { symptoms: } \\
\text { cough, purulent } \\
\text { sputum and } \\
\text { dyspnea }\end{array}$} & \multirow[t]{3}{*}{$\begin{array}{l}\text { Moderate to } \\
\text { Severe }\end{array}$} & $\begin{array}{l}\text { Management without } \\
\text { Systemic } \\
\text { Corticosteroids }\end{array}$ & $\begin{array}{l}\text { FEV1: } \\
\text { Mean } \\
36.7 \pm 11.9 \\
\end{array}$ & $\begin{array}{l}53 \text { patients } \\
\text { aged } 63.5 \pm 10.1 \text {, } \\
10 \% \text { female }\end{array}$ \\
\hline & & & & & & $\begin{array}{l}\text { Prednisolone, 40mg } \\
\text { total dose }\end{array}$ & $\begin{array}{l}\text { FEV1: } \\
\text { Mean } \\
35.3 \pm 11.7 \\
\end{array}$ & $\begin{array}{l}53 \text { patients } \\
\text { aged } 64.9 \pm 7.1 \\
18 \% \text { female }\end{array}$ \\
\hline & & & & & & $\begin{array}{l}\text { Nebulized } \\
\text { Budesonide }\end{array}$ & $\begin{array}{l}\text { FEV1: } \\
\text { Mean } \\
39.6 \pm 12.9\end{array}$ & $\begin{array}{l}53 \text { patients } \\
\text { aged } 63.9 \pm 9.7 \\
16.7 \% \text { female }\end{array}$ \\
\hline $\begin{array}{l}\text { Hamache } \\
\text { r, } 1995^{76}\end{array}$ & $\begin{array}{l}\text { Germany } \\
10 / 1992- \\
12 / 1993\end{array}$ & High risk & $\begin{array}{l}\text { American } \\
\text { Thoracic Society } \\
\text { criteria }\end{array}$ & $\begin{array}{l}\text { Rapid onset of } \\
\text { signs and } \\
\text { symptoms of }\end{array}$ & $\begin{array}{l}\text { Moderate to } \\
\text { Severe }\end{array}$ & $\begin{array}{l}\text { Meropenem, } 3 g \\
\text { overall }\end{array}$ & NR & $\begin{array}{l}86 \text { patients } \\
\text { aged } 64.0 \pm 11.5, \\
31 \% \text { female }\end{array}$ \\
\hline
\end{tabular}




\begin{tabular}{|c|c|c|c|c|c|c|c|c|}
\hline $\begin{array}{l}\text { Author, } \\
\text { Year }\end{array}$ & $\begin{array}{l}\text { Country, } \\
\text { Study } \\
\text { Period }\end{array}$ & $\begin{array}{l}\text { Risk of } \\
\text { Bias }\end{array}$ & $\begin{array}{c}\text { COPD } \\
\text { Definition }\end{array}$ & $\begin{array}{c}\text { COPD } \\
\text { Exacerbation }\end{array}$ & $\begin{array}{l}\text { Severity of } \\
\text { ECOPD }\end{array}$ & $\begin{array}{l}\text { Intervention(s) and } \\
\text { comparison }\end{array}$ & $\begin{array}{c}\text { COPD } \\
\text { Severity } \\
(\% \\
\text { predicted } \\
\text { unless } \\
\text { specified) }\end{array}$ & $\begin{array}{c}\text { Patient } \\
\text { Characteristics }\end{array}$ \\
\hline & & & & $\begin{array}{l}\text { infective } \\
\text { exacerbation with } \\
\text { aggravation or } \\
\text { development of } \\
\text { dyspnea or cough } \\
\text { with increased } \\
\text { amount and } \\
\text { purulent quality of } \\
\text { sputum. The } \\
\text { confirmation of } \\
\text { pathogenic } \\
\text { organism in } \\
\text { sputum was not } \\
\text { required } \\
\text { directly at study } \\
\text { entry, but at least } \\
\text { one adequate } \\
\text { bacteriological } \\
\text { sample had to be } \\
\text { taken before the } \\
\text { patient was } \\
\text { treated and } \\
\text { Reduced peak } \\
\text { flow (<350 } \\
\text { L/min) }\end{array}$ & & $\begin{array}{l}\text { Imipenem + } \\
\text { Cilastatin, } \\
3 \mathrm{~g} \text { imipenem overall, } \\
3 \mathrm{~g} \text { of cilastatin overall }\end{array}$ & NR & $\begin{array}{l}87 \text { patients } \\
\text { aged } 64.4 \pm 12.7 \text {, } \\
35 \% \text { female }\end{array}$ \\
\hline \multirow[t]{2}{*}{$\begin{array}{l}\text { Hasani, } \\
1998^{77}\end{array}$} & \multirow[t]{2}{*}{$\begin{array}{l}\text { United } \\
\text { Kingdom }\end{array}$} & \multirow[t]{2}{*}{ High risk } & \multirow[t]{2}{*}{ NR } & \multirow[t]{2}{*}{ NR } & \multirow[t]{2}{*}{$\begin{array}{l}\text { Mild to } \\
\text { Moderate }\end{array}$} & $\begin{array}{l}\text { Amoxicillin, 1500mg } \\
\text { total dose }\end{array}$ & NR & $\begin{array}{l}6 \text { patients aged } \\
63 \pm 7.3,33 \% \\
\text { female }\end{array}$ \\
\hline & & & & & & $\begin{array}{l}\text { Ciprofloxacin, } \\
\text { 1000mg total dose }\end{array}$ & NR & $\begin{array}{l}6 \text { patients aged } \\
63 \pm 7.3,17 \% \\
\text { female }\end{array}$ \\
\hline $\begin{array}{l}\text { Leophont } \\
\text { e, } 1998^{78}\end{array}$ & $\begin{array}{l}\text { France, } \\
\text { United }\end{array}$ & High risk & $\begin{array}{l}\text { Chronic } \\
\text { bronchitis, }\end{array}$ & $\begin{array}{l}\text { Dyspnea or } \\
\text { increased sputum }\end{array}$ & Mild & Trovafloxacin $200 \mathrm{mg}$ & NR & $\begin{array}{l}144 \text { patients } \\
\text { aged } 65.1 \pm 7.3\end{array}$ \\
\hline
\end{tabular}




\begin{tabular}{|c|c|c|c|c|c|c|c|c|}
\hline $\begin{array}{l}\text { Author, } \\
\text { Year }\end{array}$ & $\begin{array}{l}\text { Country, } \\
\text { Study } \\
\text { Period }\end{array}$ & $\begin{array}{l}\text { Risk of } \\
\text { Bias }\end{array}$ & $\begin{array}{c}\text { COPD } \\
\text { Definition }\end{array}$ & $\begin{array}{c}\text { COPD } \\
\text { Exacerbation }\end{array}$ & $\begin{array}{l}\text { Severity of } \\
\text { ECOPD }\end{array}$ & $\begin{array}{l}\text { Intervention(s) and } \\
\text { comparison }\end{array}$ & $\begin{array}{c}\text { COPD } \\
\text { Severity } \\
(\% \\
\text { predicted } \\
\text { unless } \\
\text { specified) }\end{array}$ & $\begin{array}{c}\text { Patient } \\
\text { Characteristics }\end{array}$ \\
\hline & $\begin{array}{l}\text { States of } \\
\text { America, } \\
\text { Italy, Spain, } \\
\text { South } \\
\text { Africa, } \\
\text { United } \\
\text { Kingdom, } \\
\text { Australia } \\
\text { 07/1995- } \\
\text { 08/1996 }\end{array}$ & & $\begin{array}{l}\text { FEV1/FVC } \\
<70 \% \text { predicted }\end{array}$ & $\begin{array}{l}\text { volume/purulence } \\
\text { [gram stain } \\
\text { sputum with >25 } \\
\text { PMN]) }\end{array}$ & & Trovafloxacin $100 \mathrm{mg}$ & NR & $\begin{array}{l}144 \text { patients } \\
\text { aged } 65.3 \pm 9.3\end{array}$ \\
\hline \multirow[t]{2}{*}{$\begin{array}{l}\text { Leuppi, } \\
2013^{79}\end{array}$} & \multirow[t]{2}{*}{$\begin{array}{l}\text { Switzerland } \\
\text { 03/2006- } \\
\text { 02/2011 }\end{array}$} & \multirow[t]{2}{*}{ Low risk } & \multirow{2}{*}{$\begin{array}{l}\text { At least } 20 \text { pack } \\
\text { year smoking } \\
\text { history, } \\
\text { FEV1/FVC } \\
<70 \%\end{array}$} & \multirow{2}{*}{$\begin{array}{l}\text { At least } 2 \text { of the } \\
\text { following: change } \\
\text { in baseline } \\
\text { dyspnea, cough, } \\
\text { or sputum } \\
\text { quantity or } \\
\text { purulence }\end{array}$} & \multirow[t]{2}{*}{$\begin{array}{l}\text { Moderate to } \\
\text { Severe }\end{array}$} & $\begin{array}{l}\text { Methylprednisolone } \\
\text { (day 1), Prednisone } \\
\text { (days 2-5), Placebo } \\
\text { (days 6-14) }\end{array}$ & $\begin{array}{l}\text { FEV1: } \\
\text { Mean } \\
31.3 \pm 13.2\end{array}$ & $\begin{array}{l}157 \text { patients } \\
\text { aged } 69.8 \pm 10.6 \text {, } \\
46.5 \% \text { female }\end{array}$ \\
\hline & & & & & & $\begin{array}{l}\text { Methylprednisolone } \\
\text { (day 1), Prednisone } \\
\text { (days 2-14) }\end{array}$ & $\begin{array}{l}\text { FEV1: } \\
\text { Mean } \\
31.7 \pm 15.4\end{array}$ & $\begin{array}{l}156 \text { patients } \\
\text { aged } 69.8 \pm 11.3 \text {, } \\
32.7 \% \text { female }\end{array}$ \\
\hline \multirow[t]{2}{*}{$\begin{array}{l}\text { Llor, } \\
2009^{80}\end{array}$} & \multirow[t]{2}{*}{$\begin{array}{l}\text { Spain } \\
\text { 10/2000- } \\
03 / 2005\end{array}$} & \multirow[t]{2}{*}{ High risk } & \multirow[t]{2}{*}{$\begin{array}{l}\text { FEV1/FVC < } 0.7 \\
\text { FEV1 }<80 \%\end{array}$} & \multirow[t]{2}{*}{$\begin{array}{l}\text { Anthonisen } \\
\text { criteria (increased } \\
\text { dyspnea, } \\
\text { increased sputum } \\
\text { volume and } \\
\text { purulent sputum), } \\
\text { outpatient } \\
\text { evaluation }\end{array}$} & \multirow[t]{2}{*}{ Mild } & $\begin{array}{l}\text { Amoxycillin, 1500mg } \\
\text { total dose }\end{array}$ & $\begin{array}{l}\text { Moderate: } \\
72.5 \% \\
\text { Severe: } \\
27.5 \% \\
\text { FEV1: } \\
\text { Mean } \\
62.9 \pm 11.0\end{array}$ & $\begin{array}{l}68 \text { patients } \\
\text { aged } 71.9 \pm 8.6 \\
22.1 \% \text { female }\end{array}$ \\
\hline & & & & & & $\begin{array}{l}\text { Amoxycillin + } \\
\text { clavulanate, 1500mg } \\
+375 \mathrm{mg}\end{array}$ & $\begin{array}{l}\text { Moderate: } \\
76.3 \% \\
\text { Severe: } \\
23.7 \% \\
\text { FEV1: } \\
\text { Mean } \\
60.4 \pm 11.9\end{array}$ & $\begin{array}{l}69 \text { patients } \\
\text { aged } 70.8 \pm 8.5 \\
18.8 \% \text { female }\end{array}$ \\
\hline
\end{tabular}




\begin{tabular}{|c|c|c|c|c|c|c|c|c|}
\hline $\begin{array}{l}\text { Author, } \\
\text { Year }\end{array}$ & $\begin{array}{l}\text { Country, } \\
\text { Study } \\
\text { Period }\end{array}$ & $\begin{array}{l}\text { Risk of } \\
\text { Bias }\end{array}$ & $\begin{array}{c}\text { COPD } \\
\text { Definition }\end{array}$ & $\begin{array}{c}\text { COPD } \\
\text { Exacerbation }\end{array}$ & $\begin{array}{c}\text { Severity of } \\
\text { ECOPD }\end{array}$ & $\begin{array}{l}\text { Intervention(s) and } \\
\text { comparison }\end{array}$ & $\begin{array}{c}\text { COPD } \\
\text { Severity } \\
(\% \\
\text { predicted } \\
\text { unless } \\
\text { specified) }\end{array}$ & $\begin{array}{c}\text { Patient } \\
\text { Characteristics }\end{array}$ \\
\hline $\begin{array}{l}\text { Maltais, } \\
2002^{12}\end{array}$ & \begin{tabular}{|l} 
Belgium, \\
Canada, \\
and France
\end{tabular} & High risk & $\begin{array}{l}\text { American } \\
\text { Thoracic Society } \\
\text { criteria, chronic } \\
\text { bronchitis or } \\
\text { emphysema } \\
14 \text { day history of } \\
\text { acute COPD } \\
\text { exacerbation } \\
\text { defined } \\
\text { as increased } \\
\text { breathlessness. } \\
\text { Patients were } \\
\text { included in the } \\
\text { study if they } \\
\text { were more than } \\
50 \text { years old, } \\
\text { had a smoking } \\
\text { history of at least } \\
20 \text { pack years, } \\
\text { and according to } \\
\text { the attending } \\
\text { physician had to }\end{array}$ & $\begin{array}{l}\text { Increased } \\
\text { breathlessness in } \\
\text { the past } 14 \text { days, } \\
\text { causing hospital } \\
\text { admission }\end{array}$ & $\begin{array}{l}\text { Moderate to } \\
\text { Severe }\end{array}$ & Budesonide & NR & $\begin{array}{l}71 \text { patients } \\
\text { aged } 69.1 \pm 8.7 \\
20 \% \text { female }\end{array}$ \\
\hline
\end{tabular}




\begin{tabular}{|c|c|c|c|c|c|c|c|c|}
\hline \multirow[t]{3}{*}{$\begin{array}{l}\text { Author, } \\
\text { Year }\end{array}$} & \multirow[t]{3}{*}{$\begin{array}{l}\text { Country, } \\
\text { Study } \\
\text { Period }\end{array}$} & \multirow[t]{3}{*}{$\begin{array}{l}\text { Risk of } \\
\text { Bias }\end{array}$} & $\begin{array}{c}\text { COPD } \\
\text { Definition }\end{array}$ & \multirow[t]{2}{*}{$\begin{array}{c}\text { COPD } \\
\text { Exacerbation }\end{array}$} & $\begin{array}{l}\text { Severity of } \\
\text { ECOPD }\end{array}$ & $\begin{array}{l}\text { Intervention(s) and } \\
\text { comparison }\end{array}$ & $\begin{array}{c}\text { COPD } \\
\text { Severity } \\
(\% \\
\text { predicted } \\
\text { unless } \\
\text { specified) }\end{array}$ & $\begin{array}{c}\text { Patient } \\
\text { Characteristics }\end{array}$ \\
\hline & & & $\begin{array}{l}\text { be treated in } \\
\text { hospital. }\end{array}$ & & & Prednisolone & NR & $\begin{array}{l}62 \text { patients } \\
\text { aged } 70.4 \pm 7.7 \\
16 \% \text { female }\end{array}$ \\
\hline & & & & & & Placebo & NR & $\begin{array}{l}66 \text { patients } \\
\text { aged } 70.4 \pm 8.9, \\
20 \% \text { female } \\
\end{array}$ \\
\hline \multirow[t]{2}{*}{$\begin{array}{l}\text { Mirici, } \\
2003^{81}\end{array}$} & \multirow[t]{2}{*}{ Turkey } & \multirow[t]{2}{*}{ Low risk } & \multirow[t]{2}{*}{$\begin{array}{l}\text { American } \\
\text { Thoracic Society } \\
\text { criteria }\end{array}$} & \multirow[t]{2}{*}{$\begin{array}{l}\text { Increased } \\
\text { symptoms } \\
\text { requiring } \\
\text { hospitalization }\end{array}$} & \multirow[t]{2}{*}{$\begin{array}{l}\text { Moderate to } \\
\text { Severe }\end{array}$} & $\begin{array}{l}\text { Parenteral } \\
\text { Corticosteroid } \\
\text { (prednisolone), 40mg } \\
\text { total dose }\end{array}$ & NR & $\begin{array}{l}22 \text { patients } \\
\text { aged } 64.8 \pm \text {, } \\
24 \% \text { female }\end{array}$ \\
\hline & & & & & & $\begin{array}{l}\text { Nebulized } \\
\text { Corticosteroid } \\
\text { (budesonide), 8mg } \\
\text { total dose }\end{array}$ & NR & $\begin{array}{l}22 \text { patients } \\
\text { aged } 63.06 \pm \text {, } \\
32 \% \text { female }\end{array}$ \\
\hline $\begin{array}{l}\text { Niewoehn } \\
\text { er, } 1999^{13}\end{array}$ & $\begin{array}{l}\text { United } \\
\text { States of } \\
\text { America }\end{array}$ & High risk & $\begin{array}{l}\text { Clinical } \\
\text { diagnosis of } \\
\text { COPD plus a } \\
\text { history of } 30\end{array}$ & Clinical diagnosis & $\begin{array}{l}\text { Moderate to } \\
\text { Severe }\end{array}$ & $\begin{array}{l}\text { Glucocorticoid for } 8 \\
\text { weeks }\end{array}$ & $\begin{array}{l}\text { FEV1 (L): } \\
\text { Mean } \\
0.785 \pm 0.2 \\
88\end{array}$ & $\begin{array}{l}80 \text { patients } \\
\text { aged } 68.1 \pm 6.8 \\
3.75 \% \text { female }\end{array}$ \\
\hline
\end{tabular}




\begin{tabular}{|c|c|c|c|c|c|c|c|c|}
\hline $\begin{array}{l}\text { Author, } \\
\text { Year }\end{array}$ & $\begin{array}{c}\text { Country, } \\
\text { Study } \\
\text { Period }\end{array}$ & $\begin{array}{l}\text { Risk of } \\
\text { Bias }\end{array}$ & $\begin{array}{c}\text { COPD } \\
\text { Definition }\end{array}$ & $\begin{array}{c}\text { COPD } \\
\text { Exacerbation }\end{array}$ & $\begin{array}{l}\text { Severity of } \\
\text { ECOPD }\end{array}$ & $\begin{array}{l}\text { Intervention(s) and } \\
\text { comparison }\end{array}$ & $\begin{array}{c}\text { COPD } \\
\text { Severity } \\
(\% \\
\text { predicted } \\
\text { unless } \\
\text { specified) }\end{array}$ & $\begin{array}{c}\text { Patient } \\
\text { Characteristics }\end{array}$ \\
\hline & & & \multirow{2}{*}{$\begin{array}{l}\text { pack years or } \\
\text { more of cigarette } \\
\text { smoking, and } \\
\text { either an FEV1 } \\
\text { of } 1.50 \text { liters or } \\
\text { less or an } \\
\text { inability to } \\
\text { undergo } \\
\text { spirometry } \\
\text { because of } \\
\text { dyspnea. }\end{array}$} & & & $\begin{array}{l}\text { Glucocorticoid for } 2 \\
\text { week }\end{array}$ & $\begin{array}{l}\text { FEV1 (L): } \\
\text { Mean } \\
0.772 \pm 0.2 \\
86\end{array}$ & $\begin{array}{l}80 \text { patients } \\
\text { aged } 67.1 \pm 10.6 \text {, } \\
0 \% \text { female }\end{array}$ \\
\hline & & & & & & Placebo & $\begin{array}{l}\text { FEV1 (L): } \\
\text { Mean } \\
0.750 \pm 0.2 \\
71\end{array}$ & $\begin{array}{l}\text { 111Patients, } \\
\text { aged } 67.8 \pm 10, \\
0 \% \text { female }\end{array}$ \\
\hline $\begin{array}{l}\text { Petitpretz, } \\
2007^{82}\end{array}$ & $\begin{array}{l}\text { France, } \\
\text { Germany, } \\
\text { Tunisia, } \\
\text { Belgium, } \\
\text { Austria and } \\
\text { Turkey. } \\
03 / 2003- \\
11 / 2004\end{array}$ & High risk & $\begin{array}{l}\text { A history of } \\
\text { chronic } \\
\text { bronchitis } \\
\text { characterized by } \\
\text { cough and } \\
\text { sputum } \\
\text { production on } \\
\text { most days for } 3 \\
\text { consecutive } \\
\text { months and for } \\
>2 \text { consecutive } \\
\text { years; chronic } \\
\text { obstructive } \\
\text { bronchitis } \\
\text { confirmed by } \\
\text { lung function test } \\
\text { performed in a }\end{array}$ & $\begin{array}{l}\text { The presence of } \\
\text { the three } \\
\text { Anthonisen's } \\
\text { criteria of recent } \\
\text { increase in } \\
\text { sputum volume, } \\
\text { sputum purulence } \\
\text { and dyspnea. }\end{array}$ & Mild & $\begin{array}{l}\text { Levofloxacin, 500mg } \\
\text { total dose }\end{array}$ & $\begin{array}{l}\text { FEV1: } \\
\text { Mean } \\
51.2 \pm 13.1\end{array}$ & $\begin{array}{l}340 \text { patients } \\
\text { aged } 64.3 \pm 10.1 \text {, } \\
19 \% \text { female }\end{array}$ \\
\hline
\end{tabular}




\begin{tabular}{|c|c|c|c|c|c|c|c|c|}
\hline $\begin{array}{l}\text { Author, } \\
\text { Year }\end{array}$ & $\begin{array}{c}\text { Country, } \\
\text { Study } \\
\text { Period }\end{array}$ & $\begin{array}{l}\text { Risk of } \\
\text { Bias }\end{array}$ & $\begin{array}{c}\text { COPD } \\
\text { Definition }\end{array}$ & $\begin{array}{c}\text { COPD } \\
\text { Exacerbation }\end{array}$ & $\begin{array}{l}\text { Severity of } \\
\text { ECOPD }\end{array}$ & $\begin{array}{l}\text { Intervention(s) and } \\
\text { comparison }\end{array}$ & $\begin{array}{c}\text { COPD } \\
\text { Severity } \\
(\% \\
\text { predicted } \\
\text { unless } \\
\text { specified) }\end{array}$ & $\begin{array}{c}\text { Patient } \\
\text { Characteristics }\end{array}$ \\
\hline & & & $\begin{array}{l}\text { stable condition } \\
\text { in the previous } \\
12 \text { months } \\
\text { showing a forced } \\
\text { expiratory } \\
\text { volume in } 1 \mathrm{~s} \\
\text { (FEV1)/forced } \\
\text { vital capacity } \\
\text { ratio of <70\% } \\
\text { and an FEV1 in } \\
\text { the range of 35- } \\
80 \% \text { of the } \\
\text { predicted value, } \\
\text { and no } \\
\text { significant } \\
\text { reversibility } \\
\text { following } 2- \\
\text { agonist therapy } \\
\text { (<200 ml and } \\
<15 \% \text { increase } \\
\text { in FEV1). }\end{array}$ & & & Cefuroxime, 500mg & $\begin{array}{l}\text { FEV1: } \\
\text { Mean } \\
52.6 \pm 13.3\end{array}$ & $\begin{array}{l}349 \text { patients } \\
\text { aged } 64.2 \pm 9.9 \\
18.2 \% \text { female }\end{array}$ \\
\hline \multirow[t]{2}{*}{$\begin{array}{l}\text { Phillips, } \\
1993^{83}\end{array}$} & \multirow[t]{2}{*}{$\begin{array}{l}\text { United } \\
\text { States of } \\
\text { America }\end{array}$} & \multirow[t]{2}{*}{ High risk } & \multirow[t]{2}{*}{ NR } & \multirow{2}{*}{$\begin{array}{l}\text { Cough, fever, or } \\
\text { increased sputum } \\
\text { production/purule } \\
\text { nce), absence of } \\
\text { infiltrate on chest } \\
\text { radiograph) }\end{array}$} & \multirow[t]{2}{*}{$\begin{array}{l}\text { Mild to } \\
\text { Moderate to } \\
\text { Severe }\end{array}$} & $\begin{array}{l}\text { Cefpodoxime, } 400 \mathrm{mg} \\
\text { total dose }\end{array}$ & NR & $\begin{array}{l}194 \text { patients } \\
\text { aged } 54.2 \pm 17.3 \text {, } \\
54 \% \text { female }\end{array}$ \\
\hline & & & & & & $\begin{array}{l}\text { Cefaclor, } 750 \mathrm{mg} \text { total } \\
\text { dose }\end{array}$ & NR & $\begin{array}{l}107 \text { patients } \\
\text { aged } 51.9 \pm 16.5 \text {, } \\
35 \% \text { female }\end{array}$ \\
\hline $\begin{array}{l}\text { Rhee, } \\
2015^{84}\end{array}$ & Korea & High risk & $\begin{array}{l}\text { Post- } \\
\text { bronchodilator } \\
\text { FEV1/FVC }<0.7\end{array}$ & $\begin{array}{l}\text { Worsening of the } \\
\text { respiratory } \\
\text { symptoms that is } \\
\text { beyond normal }\end{array}$ & Moderate & $\begin{array}{l}\text { Zabofloxacin, } 367 \mathrm{mg} \\
\text { total dose }\end{array}$ & $\begin{array}{l}\text { FEV1: } \\
\text { Mean } \\
50.5 \pm 18.1\end{array}$ & $\begin{array}{l}175 \text { patients } \\
\text { aged } 67.8 \pm 7.8 \\
12.6 \% \text { female }\end{array}$ \\
\hline
\end{tabular}




\begin{tabular}{|c|c|c|c|c|c|c|c|c|}
\hline $\begin{array}{l}\text { Author, } \\
\text { Year }\end{array}$ & $\begin{array}{l}\text { Country, } \\
\text { Study } \\
\text { Period }\end{array}$ & $\begin{array}{l}\text { Risk of } \\
\text { Bias }\end{array}$ & $\begin{array}{c}\text { COPD } \\
\text { Definition }\end{array}$ & $\begin{array}{c}\text { COPD } \\
\text { Exacerbation }\end{array}$ & $\begin{array}{l}\text { Severity of } \\
\text { ECOPD }\end{array}$ & $\begin{array}{l}\text { Intervention(s) and } \\
\text { comparison }\end{array}$ & $\begin{array}{c}\text { COPD } \\
\text { Severity } \\
(\% \\
\text { predicted } \\
\text { unless } \\
\text { specified) }\end{array}$ & $\begin{array}{c}\text { Patient } \\
\text { Characteristics }\end{array}$ \\
\hline & & & & $\begin{array}{l}\text { day-to-day } \\
\text { variations and } \\
\text { leads to a change } \\
\text { in medication, } \\
\text { purulent sputum } \\
\text { or increased } \\
\text { volume of } \\
\text { sputum, } \\
\text { outpatient } \\
\text { evaluation }\end{array}$ & & $\begin{array}{l}\text { Moxifloxacin, } 400 \mathrm{mg} \\
\text { total dose }\end{array}$ & $\begin{array}{l}\text { FEV1: } \\
\text { Mean: } \\
49.1 \pm 17.2\end{array}$ & $\begin{array}{l}167 \text { patients } \\
\text { aged } 68.4 \pm 8, \\
4.8 \% \text { female }\end{array}$ \\
\hline \multirow[t]{2}{*}{$\begin{array}{l}\text { Rizzato, } \\
1998^{85}\end{array}$} & \multirow[t]{2}{*}{ Italy } & \multirow[t]{2}{*}{ High risk } & \multirow[t]{2}{*}{ NR } & \multirow{2}{*}{$\begin{array}{l}\text { Admitted to } \\
\text { hospital for } \\
\text { ECOPD, } \\
\text { FEV1<70\%, } \\
\text { marked } \\
\text { respiratory } \\
\text { distress }\end{array}$} & \multirow[t]{2}{*}{$\begin{array}{l}\text { Moderate to } \\
\text { Severe }\end{array}$} & $\begin{array}{l}\text { Deflazacort } \\
\text { Hemisuccinate, 60mg } \\
\text { total dose }\end{array}$ & $\begin{array}{l}\text { FEV1 }(\mathrm{L}): \\
\text { Mean } 0.84\end{array}$ & $\begin{array}{l}30 \text { patients } \\
\text { aged } \\
74.5 \pm 15.75 \\
27 \% \text { female }\end{array}$ \\
\hline & & & & & & $\begin{array}{l}\text { Methylprednisolone, } \\
\text { 40mg total dose }\end{array}$ & $\begin{array}{l}\text { FEV1 }(\mathrm{L}): \\
\text { Mean } 0.76\end{array}$ & $\begin{array}{l}30 \text { patients } \\
\text { aged } 69.0 \pm 11 \\
33 \% \text { female }\end{array}$ \\
\hline \multirow[t]{2}{*}{$\begin{array}{l}\text { Roede, } \\
2007^{86}\end{array}$} & \multirow{2}{*}{$\begin{array}{l}\text { The } \\
\text { Netherlands } \\
\text { 11/2000- } \\
\text { 12/2003 }\end{array}$} & \multirow[t]{2}{*}{$\begin{array}{l}\text { Intermed } \\
\text { iate risk }\end{array}$} & \multirow{2}{*}{$\begin{array}{l}\text { A disorder } \\
\text { characterized by } \\
\text { abnormal tests } \\
\text { of expiratory flow } \\
\text { that did not } \\
\text { change markedly } \\
\text { during an } \\
\text { observation } \\
\text { period of several } \\
\text { months. }\end{array}$} & \multirow{2}{*}{$\begin{array}{l}\text { An increase in } \\
\text { dyspnea and the } \\
\text { volume and } \\
\text { purulence of } \\
\text { sputum. }\end{array}$} & \multirow[t]{2}{*}{$\begin{array}{l}\text { Moderate to } \\
\text { Severe }\end{array}$} & $\begin{array}{l}\text { Amoxycillin }+ \\
\text { Clavulanic for } 3 \text { days, } \\
\text { 2500mg overall }\end{array}$ & NR & $\begin{array}{l}23 \text { patients } \\
\text { aged } 69 \pm 4.25 \\
52 \% \text { female } \\
\end{array}$ \\
\hline & & & & & & $\begin{array}{l}\text { Amoxycillin }+ \\
\text { Clavulanic for } 10 \\
\text { days, } 2500 \mathrm{mg} \text { overall }\end{array}$ & NR & $\begin{array}{l}25 \text { patients } \\
\text { aged } 66 \pm 2.5 \\
36 \% \text { female }\end{array}$ \\
\hline $\begin{array}{l}\text { Ruiz- } \\
\text { Gonzalez, } \\
2007^{87}\end{array}$ & Spain & High risk & $\begin{array}{l}\text { FEV1/FVC ratio } \\
\leq 70 \% \text {, and FEV1 } \\
\leq 80 \% \text { predicted. }\end{array}$ & $\begin{array}{l}\text { Worsening of } \\
\text { dyspnea, } \\
\text { increase in } \\
\text { sputum volume } \\
\text { and increase in } \\
\text { sputum } \\
\text { purulence. }\end{array}$ & $\begin{array}{l}\text { Mild to } \\
\text { Moderate to } \\
\text { Severe }\end{array}$ & Levofloxacin & $\begin{array}{l}\text { Moderate: } \\
16.7 \% \\
\text { Severe: } \\
57.8 \% \\
\text { Very } \\
\text { Severe: } \\
24.4 \% \\
\text { FEV1: } \\
\text { Mean } \\
33.91 \pm 21 . \\
3\end{array}$ & $\begin{array}{l}50 \text { patients } \\
\text { aged } \\
64.3 \text { years, 16\% } \\
\text { female }\end{array}$ \\
\hline
\end{tabular}




\begin{tabular}{|c|c|c|c|c|c|c|c|c|}
\hline $\begin{array}{l}\text { Author, } \\
\text { Year }\end{array}$ & $\begin{array}{l}\text { Country, } \\
\text { Study } \\
\text { Period }\end{array}$ & $\begin{array}{l}\text { Risk of } \\
\text { Bias }\end{array}$ & $\begin{array}{c}\text { COPD } \\
\text { Definition }\end{array}$ & $\begin{array}{c}\text { COPD } \\
\text { Exacerbation }\end{array}$ & $\begin{array}{l}\text { Severity of } \\
\text { ECOPD }\end{array}$ & $\begin{array}{l}\text { Intervention(s) and } \\
\text { comparison }\end{array}$ & $\begin{array}{c}\text { COPD } \\
\text { Severity } \\
(\% \\
\text { predicted } \\
\text { unless } \\
\text { specified) }\end{array}$ & $\begin{array}{c}\text { Patient } \\
\text { Characteristics }\end{array}$ \\
\hline & & & & & & $\begin{array}{l}\text { Standard care } \\
\text { (clarithromycin, } \\
\text { cefuroxime, or } \\
\text { amoxicillin/clavulanat } \\
\text { e) }\end{array}$ & $\begin{array}{l}\text { Moderate: } \\
16.7 \% \\
\text { Severe: } \\
58.3 \% \\
\text { Very } \\
\text { Severe: } \\
25.0 \% \\
\text { FEV1: } \\
\text { Mean } \\
31.48 \pm 17 . \\
1\end{array}$ & $\begin{array}{l}52 \text { patients } \\
\text { aged } 61.8,17 \% \\
\text { female }\end{array}$ \\
\hline \multirow[t]{2}{*}{$\begin{array}{l}\text { Sayiner, } \\
2001^{88}\end{array}$} & \multirow[t]{2}{*}{ Turkey } & \multirow[t]{2}{*}{$\begin{array}{l}\text { Intermed } \\
\text { iate risk }\end{array}$} & \multirow{2}{*}{$\begin{array}{l}\text { Smoking history } \\
\text { at least } 20 \text { pack- } \\
\text { years and } \\
\text { severe airway } \\
\text { obstruction } \\
\text { (FEV1 <35\% } \\
\text { predicted) }\end{array}$} & \multirow{2}{*}{$\begin{array}{l}\text { Severe dyspnea } \\
\text { preventing the } \\
\text { patient from } \\
\text { performing even } \\
\text { minor activities } \\
\text { (getting dressed } \\
\text { or eating) and } \\
\text { resulting in sleep } \\
\text { disturbances, and } \\
\text { the presence of } \\
\text { respiratory failure, } \\
\text { i.e. Pao2 less } \\
\text { than or equal to } \\
55 \text { mmhg and/or } \\
\text { Paco2 level } \\
\text { greater than or } \\
\text { equal to } 45 \\
\text { mmhg. }\end{array}$} & \multirow[t]{2}{*}{ Severe } & $\begin{array}{l}\text { Methylprednisolone } \\
\text { for } 3 \text { days }\end{array}$ & $\begin{array}{l}\text { FEV1: } \\
\text { Mean } \\
25.4 \pm 5.4\end{array}$ & $\begin{array}{l}17 \text { patients } \\
\text { aged } 67.4 \pm 1.4, \\
6 \% \text { female }\end{array}$ \\
\hline & & & & & & $\begin{array}{l}\text { Methylprednisolone } \\
\text { for } 10 \text { days }\end{array}$ & $\begin{array}{l}\text { FEV1: } \\
\text { Mean } \\
27.1 \pm 5.7\end{array}$ & $\begin{array}{l}17 \text { patients } \\
\text { aged } 64.1 \pm 2.2 \\
6 \% \text { female }\end{array}$ \\
\hline $\begin{array}{l}\text { Stallberg, } \\
2009^{89}\end{array}$ & $\begin{array}{l}\text { Sweden } \\
09 / 2005 \\
07 / 2007\end{array}$ & $\begin{array}{l}\text { Intermed } \\
\text { iate risk }\end{array}$ & $\begin{array}{l}\geq 10 \text { pack year } \\
\text { smoking history, } \\
\text { moderate COPD } \\
\text { GOLD stage IIA } \\
\text { or IIB }\end{array}$ & $\begin{array}{l}\text { Increased } \\
\text { dyspnea, sputum } \\
\text { volume, or } \\
\text { sputum } \\
\text { purulence; the } \\
\text { exacerbation had } \\
\text { to be severe } \\
\text { enough that the } \\
\text { primary care }\end{array}$ & Mild & $\begin{array}{l}\text { Budesonide + } \\
\text { Formoterol }\end{array}$ & $\begin{array}{l}\text { GOLD 1: } \\
1.8 \% \\
\text { GOLD2: } \\
98.2 \% \\
\text { FEV1: } \\
\text { Mean } \\
45.1 \pm 8.9\end{array}$ & $\begin{array}{l}55 \text { patients } \\
\text { aged } 67.2 \pm 9.7 \\
55 \% \text { female }\end{array}$ \\
\hline
\end{tabular}




\begin{tabular}{|c|c|c|c|c|c|c|c|c|}
\hline $\begin{array}{l}\text { Author, } \\
\text { Year }\end{array}$ & $\begin{array}{c}\text { Country, } \\
\text { Study } \\
\text { Period }\end{array}$ & $\begin{array}{l}\text { Risk of } \\
\text { Bias }\end{array}$ & $\begin{array}{c}\text { COPD } \\
\text { Definition }\end{array}$ & $\begin{array}{c}\text { COPD } \\
\text { Exacerbation }\end{array}$ & $\begin{array}{l}\text { Severity of } \\
\text { ECOPD }\end{array}$ & $\begin{array}{l}\text { Intervention(s) and } \\
\text { comparison }\end{array}$ & $\begin{array}{c}\text { COPD } \\
\text { Severity } \\
(\% \\
\text { predicted } \\
\text { unless } \\
\text { specified) }\end{array}$ & $\begin{array}{c}\text { Patient } \\
\text { Characteristics }\end{array}$ \\
\hline & & & & $\begin{array}{l}\text { physician thought } \\
\text { that oral steroids } \\
\text { were clinically } \\
\text { indicated, but not } \\
\text { severe enough to } \\
\text { require } \\
\text { hospitalization. }\end{array}$ & & $\begin{array}{l}\text { Prednisolone + } \\
\text { formoterol }\end{array}$ & $\begin{array}{l}\text { GOLD1: } \\
\text { 1.9\% } \\
\text { GOLD2: } \\
94.4 \% \\
\text { GOLD3: } \\
3.7 \% \\
\text { FEV1: } \\
\text { Mean } \\
45.0 \pm 9.5\end{array}$ & $\begin{array}{l}54 \text { patients } \\
\text { aged } 66.7 \pm 9.3 \\
43 \% \text { female }\end{array}$ \\
\hline \multirow[t]{2}{*}{$\begin{array}{l}\text { Sun, } \\
2015^{90}\end{array}$} & \multirow[t]{2}{*}{$\begin{array}{l}\text { China } \\
1 / 2013 \\
7 / 2013\end{array}$} & \multirow[t]{2}{*}{ High risk } & \multirow{2}{*}{$\begin{array}{l}\text { GOLD criteria, at } \\
\text { least } 20 \text { pack } \\
\text { year smoking } \\
\text { history }\end{array}$} & \multirow{2}{*}{$\begin{array}{l}\text { Clinical diagnosis, } \\
\text { ECOPD due to } \\
\text { bacterial infection } \\
\text { only }\end{array}$} & \multirow[t]{2}{*}{ Unclear } & Inhaled Budesonide & $\begin{array}{l}\text { FEV1: } \\
\text { Mean } \\
49.6 \pm 8.3\end{array}$ & $\begin{array}{l}15 \text { patients } \\
\text { aged } 62.8 \pm 7.3 \\
40 \% \text { female }\end{array}$ \\
\hline & & & & & & $\begin{array}{l}\text { Systemic } \\
\text { Methylprednisolone }\end{array}$ & $\begin{array}{l}\text { FEV1: } \\
\text { Mean } \\
48.0 \pm 8.8\end{array}$ & $\begin{array}{l}15 \text { patients } \\
\text { aged } 62 \pm 8.1 \text {, } \\
47 \% \text { female }\end{array}$ \\
\hline \multirow[t]{2}{*}{$\begin{array}{l}\text { Ucar, } \\
2014^{91}\end{array}$} & \multirow[t]{2}{*}{ Turkey } & \multirow[t]{2}{*}{ High risk } & \multirow[t]{2}{*}{ Gold NOS } & \multirow[t]{2}{*}{$\begin{array}{l}\text { "Moderate or } \\
\text { severe" } \\
\text { exacerbation } \\
\text { requiring } \\
\text { hospitalization } \\
\text { (respiratory } \\
\text { failure with mild to } \\
\text { moderate } \\
\text { hypoxemia (pao2 } \\
40-80 \mathrm{mmHg} \text { ) but } \\
\text { no carbon dioxide } \\
\text { retention or } \\
\text { acidosis }\end{array}$} & \multirow[t]{2}{*}{$\begin{array}{l}\text { Moderate to } \\
\text { Severe }\end{array}$} & Methylprednisolone & $\begin{array}{l}\text { FEV1: } \\
\text { Mean } \\
39.4 \pm 11.3\end{array}$ & $\begin{array}{l}33 \text { patients } \\
\text { aged } 66.6 \pm 9.6 \\
27 \% \text { female }\end{array}$ \\
\hline & & & & & & Budesonide $4 \mathrm{mg}$ & $\begin{array}{l}\text { FEV1: } \\
\text { Mean } \\
41.0 \pm 13.4\end{array}$ & $\begin{array}{l}27 \text { patients } \\
\text { aged } 66.7 \pm 9.7 \\
7 \% \text { female }\end{array}$ \\
\hline
\end{tabular}




\begin{tabular}{|c|c|c|c|c|c|c|c|c|}
\hline $\begin{array}{l}\text { Author, } \\
\text { Year }\end{array}$ & $\begin{array}{l}\text { Country, } \\
\text { Study } \\
\text { Period }\end{array}$ & $\begin{array}{l}\text { Risk of } \\
\text { Bias }\end{array}$ & $\begin{array}{c}\text { COPD } \\
\text { Definition }\end{array}$ & $\begin{array}{c}\text { COPD } \\
\text { Exacerbation }\end{array}$ & $\begin{array}{l}\text { Severity of } \\
\text { ECOPD }\end{array}$ & $\begin{array}{l}\text { Intervention(s) and } \\
\text { comparison }\end{array}$ & $\begin{array}{c}\text { COPD } \\
\text { Severity } \\
(\% \\
\text { predicted } \\
\text { unless } \\
\text { specified) }\end{array}$ & $\begin{array}{c}\text { Patient } \\
\text { Characteristics }\end{array}$ \\
\hline & & & & & & Budesonide 8mg & $\begin{array}{l}\text { FEV1: } \\
\text { Mean } \\
49.0 \pm 14.7\end{array}$ & $\begin{array}{l}26 \text { patients } \\
\text { aged } 69.9 \pm 8.5 \\
15 \% \text { female }\end{array}$ \\
\hline \multirow[t]{4}{*}{$\begin{array}{l}\text { Umut, } \\
1999^{92}\end{array}$} & \multirow[t]{4}{*}{ Turkey } & \multirow[t]{4}{*}{ High risk } & \multirow{4}{*}{$\begin{array}{l}\text { American } \\
\text { Thoracic Society } \\
\text { criteria }\end{array}$} & \multirow{4}{*}{$\begin{array}{l}\text { Increased } \\
\text { amounts of } \\
\text { purulent } \\
\text { secretions, } \\
\text { dyspnea, chest } \\
\text { tightness }\end{array}$} & \multirow[t]{4}{*}{ Unclear } & Azithromycin & NR & $\begin{array}{l}24 \text { patients } \\
\text { aged } 64 \pm 9\end{array}$ \\
\hline & & & & & & $\begin{array}{l}\text { Ampicillin + } \\
\text { Sulbactam }\end{array}$ & NR & $\begin{array}{l}28 \text { patients } \\
\text { aged } 64 \pm 9\end{array}$ \\
\hline & & & & & & Ciprofloxacin & NR & $\begin{array}{l}25 \text { patients } \\
\text { aged } 64 \pm 9\end{array}$ \\
\hline & & & & & & Cefaclor & NR & $\begin{array}{l}29 \text { patients } \\
\text { aged } 64 \pm 9\end{array}$ \\
\hline \multirow[t]{2}{*}{$\begin{array}{l}\text { van } \\
\text { Zanten, } \\
2007^{93}\end{array}$} & \multirow[t]{2}{*}{ Netherlands } & \multirow[t]{2}{*}{ High risk } & \multirow[t]{2}{*}{$\begin{array}{l}\text { GOLD classes 2- } \\
4 .\end{array}$} & \multirow[t]{2}{*}{$\begin{array}{l}\text { NR, hospital } \\
\text { admission }\end{array}$} & \multirow[t]{2}{*}{$\begin{array}{l}\text { Moderate to } \\
\text { Severe }\end{array}$} & $\begin{array}{l}\text { Continuous } \\
\text { Cefotaxime }\end{array}$ & NR & $\begin{array}{l}47 \text { patients } \\
\text { aged } 65.3 \pm 8.4 \text {, } \\
34.04 \% \text { female }\end{array}$ \\
\hline & & & & & & $\begin{array}{l}\text { Intermittent } \\
\text { Cefotaxime }\end{array}$ & NR & $\begin{array}{l}46 \text { patients } \\
\text { aged } 68.6 \pm 5.3, \\
28.3 \% \text { female }\end{array}$ \\
\hline \multirow[t]{2}{*}{$\begin{array}{l}\text { Whitlock, } \\
1995^{94}\end{array}$} & \multirow[t]{2}{*}{$\begin{array}{l}\text { United } \\
\text { States of } \\
\text { America }\end{array}$} & \multirow[t]{2}{*}{ High risk } & \multirow{2}{*}{$\begin{array}{l}\text { History of COPD } \\
\text { with persistent } \\
\text { cough and } \\
\text { sputum } \\
\text { production for at } \\
\text { least } 3 \text { months in } \\
\text { at least } 2 \\
\text { consecutive } \\
\text { years }\end{array}$} & \multirow{2}{*}{$\begin{array}{l}\text { Least two of the } \\
\text { following: fever } \\
\left(\sim 37.8^{\circ} \mathrm{C}\right) \text { within } \\
\text { the previous } 24 \\
\text { hours, increased } \\
\text { dyspnea, } \\
\text { increased } \\
\text { frequency of } \\
\text { cough, and } \\
\text { increased volume } \\
\text { of sputum }\end{array}$} & \multirow[t]{2}{*}{$\begin{array}{l}\text { Mild to } \\
\text { Moderate }\end{array}$} & Azithromycin & NR & $\begin{array}{l}39 \text { patients } \\
\text { aged } 61 \pm 9.5 \\
33 \% \text { female }\end{array}$ \\
\hline & & & & & & $\begin{array}{l}\text { Amoxicillin + } \\
\text { Clavulanate }\end{array}$ & NR & $\begin{array}{l}31 \text { patients } \\
\text { aged } 58.5 \pm 9.5 \\
39 \% \text { female }\end{array}$ \\
\hline $\begin{array}{l}\text { Willaert, } \\
2002^{95}\end{array}$ & $\begin{array}{l}\text { Belgium } \\
07 / 1999 \\
03 / 2000\end{array}$ & High risk & NR & $\begin{array}{l}\text { 1) increased } \\
\text { dyspnea, 2) } \\
\text { increased cough } \\
\text { frequency or }\end{array}$ & $\begin{array}{l}\text { Moderate to } \\
\text { Severe }\end{array}$ & $\begin{array}{l}\text { Intravenous Steroids } \\
+ \text { Aerosol } \\
\text { Bronchodilators }\end{array}$ & $\begin{array}{l}\text { FEV1 }(L): \\
\text { Mean } \\
1.14 \pm 0.43\end{array}$ & $\begin{array}{l}23 \text { patients } \\
\text { aged } 72 \pm 6, \\
8.7 \% \text { female }\end{array}$ \\
\hline
\end{tabular}




\begin{tabular}{|c|c|c|c|c|c|c|c|c|}
\hline $\begin{array}{l}\text { Author, } \\
\text { Year }\end{array}$ & $\begin{array}{l}\text { Country, } \\
\text { Study } \\
\text { Period }\end{array}$ & $\begin{array}{l}\text { Risk of } \\
\text { Bias }\end{array}$ & $\begin{array}{c}\text { COPD } \\
\text { Definition }\end{array}$ & $\begin{array}{c}\text { COPD } \\
\text { Exacerbation }\end{array}$ & $\begin{array}{l}\text { Severity of } \\
\text { ECOPD }\end{array}$ & $\begin{array}{c}\text { Intervention(s) and } \\
\text { comparison }\end{array}$ & $\begin{array}{c}\text { COPD } \\
\text { Severity } \\
(\% \\
\text { predicted } \\
\text { unless } \\
\text { specified) }\end{array}$ & $\begin{array}{c}\text { Patient } \\
\text { Characteristics }\end{array}$ \\
\hline & & & & $\begin{array}{l}\text { severity, 3) } \\
\text { increased } \\
\text { production or } \\
\text { purulence of } \\
\text { sputum, 4) } \\
\text { increased } \\
\text { wheeze, lasting } \\
\text { for at least } 3 \text { days } \\
\text { and for which the } \\
\text { patient sought } \\
\text { medical attention }\end{array}$ & & $\begin{array}{l}\text { Oral steroids + } \\
\text { Multiple Dose Inhaler } \\
\text { Bronchodilators }\end{array}$ & $\begin{array}{l}\text { FEV1 }(L): \\
\text { Mean } \\
1.1 \pm 0.51\end{array}$ & $\begin{array}{l}25 \text { patients } \\
\text { aged } 71 \pm 8, \\
16 \% \text { female }\end{array}$ \\
\hline \multirow[t]{2}{*}{$\begin{array}{l}\text { Wilson, } \\
2012^{96}\end{array}$} & \multirow[t]{2}{*}{30 countries } & \multirow[t]{2}{*}{ High risk } & \multirow{2}{*}{$\begin{array}{l}\text { FEV1/FVC }<0.7 \text {, } \\
\text { post- } \\
\text { bronchodilator } \\
\text { FEV1 }<60 \% \text {, at } \\
\text { least } 20 \text { pack } \\
\text { year smoking } \\
\text { history, chronic } \\
\text { bronchitis, } 2 \text { or } \\
\text { more } \\
\text { exacerbations in } \\
\text { the past year }\end{array}$} & \multirow{2}{*}{$\begin{array}{l}\text { Investigator- } \\
\text { evaluated } \\
\text { Anthonisen type I } \\
\text { exacerbation and } \\
\text { who were } \\
\text { considered by the } \\
\text { investigator to } \\
\text { require antibiotic } \\
\text { therapy, } \\
\text { outpatient } \\
\text { evaluation }\end{array}$} & \multirow[t]{2}{*}{ Mild } & Moxifloxacin & $\begin{array}{l}\text { FEV1: } \\
\text { Mean } \\
39.28 \pm 11 \\
62\end{array}$ & $\begin{array}{l}686 \text { patients } \\
\text { aged } 69.6 \pm 6.8, \\
21 \% \text { female }\end{array}$ \\
\hline & & & & & & $\begin{array}{l}\text { Amoxicillin }+ \\
\text { Clavulanic Acid }\end{array}$ & $\begin{array}{l}\text { FEV1: } \\
\text { Mean } \\
39.19 \pm 11 \\
36\end{array}$ & $\begin{array}{l}686 \text { patients } \\
\text { aged } 69.3 \pm 6.3, \\
21 \% \text { female }\end{array}$ \\
\hline \multirow[t]{2}{*}{$\begin{array}{l}\text { Yoon, } \\
2013^{97}\end{array}$} & \multirow[t]{2}{*}{$\begin{array}{l}\text { Korea } \\
11 / 2006- \\
06 / 2009\end{array}$} & \multirow[t]{2}{*}{$\begin{array}{l}\text { Intermed } \\
\text { iate risk }\end{array}$} & \multirow[t]{2}{*}{$\begin{array}{l}\text { Post- } \\
\text { bronchodilator } \\
\text { FEV1/FVC }<0.7\end{array}$} & \multirow[t]{2}{*}{$\begin{array}{l}\text { A recently } \\
\text { increased cough } \\
\text { or dyspnea, } \\
\text { recent change in } \\
\text { color or amount } \\
\text { of sputum, } \\
\text { hospital } \\
\text { admission }\end{array}$} & \multirow[t]{2}{*}{ Unclear } & Levofloxacin & NR & $\begin{array}{l}65 \text { patients } \\
\text { aged } \\
70.95 \pm 8.81 \\
9.23 \% \text { female }\end{array}$ \\
\hline & & & & & & Cefuroxime & NR & $\begin{array}{l}72 \text { patients } \\
\text { aged } 69.63 \pm 8.3 \text {, } \\
6.94 \% \text { female }\end{array}$ \\
\hline
\end{tabular}

Note: \pm denotes standard deviation

ECOPD = exacerbation of chronic obstructive pulmonary disease; COPD = chronic obstructive pulmonary disease; ED = emergency department; FEV1 = forced expiration volume in 1 second; FVC = forced vital capacity; g= gram; GOLD = global initiative for chronic obstructive lung disease; $\mathrm{h}=$ hour; $\mathrm{L}=$ liter; $\mathrm{mg}=$ milligram; min= minute; 
$\mathrm{ml}=$ milliliter; $\mathrm{mmHg}=$ millimeters of mercury; $\mathrm{NOS}=$ not otherwise specified; $\mathrm{NR}=$ not reported; $\mathrm{PaCO} 2$ = partial pressure of carbon dioxide in arterial blood; Pao2 = partial pressure of oxygen in arterial blood; PMN= polymorphonuclear leukocytes 


\section{Appendix E. Risk of Bias}

Table E.1. Risk of Bias (Cochrane ROB tool) for included studies

\begin{tabular}{|c|c|c|c|c|c|c|c|c|}
\hline Author, Year & $\begin{array}{c}\text { Sequence } \\
\text { Generation }\end{array}$ & $\begin{array}{c}\text { Allocation } \\
\text { Concealme } \\
\text { nt }\end{array}$ & $\begin{array}{l}\text { Blinding of } \\
\text { Participants } \\
\text {, Personnel }\end{array}$ & $\begin{array}{c}\text { Blinding of } \\
\text { Outcome } \\
\text { Assessors }\end{array}$ & $\begin{array}{c}\text { Incomplete } \\
\text { Outcome } \\
\text { Data }\end{array}$ & $\begin{array}{l}\text { Selective } \\
\text { Outcome } \\
\text { Reporting }\end{array}$ & $\begin{array}{c}\text { Other } \\
\text { Sources of } \\
\text { Bias } \\
\end{array}$ & Overall RoB \\
\hline Aaron, $2003^{1}$ & Low risk & Low risk & Low risk & Unknown & Low risk & Unknown & High risk & Low risk \\
\hline $\begin{array}{l}\text { Abreu } \\
\text { Gonzalez, } \\
2006^{17} \\
\end{array}$ & Unknown & Unknown & Low risk & Unknown & Unknown & Unknown & Unknown & High risk \\
\hline $\begin{array}{l}\text { Aggarwal, } \\
2011^{68}\end{array}$ & Low risk & Unknown & High risk & Unknown & Low risk & Unknown & High risk & $\begin{array}{l}\text { Intermediate } \\
\text { risk }\end{array}$ \\
\hline Albert, $1980^{2}$ & Low risk & Low risk & Low risk & Unknown & Low risk & Unknown & Unknown & Low risk \\
\hline $\begin{array}{l}\text { Andre-Alves, } \\
2007^{69}\end{array}$ & Unknown & Unknown & Unknown & Unknown & Low risk & Low risk & Unknown & High risk \\
\hline $\begin{array}{l}\text { Anthonisen, } \\
1987^{3}\end{array}$ & Unknown & Unknown & Low risk & Unknown & High risk & Unknown & High risk & High risk \\
\hline Aubier, $2002^{70}$ & Unknown & Unknown & Low risk & Unknown & Low risk & Unknown & High risk & High risk \\
\hline Austin, $2010^{18}$ & High risk & Unknown & High risk & High risk & High risk & Unknown & High risk & High risk \\
\hline $\begin{array}{l}\text { Ayfer } \\
\text { Aytemur, } \\
2015^{19}\end{array}$ & Unknown & Unknown & Low risk & Unknown & Low risk & Unknown & Low risk & High risk \\
\hline Basri, $2017^{20}$ & Low risk & Unknown & Low risk & Unknown & Unknown & Unknown & Low risk & $\begin{array}{l}\text { Intermediate } \\
\text { risk }\end{array}$ \\
\hline $\begin{array}{l}\text { Bathoorn, } \\
2008^{4}\end{array}$ & Unknown & Unknown & Low risk & Unknown & Low risk & Unknown & Unknown & High risk \\
\hline $\begin{array}{l}\text { Behnke, } \\
2000^{21}\end{array}$ & Unknown & Unknown & High risk & High risk & High risk & Unknown & Unknown & High risk \\
\hline Black, 200422 & Low risk & Unknown & Low risk & Unknown & Low risk & Unknown & High risk & $\begin{array}{l}\text { Intermediate } \\
\text { risk }\end{array}$ \\
\hline Blasi, $2013^{71}$ & Low risk & Low risk & Low risk & Unknown & High risk & Unknown & Unknown & $\begin{array}{l}\text { Intermediate } \\
\text { risk }\end{array}$ \\
\hline $\begin{array}{l}\text { Borges, } \\
2014^{23}\end{array}$ & Low risk & Low risk & Unknown & Low risk & High risk & Unknown & Low risk & $\begin{array}{l}\text { Intermediate } \\
\text { risk }\end{array}$ \\
\hline Brown, $1987^{24}$ & Unknown & Unknown & High risk & Unknown & Unknown & Unknown & Unknown & High risk \\
\hline $\begin{array}{l}\text { Brusse- } \\
\text { Keizer, } 2014^{5}\end{array}$ & Low risk & Low risk & Low risk & High risk & Low risk & Unknown & Low risk & Low risk \\
\hline $\begin{array}{l}\text { Centanni, } \\
2002^{25}\end{array}$ & Unknown & Unknown & Low risk & Unknown & Low risk & Low risk & Unknown & High risk \\
\hline Cox, $2018^{26}$ & Low risk & Low risk & High risk & Low risk & High risk & Unknown & Low risk & $\begin{array}{l}\text { Intermediate } \\
\text { risk }\end{array}$ \\
\hline
\end{tabular}




\begin{tabular}{|c|c|c|c|c|c|c|c|c|}
\hline Author, Year & $\begin{array}{c}\text { Sequence } \\
\text { Generation }\end{array}$ & $\begin{array}{c}\text { Allocation } \\
\text { Concealme } \\
\text { nt }\end{array}$ & $\begin{array}{l}\text { Blinding of } \\
\text { Participants } \\
\text {, Personnel }\end{array}$ & $\begin{array}{c}\text { Blinding of } \\
\text { Outcome } \\
\text { Assessors }\end{array}$ & $\begin{array}{c}\text { Incomplete } \\
\text { Outcome } \\
\text { Data }\end{array}$ & $\begin{array}{c}\text { Selective } \\
\text { Outcome } \\
\text { Reporting }\end{array}$ & $\begin{array}{c}\text { Other } \\
\text { Sources of } \\
\text { Bias }\end{array}$ & Overall RoB \\
\hline Cross, $2012^{27}$ & Low risk & Low risk & Unknown & Unknown & High risk & Unknown & Low risk & $\begin{array}{l}\text { Intermediate } \\
\text { risk }\end{array}$ \\
\hline $\begin{array}{l}\text { Daniels, } \\
2010^{6}\end{array}$ & Low risk & Unknown & Low risk & Unknown & High risk & Unknown & High risk & High risk \\
\hline Dark, $1993^{72}$ & Unknown & Unknown & High risk & Unknown & Low risk & Unknown & High risk & High risk \\
\hline Davies, $1999^{7}$ & Low risk & Low risk & Low risk & Low risk & High risk & Low risk & High risk & $\begin{array}{l}\text { Intermediate } \\
\text { risk }\end{array}$ \\
\hline $\begin{array}{l}\text { de Jong, } \\
2007^{98}\end{array}$ & Low risk & Unknown & Low risk & Unknown & High risk & Unknown & Low risk & High risk \\
\hline Ding, 2016 & Low risk & High risk & High risk & Unknown & Low risk & Unknown & Low risk & $\begin{array}{l}\text { Intermediate } \\
\text { risk }\end{array}$ \\
\hline Du, $2018^{28}$ & Unknown & Unknown & High risk & High risk & Low risk & Low risk & Low risk & High risk \\
\hline Duffy, $2005^{29}$ & Low risk & Low risk & Low risk & Low risk & Low risk & Unknown & Unknown & Low risk \\
\hline Eaton, $2009^{30}$ & Low risk & Unknown & High risk & Low risk & High risk & Unknown & Low risk & High risk \\
\hline $\begin{array}{l}\text { Edwards, } \\
2013^{31}\end{array}$ & Low risk & Low risk & Low risk & Unknown & Low risk & Unknown & Low risk & Low risk \\
\hline $\begin{array}{l}\text { Emami } \\
\text { Ardestani, } \\
2017^{74}\end{array}$ & Unknown & Unknown & High risk & Unknown & Unknown & Unknown & Low risk & High risk \\
\hline $\begin{array}{l}\text { Emerman, } \\
1989^{8}\end{array}$ & Low risk & Unknown & Low risk & Low risk & Low risk & Unknown & High risk & $\begin{array}{l}\text { Intermediate } \\
\text { risk }\end{array}$ \\
\hline Giusti, $2016^{75}$ & Low risk & Unknown & High risk & Low risk & High risk & Unknown & High risk & High risk \\
\hline $\begin{array}{l}\text { Goktalay, } \\
2013^{32}\end{array}$ & High risk & Unknown & Low risk & Low risk & High risk & Unknown & High risk & High risk \\
\hline $\begin{array}{l}\text { Greening, } \\
2014^{33}\end{array}$ & Unknown & Unknown & High risk & High risk & Low risk & Unknown & Low risk & High risk \\
\hline $\begin{array}{l}\text { Greulich, } \\
2014^{34}\end{array}$ & Low risk & Low risk & High risk & Low risk & High risk & Unknown & Low risk & $\begin{array}{l}\text { Intermediate } \\
\text { risk }\end{array}$ \\
\hline Gunen, $2007^{9}$ & Unknown & Unknown & Unknown & Unknown & High risk & Unknown & Low risk & High risk \\
\hline $\begin{array}{l}\text { Hamacher, } \\
1995^{76}\end{array}$ & High risk & High risk & Unknown & Unknown & Low risk & High risk & Unknown & High risk \\
\hline $\begin{array}{l}\text { Hasani, } \\
1998^{77}\end{array}$ & Unknown & Unknown & Low risk & Unknown & Unknown & Unknown & High risk & High risk \\
\hline $\begin{array}{l}\text { Hassan, } \\
2015^{10}\end{array}$ & Unknown & Unknown & Low risk & Unknown & Unknown & Unknown & High risk & High risk \\
\hline $\mathrm{He}, 2015^{35}$ & Unknown & High risk & High risk & High risk & Low risk & Unknown & Low risk & High risk \\
\hline $\begin{array}{l}\text { Kirsten, } \\
1998^{37}\end{array}$ & Unknown & Unknown & High risk & High risk & Low risk & Unknown & Unknown & High risk \\
\hline Kodric, $2009^{36}$ & Unknown & Unknown & Unknown & Unknown & High risk & Unknown & Unknown & High risk \\
\hline
\end{tabular}




\begin{tabular}{|c|c|c|c|c|c|c|c|c|}
\hline Author, Year & $\begin{array}{c}\text { Sequence } \\
\text { Generation }\end{array}$ & $\begin{array}{c}\text { Allocation } \\
\text { Concealme } \\
\text { nt }\end{array}$ & $\begin{array}{l}\text { Blinding of } \\
\text { Participants } \\
\text {, Personnel }\end{array}$ & $\begin{array}{c}\text { Blinding of } \\
\text { Outcome } \\
\text { Assessors }\end{array}$ & $\begin{array}{c}\text { Incomplete } \\
\text { Outcome } \\
\text { Data }\end{array}$ & $\begin{array}{c}\text { Selective } \\
\text { Outcome } \\
\text { Reporting }\end{array}$ & $\begin{array}{c}\text { Other } \\
\text { Sources of } \\
\text { Bias }\end{array}$ & Overall RoB \\
\hline $\begin{array}{l}\text { Koutsogiannis } \\
, 2000^{65}\end{array}$ & Low risk & Unknown & Low risk & Unknown & Unknown & Unknown & Unknown & $\begin{array}{l}\text { Intermediate } \\
\text { risk }\end{array}$ \\
\hline Kurzaj, $2013^{38}$ & Unknown & Unknown & High risk & Unknown & Unknown & Unknown & Unknown & High risk \\
\hline $\begin{array}{l}\text { Lellouche, } \\
2016^{39}\end{array}$ & Low risk & Low risk & High risk & Unknown & Low risk & Unknown & Unknown & $\begin{array}{l}\text { Intermediate } \\
\text { risk }\end{array}$ \\
\hline $\begin{array}{l}\text { Leophonte, } \\
1998^{78}\end{array}$ & Unknown & Unknown & High risk & Unknown & Low risk & Low risk & High risk & High risk \\
\hline $\begin{array}{l}\text { Leuppi, } \\
2013^{79}\end{array}$ & Low risk & Low risk & Low risk & Low risk & Low risk & Unknown & Unknown & Low risk \\
\hline Liao, $2015^{40}$ & Unknown & Unknown & Unknown & Low risk & Low risk & Unknown & Low risk & High risk \\
\hline Llor, $2009^{80}$ & Unknown & Unknown & Low risk & Unknown & Low risk & Unknown & Low risk & High risk \\
\hline Llor, $2012^{11}$ & Low risk & Low risk & Low risk & Unknown & Low risk & Low risk & Unknown & Low risk \\
\hline $\begin{array}{l}\text { Mahmoud } \\
\text { Abd El Hafiz, } \\
2013^{41}\end{array}$ & Unknown & Unknown & Low risk & Unknown & Unknown & Unknown & Unknown & High risk \\
\hline $\begin{array}{l}\text { Maltais, } \\
2002^{12}\end{array}$ & Unknown & Unknown & Low risk & Unknown & High risk & Unknown & High risk & High risk \\
\hline Mirici, $2003^{81}$ & Low risk & Low risk & Low risk & Unknown & Low risk & Unknown & Unknown & Low risk \\
\hline $\begin{array}{l}\text { Moayyedi, } \\
1995^{66}\end{array}$ & Unknown & Unknown & Low risk & Unknown & High risk & Unknown & Low risk & High risk \\
\hline $\begin{array}{l}\text { Moretti, } \\
2015^{42}\end{array}$ & Unknown & Unknown & High risk & Unknown & Low risk & Low risk & High risk & High risk \\
\hline $\begin{array}{l}\text { Mukerji, } \\
2015^{43}\end{array}$ & Low risk & Low risk & Low risk & Unknown & Low risk & Unknown & Low risk & Low risk \\
\hline $\begin{array}{l}\text { Niewoehner, } \\
1999^{13}\end{array}$ & Unknown & Unknown & Low risk & Unknown & Low risk & Low risk & High risk & High risk \\
\hline $\begin{array}{l}\text { Ogasawara, } \\
2018^{44}\end{array}$ & Low risk & Low risk & Unknown & Unknown & Low risk & Unknown & Low risk & Low risk \\
\hline Oncu, $2017^{45}$ & Low risk & Unknown & High risk & Unknown & High risk & Unknown & Low risk & High risk \\
\hline $\begin{array}{l}\text { Osadnik, } \\
2014^{46}\end{array}$ & Low risk & Low risk & High risk & Low risk & Low risk & Unknown & Low risk & Low risk \\
\hline Perri, $1985^{67}$ & Unknown & Unknown & Unknown & Unknown & Unknown & Unknown & Unknown & High risk \\
\hline $\begin{array}{l}\text { Petitpretz, } \\
2007^{82}\end{array}$ & Low risk & Unknown & High risk & High risk & High risk & Unknown & High risk & High risk \\
\hline $\begin{array}{l}\text { Phillips, } \\
1993^{83}\end{array}$ & Unknown & Unknown & High risk & Low risk & High risk & Unknown & Unknown & High risk \\
\hline $\begin{array}{l}\text { Pourrashid, } \\
2018^{47}\end{array}$ & Low risk & Unknown & Low risk & Unknown & High risk & Unknown & Low risk & High risk \\
\hline Rhee, $2015^{84}$ & Low risk & Unknown & Low risk & Low risk & High risk & Unknown & High risk & High risk \\
\hline Rice, $1987^{48}$ & Low risk & Low risk & Low risk & Low risk & Low risk & Unknown & Unknown & Low risk \\
\hline
\end{tabular}




\begin{tabular}{|c|c|c|c|c|c|c|c|c|}
\hline Author, Year & $\begin{array}{c}\text { Sequence } \\
\text { Generation }\end{array}$ & $\begin{array}{c}\text { Allocation } \\
\text { Concealme } \\
\text { nt }\end{array}$ & $\begin{array}{l}\text { Blinding of } \\
\text { Participants } \\
\text {, Personnel }\end{array}$ & $\begin{array}{l}\text { Blinding of } \\
\text { Outcome } \\
\text { Assessors }\end{array}$ & $\begin{array}{c}\text { Incomplete } \\
\text { Outcome } \\
\text { Data }\end{array}$ & $\begin{array}{l}\text { Selective } \\
\text { Outcome } \\
\text { Reporting }\end{array}$ & $\begin{array}{c}\text { Other } \\
\text { Sources of } \\
\text { Bias }\end{array}$ & Overall RoB \\
\hline $\begin{array}{l}\text { Rizzato, } \\
1998^{85}\end{array}$ & Unknown & Unknown & Low risk & Unknown & Unknown & Unknown & High risk & High risk \\
\hline $\begin{array}{l}\text { Roede, } \\
2007^{86}\end{array}$ & Low risk & Unknown & Low risk & Unknown & Low risk & Unknown & Unknown & $\begin{array}{l}\text { Intermediate } \\
\text { risk }\end{array}$ \\
\hline $\begin{array}{l}\text { Ruiz- } \\
\text { Gonzalez, } \\
2007^{87} \\
\end{array}$ & Low risk & Unknown & Unknown & Unknown & Unknown & Unknown & Unknown & High risk \\
\hline $\begin{array}{l}\text { Sanjari, } \\
2015^{49} \\
\end{array}$ & Low risk & Low risk & Low risk & Low risk & High risk & Low risk & High risk & $\begin{array}{l}\text { Intermediate } \\
\text { risk }\end{array}$ \\
\hline $\begin{array}{l}\text { Saudny- } \\
\text { Unterberger, } \\
1997^{50}\end{array}$ & Unknown & Unknown & High risk & Low risk & High risk & Unknown & Unknown & High risk \\
\hline $\begin{array}{l}\text { Sayiner, } \\
2001^{88}\end{array}$ & Low risk & Low risk & High risk & Unknown & Low risk & Unknown & Unknown & $\begin{array}{l}\text { Intermediate } \\
\text { risk }\end{array}$ \\
\hline $\begin{array}{l}\text { Seidenfeld, } \\
1984^{51}\end{array}$ & Low risk & Low risk & Low risk & Unknown & Unknown & Unknown & Unknown & $\begin{array}{l}\text { Intermediate } \\
\text { risk }\end{array}$ \\
\hline $\begin{array}{l}\text { Skorodin, } \\
1995^{52}\end{array}$ & Unknown & Unknown & Low risk & Unknown & Unknown & Unknown & Unknown & High risk \\
\hline $\begin{array}{l}\text { Solooki, } \\
2014^{53}\end{array}$ & Unknown & Unknown & Low risk & Unknown & Unknown & Unknown & Low risk & High risk \\
\hline $\begin{array}{l}\text { Soltaninejad, } \\
2016^{54}\end{array}$ & Unknown & Unknown & Low risk & Unknown & Unknown & Unknown & Low risk & High risk \\
\hline $\begin{array}{l}\text { Stallberg, } \\
2009^{89}\end{array}$ & Low risk & Low risk & Low risk & High risk & Low risk & Unknown & High risk & $\begin{array}{l}\text { Intermediate } \\
\text { risk }\end{array}$ \\
\hline Sun, $2015^{90}$ & Unknown & Unknown & Unknown & Unknown & Low risk & Unknown & Low risk & High risk \\
\hline Tang, $2012^{55}$ & Low risk & Low risk & High risk & Low risk & Low risk & Unknown & Unknown & Low risk \\
\hline $\begin{array}{l}\text { Thompson, } \\
1996^{14}\end{array}$ & Unknown & Unknown & Low risk & Unknown & Unknown & Unknown & Unknown & High risk \\
\hline $\begin{array}{l}\text { Torres- } \\
\text { Sanchez, } \\
2017^{56}\end{array}$ & Low risk & High risk & High risk & Low risk & Low risk & Low risk & Low risk & $\begin{array}{l}\text { Intermediate } \\
\text { risk }\end{array}$ \\
\hline $\begin{array}{l}\text { Torres- } \\
\text { Sanchez, } \\
2017^{57} \\
\end{array}$ & Low risk & Low risk & High risk & Unknown & Low risk & High risk & Low risk & Low risk \\
\hline $\begin{array}{l}\text { Troosters, } \\
2010^{58} \\
\end{array}$ & Low risk & Unknown & High risk & High risk & High risk & Unknown & High risk & High risk \\
\hline Tumer, $2009^{59}$ & High risk & Unknown & High risk & High risk & Low risk & Unknown & Unknown & High risk \\
\hline Ucar, $2014^{91}$ & Low risk & Unknown & Unknown & Unknown & High risk & Low risk & Unknown & High risk \\
\hline Umut, $1999^{92}$ & Unknown & Unknown & Unknown & Unknown & Unknown & Unknown & Unknown & High risk \\
\hline $\begin{array}{l}\text { van Velzen, } \\
2017^{15}\end{array}$ & Low risk & Low risk & Low risk & Low risk & Low risk & Low risk & Low risk & Low risk \\
\hline
\end{tabular}




\begin{tabular}{|c|c|c|c|c|c|c|c|c|}
\hline Author, Year & $\begin{array}{l}\text { Sequence } \\
\text { Generation }\end{array}$ & $\begin{array}{c}\text { Allocation } \\
\text { Concealme } \\
\text { nt }\end{array}$ & $\begin{array}{l}\text { Blinding of } \\
\text { Participants } \\
\text {, Personnel }\end{array}$ & $\begin{array}{c}\text { Blinding of } \\
\text { Outcome } \\
\text { Assessors }\end{array}$ & $\begin{array}{c}\text { Incomplete } \\
\text { Outcome } \\
\text { Data }\end{array}$ & $\begin{array}{l}\text { Selective } \\
\text { Outcome } \\
\text { Reporting }\end{array}$ & $\begin{array}{c}\text { Other } \\
\text { Sources of } \\
\text { Bias }\end{array}$ & Overall RoB \\
\hline $\begin{array}{l}\text { van Zanten, } \\
2007^{93}\end{array}$ & Unknown & Unknown & High risk & High risk & Low risk & Unknown & High risk & High risk \\
\hline $\begin{array}{l}\text { Vermeeren, } \\
2004^{60}\end{array}$ & Unknown & Unknown & Low risk & Unknown & High risk & Unknown & Low risk & High risk \\
\hline Wang, $2016^{16}$ & Low risk & Low risk & Unknown & Low risk & Low risk & Unknown & Low risk & Low risk \\
\hline $\begin{array}{l}\text { Whitlock, } \\
1995^{94}\end{array}$ & Unknown & Unknown & High risk & Unknown & High risk & Unknown & Unknown & High risk \\
\hline $\begin{array}{l}\text { Willaert, } \\
2002^{95}\end{array}$ & Unknown & Unknown & Unknown & Unknown & High risk & Unknown & Unknown & High risk \\
\hline $\begin{array}{l}\text { Wilson, } \\
2012^{96}\end{array}$ & Low risk & Unknown & Low risk & Unknown & High risk & Unknown & Low risk & High risk \\
\hline $\begin{array}{l}\text { Woodruff, } \\
2011^{61}\end{array}$ & Unknown & Unknown & High risk & Unknown & High risk & Unknown & Low risk & High risk \\
\hline Xiong, $2008^{62}$ & Unknown & Unknown & Unknown & Unknown & Unknown & Unknown & Unknown & High risk \\
\hline $\begin{array}{l}\text { Yohannes, } \\
2003^{63}\end{array}$ & Unknown & Unknown & High risk & High risk & Low risk & Unknown & Unknown & High risk \\
\hline Yoon, $2013^{97}$ & Low risk & Unknown & Unknown & Unknown & Low risk & Unknown & Low risk & $\begin{array}{l}\text { Intermediate } \\
\text { risk }\end{array}$ \\
\hline Zuin, $2005^{64}$ & Low risk & Unknown & Low risk & Unknown & Unknown & Unknown & Unknown & $\begin{array}{l}\text { Intermediate } \\
\text { risk }\end{array}$ \\
\hline
\end{tabular}

ROB = risk of bias 


\section{Appendix F. Results From Included Studies}

Table F.1. KQ1: Intervention description and conclusions

\begin{tabular}{|c|c|c|c|c|c|c|}
\hline $\begin{array}{l}\text { Author, Year, } \\
\text { study design* }\end{array}$ & $\begin{array}{c}\text { Study setting } \\
\text { (outpatients, } \\
\text { hospitalized } \\
\text { patients) }\end{array}$ & $\begin{array}{l}\text { Length of } \\
\text { Followup } \\
\text { (months) }\end{array}$ & $\begin{array}{l}\text { Intervention(s) } \\
\text { and comparator }\end{array}$ & $\begin{array}{c}\text { Route of } \\
\text { administration }\end{array}$ & Dose and Duration & Conclusion \\
\hline \multirow[t]{2}{*}{ Aaron, $2003^{1}$} & \multirow[t]{2}{*}{$\begin{array}{l}\text { Emergency } \\
\text { department }\end{array}$} & \multirow[t]{2}{*}{1} & 1) Prednisone & Oral & $\begin{array}{l}40 \mathrm{mg}, 1 \times / 24 \text { hours } \\
\text { (total of } 40 \mathrm{mg} / 24 \\
\text { hours) for } 10 \text { days }\end{array}$ & \multirow[b]{2}{*}{$\begin{array}{l}\text { Patients in the Prednisone } \\
\text { group were found to have } \\
\text { statistically significantly } \\
\text { more improvements in } \\
\text { FEV1 absolute values, } \\
\text { and dyspnea (Chronic } \\
\text { Respiratory Disease Index } \\
\text { Questionnaire) than } \\
\text { patients in the placebo } \\
\text { group. There was no } \\
\text { statistically significant } \\
\text { difference in } 30 \text {-day } \\
\text { relapse, quality of life } \\
\text { (Chronic Respiratory } \\
\text { Disease Index } \\
\text { Questionnaire.), } \\
\text { hospitalizations, and } \\
\text { mortality. Patients in the } \\
\text { prednisone group were } \\
\text { statistically significantly } \\
\text { more likely to report } \\
\text { increase in appetite, } \\
\text { weight gain than patients } \\
\text { in the placebo group. } \\
\text { Serious adverse events } \\
\text { requiring hospitalization } \\
\text { were reported two cases } \\
\text { in the prednisone group (1 } \\
\text { for epistaxis, } 1 \text { for seizure } \\
\text { and } 1 \text { case in the placebo } \\
\text { group ( } 1 \text { for } \\
\text { schizophrenia). }\end{array}$} \\
\hline & & & 2) Placebo & Oral & $\begin{array}{l}\text { Placebo, } 1 x / 24 \text { hours } \\
\text { for } 10 \text { days }\end{array}$ & \\
\hline
\end{tabular}




\begin{tabular}{|c|c|c|c|c|c|c|}
\hline $\begin{array}{l}\text { Author, Year, } \\
\text { study design* }\end{array}$ & $\begin{array}{c}\text { Study setting } \\
\text { (outpatients, } \\
\text { hospitalized } \\
\text { patients) }\end{array}$ & $\begin{array}{l}\text { Length of } \\
\text { Followup } \\
\text { (months) }\end{array}$ & $\begin{array}{l}\text { Intervention(s) } \\
\text { and comparator }\end{array}$ & $\begin{array}{c}\text { Route of } \\
\text { administration }\end{array}$ & Dose and Duration & Conclusion \\
\hline \multirow[t]{2}{*}{ Albert, $1980^{2}$} & \multirow[t]{2}{*}{$\begin{array}{l}\text { Inpatient } \\
\text { hospital floor }\end{array}$} & \multirow[t]{2}{*}{ N/A } & $\begin{array}{l}\text { 1) } \\
\text { Methylprednisolo } \\
\text { ne }\end{array}$ & IV & $\begin{array}{l}0.5 \mathrm{mg} / \mathrm{kg}, 4 \mathrm{x} / 24 \text { hours } \\
\text { (total of } \\
2 \mathrm{mg} / \mathrm{kg} / 24 \text { hours) for } 3 \\
\text { days }\end{array}$ & \multirow{2}{*}{$\begin{array}{l}\text { The methylprednisolone } \\
\text { group had a significantly } \\
\text { larger increase in pre- and } \\
\text { post-bronchodilator FEV1 } \\
\text { than the placebo group. } \\
\text { There was no statistically } \\
\text { significant difference in } \\
\text { mortality. }\end{array}$} \\
\hline & & & 2) Placebo & IV & $\begin{array}{l}\text { Placebo } 4 x / 24 \text { hours } \\
\text { for } 3 \text { days }\end{array}$ & \\
\hline \multirow[t]{2}{*}{$\begin{array}{l}\text { Anthonisen, } \\
1987^{3}\end{array}$} & \multirow[t]{2}{*}{ Outpatient } & \multirow[t]{2}{*}{ N/A } & 1) Placebo & Oral & $\begin{array}{l}\text { placebo matching } \\
\text { prescribed antibiotic } \\
10 \text { days }\end{array}$ & \multirow{2}{*}{$\begin{array}{l}\text { The antibiotic group was } \\
\text { found to have significantly } \\
\text { more treatment success } \\
\text { and less treatment failures } \\
\text { than the placebo group. } \\
\text { There was no statistically } \\
\text { significant difference in } \\
\text { the incidence of adverse } \\
\text { events. }\end{array}$} \\
\hline & & & 2) Antibiotics & Oral & $\begin{array}{l}\text { Trimethoprim- } \\
\text { sulfamethoxazole: } \\
\text { 160mg,800mg, } \\
2 \times / 24 \text { hours (total of } \\
320 \mathrm{mg} / 24 \text { hours, } \\
1600 \mathrm{mg} / 24 \mathrm{hours} \text { ) for } \\
10 \text { days. } \\
\text { or } \\
\text { Amoxicillin: } 0.25 \mathrm{~g}, \\
4 \times / 24 \text { hours (total of } \\
1 \mathrm{~g} / 24 \text { hours) for } 10 \\
\text { days } \\
\text { or } \\
\text { Doxycycline: } 200 \mathrm{mg} \\
\text { initially, followed by } \\
0.1 \mathrm{~g}, 1 \times 24 \mathrm{hours} \text { (total } \\
\text { of } 0.1 \mathrm{~g} / 24 \mathrm{hours} \text { ) for } 10 \\
\text { days }\end{array}$ & \\
\hline \multirow[t]{2}{*}{$\begin{array}{l}\text { Bathoorn, } \\
2008^{4} \\
\text { Non-inferiority } \\
\text { trial }\end{array}$} & \multirow[t]{2}{*}{$\begin{array}{l}\text { Outpatient } \\
\text { Inpatient } \\
\text { hospital floor }\end{array}$} & \multirow[t]{2}{*}{3} & $\begin{array}{l}\text { 1) Budesonide + } \\
\text { Formoterol }\end{array}$ & $\begin{array}{l}\text { Inhalation and } \\
\text { Oral }\end{array}$ & $\begin{array}{l}160 \mu \mathrm{g}, 4.5 \mu \mathrm{g}, \\
4 \times / 24 \mathrm{hours} \text { (total of } \\
640 \mu \mathrm{g}, 189 \mu \mathrm{g} / 24 \mathrm{hours} \text { ) } \\
\text { for } 14 \text { days. }\end{array}$ & \multirow{2}{*}{$\begin{array}{l}\text { Patients in the budesonide } \\
+ \text { formoterol group and } \\
\text { patients in the } \\
\text { prednisolone group had } \\
\text { significantly better } \\
\text { symptom scores (Total } \\
\text { Symptom Score) than }\end{array}$} \\
\hline & & & 2) Prednisolone & $\begin{array}{l}\text { Inhalation and } \\
\text { Oral }\end{array}$ & $\begin{array}{l}30 \mathrm{mg}, 1 \times / 24 \text { hours } \\
\text { (total of }\end{array}$ & \\
\hline
\end{tabular}




\begin{tabular}{|c|c|c|c|c|c|c|}
\hline $\begin{array}{l}\text { Author, Year, } \\
\text { study design* }\end{array}$ & $\begin{array}{c}\text { Study setting } \\
\text { (outpatients, } \\
\text { hospitalized } \\
\text { patients) }\end{array}$ & $\begin{array}{l}\text { Length of } \\
\text { Followup } \\
\text { (months) }\end{array}$ & $\begin{array}{l}\text { Intervention(s) } \\
\text { and comparator }\end{array}$ & $\begin{array}{c}\text { Route of } \\
\text { administration }\end{array}$ & Dose and Duration & Conclusion \\
\hline & & & & & $\begin{array}{l}\text { 30mg/24hours) for } 14 \\
\text { days }\end{array}$ & \multirow[b]{2}{*}{$\begin{array}{l}\text { those in the placebo } \\
\text { group. } \\
\text { Patients in the } \\
\text { Budesonide + Formoterol } \\
\text { group had significantly } \\
\text { better quality of life } \\
\text { (Chronic Respiratory } \\
\text { Disease Questionnaire) } \\
\text { than those in the } \\
\text { prednisolone group } \\
\text { (p=0.02); however the } \\
\text { difference between the } \\
\text { budesonide + formoterol } \\
\text { group and the placebo } \\
\text { group was not significant. } \\
\text { There was no statistically } \\
\text { significant difference in } \\
\text { FEV1, treatment failures } \\
\text { between the groups. No } \\
\text { hospital admission was } \\
\text { reported in any of the } \\
\text { three groups. }\end{array}$} \\
\hline & & & 3) Placebo & $\begin{array}{l}\text { Inhalation and } \\
\text { Oral }\end{array}$ & 14 days & \\
\hline \multirow[t]{2}{*}{$\begin{array}{l}\text { Brusse-Keizer, } \\
2014^{5}\end{array}$} & \multirow[t]{2}{*}{ Outpatient } & \multirow[t]{2}{*}{4} & 1) Antibiotics & Oral & $\begin{array}{l}\text { Amoxicillin/Clavulanic } \\
\text { Acid: } 500 \mathrm{mg}, 125 \mathrm{mg} \text {, } \\
3 \times / 24 \text { hours (total of } \\
\text { 1500mg,375mg/24hou } \\
\text { rs) for } 7 \text { days }\end{array}$ & \multirow{2}{*}{$\begin{array}{l}\text { There was no statistically } \\
\text { significant difference } \\
\text { between the two groups in } \\
\text { dyspnea (Chronic } \\
\text { Respiratory Disease } \\
\text { Questionnaire), repeat } \\
\text { exacerbation, quality of } \\
\text { life (Chronic Respiratory } \\
\text { Disease Questionnaire), } \\
\text { FEV1, total symptom } \\
\text { scores (Clinical COPD } \\
\text { Questionnaire), and } \\
\text { relapse of ECOPD. }\end{array}$} \\
\hline & & & 2) Placebo & Oral & $3 x / 24$ hours for 7 days & \\
\hline
\end{tabular}




\begin{tabular}{|c|c|c|c|c|c|c|}
\hline $\begin{array}{l}\text { Author, Year, } \\
\text { study design* }\end{array}$ & $\begin{array}{c}\text { Study setting } \\
\text { (outpatients, } \\
\text { hospitalized } \\
\text { patients) }\end{array}$ & $\begin{array}{l}\text { Length of } \\
\text { Followup } \\
\text { (months) }\end{array}$ & $\begin{array}{l}\text { Intervention(s) } \\
\text { and comparator }\end{array}$ & $\begin{array}{c}\text { Route of } \\
\text { administration }\end{array}$ & Dose and Duration & Conclusion \\
\hline \multirow[t]{2}{*}{ Daniels, $2010^{6}$} & $\begin{array}{l}\text { Inpatient } \\
\text { hospital floor }\end{array}$ & 1 & 1) Doxycycline & Oral & $\begin{array}{l}\text { 200mg, } 1 \times / 24 \text { hours } \\
\text { (total of } \\
200 \mathrm{mg} / 24 \text { hours) for } 7 \\
\text { days }\end{array}$ & \multirow{2}{*}{$\begin{array}{l}\text { At the end of treatment, } \\
\text { patients in the doxycycline } \\
\text { group were more likely to } \\
\text { have clinical cure, better } \\
\text { cough score (VAS), better } \\
\text { total symptom score (Tota } \\
\text { Symptom Score), and } \\
\text { better dyspnea (VAS) than } \\
\text { those in the placebo } \\
\text { group. At the end of the } \\
\text { followup, there was no } \\
\text { statistically significant } \\
\text { difference between } \\
\text { doxycycline and placebo } \\
\text { in clinical cure, FEV1, tota } \\
\text { symptom scores, } \\
\text { dyspnea, cough, and } \\
\text { death. }\end{array}$} \\
\hline & & & 2) Placebo & Oral & $\begin{array}{l}\text { 200mg, } 1 \times / 24 \text { hours } \\
\text { (total of } \\
200 \mathrm{mg} / 24 \text { hours) for } 7 \\
\text { days }\end{array}$ & \\
\hline \multirow[t]{2}{*}{ Davies, $1999^{7}$} & \multirow[t]{2}{*}{$\begin{array}{l}\text { Emergency } \\
\text { department, } \\
\text { Inpatient } \\
\text { hospital floor }\end{array}$} & \multirow[t]{2}{*}{1.5} & 1) Prednisolone & Oral & $\begin{array}{l}\text { 30mg, 1x/24hours } \\
\text { (total of } \\
\text { 30mg/24hours) for } 14 \\
\text { days }\end{array}$ & \multirow{2}{*}{$\begin{array}{l}\text { Upon hospital discharge, } \\
\text { the patients in the } \\
\text { prednisolone group had } \\
\text { significantly more increase } \\
\text { in \% predicted FEV1 than } \\
\text { the placebo group. There } \\
\text { was no statistically } \\
\text { significant difference in } \\
\text { repeat exacerbation and } \\
\text { hospital readmissions. }\end{array}$} \\
\hline & & & 2) Placebo & Oral & & \\
\hline \multirow[t]{2}{*}{$\begin{array}{l}\text { Emerman, } \\
1989^{8}\end{array}$} & \multirow[t]{2}{*}{$\begin{array}{l}\text { Emergency } \\
\text { department }\end{array}$} & \multirow[t]{2}{*}{ N/A } & $\begin{array}{l}\text { 1) } \\
\text { Methylprednisolo } \\
\text { ne }\end{array}$ & IV & $\begin{array}{l}\text { 100mg for one time } \\
\text { treatment for } 1 \text { day. }\end{array}$ & \multirow[t]{2}{*}{$\begin{array}{l}\text { There was no statistically } \\
\text { significant difference in \% } \\
\text { predicted FEV1 values } \\
\text { and repeat exacerbation. }\end{array}$} \\
\hline & & & 2) Placebo & IV & $\begin{array}{l}\text { 100mg for one time } \\
\text { treatment for } 1 \text { day. }\end{array}$ & \\
\hline Gunen, $2007^{9}$ & $\begin{array}{l}\text { Inpatient } \\
\text { hospital floor }\end{array}$ & 1 & $\begin{array}{l}\text { 1) } \\
\text { Management } \\
\text { without Systemic } \\
\text { Corticosteroids }\end{array}$ & Inhalation & & $\begin{array}{l}\text { Patients in the nebulized } \\
\text { budesonide group had } \\
\text { statistically significant } \\
\text { higher FEV1 \% predicted }\end{array}$ \\
\hline
\end{tabular}




\begin{tabular}{|c|c|c|c|c|c|c|}
\hline $\begin{array}{l}\text { Author, Year, } \\
\text { study design* }\end{array}$ & $\begin{array}{c}\text { Study setting } \\
\text { (outpatients, } \\
\text { hospitalized } \\
\text { patients) }\end{array}$ & $\begin{array}{l}\text { Length of } \\
\text { Followup } \\
\text { (months) }\end{array}$ & $\begin{array}{l}\text { Intervention(s) } \\
\text { and comparator }\end{array}$ & $\begin{array}{c}\text { Route of } \\
\text { administration }\end{array}$ & Dose and Duration & Conclusion \\
\hline & & & $\begin{array}{l}\text { 2) } \\
\text { Prednisolone }\end{array}$ & IV & $\begin{array}{l}40 \mathrm{mg}, 1 \times / 24 \text { hours } \\
\text { (total of } \\
40 \mathrm{mg} / 24 \text { hours) for } \\
\text { more than } 10 \text { days }\end{array}$ & \multirow{2}{*}{$\begin{array}{l}\text { than those in the } \\
\text { prednisolone group or the } \\
\text { management without } \\
\text { systemic corticosteroids } \\
\text { group. There was no } \\
\text { statistically significant } \\
\text { difference in hospital } \\
\text { readmissions, repeat } \\
\text { exacerbations, \% } \\
\text { predicted FEV1, and } \\
\text { death. }\end{array}$} \\
\hline & & & $\begin{array}{l}\text { 3) Nebulized } \\
\text { Budesonide }\end{array}$ & Nebulized & $\begin{array}{l}1500 \mu g, 4 \times / 24 \text { hours } \\
\text { (total of } \\
0.5 \mathrm{mg} / 24 \text { hours) }\end{array}$ & \\
\hline \multirow[t]{2}{*}{ Hassan, $2015^{10}$} & \multirow[t]{2}{*}{ Outpatient } & \multirow[t]{2}{*}{21 days } & 1)Placebo & N/A & Matching placebo & \multirow{2}{*}{$\begin{array}{l}\text { Patients in the antibiotics } \\
\text { group were found to have } \\
\text { significantly higher clinical } \\
\text { cures and lower clinical } \\
\text { failures than patients in } \\
\text { the placebo group. There } \\
\text { was no statistically } \\
\text { significant difference in } \\
\text { FEV1 values. }\end{array}$} \\
\hline & & & 2) Antibiotic & $\mathrm{N} / \mathrm{A}$ & $\begin{array}{l}\text { Quinolone: } 500 \mathrm{mg}, \\
2 \times / 24 \text { hours (total of } \\
1000 \mathrm{mg} / 24 \text { hours) } \\
\text { or Amoxicillin: } 500 \mathrm{mg} \text {, } \\
3 \times / 24 \text { hours (total of } \\
1500 \mathrm{mg} / 24 \text { hours) for } \\
10 \text { days }\end{array}$ & \\
\hline \multirow[t]{2}{*}{ Llor, $2012^{11}$} & \multirow[t]{2}{*}{ Outpatient } & \multirow[t]{2}{*}{12} & $\begin{array}{l}\text { 1Amoxicillin + } \\
\text { Clavulanate }\end{array}$ & Oral & $\begin{array}{l}500 \mathrm{mg}, 125 \mathrm{mg} \text {, } \\
3 \times / 24 \text { hours (total of } \\
1500 \mathrm{mg} / 24 \text { hours, } \\
375 \mathrm{mg} / 24 \text { hours) for } 8 \\
\text { days }\end{array}$ & \multirow[t]{2}{*}{$\begin{array}{l}\text { The amoxicillin }+ \\
\text { clavulanate group had } \\
\text { statistically significant } \\
\text { more clinical cure rates } \\
\text { and less clinical failures } \\
\text { than the placebo group. }\end{array}$} \\
\hline & & & 1)Placebo & Oral & & \\
\hline \multirow[t]{2}{*}{ Maltais, $2002^{12}$} & \multirow[t]{2}{*}{$\begin{array}{l}\text { Inpatient } \\
\text { hospital floor }\end{array}$} & \multirow[t]{2}{*}{0.25} & \multirow[t]{2}{*}{ 1) Budesonide } & Nebulized & $\begin{array}{l}2 \mathrm{mg}, 4 \times / 24 \text { hours (total } \\
\text { of } 8 \mathrm{mg} / 24 \text { hours for } 3 \\
\text { days }\end{array}$ & \multirow{2}{*}{$\begin{array}{l}\text { The budesonide group } \\
\text { and the prednisolone } \\
\text { group had a significantly } \\
\text { larger increase in absolute } \\
\text { FEV1 than the placebo } \\
\text { group. Increases in FEV1 } \\
\text { were not significantly } \\
\text { different in the budesonide } \\
\text { group and the } \\
\text { prednisolone group. There } \\
\text { was no statistically }\end{array}$} \\
\hline & & & & Inhaled & $\begin{array}{l}\text { Then: } \\
2000 \mu \mathrm{g}, 1 \times / 24 \text { hours } \\
\text { (total of } \\
2000 \mu \mathrm{g} / 24 \text { hours) for } 7 \\
\text { days }\end{array}$ & \\
\hline
\end{tabular}




\begin{tabular}{|c|c|c|c|c|c|c|}
\hline $\begin{array}{l}\text { Author, Year, } \\
\text { study design* }\end{array}$ & $\begin{array}{c}\text { Study setting } \\
\text { (outpatients, } \\
\text { hospitalized } \\
\text { patients) }\end{array}$ & $\begin{array}{l}\text { Length of } \\
\text { Followup } \\
\text { (months) }\end{array}$ & $\begin{array}{l}\text { Intervention(s) } \\
\text { and comparator }\end{array}$ & $\begin{array}{c}\text { Route of } \\
\text { administration }\end{array}$ & Dose and Duration & Conclusion \\
\hline & & & 2) Prednisolone & Oral & $\begin{array}{l}\text { 30mg, } 2 \times / 24 \text { hours } \\
\text { (total of } \\
60 \mathrm{mg} / 24 \text { hours) for } 3 \\
\text { days, then } \\
40 \mathrm{mg} / 24 \text { hours for } 7 \\
\text { days }\end{array}$ & \multirow[t]{4}{*}{$\begin{array}{l}\text { significant difference in } \\
\text { dyspnea (Modified Borg } \\
\text { Scale), need for } \\
\text { intubation, and death } \\
\text { between the three groups. }\end{array}$} \\
\hline & & & \multirow[t]{3}{*}{ 3) Placebo } & Nebulized & For 72 hours & \\
\hline & & & & Inhaled & For 7 days & \\
\hline & & & & Oral & $\begin{array}{l}\text { For } 72 \text { hours, then for } \\
7 \text { days }\end{array}$ & \\
\hline \multirow[t]{5}{*}{$\begin{array}{l}\text { Niewoehner, } \\
1999^{13}\end{array}$} & \multirow[t]{5}{*}{$\begin{array}{l}\text { Emergency } \\
\text { department }\end{array}$} & \multirow[t]{5}{*}{6} & \multirow[t]{2}{*}{$\begin{array}{l}\text { 1) Glucocorticoid } \\
\text { for } 8 \text { weeks }\end{array}$} & IV & $\begin{array}{l}\text { Methylprednisolone: } \\
125 \mathrm{mg}, 4 \times / 24 \text { hours } \\
\text { (total of } \\
500 \mathrm{mg} / 24 \text { hours) for } 3 \\
\text { days }\end{array}$ & \multirow{5}{*}{$\begin{array}{l}\text { Patients in the 8-week } \\
\text { glucocorticoid group and } \\
\text { 2-week glucocorticoid } \\
\text { group had significantly } \\
\text { less clinical failures than } \\
\text { the placebo group. There } \\
\text { was no statistically } \\
\text { significant difference in } \\
\text { death, FEV1, need for } \\
\text { intubations, and hospital } \\
\text { readmissions. }\end{array}$} \\
\hline & & & & Oral & $\begin{array}{l}\text { Prednisone: } 60 \mathrm{mg} \text { on } \\
\text { days } 4 \text { through } 7 \text {, } \\
40 \mathrm{mg} \text { on days } 8 \\
\text { through } 11,20 \mathrm{mg} \text { on } \\
\text { days } 12 \text { through } 43 \text {, } \\
10 \mathrm{mg} \text { on days } 44 \\
\text { through } 50 \text {, and } 5 \mathrm{mg} \\
\text { on days } 51 \text { through } 57\end{array}$ & \\
\hline & & & \multirow[t]{2}{*}{$\begin{array}{l}\text { 2) Glucocorticoid } \\
\text { for } 2 \text { week }\end{array}$} & IV & $\begin{array}{l}\text { Methylprednisolone: } \\
125 \mathrm{mg}, 4 \times / 24 \mathrm{hours} \\
\text { (total of } 500 \mathrm{mg} / 24 \mathrm{hrs} \text { ) } \\
\text { for } 3 \text { days }\end{array}$ & \\
\hline & & & & Oral & $\begin{array}{l}\text { Prednisone: } 60 \mathrm{mg} \text { on } \\
\text { days } 4 \text { through } 7, \\
40 \mathrm{mg} \text { on days } 8 \\
\text { through } 11 \text {, and } 20 \mathrm{mg} \\
\text { on days } 12 \text { through } 15 \\
\text { Placebo capsules on } \\
\text { days } 16 \text { through } 57\end{array}$ & \\
\hline & & & 3) Placebo & IV & $\begin{array}{l}5 \% \text { dextrose solution: } \\
125 \mathrm{mg}, 4 \times / 24 \mathrm{hrs} \text { (total } \\
\text { of } 500 \mathrm{mg} / 24 \mathrm{hrs} \text { ) for } 3 \\
\text { days }\end{array}$ & \\
\hline
\end{tabular}




\begin{tabular}{|c|c|c|c|c|c|c|}
\hline $\begin{array}{l}\text { Author, Year, } \\
\text { study design* }\end{array}$ & $\begin{array}{c}\text { Study setting } \\
\text { (outpatients, } \\
\text { hospitalized } \\
\text { patients) }\end{array}$ & $\begin{array}{l}\text { Length of } \\
\text { Followup } \\
\text { (months) }\end{array}$ & $\begin{array}{l}\text { Intervention(s) } \\
\text { and comparator }\end{array}$ & $\begin{array}{c}\text { Route of } \\
\text { administration }\end{array}$ & Dose and Duration & Conclusion \\
\hline & & & & Oral & $\begin{array}{l}\text { Placebo capsules on } \\
\text { days } 4 \text { through } 57\end{array}$ & \\
\hline \multirow[t]{2}{*}{$\begin{array}{l}\text { Thompson, } \\
1996^{14}\end{array}$} & \multirow[t]{2}{*}{ Outpatient } & \multirow[t]{2}{*}{$\mathrm{N} / \mathrm{A}$} & Prednisone & Oral & $\begin{array}{l}60 \mathrm{mg}, 1 \times / 24 \mathrm{hrs} \text { (total } \\
\text { of } 60 \mathrm{mg} / 24 \mathrm{hrs} \text { ) for } 3 \\
\text { days } \\
40 \mathrm{mg}, 1 \times / 24 \mathrm{hrs} \text { (total } \\
\text { of } 40 \mathrm{mg} / 24 \mathrm{hrs} \text { ) for } 3 \\
\text { days } \\
20 \mathrm{mg} 1 \times / 24 \mathrm{hrs} \text { (total of } \\
20 \mathrm{mg} / 24 \mathrm{hrs} \text { ) for } 3 \\
\text { days }\end{array}$ & \multirow[t]{2}{*}{$\begin{array}{l}\text { Patients in the prednisone } \\
\text { group had statistically } \\
\text { significant more increase } \\
\text { in absolute FEV1 values } \\
\text { and lower clinical failures } \\
\text { than patients in the } \\
\text { placebo group. There was } \\
\text { no statistically significant } \\
\text { difference in dyspnea } \\
\text { scale (VAS). }\end{array}$} \\
\hline & & & Placebo & Oral & Vitamin B6 for 9 days. & \\
\hline \multirow[t]{2}{*}{$\begin{array}{l}\text { van Velzen, } \\
2017^{15}\end{array}$} & \multirow[t]{2}{*}{ Outpatient } & \multirow[t]{2}{*}{24} & Doxycycline & Oral & $\begin{array}{l}100 \mathrm{mg}, 2 \mathrm{x} / 24 \mathrm{hrs} \text { (total } \\
\text { of } 200 \mathrm{mg} / 24 \mathrm{hrs} \text { ) for } 1 \\
\text { day. } \\
\text { Then, } 100 \mathrm{mg} \text {, } \\
1 \times 124 \mathrm{hrs} \text { (total of } \\
100 \mathrm{mg} / 24 \mathrm{hrs}) 6 \text { days. }\end{array}$ & \multirow[t]{2}{*}{$\begin{array}{l}\text { There was no statistically } \\
\text { significant difference in } \\
\text { death, clinical failures, } \\
\text { incidence of adverse } \\
\text { events, or serious adverse } \\
\text { events. }\end{array}$} \\
\hline & & & Placebo & Oral & & \\
\hline \multirow[t]{2}{*}{ Wang, $2016^{16}$} & \multirow[t]{2}{*}{$\begin{array}{l}\text { Inpatient } \\
\text { hospital floor }\end{array}$} & \multirow[t]{2}{*}{1} & Antibiotics & & $\begin{array}{l}\text { Variable and } \\
\text { determined by } \\
\text { physicians }\end{array}$ & \multirow{2}{*}{$\begin{array}{l}\text { There was no statistically } \\
\text { significant difference in } \\
\text { clinical cure, 30-day } \\
\text { mortality, FEV1 \% } \\
\text { predicted, need for } \\
\text { intubations, 30-day repeat } \\
\text { exacerbation, 30-day } \\
\text { hospital readmissions, } \\
\text { and symptom scores } \\
\text { (VAS). }\end{array}$} \\
\hline & & & $\begin{array}{l}\text { Management } \\
\text { without } \\
\text { Antibiotics }\end{array}$ & & & \\
\hline
\end{tabular}

* Study provided different numbers and conclusions. We used the numbers.

ECOPD = acute exacerbation of chronic obstructive pulmonary disease; COPD = chronic obstructive pulmonary disease; FEV1 = forced expiration volume in 1 second; $\mathrm{g}=$ gram; $\mathrm{IV}=$ intravenous; $\mathrm{Kg}=$ kilogram; $\mathrm{mg}=$ milligram; $\mu \mathrm{g}=$ microgram; $\mathrm{N} / \mathrm{A}=$ not applicable; VAS = Visual Analog Scale 
Table F.2. KQ2: Intervention description and conclusions

\begin{tabular}{|c|c|c|c|c|c|c|}
\hline $\begin{array}{l}\text { Author, Year, } \\
\text { study design* }\end{array}$ & $\begin{array}{c}\text { Study setting } \\
\text { (outpatients, hospitalized } \\
\text { patients) }\end{array}$ & 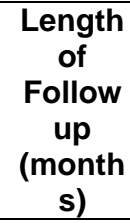 & $\begin{array}{l}\text { Intervention(s) and } \\
\text { comparator }\end{array}$ & $\begin{array}{c}\text { Route of } \\
\text { administration }\end{array}$ & Dose and Duration & Conclusion \\
\hline \multirow[t]{2}{*}{$\begin{array}{l}\text { Abreu Gonzalez, } \\
2006^{17}\end{array}$} & \multirow[t]{2}{*}{ Inpatient hospital floor } & \multirow[t]{2}{*}{$\mathrm{N} / \mathrm{A}$} & $\begin{array}{l}\text { 1) } \\
\text { Magnesium Sulfate }\end{array}$ & IV & $\begin{array}{l}1.5 \mathrm{~g} \text { dissolved in } 100 \mathrm{~mL} \text { of } \\
0.9 \% \text { saline solution over } \\
20 \mathrm{~min} \text { infusion, } 1 \times / 24 \mathrm{hrs} \text { (total } \\
\text { of } 1.5 \mathrm{~g} / 24 \mathrm{hrs} \text { ) for } 1 \text { day }\end{array}$ & \multirow{2}{*}{$\begin{array}{l}\text { There was no statistically } \\
\text { significant difference } \\
\text { between magnesium } \\
\text { sulfate and placebo in } \\
\text { FEV1 values after } 45 \\
\text { mins of application. }\end{array}$} \\
\hline & & & 2) Placebo & IV & $\begin{array}{l}100 \mathrm{~mL} \text { of } 0.9 \% \text { saline solution } \\
\text { over } 20 \mathrm{~min} \text { infusion, } 1 \times / 24 \mathrm{hrs} \\
\text { (total of } 100 \mathrm{~mL} / 24 \mathrm{hrs} \text { ) for } 1 \\
\text { day }\end{array}$ & \\
\hline \multirow[t]{2}{*}{ Austin, $2010^{18}$} & \multirow[t]{2}{*}{ Ambulance to hospital } & \multirow[t]{2}{*}{$\mathrm{N} / \mathrm{A}$} & $\begin{array}{l}\text { 1) High Flow /Free } \\
\text { Flow Oxygen }\end{array}$ & $\mathrm{N} / \mathrm{A}$ & $\begin{array}{l}\text { 8-10liters/min } \\
\text { during ambulance ride and up } \\
\text { to } 30 \text { mins in the ER (until } \\
\text { blood gas analysis was taken) }\end{array}$ & \multirow{2}{*}{$\begin{array}{l}\text { Patients in the titrated } \\
\text { oxygen group had } \\
\text { significantly lower } \\
\text { mortality than those in the } \\
\text { high flow oxygen group } \\
\text { (RR=0.22, } 95 \% \mathrm{Cl}: 0.05 \\
\text { to } 0.91) \text {. There was no } \\
\text { statistically significant } \\
\text { difference in need for } \\
\text { intubation. }\end{array}$} \\
\hline & & & 2) Titrated Oxygen & $\mathrm{N} / \mathrm{A}$ & $\begin{array}{l}\text { During ambulance ride and up } \\
\text { to } 30 \text { mins in the ER (until } \\
\text { blood gas analysis was taken) }\end{array}$ & \\
\hline \multirow[t]{2}{*}{$\begin{array}{l}\text { Ayfer Aytemur, } \\
2015^{19}\end{array}$} & \multirow[t]{2}{*}{ Inpatient hospital floor } & \multirow[t]{2}{*}{6} & $\begin{array}{l}\text { 1) } \\
\text { N-Acetylcysteine }\end{array}$ & Oral & $\begin{array}{l}200 \mathrm{mg}, 3 \mathrm{x} / 24 \mathrm{hrs} \text { (total of } \\
600 \mathrm{mg} / 24 \mathrm{hrs} \text { ) for } 30 \text { days }\end{array}$ & \multirow{2}{*}{$\begin{array}{l}\text { There was no statistically } \\
\text { significant difference in } \\
\text { FEV1, dyspnea (1-7 } \\
\text { Scale), hospital } \\
\text { admission, and recurrent } \\
\text { exacerbations. }\end{array}$} \\
\hline & & & 2) Placebo & Oral & $\begin{array}{l}\text { Identical-looking placebo for } \\
30 \text { days }\end{array}$ & \\
\hline \multirow[t]{2}{*}{ Basri, $2017^{20}$} & \multirow[t]{2}{*}{ Inpatient hospital floor } & \multirow[t]{2}{*}{$\mathrm{N} / \mathrm{A}$} & $\begin{array}{l}\text { 1) No Chest } \\
\text { Physiotherapy }\end{array}$ & $\mathrm{N} / \mathrm{A}$ & N/A & \multirow{2}{*}{$\begin{array}{l}\text { Patients in the chest } \\
\text { physiotherapy group had } \\
\text { significantly better } \\
\text { dyspnea scores (VAS) } \\
\text { than those without chest } \\
\text { physiotherapy. }\end{array}$} \\
\hline & & & $\begin{array}{l}\text { 2) Chest } \\
\text { Physiotherapy }\end{array}$ & $\mathrm{N} / \mathrm{A}$ & For 14 days. & \\
\hline \multirow[t]{2}{*}{$\begin{array}{l}\text { Bathoorn, } 2008^{4} \\
\text { Non-inferiority trial }\end{array}$} & \multirow[t]{2}{*}{$\begin{array}{l}\text { Outpatient } \\
\text { Inpatient hospital floor }\end{array}$} & \multirow[t]{2}{*}{3} & $\begin{array}{l}\text { 1) Budesonide + } \\
\text { Formoterol }\end{array}$ & $\begin{array}{l}\text { Inhalation and } \\
\text { Oral }\end{array}$ & $\begin{array}{l}160 \mu \mathrm{g}, 4.5 \mu \mathrm{g}, 4 \mathrm{x} / 24 \mathrm{hrs} \text { (total } \\
\text { of } 640 \mu \mathrm{g}, 189 \mu \mathrm{g} / 24 \mathrm{hrs} \text { ) for } 14 \\
\text { days. }\end{array}$ & \multirow{2}{*}{$\begin{array}{l}\text { Patients in the } \\
\text { budesonide }+ \text { formoterol } \\
\text { group and patients in the } \\
\text { prednisolone group had } \\
\text { significantly better } \\
\text { symptom scores (Total }\end{array}$} \\
\hline & & & 2) Prednisolone & $\begin{array}{l}\text { Inhalation and } \\
\text { Oral }\end{array}$ & $\begin{array}{l}30 \mathrm{mg}, 1 \times / 24 \mathrm{hrs} \text { (total of } \\
30 \mathrm{mg} / 24 \mathrm{hrs} \text { ) for } 14 \text { days }\end{array}$ & \\
\hline
\end{tabular}




\begin{tabular}{|c|c|c|c|c|c|c|}
\hline $\begin{array}{l}\text { Author, Year, } \\
\text { study design* }\end{array}$ & $\begin{array}{c}\text { Study setting } \\
\text { (outpatients, hospitalized } \\
\text { patients) }\end{array}$ & $\begin{array}{l}\text { Length } \\
\text { of } \\
\text { Follow } \\
\text { up } \\
\text { (month } \\
\text { s) }\end{array}$ & $\begin{array}{l}\text { Intervention(s) and } \\
\text { comparator }\end{array}$ & $\begin{array}{c}\text { Route of } \\
\text { administration }\end{array}$ & Dose and Duration & Conclusion \\
\hline & & & 3) Placebo & $\begin{array}{l}\text { Inhalation and } \\
\text { Oral }\end{array}$ & For 14 days. & $\begin{array}{l}\text { Symptom Score) than } \\
\text { those in the placebo } \\
\text { group. } \\
\text { Patients in the } \\
\text { Budesonide + Formoterol } \\
\text { group had significantly } \\
\text { better quality of life } \\
\text { (Clinical COPD } \\
\text { Questionnaire) than those } \\
\text { in the prednisolone group } \\
\text { (p=0.02); however the } \\
\text { difference between the } \\
\text { budesonide + formoterol } \\
\text { group and the placebo } \\
\text { group was not significant. } \\
\text { There was no statistically } \\
\text { significant difference in } \\
\text { FEV1, treatment failures } \\
\text { between the groups. No } \\
\text { hospital admission was } \\
\text { reported in any of the } \\
\text { three groups. }\end{array}$ \\
\hline Behnke, $2000^{21}$ & Inpatient hospital floor & 6 & $\begin{array}{l}\text { 1) } \\
\text { Aerobic Exercise }\end{array}$ & $\mathrm{N} / \mathrm{A}$ & $\begin{array}{l}10 \text { day walking training } \\
\text { program in hospital followed } \\
\text { by } 60 \text { day at home }\end{array}$ & $\begin{array}{l}\text { The aerobic exercise } \\
\text { group was found to have } \\
\text { statistically significant }\end{array}$ \\
\hline
\end{tabular}




\begin{tabular}{|c|c|c|c|c|c|c|}
\hline \multirow[t]{2}{*}{$\begin{array}{l}\text { Author, Year, } \\
\text { study design* }\end{array}$} & $\begin{array}{c}\text { Study setting } \\
\text { (outpatients, hospitalized } \\
\text { patients) }\end{array}$ & $\begin{array}{l}\text { Length } \\
\text { of } \\
\text { Follow } \\
\text { up } \\
\text { (month } \\
\text { s) }\end{array}$ & $\begin{array}{l}\text { Intervention(s) and } \\
\text { comparator }\end{array}$ & $\begin{array}{c}\text { Route of } \\
\text { administration }\end{array}$ & Dose and Duration & Conclusion \\
\hline & & & $\begin{array}{l}\text { 2) Management } \\
\text { without Aerobic } \\
\text { Exercise }\end{array}$ & N/A & N/A & $\begin{array}{l}\text { more improvement in 6- } \\
\text { minute walking distance } \\
\text { test, and dyspnea } \\
\text { (measured by the } \\
\text { modified categorical Borg } \\
\text { Scale and the Transitional } \\
\text { Dyspnea Index), and } \\
\text { quality of life (measured } \\
\text { by the Chronic } \\
\text { Respiratory Disease } \\
\text { Questionnaire). There } \\
\text { was no significant } \\
\text { difference in mortality } \\
\text { (one death in each } \\
\text { group), FEV1 absolute, } \\
\text { repeat exacerbation, and } \\
\text { number of withdrawals. }\end{array}$ \\
\hline Black, 2004 22 & Inpatient hospital floor & $\mathrm{N} / \mathrm{A}$ & 2) Placebo & Oral & $\begin{array}{l}600 \mathrm{mg}, 2 \times / 24 \mathrm{hrs} \text { (total of } \\
1200 \mathrm{mg} / 24 \mathrm{hrs} \text { ) for } 7 \text { days or } \\
\text { until discharge whichever } \\
\text { occurred first }\end{array}$ & $\begin{array}{l}\text { There was no statistically } \\
\text { significant difference in } \\
\text { FEV1 values and } \\
\text { dyspnea (Breathlessness } \\
\text { Likert Scale) between the } \\
\text { two groups. }\end{array}$ \\
\hline \multirow[t]{2}{*}{ Borges, $2014^{23}$} & \multirow[t]{2}{*}{ Inpatient hospital floor } & \multirow[t]{2}{*}{1} & $\begin{array}{l}\text { 1) Resistance } \\
\text { Training }\end{array}$ & $\mathrm{N} / \mathrm{A}$ & $\begin{array}{l}\text { A minimum of } 3 \text { sessions of } \\
\text { whole-body resistance } \\
\text { training program over } 3 \text { days. }\end{array}$ & \multirow{2}{*}{$\begin{array}{l}\text { The exercise training } \\
\text { group was found to have } \\
\text { significantly more } \\
\text { improvements in 6-min } \\
\text { walking distance test than } \\
\text { the management without } \\
\text { resistance training group. } \\
\text { There was no statistically } \\
\text { significant difference in } \\
\text { FEV1, quality of life } \\
\text { (Health-related Quality of } \\
\text { Life), hospital } \\
\text { readmission, and death. }\end{array}$} \\
\hline & & & $\begin{array}{l}\text { 2) Management } \\
\text { without Resistance } \\
\text { Training }\end{array}$ & $\mathrm{N} / \mathrm{A}$ & $\mathrm{N} / \mathrm{A}$ & \\
\hline
\end{tabular}




\begin{tabular}{|c|c|c|c|c|c|c|}
\hline $\begin{array}{l}\text { Author, Year, } \\
\text { study design* }\end{array}$ & $\begin{array}{c}\text { Study setting } \\
\text { (outpatients, hospitalized } \\
\text { patients) }\end{array}$ & $\begin{array}{l}\text { Length } \\
\text { of } \\
\text { Follow } \\
\text { up } \\
\text { (month } \\
\text { s) }\end{array}$ & $\begin{array}{l}\text { Intervention(s) and } \\
\text { comparator }\end{array}$ & $\begin{array}{c}\text { Route of } \\
\text { administration }\end{array}$ & Dose and Duration & Conclusion \\
\hline \multirow[t]{2}{*}{ Brown, $1987^{24}$} & \multirow[t]{2}{*}{$\begin{array}{l}\text { Outpatient } \\
\text { Inpatient hospital floor }\end{array}$} & \multirow[t]{2}{*}{ N/A } & $\begin{array}{l}\text { 1) Chest Wall } \\
\text { Vibration }\end{array}$ & N/A & $\begin{array}{l}\text { Mechanical vibration for } 15 \\
\text { mins on day } 1 . \\
\text { Positioning on day } 2 \text { over } 2 \\
\text { days. }\end{array}$ & \multirow[t]{2}{*}{$\begin{array}{l}\text { No statistically significant } \\
\text { difference in FEV1 values } \\
\text { were observed between } \\
\text { the two groups. }\end{array}$} \\
\hline & & & 2) Positioning & N/A & $\begin{array}{l}\text { Positioning on day } 1 . \\
\text { Mechanical vibration for } 15 \\
\text { mins on day } 2 \text { over } 2 \text { days. }\end{array}$ & \\
\hline \multirow[t]{2}{*}{$\begin{array}{l}\text { Centanni, } 2002 \\
{ }^{25}, \text { Crossover RCT }\end{array}$} & \multirow[t]{2}{*}{ Outpatient } & \multirow[t]{2}{*}{$\mathrm{N} / \mathrm{A}$} & 1) Oxitropium & Inhaled & $\begin{array}{l}100 \mu \mathrm{g} \text { puff, } 2 \text { puffs (total of } \\
200 \mu \mathrm{g} \text { ) for } 2 \text { days }\end{array}$ & \multirow{2}{*}{$\begin{array}{l}\text { There was a statistically } \\
\text { significant improvement in } \\
\text { FEV1 values } 30 \text { mins } \\
\text { after inhalation of } \\
\text { oxitropium. No significant } \\
\text { improvements were found } \\
\text { after inhalation of } \\
\text { placebo. }\end{array}$} \\
\hline & & & 2) Placebo & Inhaled & $\begin{array}{l}100 \mu g \text { puff, } 2 \text { puffs (total of } \\
200 \mu \mathrm{g} \text { ) for } 2 \text { days }\end{array}$ & \\
\hline \multirow[t]{2}{*}{ Cox, $2018^{26}$} & \multirow[t]{2}{*}{$\begin{array}{l}\text { Outpatient } \\
\text { Inpatient hospital floor }\end{array}$} & \multirow[t]{2}{*}{3} & 1) Aerobic Exercise & $\mathrm{N} / \mathrm{A}$ & $\begin{array}{l}\text { Four exercise sessions over } \\
14 \text { days in the patient's home }\end{array}$ & \multirow{2}{*}{$\begin{array}{l}\text { There was no significant } \\
\text { difference between } \\
\text { hospital pulmonary } \\
\text { rehabilitation and usual } \\
\text { care in 6-mins walking } \\
\text { distance test, quality of } \\
\text { life (COPD Assessment } \\
\text { Test), repeat } \\
\text { exacerbations, and } \\
\text { hospital readmissions. No } \\
\text { death was reported. }\end{array}$} \\
\hline & & & $\begin{array}{l}\text { 2) Management } \\
\text { without Aerobic } \\
\text { Exercise }\end{array}$ & $\mathrm{N} / \mathrm{A}$ & $\begin{array}{l}16 \text { revolutions of the bike on } \\
\text { both set of limbs, three times } \\
\text { a day for } 5 \text { consecutive days }\end{array}$ & \\
\hline \multirow[t]{2}{*}{ Cross, $2012^{27}$} & \multirow[t]{2}{*}{ Inpatient hospital floor } & \multirow[t]{2}{*}{6} & $\begin{array}{l}\text { 1) Manual Chest } \\
\text { Physiotherapy }\end{array}$ & $\mathrm{N} / \mathrm{A}$ & $\begin{array}{l}\text { Active Cycle of Breathing } \\
\text { Technique: Mean: } 2.53 \\
\text { sessions/ } 11.9 \text { mins per } \\
\text { session }\end{array}$ & \multirow{2}{*}{$\begin{array}{l}\text { There was no significant } \\
\text { difference in the SGRQ } \\
\text { total score, the SGRQ } \\
\text { symptom score, } \\
\text { Breathlessness Cough } \\
\text { and Sputum Scale } \\
\text { (BCSS), and death. }\end{array}$} \\
\hline & & & $\begin{array}{l}\text { 2) Management } \\
\text { without Manual } \\
\text { Chest } \\
\text { Physiotherapy }\end{array}$ & $\mathrm{N} / \mathrm{A}$ & $\mathrm{N} / \mathrm{A}$ & \\
\hline $\begin{array}{l}\text { Du, } 2018^{28} \\
10254\end{array}$ & Inpatient hospital floor & N/A & 1) Simvastatin & Oral & $\begin{array}{l}20 \mathrm{mg}, 1 \times / 24 \text { hours (total of } \\
20 \mathrm{mg} / 24 \text { hours) for } 14 \text { days }\end{array}$ & $\begin{array}{l}\text { Patients in the } \\
\text { simvastatin group }\end{array}$ \\
\hline
\end{tabular}




\begin{tabular}{|c|c|c|c|c|c|c|}
\hline $\begin{array}{l}\text { Author, Year, } \\
\text { study design* }\end{array}$ & $\begin{array}{c}\text { Study setting } \\
\text { (outpatients, hospitalized } \\
\text { patients) }\end{array}$ & $\begin{array}{l}\text { Length } \\
\text { of } \\
\text { Follow } \\
\text { up } \\
\text { (month } \\
\text { s) }\end{array}$ & $\begin{array}{l}\text { Intervention(s) and } \\
\text { comparator }\end{array}$ & $\begin{array}{c}\text { Route of } \\
\text { administration }\end{array}$ & Dose and Duration & Conclusion \\
\hline & & & 2) Placebo & NR & NR & $\begin{array}{l}\text { reported significantly } \\
\text { higher FEV1\% values } \\
\text { after the intervention than } \\
\text { those in the placebo } \\
\text { group. }\end{array}$ \\
\hline \multirow[t]{2}{*}{ Duffy, $2005^{29}$} & \multirow[t]{2}{*}{ Emergency department } & \multirow[t]{2}{*}{1.5} & 1)Placebo & IV & & \multirow{2}{*}{$\begin{array}{l}\text { There was no statistically } \\
\text { significant difference in } \\
\text { FEV1, dyspnea, (BORG } \\
\text { Scale) death, and } \\
\text { symptom scores (VAS). }\end{array}$} \\
\hline & & & 2)Aminophylline & IV & $\begin{array}{l}5 \mathrm{mg} / \mathrm{kg} \text { over } 30 \text { mins for } 5 \\
\text { days. }\end{array}$ & \\
\hline \multirow[t]{2}{*}{ Eaton, $2009^{30}$} & \multirow[t]{2}{*}{$\begin{array}{l}\text { Outpatient, } \\
\text { Inpatient hospital floor }\end{array}$} & \multirow[t]{2}{*}{3} & $\begin{array}{l}\text { 1) Early Pulmonary } \\
\text { Rehabilitation }\end{array}$ & $\mathrm{N} / \mathrm{A}$ & $\begin{array}{l}\text { 1-h sessions of supervised } \\
\text { exercise training, twice } \\
\text { weekly for } 56 \text { days. }\end{array}$ & \multirow{2}{*}{$\begin{array}{l}\text { There was no statistically } \\
\text { significant difference in } \\
\text { hospital readmission, } \\
\text { dyspnea score (Modified } \\
\text { Medical Research Council } \\
\text { Questionnaire), and 6-min } \\
\text { walking distance test. No } \\
\text { adverse events were } \\
\text { reported in any group. }\end{array}$} \\
\hline & & & $\begin{array}{l}\text { 2) Management } \\
\text { without Early } \\
\text { Rehabilitation }\end{array}$ & $\mathrm{N} / \mathrm{A}$ & & \\
\hline \multirow[t]{2}{*}{ Edwards, $2013^{31}$} & \multirow[t]{2}{*}{$\mathrm{N} / \mathrm{A}$} & \multirow[t]{2}{*}{$\mathrm{N} / \mathrm{A}$} & 1)Placebo & Nebulized & $\begin{array}{l}\text { Isotonic saline: } 2.5 \mathrm{ml} 3 \text { times } \\
\text { at } 30 \text { min intervals for } 1 \text { day. }\end{array}$ & \multirow{2}{*}{$\begin{array}{l}\text { There was no statistically } \\
\text { significant difference in } \\
\text { FEV1. No clinically } \\
\text { significant adverse events } \\
\text { were reported. }\end{array}$} \\
\hline & & & $\begin{array}{l}\text { 2)Magnesium } \\
\text { Sulfate }\end{array}$ & Nebulized & $\begin{array}{l}2.5 \mathrm{mg} \text { salbutamol mixed with } \\
2.5 \mathrm{ml} \text { isotonic magnesium } \\
\text { sulphate (151mg) } 3 \text { times at } \\
30 \text { min intervals for } 1 \text { day. }\end{array}$ & \\
\hline \multirow[t]{2}{*}{ Goktalay, $2013^{32}$} & \multirow[t]{2}{*}{ Inpatient hospital floor } & \multirow[t]{2}{*}{$\mathrm{N} / \mathrm{A}$} & $\begin{array}{l}\text { 1) Management } \\
\text { without High- } \\
\text { frequency Chest } \\
\text { Wall Oscillation } \\
\text { Therapy }\end{array}$ & $\mathrm{N} / \mathrm{A}$ & N/A & \multirow[t]{2}{*}{$\begin{array}{l}\text { There was no statistically } \\
\text { significant difference in } \\
\text { FEV1, Modified Medical } \\
\text { Research Council } \\
\text { dyspnea scale, and 6-min } \\
\text { walking distance test. }\end{array}$} \\
\hline & & & $\begin{array}{l}\text { 2)High-frequency } \\
\text { Chest Wall } \\
\text { Oscillation Therapy }\end{array}$ & N/A & $\begin{array}{l}20 \text { mins, } 3 \times / 24 \mathrm{hrs} \text { (total of } 60 \\
\text { mins) for } 5 \text { days } \\
\text { Application and oscillation } \\
\text { frequency were standardized } \\
\text { at } 20 \mathrm{~Hz} \text { and } 10 \mathrm{~Hz}\end{array}$ & \\
\hline
\end{tabular}




\begin{tabular}{|c|c|c|c|c|c|c|}
\hline $\begin{array}{l}\text { Author, Year, } \\
\text { study design* }\end{array}$ & $\begin{array}{c}\text { Study setting } \\
\text { (outpatients, hospitalized } \\
\text { patients) }\end{array}$ & $\begin{array}{l}\text { Length } \\
\text { of } \\
\text { Follow } \\
\text { up } \\
\text { (month } \\
\text { s) }\end{array}$ & $\begin{array}{l}\text { Intervention(s) and } \\
\text { comparator }\end{array}$ & $\begin{array}{c}\text { Route of } \\
\text { administration }\end{array}$ & Dose and Duration & Conclusion \\
\hline \multirow[t]{2}{*}{ Greening, $2014^{33}$} & \multirow[t]{2}{*}{ Inpatient hospital floor } & \multirow[t]{2}{*}{12} & $\begin{array}{l}\text { 1) Early Pulmonary } \\
\text { Rehabilitation }\end{array}$ & $\mathrm{N} / \mathrm{A}$ & \multirow{2}{*}{$\begin{array}{l}\text { Daily timed walks and Daily } \\
\text { strength training, comprising } \\
\text { three sets of eight repetitions } \\
\text { resistance training exercises } \\
\text { with weights for } 10 \text { days. }\end{array}$} & \multirow{2}{*}{$\begin{array}{l}\text { There was no significant } \\
\text { difference between the } \\
\text { two groups in hospital } \\
\text { readmissions. }\end{array}$} \\
\hline & & & $\begin{array}{l}\text { 2) Management } \\
\text { without Pulmonary } \\
\text { Rehabilitation }\end{array}$ & N/A & & \\
\hline \multirow[t]{2}{*}{ Greulich, $2014^{34}$} & \multirow[t]{2}{*}{ Inpatient hospital floor } & \multirow[t]{2}{*}{$\mathrm{N} / \mathrm{A}$} & $\begin{array}{l}\text { 1) Management } \\
\text { without Whole Body } \\
\text { Vibration }\end{array}$ & N/AN/A & $\begin{array}{l}\text { Standard program: } 5 \text { min } \\
\text { mobilization, } 5 \text { min passive } \\
\text { movement, and } 10 \text { min } \\
\text { respiratory exercises }\end{array}$ & \multirow{2}{*}{$\begin{array}{l}\text { Patients in the Whole } \\
\text { Body Vibration group had } \\
\text { statistically significant } \\
\text { better outcomes in 6- } \\
\text { minute walking distance } \\
\text { test, and quality of life } \\
\text { (SGRQ). There was no } \\
\text { statistically significant } \\
\text { difference in \% predicted } \\
\text { FEV1 values. }\end{array}$} \\
\hline & & & $\begin{array}{l}\text { 2)Whole Body } \\
\text { Vibration }\end{array}$ & N/A & $\begin{array}{l}\text { Standard program: } 5 \text { min } \\
\text { mobilization, } 5 \text { min passive } \\
\text { movement, and } 10 \text { min } \\
\text { respiratory exercises } \\
\text { complemented with sessions } \\
\text { on the WBV device }\end{array}$ & \\
\hline \multirow[t]{3}{*}{ Gunen, $2007^{9}$} & \multirow[t]{3}{*}{ Inpatient hospital floor } & \multirow[t]{3}{*}{1} & $\begin{array}{l}\text { 1) Management } \\
\text { without Systemic } \\
\text { Corticosteroids }\end{array}$ & Inhalation & For 10 days. & \multirow{3}{*}{$\begin{array}{l}\text { Patients in the nebulized } \\
\text { budesonide group had } \\
\text { statistically significant } \\
\text { higher FEV1 \% predicted } \\
\text { than those in the } \\
\text { prednisolone group or the } \\
\text { management without } \\
\text { systemic corticosteroids } \\
\text { group. There was no } \\
\text { statistically significant } \\
\text { difference in hospital } \\
\text { readmissions, repeat } \\
\text { exacerbations, \% } \\
\text { predicted FEV1, and } \\
\text { death. }\end{array}$} \\
\hline & & & 2)Prednisolone & IV & $\begin{array}{l}40 \mathrm{mg}, 1 \times / 24 \mathrm{hrs} \text { (total of } \\
40 \mathrm{mg} / 24 \mathrm{hrs} \text { ) for } 10 \text { days }\end{array}$ & \\
\hline & & & $\begin{array}{l}\text { 3)Nebulized } \\
\text { Budesonide }\end{array}$ & Nebulized & $\begin{array}{l}1500 \mu \mathrm{g}, 4 \times / 24 \mathrm{hrs} \text { (total of } \\
0.5 \mathrm{mg} / 24 \mathrm{hrs} \text { ) for } 10 \text { days. }\end{array}$ & \\
\hline
\end{tabular}




\begin{tabular}{|c|c|c|c|c|c|c|}
\hline $\begin{array}{l}\text { Author, Year, } \\
\text { study design* }\end{array}$ & $\begin{array}{c}\text { Study setting } \\
\text { (outpatients, hospitalized } \\
\text { patients) }\end{array}$ & $\begin{array}{l}\text { Length } \\
\text { of } \\
\text { Follow } \\
\text { up } \\
\text { (month } \\
\text { s) }\end{array}$ & $\begin{array}{l}\text { Intervention(s) and } \\
\text { comparator }\end{array}$ & $\begin{array}{c}\text { Route of } \\
\text { administration }\end{array}$ & Dose and Duration & Conclusion \\
\hline \multirow[t]{2}{*}{$\mathrm{He}, 2015^{35}$} & \multirow[t]{2}{*}{ Inpatient hospital floor } & \multirow[t]{2}{*}{$\mathrm{N} / \mathrm{A}$} & $\begin{array}{l}\text { 1) Early Pulmonary } \\
\text { Rehabilitation }\end{array}$ & $\mathrm{N} / \mathrm{A}$ & $\begin{array}{l}\text { Each PR session included } \\
\text { exercise training, relaxation, } \\
\text { breathing retraining and } \\
\text { education (from the second } \\
\text { day of admission until } \\
\text { discharge) for a mean of } 10 \\
\text { days }\end{array}$ & \multirow{2}{*}{$\begin{array}{l}\text { Patients in the pulmonary } \\
\text { rehabilitation group were } \\
\text { found to have significantly } \\
\text { more improvements in 6- } \\
\text { minute walking distance } \\
\text { test }(P<0.001) \text {, dyspnea } \\
\text { (Modified Medical } \\
\text { Research Council } \\
\text { Dyspnea Scale) than } \\
\text { patients in the routine } \\
\text { care group. }\end{array}$} \\
\hline & & & $\begin{array}{l}\text { 2) Management } \\
\text { without Early } \\
\text { Rehabilitation }\end{array}$ & $\mathrm{N} / \mathrm{A}$ & Mean of 10 days & \\
\hline \multirow[t]{2}{*}{ Kirsten, $1998^{37}$} & \multirow[t]{2}{*}{ Inpatient hospital floor } & \multirow[t]{2}{*}{$\mathrm{N} / \mathrm{A}$} & 1) Aerobic Exercise & $\mathrm{N} / \mathrm{A}$ & \multirow{2}{*}{$\begin{array}{l}\text { 6-min treadmill walking test } \\
\text { and five walking sessions per } \\
\text { day for } 10 \text { days }\end{array}$} & \multirow{2}{*}{$\begin{array}{l}\text { There was no statistical } \\
\text { difference between the } \\
\text { two groups in 6-minute } \\
\text { walking distance test, } \\
\text { FEV1 \% predicted, }\end{array}$} \\
\hline & & & $\begin{array}{l}\text { 2) Management } \\
\text { without Aerobic } \\
\text { Exercise }\end{array}$ & $\mathrm{N} / \mathrm{A}$ & & \\
\hline \multirow[t]{2}{*}{ Kodric, $2009^{36}$} & \multirow[t]{2}{*}{ Inpatient hospital floor } & \multirow[t]{2}{*}{6} & $\begin{array}{l}\text { 1) Management } \\
\text { without Chest } \\
\text { Physiotherapy }\end{array}$ & $\mathrm{N} / \mathrm{A}$ & $\mathrm{N} / \mathrm{A}$ & \multirow{2}{*}{$\begin{array}{l}\text { The chest physiotherapy } \\
\text { group had significantly } \\
\text { more improvements in the } \\
\text { Borg scale ( } p<0.01) \text { than } \\
\text { the management without } \\
\text { chest physiotherapy } \\
\text { group. There was no } \\
\text { statistically significant } \\
\text { difference in repeat } \\
\text { exacerbations, hospital } \\
\text { readmissions, \% } \\
\text { predicted FEV1 value, } \\
\text { quality of life, or the } \\
\text { Medical Research Council } \\
\text { Dyspnea scale. }\end{array}$} \\
\hline & & & $\begin{array}{l}\text { 2) Chest } \\
\text { Physiotherapy } \\
\text { ELTGOL (expiration } \\
\text { with the glottis open } \\
\text { in the lateral } \\
\text { posture) }\end{array}$ & N/A & For 7 days & \\
\hline Kurzaj, $2013^{38}$ & Inpatient hospital floor & $\mathrm{N} / \mathrm{A}$ & $\begin{array}{l}\text { 1)Specialized } \\
\text { Physiotherapy }\end{array}$ & $\mathrm{N} / \mathrm{A}$ & $\begin{array}{l}\text { Series of } 6 \text { massages, each } \\
\text { lasting for } 30 \text { mins for } 7 \text { days. }\end{array}$ & $\begin{array}{l}\text { Patients in the specialized } \\
\text { physiotherapy group had } \\
\text { significantly more }\end{array}$ \\
\hline
\end{tabular}




\begin{tabular}{|c|c|c|c|c|c|c|}
\hline $\begin{array}{l}\text { Author, Year, } \\
\text { study design* }\end{array}$ & $\begin{array}{c}\text { Study setting } \\
\text { (outpatients, hospitalized } \\
\text { patients) }\end{array}$ & $\begin{array}{l}\text { Length } \\
\text { of } \\
\text { Follow } \\
\text { up } \\
\text { (month } \\
\text { s) }\end{array}$ & $\begin{array}{l}\text { Intervention(s) and } \\
\text { comparator }\end{array}$ & $\begin{array}{c}\text { Route of } \\
\text { administration }\end{array}$ & Dose and Duration & Conclusion \\
\hline & & & $\begin{array}{l}\text { 2) Management } \\
\text { without Specialized } \\
\text { Physiotherapy }\end{array}$ & $\mathrm{N} / \mathrm{A}$ & $\mathrm{N} / \mathrm{A}$ & $\begin{array}{l}\text { improvements in \% } \\
\text { predicted FEV1 values. } \\
\text { There was no statistically } \\
\text { significant difference in } 6 \\
\text {-minute walking distance } \\
\text { tests, and dyspnea } \\
\text { (Modified Medical } \\
\text { Research Council } \\
\text { Dyspnea Scale). }\end{array}$ \\
\hline \multirow[t]{2}{*}{ Lellouche, $2016^{39}$} & \multirow[t]{2}{*}{ Inpatient hospital floor } & \multirow[t]{2}{*}{6} & $\begin{array}{l}\text { 1)Free02 device } \\
\text { oxygen titration }\end{array}$ & Inhalation & Oxygen flow 0-20 liters /min & \multirow{2}{*}{$\begin{array}{l}\text { There was no statistically } \\
\text { significant difference in } \\
\text { need for intubation, ICU } \\
\text { admission, and hospital } \\
\text { readmissions. }\end{array}$} \\
\hline & & & $\begin{array}{l}\text { 2)Manual Oxygen } \\
\text { Titration }\end{array}$ & Inhalation & N/A & \\
\hline \multirow[t]{2}{*}{ Liao, $2015^{40}$} & \multirow[t]{2}{*}{ Inpatient hospital floor } & \multirow[t]{2}{*}{$\mathrm{N} / \mathrm{A}$} & $\begin{array}{l}\text { 1) Early Pulmonary } \\
\text { Rehabilitation }\end{array}$ & $\mathrm{N} / \mathrm{A}$ & $\begin{array}{l}\text { RP sessions were conducted } \\
\text { a minimum of twice per day } \\
\text { for } 10 \text { mins per session for } 4 \\
\text { days. }\end{array}$ & \multirow{2}{*}{$\begin{array}{l}\text { The respiratory } \\
\text { rehabilitation group had } \\
\text { statistically significant } \\
\text { better outcomes in } \\
\text { dyspnea (Modified Borg } \\
\text { Scale), 6-minute walking } \\
\text { distance test, and } \\
\text { frequency of coughing. }\end{array}$} \\
\hline & & & $\begin{array}{l}\text { 2) Management } \\
\text { without Early } \\
\text { Rehabilitation }\end{array}$ & $\mathrm{N} / \mathrm{A}$ & $\begin{array}{l}\text { Usual care and health } \\
\text { education for } 4 \text { days. }\end{array}$ & \\
\hline \multirow[t]{2}{*}{$\begin{array}{l}\text { Mahmoud Abd El } \\
\text { Hafiz, } 2013^{41}\end{array}$} & \multirow[t]{2}{*}{ Inpatient hospital floor } & \multirow[t]{2}{*}{ N/A } & $\begin{array}{l}\text { 1) Management } \\
\text { without } \mathrm{N} \text { - } \\
\text { acetylcysteine }\end{array}$ & $\mathrm{N} / \mathrm{A}$ & $\begin{array}{l}\text { Standard COPD exacerbation } \\
\text { treatment : according to } \\
\text { GOLD } 2011 \text { ( systemic } \\
\text { glucocorticoids oral } \\
\text { prednisolone } 30-40 \mathrm{mg} / \text { day, } \\
\text { short acting B2 agonist } \\
\text { (SABA) salbutamol and } \\
\text { antibiotics)for } 10 \text { days }\end{array}$ & \multirow{2}{*}{$\begin{array}{l}\text { Patients in the high dose } \\
\mathrm{N} \text {-acetyl Cysteine group } \\
\text { had significantly higher \% } \\
\text { predicted FEV1 than } \\
\text { those in the low dose } \\
\text { group and the } \\
\text { management without } \mathrm{N}- \\
\text { acetylcysteine group. \% } \\
\text { predicted FEV1 was not } \\
\text { statistically significant } \\
\text { difference between the } \\
\text { low dose group and the }\end{array}$} \\
\hline & & & $\begin{array}{l}\text { 2) Low Dose N- } \\
\text { acetylcysteine }\end{array}$ & N/A & $\begin{array}{l}\text { Standard treatment }+ \\
\mathrm{N} \text {-acetyl Cysteine: } 200 \mathrm{mg} \text {, } \\
3 \times / 24 \mathrm{hrs} \text { (total of } \\
600 \mathrm{mg} / 24 \mathrm{hrs} \text { ) for } 10 \text { days }\end{array}$ & \\
\hline
\end{tabular}




\begin{tabular}{|c|c|c|c|c|c|c|}
\hline $\begin{array}{l}\text { Author, Year, } \\
\text { study design* }\end{array}$ & $\begin{array}{c}\text { Study setting } \\
\text { (outpatients, hospitalized } \\
\text { patients) }\end{array}$ & $\begin{array}{l}\text { Length } \\
\text { of } \\
\text { Follow } \\
\text { up } \\
\text { (month } \\
\text { s) }\end{array}$ & $\begin{array}{l}\text { Intervention(s) and } \\
\text { comparator }\end{array}$ & $\begin{array}{c}\text { Route of } \\
\text { administration }\end{array}$ & Dose and Duration & Conclusion \\
\hline & & & $\begin{array}{l}\text { 3) High Dose N- } \\
\text { acetylcysteine }\end{array}$ & $\mathrm{N} / \mathrm{A}$ & $\begin{array}{l}\text { Standard treatment }+ \\
\mathrm{N} \text {-acetyl Cysteine: } 400 \mathrm{mg} \text {, } \\
3 \times / 24 \mathrm{hrs} \text { (total of } \\
1200 \mathrm{mg} / 24 \mathrm{hrs} \text { ) for } 10 \text { days }\end{array}$ & $\begin{array}{l}\text { Management without N- } \\
\text { acetylcysteine group. }\end{array}$ \\
\hline \multirow[t]{6}{*}{ Maltais, $2002^{12}$} & \multirow[t]{6}{*}{ Inpatient hospital floor } & \multirow[t]{6}{*}{0.25} & \multirow[t]{2}{*}{ 1) Budesonide } & Nebulized & $\begin{array}{l}2 \mathrm{mg}, 4 \mathrm{x} / 24 \mathrm{hrs} \text { (total of } \\
8 \mathrm{mg} / 24 \mathrm{hrs} \text { for } 3 \text { days }\end{array}$ & \multirow{6}{*}{$\begin{array}{l}\text { The budesonide group } \\
\text { and the prednisolone } \\
\text { group had a significantly } \\
\text { larger increase in } \\
\text { absolute FEV1 than the } \\
\text { placebo group. Increases } \\
\text { in FEV1 were not } \\
\text { significantly different in } \\
\text { the budesonide group } \\
\text { and the prednisolone } \\
\text { group. There was no } \\
\text { statistically significant } \\
\text { difference in dyspnea } \\
\text { (Modified Borg Scale), } \\
\text { need for intubation, and } \\
\text { death between the three } \\
\text { groups. }\end{array}$} \\
\hline & & & & Inhaled & $\begin{array}{l}\text { Then: } \\
2000 \mu \mathrm{g}, 1 \mathrm{x} / 24 \mathrm{hrs} \text { (total of } \\
2000 \mu \mathrm{g} / 24 \mathrm{hrs} \text { ) for } 7 \text { days }\end{array}$ & \\
\hline & & & 2) Prednisolone & Oral & $\begin{array}{l}30 \mathrm{mg}, 2 \mathrm{x} / 24 \mathrm{hrs} \text { (total of } \\
60 \mathrm{mg} / 24 \mathrm{hrs} \text { ) for } 3 \text { days, then } \\
40 \mathrm{mg} / 24 \mathrm{hrs} \text { for } 7 \text { days }\end{array}$ & \\
\hline & & & \multirow[t]{3}{*}{ 3) Placebo } & Nebulized & For 72 hours & \\
\hline & & & & Inhaled & For 7 days & \\
\hline & & & & Oral & For 72 hours, then for 7 days & \\
\hline Moretti, $2015^{42}$ & Inpatient hospital floor & 2 & 1) Erdosteine & Oral & $\begin{array}{l}300 \mathrm{mg}, 3 \times / 24 \mathrm{hrs} \text { (total of } \\
900 \mathrm{mg} / 24 \mathrm{hrs} \text { ) for } 10 \text { days }\end{array}$ & $\begin{array}{l}\text { After intervention, the } \\
\text { erdosteine group had }\end{array}$ \\
\hline
\end{tabular}




\begin{tabular}{|c|c|c|c|c|c|c|}
\hline \multirow[t]{2}{*}{$\begin{array}{l}\text { Author, Year, } \\
\text { study design* }\end{array}$} & $\begin{array}{c}\text { Study setting } \\
\text { (outpatients, hospitalized } \\
\text { patients) }\end{array}$ & $\begin{array}{l}\text { Length } \\
\text { of } \\
\text { Follow } \\
\text { up } \\
\text { (month } \\
\text { s) }\end{array}$ & $\begin{array}{l}\text { Intervention(s) and } \\
\text { comparator }\end{array}$ & $\begin{array}{c}\text { Route of } \\
\text { administration }\end{array}$ & Dose and Duration & Conclusion \\
\hline & & & $\begin{array}{l}\text { 2) Management } \\
\text { without Erdosteine }\end{array}$ & $\mathrm{N} / \mathrm{A}$ & N/A & $\begin{array}{l}\text { significantly more } \\
\text { improvements in \% } \\
\text { predicted FEV1 and } \\
\text { Breathlessness-Sputum- } \\
\text { Cough Scale than the } \\
\text { management without } \\
\text { erdosteine group. } \\
\text { However, the difference } \\
\text { was not significantly in } \\
\text { the followup. After the 2- } \\
\text { month followup, the } \\
\text { erdosteine group had } \\
\text { significantly less repeat } \\
\text { exacerbations than those } \\
\text { in the placebo group. }\end{array}$ \\
\hline \multirow[t]{2}{*}{ Mukerji, $2015^{43}$} & \multirow[t]{2}{*}{ Emergency department } & \multirow[t]{2}{*}{ N/A } & $\begin{array}{l}\text { 1) Magnesium } \\
\text { Sulfate }\end{array}$ & IV & $\begin{array}{l}2 \mathrm{~g} \text { magnesium sulphate made } \\
\text { up to } 20 \text { mins in } 0.9 \% \text { sodium } \\
\text { chloride solution (saline) over } \\
15 \text { mins for } 1 \text { day. }\end{array}$ & \multirow{2}{*}{$\begin{array}{l}\text { Patients in the } \\
\text { magnesium sulphate } \\
\text { group had significantly } \\
\text { more increase in absolute } \\
\text { FEV1 values than those } \\
\text { in the placebo group. } \\
\text { There was no statistically } \\
\text { significant difference in } \\
\text { need for intubation. }\end{array}$} \\
\hline & & & 2) Placebo & IV & $\begin{array}{l}20 \mathrm{ml} \text { of saline over } 15 \mathrm{mins} \\
\text { for } 1 \text { day. }\end{array}$ & \\
\hline \multirow[t]{2}{*}{$\begin{array}{l}\text { Ogasawar, } 2018^{44} \\
10048\end{array}$} & \multirow[t]{2}{*}{ Inpatient hospital floor } & \multirow[t]{2}{*}{$\mathrm{N} / \mathrm{A}$} & $\begin{array}{l}\text { 1) Omega-3 fatty } \\
\text { acid anriched diet } \\
\text { (Eicosapentaenoic } \\
\text { acid) acid). }\end{array}$ & Oral & $\begin{array}{l}1 \mathrm{~g}, 1 \mathrm{x} / 24 \text { hours (total of } 1 \mathrm{~g} / 24 \\
\text { hours) }\end{array}$ & \multirow{2}{*}{$\begin{array}{l}\text { There was no statistically } \\
\text { difference between the } \\
\text { two groups in physical } \\
\text { activities (steps per day), } \\
\text { dyspnea (Modified } \\
\text { Medical Research Council } \\
\text { Dyspnea Scale), and } \\
\text { quality of life (COPD } \\
\text { Assessment Test). }\end{array}$} \\
\hline & & & 2) Usual Diet & Oral & $\begin{array}{l}1 \mathrm{~g}, 1 \mathrm{x} / 24 \text { hours (total of } 1 \mathrm{~g} / 24 \\
\text { hours) }\end{array}$ & \\
\hline Oncu, $2017^{45}$ & $\begin{array}{l}\text { Outpatient, Inpatient } \\
\text { hospital floor }\end{array}$ & $\mathrm{N} / \mathrm{A}$ & $\begin{array}{l}\text { 1) Transcutaneous } \\
\text { Electrical Nerve } \\
\text { Stimulation } \\
\end{array}$ & Transcutaneous & $\begin{array}{l}20 \text { sessions (TENS device) } \\
\text { each for } 45 \text { minute application } \\
\text { once a day for } 20 \text { days. }\end{array}$ & $\begin{array}{l}\text { Patients in the stimulation } \\
\text { group had significantly } \\
\text { more improvements in 6- }\end{array}$ \\
\hline
\end{tabular}




\begin{tabular}{|c|c|c|c|c|c|c|}
\hline \multirow[t]{2}{*}{$\begin{array}{l}\text { Author, Year, } \\
\text { study design* }\end{array}$} & $\begin{array}{c}\text { Study setting } \\
\text { (outpatients, hospitalized } \\
\text { patients) }\end{array}$ & $\begin{array}{l}\text { Length } \\
\text { of } \\
\text { Follow } \\
\text { up } \\
\text { (month } \\
\text { s) }\end{array}$ & $\begin{array}{l}\text { Intervention(s) and } \\
\text { comparator }\end{array}$ & $\begin{array}{c}\text { Route of } \\
\text { administration }\end{array}$ & Dose and Duration & Conclusion \\
\hline & & & $\begin{array}{l}\text { 2) Management } \\
\text { without } \\
\text { Transcutaneous } \\
\text { Electrical Nerve } \\
\text { Stimulation }\end{array}$ & Transcutaneous & $\begin{array}{l}20 \text { sessions (TENS device } \\
\text { without electrical output) each } \\
\text { for } 45-\text { minute application } \\
\text { once a day for } 20 \text { days. }\end{array}$ & $\begin{array}{l}\text { minute walking distance } \\
\text { test than patients in the } \\
\text { placebo group. There was } \\
\text { no statistically significant } \\
\text { difference in absolute } \\
\text { FEV1 values, quality of } \\
\text { life (St George's } \\
\text { Respiratory } \\
\text { Questionnaire), and } \\
\text { dyspnea (Medical } \\
\text { Research Council } \\
\text { Dyspnea Scale). }\end{array}$ \\
\hline \multirow[t]{3}{*}{ Osadnik, $2014^{46}$} & Inpatient hospital floor & 6 & $\begin{array}{l}\text { 1) Management } \\
\text { without Positive } \\
\text { Expiratory Pressure }\end{array}$ & $\mathrm{N} / \mathrm{A}$ & $\mathrm{N} / \mathrm{A}$ & \multirow{2}{*}{$\begin{array}{l}\text { There was no statistically } \\
\text { significant difference in } \\
\text { modified Medical } \\
\text { Research Council } \\
\text { dyspnea scale, FEV1 \% } \\
\text { predicted, the } \\
\text { Breathlessness, Cough } \\
\text { and Sputum Scale, } \\
\text { quality of life (St George's } \\
\text { Respiratory } \\
\text { Questionnaire), repeat } \\
\text { exacerbations, 6-minute } \\
\text { walking distance test, and } \\
\text { hospital admissions. }\end{array}$} \\
\hline & & & $\begin{array}{l}\text { 2) Positive } \\
\text { Expiratory Pressure }\end{array}$ & Mask & $\begin{array}{l}\text { 3session/ day, (five } \\
\text { repetitions for each session, } \\
\text { as tolerated for } \\
\text { approximately } 20 \text { mins } \\
\text { duration, one session was } \\
\text { supervised), daily therapy } \\
\text { continued until hospital } \\
\text { discharge or } 24 \text { h without } \\
\text { sputum expectoration, } \\
\text { whichever came first }\end{array}$ & \\
\hline & Inpatient hospital floor & 4 & 1) Placebo & IM & Single injection & \\
\hline
\end{tabular}




\begin{tabular}{|c|c|c|c|c|c|c|}
\hline $\begin{array}{l}\text { Author, Year, } \\
\text { study design* }\end{array}$ & $\begin{array}{c}\text { Study setting } \\
\text { (outpatients, hospitalized } \\
\text { patients) }\end{array}$ & $\begin{array}{l}\text { Length } \\
\text { of } \\
\text { Follow } \\
\text { up } \\
\text { (month } \\
\text { s) }\end{array}$ & $\begin{array}{l}\text { Intervention(s) and } \\
\text { comparator }\end{array}$ & $\begin{array}{c}\text { Route of } \\
\text { administration }\end{array}$ & Dose and Duration & Conclusion \\
\hline $\begin{array}{l}\text { Pourrashid, } \\
2018^{47}\end{array}$ & & & 2) Vitamin $D 3$ & IM & Single injection of $300,000 \mathrm{IU}$ & $\begin{array}{l}\text { At the end of followup, the } \\
\text { Vitamin D3 group had } \\
\text { significantly more } \\
\text { improvements in quality of } \\
\text { life (St George's } \\
\text { Respiratory } \\
\text { Questionnaire, } p<0.001 \text { ). } \\
\text { There was no statistically } \\
\text { significant difference in } \\
\text { mortality, hospital } \\
\text { readmissions, and } \\
\text { dyspnea (Modified } \\
\text { Medical Research Council } \\
\text { Dyspnea Scale). }\end{array}$ \\
\hline \multirow[t]{2}{*}{ Rice, $1987^{48}$} & \multirow[t]{2}{*}{$\begin{array}{l}\text { Emergency department, } \\
\text { outpatient }\end{array}$} & \multirow[t]{2}{*}{ N/A } & 1) Aminophylline & IV & $\begin{array}{l}\text { Loading dose: } 6 \mathrm{mg} / \mathrm{kg} \text { or } \\
3 \mathrm{mg} / \mathrm{kg} \text { based on time of last } \\
\text { administered dose of } \\
\text { standard or sustained release } \\
\text { Theophylline. } \\
\text { Maintenance dose: } 0.5 \mathrm{mg} / \mathrm{kg} \text {, } \\
\text { or } 0.3 \mathrm{mg} / \mathrm{kg} \text { to achieve the } \\
\text { desired serum theophylline } \\
\text { levels of } 72 \text { to } 94 \mu \mathrm{mol} / \mathrm{L} \\
\text { An additional loading dose of } \\
1 \mathrm{mg} / \mathrm{kg} \text { body weight was } \\
\text { given for each desired } \\
\text { increase of } 11 \mu \mathrm{mol} / \mathrm{L} \text { in the } \\
\text { Theophylline level for } 3 \text { days. }\end{array}$ & \multirow[t]{2}{*}{$\begin{array}{l}\text { There was no statistically } \\
\text { significant difference in } \\
\text { absolute FEV1 values, } \\
\text { dyspnea (Verbal dyspnea } \\
\text { index), need for } \\
\text { intubations, and number } \\
\text { of adverse events. }\end{array}$} \\
\hline & & & 2) Placebo & IV & $\begin{array}{l}\text { Frequent adjustments were } \\
\text { made to maintain the double- } \\
\text { blinded nature of the study for } \\
3 \text { days. }\end{array}$ & \\
\hline \multirow[t]{2}{*}{ Sanjari, $2015^{49}$} & \multirow[t]{2}{*}{ Inpatient hospital floor } & \multirow[t]{2}{*}{$\mathrm{N} / \mathrm{A}$} & 1) Placebo & Oral & Daily for 7 days & \multirow{2}{*}{$\begin{array}{l}\text { Patients in the calcitriol } \\
\text { had significantly } \\
\text { improvements in dyspnea }\end{array}$} \\
\hline & & & 2) Vitamin $D$ & Oral & $\begin{array}{l}50000 \mathrm{IU}, 1 \mathrm{x} / 24 \mathrm{hrs} \text { (total of } \\
50000 \mathrm{IU} / 24 \mathrm{hrs} \text { ) for } 7 \text { days }\end{array}$ & \\
\hline
\end{tabular}




\begin{tabular}{|c|c|c|c|c|c|c|}
\hline $\begin{array}{l}\text { Author, Year, } \\
\text { study design* }\end{array}$ & $\begin{array}{c}\text { Study setting } \\
\text { (outpatients, hospitalized } \\
\text { patients) }\end{array}$ & $\begin{array}{l}\text { Length } \\
\text { of } \\
\text { Follow } \\
\text { up } \\
\text { (month } \\
\text { s) }\end{array}$ & $\begin{array}{l}\text { Intervention(s) and } \\
\text { comparator }\end{array}$ & $\begin{array}{c}\text { Route of } \\
\text { administration }\end{array}$ & Dose and Duration & Conclusion \\
\hline & & & 3) Calcitriol & Oral & $\begin{array}{l}0.25 \mu \mathrm{g}, 1 \times / 24 \mathrm{hrs} \text { (total of } \\
0.25 \mu \mathrm{g} / 24 \mathrm{hrs} \text { ) for } 7 \text { days }\end{array}$ & $\begin{array}{l}\text { (Modified Medical } \\
\text { Research Council } \\
\text { Dyspnea Scale) than } \\
\text { those in the placebo } \\
\text { group or the Vitamin D } \\
\text { group. No statistically } \\
\text { significant difference was } \\
\text { observed between the } \\
\text { Vitamin D group and the } \\
\text { placebo group in } \\
\text { dyspnea. There was no } \\
\text { statistically significant } \\
\text { difference in \% predicted } \\
\text { FEV1. }\end{array}$ \\
\hline \multirow[t]{2}{*}{$\begin{array}{l}\text { Saudny- } \\
\text { Unterberger, } \\
1997^{50}\end{array}$} & \multirow[t]{2}{*}{ Inpatient hospital floor } & \multirow[t]{2}{*}{$\mathrm{N} / \mathrm{A}$} & $\begin{array}{l}\text { 1) Nutritional } \\
\text { Support }\end{array}$ & Oral & $\begin{array}{l}\text { An additional } 10 \mathrm{kcal} / \mathrm{kg} / \mathrm{day} \\
\text { for } 14 \text { days. }\end{array}$ & \multirow{2}{*}{$\begin{array}{l}\text { There was no statistically } \\
\text { significant difference in } \\
\text { death, \% predicted FEV1, } \\
\text { dyspnea (Oxygen-cost } \\
\text { diagram), 6-minute } \\
\text { walking distance test, and } \\
\text { quality of life (Genreal } \\
\text { Well-being). }\end{array}$} \\
\hline & & & 2) Usual Diet & Oral & Usual feeding for 14 days. & \\
\hline \multirow[t]{2}{*}{ Seidenfeld, $1984^{51}$} & \multirow[t]{2}{*}{ Emergency department } & \multirow[t]{2}{*}{6} & 1) Aminophylline & IV & $\begin{array}{l}5.6 \mathrm{mg} / \mathrm{kg} \text { over one hour by } \\
\text { constant infusion pump, or } \\
\text { reduced the dosage to } \\
2.8 \mathrm{mg} / \mathrm{kg} \text { if the patient had } \\
\text { received a theophylline- } \\
\text { containing preparation within } \\
\text { the previous six hours for } 1 \\
\text { day. }\end{array}$ & \multirow[t]{2}{*}{$\begin{array}{l}\text { There was no statistically } \\
\text { significant difference in } \\
\text { death, \% predicted FEV1, } \\
\text { dyspnea ( } 1-5 \text { scale), and } \\
\text { cough ( } 1-5 \text { scale). }\end{array}$} \\
\hline & & & 2) Placebo & IV & For 1 day. & \\
\hline \multirow[t]{2}{*}{ Skorodin, $1995^{52}$} & \multirow[t]{2}{*}{ Emergency department } & \multirow[t]{2}{*}{0.5} & $\begin{array}{l}\text { 1) Magnesium } \\
\text { Sulfate }\end{array}$ & IV & $\begin{array}{l}1.2 \mathrm{~g} \text { in } 150 \mathrm{ml} \text { of saline over } \\
20 \text { mins for } 1 \text { day. }\end{array}$ & \multirow{2}{*}{$\begin{array}{l}\text { There was no statistically } \\
\text { significant difference in } \\
\text { hospital admission and } \\
\text { dyspnea score (Ordinal } \\
\text { Scale) between the two } \\
\text { groups. }\end{array}$} \\
\hline & & & 2)Placebo & IV & $2.4 \mathrm{ml}$ over 20 mins for 1 day. & \\
\hline
\end{tabular}




\begin{tabular}{|c|c|c|c|c|c|c|}
\hline $\begin{array}{l}\text { Author, Year, } \\
\text { study design* }\end{array}$ & $\begin{array}{c}\text { Study setting } \\
\text { (outpatients, hospitalized } \\
\text { patients) }\end{array}$ & $\begin{array}{l}\text { Length } \\
\text { of } \\
\text { Follow } \\
\text { up } \\
\text { (month } \\
\text { s) }\end{array}$ & $\begin{array}{l}\text { Intervention(s) and } \\
\text { comparator }\end{array}$ & $\begin{array}{c}\text { Route of } \\
\text { administration }\end{array}$ & Dose and Duration & Conclusion \\
\hline \multirow[t]{2}{*}{ Solooki, $2014^{53}$} & \multirow[t]{2}{*}{$\begin{array}{l}\text { Emergency department, } \\
\text { Inpatient hospital floor }\end{array}$} & \multirow[t]{2}{*}{$\mathrm{N} / \mathrm{A}$} & $\begin{array}{l}\text { 1)Magnesium } \\
\text { Sulfate }\end{array}$ & IV & $\begin{array}{l}2 \mathrm{~g} \text { diluted in } 100 \mathrm{ml} \text { saline } \\
\text { over } 20 \text { mins for } 4 \text { days. }\end{array}$ & \multirow[t]{2}{*}{$\begin{array}{l}\text { There was no statistically } \\
\text { significant difference in \% } \\
\text { predicted FEV1. }\end{array}$} \\
\hline & & & 2)Placebo & IV & 100cc over 20 min for 4 days. & \\
\hline \multirow[t]{2}{*}{$\begin{array}{l}\text { Soltaninejad, } \\
2016^{54}\end{array}$} & \multirow[t]{2}{*}{ Inpatient Hospital } & \multirow[t]{2}{*}{$\mathrm{N} / \mathrm{A}$} & 1)Gentamicin & Nebulized & $\begin{array}{l}\text { 80mg, } 2 \times / 24 \mathrm{hrs} \text { (total of } \\
160 \mathrm{mg} / 24 \mathrm{hrs} \text { ) for } 5 \text { days }\end{array}$ & \multirow{2}{*}{$\begin{array}{l}\text { The gentamicin group } \\
\text { had significantly more } \\
\text { increase in absolute } \\
\text { FEV1 values than the } \\
\text { placebo group. }\end{array}$} \\
\hline & & & 2)Placebo & Nebulized & $\begin{array}{l}\text { 2cc distilled water, } 2 \times / 24 \mathrm{hrs} \\
\text { for } 5 \text { days }\end{array}$ & \\
\hline \multirow[t]{4}{*}{ Tang, $2012^{55}$} & \multirow[t]{3}{*}{ Inpatient hospital floor } & \multirow[t]{3}{*}{ N/A } & $\begin{array}{l}\text { 1)Low-intensity } \\
\text { Exercise Group }\end{array}$ & N/A & $\begin{array}{l}\text { 15-minute exercise sessions } \\
2 \text { times a day: walking at } 40 \% \\
\text { of } 3 \text {-min walk test for } 7.5 \text { min } \\
\text { and completing } 2 \text { sets of an } \\
\text { upper and lower limb } \\
\text { resistance exercise with } \\
\text { elasticized bands at each } \\
\text { session }\end{array}$ & \multirow[t]{3}{*}{$\begin{array}{l}\text { There was no statistically } \\
\text { significant difference } \\
\text { between the } 3 \text { groups in } \\
\text { the } 3 \text {-muntue walking } \\
\text { distance test, upper limb } \\
\text { muscle strength, FEV1 \% } \\
\text { predicted, and the } \\
\text { incidence of adverse } \\
\text { events. }\end{array}$} \\
\hline & & & $\begin{array}{l}\text { 2)Moderate to high- } \\
\text { intensity Exercise } \\
\text { group }\end{array}$ & $\mathrm{N} / \mathrm{A}$ & $\begin{array}{l}\text { 15-minute exercise sessions } \\
2 \text { times a day: walking at } 70 \% \\
\text { of their 3-minute walk test for } \\
7.5 \text { mins and completing } 2 \\
\text { sets of an upper and lower } \\
\text { limb resistance exercise with } \\
\text { elasticized bands at each } \\
\text { session }\end{array}$ & \\
\hline & & & $\begin{array}{l}\text { 3) Management } \\
\text { without Exercise } \\
\text { Training }\end{array}$ & N/A & $\begin{array}{l}\text { Once-daily physical therapy, } \\
\text { including sputum clearance } \\
\text { techniques, mobility } \\
\text { assessments, and functional } \\
\text { training required for safe } \\
\text { discharge }\end{array}$ & \\
\hline & Inpatient hospital floor & $\mathrm{N} / \mathrm{A}$ & $\begin{array}{l}\text { 1) Resistance } \\
\text { Training }\end{array}$ & $\mathrm{N} / \mathrm{A}$ & $\begin{array}{l}\text { Cycling exercise intervention } \\
\text { using a pedal exerciser }\end{array}$ & $\begin{array}{l}\text { The exercise group had } \\
\text { statistically significant }\end{array}$ \\
\hline
\end{tabular}




\begin{tabular}{|c|c|c|c|c|c|c|}
\hline $\begin{array}{l}\text { Author, Year, } \\
\text { study design* }\end{array}$ & $\begin{array}{c}\text { Study setting } \\
\text { (outpatients, hospitalized } \\
\text { patients) }\end{array}$ & $\begin{array}{l}\text { Length } \\
\text { of } \\
\text { Follow } \\
\text { up } \\
\text { (month } \\
\text { s) }\end{array}$ & $\begin{array}{l}\text { Intervention(s) and } \\
\text { comparator }\end{array}$ & $\begin{array}{c}\text { Route of } \\
\text { administration }\end{array}$ & Dose and Duration & Conclusion \\
\hline $\begin{array}{l}\text { Torres-Sanchez, } \\
2017^{56}\end{array}$ & & & $\begin{array}{l}\text { 2) Management } \\
\text { without Resistance } \\
\text { Training }\end{array}$ & $\mathrm{N} / \mathrm{A}$ & Standard Care & $\begin{array}{l}\text { more improvements in } \\
\text { lower-limb strength, } \\
\text { balance, and number of } \\
\text { steps than the } \\
\text { management without } \\
\text { exercise training group. }\end{array}$ \\
\hline $\begin{array}{l}\text { Torres-Sanchez, } \\
2017^{57}\end{array}$ & Inpatient hospital floor & $\mathrm{N} / \mathrm{A}$ & $\begin{array}{l}\text { 2)Controlled } \\
\text { breathing + Range } \\
\text { of motion exercises }\end{array}$ & $\mathrm{N} / \mathrm{A}$ & $\begin{array}{l}\text { Daily 30-40min sessions of } \\
\text { physical therapy (relaxation, } \\
\text { pursed lips breathing, active } \\
\text { expiration) plus active range } \\
\text { of motion exercises for } 9 \\
\text { days. }\end{array}$ & $\begin{array}{l}\text { The resistance exercise } \\
\text { group had significantly } \\
\text { more improvement in } \\
\text { dyspnea (Modified Borg } \\
\text { scale) than the } \\
\text { management without } \\
\text { exercise Training group. } \\
\text { The resistance exercise } \\
\text { group and the controlled } \\
\text { breathing + range of } \\
\text { motion exercises group } \\
\text { had significantly more } \\
\text { improvements in quality of } \\
\text { life (EQ-5D VAS) than the } \\
\text { placebo. No significant } \\
\text { difference was found in } \\
\text { quality of life between the } \\
\text { resistance exercise group } \\
\text { and the controlled } \\
\text { breathing + range of } \\
\text { motion exercises group. } \\
\text { There was no statistically } \\
\text { significant difference in } \\
\text { FEV1 \% predicted } \\
\text { between the } 3 \text { groups. }\end{array}$ \\
\hline Troosters, $2010^{58}$ & Inpatient hospital floor & 1 & $\begin{array}{l}\text { 1) Management } \\
\text { without Resistance } \\
\text { Training }\end{array}$ & $\mathrm{N} / \mathrm{A}$ & $\begin{array}{l}\text { Standard doses of oral } \\
\text { corticosteroids to treat the } \\
\text { exacerbation for } 7 \text { days. }\end{array}$ & $\begin{array}{l}\text { There was no statistically } \\
\text { significant difference in 6- } \\
\text { minute walking distance } \\
\text { test and hospital } \\
\text { readmission between the } \\
\text { groups. }\end{array}$ \\
\hline
\end{tabular}




\begin{tabular}{|c|c|c|c|c|c|c|}
\hline $\begin{array}{l}\text { Author, Year, } \\
\text { study design* }\end{array}$ & $\begin{array}{c}\text { Study setting } \\
\text { (outpatients, hospitalized } \\
\text { patients) }\end{array}$ & $\begin{array}{l}\text { Length } \\
\text { of } \\
\text { Follow } \\
\text { up } \\
\text { (month } \\
\text { s) }\end{array}$ & $\begin{array}{l}\text { Intervention(s) and } \\
\text { comparator }\end{array}$ & $\begin{array}{c}\text { Route of } \\
\text { administration }\end{array}$ & Dose and Duration & Conclusion \\
\hline & & & $\begin{array}{l}\text { 2)Resistance } \\
\text { training }\end{array}$ & $\mathrm{N} / \mathrm{A}$ & $\begin{array}{l}3 \text { sets of } 8 \text { repetitions } \\
\text { quadriceps resistance training } \\
\text { once a day for } 7 \text { days }\end{array}$ & \\
\hline \multirow[t]{2}{*}{ Tumer, $2009^{59}$} & \multirow[t]{2}{*}{ Inpatient hospital floor } & \multirow[t]{2}{*}{$\mathrm{N} / \mathrm{A}$} & 1)Usual Diet & $\mathrm{N} / \mathrm{A}$ & $\begin{array}{l}\text { Standard hospital diet, } 1800 \\
\text { kcal/day for } 10 \text { days. }\end{array}$ & \multirow{2}{*}{$\begin{array}{l}\text { There was no statistically } \\
\text { significant difference in } \\
\text { FEV1 values. }\end{array}$} \\
\hline & & & $\begin{array}{l}\text { 2)High-fat, Low- } \\
\text { carbohydrate Diet }\end{array}$ & $\mathrm{N} / \mathrm{A}$ & $\begin{array}{l}50 \% \text { hospital diet and } 50 \% \text { a } \\
\text { specific enteral product } \\
\text { (pulmocare and hospital diet } \\
\text { composed of } 50 \% \text { fat and } \\
28 \% \text { CHO) for } 10 \text { days. }\end{array}$ & \\
\hline \multirow[t]{2}{*}{$\begin{array}{l}\text { Vermeeren, } \\
2004^{60}\end{array}$} & \multirow[t]{2}{*}{ Inpatient hospital floor } & \multirow[t]{2}{*}{3} & $\begin{array}{l}\text { 1)Nutritional } \\
\text { Intervention }\end{array}$ & $\mathrm{N} / \mathrm{A}$ & $\begin{array}{l}\text { Respifor: } 125 \mathrm{~mL}, 3 \times / 24 \mathrm{hours} \\
\text { (total of } 375 \mathrm{~mL} / 24 \mathrm{hours} \text { ) } \\
2.38 \mathrm{MJ} / \mathrm{day}, 20 \text { energy } \% \\
\text { protein, } 20 \text { energy } \% \text { fat and } \\
60 \text { energy } \% \text { carbohydrate for } \\
9 \text { days. }\end{array}$ & \multirow[t]{2}{*}{$\begin{array}{l}\text { There was no statistically } \\
\text { significant difference in } \\
\text { hospital admissions, } \\
\text { FEV1 \% predicted and } \\
\text { dyspnea scores (VAS) } \\
\text { between the two groups. }\end{array}$} \\
\hline & & & $\begin{array}{l}\text { 2) Placebo(non- } \\
\text { caloric fluid, vanilla } \\
\text { flavored water) }\end{array}$ & $\mathrm{N} / \mathrm{A}$ & $\begin{array}{l}\text { vanilla flavored water: } \\
125 \mathrm{~mL}, 3 \times / 24 \text { hours (total of } \\
375 \mathrm{~mL} / 24 \text { hours) with } 0 \\
\text { MJ/day for } 9 \text { days. }\end{array}$ & \\
\hline Woodruff, $2011^{61}$ & Inpatient hospital floor & 1 & 1) Zileuton & $\mathrm{N} / \mathrm{A}$ & $\begin{array}{l}600 \mathrm{mg}, 4 \times / 24 \text { hours (total of } \\
2400 \mathrm{mg} / 24 \text { hours) for } 14 \text { days }\end{array}$ & $\begin{array}{l}\text { Based on the intention-to- } \\
\text { treat principle, patients in }\end{array}$ \\
\hline
\end{tabular}




\begin{tabular}{|c|c|c|c|c|c|c|}
\hline $\begin{array}{l}\text { Author, Year, } \\
\text { study design* }\end{array}$ & $\begin{array}{c}\text { Study setting } \\
\text { (outpatients, hospitalized } \\
\text { patients) }\end{array}$ & $\begin{array}{l}\text { Length } \\
\text { of } \\
\text { Follow } \\
\text { up } \\
\text { (month } \\
\text { s) }\end{array}$ & $\begin{array}{l}\text { Intervention(s) and } \\
\text { comparator }\end{array}$ & $\begin{array}{c}\text { Route of } \\
\text { administration }\end{array}$ & Dose and Duration & Conclusion \\
\hline & & & 2)Placebo & N/A & For 14 days & $\begin{array}{l}\text { the zileuton group had } \\
\text { significantly higher FEV1 } \\
\text { absolute values than } \\
\text { those in the placebo } \\
\text { group. However, the } \\
\text { difference was not } \\
\text { significant based on per- } \\
\text { protocol analysis. There } \\
\text { was no statistically } \\
\text { significant difference in } \\
\text { clinical failures, death, } \\
\text { need for intubations, } \\
\text { hospital 30-day hospital } \\
\text { readmission, FEV1 \% } \\
\text { predicted, and adverse } \\
\text { events. }\end{array}$ \\
\hline Xiong, $2008^{62}$ & Inpatient hospital floor & $\mathrm{N} / \mathrm{A}$ & $\begin{array}{l}\text { 2) Management } \\
\text { without Atomization } \\
\text { Inhalation }\end{array}$ & $\mathrm{N} / \mathrm{A}$ & For 14 days. & $\begin{array}{l}\text { Patients in the } \\
\text { budesonide+ terbutaline, } \\
\text { atomization inhalation } \\
\text { group had statistically } \\
\text { significant more } \\
\text { improvements in FEV1 } \\
\text { absolute and \% predicted } \\
\text { than those in the } \\
\text { conventional group } \\
(p<0.05) \text {. }\end{array}$ \\
\hline \multirow[t]{3}{*}{ Yohannes, $2003^{63}$} & \multirow[t]{3}{*}{ Inpatient hospital floor } & \multirow[t]{3}{*}{1} & $\begin{array}{l}\text { 1)Gutter Frame + } \\
\text { Supplemental } \\
\text { Oxygen }\end{array}$ & $\mathrm{N} / \mathrm{A}$ & $\begin{array}{l}\text { Exercise: } 15 \text { min sessions, } 3 \\
\text { times a day for } 10 \text { days. } \\
\text { Oxygen: } 2 \mathrm{~L} / \mathrm{min}\end{array}$ & \multirow{3}{*}{$\begin{array}{l}\text { There was no statistically } \\
\text { significant difference in } \\
\text { dyspnea (Borg Score), } \\
\text { hospital readmission, and } \\
\text { death. }\end{array}$} \\
\hline & & & $\begin{array}{l}\text { 2)Gutter Frame + } \\
\text { Supplemental Air }\end{array}$ & N/A & $\begin{array}{l}\text { Exercise: } 15 \text { min sessions, } 3 \\
\text { times a day for } 10 \text { days. } \\
\text { Supplemental Air: } 2 \mathrm{~L} / \mathrm{min}\end{array}$ & \\
\hline & & & 3)Rollator + Oxygen & $\mathrm{N} / \mathrm{A}$ & $\begin{array}{l}\text { Exercise: } 15 \text { min sessions, } 3 \\
\text { times a day for } 10 \text { days. } \\
\text { Oxygen: } 2 \mathrm{~L} / \mathrm{min}\end{array}$ & \\
\hline
\end{tabular}




\begin{tabular}{|c|c|c|c|c|c|c|}
\hline $\begin{array}{l}\text { Author, Year, } \\
\text { study design* }\end{array}$ & $\begin{array}{c}\text { Study setting } \\
\text { (outpatients, hospitalized } \\
\text { patients) }\end{array}$ & $\begin{array}{l}\text { Length } \\
\text { of } \\
\text { Follow } \\
\text { up } \\
\text { (month } \\
\text { s) }\end{array}$ & $\begin{array}{l}\text { Intervention(s) and } \\
\text { comparator }\end{array}$ & $\begin{array}{c}\text { Route of } \\
\text { administration }\end{array}$ & Dose and Duration & Conclusion \\
\hline & & & 4) Rollator + Air & N/A & $\begin{array}{l}\text { Exercise: } 15 \text { min sessions, } 3 \\
\text { times a day for } 10 \text { days. } \\
\text { Supplemental Air: } 2 \mathrm{~L} / \mathrm{min}\end{array}$ & \\
\hline \multirow[t]{3}{*}{ Zuin, $2005^{64}$} & \multirow[t]{3}{*}{ Outpatient } & \multirow[t]{3}{*}{ N/A } & $\begin{array}{l}\text { 1)N-acetylcysteine } \\
1200 \mathrm{mg}\end{array}$ & Oral & $\begin{array}{l}1200 \mathrm{mg}, 1 \mathrm{x} / 24 \mathrm{hours} \text { (total of } \\
1200 \mathrm{mg} / 24 \text { hours) for } 10 \text { days }\end{array}$ & \multirow{3}{*}{$\begin{array}{l}\text { No significant difference } \\
\text { was found in cough } \\
\text { frequency and intensity } \\
\text { between the N- } \\
\text { acetylcysteine } 1200 \mathrm{mg} \\
\text { and N-acetylcysteine } 600 \\
\text { mg group. However, } \\
\text { compared with the } \\
\text { placebo group, patients in } \\
\text { the two groups had } \\
\text { significantly more } \\
\text { improvements in cough } \\
\text { intensity and cough } \\
\text { frequency. There was no } \\
\text { statistically significant } \\
\text { difference in absolute } \\
\text { FEV1 values. }\end{array}$} \\
\hline & & & $\begin{array}{l}\text { 2)N-acetylcysteine } \\
600 \mathrm{mg}\end{array}$ & Oral & $\begin{array}{l}600 \mathrm{mg}, 1 \times / 24 \text { hours (total of } \\
600 \mathrm{mg} / 24 \text { hours) for } 10 \text { days. }\end{array}$ & \\
\hline & & & 3)Placebo & Oral & & \\
\hline
\end{tabular}

* Study provided different numbers and conclusions. We used the numbers.

$\mathrm{CI}$ = confidence interval; COPD = chronic obstructive pulmonary disease; ER = emergency room; EPA = eicosapentaenoic acid; FEV1 = forced expiration volume in 1 second; g = gram; GOLD = global initiative for chronic obstructive lung disease; Hz = hertz; ICU = intensive care unit; IM = intramuscular; IU = international unit; IV = intravenous; kcal = kilocalorie; $\mathrm{kg}$ = kilogram; L = liter; $\mathrm{mg}=$ milligram; $\mathrm{min}=$ minute; $\mathrm{mJ}=$ millijoule; $\mathrm{ml}=$ milliliter; $\mathrm{N} / \mathrm{A}=$ not applicable; ONS = oral nutrition supplementation; PR = pulmonary rehabilitation; $\mathrm{RR}=$ relative risk; TENS = transcutaneous electrical nerve stimulation; VAS = visual analog scale; $\mu \mathrm{g}=$ microgram; $\mu$ mol $=$ micromole 
Table F.3. KQ3: Intervention description and conclusions

\begin{tabular}{|c|c|c|c|c|c|c|}
\hline $\begin{array}{l}\text { Author, Year, } \\
\text { study design* }\end{array}$ & $\begin{array}{c}\text { Study setting } \\
\text { (outpatients, } \\
\text { hospitalized } \\
\text { patients) }\end{array}$ & $\begin{array}{l}\text { Length of } \\
\text { Followup }\end{array}$ & $\begin{array}{l}\text { Intervention(s) } \\
\text { and comparator }\end{array}$ & $\begin{array}{c}\text { Route of } \\
\text { administratio } \\
\mathbf{n}\end{array}$ & Dose and Duration & Conclusion \\
\hline \multirow[t]{3}{*}{$\begin{array}{l}\text { Koutsogiannis, } \\
2000^{65}\end{array}$} & \multirow[t]{3}{*}{$\begin{array}{l}\text { Emergency } \\
\text { department }\end{array}$} & \multirow[t]{3}{*}{$\mathrm{N} / \mathrm{A}$} & 1) Salbutamol & $\begin{array}{l}\text { Oral } \\
\text { salbutamol, IB } \\
\text { nebulizer }\end{array}$ & $\begin{array}{l}\text { Salbutamol: } 5 \mathrm{mg} \text { at } 0, \\
15 \mathrm{~min}, \& 30 \mathrm{~min} \\
\text { Once IB } 500 \mu \mathrm{g} \text { at time } \\
0 \text { for } 1 \text { day. }\end{array}$ & \multirow[t]{3}{*}{$\begin{array}{l}\text { There was no statistically } \\
\text { significant difference in \% } \\
\text { predicted FEV1 and } \\
\text { absolute FEV1 values. }\end{array}$} \\
\hline & & & $\begin{array}{l}\text { 2)Ipratropium } \\
\text { Bromide }\end{array}$ & $\begin{array}{l}\text { Nebulizer, oral } \\
\text { salbutamol. }\end{array}$ & $\begin{array}{l}\text { Salbutamol: } 5 \mathrm{mg} \text { at time } \\
0 \text { \& Ipratropium } \\
\text { Bromide } 500 \mu \mathrm{g} \text { at } 0,15 \text {, } \\
\text { 30min for } 1 \text { day. }\end{array}$ & \\
\hline & & & 3)Combined & Oral/nebulizer & $\begin{array}{l}\text { Salbutamol } 5 \mathrm{mg} \& \mathrm{IB} \\
500 \mu \mathrm{g} \text { at time } 0,15, \\
\text { 30min }\end{array}$ & \\
\hline \multirow[t]{2}{*}{$\begin{array}{l}\text { Moayyedi, } \\
1995^{66}\end{array}$} & \multirow[t]{2}{*}{$\begin{array}{l}\text { Inpatient } \\
\text { hospital floor }\end{array}$} & \multirow[t]{2}{*}{ N/A } & 1) Salbutamol & Nebulized & $\begin{array}{l}5 \mathrm{mg}, 4 \times / 24 \text { hours (total } \\
\text { of } 20 \mathrm{mg} / 24 \text { hours) }\end{array}$ & \multirow{2}{*}{$\begin{array}{l}\text { There was no statistically } \\
\text { significant difference in } \\
\text { FEV1 values. }\end{array}$} \\
\hline & & & $\begin{array}{l}\text { 2) Salbutamol + } \\
\text { Ipratropium } \\
\text { Bromide }\end{array}$ & Nebulized & $\begin{array}{l}\text { Salbutamol: } \\
5 \mathrm{mg}, 4 \times / 24 \text { hours (total } \\
\text { of } 20 \mathrm{mg} / 24 \text { hours) } \\
\text { IB: } 500 \mu \mathrm{g}, 4 \mathrm{x} / 24 \text { hours } \\
\text { (total of } \\
2000 \mu \mathrm{g} / 24 \text { hours) }\end{array}$ & \\
\hline \multirow[t]{2}{*}{ Perri, $1985^{67}$} & \multirow[t]{2}{*}{$\begin{array}{l}\text { Inpatient } \\
\text { hospital floor }\end{array}$} & \multirow[t]{2}{*}{ N/A } & $\begin{array}{l}\text { 1) Salbutamol + } \\
\text { Beclomethasone } \\
\text { Dipropionate }\end{array}$ & Inhaled & $\begin{array}{l}\text { Salbutamol: } 75 \mu \mathrm{g} / \mathrm{puff} \\
2 \text { puffs, } 3 \times / 24 \mathrm{hours} \text { (total } \\
\text { of } 450 \mu \mathrm{g} / 24 \mathrm{hours} \text { ) for } \\
28 \text { days } \\
\text { Beclomethasone } \\
\text { Dipropionate: } 50 \mu \mathrm{g} / \mathrm{puff}, \\
2 \text { puffs, } 3 \times / 24 \mathrm{hours} \\
\text { (total of } 300 \mu \mathrm{g} / 24 \mathrm{hours} \text { ) } \\
\text { for } 28 \text { days }\end{array}$ & \multirow[t]{2}{*}{$\begin{array}{l}\text { There was no statistically } \\
\text { significant difference in \% } \\
\text { predicted FEV1 values, } \\
\text { clinical cures, and clinical } \\
\text { failures. }\end{array}$} \\
\hline & & & 2) Fenoterol & Inhaled & $\begin{array}{l}200 \mu \mathrm{g} / \text { puff, } 2 \text { puffs, } \\
3 \times / 24 \text { hours (total of } \\
1200 \mu \mathrm{g} / 24 \text { hours) for } 28 \\
\text { days }\end{array}$ & \\
\hline
\end{tabular}


Table F.4. KQ4: Intervention description and conclusions

\begin{tabular}{|c|c|c|c|c|c|c|}
\hline $\begin{array}{l}\text { Author, Year, } \\
\text { study design* }\end{array}$ & $\begin{array}{l}\text { Study setting } \\
\text { (outpatients, } \\
\text { hospitalized } \\
\text { patients) }\end{array}$ & $\begin{array}{l}\text { Length of } \\
\text { Follow up }\end{array}$ & $\begin{array}{l}\text { Intervention(s) } \\
\text { and comparator }\end{array}$ & $\begin{array}{c}\text { Route of } \\
\text { administration }\end{array}$ & Dose and Duration & Conclusion \\
\hline \multirow[t]{2}{*}{$\begin{array}{l}\text { Aggarwal, } \\
2011^{68}\end{array}$} & \multirow[t]{2}{*}{$\begin{array}{l}\text { Emergency } \\
\text { department }\end{array}$} & \multirow[t]{2}{*}{0.5} & $\begin{array}{l}\text { 1) Hydrocortisone } \\
\text { i.v. Followed by } \\
\text { Prednisolone oral }\end{array}$ & $\begin{array}{l}\text { IV } \\
\text { hydrocortisone } \\
\text { then oral } \\
\text { prednisolone }\end{array}$ & $\begin{array}{l}\text { Hydrocortisone: } 200 \mathrm{mg} \text {, } \\
4 \times / 24 \text { hours (total of } \\
800 \mathrm{mg} / 24 \text { hours) for } 14 \\
\text { day } \\
\text { Prednisolone: } \\
0.75 \mathrm{mg} / \mathrm{kg}, 1 \times / 24 \text { hours } \\
\text { (total of } \\
0.75 \mathrm{mg} / \mathrm{kg} / 24 \text { hours) for } \\
14 \text { days }\end{array}$ & \multirow{2}{*}{$\begin{array}{l}\text { After 2-week followup, the } \\
\text { patients who received IV } \\
\text { methylprednisolone and } \\
\text { oral methylprednisolone } \\
\text { were found to have } \\
\text { significantly more } \\
\text { improvements in FEV1 } \\
\text { than the patients who } \\
\text { received IV } \\
\text { hydrocortisone and oral } \\
\text { prednisolone. } \\
\text { There was no statistically } \\
\text { significant difference in } \\
\text { mortality, need for } \\
\text { intubation, treatment } \\
\text { failure, and dyspnea } \\
\text { (Medical Research } \\
\text { Council Dyspnea Scale). }\end{array}$} \\
\hline & & & $\begin{array}{l}\text { 2) } \\
\text { Methylprednisolone } \\
\text { iv, Followed by } \\
\text { Methylprednisolone } \\
\text { oral }\end{array}$ & IV then oral & $\begin{array}{l}\text { IV Methylprednisolone: } \\
125 \mathrm{mg} \text { bolus then } \\
40 \mathrm{mg}, 4 \times / 24 \text { hours (total } \\
\text { of } 160 \mathrm{mg} / 24 \text { hours) for } \\
14 \text { days } \\
\text { Oral } \\
\text { Methylprednisolone: } \\
0.6 \mathrm{mg} / \mathrm{kg}, 1 \times / 24 \text { hours } \\
\text { (total of } \\
0.6 \mathrm{mg} / \mathrm{kg} / 24 \text { hours) for } \\
14 \text { days }\end{array}$ & \\
\hline \multirow[t]{2}{*}{$\begin{array}{l}\text { Andre-Alves, } \\
2007^{69}\end{array}$} & \multirow[t]{2}{*}{ Outpatient } & \multirow[t]{2}{*}{1} & 1) Azithromycin & Oral & $\begin{array}{l}500 \mathrm{mg}, 1 \times / 24 \text { hours } \\
\text { (total of } \\
500 \mathrm{mg} / 24 \text { hours) for } 3 \\
\text { days }\end{array}$ & \multirow{2}{*}{$\begin{array}{l}\text { Patients in the amoxicillin } \\
\text { group had significantly } \\
\text { higher FEV1 \% predicted } \\
\text { than those in the } \\
\text { azithromycin group*. } \\
\text { There was no statistically } \\
\text { significant difference } \\
\text { between azithromycin and } \\
\text { amoxicillin in clinical cure, } \\
\text { clinical failure, and } \\
\text { absolute FEV1 values.* }\end{array}$} \\
\hline & & & 2) Amoxicillin & Oral & $\begin{array}{l}\text { 500mg, } 3 \times / 24 \text { hours } \\
\text { (total of } \\
1500 \mathrm{mg} / 24 \text { hours) for } 10 \\
\text { days }\end{array}$ & \\
\hline Aubier, $2002^{70}$ & Outpatient & 1 & 1) Telithromycin & Oral & $\begin{array}{l}\text { Telithromycin: } 800 \mathrm{mg} \text {, } \\
1 \times / 24 \text { hours (total of } \\
800 \mathrm{mg} / 24 \text { hours) for } \\
5 \text { days. } \\
\text { Then Placebo for } 5 \\
\text { days. }\end{array}$ & $\begin{array}{l}\text { There was no statistically } \\
\text { significant difference in } \\
\text { clinical cure rate between } \\
\text { the two groups. However, } \\
\text { significantly fewer patients } \\
\text { in the telithromycin group }\end{array}$ \\
\hline
\end{tabular}




\begin{tabular}{|c|c|c|c|c|c|c|}
\hline $\begin{array}{l}\text { Author, Year, } \\
\text { study design* }\end{array}$ & $\begin{array}{l}\text { Study setting } \\
\text { (outpatients, } \\
\text { hospitalized } \\
\text { patients) }\end{array}$ & $\begin{array}{l}\text { Length of } \\
\text { Follow up }\end{array}$ & $\begin{array}{l}\text { Intervention(s) } \\
\text { and comparator }\end{array}$ & $\begin{array}{c}\text { Route of } \\
\text { administration }\end{array}$ & Dose and Duration & Conclusion \\
\hline & & & $\begin{array}{l}\text { 2) Amoxicillin + } \\
\text { Clavulanate }\end{array}$ & Oral & $\begin{array}{l}500 \mathrm{mg}, 125 \mathrm{mg} \text {, } \\
3 \times / 24 \mathrm{hours} \text { (total of } \\
\text { 1500mg,375mg/24hour } \\
\text { s) for } 10 \text { days. }\end{array}$ & $\begin{array}{l}\text { reported adverse events. } \\
\text { No death was reported in } \\
\text { either group. }\end{array}$ \\
\hline \multirow[t]{2}{*}{ Blasi, $2013^{71}$} & \multirow[t]{2}{*}{$\begin{array}{l}\text { Outpatient } \\
\text { Inpatient } \\
\text { hospital floor }\end{array}$} & \multirow[t]{2}{*}{6} & 1) Prulifloxacin & Oral & $\begin{array}{l}600 \mathrm{mg}, 1 \times / 24 \text { hours } \\
\text { (total of } \\
600 \mathrm{mg} / 24 \text { hours) for } 7 \\
\text { days }\end{array}$ & \multirow{2}{*}{$\begin{array}{l}\text { The prulifloxacin group } \\
\text { had statistically significant } \\
\text { more repeat } \\
\text { exacerbations than the } \\
\text { levofloxacin group after } 6 \\
\text { weeks of treatment. There } \\
\text { was no statistically } \\
\text { significant difference } \\
\text { between the two groups in } \\
\text { FEV1, symptoms scores } \\
\text { (Total Symptom Score), } \\
\text { dyspnea (Dyspnea Score } \\
\text { 0-3), rate of clinical cure, } \\
\text { rate of clinical failure, and } \\
\text { repeat exacerbations at } 6 \\
\text { months of treatment. No } \\
\text { death was reported in any } \\
\text { of the two groups. }\end{array}$} \\
\hline & & & 2) Levofloxacin & Oral & $\begin{array}{l}500 \mathrm{mg}, 1 \times / 24 \text { hours } \\
\text { (total of } \\
500 \mathrm{mg} / 24 \text { hours) for } 7 \\
\text { days }\end{array}$ & \\
\hline \multirow[t]{2}{*}{ Dark, $1993^{72}$} & \multirow[t]{2}{*}{ Outpatient } & \multirow[t]{2}{*}{1} & 1) Azithromycin & NR & $\begin{array}{l}500 \mathrm{mg} \text { on day } 1 \text {, } \\
1 \times / 24 \text { hours (total of } \\
500 \mathrm{mg} / 24 \text { hours), then } \\
250 \mathrm{mg}, 1 \times / 24 \text { hours } \\
\text { (total of } \\
250 \mathrm{mg} / 24 \text { hours) on } \\
\text { days } 2 \text { to } 5 \text {. }\end{array}$ & \multirow[t]{2}{*}{$\begin{array}{l}\text { There was no statistically } \\
\text { significant difference } \\
\text { between cefaclor and } \\
\text { azithromycin in clinical } \\
\text { cure, clinical failure, and } \\
\text { number of adverse } \\
\text { events. }\end{array}$} \\
\hline & & & 2) Cefaclor & NR & $\begin{array}{l}\text { 500mg, 3x/24hours } \\
\text { (total of } \\
1500 \mathrm{mg} / 24 \text { hours) for } 10 \\
\text { days }\end{array}$ & \\
\hline $\begin{array}{l}\text { de Jong, } \\
2007^{98}\end{array}$ & $\begin{array}{l}\text { Inpatient } \\
\text { hospital floor }\end{array}$ & 3 & $\begin{array}{l}\text { 1)Intravenous } \\
\text { Prednisolone }\end{array}$ & IV & $\begin{array}{l}60 \mathrm{mg}, 1 \times / 24 \text { hours (total } \\
\text { of } 60 \mathrm{mg} / 24 \text { hours) for } 5 \\
\text { days }\end{array}$ & $\begin{array}{l}\text { There was no statistically } \\
\text { significant difference in } \\
\text { treatment failure, quality of }\end{array}$ \\
\hline
\end{tabular}




\begin{tabular}{|c|c|c|c|c|c|c|}
\hline $\begin{array}{l}\text { Author, Year, } \\
\text { study design* }\end{array}$ & $\begin{array}{l}\text { Study setting } \\
\text { (outpatients, } \\
\text { hospitalized } \\
\text { patients) }\end{array}$ & $\begin{array}{l}\text { Length of } \\
\text { Follow up }\end{array}$ & $\begin{array}{l}\text { Intervention(s) } \\
\text { and comparator }\end{array}$ & $\begin{array}{c}\text { Route of } \\
\text { administration }\end{array}$ & Dose and Duration & Conclusion \\
\hline & & & $\begin{array}{l}\text { 2)Oral } \\
\text { Prednisolone }\end{array}$ & Oral & $\begin{array}{l}60 \mathrm{mg}, 1 \times / 24 \text { hours (total } \\
\text { of } 60 \mathrm{mg} / 24 \text { hours) for } 5 \\
\text { days }\end{array}$ & $\begin{array}{l}\text { life (SGRQ), absolute } \\
\text { FEV1 values, hospital } \\
\text { readmission for COPD, } \\
\text { and death. }\end{array}$ \\
\hline \multirow[t]{2}{*}{$\begin{array}{l}\text { Ding, } 2016^{73} \\
20000\end{array}$} & \multirow[t]{2}{*}{$\begin{array}{l}\text { Inpatient } \\
\text { hospital floor }\end{array}$} & \multirow[t]{2}{*}{12} & 1) Budesonide & Inhaled & $\begin{array}{l}2 \mathrm{mg}, 1 \times 3 / 24 \text { hours (total } \\
\text { of } 6 \mathrm{mg} / 24 \text { hours }\end{array}$ & \multirow{2}{*}{$\begin{array}{l}\text { There was no statistically } \\
\text { significantly difference in } \\
\text { quality of life (COPD } \\
\text { Assessment Test), FEV1 } \\
\text { absolute values, and } \\
\text { repeat exacerbation } \\
\text { between the groups }\end{array}$} \\
\hline & & & $\begin{array}{l}\text { 2) } \\
\text { Methylprednisolone }\end{array}$ & IV & $\begin{array}{l}40 \mathrm{mg}, 1 \times / 24 \text { hours (total } \\
\text { of } 40 \mathrm{mg} / 24 \text { hours }\end{array}$ & \\
\hline $\begin{array}{l}\text { Emami } \\
\text { Ardestani, } \\
2017^{74}\end{array}$ & $\begin{array}{l}\text { Emergency } \\
\text { department }\end{array}$ & N/A & 1) Dexamethasone & IV & $\begin{array}{l}\text { Methylprednisolone: } \\
2 \mathrm{mg} / \mathrm{kg}, 1 \times / 24 \mathrm{hours} \\
\text { (total of } 2 \mathrm{mg} / \mathrm{kg} \text { ) for } 3 \\
\text { days. } \\
\text { Then, } 40 \mathrm{mg} \text {, } \\
1 \mathrm{x} / 24 \mathrm{hours} \text { (total of } \\
40 \mathrm{mg} / 24 \mathrm{hours} \text { ) for } 3 \\
\text { days. } \\
\text { Then switched to oral } \\
\text { Prednisone: } 30 \mathrm{mg} \text {, } \\
\text { 1x/24hours (total of } \\
30 \mathrm{mg} / 24 \mathrm{hours} \text { ) tapered } \\
\text { every } 3 \text { days with } 5 \mathrm{mg} \\
\text { decrease in dosage (for } \\
15 \text { days). } \\
\text { Then inhaled } \\
\text { budesonide: } 400 \mu \mathrm{g}, \\
2 \times / 24 \mathrm{hours} \text { (total of } \\
800 \mu \mathrm{g} / 24 \mathrm{hours)} \text { for at } \\
\text { least } 3 \text { months. }\end{array}$ & $\begin{array}{l}\text { Patients in the } \\
\text { methylprednisolone group } \\
\text { were significantly more } \\
\text { likely to have } \\
\text { improvement of cough } \\
\text { than patients in the } \\
\text { dexamethasone group. } \\
\text { There was no statistically } \\
\text { significant difference } \\
\text { between groups for } \\
\text { shortness of breath and } \\
\text { general wellbeing. }\end{array}$ \\
\hline
\end{tabular}




\begin{tabular}{|c|c|c|c|c|c|c|}
\hline $\begin{array}{l}\text { Author, Year, } \\
\text { study design* }\end{array}$ & $\begin{array}{l}\text { Study setting } \\
\text { (outpatients, } \\
\text { hospitalized } \\
\text { patients) }\end{array}$ & $\begin{array}{l}\text { Length of } \\
\text { Follow up }\end{array}$ & $\begin{array}{l}\text { Intervention(s) } \\
\text { and comparator }\end{array}$ & $\begin{array}{c}\text { Route of } \\
\text { administration }\end{array}$ & Dose and Duration & Conclusion \\
\hline & & & $\begin{array}{l}\text { 2) } \\
\text { Methylprednisolone }\end{array}$ & IV & $\begin{array}{l}\text { Dexamethasone: } \\
0.375 \mathrm{mg} / \mathrm{kg}, 1 \times / 24 \mathrm{hours} \\
\text { (total of } \\
0.375 \mathrm{mg} / \mathrm{kg} / 24 \mathrm{hours} \text { ), } \\
\text { then gradually tapered } \\
\text { for } 7 \text { to } 14 \text { days. } \\
\text { Then } \\
\text { Methylprednisolone: } \\
30 \mathrm{mg}, 1 \times / 24 \text { hours (total } \\
\text { of } 30 \mathrm{mg} / 24 \text { hours), and } \\
\text { continued by the same } \\
\text { protocol as MP group } \\
\text { for } 6 \text { days. }\end{array}$ & \\
\hline \multirow[t]{2}{*}{$\begin{array}{l}\text { Giusti, } \\
2016^{75} \text { Non- } \\
\text { inferiority trial }\end{array}$} & \multirow[t]{2}{*}{$\begin{array}{l}\text { Inpatient } \\
\text { hospital floor }\end{array}$} & \multirow[t]{2}{*}{12} & 1)Levofloxacin & Oral & $\begin{array}{l}500 \mathrm{mg}, 1 \mathrm{x} / 24 \text { hours } \\
\text { (total of } \\
500 \mathrm{mg} / 24 \text { hours) for } 10 \\
\text { days (early interruption } \\
\text { at } 7 \text { days if all } \\
\text { symptoms resolved) }\end{array}$ & \multirow[t]{2}{*}{$\begin{array}{l}\text { There was no statistically } \\
\text { significant difference in } \\
\text { repeat exacerbation, } \\
\text { mortality, clinical cure, and } \\
\text { Total Symptom Scores. }\end{array}$} \\
\hline & & & 2)Prulifloxacin & Oral & $\begin{array}{l}600 \mathrm{mg}, 1 \times / 24 \text { hours } \\
\text { (total of } \\
600 \mathrm{mg} / 24 \text { hours) for } 10 \\
\text { days (early interruption } \\
\text { at } 7 \text { days if all } \\
\text { symptoms resolved) }\end{array}$ & \\
\hline \multirow[t]{3}{*}{ Gunen, $2007^{9}$} & \multirow[t]{3}{*}{$\begin{array}{l}\text { Inpatient } \\
\text { hospital floor }\end{array}$} & \multirow[t]{3}{*}{1} & $\begin{array}{l}\text { 1) Systemic } \\
\text { Corticosteroids }\end{array}$ & Inhalation & & \multirow{3}{*}{$\begin{array}{l}\text { Patients in the nebulized } \\
\text { budesonide group had } \\
\text { statistically significant } \\
\text { higher FEV1 \% predicted } \\
\text { than those in the } \\
\text { prednisolone group or the } \\
\text { control group. There was } \\
\text { no statistically significant } \\
\text { difference in hospital } \\
\text { readmissions, repeat } \\
\text { exacerbations, FEV1 \% } \\
\text { predicted, and death. }\end{array}$} \\
\hline & & & 2)Prednisolone & IV & $\begin{array}{l}40 \mathrm{mg}, 1 \times / 24 \text { hours (total } \\
\text { of } 40 \mathrm{mg} / 24 \text { hours) for } \\
\text { more than } 10 \text { days }\end{array}$ & \\
\hline & & & $\begin{array}{l}\text { 3)Nebulized } \\
\text { Budesonide }\end{array}$ & Nebulized & $\begin{array}{l}1500 \mu \mathrm{g}, 4 \times / 24 \text { hours } \\
\text { (total of } 0.5 \mathrm{mg} / 24 \text { hours) }\end{array}$ & \\
\hline
\end{tabular}




\begin{tabular}{|c|c|c|c|c|c|c|}
\hline $\begin{array}{l}\text { Author, Year, } \\
\text { study design* }\end{array}$ & $\begin{array}{l}\text { Study setting } \\
\text { (outpatients, } \\
\text { hospitalized } \\
\text { patients) }\end{array}$ & $\begin{array}{l}\text { Length of } \\
\text { Follow up }\end{array}$ & $\begin{array}{l}\text { Intervention(s) } \\
\text { and comparator }\end{array}$ & $\begin{array}{c}\text { Route of } \\
\text { administration }\end{array}$ & Dose and Duration & Conclusion \\
\hline \multirow[t]{2}{*}{$\begin{array}{l}\text { Hamacher, } \\
1995^{76}\end{array}$} & \multirow[t]{2}{*}{$\begin{array}{l}\text { Inpatient } \\
\text { hospital floor }\end{array}$} & \multirow[t]{2}{*}{1} & 1) Meropenem & IV & $\begin{array}{l}1 \mathrm{~g} \text { infused over } 20-30 \\
\text { mins, } 3 x / 24 \text { hours (total } \\
\text { of } 3 \mathrm{~g} / 24 \text { hours) for a } \\
\text { mean of } 8.9 \text { days }\end{array}$ & \multirow{2}{*}{$\begin{array}{l}\text { There was no statistically } \\
\text { significant difference in } \\
\text { clinical cure, clinical } \\
\text { failure, and repeated } \\
\text { exacerbation. }\end{array}$} \\
\hline & & & $\begin{array}{l}\text { 2)Imipenem + } \\
\text { Cilastatin }\end{array}$ & IV & $\begin{array}{l}1 \mathrm{~g} \text { of each infused over } \\
30 \text { mins, } 3 x / 24 \text { hours } \\
\text { (total of } 3 g / 24 \text { hours) for } \\
\text { a mean of } 9 \text { days }\end{array}$ & \\
\hline \multirow[t]{2}{*}{ Hasani, $1998^{77}$} & \multirow[t]{2}{*}{ Outpatient } & \multirow[t]{2}{*}{1} & 1)Amoxicillin & Oral & $\begin{array}{l}\text { 500mg, } 3 \times / 24 \text { hours } \\
\text { (total of } \\
1500 \mathrm{mg} / 24 \text { hours) for } 7 \\
\text { days }\end{array}$ & \multirow[t]{2}{*}{$\begin{array}{l}\text { There was no statistically } \\
\text { significant difference in } \\
\text { FEV1 values. }\end{array}$} \\
\hline & & & 2)Ciprofloxacin & Oral & $\begin{array}{l}\text { 500mg, } 2 \times / 24 \text { hours } \\
\text { (total of } \\
1000 \mathrm{mg} / 24 \text { hours) for } 7 \\
\text { days }\end{array}$ & \\
\hline \multirow[t]{2}{*}{$\begin{array}{l}\text { Leophonte, } \\
1998^{78}\end{array}$} & \multirow[t]{2}{*}{ N/A } & \multirow[t]{2}{*}{0.75} & $\begin{array}{l}\text { 1)Trovafloxacin } 200 \\
\text { mg }\end{array}$ & N/A & $\begin{array}{l}\text { 200mg, 1x/24hours } \\
\text { (total of } \\
200 \mathrm{mg} / 24 \text { hours) for } 5 \\
\text { days }\end{array}$ & \multirow[t]{2}{*}{$\begin{array}{l}\text { There was no statistically } \\
\text { significant difference in } \\
\text { clinical cure and death } \\
\text { between the groups. }\end{array}$} \\
\hline & & & $\begin{array}{l}\text { 2)Trovafloxacin } 100 \\
\text { mg }\end{array}$ & $\mathrm{N} / \mathrm{A}$ & $\begin{array}{l}\text { 100mg, 1x/24hours } \\
\text { (total of } \\
100 \mathrm{mg} / 24 \mathrm{hours} \text { ) for } 5 \\
\text { days }\end{array}$ & \\
\hline Leuppi, $2013^{79}$ & $\begin{array}{l}\text { Emergency } \\
\text { department, } \\
\text { Inpatient } \\
\text { hospital floor }\end{array}$ & 6 & $\begin{array}{l}\text { 1) } \\
\text { Methylprednisolone } \\
\text { (day 1), Prednisone } \\
\text { (days 2-5), Placebo } \\
\text { (days 6-14) }\end{array}$ & $\begin{array}{l}\text { Methylprednisol } \\
\text { one IV } \\
\text { Prednisone PO } \\
\text { Placebo PO }\end{array}$ & $\begin{array}{l}\text { Methylprednisolone: } \\
40 \mathrm{mg}, 1 \times / 24 \text { hours (total } \\
\text { of } 40 \mathrm{mg} / 24 \text { hours) for } 1 \\
\text { day } \\
\text { Prednisone: } 40 \mathrm{mg} \text {, } \\
\text { 1x/24hours (total of } \\
40 \mathrm{mg} / 24 \text { hours) during } \\
\text { days } 2-5 \\
\text { Placebo days } 6-14\end{array}$ & $\begin{array}{l}\text { There was no statistically } \\
\text { significant difference in } \\
\text { repeat exacerbations, } \\
\text { death, need for } \\
\text { intubations, and \% } \\
\text { predicted FEV1 values, } \\
\text { dyspnea score (Medical } \\
\text { Research Council } \\
\text { Dyspnea Scale), quality of }\end{array}$ \\
\hline
\end{tabular}




\begin{tabular}{|c|c|c|c|c|c|c|}
\hline $\begin{array}{l}\text { Author, Year, } \\
\text { study design* }\end{array}$ & $\begin{array}{l}\text { Study setting } \\
\text { (outpatients, } \\
\text { hospitalized } \\
\text { patients) }\end{array}$ & $\begin{array}{l}\text { Length of } \\
\text { Follow up }\end{array}$ & $\begin{array}{l}\text { Intervention(s) } \\
\text { and comparator }\end{array}$ & $\begin{array}{c}\text { Route of } \\
\text { administration }\end{array}$ & Dose and Duration & Conclusion \\
\hline & & & $\begin{array}{l}\text { 2) } \\
\text { Methylprednisolone } \\
\text { (day 1), Prednisone } \\
\text { (days 2-14) }\end{array}$ & $\begin{array}{l}\text { Methylprednisol } \\
\text { one IV } \\
\text { Prednisone PO }\end{array}$ & $\begin{array}{l}\text { Methylprednisolone: } \\
40 \mathrm{mg}, 1 \times / 24 \text { hours (total } \\
\text { of } 40 \mathrm{mg} / 24 \text { hours) for } 1 \\
\text { day } \\
\text { Prednisone: } 40 \mathrm{mg} \text {, } \\
\text { 1x/24hours (total of } \\
40 \mathrm{mg} / 24 \text { hours) during } \\
\text { days } 2-14\end{array}$ & $\begin{array}{l}\text { life (Bronchitis-Associated } \\
\text { Quality of Life). }\end{array}$ \\
\hline \multirow[t]{2}{*}{$\begin{array}{l}\text { Llor, } \\
2009^{80} \text { Non- } \\
\text { inferiority trial }\end{array}$} & \multirow[t]{2}{*}{ Outpatient } & \multirow[t]{2}{*}{1} & 1)Amoxicillin & Oral & $\begin{array}{l}500 \mathrm{mg}, 3 \times / 24 \text { hours } \\
\text { (total of } \\
1500 \mathrm{mg} / 24 \text { hours) for } \\
10 \text { days }\end{array}$ & \multirow[t]{2}{*}{$\begin{array}{l}\text { There was no statistically } \\
\text { significant difference } \\
\text { between the two groups in } \\
\text { clinical cure rates. }\end{array}$} \\
\hline & & & $\begin{array}{l}\text { 2)Amoxicillin + } \\
\text { Clavulanate }\end{array}$ & Oral & $\begin{array}{l}500 \mathrm{mg}, 125 \mathrm{mg}, \\
3 \times / 24 \text { hours (total of } \\
\text { 1500mg/24hours, } \\
375 \mathrm{mg} / 24 \text { hours) for } 10 \\
\text { days }\end{array}$ & \\
\hline \multirow[t]{6}{*}{ Maltais, $2002^{12}$} & \multirow[t]{6}{*}{$\begin{array}{l}\text { Inpatient } \\
\text { hospital floor }\end{array}$} & \multirow[t]{6}{*}{10 days } & \multirow[t]{2}{*}{ 1) Budesonide } & Nebulized & $\begin{array}{l}2 \mathrm{mg}, 4 \mathrm{x} / 24 \text { hours (total } \\
\text { of } 8 \mathrm{mg} / 24 \text { hours for } 3 \\
\text { days }\end{array}$ & \multirow{6}{*}{$\begin{array}{l}\text { The budesonide group } \\
\text { and the prednisolone } \\
\text { group had a significantly } \\
\text { larger increase in absolute } \\
\text { FEV1 than the placebo } \\
\text { group. Increases in FEV1 } \\
\text { were not significantly } \\
\text { different in the budesonide } \\
\text { group and the } \\
\text { prednisolone group. There } \\
\text { was no statistically } \\
\text { significant difference in } \\
\text { dyspnea (Modified Borg } \\
\text { Scale), need for } \\
\text { intubation, and death } \\
\text { between the three groups. }\end{array}$} \\
\hline & & & & Inhaled & $\begin{array}{l}\text { Then: } \\
2000 \mu \mathrm{g}, 1 \times / 24 \text { hours } \\
\text { (total of } \\
2000 \mu \mathrm{g} / 24 \text { hours) for } 7 \\
\text { days }\end{array}$ & \\
\hline & & & 2) Prednisolone & Oral & $\begin{array}{l}30 \mathrm{mg}, 2 \times / 24 \text { hours (total } \\
\text { of } 60 \mathrm{mg} / 24 \text { hours) for } 3 \\
\text { days, then } \\
40 \mathrm{mg} / 24 \text { hours for } 7 \\
\text { days }\end{array}$ & \\
\hline & & & \multirow[t]{3}{*}{ 3) Placebo } & Nebulized & For 72 hours & \\
\hline & & & & Inhaled & For 7 days & \\
\hline & & & & Oral & $\begin{array}{l}\text { For } 72 \text { hours, then for } 7 \\
\text { days }\end{array}$ & \\
\hline Mirici, $2003^{81}$ & $\begin{array}{l}\text { Emergency } \\
\text { department, } \\
\text { Inpatient } \\
\text { hospital floor }\end{array}$ & $\mathrm{N} / \mathrm{A}$ & $\begin{array}{l}\text { 1) Parenteral } \\
\text { Corticosteroid } \\
\text { (prednisolone) }\end{array}$ & IV & $\begin{array}{l}40 \mathrm{mg}, 1 \times / 24 \text { hours (total } \\
\text { of } 40 \mathrm{mg} / 24 \text { hours) for } 10 \\
\text { days }\end{array}$ & $\begin{array}{l}\text { No adverse events were } \\
\text { reported in any of the two } \\
\text { groups. }\end{array}$ \\
\hline
\end{tabular}




\begin{tabular}{|c|c|c|c|c|c|c|}
\hline $\begin{array}{l}\text { Author, Year, } \\
\text { study design* }\end{array}$ & $\begin{array}{c}\text { Study setting } \\
\text { (outpatients, } \\
\text { hospitalized } \\
\text { patients) }\end{array}$ & $\begin{array}{l}\text { Length of } \\
\text { Follow up }\end{array}$ & $\begin{array}{l}\text { Intervention(s) } \\
\text { and comparator }\end{array}$ & $\begin{array}{c}\text { Route of } \\
\text { administration }\end{array}$ & Dose and Duration & Conclusion \\
\hline & & & $\begin{array}{l}\text { 2) Nebulized } \\
\text { Corticosteroid } \\
\text { (budesonide) }\end{array}$ & Nebulized & $\begin{array}{l}4 \mathrm{mg}, 2 \times / 24 \text { hours (total } \\
\text { of } 8 \mathrm{mg} / 24 \text { hours) for } 10 \\
\text { days }\end{array}$ & \\
\hline \multirow[t]{6}{*}{$\begin{array}{l}\text { Niewoehner, } \\
1999^{13}\end{array}$} & \multirow[t]{6}{*}{$\begin{array}{l}\text { Emergency } \\
\text { department }\end{array}$} & \multirow[t]{6}{*}{6} & \multirow[t]{2}{*}{$\begin{array}{l}\text { 1) Glucocorticoid } \\
\text { for } 8 \text { weeks }\end{array}$} & IV & $\begin{array}{l}\text { Methylprednisolone: } \\
125 \mathrm{mg}, 4 \times / 24 \text { hours } \\
\text { (total of } \\
500 \mathrm{mg} / 24 \text { hours) for } 3 \\
\text { days }\end{array}$ & \multirow{6}{*}{$\begin{array}{l}\text { Patients in the 8-week } \\
\text { glucocorticoid group and } \\
\text { 2-week glucocorticoid } \\
\text { group had significantly } \\
\text { less clinical failures than } \\
\text { the placebo group. There } \\
\text { was no statistically } \\
\text { significant difference in } \\
\text { death, FEV1, need for } \\
\text { intubations, and hospital } \\
\text { readmissions. }\end{array}$} \\
\hline & & & & Oral & $\begin{array}{l}\text { Prednisone: } 60 \mathrm{mg} \text { on } \\
\text { days } 4 \text { through } 7,40 \mathrm{mg} \\
\text { on days } 8 \text { through } 11 \text {, } \\
20 \mathrm{mg} \text { on days } 12 \\
\text { through } 43,10 \mathrm{mg} \text { on } \\
\text { days } 44 \text { through } 50 \text {, and } \\
5 \mathrm{mg} \text { on days } 51 \text { through } \\
57\end{array}$ & \\
\hline & & & \multirow[t]{2}{*}{$\begin{array}{l}\text { 2) Glucocorticoid } \\
\text { for } 2 \text { week }\end{array}$} & IV & $\begin{array}{l}\text { Methylprednisolone: } \\
125 \mathrm{mg}, 4 \times / 24 \text { hours } \\
\text { (total of } \\
500 \mathrm{mg} / 24 \text { hours) for } 3 \\
\text { days }\end{array}$ & \\
\hline & & & & Oral & $\begin{array}{l}\text { Prednisone: } 60 \mathrm{mg} \text { on } \\
\text { days } 4 \text { through } 7,40 \mathrm{mg} \\
\text { on days } 8 \text { through } 11, \\
\text { and } 20 \mathrm{mg} \text { on days } 12 \\
\text { through } 15 \\
\text { Placebo capsules on } \\
\text { days } 16 \text { through } 57\end{array}$ & \\
\hline & & & \multirow[t]{2}{*}{ 3) Placebo } & IV & $\begin{array}{l}5 \% \text { dextrose solution: } \\
125 \mathrm{mg}, 4 \times / 24 \text { hours } \\
\text { (total of } \\
500 \mathrm{mg} / 24 \mathrm{hours} \text { ) for } 3 \\
\text { days }\end{array}$ & \\
\hline & & & & Oral & $\begin{array}{l}\text { Placebo capsules on } \\
\text { days } 4 \text { through } 57\end{array}$ & \\
\hline
\end{tabular}




\begin{tabular}{|c|c|c|c|c|c|c|}
\hline $\begin{array}{l}\text { Author, Year, } \\
\text { study design* }\end{array}$ & $\begin{array}{l}\text { Study setting } \\
\text { (outpatients, } \\
\text { hospitalized } \\
\text { patients) }\end{array}$ & $\begin{array}{l}\text { Length of } \\
\text { Follow up }\end{array}$ & $\begin{array}{l}\text { Intervention(s) } \\
\text { and comparator }\end{array}$ & $\begin{array}{c}\text { Route of } \\
\text { administration }\end{array}$ & Dose and Duration & Conclusion \\
\hline \multirow[t]{2}{*}{$\begin{array}{l}\text { Petitpretz, } \\
2007^{82} \text { Non- } \\
\text { inferiority trial }\end{array}$} & \multirow[t]{2}{*}{ Outpatient } & \multirow[t]{2}{*}{6} & 1) Levofloxacin & Oral & $\begin{array}{l}500 \mathrm{mg}, 1 \times / 24 \text { hours } \\
\text { (total of } \\
500 \mathrm{mg} / 24 \text { hours) for } 10 \\
\text { days }\end{array}$ & \multirow{2}{*}{$\begin{array}{l}\text { There was no statistically } \\
\text { significant difference in } \\
\text { clinical cure rates, repeat } \\
\text { exacerbation, and number } \\
\text { of adverse events } \\
\text { between the two groups. }\end{array}$} \\
\hline & & & $\begin{array}{l}\text { 2) } \\
\text { Cefuroxime/axetil }\end{array}$ & Oral & $\begin{array}{l}\text { cefuroxime/axetil: } \\
300.72 \mathrm{mg}(250 \mathrm{mg} \\
\text { cefuroxime), } 2 \times / 24 \text { hours } \\
\text { (total of } \\
601.44 \mathrm{mg} / 24 \text { hours } \\
\text { (500mg/24hours)) for } 10 \\
\text { days }\end{array}$ & \\
\hline \multirow[t]{2}{*}{ Phillips, $1993^{83}$} & \multirow[t]{2}{*}{$\begin{array}{l}\text { Outpatient, } \\
\text { Inpatient } \\
\text { hospital floor }\end{array}$} & \multirow[t]{2}{*}{1} & 1) Cefpodoxime & Oral & $\begin{array}{l}\text { 200mg, 2x/24hours } \\
\text { (total of } \\
400 \mathrm{mg} / 24 \text { hours) for } 10 \\
\text { days }\end{array}$ & \multirow{2}{*}{$\begin{array}{l}\text { There was no statistically } \\
\text { significant difference in } \\
\text { clinical cures, clinical } \\
\text { failures, repeat } \\
\text { exacerbations, and } \\
\text { incidence of adverse } \\
\text { events. }\end{array}$} \\
\hline & & & 2) Cefaclor & Oral & $\begin{array}{l}\text { 250mg, 3x/24hours } \\
\text { (total of } \\
750 \mathrm{mg} / 24 \text { hours) for } 10 \\
\text { days }\end{array}$ & \\
\hline \multirow[t]{2}{*}{$\begin{array}{l}\text { Rhee, } \\
2015^{84} \text { Non- } \\
\text { inferiority trial }\end{array}$} & \multirow[t]{2}{*}{ Outpatient } & \multirow[t]{2}{*}{1} & 1) Zabofloxacin & Oral & $\begin{array}{l}367 \mathrm{mg}, 1 \times / 24 \text { hours } \\
\text { (total of } \\
367 \mathrm{mg} / 24 \text { hours) for } 5 \\
\text { days }\end{array}$ & \multirow{2}{*}{$\begin{array}{l}\text { Patients in the } \\
\text { zabofloxacin group had } \\
\text { significantly better } \\
\text { outcomes in symptoms } \\
\text { (Exacerbations of Chronic } \\
\text { Pulmonary Disease Tool, } \\
\text { p<0.01) and quality of life } \\
\text { (COPD Assessment Test, } \\
\text { p<0.01). There was no } \\
\text { statistically significant } \\
\text { difference in clinical cures, } \\
\text { and clinical failures, } \\
\text { suggesting Zabofloxacin is } \\
\text { not inferior to } \\
\text { Moxifloxacin. }\end{array}$} \\
\hline & & & 2) Moxifloxacin & Oral & $\begin{array}{l}\text { 400mg, 1x/24hours } \\
\text { (total of } \\
400 \mathrm{mg} / 24 \text { hours) for } 7 \\
\text { days }\end{array}$ & \\
\hline Rizzato, $1998^{85}$ & $\begin{array}{l}\text { Inpatient } \\
\text { hospital floor }\end{array}$ & $\mathrm{N} / \mathrm{a}$ & $\begin{array}{l}\text { 1) Deflazacort } \\
\text { Hemisuccinate }\end{array}$ & IV & $\begin{array}{l}60 \mathrm{mg}, 1 \times / 24 \text { hours (total } \\
\text { of } 60 \mathrm{mg} / 24 \text { hours) for } 7 \\
\text { days }\end{array}$ & $\begin{array}{l}\text { There was no statistically } \\
\text { significant difference in }\end{array}$ \\
\hline
\end{tabular}




\begin{tabular}{|c|c|c|c|c|c|c|}
\hline $\begin{array}{l}\text { Author, Year, } \\
\text { study design* }\end{array}$ & $\begin{array}{l}\text { Study setting } \\
\text { (outpatients, } \\
\text { hospitalized } \\
\text { patients) }\end{array}$ & $\begin{array}{l}\text { Length of } \\
\text { Follow up }\end{array}$ & $\begin{array}{l}\text { Intervention(s) } \\
\text { and comparator }\end{array}$ & $\begin{array}{c}\text { Route of } \\
\text { administration }\end{array}$ & Dose and Duration & Conclusion \\
\hline & & & $\begin{array}{l}\text { 2) } \\
\text { Methylprednisolone } \\
\text {-ne }\end{array}$ & IV & $\begin{array}{l}40 \mathrm{mg}, 1 \times / 24 \text { hours (total } \\
\text { of } 40 \mathrm{mg} / 24 \text { hours) for } 7 \\
\text { days }\end{array}$ & $\begin{array}{l}\text { absolute FEV1 values and } \\
\text { death. }\end{array}$ \\
\hline \multirow[t]{2}{*}{$\begin{array}{l}\text { Roede, } \\
2007^{86} \text { Non- } \\
\text { inferiority trial }\end{array}$} & \multirow[t]{2}{*}{$\begin{array}{l}\text { Inpatient } \\
\text { hospital floor }\end{array}$} & \multirow[t]{2}{*}{3} & $\begin{array}{l}\text { 1) Amoxycillin }+ \\
\text { Clavulanic acid for } \\
3 \text { days }\end{array}$ & Oral/ IV & $\begin{array}{l}\text { 625mg, } 4 \times / 24 \text { hours } \\
\text { (total of } \\
2500 \mathrm{mg} / 24 \text { hours) for } 3 \\
\text { days. } \\
\text { Then Placebo: } \\
625 \mathrm{mg}, 4 \times / 24 \text { hours } \\
\text { (total of } \\
2500 \mathrm{mg} / 24 \text { hours) for } 7 \\
\text { days. }\end{array}$ & \multirow{2}{*}{$\begin{array}{l}\text { There was no statistically } \\
\text { significant difference in } \\
\text { clinical cure, clinical } \\
\text { failure, symptom scores } \\
\text { (combined with shortness } \\
\text { of breath, sputum volume, } \\
\text { and sputum color), } \\
\text { hospital readmissions, } \\
\text { death, repeat } \\
\text { exacerbations, and } \\
\text { adverse events. }\end{array}$} \\
\hline & & & $\begin{array}{l}\text { 2) Amoxycillin }+ \\
\text { Clavulanic acid for } \\
10 \text { days }\end{array}$ & Oral/ IV & $\begin{array}{l}\text { Oral/ IV: } \\
625 \mathrm{mg}, 4 \times / 24 \text { hours } \\
\text { (total of } \\
2500 \mathrm{mg} / 24 \text { hours) for } 3 \\
\text { days. } \\
\text { Then oral: } \\
625 \mathrm{mg}, 4 \times / 24 \text { hours } \\
\text { (total of } \\
2500 \mathrm{mg} / 24 \text { hours) for } 7 \\
\text { days. }\end{array}$ & \\
\hline $\begin{array}{l}\text { Ruiz-Gonzalez, } \\
2007^{87}\end{array}$ & $\begin{array}{l}\text { Emergency } \\
\text { department, } \\
\text { Inpatient } \\
\text { hospital floor }\end{array}$ & 6 & 1) Levofloxacin & Unclear & $\begin{array}{l}500 \mathrm{mg}, 1 \times / 24 \text { hours } \\
\text { (total of } \\
500 \mathrm{mg} / 24 \text { hours) for } 10 \\
\text { days }\end{array}$ & $\begin{array}{l}\text { Patients in the } \\
\text { levofloxacin group had } \\
\text { significantly less hospital } \\
\text { admissions than patients }\end{array}$ \\
\hline
\end{tabular}




\begin{tabular}{|c|c|c|c|c|c|c|}
\hline $\begin{array}{l}\text { Author, Year, } \\
\text { study design* }\end{array}$ & $\begin{array}{l}\text { Study setting } \\
\text { (outpatients, } \\
\text { hospitalized } \\
\text { patients) }\end{array}$ & $\begin{array}{l}\text { Length of } \\
\text { Follow up }\end{array}$ & $\begin{array}{l}\text { Intervention(s) } \\
\text { and comparator }\end{array}$ & $\begin{array}{c}\text { Route of } \\
\text { administration }\end{array}$ & Dose and Duration & Conclusion \\
\hline & & & $\begin{array}{l}\text { 2) Standard care } \\
\text { (Clarithromycin, } \\
\text { Cefuroxime, or } \\
\text { Amoxicillin } \\
\text { +Clavulanate) }\end{array}$ & Unclear & $\begin{array}{l}\text { One of the following: } \\
\text { Clarithromycin: } 500 \mathrm{mg} \text {, } \\
\text { 2x/24hours (total of } \\
\text { 1000mg/24hours). } \\
\text { Cefuroxime axetil: } \\
\text { 500mg, } 2 \times / 24 \text { hours } \\
\text { (total of } \\
\text { 1000mg/24hours). } \\
\text { Amoxicillin/ } \\
\text { Clavulanate: } \\
\text { 875mg, 125mg, } \\
3 \times / 24 \text { hours (total of } \\
2625 \mathrm{mg} \text {, } \\
375 \mathrm{mg} / 24 \text { hours). }\end{array}$ & $\begin{array}{l}\text { in the standard care } \\
\text { group. } \\
\text { There was no statistically } \\
\text { significant difference in } \\
\text { mortality, \% predicted } \\
\text { FEV1 and quality of life } \\
\text { (Airways Questionnaire } \\
\text { 20), and repeat } \\
\text { exacerbations. }\end{array}$ \\
\hline \multirow[t]{2}{*}{ Sayiner, $2001^{88}$} & \multirow[t]{2}{*}{$\begin{array}{l}\text { Inpatient } \\
\text { hospital floor }\end{array}$} & \multirow[t]{2}{*}{6} & $\begin{array}{l}\text { 1) } \\
\text { Methylprednisolone } \\
\text { for } 3 \text { days }\end{array}$ & IV & $\begin{array}{l}0.5 \mathrm{mg} / \mathrm{kg}, 4 \times / 24 \text { hours } \\
\text { (total of } 2 \mathrm{mg} / \mathrm{kg} / 24 \mathrm{jrs} \text { ) } \\
\text { for } 3 \text { days }\end{array}$ & \multirow{2}{*}{$\begin{array}{l}\text { Patients in the 10-day } \\
\text { methylprednisolone group } \\
\text { had significantly more } \\
\text { increase in absolute FEV1 } \\
\text { values than those in the 3- } \\
\text { day group immediately } \\
\text { after treatment. There was } \\
\text { no statistically significant } \\
\text { difference in repeat } \\
\text { exacerbations, dyspnea } \\
\text { (1-7 scale), and cough (1- } \\
7 \text { scale). }\end{array}$} \\
\hline & & & $\begin{array}{l}\text { 2) } \\
\text { Methylprednisolone } \\
\text { for } 10 \text { days }\end{array}$ & IV & $\begin{array}{l}0.5 \mathrm{mg} / \mathrm{kg}, 4 \mathrm{x} / 24 \mathrm{hours} \\
\text { (total of } 2 \mathrm{mg} / \mathrm{kg} / 24 \mathrm{jrs} \text { ) } \\
\text { for } 3 \text { days } \\
\text { Then } 0.5 \mathrm{mg} / \mathrm{kg} \text {, } \\
2 \times / 24 \mathrm{hours} \text { (total of } \\
1 \mathrm{mg} / \mathrm{kg} / 24 \mathrm{hours} \text { ) for } 3 \\
\text { days and } 0.5 \mathrm{mg} / \mathrm{kg}, \\
1 \times / 24 \mathrm{hours} \text { (total of } \\
0.5 \mathrm{mg} / \mathrm{kg} / 24 \text { hours) for } 4 \\
\text { more days. }\end{array}$ & \\
\hline $\begin{array}{l}\text { Stallberg, } \\
\text { 2009 }{ }^{89} \text { Non- } \\
\text { inferiority trial }\end{array}$ & Outpatient & 3 & $\begin{array}{l}\text { 1)Budesonide + } \\
\text { Formoterol }\end{array}$ & Inhalation & $\begin{array}{l}\text { Budesonide: } 320 \mu \mathrm{g}, \\
4 \times / 24 \text { hours (total of } \\
\text { 1280 } \mu \mathrm{g} / 24 \text { hours) for } 14 \\
\text { days Formoterol: } 9 \mu \mathrm{g}, \\
4 \mathrm{x} / 24 \text { hours (total of } \\
36 \mu \mathrm{g} / 24 \text { hours) for } 14 \\
\text { days }\end{array}$ & $\begin{array}{l}\text { There was no statistically } \\
\text { significant difference in \% } \\
\text { predicted and absolute } \\
\text { FEV1 values, quality of life } \\
\text { (Clinical COPD } \\
\text { Questionnaire), treatment } \\
\text { failures, repeat }\end{array}$ \\
\hline
\end{tabular}




\begin{tabular}{|c|c|c|c|c|c|c|}
\hline $\begin{array}{l}\text { Author, Year, } \\
\text { study design* }\end{array}$ & $\begin{array}{l}\text { Study setting } \\
\text { (outpatients, } \\
\text { hospitalized } \\
\text { patients) }\end{array}$ & $\begin{array}{l}\text { Length of } \\
\text { Follow up }\end{array}$ & $\begin{array}{l}\text { Intervention(s) } \\
\text { and comparator }\end{array}$ & $\begin{array}{c}\text { Route of } \\
\text { administration }\end{array}$ & Dose and Duration & Conclusion \\
\hline & & & $\begin{array}{l}\text { 2)Prednisolone + } \\
\text { Formoterol }\end{array}$ & Inhalation & $\begin{array}{l}\text { Prednisone: } 30 \mathrm{mg} \text {, } \\
1 \times / 24 \text { hours (total of } \\
30 \mathrm{mg} / 24 \text { hours) } \\
\text { Formoterol } 9 \mu \mathrm{g}, \\
2 \times / 24 \text { hours (total of } \\
18 \mu \mathrm{g} \text { ) for } 14 \text { days. }\end{array}$ & $\begin{array}{l}\text { exacerbations, } \\
\text { hospitalization, dyspnea } \\
\text { (Difficulty Breathing), } \\
\text { cough ( } 0-4 \text { Scale), and } \\
\text { incidence of adverse } \\
\text { events. }\end{array}$ \\
\hline \multirow[t]{2}{*}{ Sun, $2015^{90}$} & \multirow[t]{2}{*}{$\begin{array}{l}\text { Inpatient } \\
\text { hospital floor }\end{array}$} & \multirow[t]{2}{*}{0.25} & $\begin{array}{l}\text { 1)Inhaled } \\
\text { Budesonide }\end{array}$ & Inhalation & $\begin{array}{l}3 \mathrm{mg}, 2 \times / 24 \text { hours (total } \\
\text { of } 6 \mathrm{mg} / 24 \text { hours) for } 10 \\
\text { days }\end{array}$ & \multirow{2}{*}{$\begin{array}{l}\text { Patients in the inhaled } \\
\text { budesonide group had } \\
\text { significantly lower } \\
\text { incidence of adverse } \\
\text { events than those in the } \\
\text { systemic } \\
\text { methylprednisolone group. } \\
\text { There was no statistically } \\
\text { significant difference in \% } \\
\text { predicted FEV1 values. }\end{array}$} \\
\hline & & & $\begin{array}{l}\text { 2)Systemic } \\
\text { Methylprednisolone }\end{array}$ & $\begin{array}{l}\text { Injection and } \\
\text { oral }\end{array}$ & $\begin{array}{l}\text { Methylprednisolone } \\
\text { acetate injectable } \\
\text { suspension: } \\
40 \mathrm{mg}, 4 \times / 24 \text { hours (total } \\
\text { of } 160 \mathrm{mg} / 24 \text { hours) for } 3 \\
\text { days. } \\
\text { Methylprednisolone } \\
\text { tablets: } \\
8 \mathrm{mg}, 2 \times / 24 \text { hours (total } \\
\text { of } 16 \mathrm{mg} / 24 \text { hours) for } 7 \\
\text { days. }\end{array}$ & \\
\hline \multirow[t]{3}{*}{ Ucar, $2014^{91}$} & \multirow[t]{3}{*}{$\begin{array}{l}\text { Inpatient } \\
\text { hospital floor }\end{array}$} & \multirow[t]{3}{*}{ N/A } & $\begin{array}{l}\text { 1)Methylprednisolo } \\
\text { ne }\end{array}$ & IV & $\begin{array}{l}40 \mathrm{mg}, 1 \times / 24 \text { hours (total } \\
\text { of } 40 \mathrm{mg} / 24 \text { hours) }\end{array}$ & \multirow{3}{*}{$\begin{array}{l}\text { There was no statistically } \\
\text { significant difference in } \\
\text { FEV1 \% predicted, and } \\
\text { dyspnea (Borg Scale) } \\
\text { between the groups. }\end{array}$} \\
\hline & & & 2)Budesonide 4mg & Nebulized & $\begin{array}{l}2 \mathrm{mg}, 2 \times / 24 \text { hours (total } \\
\text { of } 4 \mathrm{mg} / 24 \text { hours) }\end{array}$ & \\
\hline & & & 3)Budesonide 8mg & Nebulized & $\begin{array}{l}4 \mathrm{mg}, 2 \mathrm{x} / 24 \text { hours(total } \\
8 / 24 \text { hours) }\end{array}$ & \\
\hline \multirow[t]{4}{*}{ Umut, $1999^{92}$} & \multirow[t]{4}{*}{$\begin{array}{l}\text { Inpatient } \\
\text { hospital floor }\end{array}$} & \multirow[t]{4}{*}{ N/A } & 1)Azithromycin & $\mathrm{N} / \mathrm{A}$ & $\begin{array}{l}500 \mathrm{mg}, 1 \times / 24 \mathrm{hours} \\
\text { (total of } 500 \mathrm{mg} / 24 \mathrm{hrs} \text { ) } \\
\text { for } 3 \text { days }\end{array}$ & \multirow{4}{*}{$\begin{array}{l}\text { There was no statistically } \\
\text { significant difference in } \\
\text { absolute FEV1 and clinical } \\
\text { cure between the groups. }\end{array}$} \\
\hline & & & $\begin{array}{l}\text { 2)Ampicillin + } \\
\text { Sulbactam }\end{array}$ & N/A & $\begin{array}{l}1.5 \mathrm{~g}, 1 \mathrm{x} / 24 \mathrm{hrs} \text { (total of } \\
1.5 \mathrm{~g} / 24 \mathrm{hrs} \text { ) for } 10 \text { days }\end{array}$ & \\
\hline & & & 3)Ciprofloxacin & $\mathrm{N} / \mathrm{A}$ & $\begin{array}{l}1 \mathrm{~g}, 1 \mathrm{x} / 24 \mathrm{hrs} \text { (total of } \\
1 \mathrm{~g} / 24 \mathrm{hrs} \text { ) for } 10 \text { days }\end{array}$ & \\
\hline & & & 4)Cefaclor & $\mathrm{N} / \mathrm{A}$ & $\begin{array}{l}1.5 \mathrm{~g}, 1 \mathrm{x} / 24 \mathrm{hours} \text { (total } \\
\text { of } 1.5 \mathrm{~g} / 24 \text { hours) for } 10 \\
\text { days }\end{array}$ & \\
\hline
\end{tabular}




\begin{tabular}{|c|c|c|c|c|c|c|}
\hline $\begin{array}{l}\text { Author, Year, } \\
\text { study design* }\end{array}$ & $\begin{array}{l}\text { Study setting } \\
\text { (outpatients, } \\
\text { hospitalized } \\
\text { patients) }\end{array}$ & $\begin{array}{l}\text { Length of } \\
\text { Follow up }\end{array}$ & $\begin{array}{l}\text { Intervention(s) } \\
\text { and comparator }\end{array}$ & $\begin{array}{c}\text { Route of } \\
\text { administration }\end{array}$ & Dose and Duration & Conclusion \\
\hline \multirow[t]{2}{*}{$\begin{array}{l}\text { van Zanten, } \\
2007^{93}\end{array}$} & \multirow[t]{2}{*}{$\begin{array}{l}\text { Inpatient } \\
\text { hospital floor }\end{array}$} & \multirow[t]{2}{*}{ N/A } & $\begin{array}{l}\text { 1)Continuous } \\
\text { Cefotaxime }\end{array}$ & IV & $\begin{array}{l}\text { An initial loading dose } \\
\text { of } 1 \mathrm{~g} \text { given over } 30 \mathrm{~min} \text {. } \\
\text { Then, a continuous } \\
\text { infusion of cefotaxime } \\
\text { ( } 2 \mathrm{~g} / 24 \text { hours) for } 7 \\
\text { days }\end{array}$ & \multirow[t]{2}{*}{$\begin{array}{l}\text { There was no statistically } \\
\text { significant difference in } \\
\text { clinical cures and clinical } \\
\text { failures. }\end{array}$} \\
\hline & & & $\begin{array}{l}\text { 2)Intermittent } \\
\text { Cefotaxime }\end{array}$ & IV & $\begin{array}{l}1 \mathrm{~g}, 3 x / 24 \text { hours (total of } \\
3 \mathrm{~g} / 24 \text { hours) for } 7 \text { days. }\end{array}$ & \\
\hline \multirow[t]{2}{*}{$\begin{array}{l}\text { Whitlock, } \\
1995^{94}\end{array}$} & \multirow[t]{2}{*}{ Outpatient } & \multirow[t]{2}{*}{0.5} & 1)Azithromycin & Oral & $\begin{array}{l}\text { 250mg, } 2 x / 24 \text { hours } \\
\text { (total of } \\
500 \mathrm{mg} / 24 \text { hours) for } 1 \\
\text { day } \\
\text { Then, } 250 \mathrm{mg} \text {, } \\
1 \times / 24 \text { hours (total of } \\
250 \mathrm{mg} / 24 \text { hours) for } 4 \\
\text { days. }\end{array}$ & \multirow[t]{2}{*}{$\begin{array}{l}\text { There was no statistically } \\
\text { significant difference in } \\
\text { clinical cures, clinical } \\
\text { failures, Incomplete cure } \\
\text { and incidence of adverse } \\
\text { events between the two } \\
\text { groups. }\end{array}$} \\
\hline & & & $\begin{array}{l}\text { 2)Amoxicillin + } \\
\text { Clavulanate }\end{array}$ & Oral & $\begin{array}{l}\text { 500mg, } 3 x / 24 \text { hours } \\
\text { (total of } \\
1500 \mathrm{mg} / 24 \text { hours) for } 10 \\
\text { days }\end{array}$ & \\
\hline
\end{tabular}




\begin{tabular}{|c|c|c|c|c|c|c|}
\hline $\begin{array}{l}\text { Author, Year, } \\
\text { study design* }\end{array}$ & $\begin{array}{c}\text { Study setting } \\
\text { (outpatients, } \\
\text { hospitalized } \\
\text { patients) }\end{array}$ & $\begin{array}{l}\text { Length of } \\
\text { Follow up }\end{array}$ & $\begin{array}{l}\text { Intervention(s) } \\
\text { and comparator }\end{array}$ & $\begin{array}{c}\text { Route of } \\
\text { administration }\end{array}$ & Dose and Duration & Conclusion \\
\hline \multirow[t]{2}{*}{ Willaert, $2002^{95}$} & $\begin{array}{l}\text { Inpatient } \\
\text { hospital floor }\end{array}$ & 1 & $\begin{array}{l}\text { 1)Intravenous } \\
\text { Steroids + Aerosol } \\
\text { Bronchodilators }\end{array}$ & $\mathrm{N} / \mathrm{A}$ & $\begin{array}{l}\text { Methylprednisolone IV: } \\
40 \mathrm{mg}, 1 \times / 24 \text { hours (total } \\
\text { of } 40 \mathrm{mg} / 24 \text { hours) for } 10 \\
\text { days. } \\
\text { Then, } 20 \mathrm{mg} \text {, } \\
\text { 1x/24hours (total of } \\
20 \mathrm{mg} / 24 \text { hours). } \\
\text { Then } \\
\text { Methylprednisolone PO: } \\
4 \mathrm{mg}, 1 \times / 24 \text { hours (total } \\
\text { of } 4 \mathrm{mg} / 24 \text { hours) for } 4 \\
\text { days } \\
\text { Aerosol Salbutamol: } \\
\text { 10mg, } 1 \times / 24 \text { hours (total } \\
\text { of } 10 \mathrm{mg} / 24 \text { hours) } \\
\text { Ipratropium bromide: } 1 \\
\text { mg, } 1 \times / 24 \text { hours (total of } \\
1 \mathrm{mg} / 24 \mathrm{hours)} \\
\text { administered in } 4 \\
\text { aerosols. }\end{array}$ & \multirow[t]{2}{*}{$\begin{array}{l}\text { There was no statistically } \\
\text { significant difference in } \\
\text { FEV1 absolute, quality of } \\
\text { life (Chronic Respiratory } \\
\text { Disease Index } \\
\text { Questionnaire), clinical } \\
\text { failures, death, hospital } \\
\text { readmission, and ICU } \\
\text { admissions. }\end{array}$} \\
\hline & & & $\begin{array}{l}\text { 2)Oral steroids + } \\
\text { Multiple Dose } \\
\text { Inhaler } \\
\text { Bronchodilators }\end{array}$ & $\mathrm{N} / \mathrm{A}$ & $\begin{array}{l}\text { Oral } \\
\text { Methylprednisolone: } \\
32 \mathrm{mg}, 1 \times / 24 \text { hours (total } \\
\text { of } 32 \mathrm{mg} / 24 \text { hours) for } 1 \\
\text { week } \\
\text { Then, } 24 \mathrm{mg} \text {, } \\
1 \times / 24 \mathrm{hours} \text { (total of } \\
24 \mathrm{mg} / 24 \text { hours) for } 4 \\
\text { days } \\
\text { Then } 20 \mathrm{mg}, 1 \times / 24 \text { hours } \\
\text { (total of } 20 \mathrm{mg} / 24 \text { hours) } \\
\text { for } 1 \text { week } \\
\text { Duovent: } 4 \times 4 \text { puffs/day } \\
\text { with a cumulative } \\
\text { dose of } 1.6 \mathrm{mg} / 24 \mathrm{hours} \\
\text { Fenoterol and } \\
640 \mathrm{mg} / 24 \mathrm{hours} \\
\text { Ipratropium Bromide }\end{array}$ & \\
\hline
\end{tabular}




\begin{tabular}{|c|c|c|c|c|c|c|}
\hline $\begin{array}{l}\text { Author, Year, } \\
\text { study design* }\end{array}$ & $\begin{array}{c}\text { Study setting } \\
\text { (outpatients, } \\
\text { hospitalized } \\
\text { patients) }\end{array}$ & $\begin{array}{l}\text { Length of } \\
\text { Follow up }\end{array}$ & $\begin{array}{l}\text { Intervention(s) } \\
\text { and comparator }\end{array}$ & $\begin{array}{c}\text { Route of } \\
\text { administration }\end{array}$ & Dose and Duration & Conclusion \\
\hline \multirow[t]{2}{*}{$\begin{array}{l}\text { Wilson, } \\
2012^{96} \text { Non- } \\
\text { inferiority trial }\end{array}$} & \multirow[t]{2}{*}{ Outpatient } & \multirow[t]{2}{*}{2} & Moxifloxacin & Oral & $\begin{array}{l}400 \mathrm{mg}, 4 \times / 24 \text { hours } \\
\text { (total of } \\
1600 \mathrm{mg} / 24 \text { hours) for } 5 \\
\text { days. }\end{array}$ & \multirow{2}{*}{$\begin{array}{l}\text { There was no statistically } \\
\text { significant difference in } \\
\text { clinical failures, death, } \\
\text { hospital admissions, } \\
\text { quality of life (SGRQ), } \\
\text { FEV1 absolute and \% } \\
\text { predicted, and incidence } \\
\text { of adverse events. }\end{array}$} \\
\hline & & & $\begin{array}{l}\text { Amoxicillin + } \\
\text { Clavulanic Acid }\end{array}$ & Oral & $\begin{array}{l}875 \mathrm{mg}, 125 \mathrm{mg} \text {, } \\
2 \times / 24 \mathrm{hours} \text { (total of } \\
1750 \mathrm{mg}, 250 \mathrm{mg} / 24 \mathrm{hour} \\
\text { s) for } 7 \text { days }\end{array}$ & \\
\hline \multirow[t]{2}{*}{ Yoon, $2013^{97}$} & \multirow[t]{2}{*}{ Outpatient } & \multirow[t]{2}{*}{0.25} & Levofloxacin & $\mathrm{N} / \mathrm{A}$ & $\begin{array}{l}500 \mathrm{mg}, 1 \times / 24 \text { hours } \\
\text { (total of } \\
500 \mathrm{mg} / 24 \text { hours) for } 7 \\
\text { days }\end{array}$ & \multirow{2}{*}{$\begin{array}{l}\text { There was no statistically } \\
\text { significant difference in } \\
\text { clinical cures, clinical } \\
\text { failures, and adverse } \\
\text { events between the two } \\
\text { groups. No death was } \\
\text { reported. }\end{array}$} \\
\hline & & & Cefuroxime & $\mathrm{N} / \mathrm{A}$ & $\begin{array}{l}\text { Moderate } \\
\text { exacerbations: } \\
250 \mathrm{mg}, 2 \times / 24 \text { hours } \\
\text { (total of } \\
500 \mathrm{mg} / 24 \text { hours) for } 7 \\
\text { days. } \\
\text { Sever exacerbations: } \\
500 \mathrm{mg}, 2 \times / 24 \text { hours } \\
\text { (total of } \\
\text { 1000mg/24hours) for } 7 \\
\text { days. }\end{array}$ & \\
\hline
\end{tabular}

* Study provided different numbers and conclusions. We used the numbers.

COPD = chronic obstructive pulmonary disease; FEV1 = forced expiration volume in 1 second; $\mathrm{g}$ = gram; ICU = intensive care unit; $\mathrm{IV}=$ intravenous; Kg = kilogram; $\mathrm{mg}=$ milligram; $\mu \mathrm{g}=$ microgram; Min/s = minutes; MP = Methylprednisolone; N/A = not applicable; N/A = not reported; SGRQ = St. George Respiratory Questionnaire; VAS = Visual Analog Scale 


\section{Appendix G. Results by Severity}

Table G.1. KQ1 results: mild ECOPD severity- antibiotics vs. placebo

\begin{tabular}{|c|c|c|}
\hline Outcome & Findings & $\begin{array}{c}\text { Study Design and Sample } \\
\text { Size }\end{array}$ \\
\hline $\begin{array}{l}\text { Mortality } \\
\text { Longest Followup }\end{array}$ & OR: $1.28 ; 95 \% \mathrm{Cl}: 0.49$ to $3.33, \mathrm{I}^{2}=\mathrm{N} / \mathrm{A}$ & $1 \mathrm{RCT}^{15}$ with 305 patients \\
\hline $\begin{array}{l}\text { Dyspnea } \\
\text { (questionnaire: CRQ, } \\
\text { dyspnea) } \\
\text { Longest Followup }\end{array}$ & $\begin{array}{l}\text { WMD: } 0.00 ; 95 \% \mathrm{Cl}:-0.97 \text { to } 0.97 \text {, } \\
\mathrm{I}^{2}=\mathrm{N} / \mathrm{A}\end{array}$ & $1 \mathrm{RCT}^{5}$ with 35 patients \\
\hline $\begin{array}{l}\text { Other Symptoms } \\
\text { CCQ symptom score } \\
\text { End of Intervention }\end{array}$ & $\begin{array}{l}\text { WMD: } 0.00 ; 95 \% \mathrm{Cl}:-0.66 \text { to } 0.66 \text {, } \\
\mathrm{I}^{2}=\mathrm{N} / \mathrm{A}\end{array}$ & $1 \mathrm{RCT}^{5}$ with 35 patients \\
\hline $\begin{array}{l}\text { Quality of Life } \\
\text { CRQ } \\
\text { Longest Followup }\end{array}$ & $\begin{array}{l}\text { WMD: } 0.00 ; 95 \% \mathrm{Cl}:-1.79 \text { to } 1.79 \text {, } \\
\mathrm{I}^{2}=\mathrm{N} / \mathrm{A}\end{array}$ & $1 \mathrm{RCT}^{5}$ with 35 patients \\
\hline $\begin{array}{l}\text { FEV1 \% Predicted } \\
\text { Longest Followup }\end{array}$ & $\begin{array}{l}\text { WMD: }-0.80 ; 95 \% \mathrm{Cl}:-6.67 \text { to } 5.07 \text {, } \\
\mathrm{I}^{2}=\mathrm{N} / \mathrm{A}\end{array}$ & $1 \mathrm{RCT}^{5}$ with 35 patients \\
\hline $\begin{array}{l}\text { FEV1 Absolute } \\
\text { End of Intervention }\end{array}$ & $\begin{array}{l}\text { WMD: } 1.30 ; 95 \% \mathrm{Cl}:-3.83 \text { to } 6.43 \text {, } \\
\mathrm{I}^{2}=\mathrm{N} / \mathrm{A}\end{array}$ & $1 \mathrm{RCT}^{10}$ with 100 patients \\
\hline $\begin{array}{l}\text { FEV1 Absolute } \\
\text { Longest Followup }\end{array}$ & $\begin{array}{l}\text { WMD: }-0.05 ; 95 \% \mathrm{Cl}:-0.21 \text { to } 0.11 \text {, } \\
\mathrm{I}^{2}=\mathrm{N} / \mathrm{A}\end{array}$ & $1 \mathrm{RCT}^{5}$ with 35 patients \\
\hline $\begin{array}{l}\text { Repeat Exacerbation } \\
\text { End of Intervention }\end{array}$ & OR: $2.24 ; 95 \% \mathrm{Cl}: 0.58$ to $8.69, \mathrm{I}^{2}=\mathrm{N} / \mathrm{A}$ & $1 \mathrm{RCT}^{5}$ with 35 patients \\
\hline $\begin{array}{l}\text { Repeat Exacerbation } \\
30 \text { days }\end{array}$ & OR: $2.00 ; 95 \% \mathrm{Cl}: 0.16$ to $24.33, \mathrm{I}^{2}=\mathrm{N} / \mathrm{A}$ & $1 \mathrm{RCT}^{5}$ with 35 patients \\
\hline $\begin{array}{l}\text { Repeat Exacerbation } \\
6 \text { Month }\end{array}$ & OR: $2.24 ; 95 \% \mathrm{Cl}: 0.58$ to $8.69, \mathrm{I}^{2}=\mathrm{N} / \mathrm{A}$ & $1 \mathrm{RCT}^{5}$ with 35 patients \\
\hline $\begin{array}{l}\text { Repeat Exacerbation } \\
\text { Longest Followup }\end{array}$ & OR: $2.24 ; 95 \% \mathrm{Cl}: 0.58$ to $8.69, \mathrm{I}^{2}=\mathrm{N} / \mathrm{A}$ & $1 \mathrm{RCT}^{5}$ with 35 patients \\
\hline $\begin{array}{l}\text { Clinical Cure } \\
\text { End of Intervention }\end{array}$ & OR: $2.04 ; 95 \% \mathrm{Cl}: 1.33$ to $3.12, \mathrm{I}^{2}=0.0$ & 2 RCTs $^{10,11}$ with 418 patients \\
\hline $\begin{array}{l}\text { Clinical Cure } \\
\text { Longest Followup }\end{array}$ & OR: $1.92 ; 95 \% \mathrm{Cl}: 1.18$ to $3.11, \mathrm{I}^{2}=\mathrm{N} / \mathrm{A}$ & $2 \mathrm{RCTs}^{5,11}$ with 353 patients \\
\hline $\begin{array}{l}\text { Clinical Failure } \\
\text { End of Intervention }\end{array}$ & $\begin{array}{l}\text { OR: } 0.54 ; 95 \% \mathrm{Cl}: 0.34 \text { to } 0.86 \text {, } \\
\mathrm{I}^{2}=20.32\end{array}$ & 2 RCTs $^{10,15}$ with 405 patients \\
\hline $\begin{array}{l}\text { Clinical Failure } \\
\text { Longest Followup }\end{array}$ & OR: $0.96 ; 95 \% \mathrm{Cl}: 0.6$ to $1.51, \mathrm{I}^{2}=\mathrm{N} / \mathrm{A}$ & $1 \mathrm{RCT}^{15}$ with 305 patients \\
\hline
\end{tabular}

CCQ = clinical COPD question are; $\mathrm{CI}$ = confidence interval; $\mathrm{CRQ}$ = clinical respiratory questionnaire; N/A = not applicable; $\mathrm{OR}=$ odds ratio; $\mathrm{RCT}=$ randomized controlled trial; $\mathrm{SMD}=$ standardized mean difference; $\mathrm{WMD}=$ weighted mean difference 
Table G.2. KQ1 results: moderate-severe ECOPD severity-antibiotics vs. control

\begin{tabular}{|c|c|c|c|}
\hline Comparison & Outcome & Findings & $\begin{array}{l}\text { Study Design and } \\
\text { Sample Size }\end{array}$ \\
\hline $\begin{array}{l}\text { Antibiotics vs. } \\
\text { Management } \\
\text { without Antibiotics }\end{array}$ & $\begin{array}{l}\text { Mortality } \\
\text { End of Intervention }\end{array}$ & $\begin{array}{l}\text { OR: } 2.02 ; 95 \% \mathrm{Cl}: 0.18 \text { to } 22.66 \text {, } \\
\mathrm{I}^{2}=\mathrm{N} / \mathrm{A}\end{array}$ & $\begin{array}{l}1 \mathrm{RCT}^{16} \text { with } 194 \\
\text { patients }\end{array}$ \\
\hline $\begin{array}{l}\text { Antibiotics vs. } \\
\text { Placebo or } \\
\text { Management } \\
\text { without Antibiotics }\end{array}$ & $\begin{array}{l}\text { Mortality } \\
\text { Longest Followup }\end{array}$ & OR: $2.58 ; 95 \% \mathrm{Cl}: 0.89$ to $7.46, \mathrm{I}^{2}=0.0$ & $\begin{array}{l}2 \mathrm{RCTS}^{6,16} \text { with } 459 \\
\text { patients }\end{array}$ \\
\hline $\begin{array}{l}\text { Antibiotics vs. } \\
\text { Placebo }\end{array}$ & $\begin{array}{l}\text { Dyspnea } \\
\text { (numeric scale: VAS) } \\
\text { End of Intervention }\end{array}$ & $\begin{array}{l}\text { WMD: }-0.80 ; 95 \% \mathrm{Cl}:-1.49 \text { to }-0.11 \text {, } \\
\mathrm{I}^{2}=\mathrm{N} / \mathrm{A}\end{array}$ & $\begin{array}{l}1 \mathrm{RCT}^{6} \text { with } 265 \\
\text { patients }\end{array}$ \\
\hline $\begin{array}{l}\text { Antibiotics vs. } \\
\text { Placebo }\end{array}$ & $\begin{array}{l}\text { Dyspnea } \\
\text { (numeric scale: VAS) } \\
\text { Longest Followup }\end{array}$ & $\begin{array}{l}\text { WMD: }-0.60 ; 95 \% \mathrm{Cl}:-1.27 \text { to } 0.07 \text {, } \\
\mathrm{I}^{2}=\mathrm{N} / \mathrm{A}\end{array}$ & $\begin{array}{l}1 \mathrm{RCT}^{6} \text { with } 265 \\
\text { patients }\end{array}$ \\
\hline $\begin{array}{l}\text { Antibiotics vs. } \\
\text { Placebo }\end{array}$ & $\begin{array}{l}\text { Cough (VAS) } \\
\text { End of Intervention }\end{array}$ & $\begin{array}{l}\text { WMD: }-1.10 ; 95 \% \mathrm{Cl}:-1.80 \text { to }-0.40 \text {, } \\
\mathrm{I}^{2}=\mathrm{N} / \mathrm{A}\end{array}$ & $\begin{array}{l}1 \mathrm{RCT}^{6} \text { with } 265 \\
\text { patients }\end{array}$ \\
\hline $\begin{array}{l}\text { Antibiotics vs. } \\
\text { Placebo }\end{array}$ & $\begin{array}{l}\text { Cough (VAS) } \\
\text { Longest Followup }\end{array}$ & $\begin{array}{l}\text { WMD: }-0.40 ; 95 \% \mathrm{Cl}:-1.13 \text { to } 0.33 \text {, } \\
\mathrm{I}^{2}=\mathrm{N} / \mathrm{A}\end{array}$ & $\begin{array}{l}1 \mathrm{RCT}^{6} \text { with } 265 \\
\text { patients }\end{array}$ \\
\hline $\begin{array}{l}\text { Antibiotics vs. } \\
\text { Placebo or } \\
\text { Management } \\
\text { without Antibiotics }\end{array}$ & $\begin{array}{l}\text { Other Symptoms (Total } \\
\text { Symptom Score) } \\
\text { End of Intervention }\end{array}$ & $\begin{array}{l}\text { WMD: }-0.54 ; 95 \% \mathrm{Cl}:-1.60 \text { to } 0.53 \text {, } \\
\mathrm{I}^{2}=61.53\end{array}$ & $\begin{array}{l}2 \mathrm{RCTs}^{6,16} \text { with } 459 \\
\text { patients }\end{array}$ \\
\hline $\begin{array}{l}\text { Antibiotics vs. } \\
\text { Placebo }\end{array}$ & $\begin{array}{l}\text { Other Symptoms (Total } \\
\text { Symptom Score) } \\
\text { Longest Followup }\end{array}$ & $\begin{array}{l}\text { WMD: }-1.10 ; 95 \% \mathrm{Cl}:-3.13 \text { to } 1.11 \text {, } \\
\mathrm{I}^{2}=\mathrm{N} / \mathrm{A}\end{array}$ & $\begin{array}{l}1 \mathrm{RCT}^{6} \text { with } 265 \\
\text { patients }\end{array}$ \\
\hline $\begin{array}{l}\text { Antibiotics vs. } \\
\text { Management } \\
\text { without Antibiotics }\end{array}$ & $\begin{array}{l}\text { FEV1 \% Predicted } \\
\text { End of Intervention }\end{array}$ & $\begin{array}{l}\text { WMD: }-1.70 ; 95 \% \mathrm{Cl}:-7.31 \text { to } 3.91 \text {, } \\
\mathrm{I}^{2}=\mathrm{N} / \mathrm{A}\end{array}$ & $\begin{array}{l}1 \mathrm{RCT}^{16} \text { with } 194 \\
\text { patients }\end{array}$ \\
\hline $\begin{array}{l}\text { Antibiotics vs. } \\
\text { Placebo }\end{array}$ & $\begin{array}{l}\text { FEV1 Absolute } \\
\text { End of Intervention }\end{array}$ & $\begin{array}{l}\text { WMD: } 0.05 ; 95 \% \mathrm{Cl}:-0.01 \text { to } 0.11 \text {, } \\
\mathrm{I}^{2}=\mathrm{N} / \mathrm{A}\end{array}$ & $\begin{array}{l}1 \mathrm{RCT}^{6} \text { with } 265 \\
\text { patients }\end{array}$ \\
\hline $\begin{array}{l}\text { Antibiotics vs. } \\
\text { Placebo }\end{array}$ & $\begin{array}{l}\text { FEV1 Absolute } \\
\text { Longest Followup }\end{array}$ & WMD: $0.07 ; 95 \% \mathrm{Cl}: 0$ to $0.14, \mathrm{I}^{2}=\mathrm{N} / \mathrm{A}$ & $\begin{array}{l}1 \mathrm{RCT}^{6} \text { with } 265 \\
\text { patients }\end{array}$ \\
\hline $\begin{array}{l}\text { Antibiotics vs. } \\
\text { Management } \\
\text { without Antibiotics }\end{array}$ & $\begin{array}{l}\text { Hospital Readmission } \\
30 \text { days }\end{array}$ & OR: $1.72 ; 95 \% \mathrm{Cl}: 0.68$ to $4.36, \mathrm{I}^{2}=\mathrm{N} / \mathrm{A}$ & $\begin{array}{l}1 \mathrm{RCT}^{16} \text { with } 194 \\
\text { patients }\end{array}$ \\
\hline $\begin{array}{l}\text { Antibiotics vs. } \\
\text { Management } \\
\text { without Antibiotics } \\
\text { vs. Management } \\
\text { without Antibiotics }\end{array}$ & $\begin{array}{l}\text { Repeat Exacerbation } \\
\text { End of Intervention }\end{array}$ & OR: $1.66 ; 95 \% \mathrm{Cl}: 0.73$ to $3.76, \mathrm{I}^{2}=\mathrm{N} / \mathrm{A}$ & $\begin{array}{l}1 \mathrm{RCT}^{16} \text { with } 194 \\
\text { patients }\end{array}$ \\
\hline $\begin{array}{l}\text { Antibiotics vs. } \\
\text { Management } \\
\text { without Antibiotics }\end{array}$ & $\begin{array}{l}\text { Repeat Exacerbation } \\
30 \text { days }\end{array}$ & OR: $1.66 ; 95 \% \mathrm{Cl}: 0.73$ to $3.76, \mathrm{I}^{2}=\mathrm{N} / \mathrm{A}$ & $\begin{array}{l}1 \mathrm{RCT}^{16} \text { with } 194 \\
\text { patients }\end{array}$ \\
\hline $\begin{array}{l}\text { Antibiotics vs. } \\
\text { Management } \\
\text { without Antibiotics }\end{array}$ & $\begin{array}{l}\text { Repeat Exacerbation } \\
\text { Longest Followup }\end{array}$ & OR: $1.66 ; 95 \% \mathrm{Cl}: 0.73$ to $3.76, \mathrm{I}^{2}=\mathrm{N} / \mathrm{A}$ & $\begin{array}{l}1 \text { RCT }{ }^{16} \text { with } 194 \\
\text { patients }\end{array}$ \\
\hline $\begin{array}{l}\text { Antibiotics vs. } \\
\text { Management } \\
\text { without Antibiotics }\end{array}$ & $\begin{array}{l}\text { Intubation } \\
\text { End of Intervention }\end{array}$ & OR: $0.49 ; 95 \% \mathrm{Cl}: 0.04$ to $5.55, \mathrm{I}^{2}=\mathrm{N} / \mathrm{A}$ & $\begin{array}{l}1 \mathrm{RCT}^{16} \text { with } 194 \\
\text { patients }\end{array}$ \\
\hline $\begin{array}{l}\text { Antibiotics vs. } \\
\text { Placebo }\end{array}$ & $\begin{array}{l}\text { Clinical Cure } \\
\text { End of Intervention }\end{array}$ & OR: $2.02 ; 95 \% \mathrm{Cl}: 1.23$ to $3.32, \mathrm{I}^{2}=\mathrm{N} / \mathrm{A}$ & $\begin{array}{l}1 \mathrm{RCT}^{6} \text { with } 265 \\
\text { patients }\end{array}$ \\
\hline $\begin{array}{l}\text { Antibiotics vs. } \\
\text { Management } \\
\text { without Antibiotics }\end{array}$ & $\begin{array}{l}\text { Clinical Cure } \\
\text { Longest Followup }\end{array}$ & $\begin{array}{l}\text { OR: } 1.30 ; 95 \% \mathrm{Cl}: 0.83 \text { to } 2.02 \text {, } \\
\mathrm{I}^{2}=49.97\end{array}$ & $\begin{array}{l}2 \mathrm{RCTs}^{6,16} \text { with } 459 \\
\text { patients }\end{array}$ \\
\hline $\begin{array}{l}\text { Antibiotics vs. } \\
\text { Placebo }\end{array}$ & $\begin{array}{l}\text { Clinical Failure } \\
\text { Longest Follow-up }\end{array}$ & OR: $0.68 ; 95 \% \mathrm{Cl}: 0.41$ to $1.11, \mathrm{I}^{2}=\mathrm{N} / \mathrm{A}$ & $\begin{array}{l}1 \mathrm{RCT}^{6} \text { with } 265 \\
\text { patients }\end{array}$ \\
\hline
\end{tabular}

$\mathrm{CI}$ = confidence interval; N/A = not applicable; OR = odds ratio; RCT = randomized controlled trial; SMD = standardized mean difference; $\mathrm{WMD}=$ weighted mean difference; $\mathrm{VAS}=$ visual analog scale 
Table G.3. KQ1 results: Mild Severity-systemic corticosteroids vs. placebo

\begin{tabular}{|c|c|c|}
\hline Outcome & Findings & $\begin{array}{c}\text { Study Design and Sample } \\
\text { Size }\end{array}$ \\
\hline $\begin{array}{l}\text { Mortality } \\
\text { End of Intervention }\end{array}$ & OR: $2.00 ; 95 \% \mathrm{Cl}: 0.18$ to $22.55, \mathrm{I}^{2}=\mathrm{N} / \mathrm{A}$ & $1 \mathrm{RCT}^{1}$ with 147 patients \\
\hline $\begin{array}{l}\text { Dyspnea } \\
\text { (numeric scale: VAS) } \\
\text { End of Intervention }\end{array}$ & WMD: $-13.10 ; 95 \% \mathrm{Cl}:-28.55$ to $2.35, \mathrm{I}^{2}=\mathrm{N} / \mathrm{A}$ & $1 \mathrm{RCT}^{14}$ with 26 patients \\
\hline $\begin{array}{l}\text { FEV1 Absolute } \\
\text { End of Intervention }\end{array}$ & WMD: $0.42 ; 95 \% \mathrm{Cl}: 0.3$ to $0.54, \mathrm{I}^{2}=92.95$ & $2 \mathrm{RCTs}^{4,14}$ with 56 patients \\
\hline $\begin{array}{l}\text { Clinical Failure } \\
\text { End of Intervention }\end{array}$ & OR: $0.02 ; 95 \% \mathrm{Cl}: 0$ to $0.49, \mathrm{I}^{2}=\mathrm{N} / \mathrm{A}$ & $1 \mathrm{RCT}^{14}$ with 26 patients \\
\hline $\begin{array}{l}\text { Clinical Failure } \\
\text { Longest followup }\end{array}$ & OR: $0.50 ; 95 \% \mathrm{Cl}: 0.25$ to $1, \mathrm{I}^{2}=\mathrm{N} / \mathrm{A}$ & $1 \mathrm{RCT}^{1}$ with 147 patients \\
\hline $\begin{array}{l}\text { Hospital admission } \\
\text { Longest followup }\end{array}$ & OR: $0.47 ; 95 \% \mathrm{Cl}: 0.19$ to $1.19, \mathrm{I}^{2}=\mathrm{N} / \mathrm{A}$ & $1 \mathrm{RCT}^{1}$ with 147 patients \\
\hline $\begin{array}{l}\text { QoL } \\
\text { (CRQ) } \\
\text { End of Intervention }\end{array}$ & WMD: $0.38 ; 95 \% \mathrm{Cl}:-0.09$ to $0.85, \mathrm{I}^{2}=\mathrm{N} / \mathrm{A}$ & $1 \mathrm{RCT}^{1}$ with 147 patients \\
\hline
\end{tabular}

CI = confidence interval; CRQ = chronic respiratory questioner; N/A = not applicable; OR = odds ratio; QoL = quality of life; $\mathrm{RCT}$ = randomized controlled trial; SMD = standardized mean difference; WMD = weighted mean difference; VAS = visual analog scale

Table G.4. KQ1 results: Moderate-severe severity- systemic corticosteroids vs. control

\begin{tabular}{|c|c|c|c|}
\hline Comparison & Outcome & Findings & $\begin{array}{l}\text { Study Design and } \\
\text { Sample Size }\end{array}$ \\
\hline $\begin{array}{l}\text { Systemic } \\
\text { Corticosteroids vs. } \\
\text { Placebo or } \\
\text { Management } \\
\text { without Systemic } \\
\text { Corticosteroids }\end{array}$ & $\begin{array}{l}\text { Mortality } \\
\text { End of Intervention }\end{array}$ & OR: $1.55 ; 95 \% \mathrm{Cl}: 0.4$ to $5.98, \mathrm{I}^{2}=0.0$ & $\begin{array}{l}3 \text { RCTs }^{9,12,13} \text { with } \\
363 \text { patients }\end{array}$ \\
\hline $\begin{array}{l}\text { Systemic } \\
\text { Corticosteroids vs. } \\
\text { Placebo or } \\
\text { Management } \\
\text { without Systemic } \\
\text { Corticosteroids }\end{array}$ & $\begin{array}{l}\text { Mortality } \\
\text { Longest Followup }\end{array}$ & OR: $0.89 ; 95 \%$ Cl: 0.37 to $2.16, I^{2}=0.0$ & $\begin{array}{l}3 \mathrm{RCTs}^{7,9,13} \text { with } \\
353 \text { patients }\end{array}$ \\
\hline $\begin{array}{l}\text { Systemic } \\
\text { Corticosteroids vs. } \\
\text { Placebo }\end{array}$ & $\begin{array}{l}\text { Dyspnea } \\
\text { (numeric scale: Modified } \\
\text { Borg Scale) } \\
\text { End of Intervention }\end{array}$ & $\begin{array}{l}\text { WMD: }-0.80 ; 95 \% \mathrm{Cl}:-1.65 \text { to } 0.05 \text {, } \\
\mathrm{I}^{2}=\mathrm{N} / \mathrm{A}\end{array}$ & $\begin{array}{l}1 \mathrm{RCT}^{12} \text { with } 128 \\
\text { patients }\end{array}$ \\
\hline $\begin{array}{l}\text { Systemic } \\
\text { Corticosteroids vs. } \\
\text { Placebo or } \\
\text { Management } \\
\text { without Systemic } \\
\text { Corticosteroids }\end{array}$ & $\begin{array}{l}\text { FEV1 \% Predicted } \\
\text { End of Intervention }\end{array}$ & $\begin{array}{l}\text { WMD: } 5.71 ; 95 \% \text { Cl: } 1.40 \text { to } 10.03 \text {, } \\
I^{2}=0.0\end{array}$ & $\begin{array}{l}3 \mathrm{RCTs}^{2,7,9} \text { with } 206 \\
\text { patients }\end{array}$ \\
\hline $\begin{array}{l}\text { Systemic } \\
\text { Corticosteroids vs. } \\
\text { Placebo }\end{array}$ & $\begin{array}{l}\text { FEV1 \% Predicted } \\
\text { Longest Followup }\end{array}$ & $\begin{array}{l}\text { WMD: } 7.75 ; 95 \% \text { Cl: }-0.30 \text { to } 15.80 \text {, } \\
\mathrm{I}^{2}=0.62\end{array}$ & $\begin{array}{l}2 \mathrm{RCTs}^{2,7} \text { with } 100 \\
\text { patients }\end{array}$ \\
\hline $\begin{array}{l}\text { Systemic } \\
\text { Corticosteroids vs. } \\
\text { Placebo }\end{array}$ & $\begin{array}{l}\text { FEV1 Absolute } \\
\text { End of Intervention }\end{array}$ & 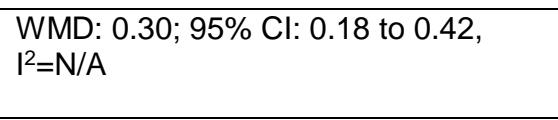 & $\begin{array}{l}1 \mathrm{RCT}^{7} \text { with } 56 \\
\text { patients }\end{array}$ \\
\hline $\begin{array}{l}\text { Systemic } \\
\text { Corticosteroids vs. } \\
\text { Placebo }\end{array}$ & $\begin{array}{l}\text { Hospital Readmission } \\
30 \text { days }\end{array}$ & OR: $0.54 ; 95 \% \mathrm{Cl}: 0.10$ to $2.88, \mathrm{I}^{2}=\mathrm{N} / \mathrm{A}$ & $\begin{array}{l}1 \mathrm{RCT}^{13} \text { with } 191 \\
\text { patients }\end{array}$ \\
\hline
\end{tabular}




\begin{tabular}{|c|c|c|c|}
\hline Comparison & Outcome & Findings & $\begin{array}{l}\text { Study Design and } \\
\text { Sample Size }\end{array}$ \\
\hline $\begin{array}{l}\text { Systemic } \\
\text { Corticosteroids vs. } \\
\text { Placebo }\end{array}$ & $\begin{array}{l}\text { Hospital Readmission } \\
\text { Longest Followup }\end{array}$ & OR: $1.07 ; 95 \% \mathrm{Cl}: 0.49$ to $2.36, \mathrm{I}^{2}=\mathrm{N} / \mathrm{A}$ & $\begin{array}{l}1 \mathrm{RCT}^{13} \text { with } 191 \\
\text { patients }\end{array}$ \\
\hline $\begin{array}{l}\text { Systemic } \\
\text { Corticosteroids vs. } \\
\text { Placebo or } \\
\text { Management } \\
\text { without Systemic } \\
\text { Corticosteroids }\end{array}$ & $\begin{array}{l}\text { Repeat Exacerbation } \\
\text { End of Intervention }\end{array}$ & $\begin{array}{l}\text { OR: } 0.73 ; 95 \% \mathrm{Cl}: 0.35 \text { to } 1.52 \text {, } \\
\mathrm{I}^{2}=34.27\end{array}$ & $\begin{array}{l}2 \mathrm{RCTs}^{7,9} \text { with } 162 \\
\text { patients }\end{array}$ \\
\hline $\begin{array}{l}\text { Systemic } \\
\text { Corticosteroids vs. } \\
\text { Placebo }\end{array}$ & $\begin{array}{l}\text { Repeat Exacerbation } \\
3 \text { months }\end{array}$ & OR: $1.29 ; 95 \% \mathrm{Cl}: 0.40$ to $4.13, \mathrm{I}^{2}=\mathrm{N} / \mathrm{A}$ & $\begin{array}{l}1 \mathrm{RCT}^{13} \text { with } 56 \\
\text { patients }\end{array}$ \\
\hline $\begin{array}{l}\text { Systemic } \\
\text { Corticosteroids vs. } \\
\text { Placebo }\end{array}$ & $\begin{array}{l}\text { Repeat Exacerbation } \\
\text { Longest Followup }\end{array}$ & OR: $1.29 ; 95 \% \mathrm{Cl}: 0.40$ to $4.13, \mathrm{I}^{2}=\mathrm{N} / \mathrm{A}$ & $\begin{array}{l}1 \mathrm{RCT}^{13} \text { with } 56 \\
\text { patients }\end{array}$ \\
\hline $\begin{array}{l}\text { Systemic } \\
\text { Corticosteroids vs. } \\
\text { Placebo }\end{array}$ & $\begin{array}{l}\text { Intubation } \\
\text { End of Intervention }\end{array}$ & OR: $0.42 ; 95 \% \mathrm{Cl}: 0.06$ to $2.68, \mathrm{I}^{2}=0.0$ & $\begin{array}{l}2 \mathrm{RCTs}^{12,13} \text { with } 319 \\
\text { patients }\end{array}$ \\
\hline $\begin{array}{l}\text { Systemic } \\
\text { Corticosteroids vs. } \\
\text { Placebo }\end{array}$ & $\begin{array}{l}\text { Intubation } \\
\text { Longest Followup }\end{array}$ & OR: $0.92 ; 95 \% \mathrm{Cl}: 0.15$ to $5.66, \mathrm{I}^{2}=\mathrm{N} / \mathrm{A}$ & $\begin{array}{l}1 \mathrm{RCT}^{13} \text { with } 191 \\
\text { patients }\end{array}$ \\
\hline $\begin{array}{l}\text { Systemic } \\
\text { Corticosteroids vs. } \\
\text { Placebo }\end{array}$ & $\begin{array}{l}\text { Clinical Failure } \\
\text { Longest Followup }\end{array}$ & OR: $1.52 ; 95 \% \mathrm{Cl}: 0.81$ to $2.86, \mathrm{I}^{2}=\mathrm{N} / \mathrm{A}$ & $\begin{array}{l}1 \mathrm{RCT}^{13} \text { with } 191 \\
\text { patients }\end{array}$ \\
\hline
\end{tabular}

$\mathrm{CI}$ = confidence interval; N/A = not applicable; OR = odds ratio; RCT = randomized controlled trial; SMD = standardized mean difference; $\mathrm{WMD}=$ weighted mean difference

Table G.5. KQ2 results: Mild severity- mucolytics vs management without mucolytics.

\begin{tabular}{|l|l|l|}
\hline \multicolumn{1}{|c|}{ Outcome } & \multicolumn{1}{|c|}{ Findings } & $\begin{array}{c}\text { Study Design and Sample } \\
\text { Size }\end{array}$ \\
\hline $\begin{array}{l}\text { FEV1 Absolute } \\
\text { End of Intervention }\end{array}$ & WMD: $0.07 ; 95 \% \mathrm{Cl}:-0.14$ to $0.28, \mathrm{I}^{2}=\mathrm{N} / \mathrm{A}$ & $1 \mathrm{RCT}^{64}$ with 83 patients \\
\hline
\end{tabular}

CI = confidence interval; N/A = not applicable; OR = odds ratio; RCT = randomized controlled trial; SMD = standardized mean difference; $\mathrm{WMD}=$ weighted mean difference 
Table G.6. KQ2 results: Moderate-severe severity - mucolytics vs placebo.

\begin{tabular}{|c|c|c|}
\hline Outcome & Findings & $\begin{array}{c}\text { Study Design and Sample } \\
\text { Size }\end{array}$ \\
\hline $\begin{array}{l}\text { Dyspnea BCSS } \\
\text { End of Intervention }\end{array}$ & WMD: $-1.00 ; 95 \% \mathrm{Cl}:-0.56$ to $0.44, \mathrm{I}^{2}=\mathrm{N} / \mathrm{A}$ & $1 \mathrm{RCT}^{58}$ with 40 patients \\
\hline $\begin{array}{l}\text { Dyspnea BCSS } \\
\text { Longest Followup }\end{array}$ & WMD: $-0.90 ; 95 \% \mathrm{Cl}:-1.80$ to $0.02, \mathrm{I}^{2}=\mathrm{N} / \mathrm{A}$ & $1 \mathrm{RCT}^{58}$ with 40 patients \\
\hline $\begin{array}{l}\text { Dyspnea } \\
\text { (numeric scale: Dyspnea, } \\
\text { Breathlessness Likert Scale) } \\
\text { End of intervention }\end{array}$ & WMD: $-0.18 ; 95 \% \mathrm{Cl}:-0.43$ to $0.06, \mathrm{I}^{2}=0.00 \%$ & $2 \mathrm{RCTs}^{19,22}$ with 92 patients \\
\hline $\begin{array}{l}\text { FEV1 \% Predicted } \\
\text { End of Intervention }\end{array}$ & WMD: $1.98 ; 95 \% \mathrm{Cl}: 0.51$ to $3.44, \mathrm{I}^{2}=83.21 \%$ & $2 \mathrm{RCTs}^{22,42}$ patients 70 pts \\
\hline $\begin{array}{l}\text { FEV1 \% Predicted } \\
\text { Longest Followup }\end{array}$ & WMD: $8.30 ; 95 \% \mathrm{Cl}:-0.69$ to $17.29, \mathrm{I}^{2}=\mathrm{N} / \mathrm{A}$ & $1 \mathrm{RCT}^{42}$ with 40 patients \\
\hline $\begin{array}{l}\text { FEV1 Absolute } \\
\text { End of Intervention }\end{array}$ & WMD: $0.06 ; 95 \% \mathrm{Cl}:-0.27$ to $0.39, \mathrm{I}^{2}=\mathrm{N} / \mathrm{A}$ & $1 \mathrm{RCT}^{19}$ with 42 patients \\
\hline $\begin{array}{l}\text { Repeat Exacerbation } \\
1 \text { month }\end{array}$ & OR: $0.05 ; 95 \% \mathrm{Cl}: 0$ to $1.04, \mathrm{I}^{2}=\mathrm{N} / \mathrm{A}$ & $1 \mathrm{RCT}^{42}$ with 40 patients \\
\hline $\begin{array}{l}\text { Repeat Exacerbation } \\
3 \text { months }\end{array}$ & OR: $0.14 ; 95 \% \mathrm{Cl}: 0.03$ to $0.65, \mathrm{I}^{2}=\mathrm{N} / \mathrm{A}$ & $1 \mathrm{RCT}^{42}$ with 40 patients \\
\hline $\begin{array}{l}\text { Repeat Exacerbation } \\
\text { Longest Followup }\end{array}$ & OR: $0.14 ; 95 \% \mathrm{Cl}: 0.03$ to $0.65, \mathrm{I}^{2}=\mathrm{N} / \mathrm{A}$ & $1 \mathrm{RCT}^{42}$ with 40 patients \\
\hline
\end{tabular}

BCSS = breathlessness, cough, and sputum scale; $\mathrm{CI}=$ confidence interval; N/A = not applicable; OR = odds ratio; RCT = randomized controlled trial; $\mathrm{SMD}=$ standardized mean difference; $\mathrm{WMD}=$ weighted mean difference

Table G.7. KQ2 results: Moderate-severe severity-inhaled corticosteroids vs control

\begin{tabular}{|c|c|c|c|}
\hline Comparison & Outcome & Findings & $\begin{array}{l}\text { Study Design and } \\
\text { Sample Size }\end{array}$ \\
\hline $\begin{array}{l}\text { ICS (Budesonide)vs } \\
\text { Placebo }\end{array}$ & $\begin{array}{l}\text { Dyspnea } \\
\text { (numeric scale: MRC) } \\
\text { End of intervention }\end{array}$ & $\begin{array}{l}\text { WMD: } 0.04 ; 95 \% \mathrm{Cl}:-0.29 \text { to } 0.38, \mathrm{I}^{2}= \\
\text { N/A }\end{array}$ & $\begin{array}{l}1 \mathrm{RCT}^{12} \text { with } 137 \\
\text { patients }\end{array}$ \\
\hline $\begin{array}{l}\text { ICS (Budesonide) vs } \\
\text { Management without } \\
\text { ICS }\end{array}$ & $\begin{array}{l}\text { FEV1 \% Predicted } \\
\text { End of intervention }\end{array}$ & $\begin{array}{l}\text { WMD: } 10.10 ; 95 \% \mathrm{Cl}: 4.23 \text { to } 15.97 \text {, } \\
\mathrm{l}^{2}=\mathrm{N} / \mathrm{A}\end{array}$ & $\begin{array}{l}1 \mathrm{RCT}^{9} \text { with } 106 \\
\text { patients }\end{array}$ \\
\hline $\begin{array}{l}\text { ICS (Budesonide) vs } \\
\text { Placebo }\end{array}$ & $\begin{array}{l}\text { Intubation } \\
\text { End of Intervention }\end{array}$ & OR: $0.31 ; 95 \% \mathrm{Cl}: 0.01$ to $7.63, \mathrm{I}^{2}=\mathrm{N} / \mathrm{A}$ & $\begin{array}{l}1 \mathrm{RCT}^{12} \text { with } 137 \\
\text { patients }\end{array}$ \\
\hline $\begin{array}{l}\text { ICS (Budesonide) vS } \\
\text { Management without } \\
\text { ICS }\end{array}$ & $\begin{array}{l}\text { Mortality } \\
\text { Longest Followup }\end{array}$ & OR: $0.33 ; 95 \% \mathrm{Cl}: 0.01$ to $8.21, \mathrm{I}^{2}=\mathrm{N} / \mathrm{A}$ & $\begin{array}{l}1 \mathrm{RCT}^{9} \text { with } 106 \\
\text { patients }\end{array}$ \\
\hline
\end{tabular}

CI = confidence interval; ICS = inhaled corticosteroids; $\mathrm{MRC}=$ medical research council scale; $\mathrm{N} / \mathrm{A}=$ not applicable; $\mathrm{OR}=$ odds ratio; $\mathrm{RCT}=$ randomized controlled trial; $\mathrm{SMD}=$ standardized mean difference; $\mathrm{WMD}=$ weighted mean difference 
Table G.8. KQ2 results: Moderate-severe severity-inhaled corticosteroids with inhaled shortacting bronchodilators vs management without inhaled corticosteroids

\begin{tabular}{|l|l|l|}
\hline \multicolumn{1}{|c|}{ Outcome } & \multicolumn{1}{|c|}{ Findings } & $\begin{array}{l}\text { Study Design and Sample } \\
\text { Size }\end{array}$ \\
\hline $\begin{array}{l}\text { FEV1 \% Predicted } \\
\text { End of Intervention }\end{array}$ & WMD: $8.30 ; 95 \% \mathrm{Cl}: 2.92$ to $13.68, \mathrm{I}^{2}=\mathrm{N} / \mathrm{A}$ & $1 \mathrm{RCT}^{62}$ with 40 patients \\
\hline $\begin{array}{l}\text { FEV1 Absolute } \\
\text { End of Intervention }\end{array}$ & WMD: $0.35 ; 95 \% \mathrm{Cl}: 0.05$ to $0.65, \mathrm{I}^{2}=\mathrm{N} / \mathrm{A}$ & $1 \mathrm{RCT}^{62}$ with 40 patients \\
\hline
\end{tabular}

$\mathrm{CI}$ = confidence interval; N/A = not applicable; $\mathrm{OR}$ = odds ratio; RCT = randomized controlled trial; SMD = standardized mean difference; $\mathrm{WMD}$ = weighted mean difference

Table G.9. KQ2 results: Mild severity-inhaled corticosteroids with inhaled long acting bronchodilators vs placebo

\begin{tabular}{|l|l|l|}
\hline \multicolumn{1}{|c|}{ Outcome } & \multicolumn{1}{|c|}{ Findings } & $\begin{array}{c}\text { Study Design and Sample } \\
\text { Size }\end{array}$ \\
\hline $\begin{array}{l}\text { FEV1 Absolute } \\
\text { End of intervention }\end{array}$ & WMD: $0.18 ; 95 \% \mathrm{Cl}:-0.17$ to $0.53, \mathrm{I}^{2}=\mathrm{N} / \mathrm{A}$ & $1 \mathrm{RCT}^{4}$ with 30 patients \\
\hline $\begin{array}{l}\text { Clinical Failure } \\
\text { End of Intervention }\end{array}$ & OR: $1.00 ; 95 \% \mathrm{Cl}: 0.06$ to $17.62, \mathrm{I}^{2}=\mathrm{N} / \mathrm{A}$ & $1 \mathrm{RCT}^{4}$ with 30 patients \\
\hline
\end{tabular}

CI = confidence interval; N/A = not applicable; OR = odds ratio; RCT = randomized controlled trial; SMD = standardized mean difference; WMD = weighted mean difference 


\section{Appendix H. Adverse Events}

Table H.1. KQ1: Adverse events. Antibiotics compared with control

\begin{tabular}{|c|c|c|c|}
\hline Comparison & Outcome & Findings & Study Design \\
\hline Antibiotics vs. Placebo & Cardiovascular AE & $\begin{array}{l}\text { Rate Ratio: } 1.92 ; 95 \% \mathrm{Cl} \text { : } \\
0.93 \text { to } 3.99, \mathrm{I}^{2}=\mathrm{N} / \mathrm{A}\end{array}$ & $1 \mathrm{RCT}^{15}$ \\
\hline Antibiotics vs. Placebo & Dermatological $\mathrm{AE}$ & $\begin{array}{l}\text { Rate Ratio: } 1.00 ; 95 \% \mathrm{Cl} \text { : } \\
0.14 \text { to } 7.10, \mathrm{I}^{2}=\mathrm{N} / \mathrm{A}\end{array}$ & $1 \mathrm{RCT}^{10}$ \\
\hline Antibiotics vs. Placebo & Gastrointestinal AE & $\begin{array}{l}\text { Rate Ratio: } 1.02 ; 95 \% \mathrm{Cl} \text { : } \\
0.58 \text { to } 1.80, \mathrm{I}^{2}=0.00 \%\end{array}$ & $3 \mathrm{RCTS}^{6,10,15}$ \\
\hline Antibiotics vs. Placebo & General Internal Medicine AE & $\begin{array}{l}\text { Rate Ratio: } 0.42 ; 95 \% \mathrm{Cl} \text { : } \\
0.15 \text { to } 1.19, \mathrm{I}^{2}=\mathrm{N} / \mathrm{A}\end{array}$ & $1 \mathrm{RCT}^{15}$ \\
\hline Antibiotics vs. Placebo & Musculoskeletal AE & $\begin{array}{l}\text { Rate Ratio: } 1.38 ; 95 \% \mathrm{Cl} \text { : } \\
0.56 \text { to } 3.44, \mathrm{I}^{2}=\mathrm{N} / \mathrm{A}\end{array}$ & $1 \mathrm{RCT}^{15}$ \\
\hline Antibiotics vs. Placebo & Oncological AE & $\begin{array}{l}\text { Rate Ratio: } 0.60 ; 95 \% \mathrm{Cl} \text { : } \\
0.22 \text { to } 1.66, \mathrm{I}^{2}=\mathrm{N} / \mathrm{A}\end{array}$ & $1 \mathrm{RCT}^{15}$ \\
\hline Antibiotics vs. Placebo & Serious $A E$ & $\begin{array}{l}\text { Rate Ratio: } 1.095 ; 95 \% \mathrm{Cl} \text { : } \\
0.70 \text { to } 1.71, \mathrm{I}^{2}=0.0 \%\end{array}$ & $2 \mathrm{RCTs}^{6,15}$ \\
\hline Antibiotics vs. Placebo & Total number of AEs & $\begin{array}{l}\text { Rate Ratio: } 1.09 ; 95 \% \mathrm{Cl} \text { : } \\
0.88 \text { to } 1.35, \mathrm{I}^{2}=0.0 \%\end{array}$ & $4 \mathrm{RCTS}^{6,10,11,15}$ \\
\hline $\begin{array}{l}\text { Antibiotics vs. Placebo } \\
\text { or Management without } \\
\text { Antibiotics }\end{array}$ & Withdrawal & $\begin{array}{l}\text { OR: } 0.89 ; 95 \% \mathrm{Cl}: 0.55 \text { to } \\
1.40,1^{2}=7.37 \%\end{array}$ & $4 \mathrm{RCTS}^{6,10,11,15,16}$ \\
\hline $\begin{array}{l}\text { Antibiotics vs. Placebo } \\
\text { or Management without } \\
\text { Antibiotics }\end{array}$ & Withdrawal due to $\mathrm{AE}$ & $\begin{array}{l}\text { OR: } 1.93 ; 95 \% \mathrm{Cl}: 0.94 \text { to } \\
3.96,1^{2}=0.00 \%\end{array}$ & $3 \mathrm{RCTS}^{6,15,16}$ \\
\hline
\end{tabular}

$\mathrm{AE}$ = adverse event; $\mathrm{CI}$ = confidence interval; N/A = not applicable; $\mathrm{OR}$ = odds ratio; $\mathrm{RCT}$ = randomized controlled trial

Table H.2. KQ1: Adverse events. Systemic corticosteroids compared with control

\begin{tabular}{|c|c|c|c|}
\hline Comparison & Outcome & Findings & Study Design \\
\hline $\begin{array}{l}\text { Systemic Corticosteroids } \\
\text { vs. Placebo }\end{array}$ & Endocrine $\mathrm{AE}$ & $\begin{array}{l}\text { Rate Ratio: } 4.86 ; 95 \% \mathrm{Cl} \text { : } \\
1.60 \text { to } 14.75, \mathrm{I}^{2}=\mathrm{N} / \mathrm{A}\end{array}$ & $1 \mathrm{RCT}^{13}$ \\
\hline $\begin{array}{l}\text { Systemic Corticosteroids } \\
\text { vs. Placebo }\end{array}$ & Infectious AE & $\begin{array}{l}\text { Rate Ratio: } 0.88 ; 95 \% \mathrm{Cl} \text { : } \\
0.43 \text { to } 1.81, \mathrm{I}^{2}=\mathrm{N} / \mathrm{A}\end{array}$ & $1 \mathrm{RCT}^{13}$ \\
\hline $\begin{array}{l}\text { Systemic Corticosteroids } \\
\text { vs. Placebo }\end{array}$ & Psychiatric AE & $\begin{array}{l}\text { Rate Ratio:1.66; } 95 \% \\
\text { Cl:0.99 to } 2.79, I^{2}=0.0 \%\end{array}$ & $2 \mathrm{RCTs}^{1,13}$ \\
\hline $\begin{array}{l}\text { Systemic Corticosteroids } \\
\text { vs. Placebo }\end{array}$ & Serious $\mathrm{AE}$ & $\begin{array}{l}\text { Rate Ratio: } 0.73 ; 95 \% \mathrm{Cl} \text { : } \\
0.27 \text { to } 1.97, \mathrm{l}^{2}=0.0 \%\end{array}$ & $2 \mathrm{RCTS}^{1,12}$ \\
\hline $\begin{array}{l}\text { Systemic Corticosteroids } \\
\text { vs. Placebo }\end{array}$ & Total number of AEs & $\begin{array}{l}\text { Rate Ratio: } 1.55 ; 95 \% \mathrm{Cl} \text { : } \\
1.14 \text { to } 2.10, \mathrm{I}^{2}=39.8 \%\end{array}$ & $4 \mathrm{RCTS}^{1,2,12,13}$ \\
\hline $\begin{array}{l}\text { Systemic Corticosteroids } \\
\text { vs. Placebo or } \\
\text { Management without } \\
\text { Systemic Corticosteroids }\end{array}$ & Withdrawal & $\begin{array}{l}\text { OR: } 0.67 ; 95 \% \text { Cl: } 0.39 \text { to } \\
1.16, \mathrm{I}^{2}=0.65 \%\end{array}$ & $4 \mathrm{RCTS}^{1,2,9,12}$ \\
\hline $\begin{array}{l}\text { Systemic Corticosteroids } \\
\text { vs. Placebo }\end{array}$ & Withdrawal due to $\mathrm{AE}$ & $\begin{array}{l}\text { OR: } 0.47 ; 95 \% \mathrm{Cl}: 0.20 \text { to } \\
1.10, \mathrm{I}^{2}=14.53\end{array}$ & $3 \mathrm{RCTS}^{2,7,12}$ \\
\hline
\end{tabular}

$\mathrm{AE}$ = adverse event; $\mathrm{CI}$ = confidence interval; N/A = not applicable; $\mathrm{OR}$ = odds ratio; $\mathrm{RCT}$ = randomized controlled trial 
Table H.3. KQ2: Adverse events. Intravenous aminophyllines compared with placebo

\begin{tabular}{|c|c|c|}
\hline Outcome & Findings & Study Design \\
\hline Cardiovascular $\mathrm{AE}$ & $\begin{array}{l}\text { Rate Ratio: } 1.65 ; 95 \% \mathrm{Cl}: 0.64 \text { to } \\
4.26, \mathrm{I}^{2}=\mathrm{N} / \mathrm{A}\end{array}$ & $1 \mathrm{RCT}^{29}$ \\
\hline Gastrointestinal AE & $\begin{array}{l}\text { Rate Ratio: } 2.10 ; 95 \% \mathrm{Cl}: 1.30 \text { to } \\
3.40, I^{2}=\mathrm{N} / \mathrm{A}\end{array}$ & $1 \mathrm{RCT}^{29}$ \\
\hline Neurological AE & $\begin{array}{l}\text { Rate Ratio: } 1.11 ; 95 \% \mathrm{Cl}: 0.55 \text { to } \\
2.24,\left.\right|^{2}=0.00 \%\end{array}$ & $2 \mathrm{RCT}^{29,48}$ \\
\hline Total number of AEs & $\begin{array}{l}\text { Rate Ratio: } 1.80 ; 95 \% \mathrm{Cl}: 1.26 \text { to } \\
2.59, \mathrm{I}^{2}=25.80 \%\end{array}$ & $2 \mathrm{RCT}^{29,48}$ \\
\hline Withdrawal & $\begin{array}{l}\text { OR: } 0.94 ; 95 \% \mathrm{Cl}: 0.35 \text { to } 2.55 \\
\mathrm{I}^{2}=\mathrm{N} / \mathrm{A}\end{array}$ & $1 \mathrm{RCT}^{29}$ \\
\hline Withdrawal due to $\mathrm{AE}$ & $\begin{array}{l}\text { OR: } 0.56 ; 95 \% \mathrm{Cl}: 0.15 \text { to } 2.07 \text {, } \\
\mathrm{I}^{2}=\mathrm{N} / \mathrm{A}\end{array}$ & $1 \mathrm{RCT}^{29}$ \\
\hline
\end{tabular}

AE = adverse event; $\mathrm{CI}$ = confidence interval; N/A = not applicable; $\mathrm{OR}$ = odds ratio; $\mathrm{RCT}$ = randomized controlled trial

Table H.4. KQ2: Adverse events. Oral mucolytics compared with placebo

\begin{tabular}{|l|l|l|}
\hline \multicolumn{1}{|c|}{ Outcome } & \multicolumn{1}{|c|}{ Findings } & \multicolumn{1}{c|}{ Study Design } \\
\hline Gastrointestinal AE & $\begin{array}{l}\text { Rate Ratio: } 0.50 ; 95 \% \mathrm{Cl}: 0.05 \text { to } \\
5.51, \mathrm{I}^{2}=\mathrm{N} / \mathrm{A}\end{array}$ & $1 \mathrm{RCT}^{22}$ \\
& & \\
\hline Total number of AEs & $\begin{array}{l}\text { Rate Ratio: } 1.00 ; 95 \% \mathrm{Cl}: 0.20 \text { to } \\
4.95, \mathrm{I}^{2}=\mathrm{N} / \mathrm{A}\end{array}$ & $1 \mathrm{RCT}^{22}$ \\
& & \\
\hline
\end{tabular}

AE = adverse event; $\mathrm{CI}$ = confidence interval; N/A = not applicable; $\mathrm{RCT}$ = randomized controlled trial

Table H.5. KQ2: Adverse events. Inhaled corticosteroids with or without inhaled short- and long acting bronchodilators compared with placebo

\begin{tabular}{|l|l|l|}
\hline \multicolumn{1}{|c|}{ Outcome } & \multicolumn{1}{|c|}{ Findings } & \multicolumn{1}{|c|}{ Study Design } \\
\hline Severe AE & $\begin{array}{l}\text { Rate Ratio: } 0.83 ; 95 \% \mathrm{Cl}: 0.32 \text { to } \\
2.14, \mathrm{I}^{2}=\mathrm{N} / \mathrm{A}\end{array}$ & $1 \mathrm{RCT}^{12}$ \\
\hline Total number of AEs & $\begin{array}{l}\text { Rate Ratio: } 0.88 ; 95 \% \mathrm{Cl}: 0.57 \text { to } \\
1.38, \mathrm{I}^{2}=\mathrm{N} / \mathrm{A}\end{array}$ & $1 \mathrm{RCT}^{12}$ \\
\hline Withdrawal & $\begin{array}{l}\text { OR: } 0.76 ; 95 \% \mathrm{Cl}: 0.43 \text { to } 1.34, \\
\mathrm{I}^{2}=73.73 \% \mathrm{I}^{2}\end{array}$ & $2 \mathrm{RCTs}^{9,12}$ \\
\hline Withdrawal due to AE & $\begin{array}{l}\text { OR: } 0.92 ; 95 \% \mathrm{Cl}: 0.38 \text { to } 2.21, \\
\mathrm{I}^{2}=\mathrm{N} / \mathrm{A}\end{array}$ & $1 \mathrm{RCT}^{12}$ \\
\hline
\end{tabular}

AE = adverse event; $\mathrm{CI}$ = confidence interval; N/A = not applicable; $\mathrm{OR}$ = odds ratio; $\mathrm{RCT}$ = randomized controlled trial

Table H.6. KQ2: Adverse events. 5-lipoxygenase inhibitor (zileuton) compared with placebo

\begin{tabular}{|l|l|l|}
\hline \multicolumn{1}{|c|}{ Outcome } & \multicolumn{1}{|c|}{ Findings } & \multicolumn{1}{c|}{ Study Design } \\
\hline Respiratory AE & $\begin{array}{l}\text { Rate Ratio: 0.68; } 95 \% \mathrm{Cl}: 0.29 \text { to } \\
1.59, \mathrm{I}^{2}=\mathrm{N} / \mathrm{A}\end{array}$ & $1 \mathrm{RCT}^{61}$ \\
\hline Severe AE & $\begin{array}{l}\text { Rate Ratio: } 0.93 ; 95 \% \mathrm{Cl}: 0.48 \text { to } \\
1.80, \mathrm{I}^{2}=\mathrm{N} / \mathrm{A}\end{array}$ & $1 \mathrm{RCT}^{61}$ \\
\hline Total number of AEs & $\begin{array}{l}\text { Rate Ratio: } 0.82 ; 95 \% \mathrm{Cl}: 0.49 \text { to } \\
1.39, \mathrm{I}^{2}=\mathrm{N} / \mathrm{A}\end{array}$ & $1 \mathrm{RCT}^{61}$ \\
\hline Withdrawal & $\begin{array}{l}\text { OR: } 0.89 ; 95 \% \mathrm{Cl}: 0.38 \text { to } 2.10, \\
\mathrm{I}^{2}=\mathrm{N} / \mathrm{A}\end{array}$ & $1 \mathrm{RCT}^{61}$ \\
\hline Withdrawal due to AE & $\begin{array}{l}\text { OR: } 0.48 ; 95 \% \mathrm{Cl}: 0.04 \text { to } 5.48, \\
\mathrm{I}^{2}=\mathrm{N} / \mathrm{A}\end{array}$ & $1 \mathrm{RCT}^{61}$ \\
\hline
\end{tabular}

$\mathrm{AE}$ = adverse event; $\mathrm{CI}$ = confidence interval; N/A = not applicable; $\mathrm{OR}$ = odds ratio; $\mathrm{RCT}$ = randomized controlled trial 
Table H.7. KQ2: Adverse events. Chest physiotherapy using breathing technique compared with management without chest physiotherapy

\begin{tabular}{|l|c|l|}
\hline \multicolumn{1}{|c|}{ Outcome } & \multicolumn{1}{c|}{ Findings } & \multicolumn{1}{c|}{ Study Design } \\
\hline Withdrawal & OR: $0.94 ; 95 \% \mathrm{Cl}: 0.66$ to1.34, $\mathrm{I}^{2}=0.00 \%$ & $2 \mathrm{RCT}^{27,36}$ \\
\hline Withdrawal due to AE & OR: $0.90 ; 95 \% \mathrm{Cl}: 0.55$ to1.48, $\mathrm{I}^{2}=0.00 \%$ & $2 \mathrm{RCT}^{27,36}$ \\
\hline
\end{tabular}

$\mathrm{AE}$ = adverse event; $\mathrm{CI}$ = confidence interval; $\mathrm{OR}$ = odds ratio; $\mathrm{RCT}$ = randomized controlled trial

Table H.8. KQ2: Adverse events. Chest physiotherapy using vibration/percussion/massage compared with management without chest physiotherapy

\begin{tabular}{|c|l|l|}
\hline \multicolumn{1}{|c|}{ Outcome } & \multicolumn{1}{c|}{ Findings } & \multicolumn{1}{c|}{ Study Design } \\
\hline Withdrawal & 0 events in each arm & $1 \mathrm{RCT}^{24}$ \\
\hline
\end{tabular}

RCT $=$ randomized controlled trial

Table H.9. KQ2: Adverse events. Chest physiotherapy using positive expiratory pressure compared with management without positive expiratory pressure

\begin{tabular}{|l|l|l|}
\hline \multicolumn{1}{|c|}{ Outcome } & \multicolumn{1}{|c|}{ Findings } & \multicolumn{1}{|c|}{ Study Design } \\
\hline Severe $\mathrm{AE}$ & $\begin{array}{l}\text { Rate Ratio: } 1.50 ; 95 \% \mathrm{Cl}: 0.53 \text { to } 4.21, \\
\mathrm{I}^{2}=\mathrm{N} / \mathrm{A}\end{array}$ & $1 \mathrm{RCT}^{46}$ \\
\hline Total number of AEs & $\begin{array}{l}\text { Rate Ratio: } 1.13 ; 95 \% \mathrm{Cl}: 0.43 \text { to } 2.92, \\
\mathrm{I}^{2}=\mathrm{N} / \mathrm{A}\end{array}$ & $1 \mathrm{RCT}^{46}$ \\
\hline Withdrawal & OR: $0.68 ; 95 \% \mathrm{Cl}: 0.20$ to $2.32, \mathrm{I}^{2}=\mathrm{N} / \mathrm{A}$ & $1 \mathrm{RCT}^{46}$ \\
\hline Withdrawal due to AE & OR: $0.63 ; 95 \% \mathrm{Cl}: 0.17$ to $2.42, \mathrm{I}^{2}=\mathrm{N} / \mathrm{A}$ & $1 \mathrm{RCT}^{46}$ \\
\hline
\end{tabular}

$\mathrm{AE}$ = adverse event; $\mathrm{CI}$ = confidence interval; N/A = not applicable; $\mathrm{OR}$ = odds ratio; $\mathrm{RCT}$ = randomized controlled trial

Table H.10. KQ2: Adverse events. Exercise using resistance training compared with management without resistance training

\begin{tabular}{|l|c|l|}
\hline \multicolumn{1}{|c|}{ Outcome } & \multicolumn{1}{|c|}{ Findings } & \multicolumn{1}{c|}{ Study Design } \\
\hline Withdrawal & OR: $0.72 ; 95 \% \mathrm{Cl}: 0.30$ to $1.69, \mathrm{I}^{2}=0.0 \%$ & $2 \mathrm{RCTs}^{21,23}$ \\
\hline
\end{tabular}

$\mathrm{CI}=$ confidence interval; N/A = not applicable; $\mathrm{OR}$ = odds ratio; $\mathrm{RCT}$ = randomized controlled trial

Table H.11. KQ2: Adverse events. Exercise using combined aerobic + resistance training compared with management without exercise training.

\begin{tabular}{|l|l|l|}
\hline Outcome & \multicolumn{1}{|c|}{ Findings } & \multicolumn{1}{c|}{ Study Design } \\
\hline Total number of AEs & $\begin{array}{l}\text { Rate Ratio: 1.00; 95\% Cl: 0.14 to 7.10, } \\
\mathrm{I}^{2}=\mathrm{N} / \mathrm{A}\end{array}$ & $1 \mathrm{RCT}^{55}$ \\
\hline
\end{tabular}

AE = adverse event; $\mathrm{CI}$ = confidence interval; N/A = not applicable; $\mathrm{RCT}$ = randomized controlled trial 
Table H.12. KQ2: Adverse events. Early pulmonary rehabilitation compared with management without early pulmonary rehabilitation

\begin{tabular}{|l|l|l|}
\hline \multicolumn{1}{|c|}{ Outcome } & \multicolumn{1}{c|}{ Findings } & \multicolumn{1}{c|}{ Study Design } \\
\hline Infectious AE & $\begin{array}{l}\text { Rate Ratio: } 1.07 ; 95 \% \mathrm{Cl}: 0.07 \text { to } 17.13, \\
\mathrm{I}^{2}=\mathrm{N} / \mathrm{A}\end{array}$ & $1 \mathrm{RCT}^{26}$ \\
\hline General AE & $\begin{array}{l}\text { Rate Ratio: } 1.07 ; 95 \% \mathrm{Cl}: 0.07 \text { to } 17.13, \\
\mathrm{I}^{2}=\mathrm{N} / \mathrm{A}\end{array}$ & $1 \mathrm{RCT}^{26}$ \\
\hline Musculoskeletal AE & $\begin{array}{l}\text { Rate Ratio: } 1.07 ; 95 \% \mathrm{Cl}: 0.07 \text { to } 17.13, \\
\mathrm{I}^{2}=\mathrm{N} / \mathrm{A}\end{array}$ & $1 \mathrm{RCT}^{26}$ \\
\hline Respiratory AE & $\begin{array}{l}\text { Rate Ratio: } 1.16 ; 95 \% \mathrm{Cl}: 0.53 \text { to } 2.54, \\
\mathrm{I}^{2}=\mathrm{N} / \mathrm{A}\end{array}$ & $1 \mathrm{RCT}^{26}$ \\
\hline Severe AE & $\begin{array}{l}\text { Rate Ratio: } 0.80 ; 95 \% \mathrm{Cl}: 0.28 \text { to } 2.32, \\
\mathrm{I}^{2}=\mathrm{N} / \mathrm{A}\end{array}$ & $1 \mathrm{RCT}^{26}$ \\
\hline Total number of AEs & $\begin{array}{l}\text { Rate Ratio: } 1.03 ; 95 \% \mathrm{Cl}: 0.62 \text { to } 1.74, \\
\mathrm{I}^{2}=\mathrm{N} / \mathrm{A}\end{array}$ & $1 \mathrm{RCT}^{26}$ \\
\hline Withdrawal & $\begin{array}{l}\text { OR: } 1.21 ; 95 \% \mathrm{Cl}: 0.48 \text { to } 3.06, \\
\mathrm{I}^{2}=20.79 \%\end{array}$ & $2 \mathrm{RCT}^{26} 30$ \\
\hline Withdrawal due to AE & OR: $1.68 ; 95 \% \mathrm{Cl}: 0.44$ to $6.38, \mathrm{I}^{2}=\mathrm{N} / \mathrm{A}$ & $1 \mathrm{RCT}^{30}$ \\
\hline
\end{tabular}

$\mathrm{AE}$ = adverse event; $\mathrm{CI}$ = confidence interval; N/A = not applicable; $\mathrm{OR}$ = odds ratio; $\mathrm{RCT}$ = randomized controlled trial

Table H.13. KQ2: Adverse events. Whole body vibration training during ECOPD compared with management without whole body vibration

\begin{tabular}{|l|c|c|}
\hline \multicolumn{1}{|c|}{ Outcome } & \multicolumn{1}{c|}{ Findings } & \multicolumn{1}{c|}{ Study Design } \\
\hline Withdrawal & OR: $0.50 ; 95 \% \mathrm{Cl}: 0.11$ to $2.28, \mathrm{I}^{2}=\mathrm{N} / \mathrm{A}$ & $1 \mathrm{RCT}{ }^{4}$ \\
\hline
\end{tabular}

$\mathrm{CI}$ = confidence interval; N/A = not applicable; $\mathrm{OR}=$ odds ratio; $\mathrm{RCT}$ = randomized controlled trial

Table H.14. KQ2: Adverse events. Transcutaneous electrical nerve stimulation (TENS) during ECOPD compared with vs management without Transcutaneous Electrical Nerve Stimulation

\begin{tabular}{|c|c|c|}
\hline \multicolumn{1}{|c|}{ Outcome } & \multicolumn{1}{c|}{ Findings } & \multicolumn{1}{c|}{ Study Design } \\
\hline Withdrawal & OR: $0.81 ; 95 \% \mathrm{Cl}: 0.23$ to $2.90, \mathrm{I}^{2}=\mathrm{N} / \mathrm{A}$ & $1 \mathrm{RCT}{ }^{45}$ \\
\hline
\end{tabular}

$\mathrm{CI}=$ confidence interval; N/A = not applicable; $\mathrm{OR}$ = odds ratio; RCT = randomized controlled trial

Table H.15. KQ2: Adverse events. Gutter frame with supplemental oxygen compared with gutter frame supplemental air

\begin{tabular}{|l|l|l|}
\hline \multicolumn{1}{|c|}{ Outcome } & \multicolumn{1}{|c|}{ Findings } & \multicolumn{1}{|c|}{ Study Design } \\
\hline Respiratory AE & $\begin{array}{l}\text { Rate Ratio: } 0.33 ; 95 \% \mathrm{Cl}: 0.03 \text { to } 3.20, \\
\mathrm{I}^{2}=\mathrm{N} / \mathrm{Al}^{2}\end{array}$ & $1 \mathrm{RCT}^{63}$ \\
\hline Withdrawal & OR: $0.46 ; 95 \% \mathrm{Cl}: 0.78$ to $2.75, \mathrm{I}^{2}=\mathrm{N} / \mathrm{A}$ & $1 \mathrm{RCT}^{63}$ \\
\hline Withdrawal due to AE & OR: $0.22 ; 95 \% \mathrm{Cl}: 0.02$ to $2.14, \mathrm{I}^{2}=\mathrm{N} / \mathrm{A}$ & $1 \mathrm{RCT}^{63}$ \\
\hline Total number of AEs & $\begin{array}{l}\text { Rate Ratio: } 0.33 ; 95 \% \mathrm{Cl}: 0.03 \text { to } 3.20, \\
\mathrm{I}^{2}=\mathrm{N} / \mathrm{Al}^{2}\end{array}$ & $1 \mathrm{RCT}^{63}$ \\
\hline
\end{tabular}

$\mathrm{AE}$ = adverse event; $\mathrm{CI}$ = confidence interval; N/A = not applicable; $\mathrm{OR}$ = odds ratio; $\mathrm{RCT}$ = randomized controlled trial

Table H.16. KQ2: Adverse events. Rollator with supplemental oxygen compared with gutter frame supplemental air

\begin{tabular}{|l|l|l|}
\hline \multicolumn{1}{|c|}{ Outcome } & \multicolumn{1}{|c|}{ Findings } & \multicolumn{1}{|c|}{ Study Design } \\
\hline Respiratory AE & $\begin{array}{l}\text { Rate Ratio: } 1.00 ; 95 \% \mathrm{Cl}: 0.14 \text { to } 7.10, \\
\text { I }^{2}=\mathrm{N} / \mathrm{A}\end{array}$ & $1 \mathrm{RCT}^{63}$ \\
\hline Withdrawal & OR: $1.00 ; 95 \% \mathrm{Cl}: 0.13$ to $7.61, \mathrm{I}^{2}=\mathrm{N} / \mathrm{A}$ & $1 \mathrm{RCT}^{63}$ \\
\hline Withdrawal due to AE & OR: $1.00 ; 95 \% \mathrm{Cl}: 0.13$ to $7.61, \mathrm{I}^{2}=\mathrm{N} / \mathrm{A}$ & $1 \mathrm{RCT}^{63}$ \\
\hline Total number of AES & $\begin{array}{l}\text { Rate Ratio: } 1.00 ; 95 \% \mathrm{Cl}: 0.14 \text { to } 7.10, \\
\mathrm{I}^{2}=\mathrm{N} / \mathrm{A}\end{array}$ & $1 \mathrm{RCT}^{63}$ \\
\hline
\end{tabular}

$\mathrm{AE}$ = adverse event; $\mathrm{CI}$ = confidence interval; N/A = not applicable; $\mathrm{OR}$ = odds ratio; $\mathrm{RCT}$ = randomized controlled trial 
Table H.17. KQ2: Adverse events. Dietary intervention using a caloric supplement during ECOPD compared with usual diet

\begin{tabular}{|c|c|c|}
\hline \multicolumn{1}{|c|}{ Outcome } & \multicolumn{1}{|c|}{ Findings } & \multicolumn{1}{c|}{ Study Design } \\
\hline Withdrawal & OR: $0.39 ; 95 \%$ Cl: 0.07 to $2.03, \mathrm{I}^{2}=\mathrm{N} / \mathrm{A}$ & $1 \mathrm{RCT}^{50}$ \\
\hline
\end{tabular}

$\mathrm{CI}=$ confidence interval; N/A = not applicable; $\mathrm{OR}$ = odds ratio; $\mathrm{RCT}$ = randomized controlled trial

Table H.18. KQ2: Adverse events. Dietary intervention using a caloric and a protein supplement during ECOPD compared with Placebo (non-caloric fluid, vanilla flavored water)

\begin{tabular}{|l|l|l|}
\hline \multicolumn{1}{|c|}{ Outcome } & \multicolumn{1}{|c|}{ Findings } & \multicolumn{1}{|c|}{ Study Design } \\
\hline Gastrointestinal AE & $\begin{array}{l}\text { Rate Ratio: } 3.13 ; 95 \% \mathrm{Cl}: 0.33 \text { to } 30.09, \\
\mathrm{I}^{2}=\mathrm{N} / \mathrm{A}\end{array}$ & $1 \mathrm{RCT}^{60}$ \\
\hline Total number of AEs & $\begin{array}{l}\text { Rate Ratio: } 3.13 ; 95 \% \mathrm{Cl}: 0.33 \text { to } 30.09, \\
\mathrm{I}^{2}=\mathrm{N} / \mathrm{A}\end{array}$ & $1 \mathrm{RCT}^{60}$ \\
\hline Withdrawal due to AE & OR: $2.47 ; 95 \% \mathrm{Cl}: 0.54$ to $11.37, \mathrm{I}^{2}=\mathrm{N} / \mathrm{A}$ & $1 \mathrm{RCT}^{60}$ \\
\hline
\end{tabular}

$\mathrm{AE}$ = adverse event; $\mathrm{CI}$ = confidence interval; N/A = not applicable; $\mathrm{OR}$ = odds ratio; $\mathrm{RCT}$ = randomized controlled trial

Table. H.19. KQ. Adverse events. Dietary intervention using omega-3 fatty acid compared with usual diet

\begin{tabular}{|l|l|l|}
\hline \multicolumn{1}{|c|}{ Outcome } & \multicolumn{1}{|c|}{ Findings } & \multicolumn{1}{c|}{ Study Design } \\
\hline Withdrawal & $\begin{array}{l}\text { OR: } 0.22 ; 95 \% \mathrm{Cl}: 0.02 \text { to } 2.11, \\
\mathrm{I}^{2}=\mathrm{N} / \mathrm{A}\end{array}$ & $1 \mathrm{RCT}^{44}$ \\
\hline
\end{tabular}

AE = adverse event; $\mathrm{CI}$ = confidence interval; N/A = not applicable; $\mathrm{OR}$ = odds ratio; $\mathrm{RCT}$ = randomized controlled trial

Table H.20. KQ2: Adverse events. Dietary intervention using vitamin D during ECOPD compared with placebo

\begin{tabular}{|l|l|l|}
\hline \multicolumn{1}{|c|}{ Outcome } & \multicolumn{1}{|c|}{ Findings } & \multicolumn{1}{c|}{ Study Design } \\
\hline Withdrawal due to $\mathrm{AE}$ & OR: $2.13 ; 95 \% \mathrm{Cl}: 0.61$ to $7.43, \mathrm{I}^{2}=\mathrm{N} / \mathrm{A}$ & $2 \mathrm{RCTs}^{47,49}$ \\
\hline
\end{tabular}

AE = adverse event; $\mathrm{CI}$ = confidence interval; N/A = not applicable; OR = odds ratio; RCT = randomized controlled trial

Table H.21. KQ3: Adverse events. ICS+ SABA (beclomethasone+ salbutamol compared with SABA (Fenoterol)

\begin{tabular}{|l|l|l|}
\hline \multicolumn{1}{|c|}{ Outcome } & \multicolumn{1}{|c|}{ Finding } & \multicolumn{1}{c|}{ Study Design } \\
\hline General Internal Medicine AE & $\begin{array}{l}\text { Rate Ratio: } 0.50 ; 95 \% \mathrm{Cl}: 0.05 \text { to } 5.51, \\
\mathrm{I}^{2}=0.00 \%\end{array}$ & $1 \mathrm{RCT}^{67}$ \\
\hline Neurological AE & $\begin{array}{l}\text { Rate Ratio: } 0.20 ; 95 \% \mathrm{Cl}: 0.02 \text { to } 1.71, \\
\mathrm{I}^{2}=0.00 \%\end{array}$ & $1 \mathrm{RCT}^{67}$ \\
\hline Total number of AEs & $\begin{array}{l}\text { Rate Ratio: } 0.29 ; 95 \% \mathrm{Cl}: 0.06 \text { to } 1.38, \\
\mathrm{I}^{2}=0.00 \%\end{array}$ & $1 \mathrm{RCT}^{67}$ \\
\hline
\end{tabular}

$\mathrm{AE}=$ adverse event; $\mathrm{CI}=$ confidence interval; $\mathrm{RCT}=$ randomized controlled trial

Table H.22. KQ4: Adverse events. Aminopenicillin plus beta-lactamase compared with fluoroquinolone

\begin{tabular}{|l|l|l|}
\hline \multicolumn{1}{|c|}{ Outcome } & \multicolumn{1}{|c|}{ Finding } & \multicolumn{1}{|c|}{ Study Design } \\
\hline Gastrointestinal AE & $\begin{array}{l}\text { Rate Ratio: } 1.31 ; 95 \% \mathrm{Cl}: 0.68 \text { to } 2.52, \\
\mathrm{I}^{2}=82.1 \% \mathrm{I}^{2}\end{array}$ & $2 \mathrm{RCTs}{ }^{78,96}$ \\
\hline Neurological AE & $\begin{array}{l}\text { Rate Ratio: } 0.60 ; 95 \% \mathrm{Cl}: 0.14 \text { to } 2.51, \\
\mathrm{I}^{2}=\mathrm{N} / \mathrm{Al}^{2}\end{array}$ & $1 \mathrm{RCT}^{96}$ \\
\hline Total number of AEs & $\begin{array}{l}\text { Rate Ratio: } 1.01 ; 95 \% \mathrm{Cl}: 0.84 \text { to } 1.20, \\
\mathrm{I}^{2}=0.0 \% \mathrm{I}^{2}\end{array}$ & $2 \mathrm{RCTs}^{78,96}$ \\
\hline Serious AE & $\begin{array}{l}\text { Rate Ratio: } 0.99 ; 95 \% \mathrm{Cl}: 0.69 \text { to } 1.43, \\
\mathrm{I}^{2}=45.8 \% \mathrm{I}^{2}\end{array}$ & $2 \mathrm{RCTs}^{78,96}$ \\
\hline
\end{tabular}

$\mathrm{AE}$ = adverse event; $\mathrm{CI}$ = confidence interval; N/A = not applicable; $\mathrm{RCT}$ = randomized controlled trial 
Table H.23. KQ4: Adverse events. Ciprofloxacin compared with amoxicillin

\begin{tabular}{|l|l|l|}
\hline \multicolumn{1}{|c|}{ Outcome } & \multicolumn{1}{|c|}{ Finding } & \multicolumn{1}{c|}{ Study Design } \\
\hline Withdrawal & $\begin{array}{l}\text { OR: 25; 95\% Cl: } 1.20 \text { to } 520.739, \\
\mathrm{I}^{2}=\mathrm{N} / \mathrm{A}\end{array}$ & $1 \mathrm{RCT}^{77}$ \\
\hline
\end{tabular}

$\mathrm{CI}=$ confidence interval; N/A = not applicable; $\mathrm{OR}$ = odds ratio; $\mathrm{RCT}$ = randomized controlled trial

Table H.24. KQ4: Adverse events. "Standard" antibiotic therapy (clarithromycin or cefuroxime or amoxicillin + clavulanic acid) compared with levofloxacin

\begin{tabular}{|l|l|l|}
\hline \multicolumn{1}{|c|}{ Outcome } & \multicolumn{1}{|c|}{ Finding } & \multicolumn{1}{c|}{ Study Design } \\
\hline Gastrointestinal AE & $\begin{array}{l}\text { Rate Ratio: } 0.35 ; 95 \% \mathrm{Cl}: 0.04 \text { to } 3.33, \\
\mathrm{I}^{2}=\mathrm{N} / \mathrm{A}\end{array}$ & $1 \mathrm{RCT}^{87}$ \\
\hline Serious AE & $\begin{array}{l}\text { Rate Ratio: } 1.28 ; 95 \% \mathrm{Cl}: 0.44 \text { to } 3.70, \\
\mathrm{I}^{2}=\mathrm{N} / \mathrm{A}\end{array}$ & $1 \mathrm{RCT}^{87}$ \\
\hline Total number of AEs & $\begin{array}{l}\text { Rate Ratio: } 1.28 ; 95 \% \mathrm{Cl}: 0.44 \text { to } 3.70, \\
\mathrm{I}^{2}=\mathrm{N} / \mathrm{A}\end{array}$ & $1 \mathrm{RCT}^{87}$ \\
\hline
\end{tabular}

$\mathrm{AE}$ = adverse event; $\mathrm{CI}$ = confidence interval; N/A = not applicable; $\mathrm{RCT}$ = randomized controlled trial

Table H.25. KQ4: Adverse events. Amoxicillin compared with azithromycin

\begin{tabular}{|l|l|l|}
\hline \multicolumn{1}{|c|}{ Outcome } & \multicolumn{1}{|c|}{ Finding } & \multicolumn{1}{|c|}{ Study Design } \\
\hline Gastrointestinal AE & $\begin{array}{l}\text { Rate Ratio: } 1.66 ; 95 \% \mathrm{Cl}: 0.56 \text { to } 4.97, \\
\mathrm{I}^{2}=\mathrm{N} / \mathrm{A}\end{array}$ & $1 \mathrm{RCT} 69$ \\
\hline Serious AE & $\begin{array}{l}\text { Rate Ratio: } 0.37 ; 95 \% \mathrm{Cl}: 0.07 \text { to } 1.91, \\
\mathrm{I}^{2}=\mathrm{N} / \mathrm{A}\end{array}$ & $1 \mathrm{RCT}^{69}$ \\
\hline Total number of AEs & $\begin{array}{l}\text { Rate Ratio: } 0.76 ; 95 \% \mathrm{Cl}: 0.45 \text { to } 1.26, \\
\mathrm{I}^{2}=\mathrm{N} / \mathrm{A}\end{array}$ & $1 \mathrm{RCT}^{69}$ \\
\hline
\end{tabular}

$\mathrm{AE}=$ adverse event; $\mathrm{CI}$ = confidence interval; N/A = not applicable; $\mathrm{RCT}$ = randomized controlled trial

Table H.26. KQ4: Adverse events. Cephalosporin compared with fluoroquinolone

\begin{tabular}{|l|l|l|}
\hline \multicolumn{1}{|c|}{ Outcome } & \multicolumn{1}{|c|}{ Finding } & \multicolumn{1}{|c|}{ Study Design } \\
\hline Dermatological AE & $\begin{array}{l}\text { Rate Ratio: } 0.97 ; 95 \% \mathrm{Cl}: 0.06 \text { to } \\
15.58, \mathrm{I}^{2}=\mathrm{N} / \mathrm{A}\end{array}$ & $1 \mathrm{RCT}^{82}$ \\
\hline Gastrointestinal AE & $\begin{array}{l}\text { Rate Ratio: } 1.30 ; 95 \% \mathrm{Cl}: 0.45 \text { to } 3.74, \\
\mathrm{I}^{2}=\mathrm{N} / \mathrm{A}\end{array}$ & $1 \mathrm{RCT}^{82}$ \\
\hline General AE & $\begin{array}{l}\text { Rate Ratio: } 0.54 ; 95 \% \mathrm{Cl}: 0.17 \text { to } 1.68, \\
\mathrm{I}^{2}=0\end{array}$ & $2 \mathrm{RCTs}^{82,97}$ \\
\hline Infectious AE & $\begin{array}{l}\text { Rate Ratio: } 0.24 ; 95 \% \mathrm{Cl}: 0.03 \text { to } 2.18, \\
\mathrm{I}^{2}=\mathrm{N} / \mathrm{A}\end{array}$ & $1 \mathrm{RCT}^{82}$ \\
\hline Musculoskeletal AE & $\begin{array}{l}\text { Rate Ratio: } 0.24 ; 95 \% \mathrm{Cl}: 0.03 \text { to } 2.18, \\
\mathrm{I}^{2}=\mathrm{N} / \mathrm{A}\end{array}$ & $1 \mathrm{RCT}^{82}$ \\
\hline Respiratory AE & $\begin{array}{l}\text { Rate Ratio: } 0.97 ; 95 \% \mathrm{Cl}: 0.06 \text { to } \\
15.58, \mathrm{I}^{2}=\mathrm{N} / \mathrm{A}\end{array}$ & $1 \mathrm{RCT}^{82}$ \\
\hline Total number of AEs & $\begin{array}{l}\text { Rate Ratio: } 0.77 ; 95 \% \mathrm{Cl}: 0.45 \text { to } 1.32, \\
\mathrm{I}^{2}=0.0 \% l^{2}\end{array}$ & $2 \mathrm{RCTS}^{82,97}$ \\
\hline Serious AE & $\begin{array}{l}\text { Rate Ratio: } 0.97 ; 95 \% \mathrm{Cl}: 0.06 \text { to } \\
15.58, \mathrm{I}^{2}=\mathrm{N} / \mathrm{Al} I^{2}\end{array}$ & $1 \mathrm{RCT}^{82}$ \\
\hline
\end{tabular}

$\mathrm{AE}$ = adverse event; $\mathrm{CI}$ = confidence interval; $\mathrm{N} / \mathrm{A}$ = not applicable; $\mathrm{RCT}$ = randomized controlled trial 
Table H.27. KQ4: Adverse events. Azithromycin compared with cefaclor

\begin{tabular}{|l|l|l|}
\hline \multicolumn{1}{|c|}{ Outcome } & \multicolumn{1}{|c|}{ Finding } & \multicolumn{1}{|c|}{ Study Design } \\
\hline Dermatological AE & $\begin{array}{l}\text { Rate Ratio: } 0.96 ; 95 \% \mathrm{Cl}: 0.09 \text { to } \\
10.54, \mathrm{I}^{2}=\mathrm{N} / \mathrm{A}\end{array}$ & $1 \mathrm{RCT}^{72}$ \\
\hline Gastrointestinal AE & $\begin{array}{l}\text { Rate Ratio: } 0.88 ; 95 \% \mathrm{Cl}: 0.43 \text { to } 1.77, \\
\mathrm{I}^{2}=\mathrm{N} / \mathrm{A}\end{array}$ & $1 \mathrm{RCT}^{72}$ \\
\hline Neurological AE & $\begin{array}{l}\text { Rate Ratio: } 1.43 ; 95 \% \mathrm{Cl}: 0.29 \text { to } 7.1, \\
\mathrm{I}^{2}=\mathrm{N} / \mathrm{A}\end{array}$ & $1 \mathrm{RCT}^{72}$ \\
\hline Psychiatric AE & $\begin{array}{l}\text { Rate Ratio: } 0.48 ; 95 \% \mathrm{Cl}: 0.07 \text { to } 3.39, \\
\mathrm{I}^{2}=\mathrm{N} / \mathrm{A}\end{array}$ & $1 \mathrm{RCT}^{72}$ \\
\hline Total number of AEs & $\begin{array}{l}\text { Rate Ratio: } 1.10 ; 95 \% \mathrm{Cl}: 0.62 \text { to } 1.94, \\
\mathrm{I}^{2}=\mathrm{N} / \mathrm{A}\end{array}$ & $1 \mathrm{RCT}^{72}$ \\
\hline Withdrawal & OR: $0.11 ; 95 \% \mathrm{Cl}: 0.01$ to $1.03, \mathrm{I}^{2}=\mathrm{N} / \mathrm{A}$ & $1 \mathrm{RCT}^{72}$ \\
\hline Withdrawal due to AE & OR: $0.11 ; 95 \% \mathrm{Cl}: 0.01$ to $1.03, \mathrm{I}^{2}=\mathrm{N} / \mathrm{A}$ & $1 \mathrm{RCT}^{72}$ \\
\hline
\end{tabular}

$\mathrm{AE}$ = adverse event; $\mathrm{CI}$ = confidence interval; N/A = not applicable; $\mathrm{OR}$ = odds ratio; $\mathrm{RCT}$ = randomized controlled trial

Table H.28. KQ4: Adverse events. Amoxicillin compared with amoxicillin plus clavulanic acid

\begin{tabular}{|l|l|l|}
\hline \multicolumn{1}{|c|}{ Outcome } & \multicolumn{1}{|c|}{ Finding } & \multicolumn{1}{c|}{ Study Design } \\
\hline Gastrointestinal AE & $\begin{array}{l}\text { Rate Ratio: } 0.38 ; 95 \% \mathrm{Cl}: 0.10 \text { to } 1.43, \\
\mathrm{I}^{2}=\mathrm{N} / \mathrm{A}\end{array}$ & $1 \mathrm{RCT}^{80}$ \\
\hline Total number of AEs & $\begin{array}{l}\text { Rate Ratio: } 0.63 ; 95 \% \mathrm{Cl}: 0.21 \text { to } 1.94, \\
\mathrm{I}^{2}=\mathrm{N} / \mathrm{A}\end{array}$ & $1 \mathrm{RCT}^{80}$ \\
\hline Withdrawal & $\begin{array}{l}\text { OR: } 5.26 ; 95 \% \mathrm{Cl}: 0.25 \text { to } 100.00, \\
\mathrm{I}^{2}=\mathrm{N} / \mathrm{A}\end{array}$ & $1 \mathrm{RCT}^{80}$ \\
\hline
\end{tabular}

AE = adverse event; $\mathrm{CI}$ = confidence interval; N/A = not applicable; $\mathrm{OR}$ = odds ratio; $\mathrm{RCT}$ = randomized controlled trial

Table H.29. KQ4: Adverse events. Amoxicillin plus clavulanic acid compared with telithromycin

\begin{tabular}{|l|l|l|}
\hline \multicolumn{1}{|c|}{ Outcome } & \multicolumn{1}{|c|}{ Finding } & \multicolumn{1}{|c|}{ Study Design } \\
\hline Gastrointestinal AE & $\begin{array}{l}\text { Rate Ratio: } 3.76 ; 95 \% \mathrm{Cl}: 1.63 \text { to } 8.66, \\
\mathrm{I}^{2}=\mathrm{N} / \mathrm{A}\end{array}$ & $1 \mathrm{RCT}^{70}$ \\
\hline Respiratory AE & $\begin{array}{l}\text { Rate Ratio: } 6.07 ; 95 \% \mathrm{Cl}: 1.36 \text { to } \\
27.14, \mathrm{I}^{2}=\mathrm{N} / \mathrm{A}\end{array}$ & $1 \mathrm{RCT}^{70}$ \\
\hline Serious AE & $\begin{array}{l}\text { Rate Ratio: } 0.87 ; 95 \% \mathrm{Cl}: 0.29 \text { to } 2.58, \\
\mathrm{I}^{2}=\mathrm{N} / \mathrm{A}\end{array}$ & $1 \mathrm{RCT}^{70}$ \\
\hline Total number of AEs & $\begin{array}{l}\text { Rate Ratio: } 1.57 ; 95 \% \mathrm{Cl}: 1.05 \text { to } 2.36, \\
\mathrm{I}^{2}=\mathrm{N} / \mathrm{A}\end{array}$ & $1 \mathrm{RCT}^{70}$ \\
\hline
\end{tabular}

$\mathrm{AE}=$ adverse event; $\mathrm{CI}$ = confidence interval; N/A = not applicable; $\mathrm{RCT}$ = randomized controlled trial

Table H.30. KQ4: Adverse events. Aminopenicillin plus beta-lactamases inhibitor plus clavulanic acid compared with macrolides

\begin{tabular}{|l|l|l|}
\hline \multicolumn{1}{|c|}{ Outcome } & \multicolumn{1}{|c|}{ Finding } & \multicolumn{1}{|c|}{ Study Design } \\
\hline Gastrointestinal AE & $\begin{array}{l}\text { Rate Ratio: } 1.62 ; 95 \% \mathrm{Cl}: 0.6 \text { to } 4.34, \\
\mathrm{I}^{2}=\mathrm{N} / \mathrm{A}\end{array}$ & $1 \mathrm{RCT}^{94}$ \\
\hline General Internal Medicine AE & $\begin{array}{l}\text { Rate Ratio: } 1.26 ; 95 \% \mathrm{Cl}: 0.08 \text { to } \\
20.11, \mathrm{I}^{2}=\mathrm{N} / \mathrm{A}\end{array}$ & $1 \mathrm{RCT}^{94}$ \\
\hline Hepatic AE & $\begin{array}{l}\text { Rate Ratio: } 1.26 ; 95 \% \mathrm{Cl}: 0.08 \text { to } \\
20.11, \mathrm{I}^{2}=\mathrm{N} / \mathrm{A}\end{array}$ & $1 \mathrm{RCT}^{94}$ \\
\hline Neurological AE & $\begin{array}{l}\text { Rate Ratio: } 0.25 ; 95 \% \mathrm{Cl}: 0.03 \text { to } 2.15, \\
\text { I }\end{array}$ & $1 \mathrm{RCT}^{24}$ \\
\hline Total number of AEs & $\begin{array}{l}\text { Rate Ratio: } 1.26 ; 95 \% \mathrm{Cl}: 0.60 \text { to } 2.64, \\
\mathrm{I}^{2}=\mathrm{N} / \mathrm{A}\end{array}$ & $1 \mathrm{RCT}^{94}$ \\
\hline
\end{tabular}

$\mathrm{AE}=$ adverse event; $\mathrm{CI}$ = confidence interval; N/A = not applicable; $\mathrm{RCT}$ = randomized controlled trial 
Table H.31. KQ4: Adverse events. Amoxicillin + Clavulanic acid compared azithromycin

\begin{tabular}{|l|l|l|}
\hline \multicolumn{1}{|c|}{ Outcome } & \multicolumn{1}{|c|}{ Finding } & \multicolumn{1}{|c|}{ Study Design } \\
\hline Gastrointestinal AE & $\begin{array}{l}\text { Rate Ratio: } 1.62 ; 95 \% \mathrm{Cl}: 0.60 \text { to } 4.34, \\
\mathrm{I}^{2}=\mathrm{N} / \mathrm{A}\end{array}$ & $1 \mathrm{RCT}^{94}$ \\
\hline General Internal Medicine AE & $\begin{array}{l}\text { Rate Ratio: } 1.26 ; 95 \% \mathrm{Cl}: 0.08 \text { to } \\
20.11, \mathrm{I}^{2}=\mathrm{N} / \mathrm{A}\end{array}$ & $1 \mathrm{RCT}{ }^{94}$ \\
\hline Hepatic AE & $\begin{array}{l}\text { Rate Ratio: } 1.26 ; 95 \% \mathrm{Cl}: 0.08 \text { to } \\
20.11, \mathrm{I}^{2}=\mathrm{N} / \mathrm{A}\end{array}$ & $1 \mathrm{RCT}^{94}$ \\
\hline Neurological AE & $\begin{array}{l}\text { Rate Ratio: } 0.25 ; 95 \% \mathrm{Cl}: 0.03 \text { to } 2.15, \\
\mathrm{I}^{2}=\mathrm{N} / \mathrm{A}\end{array}$ & $1 \mathrm{RCT}^{94}$ \\
\hline Total number of AES & $\begin{array}{l}\text { Rate Ratio: } 1.26 ; 95 \% \mathrm{Cl}: 0.6 \text { to } 2.64, \\
\mathrm{I}^{2}=\mathrm{N} / \mathrm{A}\end{array}$ & $1 \mathrm{RCT}^{94}$ \\
\hline
\end{tabular}

$\mathrm{AE}$ = adverse event; $\mathrm{CI}$ = confidence interval; N/A = not applicable; $\mathrm{RCT}$ = randomized controlled trial

Table H.32. KQ4: Adverse events. Levofloxacin compared with prulifloxacin

\begin{tabular}{|l|l|l|}
\hline \multicolumn{1}{|c|}{ Outcome } & \multicolumn{1}{|c|}{ Finding } & \multicolumn{1}{|c|}{ Study Design } \\
\hline Gastrointestinal AE & $\begin{array}{l}\text { Rate Ratio: } 0.63 ; 95 \% \mathrm{Cl}: 0.21 \text { to } 1.92, \\
\mathrm{I}^{2}=\mathrm{N} / \mathrm{A}\end{array}$ & $1 \mathrm{RCT}^{71}$ \\
\hline Neurological AE & $\begin{array}{l}\text { Rate Ratio: } 1.14 ; 95 \% \mathrm{Cl}: 0.45 \text { to } 4.44, \\
\mathrm{I}^{2}=\mathrm{N} / \mathrm{A}\end{array}$ & $1 \mathrm{RCT}^{71}$ \\
\hline Serious AE & $\begin{array}{l}\text { Rate Ratio: } 1.2 ; 95 \% \mathrm{Cl}: 0.36 \text { to } 3.98, \\
\mathrm{I}^{2}=0.0 \%\end{array}$ & $2 \mathrm{RCTs}$ \\
\hline Total number of AEs & $\begin{array}{l}\text { Rate Ratio: } 0.93 ; 95 \% \mathrm{Cl}: 0.67 \text { to } 1.29, \\
\mathrm{I}^{2}=0.0 \%\end{array}$ & $2 \mathrm{RCTs}$ \\
\hline Withdrawal & $\begin{array}{l}\text { OR: } 1.07 ; 95 \% \mathrm{Cl}: 0.74 \text { to } 1.56, \\
\mathrm{I}^{2}=0.0 \%\end{array}$ & $2 \mathrm{RCTs}$ \\
\hline Withdrawal due to AE & $\begin{array}{l}\text { OR: } 1.39 ; 95 \% \mathrm{Cl}: 0.69 \text { to } 2.78, \\
\mathrm{I}^{2}=0.00 \%\end{array}$ & $2 \mathrm{RCTs}^{71,75}$ \\
\hline
\end{tabular}

$\mathrm{AE}$ = adverse event; $\mathrm{CI}$ = confidence interval; N/A = not applicable; $\mathrm{OR}$ = odds ratio; $\mathrm{RCT}$ = randomized controlled trial

Table H.33. KQ4: Adverse events. Moxifloxacin compared with zabofloxacin

\begin{tabular}{|l|l|l|}
\hline \multicolumn{1}{|c|}{ Outcome } & \multicolumn{1}{|c|}{ Finding } & \multicolumn{1}{|c|}{ Study Design } \\
\hline Dermatological AE & $\begin{array}{l}\text { Rate Ratio: } 0.70 ; 95 \% \mathrm{Cl}: 0.12 \text { to } 4.18, \\
\mathrm{I}^{2}=\mathrm{N} / \mathrm{A}\end{array}$ & $1 \mathrm{RCT}^{84}$ \\
\hline Gastrointestinal AE & $\begin{array}{l}\text { Rate Ratio: } 0.84 ; 95 \% \mathrm{Cl}: 0.33 \text { to } 2.12, \\
\mathrm{I}^{2}=\mathrm{N} / \mathrm{A}\end{array}$ & $1 \mathrm{RCT}^{84}$ \\
\hline $\begin{array}{l}\text { Rate Ratio: } 0.84 ; 95 \% \mathrm{Cl}: 0.23 \text { to } 3.12, \\
\mathrm{I}^{2}=\mathrm{N} / \mathrm{A}\end{array}$ & $1 \mathrm{RCT}^{84}$ \\
\hline Reurological AE & $\begin{array}{l}\text { Rate Ratio: } 1.05 ; 95 \% \mathrm{Cl}: 0.07 \text { to } \\
16.75, \mathrm{I}^{2}=\mathrm{N} / \mathrm{A}\end{array}$ & $1 \mathrm{RCT}^{84}$ \\
\hline $\begin{array}{l}\text { Rate Ratio: } 1.20 ; 95 \% \mathrm{Cl}: 0.43 \text { to } 3.30, \\
\mathrm{I}^{2}=\mathrm{N} / \mathrm{A}\end{array}$ & $1 \mathrm{RCT}^{84}$ \\
\hline Serious AE & $\begin{array}{l}\text { Rate Ratio: } 0.88 ; 95 \% \mathrm{Cl}: 0.53 \text { to } \\
1.48, \mathrm{I}^{2}=\mathrm{N} / \mathrm{A}\end{array}$ & $1 \mathrm{RCT}^{84}$ \\
\hline Wotal number of AEs & $\begin{array}{l}\text { OR: } 1.05 ; 95 \% \mathrm{Cl}: 0.07 \text { to } 16.90, \\
\mathrm{I}^{2}=\mathrm{N} / \mathrm{A}\end{array}$ & $1 \mathrm{RCT}^{84}$ \\
\hline Withdrawal & OR: $1.05 ; 95 \% \mathrm{Cl}: 0.21$ to $5.27, \mathrm{I}^{2}=\mathrm{N} / \mathrm{A}$ & $1 \mathrm{RCT}^{84}$ \\
\hline
\end{tabular}

$\mathrm{AE}=$ adverse event; $\mathrm{CI}=$ confidence interval; N/A = not applicable; $\mathrm{OR}$ = odds ratio; $\mathrm{RCT}$ = randomized controlled trial

Table H.34. KQ4: Adverse events. Cefaclor compared with cefpodoxime

\begin{tabular}{|l|l|l|}
\hline \multicolumn{1}{|c|}{ Outcome } & \multicolumn{1}{|c|}{ Finding } & \multicolumn{1}{c|}{ Study Design } \\
\hline Gastrointestinal AE & $\begin{array}{l}\text { Rate Ratio: } 0.85 ; 95 \% \mathrm{Cl}: 0.37 \text { to } 1.98, \\
\mathrm{I}^{2}=\mathrm{N} / \mathrm{A}\end{array}$ & $1 \mathrm{RCT}^{83}$ \\
\hline Total number of AEs & $\begin{array}{l}\text { Rate Ratio: } 1.07 ; 95 \% \mathrm{Cl}: 0.54 \text { to } 2.13, \\
\mathrm{I}^{2}=\mathrm{N} / \mathrm{A}\end{array}$ & $1 \mathrm{RCT}^{83}$ \\
\hline Withdrawal due to AE & OR: $0.60 ; 95 \% \mathrm{Cl}: 0.06$ to $5.85, \mathrm{I}^{2}=\mathrm{N} / \mathrm{A}$ & $1 \mathrm{RCT}^{83}$ \\
\hline
\end{tabular}

$\mathrm{AE}$ = adverse event; $\mathrm{CI}$ = confidence interval; N/A = not applicable; $\mathrm{OR}$ = odds ratio; RCT = randomized controlled trial 
Table H.35. KQ4: Adverse events. Imipenem+cilastatin compared with meropenem

\begin{tabular}{|l|l|l|}
\hline \multicolumn{1}{|c|}{ Outcome } & \multicolumn{1}{|c|}{ Finding } & \multicolumn{1}{|c|}{ Study Design } \\
\hline Dermatological & $\begin{array}{l}\text { Rate Ratio: } 0.99 ; 95 \% \mathrm{Cl}: 0.06 \text { to } \\
15.80, \mathrm{I}^{2}=\mathrm{N} / \mathrm{A}\end{array}$ & $1 \mathrm{RCT}^{76}$ \\
\hline Gastrointestinal AE & $\begin{array}{l}\text { Rate Ratio: } 2.72 ; 95 \% \mathrm{Cl}: 0.87 \text { to } 8.54, \\
\mathrm{I}^{2}=\mathrm{N} / \mathrm{A}\end{array}$ & $1 \mathrm{RCT}^{76}$ \\
\hline General Internal Medicine AE & $\begin{array}{l}\text { Rate Ratio: } 0.99 ; 95 \% \mathrm{Cl}: 0.06 \text { to } \\
15.80, \mathrm{I}^{2}=\mathrm{N} / \mathrm{A}\end{array}$ & $1 \mathrm{RCT}^{76}$ \\
\hline Total number of AEs & $\begin{array}{l}\text { Rate Ratio: } 2.64 ; 95 \% \mathrm{Cl}: 1.03 \text { to } 6.74, \\
\mathrm{I}^{2}=\mathrm{N} / \mathrm{A}\end{array}$ & $1 \mathrm{RCT}^{76}$ \\
\hline Withdrawal & OR: $0.87 ; 95 \% \mathrm{Cl}: 0.32$ to $2.36, \mathrm{I}^{2}=\mathrm{N} / \mathrm{A}$ & $1 \mathrm{RCT}^{76}$ \\
\hline Withdrawal due to AE & OR: $1.67 ; 95 \% \mathrm{Cl}: 0.39$ to $7.29, \mathrm{I}^{2}=\mathrm{N} / \mathrm{A}$ & $1 \mathrm{RCT}^{76}$ \\
\hline
\end{tabular}

$\mathrm{AE}$ = adverse event; $\mathrm{CI}$ = confidence interval; N/A = not applicable; $\mathrm{OR}$ = odds ratio; $\mathrm{RCT}$ = randomized controlled trial

Table H.36. KQ4: Adverse events. Trovafloxacin $200 \mathrm{mg}$ compared with trovafloxacin $100 \mathrm{mg}$

\begin{tabular}{|l|l|l|}
\hline \multicolumn{1}{|c|}{ Outcome } & \multicolumn{1}{|c|}{ Finding } & \multicolumn{1}{|c|}{ Study Design } \\
\hline General Internal Medicine AE & $\begin{array}{l}\text { Rate Ratio: } 2.00 ; 95 \% \mathrm{Cl}: 0.37 \text { to } \\
10.92, \mathrm{I}^{2}=\mathrm{N} / \mathrm{A}\end{array}$ & $1 \mathrm{RCT}^{78}$ \\
\hline Gastrointestinal AE & $\begin{array}{l}\text { Rate Ratio: } 0.25 ; 95 \% \mathrm{Cl}: 0.03 \text { to } 2.24, \\
\mathrm{I}^{2}=\mathrm{N} / \mathrm{A}\end{array}$ & $1 \mathrm{RCT}^{78}$ \\
\hline Serious AE & $\begin{array}{l}\text { Rate Ratio: } 1.63 ; 95 \% \mathrm{Cl}: 0.67 \text { to } 3.92, \\
\mathrm{I}^{2}=\mathrm{N} / \mathrm{A}\end{array}$ & $1 \mathrm{RCT}^{78}$ \\
\hline Total number of AEs & $\begin{array}{l}\text { Rate Ratio: } 1.29 ; 95 \% \mathrm{Cl}: 0.64 \text { to } 2.59, \\
\mathrm{I}^{2}=\mathrm{N} / \mathrm{A}\end{array}$ & $1 \mathrm{RCT}^{78}$ \\
\hline
\end{tabular}

AE = adverse event; $\mathrm{CI}$ = confidence interval; N/A = not applicable; $\mathrm{RCT}$ = randomized controlled trial

Table H.37. KQ4: Adverse events. Intermittent intravenous cefotaxime compared with continuous intravenous cefotaxime

\begin{tabular}{|l|c|c|}
\hline \multicolumn{1}{|c|}{ Outcome } & Finding & Study Design \\
\hline Withdrawal & OR: $0.40 ; 95 \%$ Cl: 0.10 to $1.65, \mathrm{I}^{2}=\mathrm{N} / \mathrm{A}$ & $1 \mathrm{RCT}^{93}$ \\
\hline
\end{tabular}

$\mathrm{CI}=$ confidence interval; N/A = not applicable; $\mathrm{OR}=$ odds ratio; $\mathrm{RCT}=$ randomized controlled trial

Table H.38. KQ4: Adverse events. Amoxicillin + clavulanic for 10 days compared with amoxicillin + Clavulanic for 3 days

\begin{tabular}{|l|l|l|}
\hline \multicolumn{1}{|c|}{ Outcome } & \multicolumn{1}{|c|}{ Finding } & \multicolumn{1}{|c|}{ Study Design } \\
\hline Gastrointestinal AE & $\begin{array}{l}\text { Rate Ratio: } 4.60 ; 95 \% \mathrm{Cl}: 0.54 \text { to } \\
39.37, \mathrm{I}^{2}=\mathrm{N} / \mathrm{A}\end{array}$ & $1 \mathrm{RCT}^{86}$ \\
\hline Total number of AEs & $\begin{array}{l}\text { Rate Ratio: } 4.60 ; 95 \% \mathrm{Cl}: 0.54 \text { to } \\
39.37, \mathrm{I}^{2}=\mathrm{N} / \mathrm{A}\end{array}$ & $1 \mathrm{RCT}^{86}$ \\
\hline Withdrawal & OR: $0.44 ; 95 \% \mathrm{Cl}: 0.04$ to $5.18, \mathrm{I}^{2}=\mathrm{N} / \mathrm{A}$ & $1 \mathrm{RCT}^{86}$ \\
\hline
\end{tabular}

$\mathrm{AE}$ = adverse event; $\mathrm{CI}$ = confidence interval; N/A = not applicable; $\mathrm{OR}$ = odds ratio; $\mathrm{RCT}$ = randomized controlled trial

Table H.39. KQ4: Adverse events. Deflazacort hemisuccinate compared with methylprednisolone

\begin{tabular}{|l|l|l|}
\hline \multicolumn{1}{|c|}{ Outcome } & \multicolumn{1}{|c|}{ Finding } & \multicolumn{1}{c|}{ Study Design } \\
\hline Endocrine AE & $\begin{array}{l}\text { Rate Ratio: } 0.50 ; 95 \% \mathrm{Cl}: 0.05 \text { to } 5.51, \\
\mathrm{I}^{2}=\mathrm{N} / \mathrm{A}\end{array}$ & $1 \mathrm{RCT}^{85}$ \\
\hline Gastrointestinal AE & $\begin{array}{l}\text { Rate Ratio: 0.40; } 95 \% \mathrm{Cl}: 0.08 \text { to } 2.06, \\
\mathrm{I}^{2}=\mathrm{N} / \mathrm{A}\end{array}$ & $1 \mathrm{RCT}^{85}$ \\
\hline Total number of AEs & $\begin{array}{l}\text { Rate Ratio: } 0.27 ; 95 \% \mathrm{Cl}: 0.08 \text { to } 0.98, \\
\mathrm{I}^{2}=\mathrm{N} / \mathrm{A}\end{array}$ & $1 \mathrm{RCT}^{85}$ \\
\hline Withdrawal & OR: $0.19 ; 95 \% \mathrm{Cl}: 0.01$ to $4.06, \mathrm{I}^{2}=\mathrm{N} / \mathrm{A}$ & $1 \mathrm{RCT}^{85}$ \\
\hline
\end{tabular}

$\mathrm{AE}=$ adverse event; $\mathrm{CI}=$ confidence interval; N/A = not applicable; $\mathrm{OR}$ = odds ratio; $\mathrm{RCT}$ = randomized controlled trial 
Table H.40. KQ4: Adverse events. Hydrocortisone i.v. followed by prednisolone oral compared with methylprednisolone i.v. followed by methylprednisone oral

\begin{tabular}{|l|l|l|}
\hline \multicolumn{1}{|c|}{ Outcome } & \multicolumn{1}{|c|}{ Finding } & \multicolumn{1}{c|}{ Study Design } \\
\hline Gastrointestinal AE & $\begin{array}{l}\text { Rate Ratio: } 5.64 ; 95 \% \mathrm{Cl}: 0.68 \text { to } \\
46.85, \mathrm{I}^{2}=\mathrm{N} / \mathrm{A}\end{array}$ & $1 \mathrm{RCT}{ }^{68}$ \\
\hline General Internal Medicine AE & $\begin{array}{l}\text { Rate Ratio: } 2.19 ; 95 \% \mathrm{Cl}: 0.57 \text { to } 8.48, \\
\mathrm{I}^{2}=\mathrm{N} / \mathrm{A}\end{array}$ & $1 \mathrm{RCT}^{68}$ \\
\hline $\begin{array}{l}\text { Rate Ratio: } 3.05 ; 95 \% \mathrm{Cl}: 1.00 \text { to } 9.37, \\
\mathrm{I}^{2}=\mathrm{N} / \mathrm{A}\end{array}$ & $1 \mathrm{RCT}^{68}$ \\
\hline Total number of AEs & OR: $0.22 ; 95 \% \mathrm{Cl}: 0.02$ to $2.04, \mathrm{I}^{2}=\mathrm{N} / \mathrm{A}$ & $1 \mathrm{RCT}^{68}$ \\
\hline Withdrawal & &
\end{tabular}

$\mathrm{AE}$ = adverse event; $\mathrm{CI}$ = confidence interval; i.v = intravenous; N/A = not applicable; $\mathrm{OR}$ = odds ratio; $\mathrm{RCT}=$ randomized controlled trial

Table H.41. KQ4: Adverse events. Nebulized budesonide compared with oral prednisolone

\begin{tabular}{|l|l|l|}
\hline \multicolumn{1}{|c|}{ Outcome } & \multicolumn{1}{|c|}{ Finding } & \multicolumn{1}{|c|}{ Study Design } \\
\hline Endocrine AE & $\begin{array}{l}\text { Rate Ratio: } 0.12 ; 95 \% \mathrm{Cl}: 0.02 \text { to } 1.01, \\
\mathrm{I}^{2}=\mathrm{N} / \mathrm{A}\end{array}$ & $1 \mathrm{RCT}^{12}$ \\
\hline Total number of AEs & $\begin{array}{l}\text { Rate Ratio: } 0.77 ; 95 \% \mathrm{Cl}: 0.50 \text { to } 1.19, \\
\mathrm{I}^{2}=\mathrm{N} / \mathrm{A}\end{array}$ & $1 \mathrm{RCT}^{12}$ \\
\hline Respiratory AE & $\begin{array}{l}\text { Rate Ratio: } 1.46 ; 95 \% \mathrm{Cl}: 0.35 \text { to } 6.09, \\
\mathrm{I}^{2}=\mathrm{N} / \mathrm{A}\end{array}$ & $1 \mathrm{RCT}^{12}$ \\
\hline Serious AE & $\begin{array}{l}\text { Rate Ratio: } 1.40 ; 95 \% \mathrm{Cl}: 0.46 \text { to } 4.27, \\
\mathrm{I}^{2}=\mathrm{N} / \mathrm{A}\end{array}$ & $1 \mathrm{RCT}^{12}$ \\
\hline Withdrawal & OR; $2.04 ; 95 \% \mathrm{Cl}: 0.87$ to $7.76, \mathrm{I}^{2}=\mathrm{N} / \mathrm{A}$ & $1 \mathrm{RCT}^{12}$ \\
\hline Withdrawal due to AE & OR: $1.89 ; 96 \% \mathrm{Cl}: 0.66$ to $5.26, \mathrm{I}^{2}=\mathrm{N} / \mathrm{A}$ & $1 \mathrm{RCT}^{12}$ \\
\hline
\end{tabular}

$\mathrm{AE}$ = adverse event; $\mathrm{CI}$ = confidence interval; N/A = not applicable; $\mathrm{OR}$ = odds ratio; $\mathrm{RCT}$ = randomized controlled trial

Table H.42. KQ4: Adverse events. Oral prednisolone + inhaled formoterol compared with inhaled budesonide + formoterol

\begin{tabular}{|l|l|l|}
\hline \multicolumn{1}{|c|}{ Outcome } & \multicolumn{1}{|c|}{ Finding } & \multicolumn{1}{c|}{ Study Design } \\
\hline Total number of $\mathrm{AEs}$ & $\begin{array}{l}\text { Rate ratio: } 0.85 ; 95 \% \mathrm{Cl}: 0.43 \text { to } 1.68, \\
\mathrm{I}^{2}=\mathrm{N} / \mathrm{A}\end{array}$ & $1 \mathrm{RCT}^{89}$ \\
\hline
\end{tabular}

$\mathrm{AE}$ = adverse event; $\mathrm{CI}$ = confidence interval; N/A = not applicable; $\mathrm{RCT}$ = randomized controlled trial

Table H.43. KQ4: Adverse events. Inhaled budesonide + formoterol compared with systemic methylprednisolone

\begin{tabular}{|l|l|l|}
\hline \multicolumn{1}{|c|}{ Outcome } & \multicolumn{1}{|c|}{ Finding } & \multicolumn{1}{|c|}{ Study Design } \\
\hline Cardiovascular AE & $\begin{array}{l}\text { Rate Ratio: } 2.00 ; 95 \% \mathrm{Cl}: 0.18 \text { to } \\
22.06, \mathrm{I}^{2}=\mathrm{N} / \mathrm{A}\end{array}$ & $1 \mathrm{RCT}^{90}$ \\
\hline Gastrointestinal AE & $\begin{array}{l}\text { Rate Ratio: } 0.50 ; 95 \% \mathrm{Cl}: 0.09 \text { to } 2.73, \\
\mathrm{I}^{2}=\mathrm{N} / \mathrm{A}\end{array}$ & $1 \mathrm{RCT}^{90}$ \\
\hline General Internal Medicine AE & $\begin{array}{l}\text { Rate Ratio: } 1 ; 95 \% \mathrm{Cl}: 0.06 \text { to } 15.99, \\
\mathrm{I}^{2}=\mathrm{N} / \mathrm{A}\end{array}$ & $1 \mathrm{RCT}^{90}$ \\
\hline Total number of AEs & $\begin{array}{l}\text { Rate Ratio: } 0.56 ; 95 \% \mathrm{Cl}: 0.19 \text { to } 1.66, \\
\mathrm{I}^{2}=\mathrm{N} / \mathrm{A}\end{array}$ & $1 \mathrm{RCT}^{90}$ \\
\hline
\end{tabular}

AE = adverse event; $\mathrm{CI}$ = confidence interval; N/A = not applicable; $\mathrm{RCT}$ = randomized controlled trial

Table H.44. KQ4: Adverse events. Inhaled Budesonide 40mg compared with Intravenous Methylprednisolone (initially intravenous, then oral)

\begin{tabular}{|l|l|l|}
\hline \multicolumn{1}{|c|}{ Outcome } & \multicolumn{1}{|c|}{ Finding } & \multicolumn{1}{c|}{ Study Design } \\
\hline Endocrine AE & Rate Ratio: $0.28 ; 95 \% \mathrm{Cl}: 0.14$ to & $1 \mathrm{RCT}^{73}$ \\
& $0.57, \mathrm{I}^{2}=\mathrm{N} / \mathrm{A}$ & \\
\hline Total number of AEs & $\begin{array}{l}\text { Rate Ratio: } 0.28 ; 95 \% \mathrm{Cl}: 0.14 \text { to } \\
0.57, \mathrm{I}^{2}=\mathrm{N} / \mathrm{A}\end{array}$ & $1 \mathrm{RCT}{ }^{73}$ \\
\hline
\end{tabular}

AE = adverse event; $\mathrm{CI}=$ confidence interval; N/A = not applicable; $\mathrm{RCT}$ = randomized controlled trial 
Table H.45. KQ4: Adverse events. Inhaled Budesonide 4mg compared with Intravenous Methylprednisolone

\begin{tabular}{|l|l|l|}
\hline \multicolumn{1}{|c|}{ Outcome } & \multicolumn{1}{|c|}{ Finding } & \multicolumn{1}{|c|}{ Study Design } \\
\hline Respiratory $\mathrm{AE}$ & $\begin{array}{l}\text { Rate Ratio: } 5.78 ; 95 \% \mathrm{Cl}: 0.70 \text { to } \\
47.99, \mathrm{I}^{2}=\mathrm{N} / \mathrm{A}\end{array}$ & $1 \mathrm{RCT}^{91}$ \\
\hline Total number of AEs & $\begin{array}{l}\text { Rate Ratio: } 5.78 ; 95 \% \mathrm{Cl}: 0.70 \text { to } \\
47.99, \mathrm{I}^{2}=\mathrm{N} / \mathrm{A}\end{array}$ & $1 \mathrm{RCT}^{91}$ \\
\hline
\end{tabular}

$\mathrm{AE}$ = adverse event; $\mathrm{CI}$ = confidence interval; $\mathrm{N} / \mathrm{A}$ = not applicable; $\mathrm{RCT}$ = randomized controlled trial

Table H.46. KQ4: Adverse events. Inhaled Budesonide 8mg compared with intravenous methylprednisolone

\begin{tabular}{|l|l|l|}
\hline \multicolumn{1}{|c|}{ Outcome } & \multicolumn{1}{|c|}{ Finding } & \multicolumn{1}{c|}{ Study Design } \\
\hline Respiratory AE & $\begin{array}{l}\text { Rate Ratio: 0.63; 95\% Cl: 0.06 to 7.00, } \\
\mathrm{I}^{2}=\mathrm{N} / \mathrm{A}\end{array}$ & $1 \mathrm{RCT}^{91}$ \\
\hline Total number of AEs & $\begin{array}{l}\text { Rate Ratio: } 0.63 ; 95 \% \mathrm{Cl}: 0.06 \text { to } 7.00, \\
\mathrm{I}^{2}=\mathrm{N} / \mathrm{A}\end{array}$ & $1 \mathrm{RCT}^{91}$ \\
\hline
\end{tabular}

$\mathrm{AE}$ = adverse event; $\mathrm{CI}$ = confidence interval; N/A = not applicable; $\mathrm{RCT}$ = randomized controlled trial

Table H.47. KQ4: Adverse events. Oral prednisolone compared with intravenous prednisolone

\begin{tabular}{|c|c|c|}
\hline Outcome & \multicolumn{1}{|c|}{ Finding } & Study Design and Sample Size \\
\hline Withdrawal & OR: $1.33 ; 95 \%$ Cl: 0.60 to $2.92, \mathrm{I}^{2}=\mathrm{N} / \mathrm{A}$ & 1 RCT \\
\hline
\end{tabular}

$\mathrm{CI}=$ confidence interval; N/A = not applicable; $\mathrm{OR}$ = odds ratio; $\mathrm{RCT}=$ randomized controlled trial

Table H.48. KQ4: Adverse events. Oral methyl-prednisolone compared with intravenous methylprednisolone

\begin{tabular}{|c|c|c|}
\hline \multicolumn{1}{|c|}{ Outcome } & \multicolumn{1}{c|}{ Finding } & \multicolumn{1}{c|}{ Study Design } \\
\hline Withdrawal & OR: $1.29 ; 95 \% \mathrm{Cl}: 0.39$ to $4.30, \mathrm{I}^{2}=\mathrm{N} / \mathrm{A}$ & $1 \mathrm{RCT} 95$ \\
\hline
\end{tabular}

$\mathrm{CI}=$ confidence interval; N/A = not applicable; OR = odds ratio; RCT = randomized controlled trial

Table H.49. KQ4: Adverse events. Glucocorticoid for 2 weeks compared with glucocorticoid for 8 weeks

\begin{tabular}{|l|l|l|}
\hline \multicolumn{1}{|c|}{ Outcome } & \multicolumn{1}{|c|}{ Finding } & \multicolumn{1}{|c|}{ Study Design } \\
\hline Endocrine AE & $\begin{array}{l}\text { Rate Ratio: } 1.40 ; 95 \% \mathrm{Cl}: 0.62 \text { to } 3.15, \\
\mathrm{I}^{2}=\mathrm{N} / \mathrm{A}\end{array}$ & $1 \mathrm{RCT}^{13}$ \\
\hline General Internal Medicine AE & $\begin{array}{l}\text { Rate Ratio: } 1.50 ; 95 \% \mathrm{Cl}: 0.42 \text { to } 5.32, \\
\mathrm{I}^{2}=\mathrm{N} / \mathrm{A}\end{array}$ & $1 \mathrm{RCT}^{13}$ \\
\hline Infectious AE & $\begin{array}{l}\text { Rate Ratio: } 0.67 ; 95 \% \mathrm{Cl}: 0.32 \text { to } 1.38, \\
\mathrm{I}^{2}=\mathrm{N} / \mathrm{A}\end{array}$ & $1 \mathrm{RCT}^{13}$ \\
\hline Psychiatric AE & $\begin{array}{l}\text { Rate Ratio: } 2.5 ; 95 \% \mathrm{Cl}: 0.49 \text { to } 12.86, \\
\mathrm{I}^{2}=\mathrm{N} / \mathrm{A}\end{array}$ & $1 \mathrm{RCT}^{13}$ \\
\hline Total number of AEs & $\begin{array}{l}\text { Rate Ratio: } 0.95 ; 95 \% \mathrm{Cl}: 0.66 \text { to } 1.37, \\
\mathrm{I}^{2}=\mathrm{N} / \mathrm{A}\end{array}$ & $1 \mathrm{RCT}^{13}$ \\
\hline
\end{tabular}

$\mathrm{AE}$ = adverse event; $\mathrm{CI}$ = confidence interval; N/A = not applicable; $\mathrm{RCT}$ = randomized controlled trial

Table H.50. KQ4: Adverse events. Methylprednisolone for 5 days compared with methylprednisolone for 14 days

\begin{tabular}{|c|c|c|}
\hline Outcome & Finding & Study Design \\
\hline Endocrine $\mathrm{AE}$ & $\begin{array}{l}\text { Rate Ratio: } 1.01 ; 95 \% \mathrm{Cl} \text { : } \\
0.73 \text { to } 1.39,1^{2}=\mathrm{N} / \mathrm{A}\end{array}$ & $1 \mathrm{RCT}^{79}$ \\
\hline $\begin{array}{l}\text { General Internal Medicine } \\
\mathrm{AE}\end{array}$ & $\begin{array}{l}\text { Rate Ratio: } 1.54 ; 95 \% \mathrm{Cl} \text { : } \\
0.81 \text { to } 2.96, \mathrm{I}^{2}=\mathrm{N} / \mathrm{A}\end{array}$ & $1 \mathrm{RCT}^{79}$ \\
\hline Total number of AEs & $\begin{array}{l}\text { Rate Ratio: } 1.08 ; 95 \% \mathrm{Cl} \text { : } \\
0.83 \text { to } 1.41,1^{2}=\mathrm{N} / \mathrm{A}\end{array}$ & $1 \mathrm{RCT}^{79}$ \\
\hline Withdrawal & $\begin{array}{l}\text { OR: } 1.52 ; 95 \% \mathrm{Cl}: 0.53 \text { to } \\
4.38, \mathrm{I}^{2}=\mathrm{N} / \mathrm{A}\end{array}$ & $1 \mathrm{RCT}^{79}$ \\
\hline
\end{tabular}

$\mathrm{AE}$ = adverse event; $\mathrm{CI}$ = confidence interval; N/A = not applicable; $\mathrm{OR}$ = odds ratio; $\mathrm{RCT}$ = randomized controlled trial 
Table H.51. KQ4: Adverse events. Methylprednisolone for 3 days compared with methylprednisolone for 10 days

\begin{tabular}{|l|l|l|}
\hline \multicolumn{1}{|c|}{ Outcome } & \multicolumn{1}{|c|}{ Finding } & \multicolumn{1}{c|}{ Study Design } \\
\hline Endocrine AE & RR: $1.00 ; 95 \% \mathrm{Cl}: 0.14$ to $7.10, \mathrm{I}^{2}=\mathrm{N} / \mathrm{A}$ & $1 \mathrm{RCT}^{88}$ \\
\hline Total number of AEs & $\begin{array}{l}\text { Rate Ratio: } 1.00 ; 95 \% \mathrm{Cl}: 0.20 \text { to } 4.95, \\
\mathrm{I}^{2}=\mathrm{N} / \mathrm{A}\end{array}$ & $1 \mathrm{RCT}^{88}$ \\
\hline Withdrawal & OR: $1.00 ; 95 \% \mathrm{Cl}: 0.06$ to $17.41, \mathrm{I}^{2}=\mathrm{N} / \mathrm{A}$ & $1 \mathrm{RCT}^{88}$ \\
\hline Withdrawal due to AE & $\begin{array}{l}\text { OR: } 1.00 ; 95 \% \mathrm{Cl}: 0.06 \text { to } 17.41, \\
\mathrm{I}^{2}=\mathrm{N} / \mathrm{A}\end{array}$ & $1 \mathrm{RCT}^{88}$ \\
\hline
\end{tabular}

$\mathrm{AE}$ = adverse event; $\mathrm{CI}$ = confidence interval; N/A = not applicable; $\mathrm{OR}$ = odds ratio; $\mathrm{RCT}$ = randomized controlled trial 


\section{Appendix I. Inclusion and Exclusion Criteria of Included Studies}

Table I.1. Inclusion and exclusion criteria of included studies

\begin{tabular}{|c|c|c|}
\hline Author, Year & Inclusion Criteria & Exclusion Criteria \\
\hline Aaron, $2003^{1}$ & $\begin{array}{l}\text { Age }>35 \text { years, who presented at the ED with an } \\
\text { exacerbation of COPD (the presence of at least two of } \\
\text { the following three clinical criteria: a recent increase in } \\
\text { breathlessness, sputum volume, or sputum purulence), } \\
\text { History of } 15 \text { pack-years or more of cigarette smoking } \\
\text { and evidence of irreversible airflow obstruction defined } \\
\text { as FEV1/FVC ratio of } 0.70 \text { or less, FEV1 not more than } \\
70 \% \text { of the predicted value, and an improvement in the } \\
\text { FEV1 of }<20 \% \text { after the administration of a } \\
\text { bronchodilator. All enrolled patients either had previously } \\
\text { been given a diagnosis of COPD by a physician or had } \\
\text { at least a one-year history of chronic dyspnea or cough } \\
\text { with sputum production. }\end{array}$ & $\begin{array}{l}\text { Admitted to the hospital, had been given a diagnosis of asthma or } \\
\text { atopy, had used oral or intravenous corticosteroids within the } \\
\text { preceding } 30 \text { days, had received oral or intravenous } \\
\text { corticosteroids in the emergency department, had findings on } \\
\text { chest radiography consistent with the presence of pneumonia or } \\
\text { congestive heart failure, had had adverse reactions to oral } \\
\text { corticosteroids, or had severe uncontrolled diabetes mellitus or } \\
\text { renal, hepatic or cardiac failure. }\end{array}$ \\
\hline $\begin{array}{l}\text { Abreu Gonzalez, } \\
2006^{17}\end{array}$ & Diagnosed with an exacerbation of COPD. & $\begin{array}{l}\text { Diagnosed with pneumonia, HF, arrhythmias, or kidney failure and } \\
\text { those who did not show a sufficient level of cooperation. }\end{array}$ \\
\hline Aggarwal, $2011^{68}$ & $\begin{array}{l}\text { Aged }>50 \text { years known to have COPD as defined in } \\
\text { The GOLD guidelines, who presented to the ED with } \\
\text { moderate (FEV1 50-80\% of predicted) or severe (FEV1 } \\
30-50 \% \text { of predicted) exacerbation of COPD. }\end{array}$ & $\begin{array}{l}\text { Significant co-morbid conditions such as pneumonia, CHF or } \\
\text { interstitial lung disease, previous diagnosis of bronchial asthma, } \\
\text { and use of systemic glucocorticoids within the last30 days, } \\
\text { patients who required assisted ventilation on arrival at the ED or } \\
\text { failed to use the PEF meter, Patients who required mechanical } \\
\text { ventilation within } 1 \text { h of arrival at the ED. }\end{array}$ \\
\hline Albert, $1980^{2}$ & $\begin{array}{l}\text { Acute } \mathrm{RF}(\mathrm{PaO} 2<65 \mathrm{mmHg} \text { on room air, or } \mathrm{PaCO} 2 \geq 50 \\
\text { or } \mathrm{pH}<7.35) \text {, Chronic bronchitis (FEV1 } \leq 60 \% \text { predicted } \\
\text { or FVC } \leq 60 \%) \text {, acute bronchitis (increase in cough, } \\
\text { sputum production, and sputum color within prior } 5 \\
\text { days). }\end{array}$ & $\begin{array}{l}\text { Personal or family history of asthma, bronchodilator response } \\
\text { FEV1 }>=30 \% \text {, eczema, allergic rhinitis, consolidation on chest } \\
\text { radiograph, corticosteroid therapy in the past } 30 \text { days. }\end{array}$ \\
\hline
\end{tabular}




\begin{tabular}{|c|c|c|}
\hline Author, Year & Inclusion Criteria & Exclusion Criteria \\
\hline Andre-Alves, $2007^{69}$ & $\begin{array}{l}30 \text { and } 70 \text { years with infectious exacerbation of COPD. } \\
\text { Required to have been under outpatient treatment with } \\
\text { simple chest X-ray that showed no evidence of } \\
\text { pneumonia performed within the } 48 \text { hours. }\end{array}$ & $\begin{array}{l}\text { Females who were breastfeeding, pregnant or planning to become } \\
\text { pregnant (during the study or up to one month after the end of the } \\
\text { study), women of childbearing age who were not using some } \\
\text { efficacious method of contraception, history of hypersensitivity to } \\
\text { azithromycin or amoxicillin; undergoing treatment with systemic } \\
\text { antibiotics within the two weeks preceding the study outset; use of } \\
\text { antibiotics foreseen for other clinical condition, as well as the use } \\
\text { of allopurinol, probenecid, digoxin, warfarin or ergotamine during } \\
\text { the study; having a history of human immunodeficiency virus } \\
\text { infection, acute bronchitis or bronchiectasis; suspected of having } \\
\text { lung abscess or empyema; suspected of having active } \\
\text { tuberculosis, cystic fibrosis or lung cancer (primary or metastatic); } \\
\text { having any clinical or psychological condition deemed by the } \\
\text { examiner to potentially impair participation in the study; a history } \\
\text { of alcohol or substance abuse; undergoing treatment with } \\
\text { immunosuppressive drugs, including doses of corticosteroids } \\
\text { higher than the equivalent to } 10 \text { mg/ day of prednisone; presenting } \\
\text { any of the following laboratory test results: leukocyte count lower } \\
\text { than } 2500 / m^{3} \text { - neutrophil count lower than } 1000 / m^{3}- \\
\text { elevated transaminase levels of over twice the upper limit of } \\
\text { normality - alkaline phosphatase or bilirubin levels greater than } \\
1.5 \text { times the upper limit of normality; donating blood during the } \\
\text { study or within one month after the conclusion of the study. }\end{array}$ \\
\hline Anthonisen, $1987^{3}$ & $\begin{array}{l}\text { At least } 35 \text { years old, clinical diagnosis of COPD, live } \\
\text { close enough to the clinical center for home visits, } \\
\text { reliable (keep two consecutive outpatient appointments), } \\
\text { FEV1<70\% predicted, FEV1/FVC }<0.7 \text {, TLC }>80 \% \\
\text { predicted. }\end{array}$ & $\begin{array}{l}\text { Asthma, post-bronchodilator FEV1 increasing to }>80 \% \text { predicted, } \\
\text { other disease serious enough to influence the quality of life or } \\
\text { clinical course (e.g. cancer, left ventricular failure, stroke), other } \\
\text { disease likely to require antibiotic therapy (e.g. recurrent sinusitis, } \\
\text { urinary tract infection). }\end{array}$ \\
\hline $\begin{array}{l}\text { Emami Ardestani, } \\
2017^{74}\end{array}$ & $\begin{array}{l}\text { Adult COPD patients with acute respiratory distress, } \\
\text { increased cough frequency and severity, increased } \\
\text { sputum volume, and/or increased wheezing for } 24 \\
\text { hours or more were eligible for entry to the study. }\end{array}$ & $\begin{array}{l}\text { Patients with history of asthma or atopy, onset of respiratory } \\
\text { distress before the age of } 35 \text { years, absence of spirometric data, } \\
\text { or having received oral or intravenous steroids in the month prior } \\
\text { to presentation. }\end{array}$ \\
\hline Aubier, $2002^{70}$ & $\begin{array}{l}\text { History of chronic bronchitis and COPD } \\
\text { (FEV1/FVC<70\%), a bronchodilator response (with } 0.4 \\
\text { mg salbutamol) defined as an increase of }<12 \% \text { from } \\
\text { baseline in FEV1 from a lung function test performed } \\
\text { within } 1 \text { year prior to inclusion, and a clinical diagnosis of } \\
\text { AECB due to presumed bacterial infection (i.e. increased } \\
\text { cough and/or dyspnea, increased production of sputum, } \\
\text { and increased purulence of sputum). }\end{array}$ & $\begin{array}{l}\text { Acute bronchitis, pneumonia, asthma, bronchiectasis, cystic } \\
\text { fibrosis, lung cancer, active pulmonary tuberculosis, or acute RF. }\end{array}$ \\
\hline
\end{tabular}




\begin{tabular}{|c|c|c|}
\hline Author, Year & Inclusion Criteria & Exclusion Criteria \\
\hline Austin, $2010^{18}$ & $\begin{array}{l}\text { Aged } 35 \text { years or older with breathlessness and a history } \\
\text { or risk of chronic obstructive pulmonary disease. } \\
\text { Paramedics at the site of the emergency determined the } \\
\text { diagnosis on the basis of appropriate acute symptoms, a } \\
\text { history of COPD (or emphysema) from the patient, or a } \\
\text { greater than } 10 \text { pack year history of smoking. }\end{array}$ & $\begin{array}{l}\text { Patients with no lung function data or who did not fulfil spirometric } \\
\text { criteria for COPD. }\end{array}$ \\
\hline Ayfer Aytemur, $2015^{19}$ & $\begin{array}{l}\text { Had a spirometrically confirmed prior diagnosis of } \\
\text { COPD, had a smoking history of at least } 20 \text { pack -years, } \\
\text { hospitalized for their current exacerbation, and reported } \\
\text { increased sputum production of more than } 50 \text { ml per } \\
\text { day. }\end{array}$ & $\begin{array}{l}\text { Presence of a prior diagnosis of asthma or bronchiectasis, } \\
\text { radiographic evidence of pneumonia, and use of any mucolytic } \\
\text { drug during the preceding week. }\end{array}$ \\
\hline Basri, $2017^{20}$ & $\begin{array}{l}\text { 45-60 years age, COPD with acute exacerbation as a } \\
\text { primary diagnosis and chronic bronchitis as a secondary } \\
\text { diagnosis, pronounced symptoms of sputum retention } \\
\text { with coughing, ability to tolerate active techniques and } \\
\text { well-oriented patients. }\end{array}$ & $\begin{array}{l}\text { Sever attack with longer expected hospital stay up-to }>2 \text { weeks, } \\
\text { Cardiac or any other condition that contraindicated chest } \\
\text { physiotherapy, COPD with secondary diagnosis of emphysema, } \\
\text { any other associated lung pathological condition, pain with more } \\
\text { than } 2 \text { points on visual analogue scale while doing active } \\
\text { techniques, history of lung surgery. }\end{array}$ \\
\hline Bathoorn, $2008^{4}$ & $\begin{array}{l}\text { COPD, age }>40 \text { years, post bronchodilator FEV1 }<85 \% \\
\text { predicted but }>0.7 \text { liters, and an abnormal post } \\
\text { bronchodilator FEV1/slow inspiratory VC }<88 \% \text { predicted } \\
\text { in men and }<89 \% \text { predicted in women).Patients were not } \\
\text { allowed to use oral corticosteroids, oxygen therapy, } \\
\text { beta-blockers, or long-acting anticholinergics. All other } \\
\text { bronchodilators were allowed. }\end{array}$ & $\begin{array}{l}\text { Asthma or a history suspicious for asthma. Patients had no } \\
\text { significant other disease that could influence the results of the } \\
\text { study. }\end{array}$ \\
\hline Behnke, $2000^{21}$ & $\begin{array}{l}\text { We included patients with severe COPD according to } \\
\text { international guidelines, who were admitted to the } \\
\text { hospital due to an acute exacerbation of their disease. } \\
\text { The study was started } 4 \pm 7 \text { days after admission, when } \\
\text { the patients' condition had stabilized. }\end{array}$ & $\begin{array}{l}\text { Exclusion criteria were: evidence of unstable cardiac disease, or } \\
\text { pulmonale decompensation or other disabling diseases which } \\
\text { prevented participation in the exercise program, such as } \\
\text { orthopedic inabilities or peripheral vascular disease. }\end{array}$ \\
\hline Black, $2004^{22}$ & $\begin{array}{l}\text { A physician diagnosis of COPD, age } \geq 50 \text { years, have } \\
\text { an FEV } 1 \leq 60 \% \text { predicted, FEV } 1 / \mathrm{VC} \leq 70 \% \text { and } \geq 10 \\
\text { pack year smoking history and had been admitted to } \\
\text { hospital with an acute exacerbation of their COPD in the } \\
\text { previous } 24 \text { hours. }\end{array}$ & $\begin{array}{l}\text { Asthma (as the primary diagnosis), HF, bronchiectasis, bronchial } \\
\text { carcinoma, interstitial lung disease, pneumonia. Also if patients } \\
\text { were unable to comply with the study procedures because they } \\
\text { did not speak English or were demented or if they had any other } \\
\text { medical problems that in the opinion of the investigator would } \\
\text { interfere with the conduct of the study. }\end{array}$ \\
\hline
\end{tabular}




\begin{tabular}{|c|c|c|}
\hline Author, Year & Inclusion Criteria & Exclusion Criteria \\
\hline Blasi, $2013^{71}$ & $\begin{array}{l}\text { Aged } 40 \text { years or older, smokers, or ex-smokers }(>10 \\
\text { pack-years) with spirometrically confirmed severe COPD } \\
\text { (FEV1 } 50 \% \text { predicted and FEV1/FVC ratio }<0.7) \text { and } \\
\text { diagnosed with an AECB, requiring concomitant } \\
\text { systemic corticosteroids administration }(20-40 \mathrm{mg} / \mathrm{day} \\
\text { for } 7 \text { days), or whom an increase of the daily dosage of } \\
\text { their chronically corticosteroid treatment was required. }\end{array}$ & $\begin{array}{l}\text { Antibiotic use in the previous week, bronchial asthma, pregnancy } \\
\text { and breast feeding, recent or past history of psychiatric illness, } \\
\text { epilepsy, cardiac disease, rhythm disorders or clinically significant } \\
\text { EKG abnormalities, latent or known deficiencies for the glucose-6- } \\
\text { phospste dehydrogenase activity, pneumonia, cystic fibrosis, } \\
\text { bronchiectasis of origin other than COPD, active neoplasm, } \\
\text { tracheotomy, concomitant treatment with hypoglycemic drugs, } \\
\text { immunosuppression, hypersensitivity or allergy to } \\
\text { fluoroquinolones, history of tendinopathy, and inability to provide } \\
\text { informed consent. }\end{array}$ \\
\hline Borges, $2014^{23}$ & $\begin{array}{l}\text { COPD exacerbation (increase in sputum or cough or } \\
\text { worsening of dyspnea), no hospitalization in } 30 \text { days, } 40- \\
85 \text { years, no musculoskeletal or neurologic conditions } \\
\text { that might affect exercise performance; no participation } \\
\text { in a rehabilitation program in the last } 6 \text { months and } \\
\text { absence of any other pulmonary diseases. }\end{array}$ & $\begin{array}{l}\text { Transferred to the ICU before the second day of hospitalization, } \\
\text { exhibiting changes in mental status, worsening of hypoxemia or } \\
\text { respiratory acidosis, hospitalization time }<5 \text { days; or inability to } \\
\text { complete any of the evaluations. }\end{array}$ \\
\hline Brown, $1987^{24}$ & $\begin{array}{l}\text { Chronic productive cough with sputum expectoration of } \\
30 \mathrm{ml} \text { or more in } 24 \mathrm{hrs} \text { and had acute episode of } \\
\text { pneumonia determined by CXR or AECOPD with sputum } \\
\text { expectoration of } 30 \mathrm{ml} \text { or more in } 24 \mathrm{hrs} \text {. }\end{array}$ & NR \\
\hline Brusse-Keizer, $2014^{5}$ & $\begin{array}{l}\text { COPD according to the GOLD criteria, current or ex- } \\
\text { smoker, age } 40-80 \text { years, presenting at the outpatient } \\
\text { clinic with an AECOPD, ability to produce a spontaneous } \\
\text { sputum sample, presenting with one or two of the clinical } \\
\text { characteristics: a positive Gram's stain of sputum, a } \\
\text { clinically relevant lung function decrease (decrease in } \\
\text { FEV1 of }>200 \mathrm{~mL} \text { and }>12 \% \text { from baseline), or }>2 \\
\text { AECOPDs in the previous year. }\end{array}$ & $\begin{array}{l}\text { Requirement of hospitalization, pneumonia based on chest X-ray, } \\
\text { AECOPD or use of antibiotics or prednisolone } 4 \text { weeks prior to } \\
\text { enrolment, except for low-dose prednisolone }(\leq 5 \mathrm{mg}) \text { as } \\
\text { maintenance therapy, disease that influences bronchial symptoms } \\
\text { or lung function, maintenance therapy with antibiotics, medical } \\
\text { condition with low survival or serious psychiatric morbidity, known } \\
\text { hypersensitivity to amoxicillin/ clavulanic acid, medical condition } \\
\text { with low survival or serious psychiatric morbidity, uncontrolled DM, } \\
\text { need for domiciliary oxygen therapy, participation in another } \\
\text { clinical trial. }\end{array}$ \\
\hline
\end{tabular}




\begin{tabular}{|c|c|c|}
\hline Author, Year & Inclusion Criteria & Exclusion Criteria \\
\hline Centanni, $2002^{25}$ & $\begin{array}{l}50 \text { consecutive outpatients' patients suffering from } \\
\text { AECOPD and with a history of IHD or arrhythmias } \\
\text { supported by EKG and/or Holter EKG, or } \\
\text { echocardiogram. }>40 \text { years of age, current or previous } \\
\text { smokers ( } 410 \text { pack-years) reporting chronic cough with } \\
\text { sputum production on most days during at least } 3 \\
\text { consecutive months in consecutive years, had no } \\
\text { change in symptom severity or treatment in the } \\
\text { preceding } 4 \text { weeks, had shown no signs of a respiratory } \\
\text { tract infection in the month preceding or during the trial, } \\
\text { were not taking oral or inhaled corticosteroids for at least } \\
3 \text { months and had a FEV1 < } 65 \% \text { and FVC }<70 \% \text { of } \\
\text { predicted normal after bronchodilators had been withheld } \\
\text { for } 24 \mathrm{~h} \text { and a best post-bronchodilator FEV1/FVC of } \\
\text { less than } 0.7 \text {. }\end{array}$ & $\begin{array}{l}\text { History of asthma, allergic rhinitis, atopy, skin-test positivity or with } \\
\text { a total blood eosinophil count over } 400 \mathrm{~mm}-3 \text {, patients with co- } \\
\text { morbidities (such as CHF or PE) or complications of COPD (e.g. } \\
\text { pneumothorax) as the etiology of exacerbation of their symptoms. }\end{array}$ \\
\hline Cox, $2018^{26}$ & Aged $\geq 35$ years who were admitted with AECOPD. & $\begin{array}{l}\text { Acute myocardial infarction/HF within the last } 6 \text { weeks, } \\
\text { Suspected/confirmed PE within the last } 6 \text { weeks, known } \\
\text { abdominal aortic aneurysm of }>5.5 \mathrm{~cm} \text { (or }>4.5 \mathrm{~cm} \text { if the } \\
\text { ultrasound scan is }>3 \text { months old), Known cardiovascular } \\
\text { instability: heart rate of }>120 \text { beats per minute and/or systolic } \\
\text { blood pressure of }<100 \mathrm{mmHg} \text { at the time of screening or the } \\
\text { requirement for inotropic support or patients with an implantable } \\
\text { cardioverter defibrillator, known extensive pulmonary fibrosis, } \\
\text { Absolute contraindications to exercise or musculoskeletal } \\
\text { conditions limiting exercise capacity as assessed by a trained } \\
\text { physiotherapist, Unable to give full informed consent, Non-English } \\
\text { speaker (to allow fully informed consent and the completion of } \\
\text { questionnaires). }\end{array}$ \\
\hline Cross, $2012^{27}$ & Admitted to hospital with an exacerbation of COPD. & $\begin{array}{l}\text { Any contraindication to the use of : Manual chest physiotherapy } \\
\text { techniques or with no evidence of excess sputum production on } \\
\text { auscultation., Osteoporosis, hemoptysis, bronchial hyper- } \\
\text { reactivity, respiratory system malignancy, raised intracranial } \\
\text { pressure, uncontrolled hypertension, PE, coagulopathy, } \\
\text { bronchopleural fistula, subcutaneous emphysema and left } \\
\text { ventricular failure as primary diagnosis. }\end{array}$ \\
\hline Daniels, $2010^{6}$ & $\begin{array}{l}45 \text { years of age or older; GOLD stage I-IV COPD, an } \\
\text { acute (onset }<14 \mathrm{~d} \text { ) exacerbation (type } 1 \text { [increased } \\
\text { dyspnea, sputum volume, and sputum purulence] or type } \\
2 \text { [two of three symptoms]) that required hospitalization). }\end{array}$ & $\begin{array}{l}\text { Fever }\left(>38.5^{\circ} \mathrm{C}\right) \text { with pneumonia, antibiotic treatment for at least } \\
24 \text { hours, and radiographic signs of pneumonia, CHF (NYHA III- } \\
\text { IV), apparent immunodeficiency, impaired renal function. }\end{array}$ \\
\hline
\end{tabular}




\begin{tabular}{|c|c|c|}
\hline Author, Year & Inclusion Criteria & Exclusion Criteria \\
\hline Dark, $1993^{72}$ & $\begin{array}{l}16 \text { or older, diagnosis of acute bacterial exacerbation of } \\
\text { COPD, including acute exacerbations of concurrent } \\
\text { COPD, chronic bronchitis, emphysema, asthma, } \\
\text { bronchiectasis, or asthmatic bronchitis. }\end{array}$ & $\begin{array}{l}\text { Treated with another investigational drug within } 4 \text { weeks of study } \\
\text { entry, taken other antibiotics }<72 \text { hours before study entry, or if } \\
\text { the use of additional antibiotics was anticipated at the time of entry } \\
\text { to the study., sensitive to macrolide or cephalosporin antibiotics or } \\
\text { if they had evidence or a history of significant hematologic, renal, } \\
\text { hepatic, or cardiac disease or any other underlying condition that } \\
\text { may have affected drug absorption, cystic fibrosis, as well as } \\
\text { those with COPD, chronic bronchitis, or bronchiectasis in the } \\
\text { absence of acute infection, drug or alcohol dependence. }\end{array}$ \\
\hline Davies, $1999^{7}$ & $\begin{array}{l}\text { History of increased breathlessness and at least two of } \\
\text { the following symptoms for } 24 \mathrm{~h} \text { or more: increased } \\
\text { cough frequency or severity, increased sputum volume } \\
\text { or purulence, and increased } \\
\text { wheeze, aged } 40-80 \text { years, history of at least } 20 \text { pack- } \\
\text { years of cigarette smoking, and physiological evidence } \\
\text { of airflow limitation with initial FEV1< } 70 \% \text { predicted and } \\
\text { FEV1/FVC ratio }<75 \% \text {. }\end{array}$ & $\begin{array}{l}\text { Personal or family history of asthma or atopy, uncontrolled left- } \\
\text { ventricular failure, clinical or radiological evidence of pneumonia, } \\
\text { received oral corticosteroids within } 1 \text { month of presentation, or if } \\
\text { arterial blood pH on admission was less than } 7 \cdot 26 \text {. }\end{array}$ \\
\hline De Jong, $2007^{98}$ & $\begin{array}{l}\text { Age of }>40 \text { years, a history of at least } 10 \text { pack-years of } \\
\text { cigarette smoking, and evidence of airflow limitation. } \\
\text { Airflow limitation was defined as an FEV1/FVC ratio of }< \\
70 \% \text { and an FEV1 of }<80 \% \text { predicted (GOLD severity } \\
\text { stage II). }\end{array}$ & $\begin{array}{l}\text { Excluded were patients who had signs of a very severe } \\
\text { exacerbation on hospital admission (arterial pH }<7.26 \text { or Paco2 }> \\
9.3 \mathrm{kPa} \text { ), with significant or unstable comorbidity, who had a } \\
\text { history of asthma, had participated in another study within the } 4 \\
\text { weeks before hospital admission, were previously randomized into } \\
\text { this study, had clinically significant findings on chest radiography } \\
\text { other than fitting with signs of COPD, } \\
\text { a known hypersensitivity to prednisolone, or who were known to } \\
\text { be totally noncompliant. }\end{array}$ \\
\hline
\end{tabular}




\begin{tabular}{|c|c|c|}
\hline Author, Year & Inclusion Criteria & Exclusion Criteria \\
\hline Ding, $2016^{73}$ & $\begin{array}{l}60 \text { - } 80 \text { years old, meet the diagnostic criteria of COPD: } \\
\text { a. based on the comprehensive consideration of the } \\
\text { clinical presentations, exposure to risk factors, signs, } \\
\text { and other laboratory results ;b. show the major COPD } \\
\text { symptoms (chronic cough, expectoration, and/or } \\
\text { dyspnea) and the exposure to risk factors; c. to exhibit } \\
\text { incomplete reversible airflow ( FEV1/FVC is <70\% after } \\
\text { application of a bronchodilator), the patients will be } \\
\text { diagnosed with incomplete reversible airflow limitation; } \\
\text { and met the AECOPD diagnostic criteria: a) to possess a } \\
\text { history of COPD (the patient has been clearly diagnosed } \\
\text { with COPD; with typical clinical COPD presentations, } \\
\text { and the pulmonary functions during the hospitalization } \\
\text { confirmed COPD); b) to display unusual continuous } \\
\text { exacerbation that required a change in the routine } \\
\text { medication; c) to show cough, expectoration, dyspnea, } \\
\text { and/or wheeze exacerbated, and to have an increased } \\
\text { amount of expectoration, or to have a short-term change } \\
\text { in the sputum, which can be accompanied with evident } \\
\text { aggravation of inflammatory (infection) symptoms (such } \\
\text { as fever). }\end{array}$ & $\begin{array}{l}\text { 1) to possess serious disease that required invasive mechanical } \\
\text { ventilation (patients with excessive respiratory secretion that are } \\
\text { not able to use non-invasive mechanical ventilation, or with the } \\
\text { PaCO2 }>70 \mathrm{mmHg} \text { ) (the PaCO2 level was accessed according to } \\
\text { the safety profiles of the study); } 2 \text { ) history of acute exacerbation or } \\
\text { received systemic application of corticosteroids within the past } \\
\text { month 3)diabetes cardiac, cerebral, renal, or liver diseases that } \\
\text { required hospitalization; 4)history with pneumothorax, pulmonary } \\
\text { embolism, pneumonia, or other respiratory diseases; and 5) } \\
\text { included in this study before. }\end{array}$ \\
\hline $\mathrm{Du}, 2018^{28}$ & $\begin{array}{l}\text { A clinical diagnosis of COPD according to GOLD (Global } \\
\text { Initiative for Chronic Obstructive Lung Disease) - clinical } \\
\text { examination and FEV1/FVC }<0.70 \text {, as measured on first } \\
\text { admission. }\end{array}$ & $\begin{array}{l}\text { Presence of pulmonary diseases (except COPD for the patients), } \\
\text { infective and inflammatory diseases, neoplastic pathologies, renal, } \\
\text { gastrointestinal, endocrine and hepatic diseases, and excessive } \\
\text { alcohol consumption ( } \geq 40 \mathrm{~g} / \text { day). }\end{array}$ \\
\hline Duffy, $2005^{29}$ & $\begin{array}{l}\text { Clinical diagnosis of COPD presenting to the emergency } \\
\text { department of University Hospital Aintree were } \\
\text { considered eligible if they complained of increased } \\
\text { breathlessness and two or more of the following } \\
\text { symptoms for at least } 24 \text { hours: increased cough } \\
\text { frequency or severity, increased sputum volume or } \\
\text { purulence, increased wheeze. Patients were aged } 40-80 \\
\text { years with a smoking history of at least } 20 \text { pack years, } \\
\text { an initial FEV1 of } 70 \% \text { predicted, and FEV1/FVC ratio of } \\
70 \% \text { predicted for age. }\end{array}$ & $\begin{array}{l}\text { Clinical history of asthma or atopy, uncontrolled cardiac disease, } \\
\text { advanced malignancy, clinical or radiological evidence of } \\
\text { pneumonia, pneumothorax, or chest wall deformity. In addition, } \\
\text { those with an arterial blood pH below } 7.32 \text {. }\end{array}$ \\
\hline Eaton, $2009^{30}$ & $\begin{array}{l}\text { COPD by ATS/ERS criteria and exertional dyspnea } \\
\text { interfering with daily activity. }\end{array}$ & $\begin{array}{l}\text { Major cognitive dysfunction, comorbidities precluding ability to } \\
\text { participate. }\end{array}$ \\
\hline Edwards, $2013^{31}$ & $\begin{array}{l}\text { Age } \geq 35 \text { years, COPD, FEV } 1 / F V C \text { ratio }<70 \% \text { and an } \\
\text { FEV } 1 \leq 50 \% \text { predicted } 20 \text { min after initial treatment with } \\
2.5 \text { mg salbutamol and } 500 \mathrm{mg} \text { ipratropium bromide by } \\
\text { nebulization. }\end{array}$ & $\begin{array}{l}\text { They required intubation or NIV, were unable to perform } \\
\text { spirometry or had evidence of pneumothorax, hypotension, and } \\
\text { any other serious medical condition that would prevent their } \\
\text { participation in the trial or were pregnant. }\end{array}$ \\
\hline
\end{tabular}




\begin{tabular}{|c|c|c|}
\hline Author, Year & Inclusion Criteria & Exclusion Criteria \\
\hline Emerman, $1989^{8}$ & $\begin{array}{l}50 \text { years or older with a clinical history of emphysema or } \\
\text { chronic bronchitis presenting to ED with acute } \\
\text { respiratory distress and had initial spirometry with FEV1 } \\
<70 \% \text { predicted or FEV/FVC } \%<60 \% \text {. }\end{array}$ & $\begin{array}{l}\text { History of asthma, episodes of respiratory distress before } 35 \text { years } \\
\text { age, received oral or IV steroids within one month of presentation, } \\
\text { pneumonia, acute CHF, or other conditions mandating admission } \\
\text { to the hospital. }\end{array}$ \\
\hline Giusti, $2016^{75}$ & $\begin{array}{l}\text { Purulent sputum, plus at least two of the following } \\
\text { signs/symptoms of at least 3-day duration: increased } \\
\text { cough, increased dyspnea, and increase in sputum } \\
\text { volume, previous antibiotic treatment with any drug with } \\
\text { the exclusion of fluoroquinolones, conducted for at least } \\
3 \text { full days with persistence or worsening of symptoms } \\
\text { and consequent hospitalization, age } \geq 60 \text { years, FEV1 } \leq \\
80 \% \text { and } \geq 30 \% \text { and ratio FEV1/FVC } \leq 0.7 \text {. }\end{array}$ & $\begin{array}{l}\text { Diagnosis of asthma, pulmonary neoplasm, sepsis, tuberculosis, } \\
\text { and cystic fibrosis; renal insufficiency or hepatic dysfunction; } \\
\text { history of epilepsy, seizures, stroke (in the previous } 6 \text { months), or } \\
\text { tendinopathy; known deficiencies for the glucose-6-phosphate } \\
\text { dehydrogenase activity; drug addiction or alcohol abuse; and } \\
\text { hypersensitivity or allergy to fluoroquinolones. }\end{array}$ \\
\hline Greening, $2014^{33}$ & $\begin{array}{l}\text { Diagnosis of chronic respiratory disease (chronic } \\
\text { obstructive pulmonary disease, chronic asthma, } \\
\text { bronchiectasis, or interstitial lung disease), self reported } \\
\text { breathlessness on exertion when stable (Medical } \\
\text { Research Council dyspnea grade } 3 \text { or worse), and } \\
\text { age } 40 \text { years or greater. }\end{array}$ & $\begin{array}{l}\text { Inability to provide informed consent; concomitant acute cardiac } \\
\text { event; presence of musculoskeletal, neurological, or psychiatric } \\
\text { comorbidities that would prevent the delivery of the rehabilitation } \\
\text { intervention; and more than four emergency admissions to } \\
\text { hospital for any cause in the previous } 12 \text { months. }\end{array}$ \\
\hline Greulich, $2014^{34}$ & $\begin{array}{l}\text { COPD patients hospitalized due to severe COPD } \\
\text { exacerbations. }\end{array}$ & NR \\
\hline Gunen, $2007^{9}$ & Only patients with level II exacerbation. & $\begin{array}{l}\text { COPD hospitalized for specific reasons, e.g. pneumonia, PE, CHF } \\
\text { and pneumothorax etc., as the cause of exacerbation. With a risk } \\
\text { of imminent RF requiring mechanical ventilation or direct } \\
\text { admission to the ICU (level III exacerbation).Patients who had } \\
\text { utilized systemic corticosteroids or had an exacerbation in the } \\
\text { preceding month. }\end{array}$ \\
\hline Hamacher, $1995^{76}$ & $\begin{array}{l}18 \text { years or older with an acute bacterial exacerbation of } \\
\text { chronic bronchitis requiring a parenteral antibiotic. }\end{array}$ & $\begin{array}{l}\text { Pregnant or breastfeeding women; patients with hyper-sensitivity } \\
\text { to any beta-lactam antibiotic; patients who had received other } \\
\text { investigational agents within the previous } 28 \text { days or had entered } \\
\text { the study previously; cystic fibrosis; a history of seizures; severe } \\
\text { hepatic failure ; neutropenia (neutrophil count }<1 \times 109 / \mathrm{L} \text { ) } \\
\text { severe underlying disease such that the patient was unlikely to } \\
\text { complete at least } 48 \mathrm{~h} \text { of study drug therapy; previous antibiotics } \\
\text { in the two days before randomized treatment unless the organism } \\
\text { had been shown to be either resistant or still present. }\end{array}$ \\
\hline Hasani, $1998^{77}$ & 12 patients with COPD during exacerbation. & NR. \\
\hline Hassan, $2015^{10}$ & $\begin{array}{l}\text { Type } 1 \text { exacerbation of COPD (defined as an increase in } \\
\text { dyspnea, sputum purulence and increased sputum } \\
\text { volume). }\end{array}$ & $\begin{array}{l}\text { Received antibiotic treatment during the last } 2 \text { weeks or had } \\
\text { another disease as left ventricular failure, stroke, pneumonia, } \\
\text { pneumothorax, cancer, coma, allergy to quinolone derivatives, or } \\
\text { concomitant infection requiring systemic antibacterial therapy. }\end{array}$ \\
\hline
\end{tabular}




\begin{tabular}{|c|c|c|}
\hline Author, Year & Inclusion Criteria & Exclusion Criteria \\
\hline $\mathrm{He}, 2015^{35}$ & $\begin{array}{l}\text { Reported a limitation in daily activities due to dyspnea on } \\
\text { exertion, as categorized by mMRC dyspnea grade }>0 \text {. }\end{array}$ & $\begin{array}{l}\text { Any disease not associated with COPD (e.g., uncontrolled HF, } \\
\text { sever lower limb arthritis, and symptomatic peripheral vascular } \\
\text { disease, which may affect the outcome of dyspnea or exercise } \\
\text { tolerance), severe orthopedic or neurological disorders limiting } \\
\text { exercise performance, unstable cardiac disease. }\end{array}$ \\
\hline Kirsten, $1998^{37}$ & $\begin{array}{l}\text { Patients with severe COPD within a referral hospital after } \\
\text { an acute exacerbation of their disease. }\end{array}$ & NR. \\
\hline Kodric, $2009^{36}$ & COPD patients, hospitalized with an acute exacerbation. & $\begin{array}{l}\text { Positive bronchodilator reversibility test or any other chest disease } \\
\text { likely to bias results. }\end{array}$ \\
\hline Koutsogiannis, $2000^{65}$ & Chronic obstructive airways disease. & $\begin{array}{l}\text { Inability to perform spirometry, requirement for salbutamol or } \\
\text { adrenaline infusion, requirement for CPAP or intubation, the } \\
\text { presence of a pneumothorax, pneumonia or cardiac failure on } \\
\text { CXR and asthma. }\end{array}$ \\
\hline Kurzaj, $2013^{38}$ & $\begin{array}{l}\text { COPD and treated of worsening of the symptoms of their } \\
\text { disease. }\end{array}$ & NR. \\
\hline Lellouche, $2016^{39}$ & $\begin{array}{l}\text { Hospitalized for an acute exacerbation of COPD in } \\
\text { whom oxygen therapy was prescribed, patients aged } \geq \\
40 \text { years with a past or current smoking history of at } \\
\text { least } 10 \text { pack-years. Maintain an } \mathrm{SpO} 2 \text { of } \geq 92 \% \text { with } \\
\text { supplemental oxygen at a maximum flow rate of } 8 \mathrm{~L} / \mathrm{min} \text {. }\end{array}$ & $\begin{array}{l}\text { Admitted for } 24 \text { hours, infected with multidrug-resistant bacteria, } \\
\text { on intermittent NIV (including CPAP for obstructive sleep apnea), } \\
\text { cognitive impairment. }\end{array}$ \\
\hline Leophonte, $1998^{78}$ & $\begin{array}{l}40 \text { years or older, chronic obstructive bronchitis, } \\
\text { FEV1/FVC <70\%, sputum production during } 3 \\
\text { consecutive months for } 2 \text { or more years, acute } \\
\text { exacerbation (dyspnea or increased sputum } \\
\text { volume/purulence [gram stain sputum with }>25 \text { PMNs]). }\end{array}$ & $\begin{array}{l}\text { Hypersensitivity or intolerance to quinolone or beta lactams, } \\
\text { hospitalized, any antibiotic use longer than } 24 \text { hours in the prior } 72 \\
\text { hours, AECOPD severe enough to warrant IV antibiotics, cystic } \\
\text { fibrosis, radiograph evidence of pneumonia, significant } \\
\text { gastrointestinal condition to preclude oral absorption of pills, AIDS, } \\
\text { epilepsy, seizures, recent drug or alcohol abuse or dependence or } \\
\text { chronic prednisolone }>=10 \text { mg daily use. }\end{array}$ \\
\hline Leuppi, $2013^{79}$ & $\begin{array}{l}\text { AECOPD ( } 2 \text { of the following: change in baseline } \\
\text { dyspnea, cough, or sputum quantity or purulence); } \geq 40 \\
\text { years old, smoking } \geq 20 \text { pack years, FEV1/FVC } \leq 70 \% \text {. }\end{array}$ & $\begin{array}{l}\text { Asthma, FEV1/FVC }>70 \% \text {, radiological diagnosis of pneumonia, } \\
\text { estimated survival }<6 \text { months due to severe comorbidity, } \\
\text { pregnancy or lactation, inability to give written informed consent. }\end{array}$ \\
\hline Liao, $2015^{40}$ & $\begin{array}{l}\text { Moderate exacerbation COPD, older than } 65 \text { years, clear } \\
\text { consciousness, shortness of breath or dyspnea that was } \\
\text { not caused by heart disease, pneumothorax, or } \\
\text { pulmonary edema, received bronchodilator aerosol } \\
\text { therapy or antibiotic treatment, had not been treated with } \\
\text { an antitussive. }\end{array}$ & $\begin{array}{l}\text { Systolic blood pressure lower than } 90 \mathrm{mmHg} \text {, blood oxygen } \\
\text { concentration lower than } \mathrm{SpO} 2=90 \% \text {, unstable psychological } \\
\text { status, hemoptysis, pneumothorax, pulmonary edema. }\end{array}$ \\
\hline
\end{tabular}




\begin{tabular}{|c|c|c|}
\hline Author, Year & Inclusion Criteria & Exclusion Criteria \\
\hline Llor, $2009^{80}$ & $\begin{array}{l}\text { Spirometrically-diagnosed patients older than } 40 \text { years } \\
\text { with COPD, without criteria of hospitalization and } \\
\text { Anthonisen's types I or II exacerbations. }\end{array}$ & $\begin{array}{l}\text { Current chronic treatment with systemic steroids at any dose, } \\
\text { severe respiratory impairment requiring hospital referral, evidence } \\
\text { of a new pulmonary infiltrate on chest radiography, suspected or } \\
\text { known history of hypersensitivity to } \beta \text {-lactam antibiotics, } \\
\text { administration of antibiotics within the previous four weeks, } \\
\text { documented evidence of bronchiectasis, AIDS, another } \\
\text { immunosuppressive condition or patients receiving treatment with } \\
\text { immunosuppressive drugs, cystic fibrosis, or patients participating } \\
\text { in another clinical trial within the last year. }\end{array}$ \\
\hline Llor, $2012^{11}$ & $\begin{array}{l}\geq 40 \text { years old, mild to moderate COPD (smoking history } \\
\geq 10 \text { pack years, FEV1/FVC }<70 \% \text {, post bronchodilator } \\
\text { FEV1 }>50 \% \text { predicted) and presence of exacerbation } \\
\text { (increase of dyspnea, sputum volume or sputum } \\
\text { purulence). }\end{array}$ & $\begin{array}{l}\text { Antibiotic use in prior } 2 \text { weeks, bronchial asthma, cystic fibrosis, } \\
\text { bronchiectasis other than COPD, active neoplasm, tracheotomy, } \\
\text { need for hospital admission, immunosuppression, hypersensitivity } \\
\text { to beta lactams, clavulanate or lactose, institutionalization, or } \\
\text { inability to provide informed consent. }\end{array}$ \\
\hline $\begin{array}{l}\text { Mahmoud Abd El } \\
\text { Hafiz, 2013 }\end{array}$ & $\begin{array}{l}\text { COPD with a history of } \geq 2 \text { exacerbations/year in } 2 \text { years } \\
\text { prior to enrollment. An age between } 40 \text { and } 70 \text { years; a } \\
\text { post bronchodilator FEV1/FVC }<70 \% \text { of predicted; an } \\
\text { FEV1 reversibility }<12 \% \text { of the predicted value, } 15 \text { min } \\
\text { after } 400 \text { mcg ( } 4 \text { puffs) of Salbutamol; and to be } \\
\text { currently experiencing an AECOPD. }\end{array}$ & $\begin{array}{l}\text { Allergy or intolerance to NAC, cystic fibrosis, bronchiectasis, } \\
\text { history of infection or active infection by TB, history of active } \\
\text { peptic ulcer. }\end{array}$ \\
\hline Maltais, $2002^{12}$ & $\begin{array}{l}>50 \text { ye old, had a smoking history of at least } 20 \text { pack- } \\
\text { years, according to the attending physician had to be } \\
\text { treated in hospital. }\end{array}$ & $\begin{array}{l}\text { If they had a personal history of asthma, allergic rhinitis, or atopy; } \\
\text { were exposed to systemic corticosteroids, in the preceding month; } \\
\text { used more than } 1,500 \mathrm{~g} / \mathrm{d} \text { of inhaled beclomethasone equivalent; } \\
\text { were at risk of imminent acute respiratory failure requiring } \\
\text { mechanical ventilation or admission to the intensive care unit (pH } \\
7.30 \text { and/or PaCO2 } 70 \mathrm{~mm} \mathrm{Hg} \text {, and/or } \mathrm{PaO} 250 \mathrm{~mm} \mathrm{Hg} \text { despite } \\
\text { supplemental oxygen); or if a specific cause for the exacerbation, } \\
\text { such as pneumonia, pneumothorax, or HF. }\end{array}$ \\
\hline Mirici, $2003^{81}$ & If they had moderate to severe acute attacks of COPD. & $\begin{array}{l}\text { Exposure to systemic corticosteroids in the preceding month; } \\
\text { presence of asthma, allergic rhinitis, atopy or any systemic } \\
\text { disease (such as DM or hypertension); and being at risk of acute } \\
\text { RF requiring mechanical ventilation. }\end{array}$ \\
\hline Moayyedi, $1995^{66}$ & $\begin{array}{l}\text { Emergencies to acute medical units with diagnosis of } \\
\text { acute exacerbation of COPD, not taking regular } \\
\text { nebulized bronchodilators at home. All over } 45 \text { years and } \\
\text { had smoking history more than } 10 \text { pack-years. All had a } \\
\text { FEV1 < } 65 \% \text { predicted when well and history of } \\
\text { exertional dyspnea from respiratory disease over three } \\
\text { years. }\end{array}$ & $\begin{array}{l}\text { History suggestive of asthma (childhood respiratory disease, } \\
\text { atopy, night time wheezing) and a peripheral eosinophilia of }> \\
10 \% \text {. Patients with a }>20 \% \text { (at least } 200 \mathrm{ml} \text { ) reversibility of FEV, } \\
\text { to } 400 \text { mcg of inhaled salbutamol on the day of discharge. }\end{array}$ \\
\hline
\end{tabular}




\begin{tabular}{|c|c|c|}
\hline Author, Year & Inclusion Criteria & Exclusion Criteria \\
\hline Moretti, $2015^{42}$ & $\begin{array}{l}\text { Aged between } 38 \text { and } 75 \text { years admitted with an acute } \\
\text { exacerbation of COPD, fever, cough, and purulent } \\
\text { sputum in the previous } 24 \text { hours. }\end{array}$ & $\begin{array}{l}\text { Pneumonia, acute heart failure, bronchiectasis, asthma (as the } \\
\text { primary diagnosis), acute respiratory acidosis needing } \\
\text { noninvasive ventilation, AECOPD treated with antibiotics or } \\
\text { systemic corticosteroids in the previous } 4 \text { weeks, or any other } \\
\text { medical or personal problems that in the opinion of the } \\
\text { investigator would interfere with the conduct of the study. }\end{array}$ \\
\hline Mukerji, $2015^{43}$ & $\begin{array}{l}\text { Above age of } 35 \text { years, who had a previously } \\
\text { documented diagnosis of COPD by either their general } \\
\text { practitioner or in-hospital respiratory specialists. Non- } \\
\text { infective and infective cause of AECOPD. }\end{array}$ & $\begin{array}{l}\text { Patients requiring mechanical ventilation or NIV at presentation, } \\
\text { anyone who was unable to do spirometry or had evidence of } \\
\text { pneumothorax or hypertension or any other serious medical } \\
\text { condition that would prevent their participation. Responders or } \\
\text { 'asthma-type' COPD patients, history of asthma. }\end{array}$ \\
\hline Niewoehner, $1999^{13}$ & $\begin{array}{l}50 \text { or older, history } 30 \text { pack-years or more smoking, } \\
\text { either FEV1 } 1.50 \text { L or less or inability to undergo } \\
\text { spirometry due to dyspnea. }\end{array}$ & $\begin{array}{l}\text { Diagnosis of asthma, use of systemic glucocorticoids within the } \\
\text { preceding } 30 \text { days, coexisting medical conditions that made } \\
\text { survival for at least } 1 \text { year unlikely. }\end{array}$ \\
\hline Ogasawara, $2018^{44}$ & $\begin{array}{l}\text { Clinically diagnosed as COPD according to the GOLD } \\
\text { criteria and hospitalized for exacerbation of COPD or } \\
\text { pneumonia, planned to receive pulmonary rehabilitation } \\
\text { during the hospitalization, and able to eat and drink } \\
\text { safely without dysphagia. }\end{array}$ & $\begin{array}{l}\text { Patients who had a history of severe drug allergy, patients who } \\
\text { took oral nutritional supplements during the trial, patients regarded } \\
\text { as inadequate to receive additional nutrition therapy because of } \\
\text { uncontrolled diabetes and/or dyslipidemia, and patients who } \\
\text { refused pulmonary rehabilitation. }\end{array}$ \\
\hline Oncu, $2017^{45}$ & $\begin{array}{l}\text { Diagnosed with AECOPD by independent thoracic } \\
\text { physicians. }\end{array}$ & $\begin{array}{l}\text { Other pulmonary disease, who were not eligible as a result of } \\
\text { cardiology consultation, another chronic disease/condition drug } \\
\text { addicts, applied acupuncture/TENS previously, smoked during the } \\
\text { last four hours prior to the pulmonary function test used long-term } \\
\text { bronchodilator in the last } 12 \text { hours, and conditions that prevented } \\
\text { six-minute walk test. }\end{array}$ \\
\hline Osadnik, $2014^{46}$ & $\begin{array}{l}\text { Hospitalized due to an AECOPD were screened by study } \\
\text { personnel, and those with evidence of sputum } \\
\text { expectoration or a history of chronic sputum Production } \\
\text { ('regularly expectorated sputum on most days') were } \\
\text { recruited from respiratory units within } 48 \mathrm{~h} \text { of admission. }\end{array}$ & $\begin{array}{l}\text { A respiratory condition deemed more significant than COPD (e.g., } \\
\text { clinical history of primary bronchiectasis, asthma or lung cancer } \\
\text { requiring active therapy) even if coexistent with COPD, if they had } \\
\text { established airway clearance routines, were breathing via an } \\
\text { artificial airway or PEP therapy was contraindicated (undrained } \\
\text { pneumothorax; significant haemoptysis; recent facial, oral, } \\
\text { esophageal or skull surgery/trauma; surgical or nonsurgical lung } \\
\text { volume reduction procedures, lung transplantation or } \\
\text { pneumonectomy within the last } 6 \text { months). }\end{array}$ \\
\hline Perri, $1985^{67}$ & $\begin{array}{l}\text { Reversible chronic obstructive lung disease with at least } \\
\text { a } 15 \% \text { greater FEV1 in relation to their CECA predicted } \\
\text { values after } 200 \mathrm{mcg}(2 \text { puffs) of salbutamol } \\
\text { administered by aerosol. }\end{array}$ & NR. \\
\hline
\end{tabular}




\begin{tabular}{|c|c|c|}
\hline Author, Year & Inclusion Criteria & Exclusion Criteria \\
\hline Petitpretz, $2007^{82}$ & $\begin{array}{l}\text { Age } \geq 45 \text { years, candidate for outpatient management } \\
\text { and diagnosis of AECOPD defined as: current or former } \\
\text { smoking activity; history of chronic bronchitis } \\
\text { characterized by cough and sputum production on most } \\
\text { days for } 3 \text { consecutive months and for }>2 \text { consecutive } \\
\text { years; chronic obstructive bronchitis confirmed by lung } \\
\text { function test performed in a stable condition in the } \\
\text { previous } 12 \text { months showing a FEV1/FVC ratio of }<70 \% \\
\text { and an FEV1 in the range of } 35-80 \% \text { of the predicted } \\
\text { value, and no significant reversibility following B2-agonist } \\
\text { therapy ( }<200 \text { mL and }<15 \% \text { increase in FEV1); at least } \\
\text { three exacerbations during the previous year; a current } \\
\text { acute exacerbation characterized by the presence of the } \\
\text { three Anthonisen's criteria of recent increase in sputum } \\
\text { volume, sputum purulence and dyspnea; and no } \\
\text { evidence of pneumonia on chest radiography performed } \\
\text { within } 48 \mathrm{~h} \text { prior to or no later than } 72 \text { h after initiating } \\
\text { the study drug. }\end{array}$ & $\begin{array}{l}\text { Significant reversibility following B2-agonist therapy or failure to } \\
\text { perform this test and FEV1/FVC } \geq 70 \% \text { or failure to measure lung } \\
\text { function. }\end{array}$ \\
\hline Phillips, $1993^{83}$ & $\begin{array}{l}18 \text { years or older, } \geq 40 \mathrm{~kg}, \text { AECOPD (cough, fever, or } \\
\text { increased sputum production/purulence), absence of } \\
\text { infiltrate on chest radiograph), hospitalized and } \\
\text { outpatients were included. }\end{array}$ & $\begin{array}{l}\text { Severe respiratory infection requiring IV antibiotics, pregnant, } \\
\text { breastfeeding, hypersensitivity to cephalosporins, anaphylaxis or } \\
\text { severe reaction to penicillins, antibiotics in prior } 5 \text { days, } \\
\text { neutropenic, creatinine }>2.5 \text {, hepatic dysfunction, immune or } \\
\text { neoplastic disease, severe vascular insufficiency, gastro-intestinal } \\
\text { disorder that would impair oral absorption, enrolled in another } \\
\text { investigational protocol, prior enrollment in a cefpodoxime } \\
\text { protocol. }\end{array}$ \\
\hline Pourrashid, $2018^{47}$ & $\begin{array}{l}\text { AECOPD in the emergency ward and Vitamin D } \\
\text { deficiency (serum } 25(\mathrm{OH}) \mathrm{D}<20 \mathrm{ng} / \mathrm{mL} \text { ). }\end{array}$ & $\begin{array}{l}\text { Serum } 25(\mathrm{OH}) \mathrm{D}<5 \mathrm{ng} / \mathrm{mL} \text {, vitamin } \mathrm{D} \text { supplementation within six } \\
\text { month prior to study, use of maintenance dose of oral } \\
\text { corticosteroids within three months prior to study, diagnosed } \\
\text { asthma, osteoporosis, renal failure (serum creatinine } \geq 2.5 \\
\mathrm{mg} / \mathrm{dL}), \text { nephrolithiasis, uncompensated liver failure (Child-pugh } \\
\text { class } \mathrm{B}, \mathrm{C}) \text {, hypercalcemia (ionized calcium }>1.3 \mathrm{mmol} / \mathrm{L} \text { ), } \\
\text { conditions associated with pathological } 1 \text {-alpha hydroxylase } \\
\text { activity such as sarcoidosis, lymphoma or multiple myeloma, } \\
\text { coagulopathy (platelet count }<30,000 \text { per } \mathrm{mm}^{3} \text { or international } \\
\text { normalized ratio }>3 \text { ), contraindication for intramuscular } \\
\text { administration, pregnancy, lactation, and immuno-compromised } \\
\text { patients. }\end{array}$ \\
\hline
\end{tabular}




\begin{tabular}{|c|c|c|}
\hline Author, Year & Inclusion Criteria & Exclusion Criteria \\
\hline Rhee, $2015^{84}$ & $\begin{array}{l}40 \text { years old or older, moderate exacerbation of COPD; } \\
\text { post-bronchodilator FEV1/FVC }<0.7 ; \text { purulent sputum or } \\
\text { increased volume of sputum. }\end{array}$ & $\begin{array}{l}\text { Pregnant women, received systemic antibiotics and/or antifungal } \\
\text { agents within the last } 72 \text { hours, pneumonia, underlying septic } \\
\text { shock, bronchiectasis, lung abscess, active tuberculosis, } \\
\text { pulmonary malignancy, cystic fibrosis, empyema, or asthma; } \\
\text { kidney or liver disease with abnormal laboratory test results, } \\
\text { organic gastrointestinal disorder; absolute neutrophil count }<1,000 \\
\text { cells/ } \mathrm{mm}^{3} \text {; chronic hepatitis B or C; immunocompromised } \\
\text { patients, history of hypersensitivity to fluoroquinolone antibiotics; } \\
\text { history of seizure or anti-seizure medications; history of ventricular } \\
\text { arrhythmia; and history of corrected QT prolongation or treatment } \\
\text { with medication that prolongs the corrected QT interval. }\end{array}$ \\
\hline Rice, $1987^{48}$ & $\begin{array}{l}\text { Diagnosis of COPD consistent with FEV1 }>2 \text { SD below } \\
\text { predicted and FEV1/FVC ratio }<60 \% \text {. }\end{array}$ & $\begin{array}{l}\text { Clinical diagnosis of asthma, readily reversible episodes of } \\
\text { wheezing and dyspnea separated by asymptomatic intervals, or } \\
\text { bronchodilator response of a }>30 \% \text { increase in FEV1 from } \\
\text { previous spirometry. Left ventricular failure and pneumonia. }\end{array}$ \\
\hline Rizzato, $1998^{85}$ & $\begin{array}{l}\text { Admitted to hospital for AECOPD, max value of } \\
\text { FEV1<70\% predicted, marked respiratory distress. }\end{array}$ & $\begin{array}{l}\text { Drug sensitivity, pregnant or nursing, drug addicts, alcoholics, HIV } \\
\text { positive, cognitive impairment, clinically impaired diabetes, active } \\
\text { peptic ulcer, untreated active tuberculosis, systemic mycosis. }\end{array}$ \\
\hline Roede, $2007^{86}$ & $\begin{array}{l}\text { Aged } \geq 18 \text { years who fulfilled the clinical criteria for } \\
\text { COPD, and who presented with purulent sputum and a } \\
\text { type } 1 \text { exacerbation, increased dyspnea, increased } \\
\text { volume and purulence of sputum, or two of these criteria } \\
\text { in a patient with chronic HF and a chest radiograph that } \\
\text { showed no evidence of pneumonia. }\end{array}$ & $\begin{array}{l}\text { Patients with a history of allergy to amoxicillin-clavulanic acid, } \\
\text { neutropenia }(<1.0 \times 10 \% \mathrm{~L}) \text {, agammaglobulinemia, cystic fibrosis or } \\
\text { bronchiectasis, a life-expectancy of }<1 \text { month, previous treatment } \\
\text { with an effective antimicrobial agent for }>24 \text { h before admission, } \\
\text { or any other infection necessitating the administration of systemic } \\
\text { antibiotics. Patients admitted to an ICU and patients who required } \\
\text { ventilation. }\end{array}$ \\
\hline Ruiz Gonzalez, $2007^{87}$ & $\begin{array}{l}\text { Aged }>40 \text { years, with a diagnosis of COPD and were } \\
\text { admitted to the emergency room with a primary } \\
\text { diagnosis of an acute exacerbation. } \\
\text { Spirometry that met criteria for COPD defined as FEV1 } \\
\text { to FVC ratio } \leq 70 \% \text {, and FEV1 } \leq 80 \% \text { predicted, a severe } \\
\text { or Anthonisen's type } 1 \text { exacerbation, worsening of } \\
\text { dyspnea, increase in sputum volume and increase in } \\
\text { sputum purulence; and a CXR without any new } \\
\text { infiltrates. }\end{array}$ & $\begin{array}{l}\text { Previous adverse reaction to study drugs, pregnancy or lactation, } \\
\text { syndrome of QT prolongation, severe renal or hepatic impairment, } \\
\text { lung disease other than COPD. }\end{array}$ \\
\hline Sanjari, $2015^{49}$ & $\begin{array}{l}\text { Ex-smokers with a history of chronic cough and } \\
\text { expectoration who also had exertional dyspnea and } \\
\text { admitted with the diagnosis of COPD exacerbation. }\end{array}$ & $\begin{array}{l}\text { Unable to perform spirometry, A history of asthma symptoms, } \\
\text { existence of other respiratory disorders including bronchial } \\
\text { carcinoma, a history of hospitalization (within } 4 \text { weeks) for COPD, } \\
\text { any medical condition which needed more invasive respiratory } \\
\text { support, symptoms of lower respiratory tract infection or other } \\
\text { kinds of simultaneous systemic disease such as hypercalcemia, } \\
\text { renal failure, hyperparathyroidism, malignancy, history of renal } \\
\text { stone, cardiac arrhythmia, or patients who were using lithium. }\end{array}$ \\
\hline
\end{tabular}




\begin{tabular}{|c|c|c|}
\hline Author, Year & Inclusion Criteria & Exclusion Criteria \\
\hline $\begin{array}{l}\text { Saudny-Unterberger, } \\
1997^{50}\end{array}$ & $\begin{array}{l}\text { Diagnosis of COPD, and a FEV1 } \leq 60 \% \text { of the } \\
\text { predicted. }\end{array}$ & $\begin{array}{l}\text { Required mechanical ventilation, had a gastrointestinal tract } \\
\text { disorder, had active cancer or other conditions predisposing to } \\
\text { weight loss, terminally ill, unable to communicate in English or } \\
\text { French, suffered from mental confusion or followed a special diet. }\end{array}$ \\
\hline Sayiner, $2001^{88}$ & $\begin{array}{l}\text { COPD, all current or ex-smokers with a smoking history } \\
\geq 20 \text { pack-years and severe airway obstruction (FEV1 } \\
35 \% \text { predicted), who presented with an exacerbation } \\
\text { necessitating hospitalization. }\end{array}$ & $\begin{array}{l}\text { Personal or family history of asthma, atopy, allergic disease, } \\
\text { presence of eosinophilia, use of systemic steroids within the } \\
\text { preceding month, presence of severe hypertension, } \\
\text { uncompensated CHF or uncontrolled (or difficult to control) DM, } \\
\text { and RF necessitating mechanical ventilation therapy. }\end{array}$ \\
\hline Seidenfeld, $1984^{51}$ & Met ATS chronic bronchitis guidelines. & $\begin{array}{l}\text { Temp }>37.5^{\circ} \mathrm{C} \text {, obvious cardiac rhythm disturbance, acute } \\
\text { pneumonia, acute HF. }\end{array}$ \\
\hline Skorodin, $1995^{52}$ & $\begin{array}{l}35 \text { years or older who presented to ED with AECOPD, } \\
\text { provided they met the diagnostic criteria for chronic } \\
\text { bronchitis and/or emphysema as defined by the ATS. }\end{array}$ & $\begin{array}{l}\text { Temperature greater than } 37.9^{\circ} \mathrm{C} \text {, systolic blood pressure less } \\
\text { than } 100 \mathrm{~mm} \mathrm{Hg} \text {, history of kidney disease, and clinical evidence } \\
\text { of pneumonia. }\end{array}$ \\
\hline Solooki, $2014^{53}$ & $\begin{array}{l}40 \text { years or older, COPD exacerbation to emergency } \\
\text { department. }\end{array}$ & $\begin{array}{l}\text { Contraindication for use of IV magnesium sulfate, unable to } \\
\text { perform spirometry, presence of pneumonia, oral temperatures of } \\
38{ }^{\circ} \mathrm{C} \text { or more and systolic blood pressure less than } 100 \mathrm{mmHg} \text {. }\end{array}$ \\
\hline Soltaninejad, $2016^{54}$ & Older than 40 years and known case of COPD. & $\begin{array}{l}\text { History of } \mathrm{HF} \text { (ejection fraction }<40 \% \text { ), renal failure, ongoing } \\
\text { ischemia, hearing problems, lung cancer, lung abscess, } \\
\text { bronchiectasis, lung resection surgery, and hypersensitivity to } \\
\text { aminoglycosides. }\end{array}$ \\
\hline Stallberg, $2009^{89}$ & $\begin{array}{l}\geq 10 \text { pack year smoking history, age } \geq 40 \text { years old, } \\
\text { moderate COPD GOLD stage IIA or IIB, established } \\
\text { diagnosis of COPD for } \geq 6 \text { months at start of study, } \\
\text { exacerbation in the week prior (increased dyspnea, } \\
\text { sputum volume, or sputum purulence), primary care } \\
\text { physician thought that oral steroids were clinically } \\
\text { indicated. }\end{array}$ & $\begin{array}{l}\text { Mild exacerbation treated with antibiotics and/or increased } \\
\text { bronchodilators alone, hypoxemia, admitted to hospital, asthma, } \\
\text { FEV1/FVC }>70 \%, \text { AECOPD in prior } 30 \text { days, SaO2 }<92 \% \text { after } \\
\text { initial acute treatment, required oxygen, inhaled corticosteroid } \\
>1000 \text { mcg/day at study entry, use of non-selective beta } \\
\text { antagonist. }\end{array}$ \\
\hline Sun, $2015^{90}$ & $\begin{array}{l}\text { Patients with AECOPD meeting the GOLD diagnostic } \\
\text { criteria. }\end{array}$ & $\begin{array}{l}\text { A personal history of asthma, cystic fibrosis, diffuse pan } \\
\text { bronchiolitis (DPB), obliterative bronchiolitis, allergic rhinitis, or } \\
\text { atopy; complicated with acute RF, acidosis, cancer or serious } \\
\text { heart, liver, kidney, and gastrointestinal diseases; or were at risk } \\
\text { of admission to the ICU, exposed to corticosteroids in past } 4 \\
\text { weeks, more than } 50 \text { years. }\end{array}$ \\
\hline Tang, $2012^{55}$ & $\begin{array}{l}\text { AECOPD had to be admitted into the hospital, able to } \\
\text { walk independently before admission, have spontaneous } \\
\text { ventilation without any ventilator assistance, receive } \\
\text { medical clearance to participate, able to communicate in } \\
\text { English, and able to provide informed consent. }\end{array}$ & NR \\
\hline
\end{tabular}




\begin{tabular}{|c|c|c|}
\hline Author, Year & Inclusion Criteria & Exclusion Criteria \\
\hline Thompson, $1996^{14}$ & $\begin{array}{l}\text { History of cigarette smoking ( } \geq 20 \text { pack-year), airflow } \\
\text { obstruction, diagnosis or chronic bronchitis or } \\
\text { emphysema as defined by the ATS. }\end{array}$ & $\begin{array}{l}\text { Family or personal history of asthma; atopy, allergic rhinitis, or } \\
\text { nasal polyposis; or a history of pulmonary disease other than } \\
\text { COPD; positive skin test to common environmental allergens, } \\
\text { systemic contortionists used in last month, uncompensated CHF, } \\
\text { pneumonia, fever }>38.5^{\circ} \mathrm{C} \text {, acidemia (arterial pH< } 7.35 \text { ); hospital } \\
\text { admission for any reason; or inability to return for followup } \\
\text { appointments. }\end{array}$ \\
\hline $\begin{array}{l}\text { Torres-Sanchez, } \\
2017^{56}\end{array}$ & $\begin{array}{l}\text { AECOPD according to (ATS) criteria. Age } 65 \text { or older. } \\
\text { Patients with COPD who scored over } 3 \text { in the Brief } \\
\text { Frailty Index. }\end{array}$ & $\begin{array}{l}\text { Psychiatric or cognitive disorders, progressive neurological or } \\
\text { musculoskeletal disorders, sever orthopedic problems, organ } \\
\text { failure, cancer, or inability to cooperate. Experienced another } \\
\text { exacerbation of COPD in the previous month. }\end{array}$ \\
\hline $\begin{array}{l}\text { Torres-Sanchez, } \\
2017^{57}\end{array}$ & $\begin{array}{l}\text { COPD according to the criteria of GOLD, hospitalized } \\
\text { due to acute exacerbation of COPD. }\end{array}$ & $\begin{array}{l}\text { Severe comorbidities "unstable cardiovascular disease, } \\
\text { orthopedic diseases in the upper and lower limbs, motor sequelae } \\
\text { from neurological or visual disorders that interfere with the ability } \\
\text { to perform physical exercise, cognitive impairment that interfere } \\
\text { with the evaluation and the treatment". }\end{array}$ \\
\hline Troosters, $2010^{58}$ & Diagnosis of AECOPD, age <85 years. & $\begin{array}{l}\text { Hospitalization within the previous } 14 \text { days, current participation in } \\
\text { rehabilitation program, locomotor or neurological condition or } \\
\text { disability limiting the ability to perform exercise, lung } \\
\text { transplantation or lung volume reduction surgery foreseen within } 1 \\
\text { month after discharge. }\end{array}$ \\
\hline Goktalay, $2013^{32}$ & $\begin{array}{l}\text { Stage 3-4 COPD according to the GOLD criteria and } \\
\text { hospitalized for infective exacerbations of the COPD. }\end{array}$ & $\begin{array}{l}\text { Need for INV, bronchiectasis, active lung tuberculosis, chest wall } \\
\text { trauma, thoracic or abdominal operation in the last three months, } \\
\text { a diagnosis of PE in the last three months, myocardial infarction in } \\
\text { the last six months, and thrombocytopenia }\left(<50,000 / \mathrm{mm}^{3}\right) \text { plus } \\
\text { exacerbation secondary to cardiovascular causes and non- } \\
\text { infective exacerbations. }\end{array}$ \\
\hline Tumer, $2009^{59}$ & $\begin{array}{l}\text { Male patients hospitalized with acute exacerbation of } \\
\text { COPD. }\end{array}$ & NR \\
\hline Ucar, $2014^{91}$ & $\begin{array}{l}\text { Moderate or severe COPD exacerbation, } \geq 40 \text { years, } \geq \\
10 \text { pack-year smoking history, required hospitalization } \\
\text { for COPD exacerbation. }\end{array}$ & $\begin{array}{l}\text { Asthma, allergic rhinitis, atopy, any systemic disease (such as } \\
\text { diabetes or hypertension), prior steroid use in past month, used } \\
>1500 \mathrm{mcg} / \text { day of inhaled beclomethasone equivalent, admitted } \\
\text { to the } \mathrm{ICU}, \mathrm{pH}<7.30, \mathrm{PaCO} 2>70 \mathrm{mmHg}, \mathrm{PaO} 2<50 \mathrm{~mm} \text { Hespite } \\
\text { supplemental oxygen, or if he had a specific cause of the } \\
\text { exacerbation diagnosed (pneumonia, pneumothorax, HF, etc.) }\end{array}$ \\
\hline Umut,199992 & $\begin{array}{l}\text { COPD diagnosis was made according to the ATS } \\
\text { criteria. }\end{array}$ & $\begin{array}{l}\text { Pregnancy, known allergy to antibiotics, antibiotic therapy } 6 \text { weeks } \\
\text { prior to study, active tuberculosis, immunosuppression, liver and } \\
\text { kidney insufficiency, empyema, lung abscess, aspiration } \\
\text { pneumonia, need to use parenteral antibiotics. }\end{array}$ \\
\hline
\end{tabular}




\begin{tabular}{|c|c|c|}
\hline Author, Year & Inclusion Criteria & Exclusion Criteria \\
\hline Van Velzen, $2017^{15}$ & $\begin{array}{l}45 \text { years or older, smoking history of } 10 \text { pack-years; } \\
\text { clinical diagnosis of mild to severe COPD, last } \\
\text { exacerbation in the three years, exacerbation had to } \\
\text { have ended at least } 4 \text { weeks before and symptoms had } \\
\text { returned to baseline. }\end{array}$ & $\begin{array}{l}\text { Poor mastery of the Dutch language, poor cognitive functioning, } \\
\text { known allergy to doxycycline, pregnancy, life expectancy of } \\
\text { shorter than one month, fever, admission to hospital, current } \\
\text { antibiotics or use in previous } 3 \text { weeks. }\end{array}$ \\
\hline Van Zanten, $2007^{93}$ & $\begin{array}{l}\text { Ninety-three consecutive patients aged } \geq 18 \text { years } \\
\text { (range } 34-76 \text { years) requiring hospital admission and } \\
\text { antibiotic treatment for moderate to severe acute } \\
\text { exacerbations of COPD (GOLD classes } 2-4 \text { ). }\end{array}$ & $\begin{array}{l}\text { Suspected or proven resistance to cefotaxime, administration of } \\
\text { antibiotics in the preceding } 48 \mathrm{~h} \text {, allergy to } \beta \text {-lactam antibiotics, } \\
\text { bilirubin concentrations }>20 \mu \mathrm{mol} \mathrm{I}^{-1} \text {, serum creatinine } \\
\text { concentrations }>120 \mu \mathrm{mol} \text { and whole blood count }<3.0 \times 10^{9} \mathrm{l}^{-1} \text {. }\end{array}$ \\
\hline Vermeeren, $2004^{60}$ & $\begin{array}{l}\text { Admitted to the hospital for an exacerbation of COPD, } \\
\text { recent increase in breathlessness, cough and sputum } \\
\text { production of sufficient severity to warrant hospital } \\
\text { admission, judged by an independent chest physician. } \\
\text { Criteria for nutritional intervention were a BMI } \leq 22 \mathrm{~kg} / \mathrm{m}^{2} \\
\text { or a BMl } \leq 25 \mathrm{~kg} / \mathrm{m}^{2} \text { and }>5 \% \text { weight loss in } 1 \text { month, or } \\
>10 \% \text { weight loss in } 6 \text { months prior admission to the } \\
\text { hospital. }\end{array}$ & $\begin{array}{l}\text { Patients with DM type 1, thyroid, intestinal diseases, and } \\
\text { carcinoma. }\end{array}$ \\
\hline Wang, $2016^{16}$ & $\begin{array}{l}\text { Patients admitted to hospital with AECOPD who were } 40 \\
\text { years of age and who had a PCT level }<0.1 \mathrm{ng} / \mathrm{ml} \text {. }\end{array}$ & $\begin{array}{l}\text { Fever }\left(\geq 38.0^{\circ} \mathrm{C}\right) \text {, tracheal intubation within } 24 \mathrm{~h} \text { after hospital } \\
\text { admission, a PCT level of } \geq 0.1 \mathrm{ng} / \mathrm{ml} \text { on admission, pneumonia, } \\
\text { chronic renal failure, history of malignant disease, } \\
\text { immunosuppressive therapy. }\end{array}$ \\
\hline Whitlock, $1995^{94}$ & $\begin{array}{l}\text { Between 35-75 years old, acute bacterial exacerbation of } \\
\text { COPD. }\end{array}$ & $\begin{array}{l}\text { Pneumonia; acute bronchitis or chronic bronchitis with concurrent } \\
\text { bronchiectasis or active bronchial asthma; neutrophil function } \\
\text { disorders, cytopenias, or bleeding disorders; clinically significant } \\
\text { renal or hepatic dysfunction; clinically significant cardiovascular } \\
\text { disorders; any condition that could interfere with evaluation of } \\
\text { therapeutic response or increase the risk of an adverse event; } \\
\text { alcohol or drug dependence; significant psychiatric disorders; } \\
\text { hypersensitivity to any of } \\
\text { the study drugs; infections caused by organisms resistant or likely } \\
\text { to be resistant to study drugs; use of antibiotics within } 72 \text { hours of } \\
\text { enrollment; and use of investigational drugs within the previous } 4 \\
\text { weeks. Breastfeeding women. }\end{array}$ \\
\hline Willaert, $2002^{95}$ & $\begin{array}{l}\text { Patients with a clinical history of COPD who presented } \\
\text { with an acute exacerbation. }\end{array}$ & $\begin{array}{l}\text { There was a personal or family history of asthma (defined as } \\
\text { episodic wheezing or dyspnea that rapidly improved with } \\
\text { treatment) or atopy, INV or NIV assisted ventilation was deemed } \\
\text { necessary according to the attending casualty physician, the } \\
\text { patient was unable to successfully use a MDI as a device for } \\
\text { administering bronchodilators. }\end{array}$ \\
\hline
\end{tabular}




\begin{tabular}{|c|c|c|}
\hline Author, Year & Inclusion Criteria & Exclusion Criteria \\
\hline Wilson, $2012^{96}$ & $\begin{array}{l}\text { Outpatients with moderate-to severe COPD and chronic } \\
\text { bronchitis suffering from an investigator-evaluated } \\
\text { Anthonisen type I exacerbation and who were } \\
\text { considered by the investigator to require antibiotic } \\
\text { therapy. }\end{array}$ & NR \\
\hline Woodruff, $2011^{61}$ & $\begin{array}{l}\text { Over } 45 \text { years old, with an admitting diagnosis of } \\
\text { AECOPD, } 10 \text { or more pack-years, FEV } 1<60 \% \\
\text { predicted or inability to perform spirometry due to } \\
\text { dyspnea. }\end{array}$ & $\begin{array}{l}\text { Uncontrolled systemic disease; hypersensitivity to zileuton; } \\
\text { Asthma; Lobar pneumonia or pulmonary edema; Interstitial lung } \\
\text { disease; predicted survival of less than } 30 \text { days; History of liver } \\
\text { disease; current use of theophylline; incarceration; } \\
\text { institutionalization; pregnant; history of a suicide attempt; prior } \\
\text { inpatient admission for a psychiatric disorder; bipolar disorder. }\end{array}$ \\
\hline Xiong, $2008^{62}$ & $\begin{array}{l}\text { Diagnosis is consistent with the standard of the GOLD of } \\
2006, \text { no application of glucocorticosteroid systematically } \\
\text { in } 6 \text { months, no systemic infection in } 2 \text { weeks before the } \\
\text { study, no application of } \beta 2 \text {-receptor agonist or } \\
\text { antihistamine drugs in } 24 \text { hours, no any other chronic } \\
\text { heart or lung disease or endocrine disease, no need of } \\
\text { mechanical ventilation. }\end{array}$ & NR \\
\hline Yohannes, $2003^{63}$ & 60 years old and older, inpatient admitted with AECOPD. & $\begin{array}{l}\text { Terminal illness, current participation in any other research } \\
\text { project, uncontrolled heart failure, major cardiac arrhythmia. } \\
\text { History of nondepressive psychotic illness, history of poor } \\
\text { compliance with medical therapy, acute/chronic confusion } \\
\text { (Hodkinson Abbreviated Mental Test score }<7 / 10 \text { ), carbon dioxide } \\
\text { retention judged by the patient's supervising consultant to be } \\
\text { severe enough to prevent oxygen supplementation, limitation of } \\
\text { exercise capacity by nonrespiratory disability (e.g., } \\
\text { musculoskeletal problems), inability to use co-ordinate frame, } \\
\text { intolerance of oxygen mask or nasal cannula. }\end{array}$ \\
\hline Yoon, $2013^{97}$ & Older than 18 years with AECOPD. & $\begin{array}{l}\text { Radiographic evidence of pneumonia, bronchiectasis, cystic } \\
\text { fibrosis, tuberculosis, or lung cancer. Patients with serious renal } \\
\text { dysfunction, a history of seizure, a history of allergy or other side } \\
\text { effects to quinolone, cephalosporin, or penicillin, a history of } \\
\text { antibiotic therapy during the previous } 48 \text { hours, pregnancy or } \\
\text { possibility of pregnancy, and lactating women. }\end{array}$ \\
\hline Zuin, $2005^{64}$ & $\begin{array}{l}\text { History of COPD with at least two exacerbations in the } \\
\text { previous } 2 \text { years, post-bronchodilator FEV1 between } \\
40 \% \text { and } 70 \% \text { predicted }\end{array}$ & $\begin{array}{l}\text { Serious concomitant diseases (cardiac, hepatic, renal or cancer) } \\
\text { or } \alpha-1 \text {-antitrypsin deficiency. }\end{array}$ \\
\hline
\end{tabular}

AECB = acute exacerbation of chronic bronchitis; ECOPD = exacerbation of chronic obstructive pulmonary disease; ATS = American Thoracic Society; CHF = congestive heart failure; COPD = chronic obstructive pulmonary disease; CPAP: continuous positive airway pressure; CXR = chest $\mathrm{x}$-ray; $\mathrm{DM}=$ diabetes mellitus; ED = emergency department; EKG = electrocardiogram; EPA = eicosapentaenoic acid; ERS = European Respiratory Society; FEV1 = forced expiration volume in 1 second; FVC = forced vital capacity; GOLD = global initiative for chronic obstructive lung disease; HF = heart failure; ICU = intensive care unit; IHD = ischemic heart disease; INV = invasive ventilation; KPA = kilopascal; mMRC = modified medical research council; NIV = noninvasive ventilation; NT-proBNP = N-terminal pro b-type natriuretic peptide; NYHA = New York Heart 
Association; ONS = oral nutrition supplementation; $\mathrm{PaCO} 2=$ partial pressure of carbon dioxide in arterial blood; Pao2 = partial pressure of oxygen in arterial blood; PE = pulmonary embolism; PEF = peak expiratory flow; $\mathrm{pH}=$ potential of hydrogen; $\mathrm{RF}=$ respiratory failure; TLC = total lung capacity; VC = vital capacity; WBC = white blood cell count. 


\section{Appendix J. Sensitivity Analysis}

Table J.1. Sensitivity Analysis

\begin{tabular}{|c|c|c|c|c|c|}
\hline Comparison & Outcome & Method & OR & $95 \% \mathrm{Cl}$ & $\mathbf{I}^{2}$ \\
\hline \multirow{3}{*}{$\begin{array}{l}\text { Systemic Antibiotics versus } \\
\text { Placebo or Management without } \\
\text { Systemic Antibiotics }\end{array}$} & \multirow{3}{*}{$\begin{array}{l}\text { Clinical Cure } \\
\text { Longest Followup }\end{array}$} & Profile Likelihood & 1.56 & $0.84,2.21$ & $0.00 \%$ \\
\hline & & HKSJ & 1.53 & $0.81,2.88$ & $0.20 \%$ \\
\hline & & $\mathrm{DL}$ & 1.50 & $1.01,2.24$ & $20.33 \%$ \\
\hline \multirow{3}{*}{$\begin{array}{l}\text { Systemic Corticosteroids vs } \\
\text { Placebo }\end{array}$} & \multirow{3}{*}{$\begin{array}{l}\text { Mortality } \\
\text { End of Intervention }\end{array}$} & Profile Likelihood & 1.61 & $0.47,6.18$ & $0.00 \%$ \\
\hline & & HKSJ & 1.61 & $0.22,11.75$ & $0.00 \%$ \\
\hline & & $\mathrm{DL}$ & 1.61 & $0.47,5.47$ & $0.00 \%$ \\
\hline \multirow{2}{*}{$\begin{array}{l}\text { Systemic Corticosteroids vs } \\
\text { Placebo }\end{array}$} & \multirow{2}{*}{$\begin{array}{l}\text { FEV1 \% Predicted } \\
\text { End of Intervention }\end{array}$} & Profile Likelihood & 4.64 & $1.00,8.96$ & $0.00 \%$ \\
\hline & & HKSJ & 4.64 & $-0.81,10.09$ & $0.00 \%$ \\
\hline
\end{tabular}

DL = DerSimonian and Laird; HKSJ = Hartung-Knapp-Sidik-Jonkman; OR=odds ratio; CI=confidence interval 


\section{Appendix K. Appendix References}

1. Aaron SD, Vandemheen KL, Hebert P, et al. Outpatient oral prednisone after emergency treatment of chronic obstructive pulmonary disease. N Engl J Med. 2003 Jun 26;348(26):2618-25. doi: 10.1056/NEJMoa023161. PMID: 12826636.

2. Albert RK, Martin TR, Lewis SW. Controlled clinical trial of methylprednisolone in patients with chronic bronchitis and acute respiratory insufficiency. Annals of Internal Medicine. 1980 Jun;92(6):753-8. PMID: 6770731.

3. Anthonisen NR, Manfreda J, Warren CP, et al. Antibiotic therapy in exacerbations of chronic obstructive pulmonary disease. Annals of Internal Medicine. 1987 Feb;106(2):196-204. PMID: 3492164.

4. Bathoorn E, Liesker JJW, Postma DS, et al. Anti-inflammatory effects of combined budesonide/formoterol in COPD exacerbations. Copd: Journal of Chronic Obstructive Pulmonary Disease. 2008 Oct;5(5):282-90. PMID: 18972276.

5. Brusse-Keizer M, VanderValk P, Hendrix R, et al. Necessity of amoxicillin clavulanic acid in addition to prednisolone in mild-tomoderate COPD exacerbations. BMJ open respiratory research. 2014;1(1):e000052. PMID: 25562034.

6. Daniels JMA, Snijders D, e Graaff CS, et al. Antibiotics in addition to systemic corticosteroids for acute exacerbations of chronic obstructive pulmonary disease. American Journal of Respiratory \& Critical Care Medicine. 2010 Jan 15;181(2):150-7. PMID: 19875685.

7. Davies L, Angus RM, Calverley PM. Oral corticosteroids in patients admitted to hospital with exacerbations of chronic obstructive pulmonary disease: a prospective randomised controlled trial. Lancet. 1999 Aug 07;354(9177):456-60. PMID: 10465169.
8. Emerman CL, Connors AF, Lukens TW, et al. A randomized controlled trial of methylprednisolone in the emergency treatment of acute exacerbations of COPD. Chest. 1989 Mar;95(3):563-7. PMID: 2920584.

9. Gunen H, Hacievliyagil SS, Yetkin O, et al. The role of nebulised budesonide in the treatment of exacerbations of COPD. [Erratum appears in Eur Respir J. 2014 Jul;44(1):272]. European Respiratory Journal. 2007 Apr;29(4):660-7. PMID: 17251232.

10. Hassan WA, Shalan I, Elsobhy M. Impact of antibiotics on acute exacerbations of COPD. Egyptian Journal of Chest Diseases and Tuberculosis. 201501 Jul;64(3):579-85. PMID: 603624004.

11. Llor C, Moragas A, Hernandez S, et al. Efficacy of antibiotic therapy for acute exacerbations of mild to moderate chronic obstructive pulmonary disease. American Journal of Respiratory \& Critical Care Medicine. 2012 Oct 15;186(8):716-23. PMID: 22923662.

12. Maltais F, Ostinelli J, Bourbeau J, et al. Comparison of nebulized budesonide and oral prednisolone with placebo in the treatment of acute exacerbations of chronic obstructive pulmonary disease: a randomized controlled trial. American Journal of Respiratory \& Critical Care Medicine. 2002 Mar 01;165(5):698-703. PMID: 11874817.

13. Niewoehner DE, Erbland ML, Deupree RH, et al. Effect of systemic glucocorticoids on exacerbations of chronic obstructive pulmonary disease. Department of Veterans Affairs Cooperative Study Group. New England Journal of Medicine. 1999 Jun 24;340(25):1941-7. PMID: 10379017.

14. Thompson WH, Nielson CP, Carvalho P, et al. Controlled trial of oral prednisone in outpatients with acute COPD exacerbation. American Journal of Respiratory \& Critical Care Medicine. 1996 Aug;154(2 Pt 1):40712. PMID: 8756814. 
15. van Velzen P, ter Riet G, Bresser P, et al. Doxycycline for outpatient-treated acute exacerbations of COPD: a randomised double-blind placebo-controlled trial. The lancet respiratory medicine. 2017(pagination). PMID: CN-01374427.

16. Wang J-X, Zhang S-M, Li X-H, et al. Acute exacerbations of chronic obstructive pulmonary disease with low serum procalcitonin values do not benefit from antibiotic treatment: a prospective randomized controlled trial. International Journal of Infectious Diseases. 2016 Jul;48:40-5. PMID: 27155210.

17. Abreu Gonzalez J, Hernandez Garcia C, Abreu Gonzalez P, et al. [Effect of intravenous magnesium sulfate on chronic obstructive pulmonary disease exacerbations requiring hospitalization: a randomized placebo-controlled trial]. Archivos de Bronconeumologia. 2006 Aug;42(8):384-7. PMID: 16948990.

18. Austin MA, Wills KE, Blizzard L, et al. Effect of high flow oxygen on mortality in chronic obstructive pulmonary disease patients in prehospital setting: randomised controlled trial. BMJ. 2010 Oct 18;341:c5462. PMID: 20959284.

19. Ayfer Aytemur Z, Baysak A, Ozdemir O, et al. N-acetylcysteine in patients with COPD exacerbations associated with increased sputum. Wiener Klinische Wochenschrift. 2015 Apr;127(7-8):256-61. PMID: 25595117.

20. Basri R, Tahir M, Naseem M. Short-term effects of chest physiotherapy in acute exacerbation of chronic obstructive pulmonary disease. Journal of Medical Sciences (Peshawar). 2017 July;25(3):323-7. PMID: 620855828.

21. Behnke M, Taube C, Kirsten D, et al. Homebased exercise is capable of preserving hospital-based improvements in severe chronic obstructive pulmonary disease. Respir Med. 2000 Dec;94(12):1184-91. doi: 10.1053/rmed.2000.0949. PMID: 11192954.
22. Black PN, Morgan-Day A, McMillan TE, et al. Randomised, controlled trial of Nacetylcysteine for treatment of acute exacerbations of chronic obstructive pulmonary disease [ISRCTN21676344]. BMC Pulmonary Medicine. 2004 Dec 06;4:13. PMID: 15581425.

23. Borges RC, Carvalho CR. Impact of resistance training in chronic obstructive pulmonary disease patients during periods of acute exacerbation. Archives of Physical Medicine \& Rehabilitation. 2014 Sep;95(9):1638-45. PMID: 24879965.

24. Brown PM, Manfreda J, McCarthy DS, et al. The effect of mechanical vibration in patients with acute exacerbations of chronic obstructive pulmonary disease. Physiother Can. 1987;39(6):371-4.

25. Centanni S, Santus P, Casanova F, et al. Bronchodilating effect of oxitropium bromide in heart disease patients with exacerbations of COPD: double-blind, randomized, controlled study. Respiratory Medicine. 2002 Mar;96(3):137-41. PMID: 11905547.

26. Cox M, Connor C, Biggs K, et al. The feasibility of early pulmonary rehabilitation and activity after COPD exacerbations: External pilot randomised controlled trial, qualitative case study and exploratory economic evaluation. Health Technology Assessment. 2018;22(11).

27. Cross JL, Elender F, Barton G, et al. Evaluation of the effectiveness of manual chest physiotherapy techniques on quality of life at six months post exacerbation of COPD (MATREX): a randomised controlled equivalence trial. BMC Pulmonary Medicine. 2012 Jul 02;12:33. PMID: 22748085.

28. Du F, Liu D, He G, et al. Effect of simvastatin on serum gammaglutamyltransferase and C-reactive protein in patient with acute exacerbation chronic obstructive pulmonary disease. Acta Medica Mediterranea. 2018;34(5):1221-6. PMID: 623957543. 
29. Duffy N, Walker P, Diamantea F, et al. Intravenous aminophylline in patients admitted to hospital with non-acidotic exacerbations of chronic obstructive pulmonary disease: a prospective randomised controlled trial. Thorax. 2005 Sep;60(9):713-7. PMID: 15939732.

30. Eaton T, Young P, Fergusson W, et al. Does early pulmonary rehabilitation reduce acute health-care utilization in COPD patients admitted with an exacerbation? A randomized controlled study. Respirology. 2009 Mar;14(2):230-8. PMID: 19272084.

31. Edwards L, Shirtcliffe P, Wadsworth K, et al. Use of nebulised magnesium sulphate as an adjuvant in the treatment of acute exacerbations of COPD in adults: a randomised double-blind placebo-controlled trial. Thorax. 2013 Apr;68(4):338-43. PMID: 23299960.

32. Goktalay T, Akdemir SE, Alpaydin AO, et al. Does high-frequency chest wall oscillation therapy have any impact on the infective exacerbations of chronic obstructive pulmonary disease? A randomized controlled single-blind study. Clinical Rehabilitation. 2013 Aug;27(8):710-8. PMID: 23503735.

33. Greening NJ, Williams JEA, Hussain SF, et al. An early rehabilitation intervention to enhance recovery during hospital admission for an exacerbation of chronic respiratory disease: randomised controlled trial. BMJ : British Medical Journal. 2014;349:g4315. doi: 10.1136/bmj.g4315.

34. Greulich T, Nell C, Koepke J, et al. Benefits of whole body vibration training in patients hospitalised for COPD exacerbations - a randomized clinical trial. BMC Pulmonary Medicine. 2014 Apr 11;14:60. PMID: 24725369.

35. He M, Yu S, Wang L, et al. Efficiency and safety of pulmonary rehabilitation in acute exacerbation of chronic obstructive pulmonary disease. Medical Science Monitor. 2015 Mar 18;21:806-12. PMID: 25783889.

36. Kodric M, Garuti G, Colomban M, et al. The effectiveness of a bronchial drainage technique (ELTGOL) in COPD exacerbations. Respirology. 2009 Apr;14(3):424-8. PMID: 19192230.
37. Kirsten DK, Taube C, Lehnigk B, et al. Exercise training improves recovery in patients with COPD after an acute exacerbation. Respir Med. 1998 Oct;92(10):1191-8. PMID: 9926148.

38. Kurzaj M, Wierzejski W, Dor A, et al. The impact of specialized physiotherapy methods on BODE index in COPD patients during hospitalization. Advances in Clinical \& Experimental Medicine. 2013 SepOct;22(5):721-30. PMID: 24285458.

39. Lellouche F, Bouchard PA, Roberge M, et al. Automated oxygen titration and weaning with FreeO $<$ inf $>2<$ inf $>$ in patients with acute exacerbation of COPD: A pilot randomized trial. International Journal of COPD. 201624 Aug;11(1):1983-90. PMID: 611880937.

40. Liao L-Y, Chen K-M, Chung W-S, et al. Efficacy of a respiratory rehabilitation exercise training package in hospitalized elderly patients with acute exacerbation of COPD: a randomized control trial. International Journal of Copd. 2015;10:1703-9. PMID: 26345529.

41. Mahmoud Abd El Hafiz A, Mohammed El Wakeel L, Mohammed El Hady H, et al. High dose $\mathrm{N}$-acetyl cysteine improves inflammatory response and outcome in patients with COPD exacerbations. Egyptian Journal of Chest Diseases and Tuberculosis. 2013 January;62(1):51-7. PMID: 369315238.

42. Moretti M, Fagnani S. Erdosteine reduces inflammation and time to first exacerbation postdischarge in hospitalized patients with AECOPD. International Journal of Copd. 2015;10:2319-25. PMID: 26604731.

43. Mukerji S, Shahpuri B, Clayton-Smith B, et al. Intravenous magnesium sulphate as an adjuvant therapy in acute exacerbations of chronic obstructive pulmonary disease: a single centre, randomised, double-blinded, parallel group, placebo-controlled trial: a pilot study. New Zealand Medical Journal. 2015 Nov 20;128(1425):34-42. PMID: 26905985. 
44. Ogasawara T, Marui S, Miura E, et al. Effect of eicosapentaenoic acid on prevention of lean body mass depletion in patients with exacerbation of chronic obstructive pulmonary disease: A prospective randomized controlled trial. Clinical Nutrition ESPEN. 2018 Dec;28:67-73. PMID: 30390895.

45. Oncu E, Zincir H. The effect of transcutaneous electrical nerve stimulation in patients with acute exacerbation of chronic obstructive pulmonary disease: randomised controlled trial. Journal of Clinical Nursing. 2017 Jul;26(13-14):183444. PMID: 27325551.

46. Osadnik CR, McDonald CF, Miller BR, et al. The effect of positive expiratory pressure (PEP) therapy on symptoms, quality of life and incidence of re-exacerbation in patients with acute exacerbations of chronic obstructive pulmonary disease: a multicentre, randomised controlled trial. Thorax. 2014 Feb;69(2):137-43. PMID: 24005444.

47. 47. Pourrashid MH, Dastan F, Salamzadeh $\mathrm{J}$, et al. Role of vitamin D replacement on health related quality of life in hospitalized patients with "acute exacerbation of chronic obstructive pulmonary disease”. Iranian Journal of Pharmaceutical Research. 2018;17(2):801-10.

48. Rice KL, Leatherman JW, Duane PG, et al. Aminophylline for acute exacerbations of chronic obstructive pulmonary disease. A controlled trial. Annals of Internal Medicine. 1987 Sep;107(3):305-9. PMID: 3619219.

49. Sanjari M, Soltani A, Habibi Khorasani A, et al. The effect of vitamin D on COPD exacerbation: a double blind randomized placebo-controlled parallel clinical trial. Journal of Diabetes \& Matabolic Disorders. 2015;15(1):33. PMID: 27570748.

50. Saudny-Unterberger H, Martin JG, GrayDonald K. Impact of nutritional support on functional status during an acute exacerbation of chronic obstructive pulmonary disease. American Journal of Respiratory \& Critical Care Medicine. 1997 Sep;156(3 Pt 1):794-9. PMID: 9309995.
51. Seidenfeld JJ, Jones WN, Moss RE, et al. Intravenous aminophylline in the treatment of acute bronchospastic exacerbations of chronic obstructive pulmonary disease. Annals of Emergency Medicine. 1984 Apr;13(4):248-52. PMID: 6367554.

52. Skorodin MS, Tenholder MF, Yetter B, et al. Magnesium sulfate in exacerbations of chronic obstructive pulmonary disease. Archives of Internal Medicine. 1995 Mar 13;155(5):496-500. PMID: 7864705.

53. Solooki M, Miri M, Mokhtari M, et al. Magnesium sulfate in exacerbations of COPD in patients admitted to internal medicine ward. Iranian Journal of Pharmaceutical Research. 201401 Sep;13(4):1235-9. PMID: 600071239.

54. Soltaninejad F, Kheiri S, Habibian R, et al. Evaluation effects of nebulized gentamicin in exacerbation of chronic obstructive lung disease. Journal of Research in Medical Sciences. 2016;21 (4) (no pagination)(56). PMID: 612021136.

55. Tang CY, Blackstock FC, Clarence M, et al. Early rehabilitation exercise program for inpatients during an acute exacerbation of chronic obstructive pulmonary disease: a randomized controlled trial. Journal of Cardiopulmonary Rehabilitation \& Prevention. 2012 May-Jun;32(3):163-9. PMID: 22561417.

56. Torres-Sanchez I, Valenza MC, CabreraMartos I, et al. Effects of an Exercise Intervention in Frail Older Patients with Chronic Obstructive Pulmonary Disease Hospitalized due to an Exacerbation: A Randomized Controlled Trial. Copd: Journal of Chronic Obstructive Pulmonary Disease. 2017 Feb;14(1):37-42. PMID: 27715322.

57. Torres-Sánchez I, Valenza MC, Cebriá i Iranzo MDÀ, et al. Effects of different physical therapy programs on perceived health status in acute exacerbation of chronic obstructive pulmonary disease patients: a randomized clinical trial. Disability and Rehabilitation. 2017:1-7. 
58. Troosters T, Probst VS, Crul T, et al. Resistance training prevents deterioration in quadriceps muscle function during acute exacerbations of chronic obstructive pulmonary disease. American Journal of Respiratory \& Critical Care Medicine. 2010 May 15;181(10):1072-7. PMID: 20133927.

59. Tumer G, Mercanligil SM, Uzun O, et al. The effects of a high-fat, low-carbohydrate diet on the prognosis of patients with an acute attack of chronic obstructive pulmonary disease. Turkiye Klinikleri Journal of Medical Sciences. 2009;29(4):895-904. PMID: 358101090.

60. Vermeeren MAP, Wouters EFM, GeraertsKeeris AJW, et al. Nutritional support in patients with chronic obstructive pulmonary disease during hospitalization for an acute exacerbation; a randomized controlled feasibility trial. Clinical Nutrition. 2004 Oct;23(5):1184-92. PMID: 15380912.

61. Woodruff PG, Albert RK, Bailey WC, et al. Randomized trial of zileuton for treatment of COPD exacerbations requiring hospitalization. Copd: Journal of Chronic Obstructive Pulmonary Disease. 2011 Feb;8(1):21-9. PMID: 21299475.

62. Xiong G, Xu L, Wei L, et al. Atomization inhalation of terbutaline and budesonide efficiently improved immunity and lung function of AECOPD patients. Cellular \& Molecular Immunology. 2008 Aug;5(4):287-91. PMID: 18761816.

63. Yohannes AM, Connolly MJ. Early mobilization with walking aids following hospital admission with acute exacerbation of chronic obstructive pulmonary disease. Clinical Rehabilitation. 2003 Aug;17(5):465-71. PMID: 12952150.

64. Zuin R, Palamidese A, Negrin R, et al. High-dose $\mathrm{N}$-acetylcysteine in patients with exacerbations of chronic obstructive pulmonary disease. Clinical Drug Investigation. 2005;25(6):401-8. PMID: 41019987.

65. Koutsogiannis Z, Kelly AM. Does high dose ipratropium bromide added to salbutamol improve pulmonary function for patients with chronic obstructive airways disease in the emergency department? Australian \& New Zealand Journal of Medicine. 2000 Feb;30(1):38-40. PMID: 10800876.
66. Moayyedi P, Congleton J, Page RL, et al. Comparison of nebulised salbutamol and ipratropium bromide with salbutamol alone in the treatment of chronic obstructive pulmonary disease. Thorax. 1995 Aug;50(8):834-7. PMID: 7570433.

67. Perri G, Giovannini M, Spada E. Salbutamol plus beclomethasone dipropionate (Ventolin Flogo) vs. fenoterol (Dosberotec) in chronic obstructive lung disease therapeutic strategy: a 4-week clinical trial. International Journal of Clinical Pharmacology, Therapy, \& Toxicology. 1985 May;23(5):274-8. PMID: 3891642.

68. Aggarwal P, Wig N, Bhoi S. Efficacy of two corticosteroid regimens in acute exacerbation of chronic obstructive pulmonary disease. International Journal of Tuberculosis \& Lung Disease. 2011 May;15(5):687-92. PMID: 21756523.

69. Andre-Alves MR, Jardim JR, Frare e Silva $\mathrm{R}$, et al. Comparison between azithromycin and amoxicillin in the treatment of infectious exacerbation of chronic obstructive pulmonary disease. Jornal Brasileiro De Pneumologia: Publicacao Oficial Da Sociedade Brasileira De Pneumologia E Tisilogia. 2007 JanFeb;33(1):43-50. PMID: 17568867.

70. Aubier M, Aldons PM, Leak A, et al. Telithromycin is as effective as amoxicillin/clavulanate in acute exacerbations of chronic bronchitis. Respiratory Medicine. 2002 Nov;96(11):862-71. PMID: 12418583.

71. Blasi F, Schaberg T, Centanni S, et al. Prulifloxacin versus levofloxacin in the treatment of severe COPD patients with acute exacerbations of chronic bronchitis. Pulmonary Pharmacology \& Therapeutics. 2013 Oct;26(5):609-16. PMID: 23538168.

72. Dark D. Azithromycin versus cefaclor in the treatment of acute exacerbations of chronic obstructive pulmonary disease. Current Therapeutic Research - Clinical and Experimental. 1993;53(2):203-11. PMID: 23119468. 
73. Ding Z, Li X, Lu Y, et al. A randomized, controlled multicentric study of inhaled budesonide and intravenous methylprednisolone in the treatment on acute exacerbation of chronic obstructive pulmonary disease. Respir Med. 2016

Dec;121:39-47. doi:

10.1016/j.rmed.2016.10.013. PMID: 27888990 .

74. Ardestani ME, Kalantary E, Samaiy V, et al. Methyl prednisolone vs dexamethasone in management of COPD exacerbation; a randomized clinical trial. Emergency. 2017;5(1):195-200. PMID: 614121265.

75. Giusti M, Blasi F, Iori I, et al. Prulifloxacin vs Levofloxacin for Exacerbation of COPD after Failure of Other Antibiotics. Copd: Journal of Chronic Obstructive Pulmonary Disease. 2016 Oct;13(5):555-60. PMID: 27027547.

76. Hamacher J, Vogel F, Lichey J, et al. Treatment of acute bacterial exacerbations of chronic obstructive pulmonary disease in hospitalised patients--a comparison of meropenem and imipenem/cilastatin. COPD Study Group. Journal of Antimicrobial Chemotherapy. 1995 Jul;36 Suppl A:121-33. PMID: 8543488.

77. Hasani A, Pavia D, Rotondetto S, et al. Effect of oral antibiotics on lung mucociliary clearance during exacerbation of chronic obstructive pulmonary disease. Respiratory Medicine. 1998 Mar;92(3):4427. PMID: 9692103.

78. Leophonte P, Baldwin RJ, Pluck N. Trovafloxacin versus amoxicillin/clavulanic acid in the treatment of acute exacerbations of chronic obstructive bronchitis. European Journal of Clinical Microbiology \& Infectious Diseases. 1998 Jun;17(6):434-40. PMID: 9758289.

79. Leuppi JD, Schuetz P, Bingisser R, et al. Short-term vs conventional glucocorticoid therapy in acute exacerbations of chronic obstructive pulmonary disease: the REDUCE randomized clinical trial. Jama. 2013;309(21):2223-31.
80. Llor C, Hernandez S, Ribas A, et al. Efficacy of amoxycillin versus amoxycillin/clavulanate in acute exacerbations of chronic pulmonary obstructive disease in primary care. International Journal of Copd. 2009;4:45-53. PMID: 19436696.

81. Mirici A, Meral M, Akgun M. Comparison of the efficacy of nebulised budesonide with parenteral corticosteroids in the treatment of acute exacerbations of chronic obstructive pulmonary disease. Clinical Drug Investigation. 2003;23(1):55-62. PMID: 23319094.

82. Petitpretz P, Chone C, Tremolieres F, et al. Levofloxacin $500 \mathrm{mg}$ once daily versus cefuroxime $250 \mathrm{mg}$ twice daily in patients with acute exacerbations of chronic obstructive bronchitis: clinical efficacy and exacerbation-free interval. International Journal of Antimicrobial Agents. 2007 Jul;30(1):52-9. PMID: 17512704.

83. Phillips H, Van Hook CJ, Butler T, et al. A comparison of cefpodoxime proxetil and cefaclor in the treatment of acute exacerbation of COPD in adults. Chest. 1993 Nov;104(5):1387-92. PMID: 8222793.

84. Rhee CK, Chang JH, Choi EG, et al. Zabofloxacin versus moxifloxacin in patients with COPD exacerbation: a multicenter, double-blind, double-dummy, randomized, controlled, Phase III, noninferiority trial. International Journal of Copd. 2015;10:2265-75. PMID: 26543359.

85. Rizzato G. Effects of deflazacort hemisuccinate vs. methylprednisolone succinate in COPD exacerbations. Internista. 1998;6(1):35-40. PMID: 28122103.

86. Roede BM, Bresser P, El Moussaoui R, et al. Three vs. 10 days of amoxycillinclavulanic acid for type 1 acute exacerbations of chronic obstructive pulmonary disease: a randomised, doubleblind study. Clinical Microbiology \& Infection. 2007 Mar;13(3):284-90. PMID: 17391383. 
87. Ruiz-Gonzalez A, Gimenez A, GomezArbones X, et al. Open-label, randomized comparison trial of long-term outcomes of levofloxacin versus standard antibiotic therapy in acute exacerbations of chronic obstructive pulmonary disease. Respirology. 2007 Jan;12(1):117-21. PMID: 17207036.

88. Sayiner A, Aytemur ZA, Cirit M, et al. Systemic glucocorticoids in severe exacerbations of COPD. Chest. 2001 Mar;119(3):726-30. PMID: 11243949.

89. Stallberg B, Selroos O, Vogelmeier C, et al. Budesonide/formoterol as effective as prednisolone plus formoterol in acute exacerbations of COPD. A double-blind, randomised, non-inferiority, parallel-group, multicentre study. Respiratory Research. 2009 Feb 19;10:11. PMID: 19228428.

90. Sun X, He Z, Zhang J, et al. Compare the efficacy of inhaled budesonide and systemic methylprednisolone on systemic inflammation of AECOPD. Pulmonary Pharmacology \& Therapeutics. 2015 Apr;31:111-6. PMID: 25281894.

91. Yilmazel Ucar E, Araz O, Meral M, et al. Two different dosages of nebulized steroid versus parenteral steroid in the management of COPD exacerbations: a randomized control trial. Medical Science Monitor. 2014 Mar 28;20:513-20. PMID: 24675102.

92. Umut S, Tutluoglu B, Aydin Tosun G, et al. Determination of the etiological organism during acute exacerbations of COPD and efficacy of azithromycin, ampicillinsulbactam, ciprofloxacin and cefaclor. Turkish Thoracic Society COPD Working Group. Journal of Chemotherapy. 1999 Jun;11(3):211-4. PMID: 10435684.

93. van Zanten ARH, Oudijk M, NohlmansPaulssen MKE, et al. Continuous vs. intermittent cefotaxime administration in patients with chronic obstructive pulmonary disease and respiratory tract infections: pharmacokinetics/pharmacodynamics, bacterial susceptibility and clinical efficacy. British Journal of Clinical Pharmacology. 2007 Jan;63(1):100-9. PMID: 16869814.
94. Whitlock W. Multicenter comparison of azithromycin and amoxicillin/clavulanate in the treatment of patients with acute exacerbations of chronic obstructive pulmonary disease. Current Therapeutic Research - Clinical and Experimental. 1995;56(10):985-95. PMID: 25335828.

95. Willaert W, Daenen M, Bomans P, et al. What is the optimal treatment strategy for chronic obstructive pulmonary disease exacerbations? European Respiratory Journal. 2002 May;19(5):928-35. PMID: 12030735.

96. Wilson R, Anzueto A, Miravitlles M, et al. Moxifloxacin versus amoxicillin/clavulanic acid in outpatient acute exacerbations of COPD: MAESTRAL results. [Erratum appears in Eur Respir J. 2012 Sep;40(3):800]. European Respiratory Journal. 2012 Jul;40(1):17-27. PMID: 22135277.

97. Yoon HI, Lee C-H, Kim DK, et al. Efficacy of levofloxacin versus cefuroxime in treating acute exacerbations of chronic obstructive pulmonary disease. International Journal of Copd. 2013;8:329-34. PMID: 23874094.

98. de Jong YP, Uil SM, Grotjohan HP, et al. Oral or IV prednisolone in the treatment of COPD exacerbations: a randomized, controlled, double-blind study. Chest. 2007 Dec;132(6):1741-7. PMID: 17646228. 